DOE/WIPP-91-043-REV.

\title{
TRANSURANIC WASTE CHARACTERIZATION SAMPLING AND ANALYSIS METHODS MANUAL
}

Controlled Copy No.

U.S. Department of Energy - Carlsbad Area Office 


\section{DISCLAMMER}

Portions of this document may be illegible in electronic image products. Images are produced from the best available original document. 


\title{
TRANSURANIC WASTE CHARACTERIZATION SAMPLING AND ANALYSIS METHODS MANUAL
}

\author{
Revision 0
}

May, 1995

\section{DISCLAIMER}

This report was prepared as an account of work sponsored by an agency of the United States Government. Neither the United States Government nor any agency thereof, nor any of their employees, makes any warranty, express or implied, or assumes any legal liability or responsibility for the accuracy, completeness, or usefulness of any information, apparatus, product, or process disclosed, or represents that its use would not infringe privately owned rights. Reference herein to any specific commercial product, process, or service by trade name, trademark, manufacturer, or otherwise does not necessarily constitute or imply its endorsement, recommendation, or favoring by the United States Government or any agency thereof. The views and opinions of authors expressed herein do not necessarily state or reflect those of the United States Government or any agency thereof.

Prepared by:

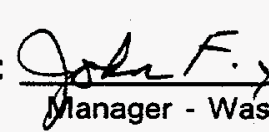
National TRU Program

Approved by: $\frac{\text { That }}{\text { Team Leader, National TRU Program }}$

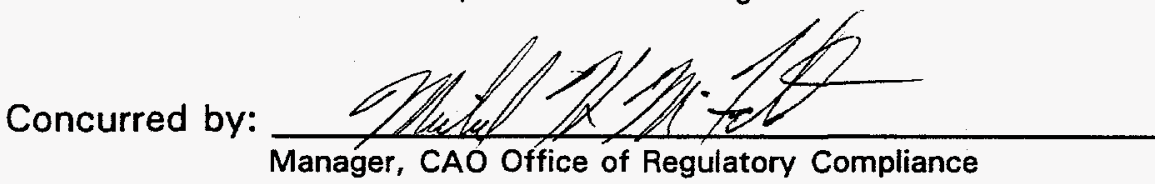

Concurred by:

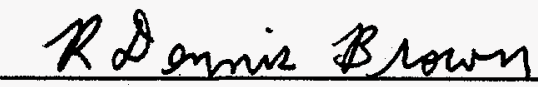

Manager, CAO Quality Assurance
Date: $5-30-95$

Date:

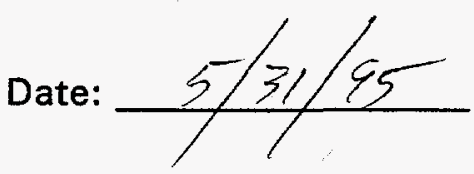

Date: May 27,1995

Date: $5 / 30 / 95$ 


\section{TABLE OF CONTENTS}

\subsection{Sampling}

110 Headspace Sampling

110.1 Sampling Manifold Method to Collect Headspace Gas Samples From a TRU Waste Drum

110.2 Direct Canister Method to Collect Headspace Gas Samples From a TRU Waste Drum

110.3 Using a Side-Port Needle to Collect Headspace Gas Samples Through a TRU Waste Drum's Carbon Composite Filter

110.4 Punching the Drum Lid to Collect Headspace Gas Samples from a TRU Waste Drum

120.1 Collecting Samples from TRU Waste Drums Containing Homogenous Solids and Soil/Gravel (Sludge)

2.0 Cleaning

210 Canister Cleaning

210.1 SUMMA ${ }^{\oplus}$ Passivated Stainless Steel Canister Certification and Cleaning

3.0 Physical Waste Form Characterization

310 Nondestructive/Destructive Characterization

310.1 Physical Waste Form Characterization Using Radiography

310.2 Physical Waste Form Characterization Using Visual Examination

4.0 Organics

430 Gas Chromatography/Mass Spectrometry Volatile Organic Compound and Semi-Volatile Organic Compound Analysis

430.1 Modified Method TO-14 for the Gas Chromatography/Mass Spectrometry Determination of Volatile Organic Compounds in Waste Container Headspace

430.2 Modified Method 8240/8260 for the Determination of Volatile Organic Compounds in Waste Container Headspace

430.3 Method 8240B for the Determination of Total Volatile Organic Compounds in Homogenous Solids and Soil/Gravel

430.4 Method 8260A for the Determination of Total Volatile Organic Compounds in Homogenous Solids and Soil/Gravel 


\section{TABLE OF CONTENTS}

(Continued)

430.5 Method 8250A for the Determination of Total Semi-Volatile Organic Compounds in Homogenous Solids and Soil/Gravel

430.6 Method 82708 for the Determination of Total Semi-Volatile Organic Compounds in Homogenous Solids and Soil/Gravel

440 Gas Chromatography Volatile Organic Compound and Semi-Volatile Organic Compound Analysis

440.1 Gas Chromatography/Flame Ionization Detector Determination of Alcohols and Ketones in Waste Container Headspace

440.2 Direct Injection Gas Chromatography/Flame Ionization Detector Determination of Nonhalogenated Volatile Organic Compounds in Homogenous Solids and Soil/Gravel

440.3 Gas Chromatography/Electron Capture Detection Determination of PCBs in Organic Sludge

5.0 Hydrogen and Methane

510 Mass Spectrometry Analysis

510.1 Mass Spectrometry Determination of Hydrogen and Methane in Waste Container Headspace

520 Gas Chromatography Analysis

520.1 Gas Chromatography Determination of Hydrogen and Methane in Waste Container Headspace

6.0 Inorganics

610 Sample Preparation

610.1 Microwave Assisted Acid Digestion of Homogenous Solids and Soil/Gravel

620 Sample Clean-up

620.1 Extraction Chromatography Cleanup of Homogenous Solids and Soil/Gravel Samples Undergoing Total Metals Analysis

630 Atomic Mass Spectrometry Analysis

630.1 Method 6020 for the Inductively Coupled Plasma-Mass Spectrometry Determination of Total Metals in Homogenous Solids and Soil/Gravel

640 Atomic Emission Spectroscopy Analysis

640.1 Method 6010A for the Inductively Coupled Plasma-Atomic Emission Spectroscopy Determination of Total Metals in Homogenous Solids and Soil/Gravel 


\section{TABLE OF CONTENTS}

(Continued)

\section{Atomic Absorption Spectroscopy Analysis}

650.1 Flame Atomic Absorption Spectroscopy Determination of Total Metals in Homogenous Solids and Soil/Gravel

650.2 Graphite Furnace Atomic Absorption Spectroscopy Determination of Total Metals in Homogenous Solids and Soil/Gravel

650.3 Cold Vapor Atomic Absorption Spectroscopy Determination of Total Mercury in Homogenous Solids and Soil/Gravel

650.4 Hydride Generation Atomic Absorption Spectroscopy Determination of Total Arsenic in Homogenous Solids and Soil/Gravel

650.5 Borohydride Generation Atomic Absorption Spectroscopy Determination of Total Antimony and Arsenic in Homogenous Solids and Soil/Gravel

650.6 Hydride Generation Atomic Absorption Spectroscopy Determination of Total Selenium in Homogenous Solids and Soil/Gravel

650.7 Borohydride Generation Atomic Absorption Spectroscopy Determination of Total Selenium in Homogenous Solids and Soil/Gravel 


\section{PREFACE}

\section{Purpose of the Manual}

The Transuranic Waste Characterization Sampling and Analysis Methods Manual (Methods Manual) provides a unified source of information on the sampling and analytical techniques that enable Department of Energy (DOE) facilities to comply with the requirements established in the current revision of the Transuranic Waste Characterization Quality Assurance Program Plan (QAPP) for the Waste Isolation Pilot Plant (WIPP) Transuranic (TRU) Waste Characterization Program (the Program). This Methods Manual includes all of the testing, sampling, and analytical methodologies accepted by DOE for use in implementing the Program requirements specified in the QAPP.

The procedures in this Methods Manual are comprehensive and detailed and are designed to provide the necessary guidance for the preparation of site-specific procedures. The use of these procedures is intended to provide the necessary precision, accuracy, and comparability of waste characterization results. The solutions to achieving specific program objectives will depend upon facility constraints, compliance with DOE Orders and DOE facilities' management and operating contractor requirements, and the knowledge and experience of the TRU waste handlers and analysts. With some analytical methods, such as Gas Chromatography/Mass Spectrometry, the Methods Manual procedures may be used directly. With other methods, such as nondestructive characterization, the Methods Manual provides guidance rather than a step-by-step procedure. Each DOE site must document the details of the procedures it will use and demonstrate the efficacy of such procedures to the Manager, National TRU Program, during Waste Characterization and Certification audits.

Many of the procedures found in this Methods Manual are based on analytical methods found in Test Methods for Evaluating Solid Waste, Physical/Chemical Methods, SW-846, Third Edition, Final Update I, and Final Update 11 . In these instances, the analyst is referred directly to the SW-846 method for the requirements of the procedure. Only information unique to the Program (e.g., target analytes, quality assurance objectives, quality control requirements) is included in the Methods Manual.

This Methods Manual is issued by the Carlsbad Area Office (CAO) and is a controlled document. Future revisions of this Methods Manual will be published to reflect requirements in future QAPP revisions. New Procedures will be added to this Methods Manual as they receive approval from CAO. For procedures to be included in this Methods Manual, they must satisfy all of the programmatic requirements and quality assurance objectives identified in the QAPP. In addition, they must undergo technical review and be organized in the appropriate format. 


\section{Organization and Format}

The format of this Methods Manual is analogous to the format of DOE Methods for Evaluating Environmental and Waste Management Samples. Major categories of techniques and analytes are divided into sections. These sections are subdivided by specific sampling or analytical technique. Each technique is indicated by a three-digit number and each procedure under a technique is uniquely identified by the addition of a decimal identifier following the technique number. For some waste samples, analysts may be required to select an appropriate sample preparation procedure and sample clean-up procedure in addition to an analytical procedure. New techniques and new procedures will be added as required to ensure that all DOE sites participating in the Program have available DOE procedures for meeting Program requirements.

\section{SECTION 1.0 SAMPLING}

This Methods Manual contains the following procedures for collecting a headspace gas sample from a waste container:

Procedure 110.1 provides the detailed requirements for the collection of headspace gas samples using a sampling manifold and SUMMA ${ }^{\oplus}$ passivated stainless steel canisters.

Procedure 110.2 provides the detailed requirements for the collection of headspace gas samples using the direct canister method and SUMMA ${ }^{\circledR}$ passivated stainless steel canisters.

Procedure 110.3 provides the detailed requirements for the collection of headspace gas samples through the carbon composite filter of a waste drum using either the manifold or direct canister procedures.

Procedure 110.4 provides the detailed requirements for the collection of headspace gas samples through the drum lid by puncturing the drum lid and using either the manifold or direct canister procedure.

This Methods Manual also contains the following procedure for collecting samples of homogenous solids and soil/gravel from a waste container:

Procedure 120.1 provides the detailed requirements for the collection of homogenous solids and soil/gravel samples using either rotational or non-rotational coring equipment. The hardness of the waste will determine whether rotational or non-rotational coring equipment is used for sample collection. This procedure also provides the requirements for cleaning and certifying coring tools and associated sampling equipment. 


\section{SECTION 2.0 CLEANING}

This Methods Manual contains the following procedures for cleaning and certifying headspace gas sample containers and homogenous solids and soil/gravel sampling equipment:

Procedure 210.1 provides the requirements for cleaning and pressure certifying SUMMA ${ }^{\oplus}$ passivated stainless steel canisters.

\section{SECTION 3.0 PHYSICAL WASTE FORM CHARACTERIZATION}

This Methods Manual contains the following procedures for the physical waste form characterization of containerized wastes:

Procedure 310.1 provides the requirements for the non-destructive, $X$-ray scanning of waste containers using radiography. Radiography can be used to provide an estimate of the types and quantities of waste items, residual materials, and packaging materials.

Procedure 310.2 provides the requirements for visually examining the type and amount of waste material in each container. Visual examination will be used to verify the results of radiography (Procedure 310.1).

\section{SECTION 4.0 ORGANICS}

This Methods Manual contains the following procedures for the analysis of organic compounds:

Procedure 430.1 provides the requirements for the Gas Chromatography/Mass Spectrometry (GC/MS) determination of volatile organic compounds (VOCs) in waste container headspace samples that have been collected using SUMMA ${ }^{\circledR}$ passivated canisters.

Procedure 430.2 provides the requirements for an additional GC/MS determination of VOCs in waste container headspace samples that have been collected using SUMMA ${ }^{\circledR}$ passivated canisters.

Procedure 430.3 provides the requirements for the GC/MS determination of total VOCs in samples of homogenous solids and soil/gravel that have been collected using either rotational or non-rotational coring equipment.

Procedure 430.4 provides the requirements for an additional GC/MS determination of total VOCs in samples of homogenous solids and soil/gravel that have been collected using either rotational or nonrotational coring equipment. 
Procedure 430.5 provides the requirements for the GC/MS determination of total semi-volatile organic compounds (semi-VOCs) in samples of homogenous solids and soil/gravel that have been collected using either rotational or non-rotational coring equipment.

Procedure 430.6 provides the requirements for an additional GC/MS determination of total semi-VOCs in samples of homogenous solids and soil/gravel that have been collected using either rotational or nonrotational coring equipment.

Procedure 440.1 provides the requirements for the Gas Chromatography/Flame lonization Detector (GC/FID) determination of total alcohols and ketones in waste container headspace samples that have been collected using SUMMA ${ }^{\oplus}$ passivated canisters.

Procedure 440.2 provides the requirements for the GC/FID determination of total alcohols and ketones in homogenous solids and soil/gravel samples that have been collected using either rotational or nonrotational coring equipment.

Procedure 440.3 provides the requirements for the Gas Chromatography/Electron Capture Detector (GC/ECD) determination of total polychlorinated biphenyls (PCBs) in organic sludge samples collected using either rotational or non-rotational coring equipment.

\section{SECTION 5.0 HYDROGEN AND METHANE}

This Methods Manual contains the following procedures for the analysis of waste container headspace hydrogen and methane:

Procedure 510.1 provides the requirements for the Mass Spectrometry (MS) determination of hydrogen and methane in waste container headspace samples that have been collected using SUMMA ${ }^{\circ}$ passivated canisters.

Procedure 520.1 provides a Gas Chromatography (GC) determination of hydrogen and methane in waste container headspace samples that have been collected using SUMMA ${ }^{\circledR}$ passivated canisters.

\section{SECTION 6.0 INORGANICS}

This Methods Manual contains the following procedures for the analysis of inorganics in homogenous solids and soil/gravel: 
Procedure 630.1 provides the requirements for the Inductively Coupled Plasma-Mass Spectrometry (ICP-MS) determination of total metals in homogenous solids and soil/gravel samples collected using either rotational or non-rotational coring equipment.

Procedure 640.1 provides the requirements for the Inductively Coupled Plasma-Atomic Emission Spectroscopy (ICP-AES) determination of total metals in homogenous solids and soil/gravel samples collected using either rotational or non-rotational coring equipment.

Procedure 650.1 provides the requirements for the Flame Atomic Absorption Spectroscopy (FLAA) determination of total metals in homogenous solids and soil/gravel samples collected using either rotational or non-rotational coring equipment.

Procedure 650.2 provides the requirements for the Graphite Furnace Atomic Absorption Spectroscopy (GFAA) determination of total metals in homogenous solids and soil/gravel samples collected using either rotational or non-rotational coring equipment.

Procedure 650.3 provides the requirements for the Cold Vapor Atomic Absorption Spectroscopy (CVAA) determination of total mercury in homogenous solids and soil/gravel samples collected using either rotational or non-rotational coring equipment.

Procedure 650.4 provides the requirement for the Hydride Generation Atomic Absorption Spectroscopy (HAA) determination of total arsenic in homogenous solids and soil/gravel samples collected using either rotational or non-rotational coring equipment.

Procedure 650.5 provides the requirement for the Borohydride Generation Atomic Absorption Spectroscopy (HAA) determination of total arsenic in homogenous solids and soil/gravel samples collected using either rotational or non-rotational coring equipment.

Procedure 650.6 provides the requirements for the Hydride Generation Atomic Absorption Spectroscopy (HAA) determination of total selenium in homogenous solids and soil/gravel samples collected using either rotational or non-rotational coring equipment.

Procedure 650.7 provides the requirements for the Borohydride Generation Atomic Absorption Spectroscopy (HAA) determination of total selenium in homogenous solids and soil/gravel samples collected using either rotational or non-rotational coring equipment. 
PROCEDURE 110.1

\section{SAMPLING MANIFOLD METHOD TO COLLECT \\ HEADSPACE GAS SAMPLES FROM A TRU WASTE DRUM}

\subsection{Scope and Application}

1.1 This procedure may be used to collect representative headspace gas samples from within a TRU waste drum. This sampling procedure is based on guidelines in "Compendium Method TO-14, The Determination of Volatile Organic Compounds (VOC) in Ambient Air Using SUMMA ${ }^{\circledR}$ Passivated Canister Sampling and Gas Chromatographic Analyses" (EPA 1988) and Test Methods for Evaluating Solid Waste, Physical/Chemical Methods, SW-846, Third Edition, Final Update I, and Final Update II (SW-846) (EPA 1995). As such, it is applicable to sampling the headspace gases in drums containing all types of waste. The procedure is used to sample for hydrogen, methane, and specific VOCs that have a vapor phase component at room temperature and pressure. The quality assurance requirements for this procedure are described in the Transuranic Waste Characterization Quality Assurance Program Plan (QAPP) (DOE 1995). This procedure must be implemented with a site-specific standard operating procedure (SOP).

1.2 For the purposes of the TRU Waste Characterization Program, headspace gas samples are to be collected in sampling batches. A sampling batch is defined as a suite of samples collected consecutively, using the same sampling equipment, within a specific time period. A sampling batch can be up to 20 samples (excluding field $\mathrm{OC}$ samples), all of which must be collected within 14 days of the first sample in the batch.

\subsection{Summary of Procedure}

This sampling procedure employs a multiport sampling manifold capable of collecting samples of headspace gas from within waste drums. Cleaned and evacuated sample canisters are attached to the evacuated sampling manifold. A changeable needle assembly with a valve is also connected to the sampling manifold. The needle is inserted into the headspace of the drum or poly liner bag and the valve is opened, allowing the headspace gases within the drum or poly bag to expand into the evacuated sample canisters.

\subsection{Limitations and Interferences}

No limitations or interferences have been identified.

\subsection{Safety}

This procedure may involve the use of hazardous materials, operations, and equipment. It is the responsibility of whoever uses this procedure to consult appropriate site personnel concerning health and safety issues and establish appropriate health and safety practices. Consideration should be given to safety concerns regarding chemical and radiation exposure. Training regarding proper storage, usage, and disposal of chemicals is recommended.

\subsection{Apparatus and Materials}

5.1 Collecting representative samples of headspace gas from within a TRU waste drum requires the use of a variety of components. The major components required for sampling include a sampling manifold, sample canisters, pressure sensors, a thermometer, a vacuum pump, cylinders of pressurized gas, and a rotameter. In 
addition, the use of an organic vapor analyzer (OVA) is recommended to provide immediate indications of sampling manifold cleanliness. Each major component of the sampling apparatus is described below.

5.2 Sampling Manifold. With the exceptions of the pressurized gas cylinders, the vacuum pump and the OVA, all components of the sampling manifold are designed to be panelmounted in close configuration to minimize the system's internal volume and surface area. The panel can be mounted in a standard 19-inch rack mount cabinet on a moveable cart with the pressurized gas cylinders and vacuum pump for a mobile system, or on a wall. Figure 1 illustrates the system configuration and lists the major parts required for this configuration. The numbered components in Figure 1 correspond to the numbered components described in the text below.

5.2.1 The sampling manifold is a system with two pneumatically distinct lines: an evacuated line for collecting samples and a pressurized line for providing zero air, helium or nitrogen, and field reference standard gases.

NOTE: Zero air is defined as hydrocarbon-free air containing $0.01 \mathrm{ppmv}$, or less, carbon monoxide, $1.0 \mathrm{ppmv}$, or less, carbon dioxide and $0.01 \mathrm{ppmv}$, or less, total organic carbon (measured as methane). Nitrogen, helium, or argon may be used in lieu of zero air depending on project needs.

5.2.2 The evacuated line consists of a needle assembly, capacitance manometer, six ports for attaching sample canisters, and a dry vacuum pump. The needle consists of a 6 -inch tube, 1/8-inch O.D., sharpened at one end and connected to an elbow fitting at the other (1). The elbow fitting is connected to a 0.5 micron filter (2), which is connected to the body portion of a quick-connect (3). The quick-connect allows the operator to easily connect a clean needle assembly between samples, thus reducing the potential for cross contamination.

5.2.3 A flow regulating bellows valve (4) is connected to the stem side of the quickconnect. On the other side of valve (4) is a 7 -micron filter (6) which provides additional protection against system contamination from particulate matter. Connecting valve $(4)$ and filter $(6)$ is flexible Teflon ${ }^{\oplus}$ tubing $(5)$ that allows movement of the needle from the purge assembly tee (27) to the 208-liter (55-gallon) drum.

5.2.4 After passing through filter (6), headspace gas passes through a tee connector before being swept past the sample canister ports and into the vacuum pump. The tee provides a pneumatic link to the transducer (8) of a capacitance manometer pressure sensor. Closing bellows valve (7) protects the sensor from pressure shocks and overpressurization.

CAUTION: The transducer should not be exposed to pressure greater than $1,200 \mathrm{~mm} \mathrm{Hg}$.

5.2.5 Once downstream of the pressure transducer tee, headspace gases is swept past six tees (10-15) that provide connection ports for the sample canisters. Each port has a permanent $V_{C R^{\oplus}}$ adaptor fitting for canister attachment to the sampling manifold to circumvent potential leak problems caused by reconnecting the Swagelok ${ }^{\circledR}$ fitting after cleaning. If desired, valves may be placed between the ports and the canister valves to facilitate closing the ports when sample canisters are not attached. 
5.2.6 Bellows valve (16) connects the vacuum pump (17) to the sampling manifold. When the pump is operating, opening valve (16) decreases the system pressure and closing the valve isolates the pump. The pump exhaust is directed to the containment area through a 7-micron filter (18). This filter serves as a safety precaution to prevent contaminated particulate matter from accidentally entering the system through this port. Filter $(18)$ is an optional component of the sampling manifold.

5.2.7 An OVA (32) is connected downstream of the sample port farthest from the needle assembly (15). The OVA's internal pump pulls zero air through the manifold to check for cleanliness. Exhaust from the OVA is directed to the exhaust port.

5.2.8 The pressurized line consists of a pressurized cylinder of zero air (19), and a pressurized cylinder of gas (e.g., nitrogen) containing field reference standards (20) metered through two-stage stainless steel regulators $(21,22)$ to a purge assembly where flow rates of the pressurized gases can be adjusted and monitored with a rotameter (30). Valve (25) is a flow regulating bellows valve for regulating flows from the gas cylinders through the rotameter. Valve (29) is a bellows valve that allows the operator to clean the system after collecting a field reference standard sample. Filters $(26)$ and $(28)$ are 7-micron particulate filters to prevent contaminated particulate matter from accidentally entering the system through the purge assembly tee (27). Filters (26) and (28) are optional components of the sampling manifold.

5.2.9 A bellows valve (23) connects a $250 \mathrm{~mL}$ sample canister (24) containing approximately $100 \mathrm{~mL}$ of ASTM Type II (or equivalent) water to the pressurized line. When valve $(23)$ is open, the water humidifies the dry pressurized gases from the pressurized gas cylinders. Closing valve (23) isolates the water from the sampling manifold. The dry pressurized gases are humidified for $Q A / O C$ purposes. For $O C$ purposes, the water vapor assists with the cleaning of the internal surfaces of the sampling manifold and, for QA purposes, the water vapor conditions the zero air and field reference standards so they are comparable to the headspace gas which is expected to be humid. In lieu of installing the bellows valve (23) and humidifier (24), the zero air field reference standard gases may be humidified by adding water directly to cylinders (19) and (20). A cylinder gas concentration of approximately $10,000 \mathrm{ppmv}$ water is recommended.

5.2.10 With valve (29) open and the needle assembly attached to the purge assembly tee (27), valve (25) is used to adjust the flow rate through the rotameter (30). The purge assembly tee is a $1 / 8$-inch stainless steel tee with Teflon ${ }^{\circ}$ ferrules seated by a stainless steel nut at the needle connection port. The needle port has been drilled to 1/8-inch I.D. to facilitate complete insertion of the needle. The pliable Teflon $^{\oplus}$ ferrules permit the needle to be repeatedly withdrawn from and tightened into the port. By maintaining flow through the rotameter when purging or sampling, the technician operating the system is assured that only gases from the pressurized line of the sampling manifold enter the evacuated line, thus preventing ambient air from entering the system through the rotameter, diluting the sample(s) collected and contaminating the sampling manifold.

5.2.11 The only time it is necessary to remove the Teflon ${ }^{*}$ ferrules from the purge assembly tee (27) is to facilitate attachment of the system cleaning assembly after the collection of a field reference standard sample. This assembly does 
not have the 0.5 micron particulate filter, and a port connector is used in place of the needle assembly's 6 -inch needle. This configuration allows the pressurized line, as well as the evacuated line, of the system to be evacuated, swept, and cleaned with zero air.

5.2.12 An additional $O C$ provision which should be incorporated into the design of the sampling manifold, but not shown in Figure 1 , is the ability to heat the sampling manifold during sampling and cleaning activities. This may be accomplished by wrapping the pneumatic lines, valves, and filters with resistive wire or tape in order to heat the internal surfaces of the sampling manifold (approximately $50^{\circ} \mathrm{C}$ is recommended). Heating the sampling manifold is recommended to reduce analyte adsorption on the sampling manifold's internal surfaces.

5.3 Sample Canister. A sample canister is a leak-free welded stainless steel SUMMA ${ }^{\circ}$ pressure vessel with a $\mathrm{Cr}$-NiO-passivated interior surface, bellows valve, and dial pressure/vacuum gauge. Two sizes of canisters $(100 \mathrm{~mL}$ and $250 \mathrm{~mL})$ may be used for the collection of headspace gas samples. These are presented in Figure 2.

These sample canisters have $\mathrm{VCR}^{\oplus}$ fittings for connection to sampling and analytical equipment. The dial pressure/vacuum gauge indicates if the canister has leaked, thereby preventing sampling manifold contamination by exposure to a potentially contaminated sample canister. SUMMA ${ }^{\circ}$ canisters have been used in the past for VOC sample collection and have a demonstrated sample storage stability for many specific VOCs (EPA 1988; Oliver et al. 1986).

5.4 Pressure and Temperature Measurement Apparatus. When using the sampling manifold, the sampling manifold pressure, read with the capacitance manometer, is monitored and recorded. Ambient temperature is also monitored and recorded.

5.4.1 An accurate barometer is used to monitor ambient pressure, while a capacitance manometer is used to monitor both manifold and canister pressures. A dial pressure/vacuum gauge is used to monitor sample canister pressure when a sampling manifold equipped with a capacitance manometer is not available.

5.4.1.1 Ambient Pressure Sensor: The ambient pressure sensor is a fullrange Fortin barometer, or equivalent, with a range of approximately 500 to $800 \mathrm{~mm} \mathrm{Hg}$. It is kept in the sampling area. Its resolution is $1 \mathrm{~mm} \mathrm{Hg}$ and its calibration is traceable to National Institute of Standards and Technology (NIST) standards. A dual scale thermometer should be provided with the barometer for temperature corrections.

5.4.1.2 Dial Pressure/Vacuum Gauge: This type of gauge is helium leak tested to $1.5 \times 10^{-7}$ standard $\mathrm{cc} / \mathrm{sec}$, has all stainless steel construction, and is capable of tolerating temperatures to $125^{\circ} \mathrm{C}$. The gauge is able to indicate 5 psig pressure to a vacuum of 30 inches of mercury.

5.4.1.3 Capacitance Manometer: The capacitance manometer is pneumatically connected to the sampling manifold. The pressure sensor consists of a pressure transducer and electronics/display unit. The transducer is able to measure absolute pressure in the 
range from $0.1 \mathrm{~mm} \mathrm{Hg}$ to $100 \mathrm{~mm} \mathrm{Hg}$ with a usable resolution of $\pm 0.005 \mathrm{~mm} \mathrm{Hg}$ at $0.05 \mathrm{~mm} \mathrm{Hg}$.

5.4.2 Thermometer: The thermometer is a NIST-traceable laboratory grade mercury thermometer, total immersion type, with nitrogen above the mercury. It has permanent stain markings in the approximate range of $-30^{\circ}$ to $50^{\circ} \mathrm{C}$, with a resolution of $1^{\circ} \mathrm{C}$.

5.5 Organic Vapor Analyzer. The OVA is a battery powered portable organic vapor analyzer capable of measuring volatile organic compounds in air in two ranges. In the range of $0-200 \mathrm{ppm}$, it has a resolution of $0.1 \mathrm{ppm}$. In the range of $200-2000 \mathrm{ppm}$, it has a resolution of $1.0 \mathrm{ppm}$. Gas cylinders are supplied by the manufacturer for calibrating the unit. The OVA is connected downstream of the sampling ports on the manifold and used, along with confirmatory equipment blanks, to check for manifold cleanliness.

\subsection{Preplanning}

Studies to characterize TRU waste require careful planning to prepare facilities, develop written documentation, secure laboratory support, and evaluate data.

7.0 Reagents, Preservation Measures, and Handling

7.1 All sampling equipment components that come into contact with the headspace sample gases must be constructed of relatively inert materials such as stainless steel or Teflon. Stainless steel components with passivated interior surfaces is recommended. Sample holding times and storage conditions should conform to the requirements specified in Table 1.

7.2 All sample handling and chain-of-custody procedures described in Section 6.0 of the QAPP must be followed.

\subsection{Procedure}

8.1 Cleaning the Sampling Manifold. These procedures are provided to ensure the samples collected using this apparatus are representative by reducing the possibility of contamination by manufacturing impurities and ambient air. Use of these cleaning procedures will also reduce the possibility of cross-contamination between sampling operations. Prior to use in any headspace gas sample collection activities, SUMMA canisters must be cleaned and certified in accordance with Procedure 210.1 of this Methods Manual. The procedures for cleaning the sampling manifold are as follows:

- Section 8.1.1 describes a procedure for initially cleaning and leak-checking the sampling manifold.

- Section 8.1.2 describes a procedure for routine cleaning and leak-checking the sampling manifold.

- Section 8.1.3 describes a procedure for cleaning the sampling manifold after collection of a field reference standard.

- Section 8.1.4 describes a procedure for needle assembly cleaning. 
TABLE 1

Holding Conditions and Holding Times

\begin{tabular}{|c|c|c|c|c|c|c|}
\hline Parameter & Container & $\begin{array}{c}\text { Minimum } \\
\text { Drum } \\
\text { Headspace } \\
\text { Sample } \\
\text { Volume }^{\mathrm{a}}\end{array}$ & $\begin{array}{c}\text { Holding } \\
\text { Temperatures }\end{array}$ & $\begin{array}{c}\text { Field } \\
\text { Holding } \\
\text { Time }^{\mathrm{b}}\end{array}$ & $\begin{array}{l}\text { Shipping } \\
\text { Allowance }\end{array}$ & $\begin{array}{c}\text { Laboratory } \\
\text { Holding } \\
\text { Time }^{c}\end{array}$ \\
\hline $\mathrm{H}_{2}, \mathrm{CH}_{4}$ & $\begin{array}{l}\text { SUMMA } \\
\text { Canister }\end{array}$ & $\begin{array}{c}100 \\
\text { milliliters }\end{array}$ & $0-40^{\circ} \mathrm{C}$ & 4 days & 2 days & 28 days \\
\hline VOCs & $\begin{array}{l}\text { SUMMA® } \\
\text { Canister }\end{array}$ & $\begin{array}{c}250 \\
\text { milliliters }\end{array}$ & $0-40^{\circ} \mathrm{C}$ & 4 days & 2 days & 28 days \\
\hline
\end{tabular}

alternatively, if available headspace is limited, a single $100 \mathrm{~mL}$ sample may be collected for determination of VOCs, $\mathrm{H}_{2}$, and $\mathrm{CH}_{4}$.

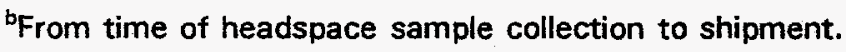

cProgrammatic-based maximum holding time. Holding time begins at validated time of sample receipt. 
8.1.1 Initial Manifold Cleaning and Leak-Free Certification: After receipt from the various manufacturers and prior to assembly, all components of the sampling manifold that will come into contact with headspace or cylinder gases are inspected and thoroughly cleaned using this procedure. Parts received free of contamination, as stated or certified by the manufacturers, may be exempted from this cleaning procedure at the discretion of the sampling personnel.

1. Disassemble nonmetallic components of the sampling manifold, rinse with HPLC-grade deionized water, and dry in an oven at $50^{\circ} \mathrm{C}$ for 12 to 24 hours. An evacuated oven is preferable, but not required.

2. Place stainless steel parts and fittings in a beaker of reagent-grade hexane in an ultrasonic bath for 15 minutes. Repeat this procedure with reagent-grade methanol. Rinse the stainless steel parts with HPLC-grade deionized water and dry in an oven at $100^{\circ} \mathrm{C}$ for 12 to 24 hours. Stainless steel parts and fittings may also be cleaned by assembling and forcing steam through the assembly.

3. Assemble the sampling manifold and leak-check by pressurizing to 1,700 mbar and placing bubble solution on all fittings and connections. Then periodically check for the production of bubbles for a 30-minute period. As an alternative, the system may be pressurized with helium and leakchecked with a helium detection device.

4. Tighten fittings and connections that leak until no leaks are observed. From clean spare parts, replace any fittings and connections that continue to leak.

5. Evacuate the sampling manifold to $0.13 \mathrm{mbar}$ and monitor the pressure for 1 hour. If the pressure increases by more than 0.03 mbar, repeat steps 3 and 4.

6. Purge the sampling manifold with humid zero air for 24 hours.

7. Evacuate and pressurize the sampling manifold with zero air three times.

8. Collect an equipment blank through the manifold as described in Section 8.2.4 and analyze for the target compounds listed in Table 2. Check for system cleanliness with the OVA and confirm by analysis of the equipment blank.

9. Pressurize the sampling manifold with zero air to 1,700 mbar when not in use.

8.1.2 Routine Manifold Cleaning and Leak Check: The following procedure assumes that a clean needle assembly (1 through 3 ) has been connected to valve (4) and inserted into purge assembly tee (27). The procedure also assumes that all sampling manifold valves are closed, the vacuum pump and OVA are operating, and all regulators for the high pressure cylinders (zero air and field reference standards, etc.) have been properly purged and set to a delivery pressure of approximately 1,700 mbar. Refer to Figure 1 for location of numbered components. 
TABLE 2

Headspace Gas Target Analyte List

Acetone

Benzene

Bromoform

Butanol

Carbon tetrachloride

Chlorobenzene

Chloroform

Cyclohexane

1,1-Dichloroethane

1,2-Dichloroethane cis-1,2-Dichloroethylene

1,1-Dichloroethylene

Ethyl benzene

Ethyl ether

Hydrogen

Methane

Methanol

Methyl ethyl ketone

Methyl isobutyl ketone

Methylene chloride

1,1,2,2-Tetrachloroethane

Tetrachloroethylene

Toluene

1,1,1-Trichloroethane

Trichloroethylene

1,1,2-Trichloro-1,2,2-trifluoroethane

1,2,4-Trimethylbenzene

1,3,5-Trimethylbenzene

$\mathrm{m}$-Xylene

o-Xylene

p-Xylene
67-64-1

$71-43-2$

$75-25-2$

$71-36-3$

56-23-5

108-90-7

67-66-3

$110-87-7$

75-34-3

107-06-2

540-59-0

75-35-4

100-41-4

60-29-7

1333-74-0

74-82-8

67-56-1

78-93-3

108-10-1

75-09-2

79-34-5

127-18-4

108-88-3

71-55-6

79-01-6

76-13-1

95-63-6

108-67-8

108-38-3

95-47-6

106-42-3
$150 \mathrm{ng}$

$10 \mathrm{ng}$

$10 \mathrm{ng}$

$150 \mathrm{ng}$

$10 \mathrm{ng}$

$10 \mathrm{ng}$

$10 \mathrm{ng}$

$10 \mathrm{ng}$

$10 \mathrm{ng}$

$10 \mathrm{ng}$

$10 \mathrm{ng}$

$10 \mathrm{ng}$

$10 \mathrm{ng}$

$10 \mathrm{ng}$

0.05 vol $\%$

0.05 vol $\%$

$150 \mathrm{ng}$

$150 \mathrm{ng}$

$150 \mathrm{ng}$

$10 \mathrm{ng}$

$10 \mathrm{ng}$

$10 \mathrm{ng}$

$10 \mathrm{ng}$

$10 \mathrm{ng}$

$10 \mathrm{ng}$

$10 \mathrm{ng}$

$10 \mathrm{ng}$

$10 \mathrm{ng}$

$10 \mathrm{ng}$

$10 \mathrm{ng}$

$10 \mathrm{ng}$ 
1. Select the sample canister(s) to be used for sampling and check each canister's dial pressure gauge reading (lightly tap on the gauge face plate to verify readingl. Record the reading on the sample canister tag. Attach the sample canister(s) to the sampling manifold and tighten connector(s). Sample canister port(s) not occupied by a canister must be closed with a cap or valve. If the port to which a canister is attached has an optional valve (not shown) attached to the manifold port tee, open this valve prior to proceeding to step 2 . Leave the sample canister valve closed.

2. Open valves (29), (23), and (21).

3. Slowly open valve (25) until rotameter $(30)$ indicates a flow rate of approximately 2 liters per minute.

4. Open valve (16) and slowly open valve (4) until rotameter $(30)$ indicates a flow rate of approximately 1 liter per minute. The sampling manifold is now being purged with zero air. Purge for approximately 3 minutes, periodically checking and adjusting valves (25) and (4), if necessary, to maintain rotameter flow rate at 1 liter per minute.

5. Close valve (4) to evacuate the sampling manifold. Wait 5 seconds, close valve (16), and again reopen valve (4) to allow the sampling manifold to fill with zero air. Repeat this 3 to 4 times. Terminate this procedure by closing valve (16), opening valve (4), and allowing the sampling manifold to fill with zero air. Open valve (31) and allow the OVA to pull zero air from the manifold. Observe the OVA display for at least 10 seconds. If the OVA indicates a VOC concentration of 0.5 ppmv or greater, continue purging and sweeping the sampling manifold with zero air until the OVA indicates less than $0.5 \mathrm{ppmv}$. When the OVA indicates less than $0.5 \mathrm{ppmv}$, the internal volume between valve (7) and transducer $(8)$ is purged by opening valve (7).

CAUTION: Care should be taken to slowly evacuate and fill the sampling manifold, since rapid fluctuations in manifold pressure may damage the manometer when valve $(7)$ is open.

6. Open valve (7) and close valves (4), (31), (21), (23), (25) and (29).

7. Open valve (16) and allow the vacuum pump to reduce the pressure in the sampling manifold to $0.13 \mathrm{mbar}$, as indicated by the manometer (9).

8. Once the sampling manifold pressure has been reduced to $0.13 \mathrm{mbar}$, or less, close valve (16).

9. Observe the manometer (9) reading for 3 to 5 seconds to verify that all sampling manifold valves have been properly closed and no observable leaks are present. If the manometer indicates a leak, cease sampling activities and leak check the manifold as described in steps 3 through 5 of Section 8.1.1.

10. Open the sample canister valve(s) and observe the manometer (9) readings to check for changes in pressure as each valve is opened. Record the pressure reading on the respective sample canister tag. 
NOTE: An increase in manifold pressure greater than $0.03 \mathrm{mbar}$ indicates a canister leak, and opening the remaining sample canister valves is not advised. All open valves are closed and the exposed sample canisters are removed and replaced. Then the manifold is purged again following steps 4 through 9 , above.

11. If no leaks are detected, the sampling manifold is ready for sample collection.

8.1.3 Manifold Cleaning After Field Reference Standard Collection: Analytes in the field-reference-standard gas have the potential to remain in the pneumatic lines after field reference standard sample collection. to ensure the pneumatic lines are clean, this procedure is performed immediately after collecting a field reference standard in accordance with Section 8.2.5. It assumes that the needle assembly ( 1 through 3 ) used for the field reference standard collection is still inserted into the purge assembly tee (27). Refer to Figure 1 for location of numbered components.

1. Close valve (23).

2. Close valve (29) and open valve (4).

3. Observe the manometer (9) reading until the pressure ceases to increase (approximately $1700 \mathrm{mbar}$ ) and then close valve (21).

CAUTION: Care should be taken to slowly evacuate and fill the sampling manifold, since rapid fluctuations in manifold pressure may damage the manometer when valve $(7)$ is open.

4. Open valve (16) and evacuate the sampling manifold for a minimum of 5 seconds.

5. Close Valve (16) and then slowly open valve (21).

6. Repeat steps 2 through 4 three more times and terminate this step by closing valve (16) and slowly opening valve (21).

7. Close valve (4), open valve (29), and adjust valve (25) until rotameter (30) indicates approximately 1 liter per minute.

8. Close valve (21).

9. Disconnect the needle assembly from the purge assembly tee (27) and connect a clean needle assembly to valve (4).

10. Insert needle assembly (1 through 3 ) into purge assembly tee (27) and proceed with the cleaning described in Section 8.1.2.

8.1.4 Needle Assembly Cleaning: This procedure describes the steps for cleaning a needle assembly after headspace gas samples or field reference standards have been collected.

1. After samples have been collected, and the sample canister valves have been closed, detach the needle assembly (1 through 3 ) at the quickconnect (3). 
2. Remove the filter assembly to an area where it can safely be disassembled and cleaned.

3. Unscrew the housing of the filter (2) and firmly grasp the filter element with a sturdy pair of forceps. Firmly pull the element to dislodge it from the housing. Properly discard the element.

4. Remove the Viton ${ }^{\oplus}$ O-ring from the body of the quick-connect (3) and discard the O-ring.

5. Place the reusable parts in a beaker of reagent-grade hexane in an ultrasonic bath for 15 minutes. Repeat this procedure with reagentgrade methanol. Rinse the parts with HPLC-grade deionized water and dry in an oven at $100^{\circ} \mathrm{C}$ for $12-24$ hours.

6. Insert a new filter element into the filter (2) and tightly screw the body together.

7. Place a new Viton ${ }^{\oplus}$ O-ring on the body of the quick-connect (3).

8. Purge the needle assembly and a resealable bag with zero air as the needle assembly is being placed inside the bag.

9. Close the resealable bag and place it in a box for storage.

8.2 Collecting Headspace Gas and Quality Control Samples using a Low Internal Volume Sampling Manifold. Headspace gas and quality control samples collected using the sampling manifold and procedures described here are as follows:

- Section 8.2.1 describes a procedure for collecting headspace gas samples.

- Section 8.2.2 describes a procedure for collecting field duplicates.

- Section 8.2.3 describes a procedure for collecting field blanks.

- Section 8.2.4 describes a procedure for collecting equipment blanks.

- Section 8.2.5 describes a procedure for collecting field reference standard samples.

NOTE: Prior to sampling, drums are allowed to equilibrate to the temperature of the sampling area for at least 72 hours.

CAUTION: Appropriate site radiological controls must be established prior to removing the drum lid.

8.2.1 Headspace Gas Sample Collection: Headspace gas samples are collected from within all innermost layers of confinement that meet the criteria specified in Section 7.1 of the QAPP. Refer to Figure 1 for location of numbered components of the sampling manifold. Samples are collected as follows.

1. Verify that the sampling manifold has been properly cleaned and leakchecked as described in Section 8.1.2.

2. Prepare the drum for sampling by removing the drum lid and the internal rigid liner lid which should expose the poly liner bags.

3. Remove the needle assembly (1 through 3) from the purge assembly tee (27) and press the needle through the poly bag and into the headspace gas. Disturbing the bag should be avoided, if possible. However, if 
handling the bag is necessary, the operator should do so in a manner that minimizes the potential for introduction of outside air into the poly bag's headspace gas.

4. Hold the needle in the headspace and open valve (4). Hold the needle steady until the canister gauge indicates zero psig.

5. Close the sample canister(s) valve(s).

6. Remove the needle (1) from the poly bag and disconnect the needle assembly from valve (4). Place the needle assembly in a collection box for items to be cleaned or dispose of it.

7. Remove a fresh needle assembly from its resealable bag and connect the needle assembly to valve (4). Insert the fresh needle assembly into the purge assembly tee (27) and hand tighten the assembly in place.

8. Remove the sample canister(s) from the sampling manifold and log the date, time, sampling manifold pressure (9), ambient pressure, and ambient temperature on the sample canister tag.

9. Record on the sample type on a QC sample record and place the sample canister in a shipping container.

10. Clean the sampling manifold according to the procedure in Section 8.1.2.

8.2.2 Field Duplicate Collection: The procedure for field duplicate collection is similar to that specified in Section 8.2.1 for headspace gas sample collection. However, when collecting field duplicates, two sample canisters are attached to the sampling manifold. One canister is attached at port $(10)$ and the other is attached at port (11). When collecting field duplicates, valves (10) and (11) are closed prior to opening valve (4). After opening valve (4), valve (10) is opened and the first sample canister filled. Then valve $(11)$ is opened and the second sample canister is filled. Finally, valve $(4)$ is closed followed by valves (10) and (11).

8.2.3 Field Blank Collection: An equipment blank (Section 8.2.4) should be collected immediately prior to collecting the field blank. Also prior to field blank collection, sampling sites should use the OVA to obtain a real time indication of sampling manifold cleanliness. The following procedure is used for collection of a field blank through the sampling manifold. Refer to Figure 1 for location of numbered components.

1. Verify that the sampling manifold has been properly cleaned and leakchecked as described in Section 8.1.2.

2. Remove the needle assembly (1 through 3 ) from the purge assembly tee (27) and hold the needle assembly near the top of the drum.

3. Open valve (4). Hold the needle steady until the manometer (9) indicates ambient pressure, or ceases to show an increase in pressure. Verify that the canister gauge indicates zero psig.

4. Close the sample canister(s) valve(s). 
5. Disconnect the needle assembly from valve (4). Place the needle assembly in a collection box for items to be cleaned or discarded.

6. Remove (from its resealable bag) a clean needle assembly and connect it to quick-connect (3). Insert the clean needle assembly into the purge assembly tee (27) and hand tighten the assembly in place.

7. Remove the sample canister(s) from the sampling manifold and log the date, time, sampling manifold pressure (9), ambient pressure, and ambient temperature on the sample canister tag.

8. Record on the sample type on a $\mathrm{OC}$ sample record and place the sample canister in a shipping container.

9. Clean the sampling manifold according to the procedure in Section 8.1.2.

8.2.4 Equipment Blank Collection: An equipment blank is collected and analyzed prior to first use of the sampling manifold, then at a frequency of one in every twenty samples consecutively collected. The following procedure is used for collecting equipment blanks. Refer to Figure 1 for location of numbered components.

1. Verify that the sampling manifold has been properly cleaned and leakchecked as described in Section 8.1.2.

2. With the needle assembly (1 through 3 ) connected to the purge assembly tee (27), adjust valve (25) until rotameter $(30)$ indicates a flow rate of approximately 10 liters per minute of zero air.

3. Open valve (4) to allow the sample canister to fill as quickly as possible without reducing the flow indicated by the rotameter $(30)$ to zero.

NOTE: If rotameter $(30)$ indicates zero flow, the sampling manifold blank is invalidated and the sampling manifold is cleaned (Section 8.1.2) prior to collection of another sampling manifold blank.

4. Continue drawing zero air from the purge assembly tee (27) until the manometer (9) indicates ambient pressure or ceases to show an increase in pressure. Verify that the sample canister gauge indicates zero psig.

5. Close valve (4) and the sample canister valve when the manometer indicates ambient pressure.

6. Adjust valve $(25)$ to reduce the flow rate through the rotameter $(30)$ to less than 1 liter per minute (to conserve zero air).

7. Remove the sample canister from the manifold.

8. Log the date, time, manifold pressure (9), ambient pressure, and ambient temperature on the sample canister tag.

9. Record on the sample type on a QC sample record and place the sample canister in a shipping container. 
8.2.5 Field Reference Standard Sample Collection: A field reference standard is collected and analyzed prior to first use of the sampling manifold, then at a frequency of one in every twenty samples consecutively collected. The following procedure is used for collecting field reference standard samples. Refer to Figure 1 for location of numbered components.

1. Verify that the sampling manifold has been properly cleaned and leakchecked as described in Section 8.1.2 and verify that canister port (11) is closed.

2. Open valves (23) and (29). Open valve (25) slightly and then open valve (22) to initiate flow of field-reference-standard gas to the purge assembly tee (27).

3. With the needle assembly $(1$ through 3$)$ connected to the purge assembly tee (27), adjust valve $(25)$ until rotameter $(30)$ indicates a flow rate of approximately 10 liters per minute.

4. Open valve (4) to allow the sample canister(s) to fill as quickly as possible without reducing the flow indicated by the rotameter $(30)$ to zero.

NOTE: If rotameter $(30)$ indicates zero flow, the field reference standard is invalidated and the sampling manifold is cleaned (Section 8.1.3) prior to collection of another field reference standard sample.

5. Continue drawing field reference standard gas from the purge assembly tee (27) until the manometer (9) indicates ambient pressure or ceases to show an increase in pressure. Verify that the sample canister gauge indicates 0 psig.

6. Close valve (4) and the sample canister valve when the manometer indicates ambient pressure.

7. Adjust valve (25) to reduce the flow rate through rotameter $(30)$ to approximately 1 liter per minute. Then, close valve (22).

8. Open valve (21) and open and close valve (25) a few times to facilitate purging residual field reference standard gas from the pneumatic lines. Terminate this step with valve (25) adjusted to deliver approximately 1 liter per minute zero air through rotameter (30).

9. Remove the sample canister from the sampling manifold.

10. Log the date, time, manifold pressure (9), ambient pressure, and ambient temperature on the sample canister tag.

11. Record on the sample type on a OC sample record and place the sample canister in a shipping container.

12. Close the sample canister port and proceed with cleaning the sampling manifold as described in Section 8.1.3.

8.3 Calculations. The potential exists for sampling or analytical personnel, upon receipt of a sample canister, to find that the dial pressure gauge on the sample canister does not 
indicate the same pressure as that which was last logged on the sample canister tag. Changes in the sample canister gauge pressure may be the result of four possible occurrences: (1) a leak in the sample canister or sample canister component (value, gauge, etc.); (2) damage to the sample canister gauge during storage or transportation; (3) an ambient pressure difference between the sampling and analysis location; and (4) an ambient temperature difference between the sampling and analysis location.

The procedures below should be followed to determine the sample canister gauge pressure change expected if ambient pressure and temperature differences exist between the sampling and analysis locations. If the change in sample canister gauge pressure can be accounted for using the procedures below, then the integrity of the sample has been maintained. If, however, the sample canister gauge pressure change cannot be accounted for by considering ambient pressure and temperature changes, the canister must be leak checked and re-certified for use.

Changes in sample canister gauge pressure that result from changes in temperature and ambient pressure are additive. Care, however, must be taken to ensure that the sign (positive or negative) of the calculated gauge pressure changes are accurately assigned.

8.3.1 Temperature Change at Constant Ambient Pressure: Sample canister gauge pressure changes resulting from a temperature change can be explained by the following equation:

$$
P_{2}=\frac{P_{1} T_{2}}{T_{1}}
$$

where

$$
\begin{aligned}
& P_{1}=\quad \begin{array}{l}
\text { sample canister internal pressure (ambient pressure } \\
\text { plus gauge pressure) at the time of sampling } \\
\text { (inches } \mathrm{Hg} \text { ) }
\end{array} \\
& P_{2}=\quad \begin{array}{l}
\text { sample canister internal pressure (ambient pressure } \\
\text { plus gauge pressure) at the time of analysis (inches } \\
\text { Hg), if the ambient temperature changes from } T_{1} \text { to } T_{2} \\
\text { at constant ambient pressure }
\end{array} \\
& T_{1}=\quad \begin{array}{l}
\text { ambient temperature at the time of sampling }\left({ }^{\circ} \mathrm{K}\right) \\
T_{2}=\quad \text { ambient temperature at the time of analysis }\left({ }^{\circ} \mathrm{K}\right)
\end{array}
\end{aligned}
$$

According to this equation, increasing the temperature of the canister $\left\langle T_{2}>T_{1}\right\rangle$ will increase the internal canister pressure, while decreasing the canister temperature $\left(T_{2}<T_{1}\right)$ will decrease the internal canister pressure.

8.3.2 Ambient Pressure Change at Constant Temperature: A sample canister's dial gauge is expected to indicate a change in pressure if the canister is transported from a high elevation to a lower elevation, or vice versa. The expected change in canister gauge pressure should be the same as the difference in ambient pressure between the high elevation site and the low elevation site, or vice versa. 


\subsection{Quality Control}

9.1 Specific sampling steps to ensure that the headspace gas samples are representative include:

- Sample canister cleaning and leak checking

- Use of sample canisters with a passivated internal surface

- Use low internal volume sampling apparatus

- Small sample volume: low sample volume to available headspace gas volume ratio

- Careful pressure regulation in sample canister

- Performance audits

- Collection of field blanks, field reference standards, equipment blanks, and field duplicates

9.2 Quality Control for Pressure and Temperature Measurements. This section pertains to the calibration and certification of equipment and instrumentation used for temperature and pressure measurements. All information related to the calibrations of the pressure and temperatures sensors is recorded.

9.2.1 Certification Check of the Ambient Pressure Sensor: The ambient pressure sensor is certified by the manufacturer using standards or equipment traceable to the NIST. The pressure indication of the sensor is corrected in accordance with temperature indicated by a dual-scale thermometer attached to its casing, if necessary.

9.2.2 Calibration of the Capacitance Manometer: The capacitance manometer is certified using standards or equipment traceable to the NIST.

9.2.3 Calibration of Sample Canister Pressure Gauge: The dial pressure gauge of each sample canister is certified using standards or equipment traceable to the NIST. Each canister gauge is recertified once per month, or as necessary.

9.2.4 Calibration of the Mercury Thermometer: The mercury thermometer is certified using standards or equipment traceable to the NIST.

9.2.5 Calibration of the Organic Vapor Analyzer: Cylinders of calibration gases, certified using standards traceable to NIST, are supplied by the manufacturer of the organic vapor analyzer for calibrating the unit.

9.3 Field blanks are used to assess background levels of the analytes listed in Table 2. Field blanks must be collected prior to sample collection, and then at the frequency specified in Table 3. An equipment blank should be collected immediately prior to collecting the field blank. Also prior to field blank collection, an OVA should be used to obtain a real time indication of sampling manifold cleanliness. Field $O C$ sample acceptance criteria is specified in Table 4. Corrective action must be implemented if acceptance criteria is not met.

9.4 Equipment blanks are used to assess the cleanliness of the sampling manifold. Equipment blanks are collected and analyzed for the analytes listed in Table 2 prior to 
TABLE 3

Summary of Field OC Sample Frequencies

Field blanks ${ }^{a}$

Equipment blanks ${ }^{b}$

Field reference standards ${ }^{\circ}$

Field duplicates
1 per sampling batch ${ }^{d}$

1 per sampling batch ${ }^{d}$

1 per sampling batch ${ }^{d}$

1 per sampling batch ${ }^{d}$

analysis of field blanks for VOCs only, is required.

bone equipment blank sample must be collected, analyzed for VOCs, and demonstrated clean prior to first use of the headspace gas sampling equipment with each of the sampling heads.

'One field reference standard must be collected, analyzed, and demonstrated to meet the acceptance criteria specified in Table 4 prior to first use.

${ }^{d}$ A sampling batch is a suite of samples collected consecutively using the same sampling equipment within a specific time period. A sampling batch can be up to 20 samples (excluding field $\mathrm{OC}$ samples), all of which must be collected within 14 days of the first sample in the batch. 
TABLE 4

Summary of Field QC

Sample Acceptance Criteria

\begin{tabular}{|c|c|c|}
\hline QC Sample & $\begin{array}{l}\text { Acceptance } \\
\text { Criteria }\end{array}$ & $\begin{array}{l}\text { Corrective } \\
\text { Action }^{\mathrm{a}}\end{array}$ \\
\hline Field blanks & $\begin{array}{l}\text { VOC amounts }<3 \times \text { MDLs in } \\
\text { Table } 2\end{array}$ & $\begin{array}{l}\text { Nonconformance if any VOC } \\
\text { amount }>3 \times \text { MDLs in Table } 2\end{array}$ \\
\hline Equipment blanks & $\begin{array}{l}\text { Hydrogen, Methane, and VOC } \\
\text { amounts }<3 \times \text { MDLs in Table } 2\end{array}$ & $\begin{array}{l}\text { Nonconformance if any analyte } \\
\text { amount }>3 \times \text { MDLs in Table } 2\end{array}$ \\
\hline $\begin{array}{l}\text { Field reference } \\
\text { standards }\end{array}$ & $70-130 \% R$ & $\begin{array}{l}\text { Nonconformance if } \% R<70 \text { or } \\
>130\end{array}$ \\
\hline Field duplicates & $R P D \leq 25$ & Nonconformance if RPD $>25$ \\
\hline
\end{tabular}

aCorrective action if acceptance criteria is not met, nonconformance procedures are outlined in Section 2.1.2.1 of the QAPP.

MDL = Method detection limit

$\% R=$ Percent recovery

RPD $=$ Relative percent difference 
first use of the sampling manifold, then at the frequency specified in Table 3. Field $\mathrm{QC}$ sample acceptance criteria is specified in Table 4 . Corrective action must be implemented if acceptance criteria is not met.

9.5 Field reference standards are used to assess the accuracy of the sampling procedure. Field reference standards are collected prior to first use of the sampling manifold, then at the frequency specified in Table 3 and submitted blind to the analytical laboratory. Field reference standards must contain at least six of the VOCs listed in Table 2 at concentrations in the range of 10 to $100 \mathrm{ppmv}$. Field reference standards must have a known valid relationship to a nationally recognized standard such as NIST. If commercially purchased gas standards are used, a Certificate of Analysis is required documenting traceability. Commercially purchased stock gases must not be used beyond their manufacturer-specified shelf life. Field QC sample acceptance criteria is specified in Table 4. Corrective action must be implemented if acceptance criteria is not met. Accuracy, as percent recovery $(\% R)$ shall be determined as follows:

$$
\% R=\frac{C_{m}}{C_{s r m}} * 100
$$

where

$$
\begin{aligned}
& C_{m}=\text { measured concentration } \\
& C_{s r m}=\text { true concentration }
\end{aligned}
$$

9.6 Field duplicates are used to assess the precision of the sampling procedure. field duplicates are collected simultaneously using a tee fitting and two separate SUMMA ${ }^{\oplus}$ canisters. Field duplicates must be collected at the frequency specified in Table 3 and submitted blind to the analytical laboratory. Field OC sample acceptance criteria is specified in Table 4. Corrective action must be implemented if acceptance criteria is not met. Precision, as relative percent difference (RPD) shall be determined as follows:

$$
R P D=\frac{C_{1}-C_{2}}{\frac{\left(C_{1}+C_{2}\right)}{2}} * 100
$$

where

$$
\begin{array}{lll}
C_{1} \text { and } C_{2}= & \text { two values obtained by analyzing the duplicate samples } \\
C_{1} & =\text { the larger of the two observed values }
\end{array}
$$

\subsection{Procedure Performance}

Since 1991, Argonne National Laboratory-West has been collecting headspace gas samples from drums of TRU waste in accordance with this procedure. Although the data set is limited, sufficient data have been collected to assess the precision and accuracy with which the headspace gas sampling manifold collects samples.

10.1 Precision. Precision for the manifold method was determined by collecting duplicate samples of headspace gas as specified in Section 8.2.2. For each pair of canisters, the RPD for each analyte detected was calculated as in Equation (3).

The RPDs were then averaged. For analytes found in three or more duplicate samples, the standard deviation (s) of the RPDs was calculated as follows: 


$$
s=\sqrt{\sum_{i=1}^{n} \frac{\left(y_{i}-\bar{y}\right)^{2}}{n-1}}
$$

where

$$
\begin{aligned}
& \bar{y}=\text { mean of the replicate measurements } \\
& y_{i}=\text { measured value of the } i^{\text {th }} \text { replicate sample analysis measurement } \\
& n=\text { the number of replicate analyses }
\end{aligned}
$$

The results are summarized in Table 5 . All average RPDs were within the procedure's precision limit of $\leq 25$, with the exception of 1,1-dichloroethane, which was found in only one duplicate sample.

10.2 Accuracy. Recovery for the manifold method was determined by collecting samples of field reference standards as specified in Section 8.2.5. Compressed gas cylinders certified to contain known concentrations of known VOCs and gases were used as the field reference standards. The \%R for each analyte was calculated as in Equation (2). The \%R was then averaged for each analyte and the $s$ of the \%Rs was calculated as in Equation (4).

The results are presented in Table 6 . All average $\%$ Rs for the analytes were within the procedure's accuracy limit of 70 to 130 .

\subsection{References}

Oliver, K.D., J.D. Pleil, and W.A. McClenny. 1986. Atmospheric Environment, Vol. 20, No. 7, pp. 1403-1411.

DOE. 1995. Transuranic Waste Characterization Quality Assurance Program Plan. CA0-94-1010, Current Revision. Carlsbad, New Mexico, Carisbad Area Office, U.S. Department of Energy.

EPA. 1988. "Compendium Method TO-14, The Determination of Volatile Organic Compounds (VOC) in Ambient Air Using SUMMA ${ }^{\circledR}$ Passivated Canister Sampling and Gas Chromatographic Analyses." Compendium of Methods for the Determination of Toxic Organic Compounds in Ambient Air, Research Triangle Park, North Carolina, Quality Assurance Division, Environmental Monitoring Systems Laboratory, U.S. Environmental Protection Agency.

EPA. 1995. Test Methods for Evaluating Solid Waste, Physical/Chemical Methods. SW-846, Third Edition, Final Update I, and Final Update II, Washington, D.C. Office of Solid Waste and Emergency Response, Environmental Protection Agency. 
TABLE 5

Procedure Precision for the Sampling Manifold Method

\begin{tabular}{lcc}
\hline Analyte & $\begin{array}{c}\text { Number of } \\
\text { Duplicate Samples }\end{array}$ & Average RPD $\pm s^{\mathbf{a}}$ \\
\hline Benzene & 1 & 12 \\
1,1-Dichloroethane & 1 & 26 \\
1,1-Dichloroethylene & 1 & 7 \\
Methylene chloride & 4 & $9 \pm 7$ \\
Toluene & 6 & $13 \pm 5$ \\
1,1,1-Trichloroethane & 7 & $11 \pm 7$ \\
Trichloroethylene & 2 & 7 \\
Cyclohexane & 1 & 7 \\
\hline
\end{tabular}

${ }^{\mathrm{a}} \mathrm{RPD}=$ Average relative percent difference between duplicate samples

$s=$ Standard deviation of the RPDs (standard deviation is not calculated for fewer than three samples) 
TABLE 6

Procedure Accuracy for the Sampling Manifold Method

\begin{tabular}{lcc}
\hline Analyte & $\begin{array}{c}\text { Number of Field Reference } \\
\text { Standard Sampies Collected }\end{array}$ & Average \% $\pm s^{\mathrm{a}}$ \\
\hline 1,1-Dichloroethane & 10 & $96 \pm 16$ \\
1,2-Dichloroethane & 10 & $104 \pm 11$ \\
cis-1,2-Dichloroethylene & 10 & $99 \pm 6$ \\
1,1-Dichloroethylene & 10 & $106 \pm 10$ \\
Tetrachloroethane & 10 & $95 \pm 11$ \\
Hydrogen & 9 & $104 \pm 7$ \\
Methane & 5 & $103 \pm 4$ \\
\end{tabular}

$\mathrm{a} \% \mathrm{R}=$ Percent recovery of a surrogate standard

$s=$ Standard deviation 


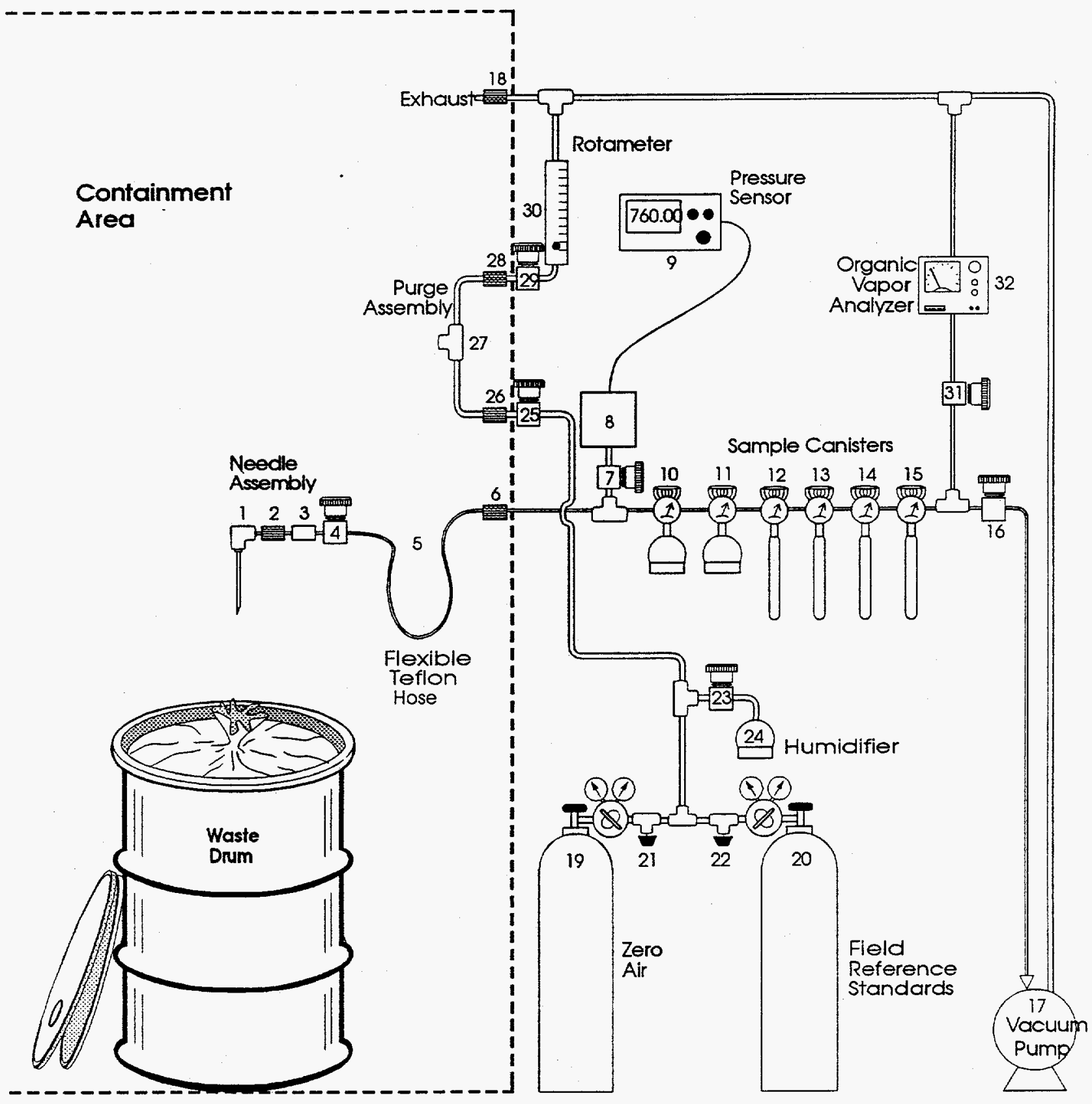

$110-1-1$ 
Component Number"

Component Description
Purpose

\section{Needle Assembly}

1

2

3

1/" SS tubing with sharpened tip

Compact in-line filter $(0.5 \mu \mathrm{m}$ sintered)

Full flow quick-connect (body)

\section{Hose Assembly}

3

4

5

6

7

8

9

$\begin{array}{ll}10-15 & 1 /{ }^{n} \text { stainless steel union tees } \\ 16 & \text { Manually operated bellows valve } \\ 17 & \text { Oil-free (dry) vacuum pump }\end{array}$

\section{8}

\section{Manifold Assembly (Pressurized Line)}

Full flow quick-connect (stem)

Manually operated bellows valve (with flow control)

1/2" PFA Teflon ${ }^{\otimes}$ tubing

Compact in-line filter ( $7 \mu \mathrm{m}$ sintered)

\section{Manifold Assembly (Evacuated Line)}

Manually operated bellows valve

Pressure transducer

Electronics/display unit

Compact in-line filter ( $7 \mu \mathrm{m}$ sintered) [OPTIONAL]

Pressurized cylinder of zero air

Pressurized cylinder of field reference standards (balance nitrogen)

Two-stage SS regulator with needle valve
Pierces drum's internal poly bag

Prevents particulate contamination from entering system

Allows multiple needle connections to be made daily

Allows multiple needle connections to be made daily

Allows controlled flow of sample gases through manifold

Allows movement of needle assembly

Provides additional particulate protection for manifold

Isolates pressure sensor when necessary

Senses pressure from $0-1000$ torr

Controls pressure transducer and displays readings

Provides connection ports for sample canisters

Isolates pump from manifold when necessary

Evacuates the sampling manifold

Provides additional particulate protection for manifold

Provides clean air for cleaning procedures and collection of equipment blanks

Provides known concentrations of known analytes for quality assurance/quality control purposes

Controls delivery pressure of zero air

aSee numbered components of Figure 1

${ }^{b}$ Components not shown in Figure 1

FIGURE 1

\section{Headspace Gas Sampling Manifold Listing of Major Components (Continued)}




\section{Component}

\section{2}

Two-stage SS regulator with needle valve

Manually operated bellows valve

$250 \mathrm{~mL}$ SUMMA ${ }^{2}$ canister containing $100 \mathrm{~mL}$ of water

Manually operated bellows valve (with flow control)

Compact in-line filter (7 $\mu \mathrm{m}$ sintered) [OPTIONAL]

$1 / s^{\prime \prime}$ union tee

Compact in-line filter ( $7 \mu \mathrm{m}$ sintered) [OPTIONAL]

Manually operated bellows valve

High-accuracy rotameter (.2-14 L/min)

Manually operated bellows valve

Organic vapor analyzer

Additional System Components ${ }^{b}$

Blank aluminum panei (19" $\left.\times 21 " \times 12^{\prime \prime}\right)$

Full range Fortin mercury barometer

Certified mercury thermometer

Resistive (heat) tape or wire

System Cleaning Assembly

Port connector ( $1 / \mathrm{s} ")$

Union elbow (1/8")

Full flow quick-connect (body)
Controls delivery pressure of helium

Isolates humidifier from manifold when necessary

Humidifies pressurized gases

Controls flowrate of pressurized gases entering purge assembly

Provides additional particulate protection for manifold

Allows evacuated line to be connected to pressurized side

Provides additional particulate protection for manifold

Allows pressurized line cleaning after field reference standard collection

Allows system operator to monitor excess flow

Isolates OVA from manifold when necessary

Provides real-time analysis for manifold cleanliness

Allows system components to be rack mounted

Provides ambient pressure measurements

Provides ambient temperature measurements

Reduces potential for analytes to adhere to internal surfaces of manifold

Provides tight connection to purge assembly tee (27)

Connects port connector to quick-connect at 90 degrees

Allows connection to evacuated line of manifold

asee numbered components of Figure 1

${ }^{b}$ Components not shown in Figure 1

FIGURE 1

\section{Headspace Gas Sampling Manifold Listing of Major Components (Continued)}




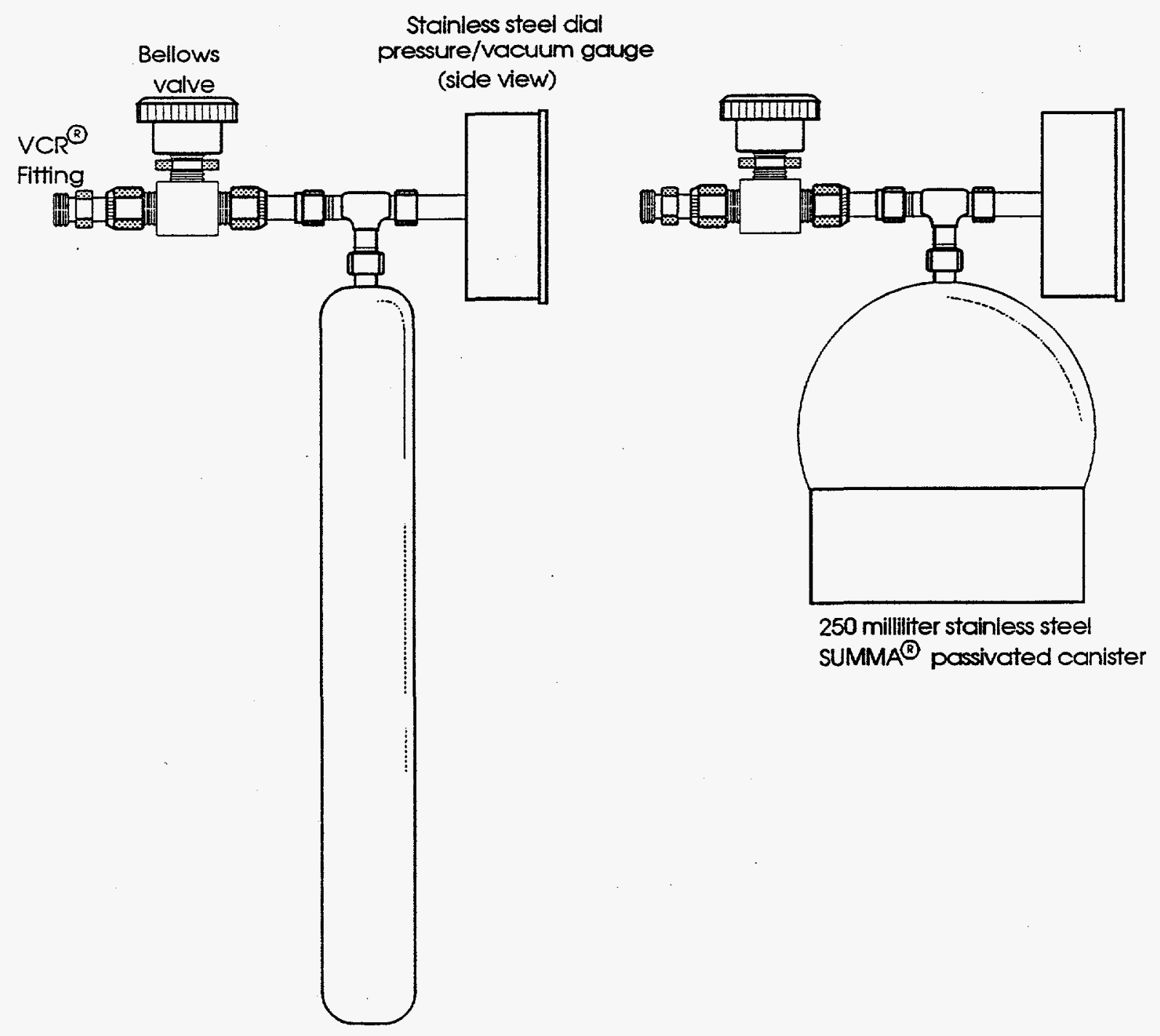

100 milliliter stainiess steel

SUMMA ${ }^{\circledR}$ passivated canister

FIGURE 2

SUMMA ${ }^{\oplus}$ Canister Components Configuration

(Not to Scale) 
PROCEDURE 110.2

\section{DIRECT CANISTER METHOD TO COLLECT \\ HEADSPACE GAS SAMPLES FROM A TRU WASTE DRUM}

\section{Scope and Application}

1.1 This procedure may be used to collect representative headspace gas samples from within a TRU waste drum. This sampling procedure is based on guidelines in "Compendium Method TO-14, The Determination of Volatile Organic Compounds (VOC) in Ambient Air Using SUMMA ${ }^{\circ}$ Passivated Canister Sampling and Gas Chromatographic Analyses" (EPA 1988) and Test Methods for Evaluating Solid Waste, Physical/Chemical Methods, SW-846, Third Edition, Final Update I, and Final Update II (SW-846) (EPA 1995). As such, it is applicable to sampling the headspace gases in drums containing all types of waste. The procedure is used to sample for hydrogen, methane, and specific VOCs that have a vapor phase component at room temperature and pressure. The quality assurance requirements for this procedure are described in the Transuranic Waste Characterization Quality Assurance Program Plan (OAPP) (DOE 1995). This procedure must be implemented with a site-specific standard operating procedure (SOP).

1.2 For the purposes of the TRU Waste Characterization Program, headspace gas samples are to be collected in sampling batches. A sampling batch is defined as a suite of samples collected consecutively using the sampling equipment within a specific time period. A sampling batch can be up to 20 samples (excluding field $\mathrm{OC}$ samples), all of which must be collected within 14 days of the first sample in the batch.

\subsection{Summary of Procedure}

This sampling procedure employs a SUMMA ${ }^{\circledR}$ canister and attachment to collect headspace gas samples for analysis and certified cylinder gases for quality control purposes. Sample canisters are evacuated prior to use and attached to a changeable filter and needle assembly capable of puncturing poly bags inside a waste drum. Field duplicates are collected by attaching two canisters to a tee fitting to which a filter and needle are attached. The needle is punched through the poly bag and the two canister valves are opened simultaneously. Field blanks are samples of room air collected in the immediate vicinity of the waste drum. Equipment blanks and field reference standard samples are collected from a purge assembly connected directly to the regulator of a cylinder of compressed gas or the purge assembly described in Procedure 110.1 of this Methods Manual. These samples are collected from the needle tip through the same components (e.g., needle, filter) the headspace gas samples pass through.

The sample canister, needle assembly and headspace sample volume requirements ensure that a representative sample is collected. When an estimate of the available headspace volume in the drum or poly bags(s) can be made, less than 10 percent of that volume should be withdrawn.

\subsection{Limitations and Interferences}

No limitations or interferences have been identified.

\subsection{Safety}

This procedure may involve the use of hazardous materials, operations, and equipment. It is the responsibility of whoever uses this procedure to consult appropriate site personnel 
concerning health and safety issues and establish appropriate health and safety practices. Consideration should be given to safety concerns regarding chemical and radiation exposure. Training regarding proper storage, usage, and disposal of chemicals is recommended.

The use of the direct canister sampling apparatus requires the transfer of sample canisters into and out of a sampling area which may be contaminated with radionuclides. The following steps are general guidelines for this transfer.

NOTE: Each sampling site must obtain approval from appropriate operations and/or transportation personnel for proper radiological controls.

1. Prepare sample canisters to be sent into a contaminated area by taping all external surfaces, except for the valve handle, with duct tape.

2. Place each sample canister inside a plastic bag and tape to seal.

3. After sample collection, wipe the plastic covering of the sample canister and obtain a radiation level reading.

4. Properly dispose of contaminated plastic and tape.

\subsection{Apparatus and Materials}

5.1 SUMMA ${ }^{\oplus}$ Canister Headspace Gas Sampling Apparatus. Figure 1 illustrates the direct canister sampling equipment and describes the numbered components. Figure 1 shows an $1 / 8$ " stainless steel needle (1) fitted via a reducer (2) to the $1 / 4$ " inlet of a 0.5 micron sintered metal filter (3). This filter is connected to a Swagelok ${ }^{\oplus}$ fitting on the SUMMA $^{\oplus}$ canister (5) valve via a Swagelok ${ }^{\oplus}$ to $V^{\circ} \mathrm{CR}^{\oplus}$ adapter $(4)$. The low internal volume of these components, in comparison to the internal volume of the canister, ensures a representative headspace gas sample is collected.

5.2 Sample Canister. A sample canister is a leak-free welded stainless steel SUMMA pressure vessel with a Cr-NiO-passivated interior surface, bellows valve, and dial pressure/vacuum gauge. The gauge indicates the status of canister pressure and provides evidence of canister leaks. All sample canisters have $\mathrm{VCR}^{\oplus}$ fittings for connection to sampling and analytical equipment.

SUMMA $^{\oplus}$ canisters have been used in the past for VOC sample collection and have a demonstrated sample storage stability for many specific VOCs (EPA 1988; Oliver et al. 1986). Two sizes of canisters $(100 \mathrm{~mL}$ and $250 \mathrm{~mL})$ are used for the collection of headspace gas samples.

5.3 Pressure and Temperature Measurement Apparatus. Canister pressure and ambient pressure are monitored and recorded during sampling operations. Ambient temperature is also monitored and recorded.

5.3.1 Pressure Gauges: A Fortin barometer is used to monitor ambient pressure, while a dial pressure/vacuum gauge is used to monitor canister pressure.

5.3.1.1 Ambient Pressure Sensor: The ambient pressure sensor is a fullrange Fortin barometer, or equivalent, with a range of approximately 500 to $800 \mathrm{~mm} \mathrm{Hg}$. It is kept in the sampling area. Its resolution is $1 \mathrm{~mm} \mathrm{Hg}$ and its calibration is traceable to National Institute of Standards and Technology (NIST) standards. A dual-scale thermometer is used for temperature corrections. 
5.3.1.2 Dial Pressure/Vacuum Gauge: This type of gauge is helium leak tested to $1.5 \times 10^{-7}$ standard $\mathrm{cc} / \mathrm{sec}$, has all stainless steel construction, and is capable of tolerating temperatures to $125^{\circ} \mathrm{C}$. The gauge is able to indicate 5 psig pressure to a vacuum of 30 inches of mercury.

5.3.2 Thermometer: The thermometer is a NIST-traceable laboratory grade mercury thermometer, total immersion type, with nitrogen above the mercury. It has permanent stain markings in the approximate range of $-30^{\circ}$ to $50^{\circ} \mathrm{C}$, with a resolution of $1^{\circ} \mathrm{C}$.

\subsection{Preplanning}

Studies to characterize TRU waste require careful planning to prepare facilities, develop written documentation, secure laboratory support, and evaluate data.

7.0 Reagents, Preservation Measures, and Handling

7.1 All sampling equipment components that come into contact with headspace sampling gases must be constructed of relatively inert materials such as stainless steel or Teflon. A passivated interior surface on the stainless steel components is recommended. Sampling holding times and storage conditions should conform to the requirements specified in Table 1.

7.2 All sample handling and chain-of-custody procedures described in Section 6.0 of the QAPP must be followed.

\subsection{Procedure}

8.1 Cleaning the SUMMA ${ }^{\circ}$ Canister Sampling Apparatus. Procedures for cleaning the sampling apparatus are provided to ensure the sample is representative. This is accomplished by reducing the possibility of contamination from manufacturing impurities and ambient air. Use of these cleaning procedures will also reduce the possibility of cross-contamination between sampling operations. Prior to use in any headspace gas sample collection activities, SUMMA ${ }^{\circledR}$ canisters must be cleaned and certified in accordance with Procedure 210.1 of this Methods Manual. The procedures for cleaning the sampling equipment are as follows:

- Section 8.1.1 is a procedure for cleaning the individual components of the sampling apparatus prior to assembly.

- Section 8.1.2 is a procedure for cleaning the needle and filter between samples.

NOTE: Needles and filters may be disposed of after each sampling operation.

8.1.1 Initial Sampling Apparatus Cleaning and Leak-Check: Prior to assembly, components of the sampling apparatus are inspected and thoroughly cleaned using the following procedure.

NOTE: Parts received free of contamination, as stated or certified by the manufacturer, may be exempted from this cleaning procedure at the discretion of sampling personnel.

1. Place stainless steel parts and fittings in a beaker of reagent grade hexane in an ultrasonic bath for 15 minutes. Repeat this procedure with 
TABLE 1

\section{Holding Conditions and Holding Times}

\begin{tabular}{|c|c|c|c|c|c|c|}
\hline Parameter & Container & $\begin{array}{c}\text { Minimum } \\
\text { Drum } \\
\text { Headspace } \\
\text { Sample } \\
\text { Volume }^{\mathbf{a}}\end{array}$ & $\begin{array}{c}\text { Holding } \\
\text { Temperatures }\end{array}$ & $\begin{array}{c}\text { Field } \\
\text { Holding } \\
\text { Time }^{\mathrm{b}}\end{array}$ & $\begin{array}{l}\text { Shipping } \\
\text { Allowance }\end{array}$ & $\begin{array}{c}\text { Laboratory } \\
\text { Holding } \\
\text { Time }^{c}\end{array}$ \\
\hline $\mathrm{H}_{2}, \mathrm{CH}_{4}$ & $\begin{array}{l}\text { SUMMA } \\
\text { Canister }\end{array}$ & $\begin{array}{c}100 \\
\text { milliliters }\end{array}$ & $0-40^{\circ} \mathrm{C}$ & 4 days & 2 days & 28 days \\
\hline VOCs & $\begin{array}{l}\text { SUMMA® } \\
\text { Canister }\end{array}$ & $\begin{array}{c}250 \\
\text { milliliters }\end{array}$ & $0-40^{\circ} \mathrm{C}$ & 4 days & 2 days & 28 days \\
\hline
\end{tabular}

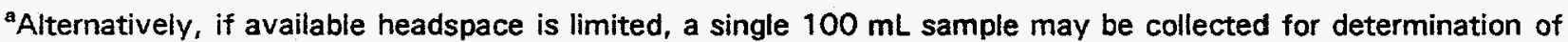
VOCs, $\mathrm{H}_{2}$ and $\mathrm{CH}_{4}$.

${ }^{b}$ From time of headspace sample collection to shipment.

'Programmatic-based maximum holding time. Holding time begins at validated time of sample receipt. 
reagent grade methanol. Rinse the stainless steel parts with HPLC grade water and dry parts in an oven at $100^{\circ} \mathrm{C}$ for at least 12 hours. Stainless steel parts and fittings may also be cleaned by assembling and forcing steam through them.

2. Assemble the sampling apparatus with one end capped and the other end connected to a cylinder of zero air. Leak-check by pressurizing the apparatus to 1700 mbar, placing soap solution on all fittings and connections, and periodically checking for bubble production for a period of 30 minutes. The assembly may also be pressurized with helium and leak-checked with a helium detection device.

3. Tighten fittings and connections that leak until no leaks are observed. From clean spare parts, replace any fittings and connections that continue to leak.

4. Purge the sampling assembly with humid zero air and cap open connections for storage, seal the assembly inside a clean plastic bag.

8.1.2 Needle and Filter Cleaning: This procedure describes the steps for cleaning the needle and filter after headspace gas, field duplicate, field blank, or field reference standards samples have been collected. With slight modifications, this procedure can also be used to batch clean the needle and filters, prior to use. In lieu of cleaning, the needle and filter may be disposed of after headspace gas sample or field reference standard collection. Refer to Figure 1 for location of numbered components of the direct canister sampling apparatus.

1. After sample collection, detach the needle (1) and filter (3) at the VCR fitting (4) and dispose of the VCR ${ }^{\circ}$ gasket.

CAUTION: Needle and filter assembly may be radiologically contaminated.

2. Remove the needle and filter assembly to an area where they can safely be disassembled and cleaned.

3. Unscrew the housing of the filter and dislodge the filter element from its housing. Properly discard the element.

4. Place the reusable parts in a beaker of reagent grade hexane in an ultrasonic bath for 15 minutes. Repeat this procedure with reagent grade methanol. The needle assembly may also be cleaned by screwing the filter housing together again and forcing steam through the entire assembly. Rinse the parts with HPLC grade water and dry in an oven at $100^{\circ} \mathrm{C}$ for at least 12 hours.

5. Insert a new filter element into the filter and tightly screw the body together.

6. Purge the needle and filter with zero air, place a Teflon plug over the needle tip, and seal the assembly inside a clean plastic bag. Then place the bag in a box for storage. 
8.2 Collecting Headspace Gas and Quality Control Samples using the Direct Canister Sampling Apparatus. Headspace gas and quality control samples are collected using the following procedures:

- Section 8.2.1 describes a procedure for collecting headspace gas samples.

- Section 8.2.2 describes a procedure for collecting field duplicates.

- Section 8.2.3 describes a procedure for collecting field blanks.

- Section 8.2.4 describes a procedure for collecting equipment blanks.

- Section 8.2.5 describes a procedure for collecting field reference standards.

NOTE: Prior to sampling, drums must be allowed to equilibrate to the temperature of the sampling area for at least 72 hours.

CAUTION: Appropriate site radiological controls must be established prior to removing the drum lid.

8.2.1 Headspace Gas Sample Collection: Headspace gas samples are collected from all layers of confinement in a waste drum that meet the criteria specified in Section 7.1 of the QAPP. Samples are collected as follows.

1. Select a sample canister and verify its gauge indicates a vacuum of 30 inches of mercury. See Section 8.3 for guidance if the canister does not indicate 30 inches of mercury.

2. After the drum lid and inner liner lid (if present) have been removed, and the poly bag has been exposed, locate an area within the poly bag from which a headspace gas sample can be withdrawn. Care should be taken to avoid placing the tip of the needle in an area where solid material or particulate matter might fill or clog the needle.

3. Remove the cap from the tip of the needle and press the needle through the poly bag and into the headspace gas. Disturbing the bag should be avoided, if possible. If pulling on the bag is necessary, do so in a manner that will minimize the potential for introducing outside air into the poly bag's internal gas space.

4. While holding the needle in the bag's headspace, open the sample canister valve to allow headspace gas to expand into the canister.

5. Observe the vacuum gauge on the sample canister while the sample is being collected. When the gauge indicates zero psig, close the sample canister valve and withdraw the needle from the bag.

6. Log the date, time, sample canister pressure, ambient pressure, and ambient temperature on the sample canister tag.

7. Note the sample type as a headspace gas sample on a QC sample record and place the sample canister in a shipping container.

8.2.2 Field Duplicate Collection: The procedure for field duplicate sample collection is similar to that specified in Section 8.2.1 for headspace gas samples. However, two SUMMA ${ }^{\oplus}$ canisters and one attachment (i.e., needle, adapter, filter) are connected to a tee fitting. After the needle has been pressed through the poly bag and into the headspace gas, the canister valves are opened simultaneously. 
8.2.3 Field Blank Collection: The following procedure is used for the collection of field blanks.

1. Select a sample canister and verify the its gauge indicates a vacuum of 30 inches of mercury. See Section 8.3 for guidance if the canister does not indicate 30 inches of mercury.

2. Place the canister near the drum in the sampling area prior to removing the drum lid. Remove the cap from the tip of the needle and open the sample canister valve.

3. Allow the sample canister to equilibrate to ambient pressure, then close the canister valve.

4. Log the date, time, sample canister pressure, ambient pressure, and ambient temperature on the canister tag.

5. Note the sample type as a field blank on a $\mathrm{QC}$ sample record and place the sample canister in a shipping container.

8.2.4 Sampling Equipment Blank Collection: An equipment blank is collected and analyzed prior to first use of the SUMMA ${ }^{\infty}$ canister sampling apparatus. A field blank may be used in lieu of an equipment blank if the analytes listed in Table 2 are not expected to be present at, or above, the method detection limits (MDLs). A purge assembly, as presented in Figure 2, can be constructed and used for collecting equipment blanks. If the headspace gas sampling manifold is available for use in the sampling area, its purge assembly may be used in lieu of this purge assembly.

The compressed gas cylinder supplies zero air and the humidifier conditions this gas before sample collection. The rotameter indicates gas flow rate and is monitored to assure ambient air is not drawn back through the rotameter and into the sample canister. The tee in the purge line is adapted for leak tight connection to the $1 / 8$ " needle of the direct canister sampling apparatus.

The following procedure assumes that all valves are closed and that the regulator on the zero air cylinder has been purged and set to a delivery pressure of 1700 mbar.

1. Select a sample canister with attached needle and filter to be used for equipment blank collection (lightly tap on the gauge face plate to verify reading). Enter the reading on the sample canister tag.

2. Insert the needle into the purge assembly and tighten the fitting.

3. Adjust the valve on the zero air cylinder until the rotameter indicates a flow rate of approximately 1 liter per minute. The flow of zero air should be swept through a container of American Society for Testing and Materials (ASTM) Type II water to humidify the air prior to the purge assembly. In lieu of installing the a humidifier upstream of the purge assembly tee, the zero air and field reference standard gases may be humidified by adding water directly to the cylinders gases. A cylinder gas concentration of approximately $10,000 \mathrm{ppmv}$ water is recommended. 
TABLE 2

Headspace VOC Target Analyte List and Program Required Quantitation Limits

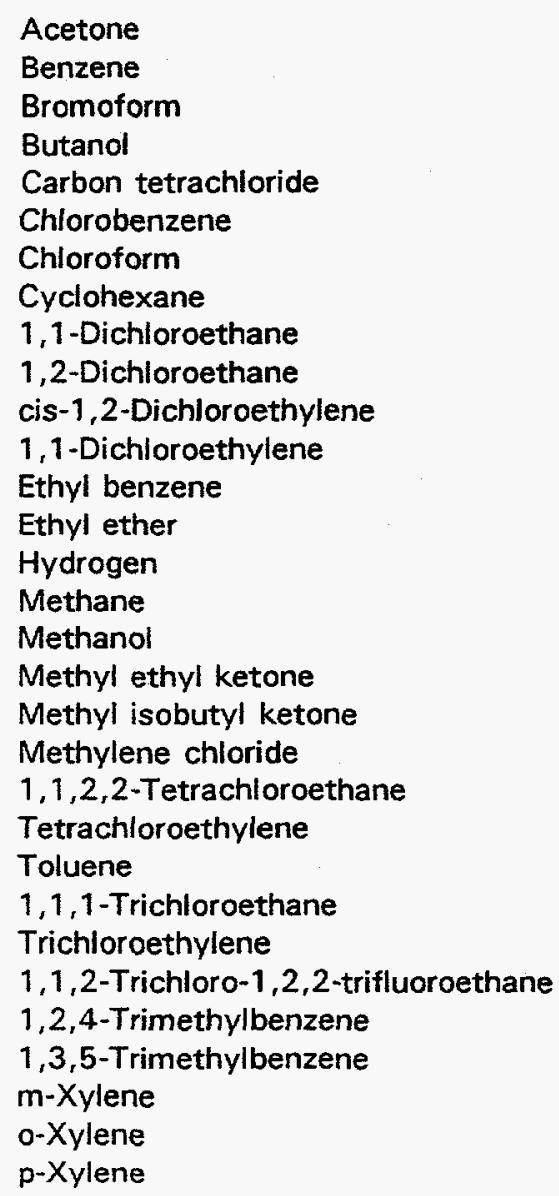

67-64-1

71-43-2

$75-25-2$

$71-36-3$

56-23-5

108-90-7

67-66-3

110-87-7

75-34-3

107-06-2

540-59-0

$75-35-4$

100-41-4

60-29-7

1333-74-0

74-82-8

67-56-1

78-93-3

108-10-1

75-09-2

79-34-5

127-18-4

108-88-3

71-55-6

79-01-6

76-13-1

95-63-6

108-67-8

108-38-3

95-47-6

106-42-3
$150 \mathrm{ng}$

$10 \mathrm{ng}$

$10 \mathrm{ng}$

$150 \mathrm{ng}$

$10 \mathrm{ng}$

$10 \mathrm{ng}$

$10 \mathrm{ng}$

$10 \mathrm{ng}$

$10 \mathrm{ng}$

$10 \mathrm{ng}$

$10 \mathrm{ng}$

$10 \mathrm{ng}$

$10 \mathrm{ng}$

$10 \mathrm{ng}$

0.05 vol $\%$

0.05 vol \%

$150 \mathrm{ng}$

$150 \mathrm{ng}$

$150 \mathrm{ng}$

$10 \mathrm{ng}$

$10 \mathrm{ng}$

$10 \mathrm{ng}$

$10 \mathrm{ng}$

$10 \mathrm{ng}$

$10 \mathrm{ng}$

$10 \mathrm{ng}$

$10 \mathrm{ng}$

$10 \mathrm{ng}$

$10 \mathrm{ng}$

$10 \mathrm{ng}$

$10 \mathrm{ng}$ 
4. Purge the line for at least one minute.

5. Adjust the valve on the zero air cylinder until the rotameter indicates a flow rate of approximately 10 liters per minute.

6. Open the sample canister valve to allow the canister to fill without reducing the flow indicated by the rotameter to zero.

NOTE: If the rotameter indicates zero flow during this process, then the sample is invalidated.

7. Continue drawing zero air from the purge assembly until the sample canister pressure gauge indicates zero psig.

8. Close the sample canister valve.

9. Close the valve on the zero air cylinder.

10. Disconnect the sampling apparatus needle from the purge tee.

11. Log on the sample canister tag the date, time, sample canister pressure, ambient pressure, and ambient temperature.

12. Note the sample type as an equipment blank on a OC sample record and place the sample canister in a shipping container.

8.2.5 Field Reference Standard Collection: A field reference standard is collected and analyzed prior to first use of the sampling apparatus. Field reference standard samples are collected in the same manner as equipment blanks (Section 8.2.4), with the following exceptions.

- The cylinder of zero air is substituted with a cylinder of gas containing field reference standards (know analytes at known concentrations).

- The sample type is noted on a $Q C$ sample record as a field reference standard sample.

8.3 Calculations. The potential exists for sampling or analytical personnel, upon receipt of a sample canister, to find that a canister's dial pressure gauge does not indicate the same pressure as that which was last logged on the canister tag. Changes in the sample canister gauge pressure may be the result of four possible occurrences: (1) a leak in the sample canister or sample canister component (value, gauge, etc.); (2) damage to the sample canister gauge during storage or transportation; (3) an ambient pressure difference between the sampling and analysis location; and (4) an ambient temperature difference between the sampling and analysis location.

The procedures below should be followed to determine the sample canister gauge pressure change expected if ambient pressure and temperature differences exist between the sampling and analysis locations. If the change in sample canister gauge pressure can be accounted for using the procedures below, then the integrity of the sample has been maintained. If, however, the sample canister gauge pressure change cannot be accounted for by considering ambient pressure and temperature changes, the canister must be leak checked and re-certified for use. 
Changes in sample canister gauge pressure that result from changes in temperature and ambient pressure are additive. Care, however, must be taken to ensure that the sign (positive or negative) of the calculated gauge pressure changes is accurately assigned.

8.3.1 Temperature Change at Constant Ambient Pressure: Sample canister gauge pressure changes resulting from a temperature change can be explained by the following equation:

$$
P_{2}=\frac{P_{1} T_{2}}{T_{1}}
$$

where

$$
\begin{aligned}
& P_{1}=\quad \text { the sample canister internal pressure lambient pressure } \\
& P_{2}= \\
& T_{1}= \\
& \text { the ambient temperature at the time of sampling, }\left({ }^{\circ} \mathrm{K}\right) \\
& T_{2}= \\
& \text { the ambient temperature at the time of analysis, }\left({ }^{\circ} \mathrm{K}\right)
\end{aligned}
$$

According to this equation, increasing the temperature of the canister $\left\langle T_{2}>T_{1}\right\rangle$ will increase the internal canister pressure, while decreasing the canister temperature $\left(T_{2}<T_{1}\right)$ will decrease the internal canister pressure.

8.3.2 Ambient Pressure Change at Constant Temperature: A sample canister's dial gauge is expected to indicate a change in pressure if the canister is transported from a high elevation to a lower elevation, or vice versa. The expected change in canister gauge pressure should be the same as the difference in ambient pressure between the high elevation site and the low elevation site, or vice versa.

9.1 Specific sampling steps to ensure that the headspace gas samples are representative include:

- Sample canister cleaning and leak checking

- Use of sample canisters with a passivated internal surface

- Use low internal volume sampling apparatus

- Small sample volume: low sample volume to available headspace gas volume ratio

- Careful pressure regulation in sample canister

- Performance audits 
- Collection of field blanks, field reference standards, equipment blanks, and field duplicates.

9.2 Quality Control for Pressure and Temperature Measurements. This section pertains to the calibration and certification of equipment and instrumentation used for temperature and pressure measurements. All information related to the calibrations of the pressure and temperatures sensors is recorded.

9.2.1 Certification Check of the Ambient Pressure Sensor: The ambient pressure sensor is certified by the manufacturer using standards or equipment traceable to NIST. The pressure indication of the sensor is corrected in accordance with temperature indicated by a dual-scale thermometer attached to its casing, if necessary.

9.2.2 Calibration of Sample Canister Pressure Gauge: The dial pressure gauge of each sample canister is certified using standards or equipment traceable to NIST. Each canister gauge is recertified once per month, or as necessary.

9.2.3 Calibration of the Mercury Thermometer: The mercury thermometer is certified using standards or equipment traceable to NIST.

9.3 Field blanks are used to assess background levels of the analytes listed in Table 2. Field blanks must be collected prior to sample collection, and then at the frequency specified in Table 3. Field OC sample acceptance criteria is specified in Table 4. Corrective action must be implemented if acceptance criteria is not met.

9.4 Equipment blanks are used to assess the cleanliness of the sampling apparatus. Equipment blanks are collected and analyzed for the analytes listed in Table 2 prior to first use of the sampling apparatus. Field QC sample acceptance criteria is specified in Table 4. Corrective action must be implemented if acceptance criteria is not met.

9.5 Field reference standards are used to assess the accuracy of the sampling procedure. Field reference standards are collected prior to first use of the sampling equipment and submitted blind to the analytical laboratory. Field reference standards must contain at least six of the VOCs listed in Table 2 at concentrations in the range of 10 to 100 ppmv. Field reference standards must have a known valid relationship to a nationally recognized standard such as NIST. If commercially purchased gas standards are used, a Certificate of Analysis is required documenting traceability. Commercially purchased stock gases must not be used beyond their manufacturer-specified shelf life. Field OC sample acceptance criteria is specified in Table 4 . Corrective action must be implemented if acceptance criteria is not met. Accuracy, as percent recovery (\%R) shall be determined as follows:

$$
\% R=\frac{C_{m}}{C_{s r m}} * 100
$$

where

$$
\begin{array}{ll}
C_{m} & =\text { measured concentration } \\
C_{s r m} & =\text { true concentration }
\end{array}
$$

9.6 Field duplicates are used to assess the precision of the sampling procedure. Field duplicates are collected simultaneously using a tee fitting and two separate SUMMA ${ }^{\circ}$ canisters. Field duplicates must be collected at the frequency specified in Table 3 and submitted blind to the analytical laboratory. Fieid QC sample acceptance criteria is 
TABLE 3

\section{Summary of Field OC Sample Frequencies}

OC Samples

Frequency

Field blanks ${ }^{a}$

Equipment blanks ${ }^{b}$

Field reference standards ${ }^{\circ}$

Field duplicates
1 per sampling batch ${ }^{d}$

once $^{e}$

once $^{\text {e }}$

1 per sampling batch ${ }^{d}$

aAnalysis of field blanks for VOCs only, is required.

${ }^{b}$ One equipment blank sample must be collected, analyzed for VOCs, and demonstrated clean prior to first use of the headspace gas sampling equipment with each of the sampling heads.

'One field reference standard must be collected, analyzed, and demonstrated to meet the acceptance criteria specified in Table 4 prior to first use.

${ }^{\mathrm{A}} \mathrm{A}$ sampling batch is a suite of samples collected consecutively using the same sampling equipment within a specific time period. A sampling batch can be up to 20 samples (excluding field $\mathrm{OC}$ samples), all of which must be collected within 14 days of the first sample in the batch.

'One equipment blank and field reference standard must be collected after equipment purchase, cleaning, and assembly. 
TABLE 4

Summary of Field QC

Sample Acceptance Criteria

OC Sample

Acceptance

Criteria

Corrective

Action $^{8}$

Field blanks

VOC amounts $<3 \times$ MDLs in Table 2

Equipment blanks

Hydrogen, Methane, and VOC amounts $<3 \times$ MDLs in Table 2

Field reference standards

Field duplicates
$70-130 \% R$

$R P D \leq 25$
Nonconformance if any $\mathrm{VOC}$ amount $>3 \times$ MDLs in Table 2

Nonconformance if any analyte amount $>3 \times$ MDLs in Table 2

Nonconformance if $\% R<70$ or $>130$

Nonconformance if RPD > 25

${ }^{a}$ Corrective action if acceptance criteria is not met, nonconformance procedures are outlined in Section 2.1.2.1 of the QAPP.

MDL = Method detection limit

$\% R=$ Percent recovery

$\mathrm{RPD}=$ Relative percent difference 
specified in Table 4. Corrective action must be implemented if acceptance criteria is not met. Precision, as relative percent difference (RPD) shall be determined as follows:

$$
R P D=\frac{C_{1}-C_{2}}{\frac{\left(C_{1}+C_{2}\right)}{2}} * 100
$$

where

$$
\begin{array}{ll}
C_{1} \text { and } C_{2} & \text { two values obtained by analyzing the duplicate samples } \\
C_{1} & =\text { the larger of the two observed values }
\end{array}
$$

\subsection{Procedure Performance}

As of June 1994, the Radioactive Waste Management Complex (RWMC) at the Idaho National Engineering Laboratory has collected headspace gas samples from directly under the lid of 495 drums of TRU waste in accordance with this procedure. These samples were collected by pressing the needle through the drum's carbon composite filter. In addition, Argonne National Laboratory-West (ANL-W) has collected headspace gas samples from directly under the lid, and from poly bags inside the drum, of 45 drums containing TRU waste in accordance with this procedure. From these sampling activities, sufficient data have been collected to assess the precision and accuracy of the direct canister headspace gas sampling procedure. These data are summarized below.

10.1 Precision. Duplicate samples were obtained at ANL-W in accordance with Section 8.2.2. For each pair of canisters, the RPD for each analyte detected was calculated as in Equation (3).

The RPDs were then averaged. For analytes found in three or more duplicate samples, the standard deviation ( $s$ ) of the RPDs was calculated as follows:

$$
s=\sqrt{\sum_{i=1}^{n} \frac{\left(y_{i}-\bar{y}\right)^{2}}{n-1}}
$$

where

$$
\begin{array}{ll}
\bar{y}= & \text { mean of the replicate measurements } \\
y_{i}= & \text { measured value of the } i^{\text {th }} \text { replicate sample analysis measurement } \\
n= & \text { number of replicate analyses }
\end{array}
$$

The results are summarized in Table 5. All average RPDs were within the procedure's precision limit of $\leq 25$, with the exception of 1,1-dichloroethane, which was found in only one duplicate sample.

10.2 Accuracy. Recovery for the manifold method was determined by collecting samples of field reference standards as specified in Section 8.2.5, with the exception that the needle was pressed through a carbon composite filter normally used for venting drums of TRU waste. Compressed gas cylinders certified to contain known concentrations of known VOCs and gases were used as the field reference standards. The \%R for each analyte was calculated as in Equation (2). The \%Rs were then averaged for each analyte and the $s$ of the \%R was calculated as in Equation (4). 
TABLE 5

Procedure Precision for the Direct Canister Sampling Method ${ }^{(a)}$

Analyte

Number of Duplicate Samples
Average

$R P D \pm s^{(b)}$
1,1-Dichloroethane

Methylene chloride

Toluene

1,1,1-Trichloroethane

Trichloroethylene

Chloroform

Carbon tetrachloride
1

1

6

8

5

1

2
14

5

$15 \pm 13$

$8 \pm 11$

$12 \pm 16$

5

4

aTwo canisters connected by tee and opened simultaneously.

${ }^{b} \mathrm{RPD}=$ Average relative percent difference between duplicate samples.

$s=$ Standard deviation of the RPDs. Standard deviation is not calculated for fewer than three samples. 
The results are presented in Table 6 . All average \%Rs for the analytes were within the procedure's accuracy limit of 70 to 130 .

\subsection{References}

EG\&G Idaho, Inc. (EG\&G). 1992. Engineering Design File Drum Headspace Sampler Design and Test - Quantitate Recovery Efficiency of Design Assembly. Project File No. WIPP-001, EDF Serial No. ECU-001, Functional File No. DHS-392-REE. Idaho Falls, Idaho, Idaho National Engineering Laboratory, U.S. Department of Energy.

Oliver, K.D., J.D. Pleil, and W.A. McClenny. 1986. Atmospheric Environment, Vol. 20 , No. 7, pp. 1403-1411.

DOE. 1995. Transuranic Waste Characterization Quality Assurance Program Plan. CA0-94-1010, Current Revision, Carlsbad, New Mexico, Carlsbad Area Office, U.S. Department of Energy.

EPA. 1988. "Compendium Method TO-14, The Determination of Volatile Organic Compounds (VOC) in Ambient Air Using SUMMA Passivated Canister Sampling and Gas Chromatographic Analyses." Compendium of Methods for the Determination of Toxic Organic Compounds in Ambient Air, Research Triangle Park, North Carolina, Quality Assurance Division, Environmental Monitoring Systems Laboratory, U.S. Environmental Protection Agency.

EPA. 1995. Test Methods for Evaluating Solid Waste, Physical/Chemical Methods, SW-846, Third Edition, Final Update I, and Final Update II, Washington, D.C., Office of Solid Waste and Emergency Response, U.S. Environmental Protection Agency. 
TABLE 6

Procedure Accuracy for the Direct Canister Sampling Method

\begin{tabular}{|c|c|c|}
\hline Analyte & $\begin{array}{l}\text { Number of Field Reference } \\
\text { Standard Samples Collected }\end{array}$ & Average $\% R^{(a)}$ \\
\hline Benzene & 2 & 123.4 \\
\hline Bromoform & 2 & 105.8 \\
\hline Carbon tetrachloride & 2 & 100.0 \\
\hline Chlorobenzene & 2 & 126.9 \\
\hline Chloroform & 2 & 106.1 \\
\hline Cyclohexane & 2 & 112.8 \\
\hline 1,1-Dichloroethane & 2 & 107.0 \\
\hline 1,2-Dichloroethane & 2 & 117.6 \\
\hline cis-1,2-Dichloroethylene & 2 & 110.9 \\
\hline 1,1-Dichloroethylene & 2 & 119.5 \\
\hline Ethyl benzene & 2 & 99.3 \\
\hline Ethyl ether & 2 & 115.1 \\
\hline Methylene chloride & 2 & 127.6 \\
\hline $1,1,2,2$-Tetrachloroethane & 2 & 92.6 \\
\hline Tetrachloroethylene & 2 & 113.6 \\
\hline Toluene & 2 & 108.0 \\
\hline $1,1,1$-Trichloroethane & 2 & 103.1 \\
\hline Trichloroethylene & 2 & 104.8 \\
\hline 1,1,2-Trichloro-1,2,2-trifluoroethane & 2 & 110.2 \\
\hline 1,2,4-Trimethylbenzene & 2 & 98.1 \\
\hline 1,3,5-Trimethylbenzene & 2 & 103.7 \\
\hline $\mathrm{m} / \mathrm{p}$-Xylene & 2 & 119.3 \\
\hline o-Xylene & 2 & 124.8 \\
\hline
\end{tabular}

${ }^{\mathrm{a}} \% \mathrm{R}=$ Average percent recovery of a surrogate standard 


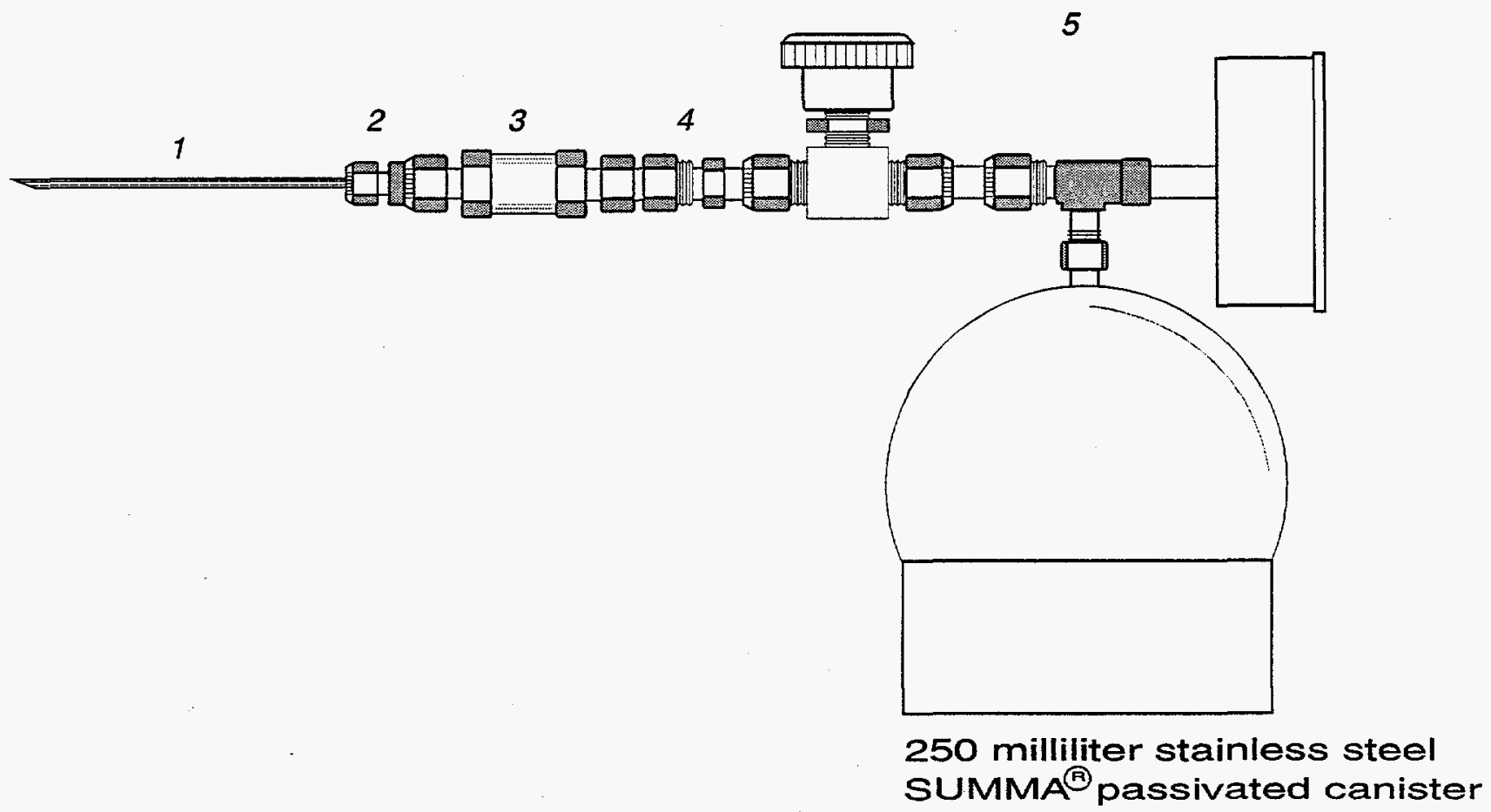

$110-2-1$

\section{FIGURE 1}

Direct Canister Headspace Sampling Apparatus

(See explanation of numbered components on following page) 


\section{Component Component Description Purpose}

$1 \quad 1 / 8^{n}$ SS tubing w/sharpened tip ${ }^{\mathrm{a}}$

$21 / 4 "$ to $1 / 8$ " reducer ${ }^{\mathrm{a}}$

$3 \quad$ Compact in-line filter $10.5 \mu \mathrm{m}$ sintered stainless steel) ${ }^{a}$

4

5

6

7

8

9

10
1/4" Swagelok ${ }^{\otimes}$ to VCR adapter $^{\circledR}$

SUMMA canister with bellows valve and dial vacuum/pressure gauge ${ }^{a}$

Pressurized cylinder of zero air and pressurized cylinder of field reference standard gas, each with a 2-stage stainless steel regulator and needle valve with $1 / 8^{\text {" }}$ port $^{b}$

Humidifier $1250 \mathrm{~mL}$ canister containing $100 \mathrm{~mL}$ ASTM Type II water and is pneumatically connected between cylinder and SS tee) ${ }^{\mathrm{b}}$

$1 / 8^{n}$ SS tee fitting with Teflon ${ }^{\mathrm{m}}$ ferrules ${ }^{\mathrm{C}}$

High-accuracy rotameter $(2-14 \mathrm{~L} / \mathrm{min})^{\text {b }}$

1/8" PFA Teflon ${ }^{\text {Tw }}$ tubing ${ }^{\circ}$
Pierce drum's internal poly bag(s)

Adapt needle to filter

Prevents particulate contamination from entering sampling apparatus

Allows repetitive leak tight connection using disposable metal gasket

Sample container

Zero air provides clean air for equipment cleaning and collection of equipment blanks. Field reference standard gas provides certified gas for collecting field reference standard samples.

Humidifies dry cylinder gas

Provide a purge assembly port for collection of quality control samples

Allows operator to monitor excess flow during collection of quality control samples

Allows purge assembly tee to be connected between cylinder and rotameter

'See numbered components of Figure 1

${ }^{b}$ Components not shown

"Components shown in Figure 2

FIGURE 1

Direct Canister Headspace Sampling Apparatus

List of Major Components

(Continued) 


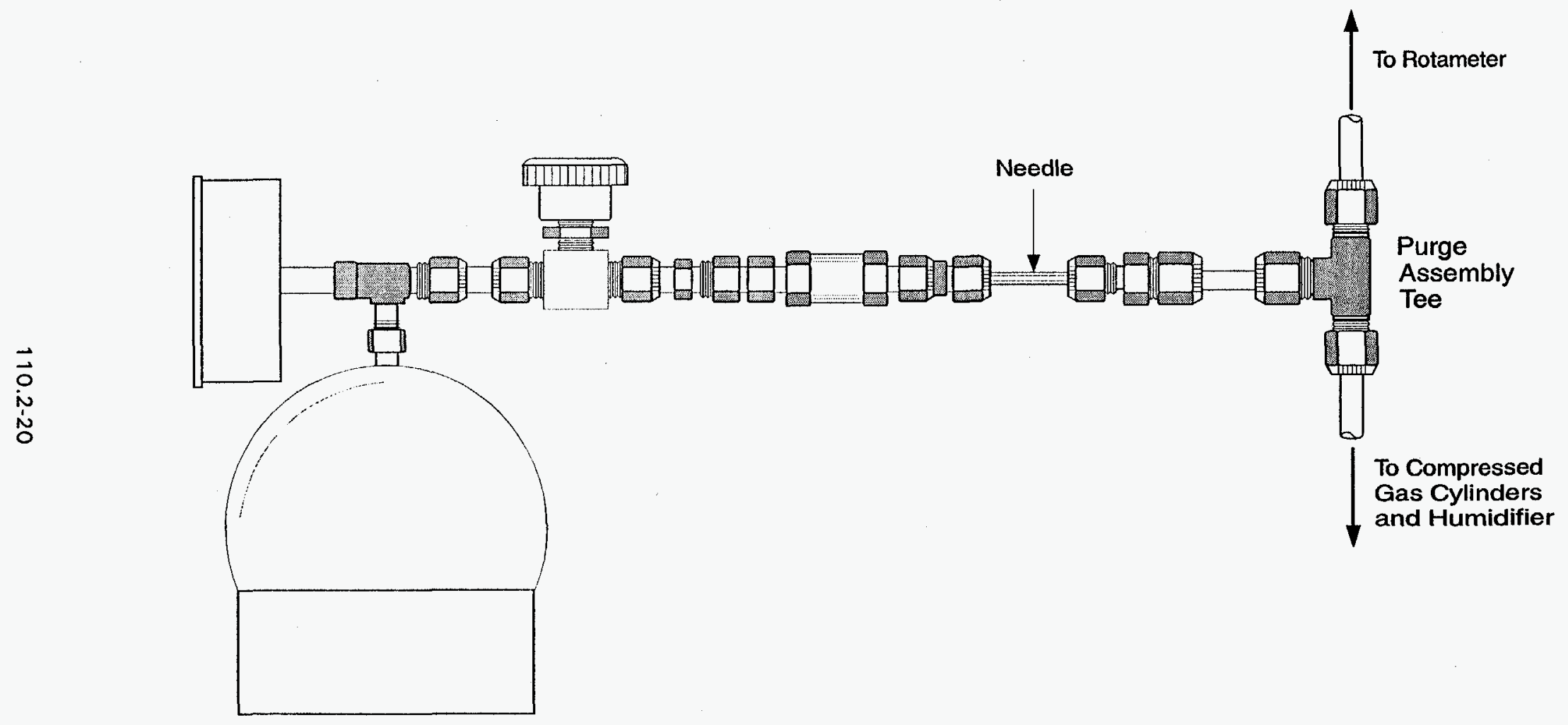


PROCEDURE 110.3

\section{USING A SIDE-PORT NEEDLE TO COLLECT HEADSPACE GAS SAMPLES THROUGH A TRU WASTE DRUM'S CARBON COMPOSITE FILTER}

\subsection{Scope and Application}

1.1 The transuranic (TRU) wastes to be sent to the WIPP are packaged in 208-liter (55-gallon) drums. All drums, prior to shipment, must be vented to atmosphere (for venting of hydrogen) through a carbon composite filter. Drum lids that do not have a carbon composite filter must have one installed before shipment is allowed.

1.2 This procedure describes equipment in use at the Idaho National Engineering Laboratory, but is suitable for use at all DOE TRU waste sites. A side-port needle assembly is used to intrusively punch through the carbon composite filters being used to vent TRU waste drums. The side-port needle assembly is then used to collect a sample of the headspace gas directly under the drum's lid. The side-port needle assembly is used in conjunction with headspace gas sampling Procedures 110.1 and 110.2 of this Methods Manual. Individuals wishing to use this device should become familiar with Procedures 110.1 and 110.2. This procedure must be implemented with a site-specific standard operating procedure (SOP).

\subsection{Summary of Procedure}

The side-port needle assembly described in this procedure is connected to the headspace gas sampling system described in Procedure 110.1 or the direct canister described in Procedure 110.2. The side-port needle is pressed through the drum's carbon composite filter and headspace gas directly underneath the drum's lid is drawn into the headspace gas sampling system through the assembly.

\subsection{Limitations and Interferences}

For the sample to be representative, the quantity of drum headspace gas removed should be no more than 10 percent of the headspace gas available between the inner walls of the drum and the 208-liter (55-gallon) poly bag. In addition, the drum's inner liner should be vented to the drum and all areas of the carbon composite filter that allow drum gases to vent to atmosphere should be sealed prior to sampling.

\subsection{Safety}

This procedure may involve the use of hazardous materials, operations, and equipment. It is the responsibility of whoever uses this procedure to consult appropriate site personnel concerning health and safety issues and establish appropriate health and safety practices. Consideration should be given to safety concerns regarding chemical and radiation exposure. Training regarding proper storage, usage, and disposal of chemicals is recommended.

\subsection{Apparatus}

5.1 Figure 1 illustrates the configuration of the carbon composite filter being used at the Idaho National Engineering Laboratory near Idaho Falls, Idaho (EG\&G 1988). The filter contains four vents in the stainless steel cap that is welded to the stainless steel body. The body houses the carbon composite filter which is designed to exhibit a filtration efficiency greater than or equal to 99.90 percent filtration of 0.3 micron, or larger, particles at a flow rate of 5 to 10 liters per minute. The filter is also waterproofed with 
silicon oil. A nitrile rubber grommet, molded to a steel washer, seals the filter housing against the drum lid to prevent leaks and the base of the filter housing is threaded to permit the filter assembly to be screwed into the drum lid.

5.2 The needle assembly required for drum headspace gas sampling is different from the assembly required for sampling a 208-liter (55-gailon) poly bag (Procedure 110.1 ) in that the needle is shorter in length, smaller in outer diameter, and requires an adapter to properly attach to the $3.18 \mathrm{~mm}(0.125 \mathrm{inch})$ union elbow. Figure 2 illustrates how the needle and adapter are connected and the approximate angle at which the needle is pressed through the carbon composite filter.

5.3 The side-port needle is a 21 gauge $5 \mathrm{~mm}$ (2-inch) long tube with an outer diameter approximately $0.8 \mathrm{~mm}(0.032$ inches) and inner diameter approximately $0.5 \mathrm{~mm}$ (0.020 inches) sealed on its leading end with a side hole located at a right angle to its long axis. The small outer diameter of this needle, in relation to the needles used for 208-liter (55-gallon) poly bag and inner layers of confinement sampling, allow it to be pressed all the way through the housing of the composite filter into the headspace gas under the drum lid.

5.4 At the other end on the needle is a metal hub, which connects to a Teflon luer adapter. The adapter connects to the elbow union of the needle assembly used for sampling 208-liter (55-gallon) poly bags and inner layers of confinement.

5.5 Also illustrated in Figure 2 is an elastomer film wrapped around the body of the filter and an $\mathrm{O}$-ring to hold the film in place. The O-ring has an inner diameter of approximately $25.4 \mathrm{~mm}$ ( 1 inch) and is made of rubber. The film seals the vents in and around the filter cap and minimizes intrusion of room air into the drum headspace during sample collection.

5.6 Another component required for drum headspace gas sampling, not shown in Figure 2, is a teflon septum assembly that connects to the headspace sampling system's purge assembly (component 27 in Figure 1 of Procedure 110.1). This septum assembly allow the needle to be inserted into the purge assembly for system cleaning and collection of sampling manifold blanks and field reference standard samples. Septums are discarded between samples of drum headspace gas.

\subsection{Preplanning}

Studies to characterize TRU waste require careful planning to prepare facilities, develop written documentation, secure laboratory support, and evaluate data.

\subsection{Reagents, Preservation Measures, and Handling}

All sampling equipment components that come into contact with the headspace sample gases must be constructed of relatively inert materials such as stainless steel or Teflon". Stainless steel components with passivated interior surfaces is recommended. Sample holding times and storage conditions should conform to the requirements specified in Table 2 of Procedure 110.1 .

\subsection{Procedure}

8.1 Collection of drum headspace gas through the carbon composite filter is conducted using the headspace sampling system described in Procedure 110.1 or by direct canister as described in Procedure 110.2. 
8.2 Individuals using the side-port needle assembly should follow the procedure described below (Note: See Figure 1 in Procedure 110.1 for assistance in locating the numbered components of the headspace sampling system).

1. Verify the drum has equilibrated to the temperature of the sampling area for at least 72 hours.

2. Verify the sampling manifold has been properly cleaned and leak-checked as described in Sections 8.1.1 and 8.1.2 of Procedure 110.1. Also verify that this cleaning was conducted with the side-port needle inserted through the septum mounted on the purge assembly.

3. Move the drum to a location that will allow the needle to access the drum's carbon composite filter.

4. Fold twice in half a 6-inch square sheet of the elastomer film and place it on top of the carbon composite filter. Then place the O-ring on top of the film, centering the O-ring on the filter cap.

5. Firmly press down on the O-ring to stretch it over the edges of the filter housing and roll it down to the housing base. Inspect the film to make sure it covers the cap of the composite filter entirely.

6. Remove the needle assembly (1) from the purge assembly (27) and place the leading end of the needle in one of the vents in the cap of the carbon composite filter.

7. Move the tip of the needle to the inner most edge of the vent, angle the needle approximately $22^{\circ}$, and slowly press the needle through the carbon composite filter. Note: The angle of the needle may have to be readjusted as it is pressed through the filter. It may also slightly wedge itself against the inner wall of the threaded portion of the filter housing. With modest pressure, the needle should press past the inside bottom edge of the filter housing and into the headspace gas of the drum.

8. Continue pressing down on the needle until the metal hub of the needle presses against the plastic film, or until the tip of the needle presses against the rigid liner inside the drum. Note: If the needle presses against the rigid liner inside the drum, pull the metal hub of the needle up and away from the filter cap approximately $6 \mathrm{~mm}(1 / 4 \mathrm{inch})$.

9. Hold the needle in the filter assembly and open valve (4). Hold the needle steady until the pressure sensor (9) indicates ambient pressure, or ceases to show an increase in pressure. Verify that the canister gauge(s), if present, indicate 0 psig.

10. Close the valves of the canisters that were used for sample collection.

11. Remove the needle assembly (1) from the carbon composite filter and disconnect the needle assembly from valve (4). Place the needle assembly in a collection box for items to be cleaned, or dispose of it.

12. Follow Steps 7 through 10 of Section 8.2.1 in Procedure 110.1. 


\subsection{Quality Control}

Quality control in the use of this needle assembly is maintained by collecting the specified number of field OC samples (i.e., field blanks, field duplicates, field reference standards, equipment blanks) as specified in Section 9.0 of Procedure 110.1 or 110.2 of this Methods Manual. Quality control is also maintained by cleaning the apparatus in accordance with the cleaning procedures specified in Section 8.0 of Procedure 110.1, with the following exceptions:

1. The needle is pressed through a septum attached to the purge assembly tee rather than tightened to the purge assembly with Teflon $^{\circledR}$ ferrules.

2. Sample canister may require additional time to fill due to the gas flow restriction caused by the small inner diameter of the side-port needle.

3. The organic vapor analyzer (OVA) may or may not operate properly as a result of the flow restriction. This must be determined on a case-by-case basis depending on the type of OVA in use.

\subsection{Procedure Performance}

See Section 10.0 in Procedures 110.1 and 110.2.

\subsection{References}

EG\&G. 1988. "RWMC/SWEPP Drum Venting System Carbon Filter Assembly", Mod. 1, Drawing No. 42240, Rev. B. EG\&G Idaho, Inc., August 26, 1988. 


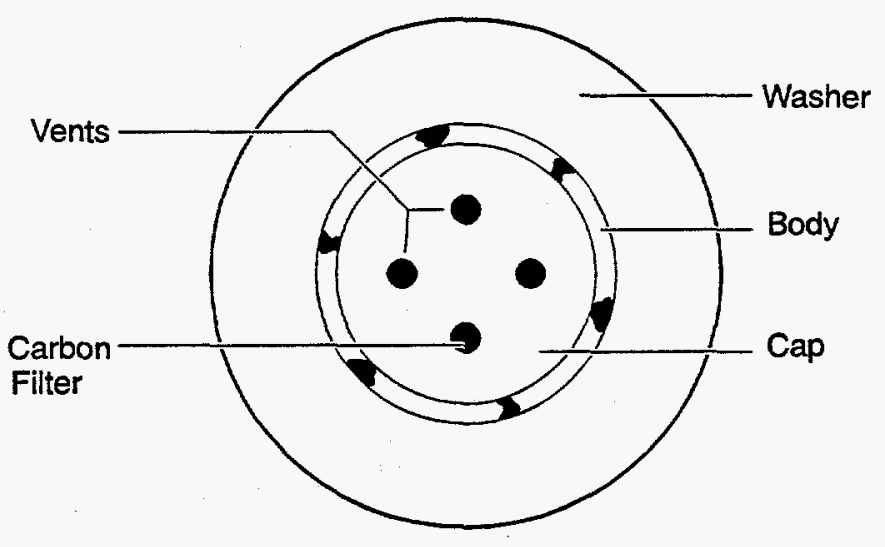

Top View

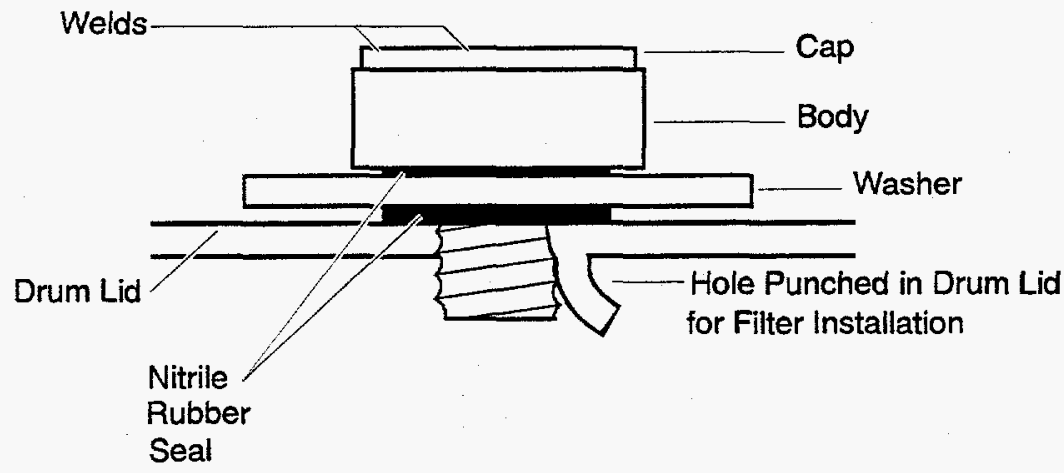

Cross-Section 


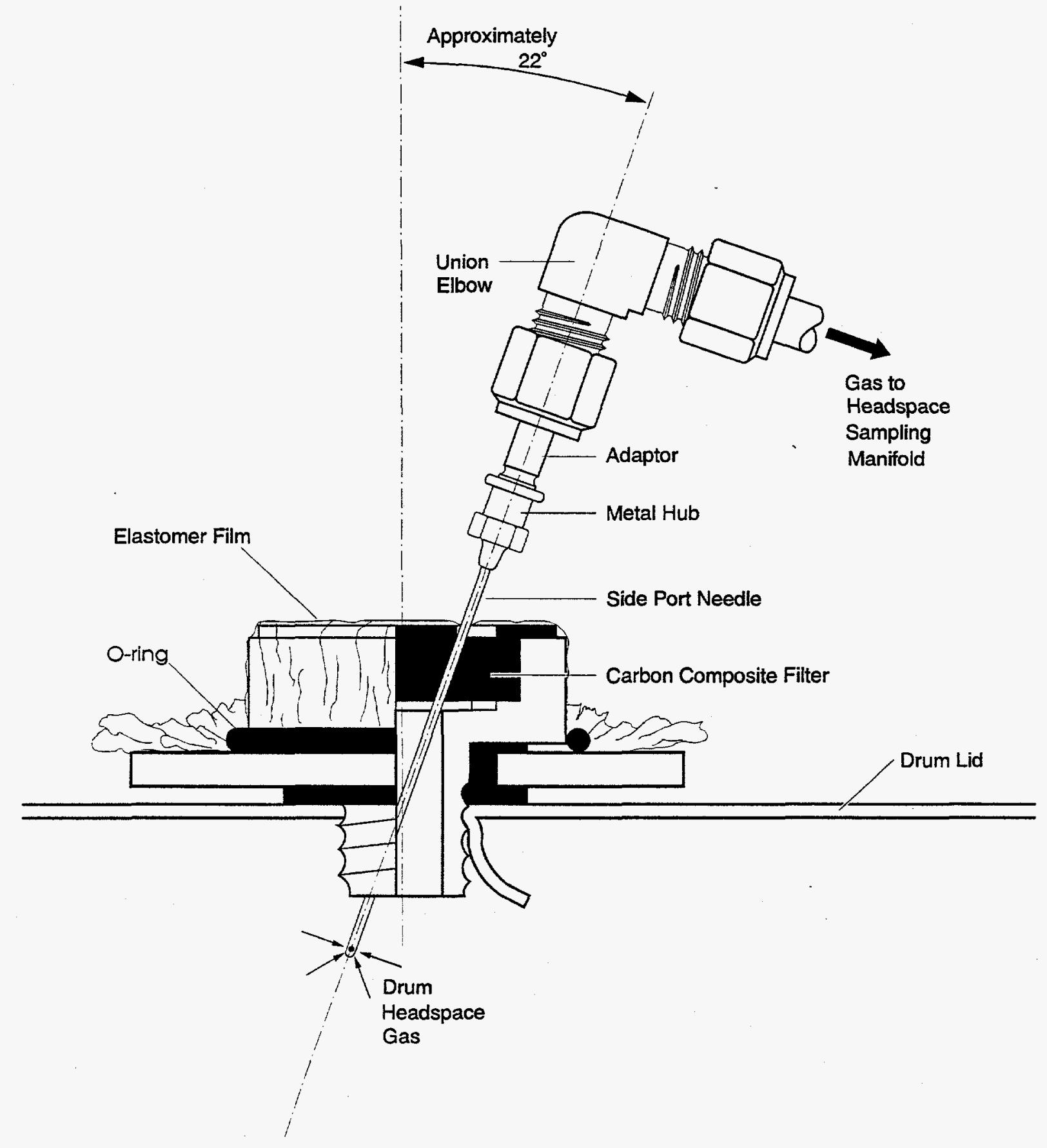


PROCEDURE 110.4

\section{PUNCHING THE DRUM LID TO COLLECT HEADSPACE GAS SAMPLES FROM A TRU WASTE DRUM}

\subsection{Scope and Application}

The transuranic (TRU) wastes to be sent to the WIPP are packaged in 208-liter (55-gallon) drums. Prior to shipment, all drums must be vented to atmosphere (for venting of hydrogen) through a carbon composite filter (see Procedure 110.3 of this Methods Manual). Drums with lids that do not have a carbon composite filter that permits insertion of a needle into the headspace gas directly under the drum lid may be sampled by puncturing the drum lid. This procedure must be implemented with a site-specific standard operating procedure (SOP).

\subsection{Summary of Procedure}

This sampling procedure may be used to collect representative headspace gas samples from within a 208-liter (55-gallon) TRU waste drum by pressing a sparkless punch through the drum lid into the headspace gas of the drum. Once the drum lid is breached, the headspace gas directly under the drum lid is withdrawn through a grooved plate pressed tight against the drum lid to a manifold type headspace gas sampling system or directly to a SUMMA'-passivated canister (see Procedures 110.1 and 110.2 respectively, of this Methods Manual). The headspace gas sampling systems require no modifications to permit use of this procedure.

\subsection{Limitations and Interferences}

3.1 For the sample to be representative, the quantity of drum headspace gas removed should be no more than 10 percent of the headspace gas available between the inner walls of the drum and the 208-liter (55-gallon) poly bag. Also, the drum's inner liner should be vented to the drum and the carbon composite filter that allows drum gases to vent to atmosphere should be sealed prior to sampling.

3.2 The drum should be allowed to equilibrate to the temperature of the sampling area for at least 72 hours prior to sampling. The center of the drum lid should be within reach of the needle assembly of the headspace sampling system (see Procedure 110.1) and the lid of the drum to be sampled should be relatively free of dents, scratches, and materials that may cause leaks to occur during sampling.

\subsection{Safety}

This procedure may involve the use of hazardous materials, operations, and equipment. It is the responsibility of whoever uses this procedure to consult appropriate site personnel concerning health and safety issues and establish appropriate health and safety practices. Consideration should be given to safety concerns regarding chemical and radiation exposure. Training regarding proper storage, usage, and disposal of chemicals is recommended.

\subsection{Apparatus}

5.1 The drum punch (Figures 1 and 2) consists of seven major components including a channel beam, two clamps, a press assembly, a punch assembly, a sampling plate, a flow indicator, and a filter cup assembly (Coughlan, 1990). The numbers in parentheses below correspond with the numbered components in Table 1 and the numbered components in the figures presented in this procedure. 
TABLE 1

List of Drum Punch Sampling System Components

\section{Component No. Component Description}

44

45

46

47

48

49

50

51

52

53

54

55

56
$610 \mathrm{~mm}$ (24 inches) channel beam

Clamp

Handle

$152.4 \mathrm{~mm}$ (6 inches) hand wheel

$19 \mathrm{~mm}(3 / 4 \mathrm{inch})$ threaded shaft

$12.7 \mathrm{~mm}(1 / 2$ inch) bolt punch driver

$12.7 \mathrm{~mm}(1 / 2$ inch) thrust bolt

$9.5 \mathrm{~mm}$ (3/8 inch) sparkless punch

$152.4 \mathrm{~mm}$ (6 inches) diameter stainless steel sampling plate, $19 \mathrm{~mm}(3 / 4$ inch) thick, machined to permit attachment of components $48,53,54,55$ 56,58 , and 60

Ultra-Torr fitting ${ }^{\circledR}$

$3.2 \mathrm{~mm}(1 / \mathrm{s}$ inch) crosssectional diameter Viton ${ }^{\circledR} \mathrm{O}$ ring, 50 durometer, $139.7 \mathrm{~mm}$ $\left(5 \frac{1}{2}\right.$ inches) inner diameter

\section{$3.2 \mathrm{~mm}(1 / \mathrm{s}$ inch) cross-} sectional diameter Viton ${ }^{\circledR} \mathrm{O}$ ring, 50 durometer, $104.8 \mathrm{~mm}$ (41/8 inches) inner diameter

Bellows valve with $3.2 \mathrm{~mm}(1 / 8$ inch) NPT fittings

$3.2 \mathrm{~mm}(1 / 8 \mathrm{inch})$ female elbow

Bellows valve with $3.2 \mathrm{~mm} \mathrm{(1/8}$ inch) NPT fittings
Purpose

Support beam for pressing sampling plate and filter cup against drum lid.

Holds channel beam to drum.

Allows turning of press assembly.

Allows turning of press assembly.

Presses down on sampling plate as press assembly hand wheel is turned.

Presses down on sparkless punch as it is turned with $12.7 \mathrm{~mm}$ ( $1 \frac{1}{2}$ inch) ratchet.

Prevents sparkless punch from turning as $12.7 \mathrm{~mm} \mathrm{(1/2} \mathrm{inch)} \mathrm{bolt} \mathrm{is} \mathrm{turned.}$

Pierces drum lid under sampling plate.

Seals against drum lid to permit collection of sample gases.

Allows vertical movement of sparkless punch while maintaining seal.

Seals groove from outside air.

Seals groove from dead space under sampling plate.

Isolates flow to sampling plate groove.

Channels zero air and field reference standard gases from purge assembly tee (27) to sampling plate groove.

Isolates flow from sampling plate groove. 
TABLE 1

List of Drum Punch Sampling System Components

(Continued)

\section{Component No. Component Description Purpose}

$3.2 \mathrm{~mm}(1 / 8 \mathrm{inch})$ female elbow

60

61

62

63

64

65

66

67

68

69

70

71
$3.2 \mathrm{~mm}(1 / 8$ inch) male elbow with Teflon ${ }^{\oplus}$ ferrules

Hand knob

$12.7 \mathrm{~mm}(1 / 2$ inch) threaded shaft

Sliding nut

$12.7 \mathrm{~mm}(1 / 2$ inch) swivel pad

$63.5 \mathrm{~mm}\left(2 \frac{1}{2}\right.$ inches $)$ diameter inverted cup with 0 -ring at base

$1.6 \mathrm{~mm}\left(1 / 16\right.$ inch) Viton ${ }^{\oplus} 0$ ring

Check valve with $3.2 \mathrm{~mm} \mathrm{(1/8}$ inch) NPT fittings

$3.2 \mathrm{~mm}\left(1 / \mathrm{s}\right.$ inch) Teflon ${ }^{\oplus}$ tubing

$3.2 \mathrm{~mm}(1 / \mathrm{s}$ inch) Teflon tubing

High-accuracy rotameter 10.2 to $14.0 \mathrm{~L} / \mathrm{min}$ )

Curved $152.4 \mathrm{~mm}$ (6 inches) metal plate
Channels zero air and field reference standard gases from sampling plate groove to rotameter.

Provides connection port for needle assembly $(1-3)$ of headspace sampling system.

Permits turning of filter cup assembly.

Presses down on filter cup as hand knob is turned.

Permits $127 \mathrm{~mm}$ (5 inches) lateral movement of filter cup assembly.

Permits angular and prevents rotational movement of filter cup.

Seals carbon composite filter from outside air.

Seals carbon composite filter from outside air.

Relieves pressure in drum as sampling plate is being pressed against drum lid and prevents outside air from entering drum during sampling.

Connects sampling plate (52) groove to purge assembly tee (27).

Connects sampling plate (52) groove to rotameter (70).

Allows system operator to monitor excess flow of zero air and field reference standard gases.

Provides additional bracing against side of drum. 
5.2 The channel beam (44), made of either $6.4 \mathrm{~mm}(1 / 4-$ inch) steel, stainless steel, or aluminum, is approximately $610 \mathrm{~mm}$ ( 24 inches) long, $102 \mathrm{~mm}$ (4 inches) wide, and 51 $\mathrm{mm}$ ( 2 inches) high. Its shape is that of an inverted " $U$ " and its weight is dependent on the type of metal used for construction. Braces at each end permit the bottom edge of the channel beam to be secured approximately $89 \mathrm{~mm}\left(3 \frac{1}{2}\right.$ inches) above the drum lid and provide support for the clamps. Welded underneath the center of the channel beam is a $38 \mathrm{~mm}\left(1 \frac{1 / 2}{2}\right.$-inch) by $127 \mathrm{~mm}$ (5-inch) by $19 \mathrm{~mm}$ (3/4-inch) thick block through which is tapped a $19 \mathrm{~mm}(3 / 4$ inch) threaded hole for the threaded shaft of the press assembly. Adjacent and to the left of this hole, also tapped through the block, is a threaded $12.7 \mathrm{~mm}$ $(1 / 2$-inch) hole for the punch driver (49). A $127 \mathrm{~mm}$ (5-inch) long, $25.4 \mathrm{~mm}$ (1-inch) wide, slot to the right of the threaded shaft permits lateral movement of the filter cup assembly for placement directly over the drum's carbon composite filter.

5.3 Clamps (45) attached to each end of the channel beam (44) are placed such that a 152 $\mathrm{mm}$ (6-inch) long, 25.4 (1-inch) wide, $6.4 \mathrm{~mm}$ (1/4-inch) thick plate (71) attached to the spindle is pressed against each side of the outside drum wall just below the upper lip of the drum. The curvature of the plate (71) corresponds with the curvature of the drum. The clamps have approximately $227 \mathrm{Kg}(500 \mathrm{lbs})$ holding capacity each. This configuration allows the channel beam (44) to be firmly clamped to the drum and permits minimal vertical slippage as pressure is placed down on the drum lid.

5.4 The press assembly consists of a $19 \mathrm{~mm}(3 / 4-$ inch) diameter threaded shaft (48) approximately $178 \mathrm{~mm}$ (7 inches) long with a $152.4 \mathrm{~mm}$ (6-inch) diameter hand wheel (47) with handle (46) attached at the top and a lubricated snap ring at its base. The cross-sectional configuration of the press assembly is shown in Figure 3 . The snap ring prevents circular movement of the sampling plate $(52)$ as it is pressed against the drum lid.

5.5 The punch assembly consists of a punch driver (49), threaded through the channel beam (44), connected to a sparkless punch $(51)$ by a thrust bearing (50). The punch driver is a threaded $12.7 \mathrm{~mm}(1 / 2$-inch) bolt that is manually turned with a $12.7 \mathrm{~mm}(1 / 2$-inch) rachet (not shown). The thrust bearing (50) prevents the punch from turning as the punch driver $(49)$ is turned, thereby, further minimizing the potential to generate a spark. The sparkless punch $(51)$ is $9.5 \mathrm{~mm}(3 / 8-i n c h)$ in diameter and approximately $95 \mathrm{~mm}(33 / 4$ inches) long. Its general composition is approximately 5 percent nickel, 10 percent aluminum, and 85 percent bronze. The remaining 5 percent is mostly iron and zinc. The tip is sharpened to a conical shape at an approximate 30 degree angle to its long axis.

5.6 The sampling plate (52) is a $19 \mathrm{~mm}(3 / 4$-inch) stainless steel plate, machined to a circular shape approximately $152 \mathrm{~mm}$ (6 inches) in diameter (Figure 4). A $38 \mathrm{~mm}\left(1 \frac{1}{2}\right.$-inch) diameter fitting in the center top attaches the plate to the threaded shaft of the press assembly. Also attached to the top of the plate is an Ultra-Torr ${ }^{\circ}$ fitting (53), a male elbow (60), and two bellows valves (56-58) with female elbows attached to each.

5.6.1 The O-ring configuration of the Ultra-Torr fitting (53) permits the sparkless punch (51) to move vertically while maintaining a seal from outside air.

5.6.2 The male elbow (60) provides as a connection port for the drum headspace sampling system's (Procedure 110.1 ) needle assembly. The compression fitting of the male elbow (60) has pliable Teflon ${ }^{\circ}$ ferrules that permit the needle to be repeatedly inserted, "finger-tightened" into place, and again removed.

5.6.3 The bellows valve on the right (56), shown in Figure 4 is, connected with a length of $3.2 \mathrm{~mm}$ ( $1 / 8$-inch) Teflon ${ }^{-}$tubing to the headspace gas sampling system's purge 
assembly tee (27) described in Procedure 110.1. The bellows valve on the left $(58)$ is exhausted to the rotameter $(70)$ mounted on the channel beam (44).

5.6.4 The bottom side of the sampling plate (Figure 5) contains two $3.2 \mathrm{~mm}(1 / \mathrm{s}$-inch) cross-sectional diameter Viton ${ }^{\otimes}$ O-rings (54-55), 50 durometer hardness (Parker, 1991), separated by a circular sampling groove (cross-hatching) approximately 9.5 $\mathrm{mm}(3 / 8$ inch) wide and $1.6 \mathrm{~mm}$ (1/16-inch) deep.

5.6.5 The O. D. of the outer O-ring is approximately $146 \mathrm{~mm}\left(5 \frac{3}{4}\right.$ inches) and its I. D. is approximately $138 \mathrm{~mm}\left(5 \frac{1}{2}\right.$ inches $)$. It is held in place in a groove approximately $2.7 \mathrm{~mm}(0.107$ inches) deep, $4.1 \mathrm{~mm}(0.163$ inches) wide, and with an I. D. of $140.5 \mathrm{~mm}$ (5.531 inches).

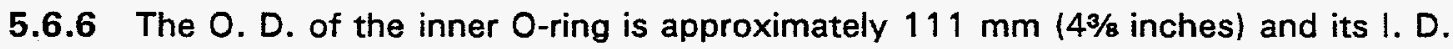
is approximately $104.7 \mathrm{~mm}\left(4 \frac{1}{8}\right.$ inches). It is held in place in a groove $2.7 \mathrm{~mm}$ (0.107 inches) deep, $4.1 \mathrm{~mm}(0.163$ inches) wide, with an I. D. of $105.5 \mathrm{~mm}$ (4.156 inches).

5.6.7 The inner diameters of the O-rings are slightly smaller than the inner diameters of the grooves in which they fit and must be slightly stretched into the groove. This gives the $\mathrm{O}$-rings a surface against which to stretch and adhere to, thus preventing them from falling out.

5.6.8 The sampling groove contains four ports that correspond respectively with the Ultra-Torr fitting (53), male elbow (60), and bellows valves $(56$ and 58).

5.7 The flow indicator (70), shown in Figure 1 , is a 0.2 to $14,000 \mathrm{ml} / \mathrm{min}$ rotameter identical to the type of flow indicator $(30)$ used with the headspace gas sampling system (Procedure 110.1). The rotameter, mounted in a vertical position on top of the channel beam (44) and connected to the outlet female elbow (59) of the sampling plate (52), is used to verify excess flow when purging the groove with zero air or field reference standard gases.

5.8 The filter cup assembly (Figure 6) consists of an inverted cup (65) with a check valve (67). The cup is connected to the hard knob (61) by a $12.7 \mathrm{~mm}(1 / 2$-inch) $O$. D. shaft (62) threaded through an adjustable block assemblage (63) that is tightened to the channel beam (44). A $12.7 \mathrm{~mm}(1 / 2$-inch) swivel pad between the cup and the shaft prevents the cup from rotating as the shaft rotates and permits the cup to seal tightly on top of sloped surfaces, such as may be the case when the sampling plate (52) is pressed against the drum lid.

5.8.1 The inverted cup (65) is approximately $63.5 \mathrm{~mm}\left(2 \frac{1}{2}\right.$ inches) $O$. D. and $25.4(1$ inch) in height. Its wall thickness is approximately $3.2 \mathrm{~mm}(1 / 8 \mathrm{inch})$. The cup has a groove at its base $1.4 \mathrm{~mm}(0.054$ inch) deep, a width of $2.2 \mathrm{~mm}(0.088$ inch), and an inner diameter of $51.6 \mathrm{~mm}\left(2.031\right.$ inches). A Viton ${ }^{\oplus} \mathrm{O}$-ring fits into this groove.

5.8.2 The 50 durometer hardness Viton ${ }^{\oplus}$ O-ring $(1.59 \mathrm{~mm}$ cross-sectional diameter) has

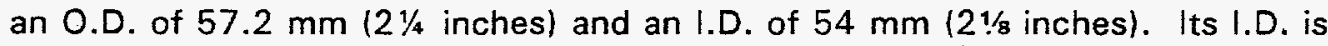
slightly smaller than the inner diameter of the groove in which it fits and must be slightly stretched into the groove. This gives the O-ring a surface against which to stretch and adhere to, thus preventing it from falling out.

5.8.3 A check valve (67) is mounted in one side of the inverted cup (65) to prevent pressure from building up in the drum as the sampling plate (52) is pressed down 
on the drum lid. The check valve (67) prevents outside air from entering the drum during headspace gas sampling.

5.8.4 The adjustable block assemblage (63) allows the filter cup assembly to be moved as needed for placement directly over drum lid carbon composite filters.

\subsection{Preplanning}

Studies to characterize TRU waste require careful planning to prepare facilities, develop written documentation, secure laboratory support, and evaluate data.

\subsection{Reagents, Preservation Measures, and Handling}

All sampling equipment components that come into contact with the headspace sample gases must be constructed of relatively inert materials such as stainless steel or Teflon". Stainless steel components with passivated interior surfaces is recommended. Sample holding times and storage conditions should conform to the requirements specified in Table 2 of Procedure 110.1.

\subsection{Procedure}

8.1 Collection of drum headspace gas with the drum punch is conducted using the headspace sampling system described in Procedure $\mathbf{1 1 0 . 1}$ or by direct canister as described in Procedure 110.2.

8.2 Individuals using the drum punch should follow the procedure described below. Note: See Figure 1 in Procedure 110.1 to locate components with numbers 32 and lower. This procedure assumes bellows valves $(56)$ and $(58)$ are closed.

1. Verify the drum has equilibrated to the temperature of the sampling area for at least 72 hours.

2. Verify the center of the drum is within reach of the needle assembly.

3. Verify the sampling manifold has been properly cleaned and leak-checked as described in Section 8.1 of Procedure 110.1 with consideration of the exceptions described below in Section 9.0.

4. Inspect the drum lid for dents scratches and dirt in the areas where the sampling plate and filter cup will be placed. Special attention should be given to leak checking the sampling plate and filter cup when dents, scratches, or other conditions exist that may compromise the seal at these locations. With a clean cloth, wipe dirt away from these areas prior to clamping the drum punch sampling system to the drum lid.

5. Hoist the drum punch sampling system onto the top of the drum and position the filter cup assembly directly over the filter.

6. Press down the clamps (45) on the channel beam (44) until they press tightly against the sides of the drum. Adjust the clamps, if necessary, to achieve a tight fit.

7. Turn the hard knob (61) of the filter cup assembly to lower and press the filter cup over the carbon composite filter. 
8. Loosen the finger nut of the Ultra-Tor ${ }^{*}$ fitting (53) and turn the hand wheel (47) until the sampling plate (52) presses flush against metal surface of the drum lid. Re-tighten the finger nut of the Ultra-Tor' fitting.

9. Again check the filter cup (65) to make sure it is pressed tightly against the drum lid and adjust if necessary.

10. Remove the needle assembly (1-3) from the purge assembly tee (27) described in Procedure 110.1 and insert it in the male elbow (60) of the sampling plate (52). Finger tighten the nut to secure the needle in place.

11. Insert the free end of the Teflon ${ }^{\infty}$ line (68) from valve (56) into the purge assembly tee (27) and finger tighten to secure the line in place. [NOTE: For a more secure fit, the free end of line (68) may be fitted with a $3.2 \mathrm{~mm}(1 / 8$-inch) compression fitting and attached to the purge assembly tee.

12. Open valves (56) and (58) and close valve (29) of the headspace gas sampling system.

13. Adjust valve (25) of the headspace gas sampling system to purge the groove of the sampling plate (52) at approximately $1 \mathrm{~L} / \mathrm{min}$, as indicated on flow indicator (70). Purge the groove with humidified zero air, nitrogen, or helium, for approximately 3 minutes.

14. After purging the groove for 3 minutes, close valve (58) and add bubble solution around the edges of the sampling plate (52), where the outer O-ring (54) is pressed against the drum lid. Observe this area for the production of bubbles. If any bubbles are detected, increase the pressure on the sampling plate (52) by turning the hand wheel (47) until the bubble(s) cease to be produced. If bubbling cannot be stopped, the drum should not be sampled by this method. Note: This leak check may also be performed using helium in place of zero air and sniffing for helium leaks at the edges of the sampling plate.

15. If no bubbles are observed, open valve (29) above the purge assembly tee and then close valve (56) on the sampling plate (52).

16. Place the $12.7 \mathrm{~mm}(1 / 2$-inch) ratchet on the punch driver (49) and slowly turn the bolt clockwise (as though looking down on bolt) until resistance from the drum lid is felt.

17. Continue turning the bolt (49) until operator is assured that the drum lid has been breached.

18. Turn the bolt (49) counterclockwise to back it out slightly.

19. Open valve (4) and monitor the manometer (9) until it indicates ambient pressure, or ceases to show an increase in pressure. Verify that the canister gauge(s), if present, indicate 0 psig.

20. Close the valves of the canisters that were used for sample collection.

21. Remove the needle assembly (1-3) from the male elbow (60) and disconnect the needle assembly from valve (4). Place the needle assembly in a collection box for items to be cleaned, or dispose of it. 
22. Attach a clean needle assembly (1-3) to valve (4) and re-insert the needle assembly back into the purge assembly tee (27).

23. Remove the drum punch sampling system from the drum.

24. Replace the O-rings (54 and 55) with clean O-rings and discard the used O-rings.

25. Turn the press assembly hand wheel (47), punch assembly bolt (49), and filter cup assembly hand knob (61) a few times counterclockwise to return these to their pre-sampling positions.

26. Follow Steps 7 through 10 of Section 8.2.1 in Procedure 110.1.

\subsection{Quality Control}

Quality control in the use of this needle assembly is maintained by collecting the specified number of field OC samples (i.e., field blanks, field duplicates, field reference standards, equipment blanks) as specified in Section 9.0 of Procedure 110.1 or 110.2 of this Methods Manual. Quality control is also maintained by cleaning the apparatus in accordance with the cleaning procedures specified in Section 8.0 of Procedure 110.1 , with the following exceptions:

1. $\mathrm{QA} / \mathrm{OC}$ samples are collected by connecting the needle assembly $(1-3)$ to the male elbow $(60)$ on the sampling plate (52) rather than the purge assembly tee (27) of the headspace sampling system.

2. A length of $3.2 \mathrm{~mm}$ (1/8-inch) Teflon tubing (68) supplies humidified zero air from the headspace sampling system to the sampling plate (52).

3. The flow indicator (70) mounted on the channel beam (44) is used for verifying excess flow during zero air and field reference standard gas collection rather than the headspace sampling system flow indicator (30).

\subsection{Procedure Performance}

No data are available to determine the performance of the drum punch.

\subsection{References}

Coughlan, J. 1990. "Drum Lid Gas Sampling Punch Concept", Drawing No, W0096-0024-ED00. Argonne National Laboratory - West, September 27, 1990.

Parker. 1991. Parker O-Ring Handbook, Parker Hannifin Corporation, Cleveland, OH, 10/91, Pp. A5-13. 
Revision: 0

Date: May, 1995

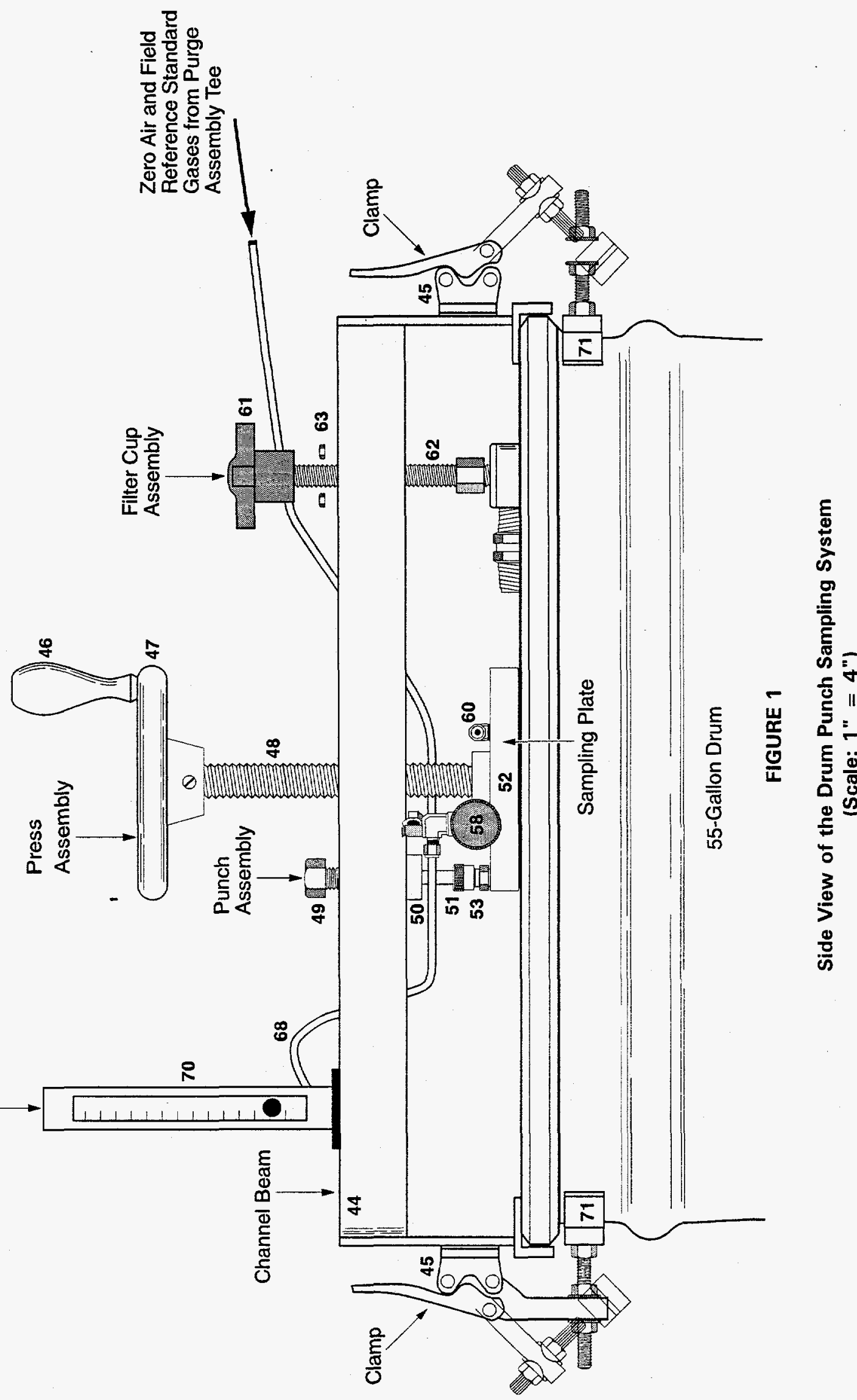




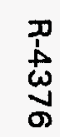

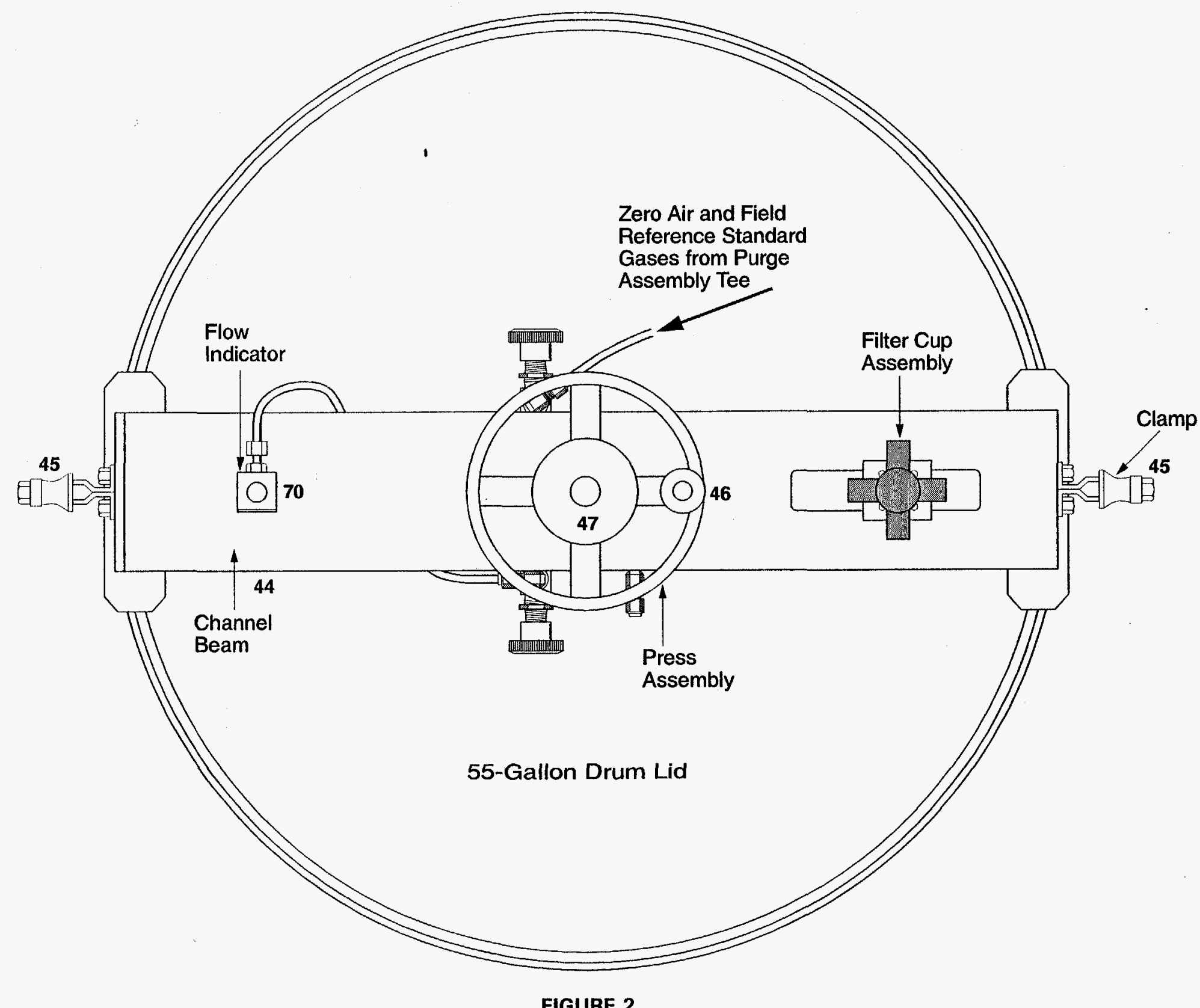

Overhead View of the Drum Punch Sampling System

(Scale: 1" = 4") 
Revision: 0

Date: May, 1995

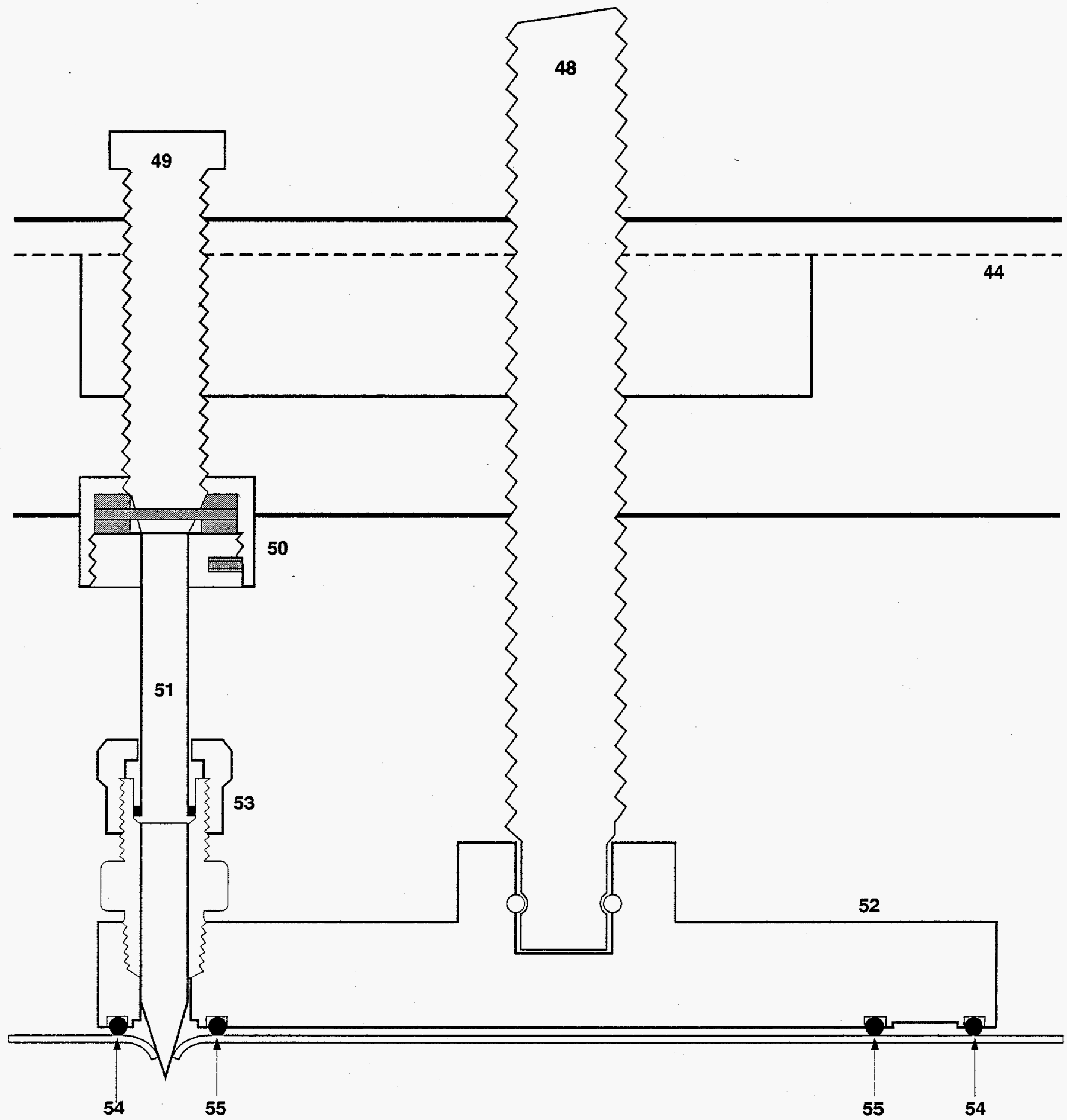

$110-4-3$

FIGURE 3

Cross Section of the Punch Assembly, Press Assembly, and Sampling Plate

(Not to Scale) 
ग

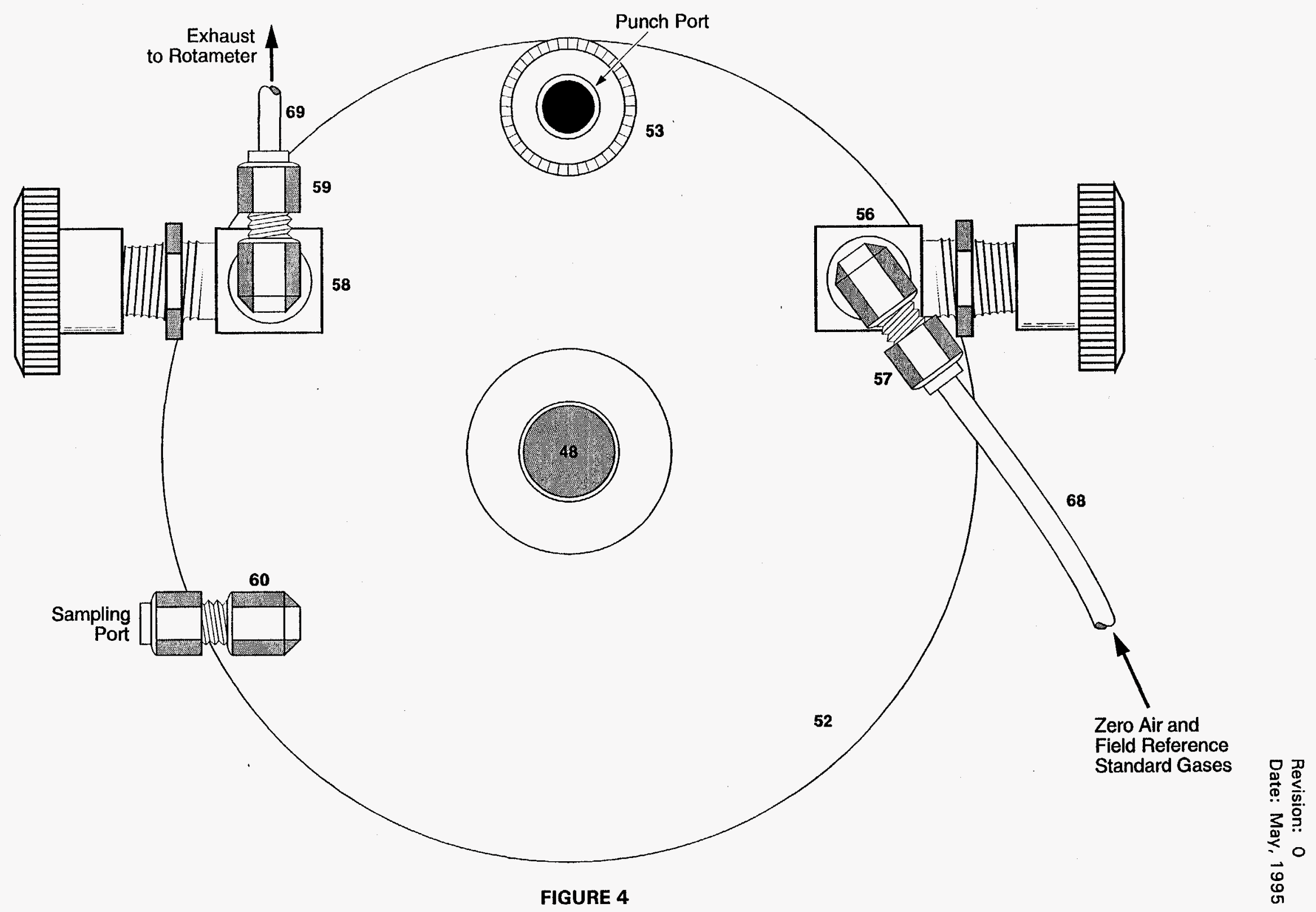

Top View of the Sampling Plate (Not to Scale) 


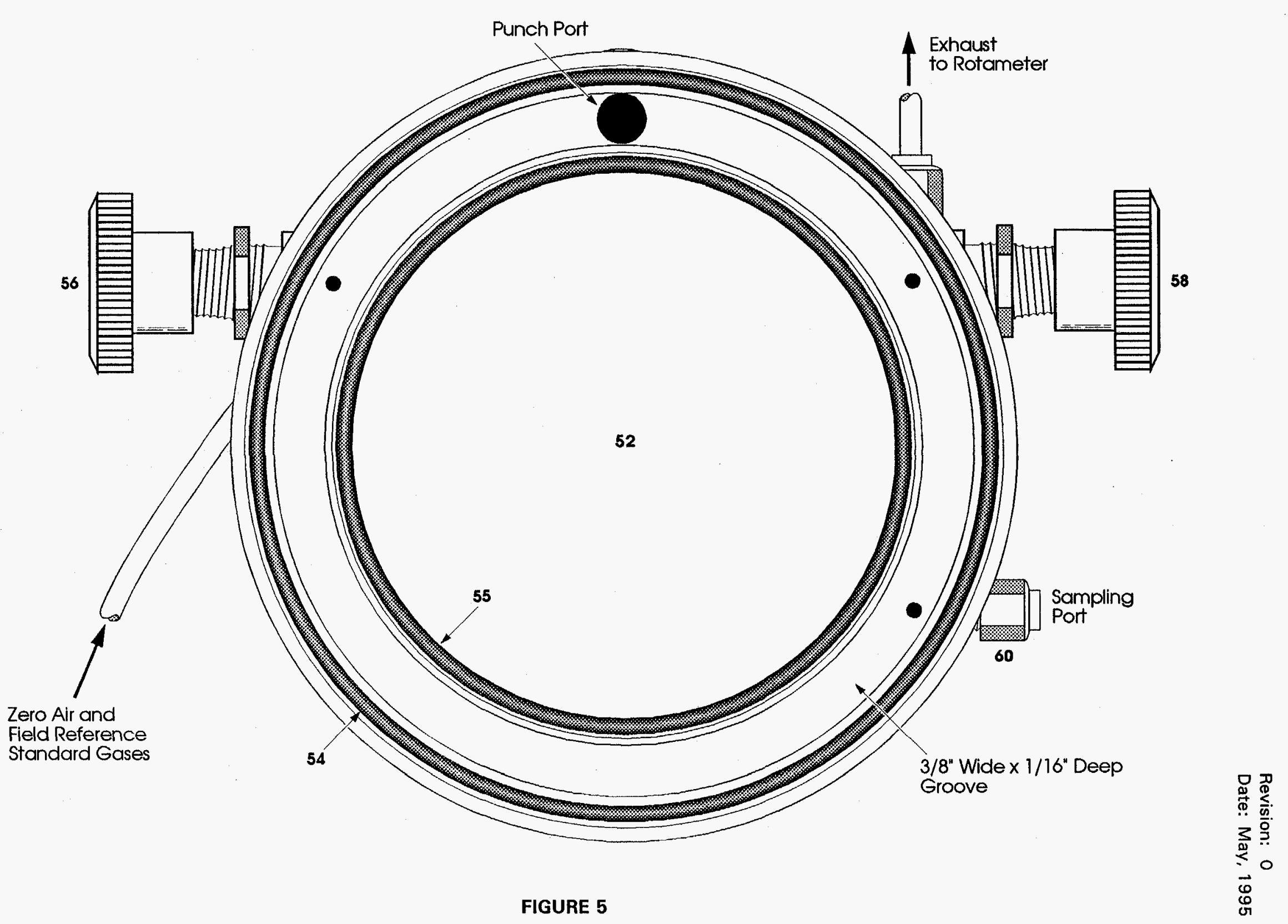

Bottom View of the Sampling Plate

(Not to Scale) 
Revision: 0

Date: May, 1995

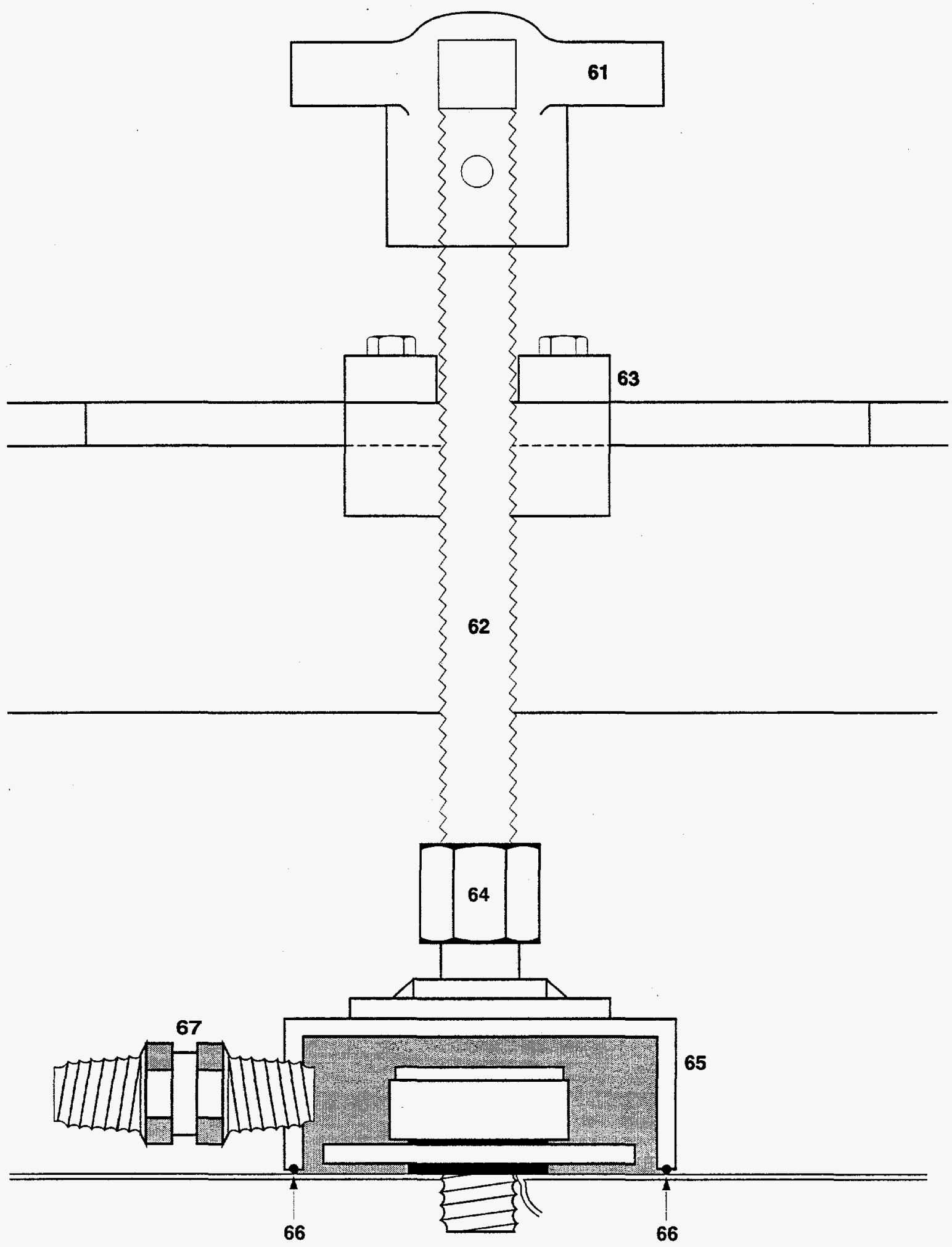

FIGURE 6

Cross Sectional View of the Filter Cup 
PROCEDURE 120.1

\section{COLLECTING SAMPLES FROM TRU WASTE DRUMS CONTAINING HOMOGENOUS SOLIDS AND SOIL/GRAVEL (SLUDGE)}

\subsection{Scope and Application}

1.1 This procedure may be used to collect samples from 208 liter (55-gallon) drums of transuranic (TRU) waste classified as homogenous solids and soil/gravel. Although the primary focus of this procedure is on 208 liter (55-gallon) drums, it may also be used to collect samples of TRU waste from smaller containers (e.g., 3.8-liter polyethylene bottles) stored in 208 liter (55-gallon) drums. For the purposes of this procedure, "homogenous solids and soil/gravel" are referred to as "sludge". The physical properties of these wastes are described in Table 1 . This procedure must be implemented with a site-specific standard operating procedure (SOP).

1.2 This procedure is applicable to characterizing a sludge on a "waste-stream" basis rather than an "individual-drum" basis. This is because the samples are collected from one randomly selected location within the sludge which may, or may not, be homogenous with the sludge in other areas of the drum. A waste stream is a waste material generated from a single process or activity that is similar in material, physical form, isotopic make-up, and hazardous constituents (DOE 1995a). To adequately characterize a waste stream, several drums in that waste stream must be sampled. The requirements for drum selection and sludge characterization are described in the Transuranic Waste Characterization Quality Assurance Program Plan (QAPP) (DOE 1995a).

1.3 For the purposes of the TRU Waste Characterization Program, sludge samples are to be collected in sampling batches. A sampling batch is defined as a suite of samples collected consecutively, using the same sampling equipment, within a specific time period. A sampling batch can be up to 20 samples (excluding field $\mathrm{OC}$ samples), all of which must be collected within 14 days of the first sample in the batch.

\subsection{Summary of Procedure}

2.1 This procedure is based on sampling methods similar to those approved by EPA for solid waste and soil sampling contained in Test Methods for Evaluating Solid Waste, Physical/Chemical Methods, SW-846, Third Edition, Final Update I, and Final Update II (SW-846) (EPA 1995), "Standard Practice for Thin-Walled Tube Sampling of Soils" (ASTM 1983) and "Standard Practice for sampling Waste and Soils for Volatile Organic Compounds" (ASTM 1991).

2.2 This procedure requires the use of drilling equipment, coring tools, and sample collection equipment. The use of this equipment is designed to provide laboratories the minimum amount of required sample. This minimizes the quantity of sample requiring transport to a laboratory and minimizes the quantity of investigation-derived waste. The quantities of sludge required for analysis and the sample handling requirements are listed in Table 2.

2.3 The drum lid and inner liner lid (if present) are removed and the surface of the sludge is exposed. The sludge is tested for hardness to determine the type of coring tool required. A non-rotational coring tool is used to core soft sludges and a rotational coring tool is used when the hardness of the sludge prevents penetration with the non-rotational coring tool. Prior to use, each coring tool must be lined inside with eight removable transparent liners to contain the sludge. 
TABLE 1

\section{Description of Waste Physical Properties a}

Homogenous Solids and Soil/Gravel

Inorganic Waste Water Treatment Sludge

Organic Liquid and Sludge

Solidified Liquid

Cation and Anion Exchange Resins

Pyrochemical Salts

Soils
Description

Soft ferric hydroxide sludge packed with cement on top and bottom, packed as alternating layer of sludge and cement, or as a hard mixture of sludge and cement

Soft sludge formed by mixing oil and grease with calcium silicate packed with adsorbent on top and bottom or as alternating layers of adsorbent and sludge

Hard, friable sludge formed by mixing alkaline aqueous waste water with portland and magnesia cement

A slurry of ion exchange resins mixed with cement to form a hard cement matrix which immobilizes the resins

Solid crystalline waste salts from a molten salt extraction process

Contaminated soil generated from cleanup of spills, decontamination and decommissioning activities

aSummarized from the Waste /solation Pilot Plant Transuranic Waste Baseline Inventory Report (DOE 1995b). 
TABLE 2

Sample Handling Requirements for Homogenous Solids and Soil/Gravel

\begin{tabular}{|c|c|c|c|c|}
\hline Parameter & $\begin{array}{c}\text { Minimum Quantity } \\
\text { Required }\end{array}$ & Preservative & Container & $\begin{array}{l}\text { Holding } \\
\text { Time }^{\mathrm{a}}\end{array}$ \\
\hline VOCs & 15 Grams & Cool to $4^{\circ} \mathrm{C}$ & Glass Vial ${ }^{\mathbf{b}}$ & $\begin{array}{l}14 \text { Days Prep/ } \\
40 \text { Days } \\
\text { Analyze }^{c}\end{array}$ \\
\hline SVOCs & 50 Grams & Cool to $4^{\circ} \mathrm{C}$ & Glass Jar ${ }^{d}$ & $\begin{array}{c}14 \text { Days Prep/ } \\
40 \text { Days } \\
\text { Analyze }\end{array}$ \\
\hline $\begin{array}{l}\text { Polychlorinated } \\
\text { Biphenyls (PCBs) }\end{array}$ & 50 Grams & Cool to $4^{\circ} \mathrm{C}$ & Glass Jar ${ }^{d}$ & $\begin{array}{c}14 \text { Days Prep/ } \\
40 \text { Days } \\
\text { Analyze }\end{array}$ \\
\hline Metals & 10 Grams & Cool to $4^{\circ} \mathrm{C}$ & Plastic Jar $^{f}$ & 180 Days $^{g}$ \\
\hline
\end{tabular}

${ }^{\text {aH }}$ Holding time begins at sample collection (holding times are consistent with SW-846 requirements).

$b_{40} \mathrm{~mL}$ VOA vial, must have septum cap.

'40-day holding time allowable only for methanol extract -- 14-day holding time for non-extracted VOCs.

${ }^{d} 250 \mathrm{~mL}$ amber jar, must have Teflon ${ }^{\oplus}$ lined cap.

${ }^{\circ}$ Analysis for PCBs is required only for waste streams in matrix parameter category S3220 (organic sludges).

${ }^{f_{2}} 250 \mathrm{~mL}$ polyethylene or polypropylene.

9Holding time for mercury analysis is 28 days. 
2.4 As presented in Figure 1, the coring tool is mounted vertically above the drum and pressed or drilled to the bottom of the sludge at a coring location randomly selected on the sludge's surface.

2.5 The coring tool is removed from the sludge and the transparent liners containing the core are extruded from the coring tool. The sludge is visually examined for evidence of nonhomogeneity (e.g., stratification), disturbance due to coring, and its length is measured. The entire depth of the waste must be cored and the core collected must have a length greater than or equal to 50-percent of the depth of the waste. This is called the core recovery and is calculated as in Section 8.0.

2.6 A location from which to collect samples is randomly selected along the length of the core and the liner at this location is separated from the other liners to expose the sludge.

2.7 If the exposed sludge is soft, the Table 2 specified weight of sludge is immediately collected, using a modified (with its tip removed) plastic syringe, and sealed in a vial for volatile organic compound (VOC) analysis. Table 2 specified weights of sludge for semivolatile organic compound (SVOC) and metals analyses are also collected from this location, with the syringe or by scoop, and placed in sample jars.

2.8 If the sludge is hard, the core is removed from the liner, broken into pieces, and the pieces are placed in sample containers to meet the Table 2 specified weights and handling requirements.

\subsection{Limitations and Interferences}

\subsection{Limitations.}

This procedure is prepared in accordance with the following limitations:

- Coring and sample collection activities must be conducted in a containment area (e.g., glove box).

- Liquids are not allowed in the containment area.

\subsection{Interferences.}

3.2.1 The parts of the coring tool that come in contact with the sludge (e.g., cutting edge of the auger) have the potential to contaminate the surface of the core with metals such as nickel, chromium, and lead (metals commonly found in coring tool components), especially through abrasion of the coring tool when coring hard sludges. To minimize or eliminate this potential interference, individuals should:

- Use a plastic basket retainer instead of a spring steel basket retainer.

Note: It is recommended that the basket retainer not be used when relatively certain that the core will not fall out as it is removed from the drum.

- Design the coring tool such that the liners are placed as close as possible to the tool's leading edge.

- Collect sludge sample (for metals analysis) from an area of the core that did not contact the coring tool (i.e., the longitudinal center of the core). 
3.2.2 The polycarbonate liners specified in this procedure have the potential to contaminate the core with organic compounds such as methylene chloride and chlorobenzene when the sludge contains solvents. To minimize or eliminate this potential interference, individuals should:

- Assess the potential for the liners to leach compounds that are on the list of analytes, especially in the presence of solvents.

- Use liners made from relatively inert material such as Teflon ${ }^{\circledR}$ or glass.

Note: Translucent Teflon ${ }^{\circledast}$ piping with a $5 \mathrm{~cm}$ ( 2 inches) outer diameter and 1.6 $\mathrm{mm}$ (0.063 inches) wall thickness may require special order.

3.2.3 Additional information regarding these potential interferences can be found in the Idaho National Engineering Laboratory Simulated Transuranic Waste Sampling Program (Connolly 1994).

\subsection{Safety}

This procedure may involve the use of hazardous materials, operations, and equipment. It is the responsibility of whoever uses this procedure to consult appropriate site personnel concerning health and safety issues and establish appropriate health and safety practices. Safety concerns regarding the chemical and radiation exposure should be considered. Training regarding proper storage, usage, and disposal of chemicals is recommended.

\subsection{Apparatus and Materials}

The apparatus and materials necessary for coring and sampling TRU waste drums containing sludge consists of drilling equipment, coring tools, sampling tools, and sample containers.

5.1 Drilling Equipment. It is not the intent of this procedure to provide detailed specifications for the drilling equipment necessary for core collection. The facilities are expected to differ between sites designated to conduct coring and sampling activities and these differences will dictate the types and configuration equipment required.

It is expected that eight major components of drilling equipment will be necessary to collect representative cores of the sludge. These components include a feed frame, rotation unit, slide frame, coring tool guide, power pack, control unit, drilling platform, and drum platform. The purpose of each component is described in Table 3.

Figure 2 provides a conceptual configuration for a hydraulically powered drilling system arranged for core collection inside a containment area. As shown, the slide frame, feed frame, rotation unit, and coring tool guide are operated inside the containment area. The power pack and control panel, and drum platform are outside of the containment area. A manipulator arm in the containment area facilitates coring operations and provides reach to areas not accessible by glove ports.

5.2 Non-Rotational Coring Tool. The non-rotational coring tool, found in "Standard Practice for Thin-Walled Sampling of Soils" (ASTM 1983), is used to collect a representative core of soft sludge. As presented in Figure 3, it consists of a stainless-steel ball check valve and a stainless-steel core barrel. The liners and basket retainer described in Section 5.4 fit inside the non-rotational coring tool.

The non-rotational coring tool is designed such that the tool's kerf width is minimized. Kerf width is defined as one-half of the difference between the outer diameter of the tool 
Feed Frame

Rotation Unit

Slide Frame

Coring Tool Guide

Power Pack

Control Unit

Drilling Platform

Drum Platform
Applies downward (push) and upward (pull) forces to the coring tool. It has a minimum feed length of $122 \mathrm{~cm}$ (48 inches) and should be equipped with an adjustable mechanism to limit travel distance up and down. It is capable of applying a minimum of $4448 \mathrm{~N}(1,000 \mathrm{lbs}$.) pressure.

Applies clockwise and counterclockwise rotation to the rotational coring tool. It can be operated at variable speeds up to $1,000 \mathrm{rpm}$. It is capable of supplying a minimum of $54.24 \mathrm{Nm}$ (40 ft. lbs.) torque.

Permits the feed frame/coring tool to be moved and locked in position over the horizontal centerline of the drum, or pushed back out of the way.

Provides lateral support to the coring tool to prevent the coring tool from "walking" during rotational coring activities.

Provides the power necessary to drive the rotational unit and feed frame.

Provides the drill operator with the ability to control and monitor the forces required to position, push, pull, and rotate the coring tool. It allows the operator to control the speed and direction of the rotation unit, control the forces of the feed frame, and energize and shutdown the power pack. The control panel does not provide rotational speeds. The rotational speeds are measured with a handheld tachometer. The control panel does not operate the feed frame tilt mechanism. This is done manually.

Supports the feed frame and rotation unit above the drum.

Supports and braces the drum to prevent movement during coring activities. 
and the inner diameter of the tool's inlet. This minimizes resistance as the tool is pressed into the sludge and enhances core recovery.

The ball check valve allows air to escape from inside the liners as the tool is pressed into the sludge. The ball check valve also provides a relatively air-tight seal above the sludge to help prevent it from slipping out of the liners when the tool is withdrawn from the drum.

The core barrel is sharpened and tapered at its leading edge to an inner diameter slightly smaller than the inner diameter of the liners that line its interior. This tapering prevents the liners from falling out and the sharpening enhances the core barrels ability to penetrate the sludge. The core barrel is constructed to allow placement of the liner's leading edge as close as possible to the core barrel's leading edge. This minimizes the core's contact with stainless steel and minimizes the surface area that must be cleaned (wiped) prior to reuse.

5.3 Rotational Coring Tool. The rotational coring tool, presented in Figure 4, is used primarily to collect samples from hard sludge. It may also be used for coring softer sludges. The rotational coring tool is designed to minimize disturbance to the core by containing the core inside an inner core barrel that remains stationary as the auger is rotated and pressed into the sludge.

Its design permits placement of the liners and basket retainer, described in Section 5.4, as close as possible to the coring tool's leading edge. This minimizes the core's contact with the metal parts of the tool.

Thin flights, made from $3 \mathrm{~mm}(0.125$ inches) square rod, are spiraled up and around the outside of a $84 \mathrm{~cm}$ ( 33 inches) long auger, lift the sludge cuttings from the borehole as the auger is pressed and rotated into the sludge.

The leading edge of the auger is sharpened and tapered to a diameter slightly smaller than the inner diameter of the liners. The flights protrude slightly below this edge where they are hardened and sharpened for cutting the sludge.

The inner core barrel, equivalent to a non-rotational coring tool without a tapered leading edge, holds the liners inside with a core barrel tip (tapped into place). This facilitates placement and removal of the liners and basket retainer.

The assembled coring tool is secured to the drill rod with a pin. A key and screws hold the auger to the thrust bearing/ball check valve.

The thrust bearing ball check valve prevents the core barrel from rotating with the auger and provides a vent to release the air inside the liners as sludge is pushed up inside. The ball check valve also provides a relatively air-tight seal above the sludge to help prevent it from slipping out of the liners when the tool is withdrawn from the drum.

\subsection{Common Components.}

Three components are common to both the rotational and non-rotational coring tools. These include liners to contain the sludge, a basket retainer to prevent the sludge from dropping out of the coring tool when it is withdrawn from the drum, and an extruder to remove the liners from the coring tool, when necessary.

5.4.1 Liners: The liners are constructed of rigid clear (transparent) polycarbonate. The liner's transparency allows visual examination of the core after sampling. 
Note: Teflon ${ }^{\oplus}$ or glass may be used in place of polycarbonate; however, the effectiveness of visual examination of the sludge through Teflon ${ }^{*}$. which is generally not as transparent as polycarbonate, may be reduced. Metal liners (e.g., stainless steel, brass) may be used if the sludge is not going to be analyzed for metals content.

The liner's outer diameter is $5 \mathrm{~cm}$ (2 inches) nominal. Liner wall thickness is 1.6 $\mathrm{mm}(0.0625$ inches) nominal.

Before cleaning, as described in Section 9.3, and use, each liner is cut to a length of $10 \mathrm{~cm}$ (4 inches). Several liners are also cut with the width of the basket retainer subtracted from the length.

Plastic or Teflon end caps should be placed over the ends of the liners if there is going to be a substantial delay ( $>60$ minutes) between core recovery and sample collection. If the liners are to remain inside the core barrel during the delay, then end caps should be placed over the exposed ends of the core barrel. End caps should fit tightly.

5.4.2 Basket Retainer: A basket retainer is used with each coring tool when the physical properties of the waste are such that the waste may fall out of the liners and back into the drum when the coring tool is removed from sludge.

Basket retainers are made from either metal or plastic. Each has very thin flexible flaps made from spring steel or plastic. The flaps are attached to a ring with an outer diameter of $5 \mathrm{~cm}$ ( 2 inches) and an inner diameter of $4.8 \mathrm{~cm}$ (1.875 inches). The ring is made of stainless steel or plastic.

The basket retainer is placed inside at the leading tip of the coring tool in front of the liners. As the coring tool is pressed into the sludge, the sludge pushes the spring steel, or plastic, flaps up against the inside wall of the first liner. When the coring tool is withdrawn from the drum, the force created by pulling pulls the flaps closed which cuts sludge and seals the inlet to prevent the sludge from falling back into the drum.

5.4.3 Extruder: An extruder may be necessary to force the liners from the inside of the tool if they do not slide freely. Use of this device is dependent on the liner's tightness of fit inside the coring tool and the quantity of sludge that may have wedged between the liners and the coring tool.

The extruder for use with the rotational coring tool is made of stainless steel approximately $76 \mathrm{~cm}$ ( 30 inches) long with a $5 \mathrm{~mm}$ (2 inches) diameter pistonshaped head.

The extruder for use with the non-rotational coring tool made of stainless steel approximately $84 \mathrm{~cm}$ ( 33 inches) long with a $4.5 \mathrm{~cm}$ ( 1.77 inches) diameter piston shaped head. A flexible ring fits in a slot that is machined near the leading edge of the head. The ring is compressed for insertion through the leading edge of the non-rotational coring tool. Once through, the ring expands to approximately $5 \mathrm{~mm}$ ( 2 inches) diameter to push against the basket retainer and liners.

\subsection{Sampling Tools.}

Small disposable tools are used to sample cores for waste characterization on a wastestream basis. The intent is to minimize equipment costs and the quantity of investigation- 
derived waste. Additional sampling tools (e.g., mixer, bowls) are necessary for waste characterization on an individual-drum basis. Sample collection tools and their respective uses for waste characterization on an individual-drum or waste-stream basis are described in Table 4.

\subsection{Sample Containers.}

Sample containers are described in Table 2.

\subsection{Preplanning}

Studies to characterize TRU waste require careful planning to prepare facilities, develop written documentation, secure laboratory support, and evaluate data.

\subsection{Reagents, Preservation Measures and Handling}

7.1 Handling requirements for sludge samples must conform to the requirements for sample quantity, container, preservation and holding times specified in Table 2. The sample quantities provided are the approximate amounts to be collected for each parameter. If other parameters are to be measured, the method should be reviewed and the laboratory conducting the analysis should be consulted to determine the proper sample size.

7.2 All sample handling and chain-of-custody procedures described in Section 6.0 of the QAPP must be followed.

\subsection{Procedure}

\subsection{Pre-Coring Preparations.}

1. The identification of the waste drum selected for sampling is recorded and the height of the drum $\left(X_{1}\right)$ is measured and recorded on the Field Log Sheet presented in Figure 5. The drum is positioned underneath the containment area and sealed (bagged in) to an opening in the containment area floor. Once the drum is braced with the containment area, and braced to the drum platform, its lid is removed.

Note: Headspace gas sample collection in accordance with requirements specified in the QAPP may be necessary prior to removal of the drum lid.

2. The lid of the drum's inner liner, if present, is also removed along with packaging materials (e.g., vermiculite) to expose the surface of the sludge. The height of the drum $\left(X_{1}\right)$ is measure and recorded on the field log sheet. The distance from the top of the drum (rim) to the surface of the sludge $\left(X_{2}\right)$ is measured and recorded on the Field Log Sheet. The distance from the bottom of the sludge to the bottom of the drum $\left(X_{3}\right)$ is estimated (based on the thickness of the rigid liner and the thickness of the rim at the bottom of the drum) and recorded on the field log sheet. The depth of the sludge $\left(X_{4}\right)$ is then estimated as indicated on the Field Log Sheet.

3. A coring location is selected along the centerline of the drum. This location is selected by measuring the diameter of the sludge in centimeters and randomly selecting a number between $5 \mathrm{~cm}$ and the diameter of the sludge minus $5 \mathrm{~cm}$. This location is recorded and the feed frame is moved horizontally along the slide frame until the drill rod is directly above this location. The feed frame is then secured to the slide frame. 
Syringe ${ }^{a}, 10$ cc capacity, high density polyethylene with tip removed.

Lab scoop, stainless steel, $16.5 \mathrm{~cm}$ (6.5 inches) long.

Lab scoop, high density polyethylene, $16.5 \mathrm{~cm}$ (6.5 inches) long.

Preparation knife $e^{b}$, hardened steel, overall length $25 \mathrm{~cm}$ (10 inches), blade length $15 \mathrm{~cm}$ (6 inches).

Mixer ${ }^{b}$, typical cake mixer, 600 watt, 3.8-liter glass or high-density polyethylene bowl, beater blades.

Mortar and Pestle, 1 liter (32 oz.) clear heavy flint glass.

Hammer, carpenter's, steel.

Chisel, hardened steel, $2.5 \mathrm{~cm}(1$ inch) blade.

Forceps, plastic or stainless steel.

Bags, tubular polyester film, $24 \mathrm{~cm}$ (9.5 inches) width, 4.5 mil thick.

Film, disposable plastic or Teflon ${ }^{\oplus}$.

Balance, top loading, readability 0.1 $\mathrm{g}$, weighing capacity $6000 \mathrm{~g}$.
Collect samples for VOC, SVOC Not used. and metals analyses.

Collect samples for SVOC and metals analyses.

Collect samples for SVOC and metals analyses.

Split (slice) core longitudinally prior to homogenization and/or sample collection.

Homogenize entire core or split of core prior to sample collection.

Not used.

Not used.

Not used.

Not used.

Not used.

Drop cloth to contain and remove sludge debris.

Measure the quantity of sample required by the lab.
Scrape longitudinally down length of core to collect samples for SVOC and metals analyses.

Not used.

Scrape longitudinally down length of core to collect samples for SVOC and metals analyses.

Not used.

Pulverize and mix core, or pieces of core prior to sample collection.

Shatter core into pieces prior to sample collection.

Shatter core into pieces prior to sample collection.

Transfer pieces of core to sample container.

Contain pieces of core during shattering process.

Drop cloth to contain and remove sludge debris.

Measure the quantity of sample required by the lab.

\footnotetext{
${ }^{a}$ Clear high-density plastic syringe with a $10 \mathrm{cc}$ volume available through laboratory suppliers. Luer-Lok ${ }^{\circledR}$ tip and plunger with rubber stopper, O.D. approximately $1.6 \mathrm{~cm}$. Graduations are marked every $0.2 \mathrm{cc}$ of volume. The syringe is modified in that the Luer-Lok ${ }^{\otimes}$ tip is removed with a razor blade and the syringe's leading edge is sharpened with a file, and the plunger's rubber tip and holding post are removed allowing the plunger free movement inside the barrel.
}

${ }^{b}$ This tool is necessary only when sampling on an individual-drum basis. 
4. The sludge is tested for hardness by placing the sharp edge of a chisel or metal punch on the surface of the exposed sludge and striking it with a hammer. Alternately, a sharp object is attached to the drill rod and the feed frame is advanced to press the sharp object into the sludge.

Note: Process knowledge indicates a hardened layer of cement may be present on the surface of the sludge in many of the TRU waste drums. With pressure from the sharp object, this layer should fracture, if present.

5. Segments of fractured cement that may obstruct core collection are moved from underneath the coring tool prior to continuing. The hardness determination is recorded on the Field Log Sheet.

6. Based on the hardness of the sludge, the appropriate coring tool is selected. The tool and its protective covering are visually inspected for cleanliness. If confirmed clean and in good condition, the tool is removed from its protective covering and mounted to the drill rod held secure by the rotation unit. The coring tool's equipment cleaning batch number is recorded on the Field Log Sheet. The protective covering is discarded.

\subsection{Collecting a Core of Soft Sludge.}

1. If the sludge is determined to be soft, the non-rotational coring tool is selected and connected to the drilling rod. The leading edge of the coring tool is lowered until it touches the surface of the sludge. The feed frame is then adjusted to deliver a stroke no greater than the depth of the sludge $\left(X_{4}\right)$.

Note: This is a safety mechanism to ensure the coring tool is not punched through the bottom of the drum or drum liner, if present.

2. The non-rotational coring tool is pressed into the sludge in a steady continuous stroke. The feed frame pressure is monitored during the stroke which should require no more than 10 seconds to complete. The stroke is complete when one of the following conditions have been met:

- The pre-set stroke length has been achieved.

- The feed frame is near the pre-set stroke length and the coring tool has encountered a hard layer halting its advancement.

Note: Process knowledge indicates a hardened layer of cement may be present at the bottom of the sludge in many of the TRU waste drums.

3. The length of the feed frame stroke is measured and recorded on the Field Log Sheet as the "borehole depth."

4. Slow clockwise rotation (approximately $30 \mathrm{rpm}$ ) is applied to the coring tool, the feed frame is reversed, and the coring tool is slowly pulled from the sludge.

Note: It is recommended that clean rags be used to remove sludge from the tool's outer surface as it emerges from the sludge. This will assist in maintaining a clean work area and reduce the potential for cross contamination if this coring tool is reused. 
5. The core barrel containing the core is detached from the ball check valve and the core recovery must be determined. The entire depth of the waste must be cored and the core collected must have a length greater than or equal to 50-percent of the depth of the waste. This is called the core recovery and is calculated as follows:

$$
\text { Core recovery }=\frac{y}{x} * 100
$$

where $x$ is the depth of the waste in the container and $y$ is the length of the core collected from the waste. If core recovery is less than 50-percent of the depth of the waste, a second coring location shall be randomly selected. The core from the second location shall be used for sample collection regardless of the core recovery. The core recovery must be recorded on the Field Log Sheet.

6. At this point, the entire core may be sent to the sample collection area for sampling in accordance with Section 8.6 if an end cap is sealed to each end. Individuals wishing to reduce the quantity of sludge requiring transfer to the sample collection area may implement, in the drilling area, the procedures described in Section 8.5.

\subsection{Collecting a Core of Hard Sludge.}

1. If the sludge is determined to be hard, the rotational coring tool is selected and connected to the drilling rod. The leading edge of the coring tool is lowered until it touches the surface of the sludge. The feed frame is then adjusted to deliver a stroke no greater than the depth of the sludge $\left(X_{4}\right)$.

Note: This is a safety mechanism to ensure the coring tool is not drilled through the bottom of the drum or drum liner, if present.

2. A constant clockwise rotation of 300 to $600 \mathrm{rpm}$ is applied to the coring tool and sufficient pressure is applied with the feed frame to complete the stroke as quickly as possible without compromising the structural integrity of the drum, drilling equipment, and containment area.

Note: The results of the Idaho National Engineering Laboratory Simulated Solidified Transuranic Waste Sampling Program (Connolly, 1994) indicate that rapid coring of hard sludge minimizes heat generation and subsequent transfer of heat to the core. Heating the core is not desirable in that it may facilitate the volatilization and loss of target analytes during coring.

3. Rotational speed is reduced, if necessary, to control the generation of visible dust.

4. The stroke should require no more than 60 seconds to complete. The feed frame pressure, rotational speed, and torque are monitored during the stroke and the stroke is complete when the pre-set stroke length has been achieved.

5. The length of the feed frame stroke is measured and recorded on the Field Log Sheet as the "borehole depth."

6. The pin connecting the auger to the thrust bearing ball check valve is removed and, using the feed frame, the core barrel is lifted from the auger.

Note: As the core barrel is lifted from the auger, it is recommended that clean rags be used to remove any sludge visible on the core barrel's outer surface. This will 
assist in maintaining a clean work area and reduce the potential for cross contamination if this coring tool is reused.

7. The core barrel containing the core is detached from the thrust bearing ball check valve and the core recovery must be determined as in Equation (1). The entire depth of the waste must be cored and the core recovery must be greater than or equal to 50 percent. If the core recovery is less than 50 percent, then a second coring location must be randomly selected. The core from the second location shall be used for sample collection regardless of the core recovery. The core recovery must be recorded on the Field Log Sheet.

8. At this point, the entire core may be sent to the sample collection area for sampling in accordance with Section 8.7 if an end cap is sealed to each end. Individuals wishing to reduce the quantity of sludge requiring transfer to the sample collection area may implement, in the drilling area, the procedures described in Section 8.5.

\subsection{Preparing the Sampling Area.}

1. Prior to receiving core barrels or individual liners containing sludge, the sample collection area and balance are covered with clean plastic film. All sample collection tools are available and contained in their protective coverings.

2. All sample containers (e.g., vials, jars) into which sludge samples will be placed are available with their caps in place.

Note: It is recommended that sludge sample information (e.g., site location, date, waste container number, type of analysis) be recorded on the sample container's label prior to placement in the sample collection area.

3. The balance is available and operating within manufacturer specifications.

4. The cap of the vial designated and pre-labeled to contain the sample for VOC analysis is removed and the vial is placed on the balance. The balance is tared and the vial's identification number is recorded le.g., equipment cleaning batch or manufacturer's lot number) on the Field Log Sheet.

\subsection{Selecting the Sampling Location.}

1. The liners are extruded from the core barrel and the core is visually examined and measured. The visual observations and core length are recorded on the Field Log Sheet.

2. A random number generator is used to randomly select a number between zero and the number that corresponds to the length of the core. This number represents the distance from the leading edge of the first liner (bottom of sludge) that the sample is to be collected.

3. The location is measured and marked on the outside surface of its respective liner. If the location falls at a break between two liners, the sample is collected toward the first liner. If the location falls near the top of the core and the quantity of sludge is insufficient for sample collection, then the bottom edge of this liner and top edge of the adjacent liner that contains sludge are selected.

4. The liner containing that location is selected and separated from the other liners. For soft sludge, a clean separation may require slicing between the liners with a sharp thin 
object (e.g., knife). If the sludge is hard, separation may require the use of a hack saw.

Note: Once the sludge at the ends of a liner are exposed to ambient air, sample collection for VOC analysis should be conducted as quickly as possible to minimize the loss of target VOCs present in the sludge. Additional guidance for this type of sampling is provided in Soil Sampling and Analysis for Volatile Organic Compounds (EPA 1991a) and Review of Current and Potential Future Sampling Practices for Volatile Organic Compounds in Soil (Hewitt 1992).

5. If a delay in sampling sludge for $V O C$ analysis is anticipated (i.e., greater than 60 seconds), the ends of the liner are sealed with end caps. Any sludge protruding from the ends of the liner is chipped or cut flush prior to sealing.

\subsection{Collecting Samples from Soft Sludge.}

1. A modified syringe is selected and its identification number is recorded on the Field Log Sheet. The syringe's barrel is pressed into the sludge at the end of the liner closest to the sampling location. It is offset from the liner's longitudinal center and pressed until the sludge reaches the $10 \mathrm{cc}$ graduation. The syringe's plunger is allowed to rise freely.

2. The syringe is removed from the sludge and the outside surface of its barrel is quickly wiped with a clean tissue.

3. The leading edge of the syringe is inserted through the mouth of the vial and the sludge is extruded by pressing down on the plunger.

4. The weight registered by the balance is observed and additional sludge is collected as necessary to obtain approximately 15 grams. As presented in Table 5 , it is expected that 1.5 to 2 syringe volumes will be required to obtain the Table 2 specified weight.

5. The mouth of the vial is quickly wiped with a clean tissue (to remove any particulate that could prevent a proper seal), and the vial's cap is tightened into place.

6. Samples for SVOC, metals, and PCB analyses are collected from the same end of the liner using the same syringe or other sample collection tools (e.g., scoop). The prelabeled sample jars are tared and the sludge is transferred from the liner to the jar until the balance indicates the Table 2 specified weight. As presented in Table 5, as much as 94 percent of the sludge in a liner may be needed for laboratory analyses. Each sample collection tool and sample jar's identification number is recorded on the Field Log Sheet.

7. The sampling tools and plastic film, core barrel tip and basket retainer, if used, are discarded. All remaining sludge is returned to the waste drum and the empty liners are discarded.

8. If the core barrel is to be reused, it is cleaned in accordance with the procedure described in 9.3, or the appropriate procedure in Section 9.4. Otherwise, the core barrel is discarded.

9. The vials and jars containing sludge are handled in accordance with the requirements specified in Table 2. 
TABLE 5

\section{Estimated Sludge Sample Availability}

\begin{tabular}{|c|c|c|c|c|c|}
\hline Waste Forma & $\begin{array}{l}\text { Quantity Required } \\
\text { for Analysis }{ }^{b}(g)\end{array}$ & $\begin{array}{c}\text { Estimated } \\
\text { Density }^{c} \\
\text { (g/cc) }\end{array}$ & $\begin{array}{l}\text { Number of Full } \\
\text { Syringes to } \\
\text { Obtain } 15 \mathrm{~g}^{\mathrm{d}}\end{array}$ & $\begin{array}{l}\text { Estimated } \\
\text { Quantity of } \\
\text { Sludge in } \\
\text { Liner }^{\circ}(g)\end{array}$ & $\begin{array}{l}\text { Percentage } \\
\text { in Liner } \\
\text { Required for } \\
\text { Analysis }\end{array}$ \\
\hline $\begin{array}{l}\text { Inorganic Waste Water } \\
\text { Treatment Sludge }\end{array}$ & 125 & 0.96 & 1.5 & 173 & $72 \%$ \\
\hline $\begin{array}{l}\text { Organic Liquid and } \\
\text { Sludge }\end{array}$ & 125 & 0.74 & 2 & 133 & $94 \%$ \\
\hline Solidified Liquid & 125 & 1.01 & NA & 182 & $69 \%$ \\
\hline
\end{tabular}

Waste forms, were simulated for the idaho National Engineering Laboratory Simulated Solidified Transuranic Waste Sampling Program (Connolly, 1994), are based on information in the Waste isolation Pilot Plant Transuranic Waste Baseline Inventory Report (DOE 1995b).

'Sum of sludge quantities required for VOC, SVOC, PCB, and metals analyses (See Table 2).

'Density averaged from tests conducted on simulated waste in the Idaho National Engineering Laboratory Simulated Solidified Transuranic Waste Sampling Program (Connolly, 1994).

${ }^{d}$ A syringe with its tip removed will hold approximately $10 \mathrm{cc}$ of sludge. Analysis for VoCs requires $15 \mathrm{~g}$ of sludge.

'The internal volume of a liner is $180 \mathrm{cc}$. 


\subsection{Collecting Samples from Hard Sludge.}

1. If the sludge inside the sleeve is solid (monolithic), it is extruded or pushed into a clean clear plastic bag. A hammer, and chisel if necessary, is used to strike the monolith to break it into pieces small enough to fit thorough the mouth of the vial for VOC analysis.

Note: The purpose of the clear plastic bag is to contain the sludge as it is broken apart. Other containers may be used provided they are disposable and not likely to affect the composition of the sludge.

2. After the monolith has been sufficiently fractured, the clear plastic bag is opened and forceps are used to select the largest pieces that will fit inside the vial. The identification number of the forceps is recorded on the Field Log Sheet. The weight registered by the balance is observed and additional pieces aerated as necessary to obtain approximately 15 grams. The mouth of the vial is wiped with a clean tissue (to remove any particulate that could prevent a proper seal) and the vial's cap is tightened into place.

Note: If the sludge inside the liner exhibits extensive fracturing and pulverization, use of the clear plastic bag may not be necessary and the largest pieces that will fit inside the vial are transferred directly from the liner to the vial.

3. Samples for SVOC and metals analyses are collected with a lab scoop. The prelabeled sample jars are tared and the sludge is transferred from the bag or liner to the jar until the balance indicates the Table 2 specified weights. As presented in Table 5 , as much as 69 percent of the sludge in a liner may be needed for laboratory analyses. Each sample collection tool and sample jar's identification number is recorded on the Field Log Sheet.

4. The sampling tools and plastic film, core barrel tip and basket retainer, if used, are discarded. All remaining sludge is returned to the waste drum and the empty liners are discarded.

5. If the core barrel is to be reused, it is cleaned in accordance with the procedure described in 9.3 , or the appropriate procedure in Section 9.4. Otherwise, the core barrel is discarded.

6. The vials and jars containing sludge are handled in accordance with the requirements specified in Table 2.

\subsection{Quality Control}

Several features inherent to the coring and sampling techniques enhance the quality with which samples are collected. The design of the coring tools maximizes core recovery and minimizes disturbance to the core. The liners that contain the core are placed close to the coring tool's leading edge to minimize contact with the tool's metal surfaces. Hard sludges are cored as quickly as possible to minimize heat generation and potential loss of target analytes. The techniques used to collect samples for VOC analysis (e.g., time constraints, use of modified syringe) are designed to minimize the potential loss of target analytes. Quality control for sludge. sampling also includes collection of co-located cores to determine procedure precision and equipment blanks to verify cleanliness of the coring and sampling tools. 


\subsection{Co-Located Cores.}

Co-located cores are collected to determine the combined precision of the coring and sampling procedures. Co-located cores are collected at a frequency of 1 per sampling batch, or once per week during sampling operations, which ever is most frequent. Colocated cores are collected side-by-side as close as feasible to one another, handled in the same manner, visually inspected through the transparent liners, and sampled in the same manner at the same randomly selected sample location.

If physical differences are observed at the sampling locations, co-located cores may again be collected at another randomly selected sampling location in the drum. Samples from co-located cores must be submitted blind to the analytical laboratory.

9.2 Equipment Blanks. A site may choose to discard liners and sampling tools after one use. In these instances, equipment cleaning and equipment blank collection is not required.

9.2.1 Equipment blanks must be collected from fully assembled coring tools (including liners) prior to first use at a frequency of one per equipment cleaning batch. An equipment cleaning batch is the number of sampling equipment items cleaned together at one time using the same cleaning method. The equipment blank must be collected from the fully assembled coring tool, in the area where the coring tools are cleaned, prior to covering with protective wrapping and storage. The equipment blank must be collected by pouring clean water (e.g., deionized water, HPLC water) down the inside of the liners of the assembled coring tool. The water must be collected in a clean sample container placed at the leading edge of the coring tool. The container is labelled with the equipment cleaning batches' unique identification number and the sample is analyzed for the analytes listed in Table 6. The results of the equipment blank will be considered acceptable if the analysis indicates no analyte at a concentration greater than the acceptance limits listed in Table 6. If analytes are detected at concentrations greater than the acceptance limits, then the associated equipment cleaning batch of coring tools must be cleaned again and another equipment blank collected.

9.2.2 Equipment blanks must be collected from liners that are cleaned separately from the coring tools. These equipment blanks must be collected at a frequency of one per equipment cleaning batch. The equipment blanks must be collected by randomly selecting a liner from the equipment cleaning batch, pouring clean water (e.g., deionized water, HPLC water) across its internal surface, collecting the water in a clean sample container, and analyzing the water for the analytes listed in Table 6. The results of the equipment blank analysis will be considered acceptable if the results indicate no analyte at a concentration greater than the acceptance limits listed in Table 6 . If analytes are detected at concentrations greater than the acceptance limits, then the associated equipment cleaning batch of liners must be cleaned again and another equipment blank collected.

9.2.3 Sampling equipment (e.g., bowls, spoons, chisel, VOC sub-sampler) must also be cleaned. Equipment blanks must be collected for the sampling equipment at a frequency of one per equipment cleaning batch. After the sampling equipment has been cleaned, one item from the equipment cleaning batch is randomly selected, water (e.g., deionized water, HPLC water) is passed over its surface, collected in clean container, and analyzed for the analytes listed in Table 6 . The results of the equipment blank will be considered acceptable if the results indicate no analyte present at a concentration greater than the acceptance limits listed in Table 6 . If analytes are detected at concentrations greater than the acceptance limits, then the associated equipment cleaning batch of sampling equipment must be cleaned again and another equipment blank collected. 
TABLE 6

Equipment Blank Acceptance Criteria

\begin{tabular}{|c|c|c|}
\hline Analyte & $\begin{array}{c}\text { CAS } \\
\text { Number }\end{array}$ & $\begin{array}{c}\text { Acceptance } \\
\text { Limit }\end{array}$ \\
\hline VOCs & & $(\mathrm{mg} / \mathrm{kg})$ \\
\hline$\overline{\text { Berizene }}$ & $71-43-2$ & 3 \\
\hline Bromoform & $75-25-2$ & 3 \\
\hline Carbon disulfide & $75-15-0$ & 3 \\
\hline Carbon tetrachloride & $56-23-5$ & 3 \\
\hline Chlorobenzene & $108-90-7$ & 3 \\
\hline Chloroform & $67-66-3$ & 3 \\
\hline 1,4-Dichlorobenzene & $106-46-7$ & 3 \\
\hline ortho-Dichlorobenzene & $95-50-1$ & 3 \\
\hline 1,2-Dichloroethane & $107-06-2$ & 3 \\
\hline 1,1-Dichloroethylene & $75-35-4$ & 3 \\
\hline Ethyl benzene & $100-41-4$ & 3 \\
\hline Methylene chloride & $75-09-2$ & 3 \\
\hline $1,1,2,2$-Tetrachloroethane & $79-34-5$ & 3 \\
\hline Tetrachloroethylene & $127-18-4$ & 3 \\
\hline Toluene & $108-88-3$ & 3 \\
\hline 1,1,1-Trichloroethane & $71-55-6$ & 3 \\
\hline $1,1,2$-Trichloroethane & $79-00-5$ & 3 \\
\hline Trichloroethylene & $79-01-6$ & 3 \\
\hline Trichlorofluoromethane & $75-69-4$ & 3 \\
\hline $\begin{array}{l}\text { 1,1,2-Trichloro-1,2,2- } \\
\text { trifluoroethane }\end{array}$ & $76-13-1$ & 3 \\
\hline Vinyl chloride & $75-01-4$ & 3 \\
\hline m-xylene & $108-38-3$ & 3 \\
\hline o-xylene & $95-47-6$ & 3 \\
\hline p-xylene & $106-42-3$ & 3 \\
\hline Acetone & $67-64-1$ & 30 \\
\hline Butanol & $71-36-3$ & 30 \\
\hline Ethyl ether & $60-29-7$ & 30 \\
\hline Formaldehyde & $50-00-0$ & 30 \\
\hline Hydrazine & $302-01-2$ & 30 \\
\hline Isobutanol & 78-83-1 & 30 \\
\hline Methanol & $67-56-1$ & 30 \\
\hline Methyl ethyl ketone & $78-93-3$ & 30 \\
\hline Pyridine & $110-86-1$ & 30 \\
\hline SvoCs & & $(\mathrm{mg} / \mathrm{kg})$ \\
\hline Cresols & $1319-77-3$ & 15 \\
\hline 1,4-Dichlorobenzene & $106-46-7$ & 15 \\
\hline ortho-Dichlorobenzene & $95-50-1$ & 15 \\
\hline 2,4-Dinitrophenol & $51-28-5$ & 15 \\
\hline 2,4-Dinitrotoluene & $121-14-2$ & 0.9 \\
\hline Hexachlorobenzene & $118-74-1$ & 0.9 \\
\hline Hexachloroethane & $67-72-1$ & 15 \\
\hline Nitrobenzene & $98-95-3$ & 15 \\
\hline \multicolumn{3}{|l|}{$\begin{array}{l}\text { Polychlorinated Biphenyls } \\
\text { (PCBs) }\end{array}$} \\
\hline (PCBs) & & \\
\hline Aroclor 1016 & $12674-11-2$ & 15 \\
\hline Arocior 1221 & $11104-28-2$ & 15 \\
\hline Arocior 1232 & $11141-16-5$ & 15 \\
\hline Aroclor 1242 & $53469-21-9$ & 15 \\
\hline Arocior 1248 & $12672-29-6$ & 15 \\
\hline Aroclor 1254 & $11097-69-1$ & 15 \\
\hline Aroclor 1260 & $11096-82-5$ & 15 \\
\hline Pentachlorophenol & $87-86-5$ & 15 \\
\hline Pyridine & $110-86-1$ & 15 \\
\hline
\end{tabular}


TABLE 6

Equipment Blank Acceptance Criteria

(Continued)

\begin{tabular}{lcc}
\hline Analyte & $\begin{array}{c}\text { CAS } \\
\text { Number }\end{array}$ & $\begin{array}{c}\text { Acceptance } \\
\text { Limit }\end{array}$ \\
\hline Metals & $7440-36-0$ & $\frac{(\mu \mathrm{g} / \mathrm{L})}{300}$ \\
\hline Antimony & $7440-38-2$ & 300 \\
Arsenic & $7440-39-3$ & 6000 \\
Barium & $7440-41-7$ & 300 \\
Beryllium & $7440-43-9$ & 60 \\
Cadmium & $7440-47-3$ & 300 \\
Chromium & $7439-92-1$ & 300 \\
Lead & $7439-97-6$ & 12 \\
Mercury & $7440-02-0$ & 100 \\
Nickel & $7782-49-2$ & 60 \\
Selenium & $7440-22-4$ & 300 \\
Silver & $7440-28-0$ & 300 \\
Thallium & $7440-62-2$ & 300 \\
Vanadium & $7440-66-6$ & 300 \\
Zinc & &
\end{tabular}


Note: The results of the equipment blanks must be traceable to the equipment cleaning batch it represents. It is recommended that the equipment blank results for the coring tools, liners, and sampling tools be reviewed prior to use. A sufficient quantity of these items should be maintained in storage to prevent disruption of sampling operations.

\subsection{Initial Coring and Sampling Tool Cleaning Procedures.}

Coring and sampling tools and components that will come into contact with the sludge should be thoroughly cleaned prior to first use. This cleaning is conducted at a location outside of the containment area. The following procedure, adapted from an EPA-approved field sampling equipment cleaning procedure (EPA 1991b), should be followed.

1. Identical tools (e.g., non-rotational coring tools, modified syringes) and components (e.g., basket retainers) are assembled into equipment cleaning batches. Assign a unique identification number to each equipment cleaning batch.

2. The tools and components are washed with a non-phosphate detergent solution (e.g., Alconox).

3. The tools and components are rinsed with tap water.

4. The tools and components are rinsed with 10 percent nitric acid.

5. The tools and components are rinsed with distilled/deionized water.

6. The metal parts are rinsed with reagent-grade hexane followed by reagent-grade methanol.

7. The tools and components are rinsed with ASTM Type II or High Performance Liquid Chromatography (HPLC) water.

8. The non-metallic tools and components are dried in an oven at $50^{\circ} \mathrm{C}$ for 12 to 24 hours. The metallic tools and components are dried in an oven at $100^{\circ} \mathrm{C}$ for 12 to 24 hours.

9. A minimum of 1 component from each equipment cleaning batch is randomly selected. An equipment blank is collected as described in Section 9.2.

10. The equipment cleaning batch is placed in a clean protective covering (e.g. clear plastic bag) and labeled with the proper equipment cleaning batch number.

Note: Prior to using any of the components in the equipment cleaning batch, it should be verified that no significant levels of target analytes were detected in the equipment blank.

\subsection{Routine Coring Tool Cleaning Procedures.}

This procedure applies to non-rotational and rotational coring tools and components that will be used more than once before being discarded or cleaned in accordance with Section 9.3. This cleaning procedure applies only when coring and sampling is conducted on a waste-stream basis and only on drums containing similar wastes le.g., wastes from the same operation). This cleaning is conducted inside the containment area. The procedure described in Section 9.3 is used if coring and sampling is conducted on an individual-drum basis. 


\subsubsection{Non-Rotational Coring Tool:}

1. The core barrel is inspected for damage (e.g., dents, deformations). If damage is abserved, the core barrel is discarded or repaired prior to further use.

2. Clean rags are used to remove as much residual sludge as possible from the outside surfaces of the coring tool.

3. A clean rag is used to remove all residual sludge within $2.5 \mathrm{~cm}(1 \mathrm{inch})$ of the outside and inside of the leading edge of the core barrel such that no residual sludge is visible.

4. A clean basket retainer is installed in the core barrel, if necessary, and the retainer's equipment cleaning batch number is recorded on the Field Log Sheet.

5. Ejght clean liners, each pre-cut to $10 \mathrm{~cm}$ ( 4 inches) in length, are placed inside the core barrel and the liners' equipment cleaning batch number is recorded on the Field Log Sheet.

6. The core barrel is connected to the ball check valve and the coring tool is attached to the drill rod.

Note: This coring tool should be used only on TRU waste drums containing sludge similar to the TRU waste drum previously cored.

\subsubsection{Rotational Coring Tool:}

1. Scrapers, brushes, and clean rags are used to remove residual sludge from the outside and inside of the auger.

2. The auger and core barrel are inspected for damage le.g., dents, deformations). If damage to the core barrel, or cutting edge of the auger, is observed, the tool is discarded or repaired prior to use.

3. Clean rags are used to remove as much residual sludge as possible from the outside surfaces of the core barrel.

4. A clean rag is used to remove all residual sludge within 2.5 ( 1 inch) of the outside and inside of the leading edge of the core barrel such that no residual sludge is visible.

5. A clean core barrel tip is tapped in place at the leading edge of the core barrel and the tip's equipment cleaning batch number is recorded on the Field Log Sheet.

6. A clean basket retainer is installed in the core barrel, if necessary, and the retainer's equipment cleaning batch number is recorded on the Field Log Sheet.

7. Eight clean liners, each pre-cut to $10 \mathrm{~cm}$ (4 inches) in length, are placed inside the core barrel and the liners' equipment cleaning batch number is recorded on the Field Log Sheet.

8. The auger and core barrel are connected to the thrust bearing ball check valve and the rotational coring tool is attached to the drill rod. 
Note: This coring tool should be used only on TRU waste drums containing sludge similar to the TRU waste drum previously cored.

\subsection{Sample Container Cleaning Procedures.}

The sample containers specified in Table 2 are cleaned in accordance with the procedures described in EPA's Specifications and Guidance for Contaminant-Free Sample Containers, (EPA 1992a). The $40 \mathrm{~mL}$ vials are cleaned in accordance with container type $B$ and the $250 \mathrm{~mL}(8 \mathrm{oz}$.) jars are cleaned in accordance with container type $A$.

\subsection{Maintenance and Calibration.}

\subsubsection{Good Housekeeping Procedures:}

Potential cross contamination problems are minimized by maintaining the containment and sample collection areas clean of residual sludge and debris (e.g., discarded plastic bags and common components). Drilling equipment is maintained as specified by the manufacturer. Hydraulic lines and fitting are maintained leak free and inspected on a routine basis. Inspection, maintenance, and repair records are maintained at the site.

Note: The routine use of a vacuum cleaner with a high efficiency particulate air (HEPA) filter is recommended in the containment and sample collection areas. Vacuum bags and HEPA filters are discarded as necessary with the coring and sampling tools.

\subsubsection{Balance:}

The balance is maintained in accordance with manufacturer's specifications and calibrated on a routine basis with weights traceable to the National Institute of Standards and Technology (NIST). Calibration and maintenance records are maintained at the site.

\subsection{Procedure Performance}

10.1 This procedure was developed from the results of a sampling demonstration program (Connolly 1994) conducted by EG\&G Idaho Inc. to identify which coring and sampling techniques and tools were most effective for collecting representative samples of sludge from drums of TRU waste. A key program objective was to develop and demonstrate coring tools that could collect undisturbed cores of soft and hard sludge with greater than 50 -percent core recovery. A core that appeared relatively undisturbed and met this recovery specification was deemed to be representative of the sludge in the drum. Core recovery was determined as in Equation (1).

10.2 The results of the sampling demonstration program found the following:

- Soft sludge core recoveries averaged 99 percent using the non-rotational coring tool specified in this procedure.

- Hard sludge core recoveries were 97 percent using the rotational coring tool specified in this procedure.

- Cores of soft sludge collected with the non-rotational coring tool specified in this procedure appeared relatively undisturbed, with the exception of cores from drums that had alternating layers of soft sludge and hard cement. 
- Cores of hard sludge collected with the rotational coring tool specified in this procedure exhibited some disturbance (e.g., fracturing, pulverization) however, the degree of disturbance was notably less than with the other hard sludge coring tools tested.

10.3 Data are not available for determining procedure precision as described in Section 9.1. The simulated sludge was not spiked nor analyzed for any analytes of concern.

10.4 Data are not available for determining procedure accuracy. To determine procedure accuracy, coring and sampling of a drum of homogenous sludge with known analytes at known concentrations will be required. No such drum was available for the sampling demonstration program.

\subsection{References}

ASTM. 1983. "Standard Practice for Thin-Walled Tube Sampling of Soils." ASTM D1587-83, Annual Book of ASTM Standards, Philadelphia, Pennsylvania, American Society for Testing and Materials.

ASTM. 1991. "Standard Practice for Sampling Waste and Soils for Volatile Organic Compounds." ASTM D4547-91, Annual Book of ASTM Standards, Philadelphia, Pennsylvania, American Society for Testing and Materials.

Connolly, M. J. 1994. Idaho National Engineering Laboratory Simulated Solidified Transuranic Waste Sampling Program. EGG-WM-11222, Idaho Falls, Idaho, EG\&G Idaho, Inc., Idaho National Engineering Laboratory, U.S. Department of Energy.

DOE. 1989. TRUPACT-/l Content Codes (TRUCON). DOE/WIPP 89-004, Revision 3, Carlsbad, New Mexico, Waste Isolation Pilot Plant, U.S. Department of Energy.

DOE. 1995a. Transuranic Waste Characterization Quality Assurance Program Plan. CAO-941010, Current Revision, Carlsbad, New Mexico, National TRU Program Office, Carlsbad Area Office, U.S. Department of Energy.

DOE. 1995b. Waste Isolation Pilot Plant Transuranic Waste Baseline Inventory Report. CAO-94-1005, Carlsbad, New Mexico, Carlsbad Area Office, U.S. Department of Energy.

EPA. 1991a. Soil Sampling and Analysis for Volatile Organic Compounds. EPA/540/4-91/001. T.E. Lewis, A.B. Crocket, R.L. Siegrist, K. Zarrabi, Office of Research and Development, U.S. Environmental Protection Agency.

EPA. 1991b. Compendium of ERT Soil Sampling and Surface Geophysics Procedures. EPA/540/P-91/006, OSWER Directive 9360.4-02, Interim Final, Washington, D.C., Environmental Response Team, Emergency Response Division, Office of Emergency and Remedial Response, U.S. Environmental Protection Agency.

EPA. 1992a. Specification and Guidance for Obtaining Contaminant-Free Sample Containers. Directive No. 9240.0-05A, Washington, D.C., Office of Solid Waste and Emergency Response, U.S. Environmental Protection Agency.

EPA. 1995. Test Methods for Evaluating Solid Waste, Physical/Chemical Methods. SW-846, Third Edition, Final Update I and Final Update II. Washington, D.C., Office of Solid Waste and Emergency Response, U.S. Environmental Protection Agency. 
Hewitt, A. D. 1992. "Review of Current and Potential Future Sampling Practices for Volatile Organic Compounds in Soils." Proceedings of the 16th Army Annual Environmental R\&D Symposium. Williamsburg, Virginia, June 23-23, 1992. 


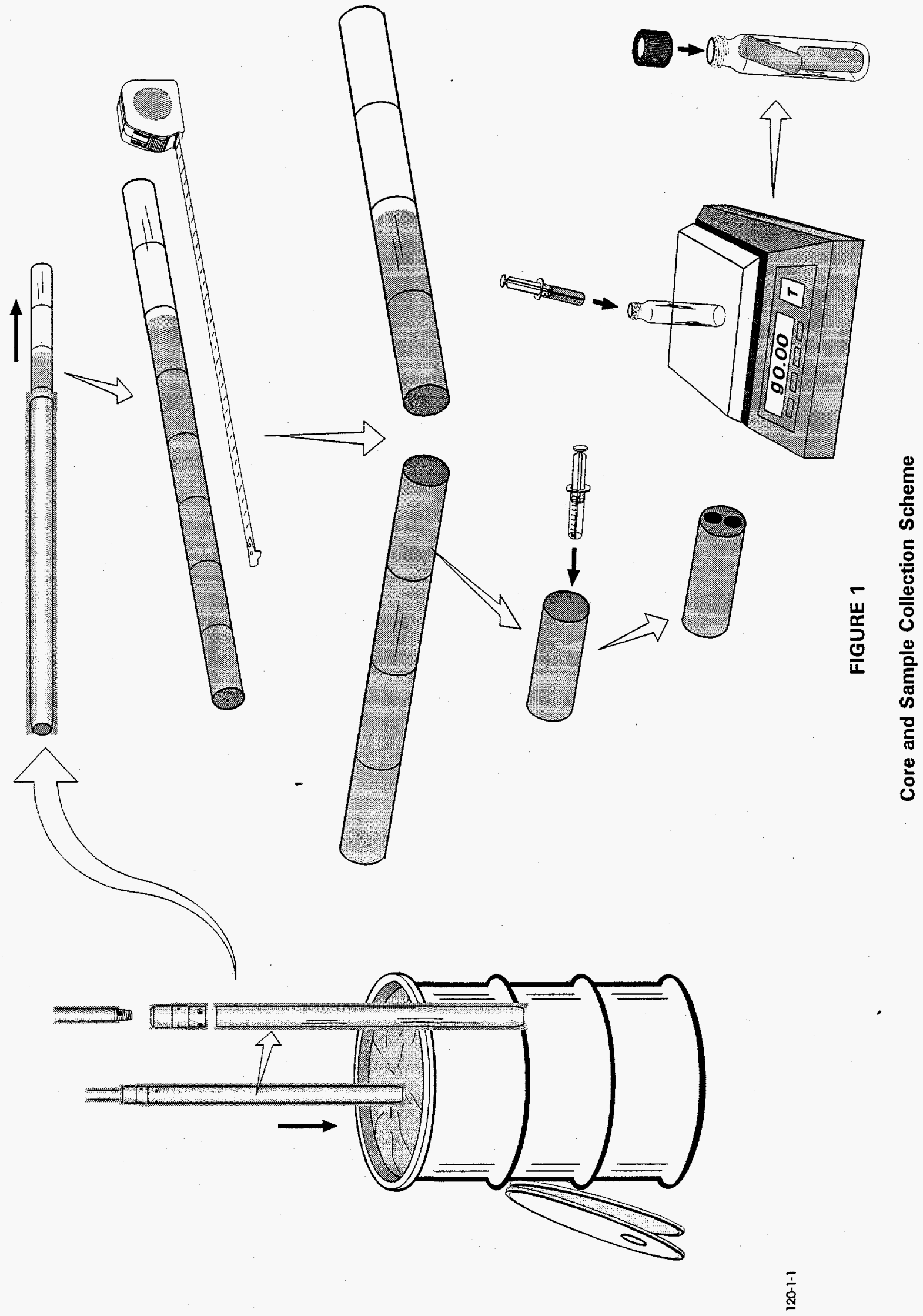




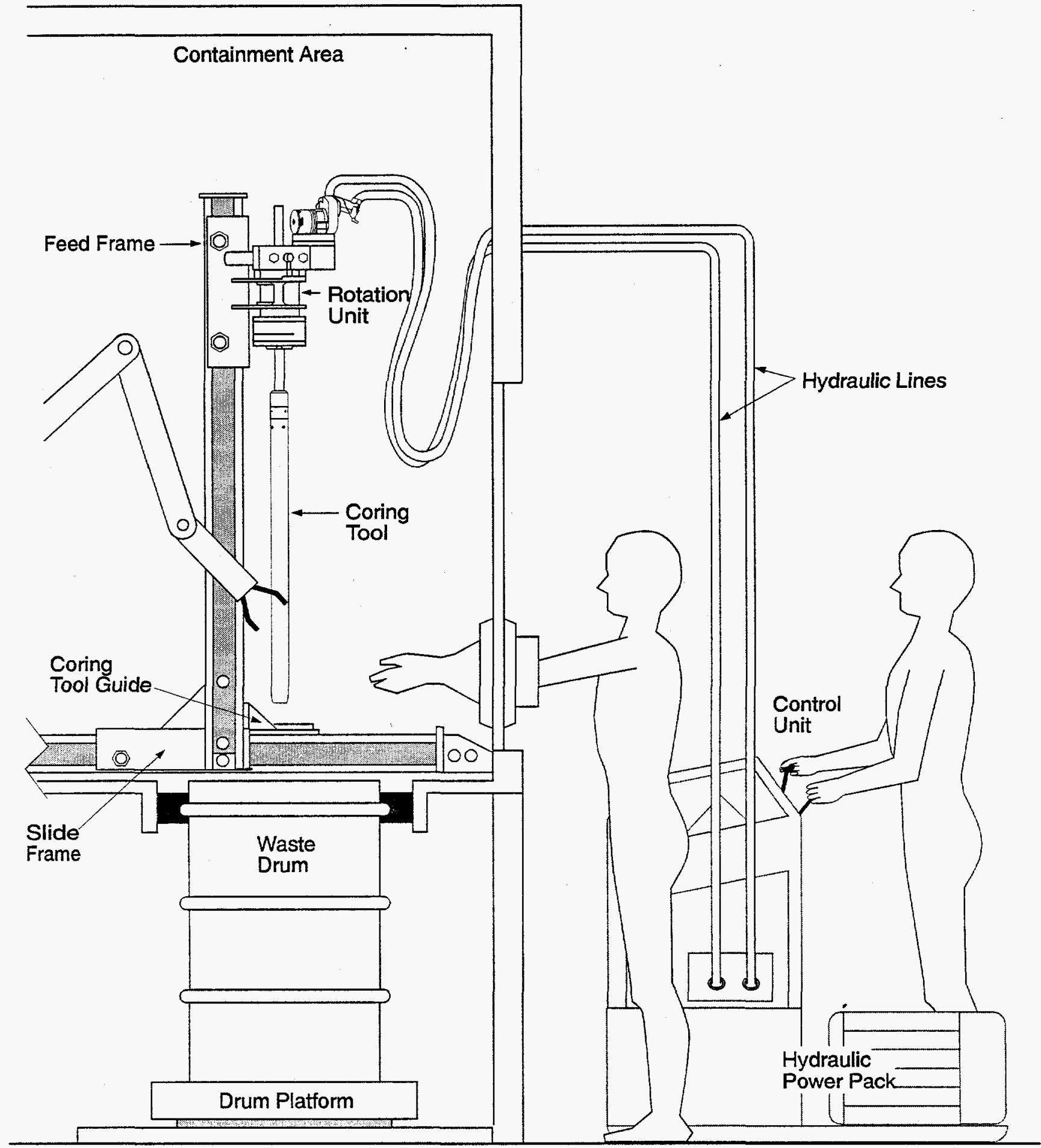

FIGURE 2 


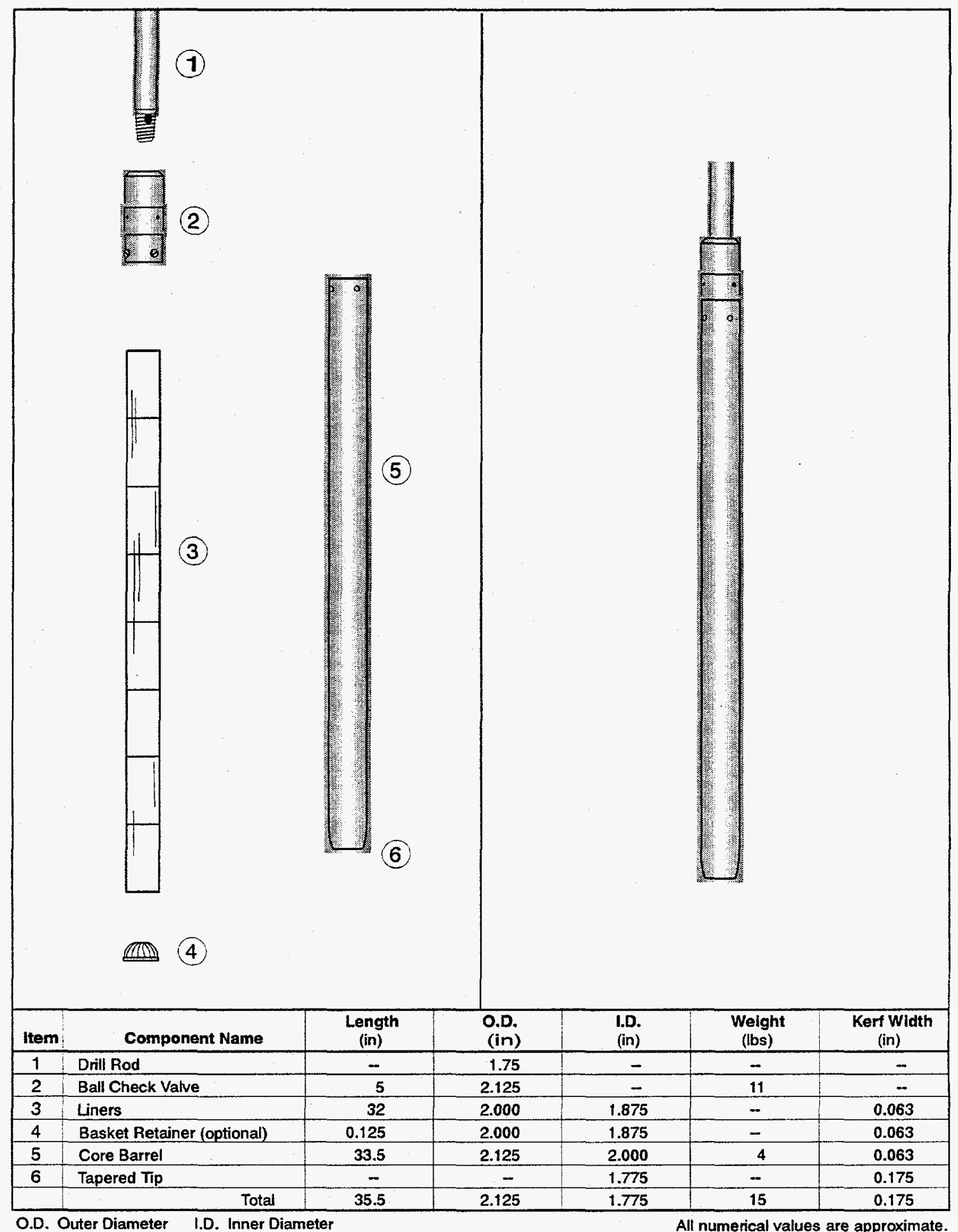

$120-1-3$

FIGURE 3

Non-Rotational Coring Tool 


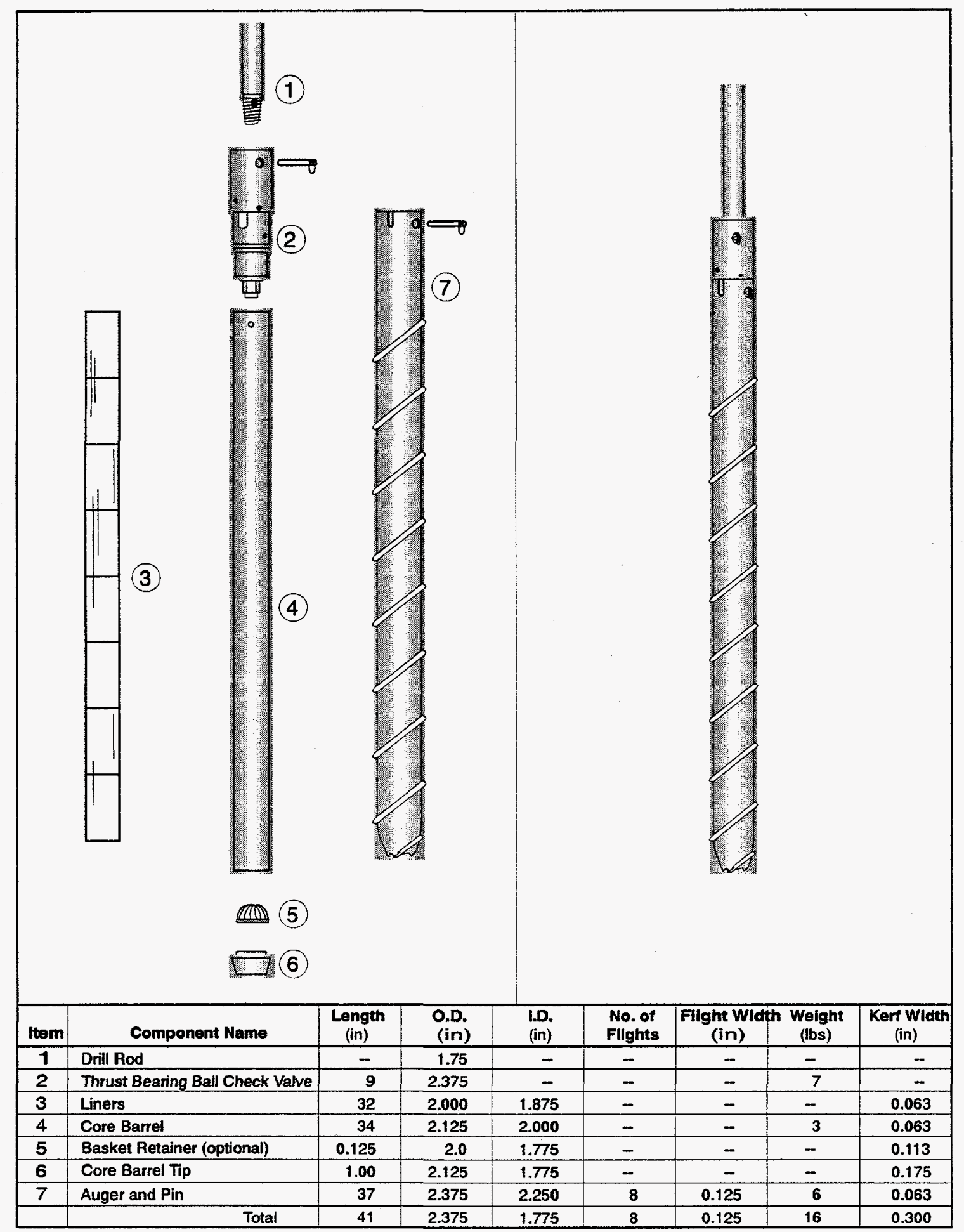


FIELD LOG SHEET

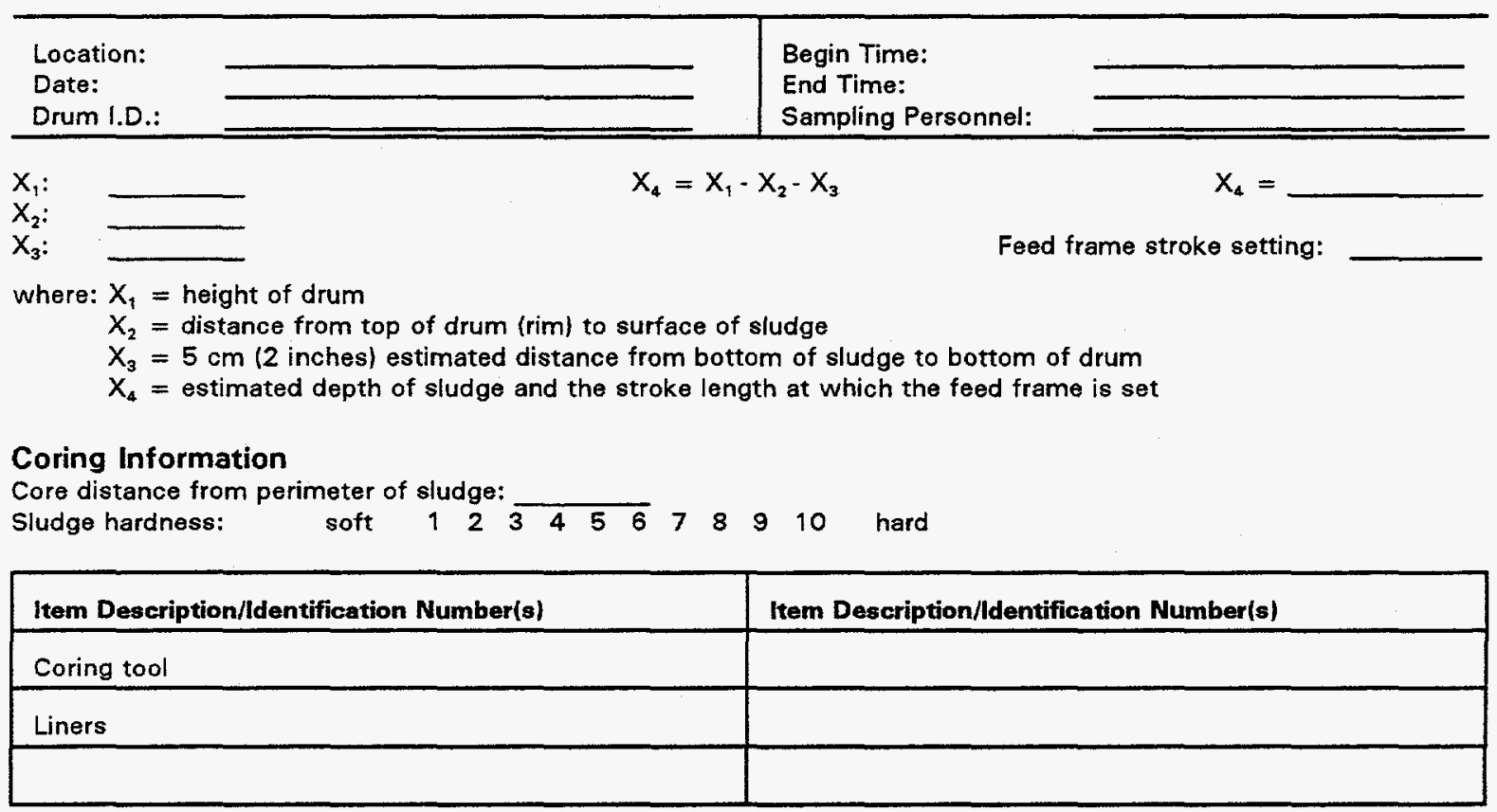

Borehole depth:

Visual observations of core:

Comments:

\section{Sample Collection Information}

Length of core:

Recovery: $\%$

Sampling location (distance from leading edge of first sleeve:

\begin{tabular}{|l|l|}
\hline Item Description/Identification Number(s) & Item Description/ldentification Number(s) \\
\hline & \\
\hline & \\
\hline & \\
\hline
\end{tabular}

Comments:

\begin{tabular}{|l|l|l|l|}
\hline \multicolumn{1}{|c|}{ Analysis } & Container/ldentification Number & Weight (g) & Identification \\
\hline VOCs & & & \\
\hline SVOCs & & & \\
\hline PCBs & & & \\
\hline Metals & & & \\
\hline
\end{tabular}

Comments:

FIGURE 5

Field Log Sheet 


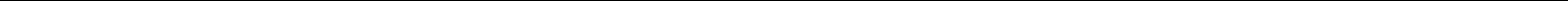


PROCEDURE 210.1

\section{SUMMA' PASSIVATED STAINLESS STEEL CANISTER CERTIFICATION AND CLEANING}

\subsection{Scope and Application}

This procedure describes how to clean and pressure certify SUMMA ${ }^{\circledR}$ passivated stainless steel canisters. This procedure is based on the Environmental Protection Agency (EPA) "Compendium Method TO-14, The Determination of Volatile Organic Compounds (VOCs) in Ambient Air Using SUMMA ${ }^{\oplus}$ Passivated Canister Sampling and Gas Chromatographic Analysis" (EPA 1988). SUMMA ${ }^{\oplus}$ canisters used for the collection of waste container headspace samples will be certified for pressure and residual contamination.

\subsection{Summary of Procedure}

Canisters will be cleaned on an equipment cleaning batch basis. An equipment cleaning batch is the number of canisters cleaned together at one time using the same cleaning method.

A cleaning system, capable of processing several canisters at a time, composed of an oven (optional) and a cryogenically trapped vacuum manifold will be used to clean the canisters. If an oil-free vacuum pump is used, the cryogenic trap is not necessary. At the end of the cleaning cycle, all canisters used for collecting hydrogen and methane samples will be evacuated to $<0.05 \mathrm{~mm} \mathrm{Hg}$ for storage. During cleaning, leak tests will be performed un all canisters. Any canister that fails will be checked for leaks and reprocessed. For canisters used to collect VOC samples, one canister per equipment cleaning batch will be filled with humid zero-air or nitrogen and analyzed for the VOCs listed in Table 1. The equipment cleaning batch will be considered clean if there are no VOCs detected above the acceptance limits listed in Table 1.

\subsection{Limitations and Interferences}

No limitations or interferences have been identified.

\subsection{Safety}

This procedure may involve the use of hazardous materials, operations, and equipment. It is the responsibility of whoever uses this procedure to consult appropriate site personnel concerning health and safety issues and establish appropriate health and safety practices. Consideration should be given to safety concerns regarding chemical and radiation exposure. Training regarding proper storage, usage, and disposal of chemicals is recommended.

\subsection{Apparatus and Materials}

5.1 Complete canister cleaning system commercially available from Scientific Instruments Specialist, Moscow, ID. Model GS-ERS-A4.

5.2 Vacuum pump capable of evacuating sample canister(s) to an absolute pressure of $<0.05$ $\mathrm{mm} \mathrm{Hg}$.

5.3 Manifold constructed of stainless steel tubing with connections for simultaneously cleaning several canisters. 
TABLE 1

Headspace VOC Target Analyte List and Equipment Cleaning Batch Blank Acceptance Limits

Acceptance

Limit

(ng)

Acetone
Benzene
Bromoform
Butanol
Carbon tetrachloride
Chlorobenzene
Chloroform
Cyclohexane
1,1-Dichloroethane
1,2-Dichloroethane
cis-1,2-Dichloroethylene
1,1-Dichloroethylene
Ethyl benzene
Ethyl ether
Methanol
Methyl ethyl ketone
Methyl isobutyl ketone
Methylene chloride
1,1,2,2-Tetrachloroethane
Tetrachloroethylene
Toluene
1,1,1-Trichloroethane
Trichloroethylene
$1,1,2-$-Trichloro-1,2,2-trifluoroethane
1,2,4-Trimethylbenzene
1,3,5-Trimethylbenzene
m-Xylene
$0-X y l e n e$
p-Xylene

67-64-1

71-43-2

$75-25-2$

$71-36-3$

$56-23-5$

108-90-7

67-66-3

$110-87-7$

75-34-3

107-06-2

540-59-0

75-35-4

$100-41-4$

60-29-7

67-56-1

78-93-3

108-10-1

75-09-2

79-34-5

127-18-4

108-88-3

71-55-6

79-01-6

76-13-1

95-63-6

108-67-8

108-38-3

95-47-6

$106-42-3$
450

450

30

30

30

30

30

30

30

30

30

30

450

450

450

30

30

30

30

30

30

30

30

30

30

30

30 
5.4 Shut-off valves made of stainless steel and of the bellows type (Nupro "H" series or equivalent).

5.5 Cryogenic trap made of stainless steel with an open tubular U-shaped trap cooled with liquid nitrogen to prevent contamination from back diffusion of oil from vacuum pump.

5.6 Humidifier - pressurizable water bubbler containing ASTM Type II water or equivalent, capable of providing moisture to the zero air or nitrogen supply.

5.7 Isothermal oven (optional) for heating canisters.

\subsection{Preplanning}

Activities associated with TRU waste characterization require careful planning to prepare facilities, develop written documentation, secure laboratory support, and evaluate data.

\subsection{Reagents, Preservation Measures, and Handling}

7.1 Gas cylinders of humid zero air, nitrogen, or helium. If helium is to be used for canister pressurization, humid zero air or nitrogen will also be required for the VOC certification step. All gases must be ultra-high purity grade.

7.2 All canister handling and chain-of-custody procedures described in Section 6.0 of the Transuranic Waste Characterization Quality Assurance Program Plan (QAPP) (DOE 1995) must be followed.

\subsection{Procedure}

8.1 Canister Cleaning and Certification. All canisters used for VOCs must be clean and free of any analytes (less than the acceptance limits) listed in Table 1 prior to use.

1. All canisters are leak tested by pressurizing them to approximately 30 psig with zero air or nitrogen. The cleaning system in Figure 1 can be used for this task. The initial canister pressure is measured, the valve is closed and the final pressure is checked after 24 hours. The pressure must not vary by more than 2 psig over the 24-hour period. When the system is equipped with Pirani vacuum controller, the canisters may be evacuated to $<0.05 \mathrm{~mm} \mathrm{Hg}$, and the equipment cleaning batch leak checked by placing the controller in "Leak Test" mode. When no rise in pressure is observed, the equipment cleaning batch may be considered leak tight. If leaks are suspected, isolate all canisters by closing the canister valves and open each one until the suspect leaking canister is identified. Remove leaking canisters from the cleaning equipment cleaning batch and reprocess after repair.

2. A canister cleaning system may be assembled as shown in Figure 1 or purchased commercially (Scientific Instrument Specialist, Moscow, ID). The canisters are connected to the manifold. Cryogen is added to the trap. The vent shut-off valve and canister valve(s) are opened to release any remaining pressure in the canister(s). The vacuum pump is started and the shut-off valve is opened. If the optional oven is used, the temperature can be set to $150^{\circ} \mathrm{C}$. For canisters with integral vacuum/pressure gauges the maximum temperature is $125^{\circ} \mathrm{C}$ after removing the plastic face plate and rubber blow-out plug. The canisters are evacuated to $<0.05$ $\mathrm{mm} \mathrm{Hg}$ for a minimum of one hour. When the optional oven is used the canisters are evacuated for a minimum of eight hours. 
3. At the end of the cleaning period, the canisters are pressurized to $30 \mathrm{psig}$ and evacuated to $.05 \mathrm{~mm} \mathrm{Hg}$ lif oven is used, allow canisters to reach ambient temperature). This pressurization/evacuation cycle is repeated 3 times for the process equipment cleaning batch.

4. At the end of the evacuation/pressurization cycle, the canister or one canister from the set is pressurized to $30 \mathrm{psig}$ with humid zero air or nitrogen. The canister is then analyzed for VOCs by Procedures 430.1, 430.2, and/or 440.1 of this Methods Manual. If no compounds are detected at levels greater than the acceptance limits (Table 1), the canister(s) is clean. If contaminants are found at levels exceeding the acceptance limits, then the cleaning procedure must be repeated until the canister(s) is clean.

5. The canister is reattached to the cleaning manifold and evacuated to $<0.05 \mathrm{~mm} \mathrm{Hg}$ and remains in this condition until used. The canister valve is closed. The canister is removed from the cleaning system.

8.2 After canister cleaning and certification, a removable tag must be securely fastened to each canister. Section 6.2.3 of the QAPP describes the sample canister tags, their use, and required informational content.

\subsection{Quality Control}

After cleaning, one canister per equipment cleaning batch shall be filled with humid zero air or nitrogen for use as a cleaning certification blank. This blank shall be analyzed for the VOCs in Table 1. The equipment cleaning batch may be certified as clean if no VOCs are detected in the blank at concentrations exceeding the acceptance limits.

\subsection{Procedure Performance}

The procedure has been found to be acceptable for canister cleaning and certification during headspace gas sampling and analysis operations at INEL.

\subsection{References}

DOE 1995. Transuranic Waste Characterization Quality Assurance Program Plan. CAO-94-1010 Current Revision, Carlsbad, New Mexico, Carlsbad Area Office, U.S. Department of Energy.

EPA. 1988. "Compendium Method TO-14, The Determination of Volatile Organic Compounds in Ambient Air Using SUMMA ${ }^{\oplus}$ Passivated Canister Sampling and Gas Chromatographic Analyses," Compendium of Methods for the Determination of Toxic Organic Compounds in Ambient Air, Research Triangle Park, North Carolina, Quality Assurance Division, Environmental Monitoring Systems Laboratory, U.S. Environmental Protection Agency. 


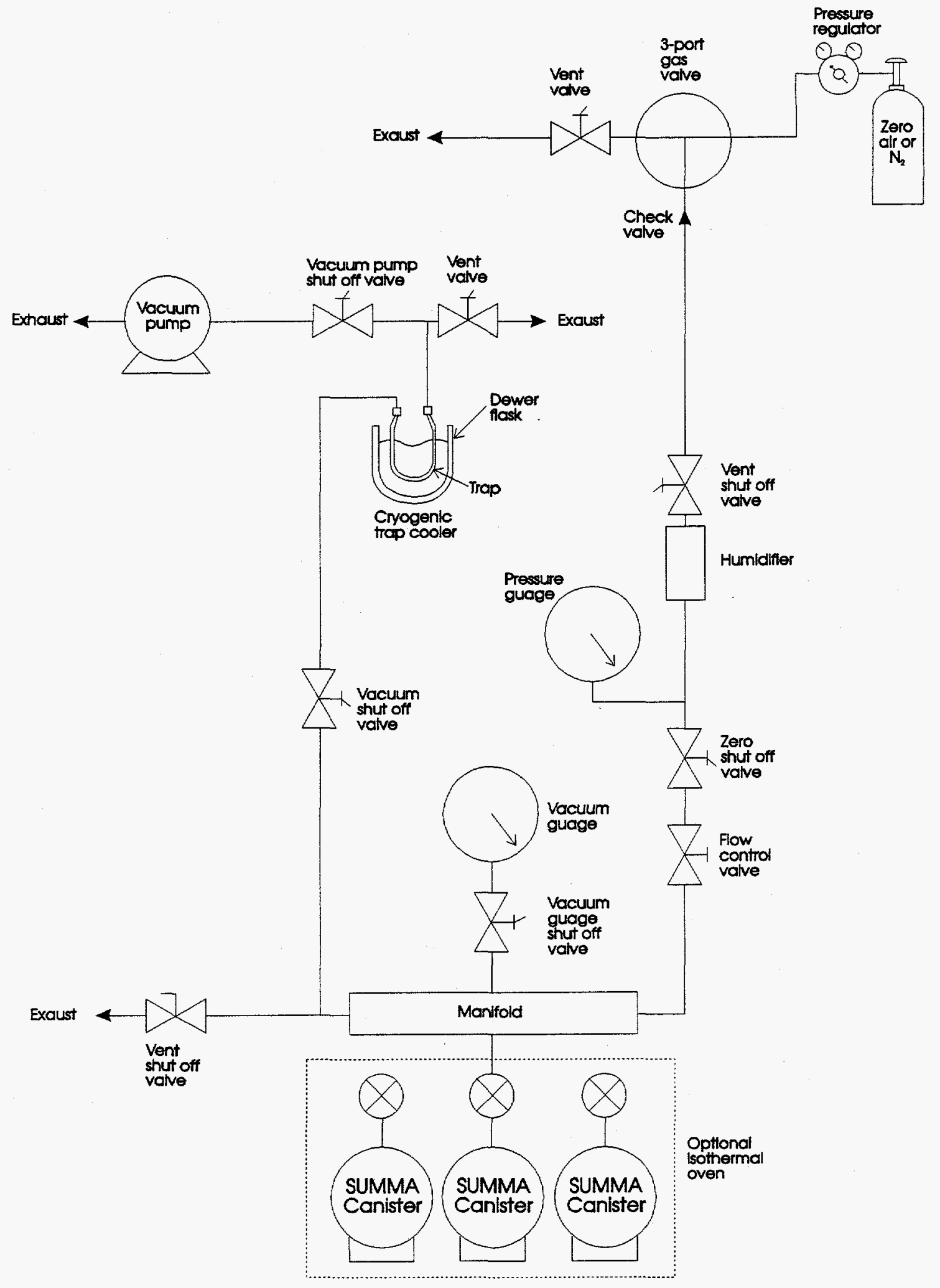

Figure 1. Canister Cleaning System 



\section{PHYSICAL WASTE FORM CHARACTERIZATION USING RADIOGRAPHY}

\subsection{Scope and Application}

1.1 Radiography is a non-destructive technique that involves $X$-ray scanning of waste containers. Radiography is used by DOE transuranic (TRU) waste generator/storage sites to demonstrate compliance with the Waste Acceptance Criteria for the Waste Isolation Pilot Plant (WIPP WAC) (DOE 1989) and with the requirements contained in "TRUPACT-II Authorized Methods for Payload Control (TRAMPAC)" (Nuclear Packaging Inc. 1989). In the WIPP TRU Waste Characterization Program (the Program), radiography will be used in conjunction with visual examination to determine the physical form of waste and the weight of the waste material parameters listed in Table 1. To achieve this, the inventories of waste container contents obtained by radiography should be as detailed as possible. This procedure must be implemented with a site-specific standard operating procedure (SOP).

1.2 In this procedure, radiography is used to evaluate the following: the waste inventory, TRUCON code, matrix parameter category, and/or Item Description Code (IDC), as appropriate; and the waste packaging configuration. This information is necessary for making weight estimates of the waste material parameters.

1.3 For the purposes of the TRU Waste Characterization Program, waste containers are to be characterized by radiography in testing batches. A testing batch is defined as suite of waste containers undergoing radiography using the same equipment. A testing batch can be up 20 waste containers without regard to waste matrix.

\subsection{Summary of Procedure}

Waste containers are moved into the shielded vault, $\mathrm{X}$-rays are projected through the container onto a fluorescent screen/image intensifier, and the resultant image is transferred by a camera to a remotely located television screen. A description of the contents of the waste container is audio/video recorded and documented on a data form. The description should clearly identify all discernable waste items, residual and packaging materials, and/or waste material parameters whenever possible so that waste can be classified according to the waste material parameters specified in Table 1. This classification based on radiography information will be confirmed by visual examination (Procedure 310.2 of this Methods Manual).

\subsection{Limitations and Interferences}

Containers of high density waste (e.g., leaded rubber, cemented sludges) can only be examined at their edges. In addition to this limitation, waste containers that are configured with a lead liner cannot be examined using radiography.

\subsection{Safety}

This procedure may involve the use of hazardous materials, operations, and equipment. It is the responsibility of whoever uses this procedure to consult appropriate site personnel concerning health and safety issues and establish appropriate health and safety practices. Consideration should be given to safety concerns regarding chemical and radiation exposure. Training regarding proper storage, usage, and disposal of chemicals is recommended. 
TABLE 1

Waste Material Parameters and Descriptions

Waste Material Parameter

Iron-based Metals/Alloys

Aluminum-based Metals/Alloys

Other Metals

Other Inorganic Materials

Cellulosics

Rubber

Plastics (waste materials)

Organic Matrix

Inorganic Matrix

Soils

Steel (packaging materials)

Plastics (packaging materials)
Description

Iron and steel alloys in the waste; does not include the waste container materials

Aluminum or aluminum-based alloys in the waste materials

All other metals found in the waste materials

Nonmetallic inorganic waste including concrete, glass, firebrick, ceramics, sand, and inorganic sorbents

Materials generally derived from high polymer plant carbohydrates; (e.g., paper, cardboard, wood, cloth)

Natural or man-made elastic Latex materials; (e.g., surgeons' gloves, leaded rubber gloves)

Generally man-made materials, often derived from petroleum feedstock; (e.g., polyethylene, polyvinylchloride)

Cemented organic resins, solidified organic liquids, and sludges

Any homogeneous materials consisting of sludge, or aqueousbased liquids which are solidified with cement, calcium silicate, or other solidification agents; (e.g., waste water treatment sludge, cemented aqueous liquids, and inorganic particulates)

Generally consists of naturally occurring soils which have been contaminated with inorganic waste materials

208-liter (55-gallon) drums

90 mil polyethylene drum liner and plastic bags

Source: Waste Isolation Pilot Plant Transuranic Waste Baseline Inventory Report (DOE 1995b). 


\subsection{Apparatus and Materials}

A typical radiography system consists of the following equipment and facilities:

- Shielded room that is properly ventilated and lighted

- X-ray head and associated equipment

- Drum turntable dolly assembly

- Fluoroscopic screen and accessories

- Closed-circuit television equipment and monitors

- Safety interlocks

- Data management system

\subsection{Preplanning}

Studies to characterize TRU waste require careful planning to prepare facilities, develop written documentation, secure laboratory support, and evaluate data.

\subsection{Reagents, Preservation Measures, and Handling}

All waste container handling and chain-of-custody procedures described in Section 6.0 of the Transuranic Waste Characterization Quality Assurance Program Plan (QAPP) (DOE 1995a) must be followed.

\subsection{Procedure}

The exact sequence of operations is determined by the equipment available at individual radiography facilities.

1. Waste containers selected for the Program (metal drums or boxes) are placed on a turntable/dolly and exposed to a collimated source of X-rays of sufficient intensity and beam geometry to permit a scan of container contents to be made for different orientations of the waste container. The contents of the waste container appear as a shadow image on a fluorescent screen that is in-line with the source of the $X$-rays and the waste container. The screen images resulting from different orientations of the waste container are recorded by videotaping the screen.

2. Drums are rotated to allow for total X-ray coverage, providing a quasi-three-dimensional image. However, boxes cannot be rotated during inspection; but are moved back and forth in front of the fluorescent screen to produce variation in the viewing angle to enhance waste identification. Boxes are first inspected from one side and then are rotated 180 degrees and are inspected from the other side.

3. During radiography, the waste container and its contents are examined, and a description of the container contents and packaging materials (e.g., container liner) is recorded according to written procedures. The description is detailed enough to determine whether the waste is composed of a single component (i.e., well-segregated/homogeneous) and to determine whether the TRUCON code, matrix parameter category, and/or IDC, as appropriate, has been properly assigned to the container. During this part of the examination, specify the height and shape of the waste in the container so that the volume utilization percentage can be determined.

4. Jog the container by starting and stopping the turntable/dolly. After jogging the container, look for wave motion, a sign that liquids are present. Describe the location, container, and estimated volume of any liquids detected. 
5. Every effort should be made to provide an inventory of the waste container contents. This inventory should include a description of identifiable waste items, residual materials, packaging materials, and/or waste material parameters. When possible, count the number of identical waste items present and estimate the amount of residual and packaging materials. Record this description of the waste container inventory and specific waste container identification data on the audio/videotape and on the radiography data form. It is understood that the amount of inventory information obtained by radiography will be limited when a heterogeneous waste form is examined.

6. The identities of the waste items, residual materials, packaging materials, and/or waste material parameters documented on the audio/videotape and on the radiography data form are used in combination with a list of standard waste material weights (e.g., Table 2) to estimate the waste item and the weight of residual materials, packaging materials, and/or waste material parameters. Sites should develop a source of standard weights (an expansion of Table 2) based on knowledge of the waste and the process that generated it. Manufacturer's data or information from visual examination may also be used for this purpose.

7. The inventory and description of waste items, residual materials, packaging materials are used to classify the waste into waste material parameters and to estimate the total weight of waste comprising each of the waste material parameters.

8. The utilized waste container volume percentage is estimated based on the highest point and shape of waste in a waste container. Figure 1 illustrates a typical waste drum and associated utilization volume percentage according to its height (assuming a flat top) within the drum. Individual sites must qualify when these percent values will not hold due to the presence of certain packaging materials (e.g., presence of fiber packs).

9. Table 3 is provided as a reference to aid in determining liquid quantities. Table 4 provides a list of radiography lens magnifications to aid in determining the physical size of waste items.

\subsection{Quality Control}

9.1 Quality control during radiography operations is assured by operator training, equipment standards, replicate scans, and subsequent independent visual examination of the waste to verify radiography results (see Procedure 310.2 of this Methods Manual).

9.2 Operator training and experience are the most important considerations for assuring the quality of the radiography data and for interpretation and disposition of radiography results. Only trained personnel are allowed to operate radiography equipment. Standardized training requirements for radiography operators are based upon existing industry standard training requirements and comply with the training and qualification requirements of ASME NOA-1. Element 2, except for Supplement 2S-2 (ASME 1994). Requalification of operators is based upon evidence of continued satisfactory performance (primarily tape reviews) and is done at least every two years. Unsatisfactory performance results in disqualification. Retraining and demonstration of satisfactory performance are required before an operator is again qualified to operate the radiography system. It is the responsibility of each participating site to determine the criteria by which an operator is considered qualified.

9.3 The radiography equipment is tuned precisely enough to allow an radiography operator to resolve a 2-2T hole in a steel block. The quality of the radiography image is also assessed by viewing an image test pattern. 
TABLE 2

\section{Example Weights of Package Materials ${ }^{a}$}

Item

Weight $^{\mathrm{b}}(\mathrm{kg})$

208 L drum (17C)

28

90 mil liner (208 L)

7.3

Drum plastic bag (208 L)

1.8

Box fiberboard liner $(122 \times 122 \times 213 \mathrm{~cm})$

Box plastic liner $(122 \times 122 \times 213 \mathrm{~cm})$

12

Lead Brick $(5.1 \times 10 \times 20 \mathrm{~cm})^{\mathrm{c}}$

Lead Lined Drum: ${ }^{c}$

(0.32 $\mathrm{cm}$ thick, $71 \mathrm{~cm}$ high by $180 \mathrm{~cm}$ long)

aThis list is not intended to be comprehensive. Sites are expected to develop their own table based on knowledge of the wastes and the processes that generate them.

${ }^{b}$ Rocky Flats Plant

${ }^{\mathrm{c}} \rho \mathrm{Pb}=11.34 \mathrm{~g} / \mathrm{cm}^{3}$ 
TABLE 3

Volume Conversions

a. Volume of Spheres

Radius

inches

centimeters

quarts

Volume ${ }^{a}$

\begin{tabular}{cccc} 
inches & centimeters & quarts & milliliters \\
\hline & & & 9.2 \\
1.0 & 1.3 & 0.01 & 65 \\
2.0 & 2.5 & 0.07 & 560 \\
3.0 & 5.1 & 0.58 & 1800 \\
\hline
\end{tabular}

Volume $=4 / 3 \pi r^{3}$

b. Volume of Half Spheres

Radius

inches

0.5

1.0

2.0

3.0

4.0
Radius

centimeters
Volume $^{b}$

quarts milliliters
0.005
0.035
0.29
0.95
2.3

4.6

32.5

280

900

2200

bolume $=2 / 3 \pi r^{3}$

c. Volume of Cylinders ${ }^{\mathrm{c}}$

\begin{tabular}{|c|c|c|c|c|}
\hline \multicolumn{5}{|c|}{ Radius (inches/cm) } \\
\hline (inches $/ \mathrm{cm}$ ) & $0.5 / 1.3$ & $1.0 / 2.5$ & $2.0 / 5.1$ & $3.0 / 7.6$ \\
\hline $\begin{array}{l}0.50 / 1.3 \\
1.0 / 2.5 \\
2.0 / 5.1 \\
3.0 / 7.6 \\
4.0 / 10\end{array}$ & $\begin{array}{l}0.007 / 6.9 \\
0.014 / 13 \\
0.029 / 27 \\
0.043 / 40 \\
0.056 / 53\end{array}$ & $\begin{array}{l}0.01 / 9.8 \\
0.052 / 49 \\
0.11 / 100 \\
0.16 / 150 \\
0.21 / 200\end{array}$ & $\begin{array}{l}0.11 / 110 \\
0.22 / 200 \\
0.44 / 420 \\
0.66 / 620 \\
0.86 / 820\end{array}$ & $\begin{array}{l}0.25 / 240 \\
0.48 / 450 \\
0.98 / 930 \\
1.5 / 1400 \\
1.9 / 1800\end{array}$ \\
\hline ume $=$ & & & & \\
\hline$R-4376$ & & 310.1 & & \\
\hline
\end{tabular}


TABLE 4

Typical Lens Magnification Factors

Lens

(mm)

Near Side

Middle

Far Side

55-gal Drums

$\begin{array}{llll}24 & 0.4 & 0.5 & 0.6 \\ 35 & 0.5 & 0.65 & 0.8 \\ 50 & 0.8 & 1.0 & 1.2 \\ 60 & 1.2 & 1.45 & 1.7 \\ 85 & 1.5 & 1.9 & 2.3\end{array}$

Boxes

$\begin{array}{llll}24 & 0.4 & 0.6 & 1.8 \\ 35 & 0.6 & 0.9 & 2.5 \\ 50 & 0.9 & 1.3 & 3.5 \\ 60 & 1.3 & 2.2 & 6.2 \\ 85 & 1.65 & 2.5 & 7.0\end{array}$

SOURCE: "RWMC/SWEPP Programs; SWEPP Certified Waste Sampling Program" (EG\&G 1989). 
9.4 Independent replicate scans are performed on one out of every 20 drums. In addition, supervisory personnel (also trained and qualified to the same standards) perform periodic tape reviews of accepted waste containers.

9.5 During the Program, radiography results are verified by visual examination (Procedure 310.2 of this Methods Manual).

\subsection{Procedure Performance}

Table 5 presents the results of a comparison of radiography and visual examination (Procedure 310.2 of this Methods Manual) determined weights of waste materials similar to the waste material parameters determined by this characterization method. Table 5 illustrates that radiography can provide a reasonable weight estimate for waste materials composed of plastic, rubber, steel, non-corroding metal, and solid inorganic wastes. However, radiography does not provide good weight estimates for waste materials composed of cellulosics and aluminum. The relative percent difference (RPD) was determined as follows:

$$
R P D=\frac{C_{1}-C_{2}}{\frac{\left(C_{1}+C_{2}\right)}{2}} * 100
$$

where:

$$
\begin{array}{lll}
C_{1} \text { and } C_{2} & =\quad \text { two values determined by the different procedures } \\
C_{1} & =\quad \text { the larger of the two values }
\end{array}
$$

\subsection{References}

ASME. 1994. Quality Assurance Program Requirements for Nuclear Facility Applications. ASME-NOA-1-1994 Edition, New York, New York, American Society of Mechanical Engineers.

DOE. 1989. Waste Acceptance Criteria for the Waste /solation Pilot Plant. DOEMIPP-069, Revision 3, Carlsbad, New Mexico, Waste Isolation Pilot Plant, U.S. Department of Energy.

DOE. 1995a. Transuranic Waste Characterization Quality Assurance-Program Plan. CAO-94-1010, Carlsbad, New Mexico, Carlsbad Area Office, U.S. Department of Energy.

DOE. 1995b. Waste Isolation Pilot Plant Transuranic Waste Baseline Inventory Report. CAO-94-1005, Carlsbad, New Mexico, Carlsbad Area Office, U.S. Department of Energy.

EG\&G. 1989. "RWMC/SWEPP Programs; SWEPP Certified Waste Sampling Program." Engineering Design File RWMC, 363, Revision 1, 1989. Idaho Falls, Idaho, EG\&G Idaho, Inc., Idaho National Engineering Laboratory, U.S. Department of Energy.

Nuclear Packaging Inc. 1989. "TRUPACT-Il Authorized Methods for Payload Control." Safety Analysis Report for the TRUPACT-II Shipping Package, Revision 4, Appendix 1.3.7, Federal Way, Washington, Nuclear Packaging, Inc. 
TABLE 5

Results of Radiography Versus Visual Examination (at the Idaho National Engineering Laboratory)

\begin{tabular}{lcccr}
\hline & $\begin{array}{c}\text { Number of } \\
\text { Drums }\end{array}$ & $\begin{array}{c}\text { Weight Determined } \\
\text { by Radiography } \\
(\mathrm{kg})\end{array}$ & $\begin{array}{c}\text { Weight Determined } \\
\text { by Visual } \\
\text { Examination } \\
(\mathrm{kg})\end{array}$ & $\begin{array}{c}\text { Relative } \\
\text { Percent } \\
\text { Difference }\end{array}$ \\
\hline Cellulosics & 33 & 3.1 & 1.6 & 64 \\
Plastics & 33 & 6.2 & 6.0 & 3 \\
Rubber & 19 & 6.5 & 5.5 & 17 \\
Corroding Metal - Steel & 33 & 10.4 & 9.6 & 8 \\
Corroding Metal - & 10 & 11.8 & 5.2 & 77 \\
Aluminum & 19 & 6.5 & 6.8 & 4 \\
Non-Corroding Metal & 33 & 22.5 & 21.9 & 3 \\
Solid Inorganic Wastes & & & & 3 \\
\hline
\end{tabular}

SOURCE: Personal Communication with Dr. Michael Connolloy, Idaho National Engineering Laboratory, Idaho Falls, Idaho. 


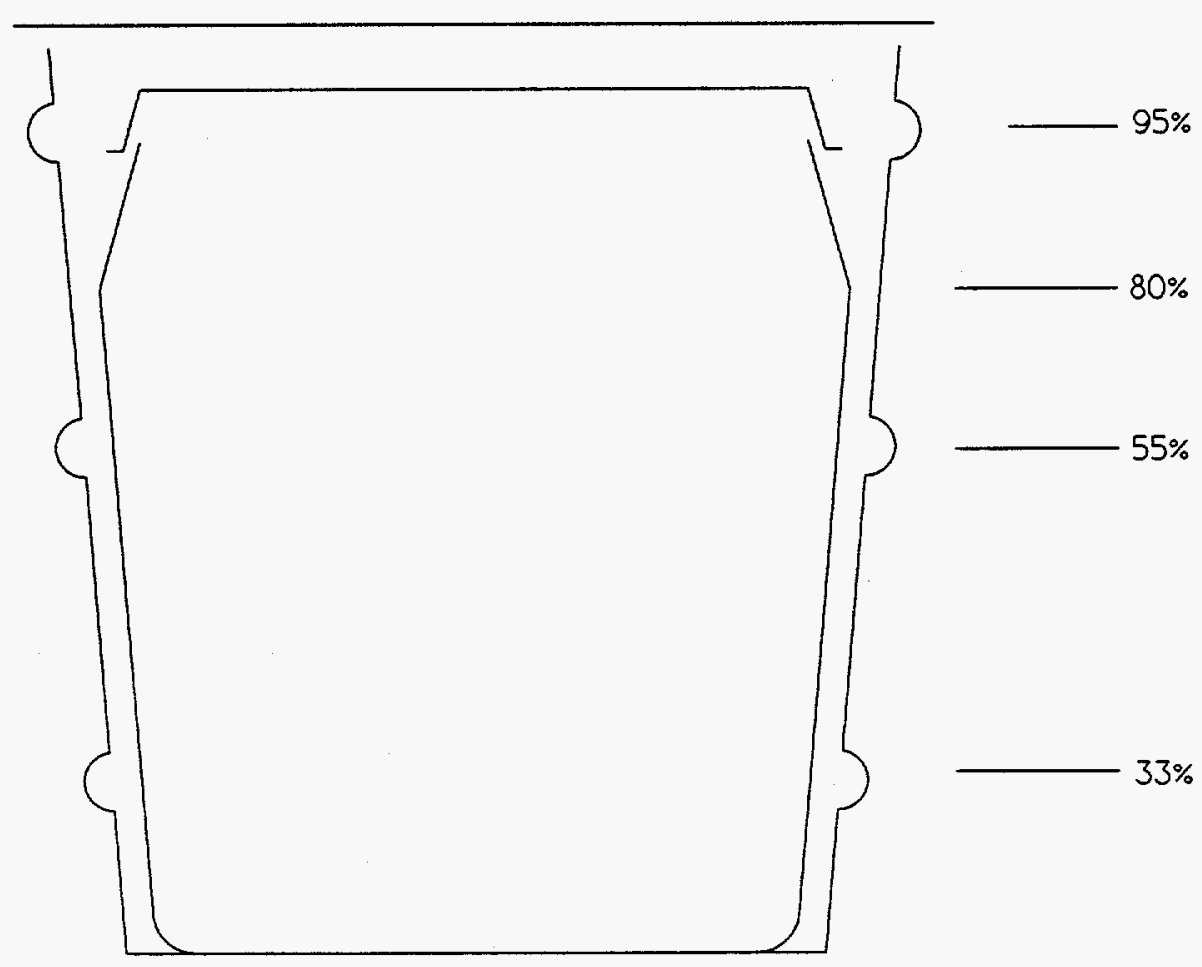

Volume utilization percentage to be based on the highest point of waste in drum (Source: "RWMC/SWEPP Programs; SWEPP Certified Waste Sampling Program." (EG\&G 1989))

FIGURE 1

\section{Volume Utilization Percentage}


PROCEDURE 310.2

\section{PHYSICAL WASTE FORM CHARACTERIZATION USING VISUAL EXAMINATION}

\subsection{Scope and Application}

1.1 This procedure for the visual examination of the contents of a waste container is designed to provide data on the type and amount of material in each of the waste containers included in the TRU Waste Characterization Program (the Program). This procedure is used as a quality control check for "Physical Waste Form Characterization Using Radiography" (Procedure 310.1 of this Methods Manual). The waste material parameters of interest for compliance activities are listed in Table 1. The estimated or measured weight of waste within these waste material parameters are determined using this procedure. This procedure must be implemented with a site-specific standard operating procedure (SOP).

1.2 The results of visual examination will be included in the testing batch data reports for radiography. For the purposes of the TRU Waste Characterization Program, waste containers are to be characterized by radiography in testing batches. A testing batch is defined as suite of waste containers undergoing radiography using the same equipment. A testing batch can be up 20 waste containers without regard to waste matrix.

\subsection{Summary of Procedure}

The transuranic (TRU) waste that is designated for the Program is visually examined, weights determined or estimated, and video recorded. A description and estimated or measured weights of the contents of each waste container is also recorded on audio/video tape and a visual examination data form. The description should clearly identify all discernible waste items, residual materials, packaging materials, or waste material parameters so that all the waste in each container can be classified according to the waste material parameters listed in Table 1. Technical experts who are experienced and trained waste operations personnel are expected to assess the need to open individual bags or packages of waste. Opening individual bags/packages may be necessary in order to ensure the quality of the examination data. If individual bags/packages are not opened, estimated weights of waste items, residual materials, packaging materials, or waste material parameters within the bags/packages are recorded. If it is necessary to open individual bags/packages, then actual weights of waste items, residual materials, packaging materials, or waste material parameters are recorded. Figure 1 illustrates the overall programmatic approach to visual characterization of the waste.

\subsection{Limitations and Interferences}

It may not be possible to see through the inner plastic bags/packages of waste because of discoloration, grease and dust adhering to the interior surface of the bags/packages or because of sealed and taped containers within the bags/packages. In these cases, knowledge of the waste materials and the processes that generate them, and/or technical experts should assess the need to open the bags/packages in order to identify all of their contents. Whether bags/ packages are opened or not, it is important that the weight estimates be the best possible values.

\subsection{Safety}

This procedure may involve the use of hazardous materials, operations, and equipment. It is the responsibility of whoever uses this procedure to consult appropriate site personnel concerning health and safety issues and establish appropriate health and safety practices. Consideration should be given to safety concerns regarding chemical and radiation exposure. Training regarding proper storage, usage, and disposal of chemicals is recommended. 
TABLE 1

Waste Material Parameters and Descriptions

Waste Material Parameter

Iron-based Metals/Alloys

Aluminum-based Metals/Alloys

Other Metals

Other Inorganic Materials

Cellulosics

Rubber

Plastics (waste materials)

Organic Matrix

Inorganic Matrix

Soils

Steel (packaging materials)

Plastics (packaging materials)

\section{Description}

Iron and steel alloys in the waste; does not include the waste container materials

Aluminum or aluminum-based alloys in the waste materials

All other metals found in the waste materials

Nonmetallic inorganic waste including concrete, glass, firebrick, ceramics, sand, and inorganic sorbents

Materials generally derived from high polymer plant carbohydrates; (e.g., paper, cardboard, wood, cloth)

Natural or man-made elastic Latex materials; (e.g., surgeons' gloves, leaded rubber gloves)

Generally man-made materials, often derived from petroleum feedstock; (e.g., polyethylene, polyvinylchloride)

Cemented organic resins, solidified organic liquids, and sludges

Any homogeneous materials consisting of sludge, or aqueousbased liquids which are solidified with cement, calcium silicate, or other solidification agents; (e.g., waste water treatment sludge, cemented aqueous liquids, and inorganic particulates)

Generally consists of naturally occurring soils which have been contaminated with inorganic waste materials

208-liter (55-gallon) drums

90 mil polyethylene drum liner and plastic bags

Source: Waste Isolation Pilot Plant Transuranic Waste Baseline Inventory Report (DOE 1995b). 


\subsection{Apparatus and Materials}

Participating sites develop radiation containment facilities and provide the following equipment:

- Drum, waste bag, and waste handling equipment

- Video cameras and audio equipment

- Mass balances and calibration standards

- Bag opening unit

- Data input station

- Safety equipment

\subsection{Preplanning}

Studies to characterize TRU waste require careful planning to prepare facilities, develop written documentation, secure laboratory support, and evaluate data.

\subsection{Reagents, Preservation Measures, and Handling}

All waste container handling and chain-of-custody procedures described in Section 6.0 of the Transuranic Waste Characterization Quality Assurance Program Plan (QAPP) (DOE 1995a) must be followed.

\subsection{Procedure}

1. Before beginning the visual examination, review all documented data related to the waste container and its contents. However, since visual examination is a quality control check of radiography results, radiography results should not be part of this review. If the available information is judged by a technical expert to be comprehensive, then the extent of the visual examination may be limited. In some cases, a decision to open all bags/packages in a waste container of a particular TRUCON code, matrix parameter category, and/or IDC, as appropriate, may be made in advance.

2. After this initial assessment, remove the lid from the waste container land the 90 mil rigid liner lid, if present) and estimate the waste container volume utilization percentage. Next, open the poly liner(s) bag(s) and document the number of liners and their type. Remove the individual inner bags/packages, if present, from the poly liner(s). Label and weigh each inner bag/package using properly calibrated mass balances. Taking care to ensure that the video camera is properly focused, begin the visual examination of an unopened inner bag/package while recording the operator's verbal description of the inner bag/package's inventory.

3. This inventory should include a description of all waste items, residual materials, packaging materials, and/or waste material parameters contained in the inner bag/package. Estimated weights of the waste items, residual materials, packaging materials, and/or waste material parameters should also be recorded, both on the audiotape and on the visual examination data form. Reference tables such as Table 2 and 3 should be developed to assist the operator in making weight estimates and assigning waste to a particular waste material parameter. These tables should be updated and refined by DOE site personnel as the Program proceeds.

4. If the identity of waste items, residual materials, packaging materials, and/or waste material parameters cannot be ascertained through the unopened bag/package, then technical experts decide if the inner bag/package must be opened and document their rationale. Technical experts also assess the accuracy of the TRUCON code, matrix parameter category, and/or IDC, as appropriate, and recommend changes, if necessary. 
TABLE 2

Example Weights of Package Materials"

Item

Weight $^{\mathrm{b}}(\mathrm{kg})$

$208 \mathrm{~L}$ drum $(17 \mathrm{C})$

90 mil liner $(208 \mathrm{~L})$

7.3

Drum plastic bag (208 L)

1.8

Box fiberboard liner $(122 \times 122 \times 213 \mathrm{~cm})$

Box plastic liner $(122 \times 122 \times 213 \mathrm{~cm})$

Lead Brick $(5.1 \times 10 \times 20 \mathrm{~cm})^{\circ}$

Lead Lined Drum: ${ }^{\circ}$

$10.32 \mathrm{~cm}$ thick, $71 \mathrm{~cm}$ high by $180 \mathrm{~cm}$ long)

$10.16 \mathrm{~cm}$ thick, $71 \mathrm{~cm}$ high by $180 \mathrm{~cm}$ long)

aThis list is not intended to be comprehensive. Sites are expected to develop their own table based on knowiedge of the wastes and the processes that generate them.

${ }^{\text {bRocky Flats Plant }}$

${ }^{\mathrm{c}} \mathrm{pPb}=11.34 \mathrm{~g} / \mathrm{cm}^{3}$ 
TẢBLE 3

Volume Conversions

a. Volume of Spheres

\begin{tabular}{cccc}
\hline & & & \\
Radius & & & Volume $^{\text {a }}$ \\
inches & centimeters & quarts & millititers \\
& & & \\
& & & \\
0.5 & 1.3 & 0.01 & 9.2 \\
1.0 & 2.5 & 0.07 & 560 \\
2.0 & 5.1 & 0.58 & 1800 \\
3.0 & 7.6 & 1.9 & \\
\hline
\end{tabular}

- Volume $=4 / 3 \pi \mathrm{r}^{3}$

b. Volume of Half Spheres

Radius

inches

centimeters

Volume $^{b}$

quarts

milliliters

$\begin{array}{rrrr}0.5 & 1.3 & 0.005 & 4.6 \\ 1.0 & 2.5 & 0.035 & 32.5 \\ 2.0 & 5.1 & 0.29 & 280 \\ 3.0 & 7.6 & 0.95 & 900 \\ 4.0 & 10 & 2.3 & 2200\end{array}$

bolume $=2 / 3 \pi r^{3}$

c. Volume of Cylinders ${ }^{\circ}$

Radius (inches $/ \mathrm{cm}$ )

Height

(inches $/ \mathrm{cm}$ )

$0.5 / 1.3$

$1.0 / 2.5$

$2.0 / 5.1$

$3.0 / 7.6$

\title{
$0.50 / 1.3$ \\ $1.0 / 2.5$ \\ 2.0/5.1 \\ $3.0 / 7.6$ \\ $4.0 / 10$
}

$0.007 / 6.9$

$0.014 / 13$

$0.029 / 27$

$0.043 / 40$

$0.056 / 53$

\begin{abstract}
$0.01 / 9.8$
$0.052 / 49$

$0.11 / 100$

$0.16 / 150$

$0.21 / 200$
\end{abstract}

$0.11 / 110$

$0.22 / 200$

$0.44 / 420$

$0.66 / 620$

$0.86 / 820$

$0.25 / 240$

$0.48 / 450$

$0.98 / 930$

$1.5 / 1400$

$1.9 / 1800$

${ }^{c}$ Volume $=\pi r^{2} h$ (quarts $/ \mathrm{mL}$ ) 
Those inner bags/packages that require opening are examined and their contents sorted, weighed, and recorded. An inventory of loose waste items, residual materials, packaging materials, and/or waste material parameters not contained in inner bags/packages is also recorded, and the waste sorted and weighed. If liquids are encountered at any time during the visual examination, then a description of their location, container, and estimated volume is recorded. Table 3 is provided as a reference to aid in determining liquid quantities by determining the volume of the containers in which they occur.

5. Once the contents of the waste container have been removed and examined, the weight of the empty waste container and the weight of its rigid poly liner, if present, is determined and recorded. The gross weight of the waste container (container plus contents) is recorded on the visual examination data form. The total number of bags/packages is also recorded on the data form.

6. The inventory of waste items, residual materials, packaging materials, and/or waste material parameters is used in conjunction with knowledge of the wastes and the processes that generate them to classify the waste container contents into the waste material parameters listed in Table 1. As much discrimination as possible between materials in a particular waste material parameter should be attempted. However, for purposes of the Program, only the total weight $(\mathrm{kg})$ of material within each of the twelve waste material parameters is required.

\subsection{Quality Control}

9.1 A specific waste recognition training program for each site is used to assess operator performance before actual waste characterization by visual examination. This training program employs exercises such as evaluation of simulated waste inon-radioactive materials), review of audio/videotapes of previous waste handling operations, including radiography, and familiarization with estimating and weighing techniques. Standard nomenclature is established, based on current site practice, to assure that all operators recognize waste by the same descriptors.

9.2 Standard error calculation procedures are developed with the concurrence of Sandia National Laboratories. Participating sites should coordinate these efforts to assure maximum consistency.

9.3 Replicate weight measurements are performed and used as a measure of the precision of the weighing process. One in twenty, or at least one waste item, residual and packaging material, or waste from a single waste material parameter from each drum, is reweighed after all other drum contents are weighed.

9.4 Mass balances, spanning a range of weights from $10 \mathrm{~g}$ to $450 \mathrm{~kg}(1000 \mathrm{lbs})$, are checked prior to use and calibrated annually. The weighing system is calibrated using standards traceable to the National Institute of Standards and Technology (NIST).

9.5 Other equipment used during visual examination includes audio/video recording apparatus. Checks of this equipment include observation of test patterns, prior to each day's use, to ensure that the video picture and record meet minimum quality requirements.

9.6 Two operators concur on the results of the visual examination by signing the data form.

10.0 Procedure Performance

Table 4 presents the results of a comparison of visual examination and radiography (Procedure 310.1 of this Methods Manual) determined weights of waste materials similar to the 
TABLE 4

Results of Radiography Versus Visual Examination (at the Idaho National Engineering Laboratory)

Waste Material

Number of

Drums
Weight Determined

by Visual

Examination

( $\mathrm{kg})$
Relative

Percent

Difference

\begin{tabular}{lrrrr}
\hline Cellulosics & 33 & 3.1 & 1.6 & 64 \\
Plastics & 33 & 6.2 & 6.0 & 3 \\
Rubber & 19 & 6.5 & 5.5 & 17 \\
Corroding Metal - Steel & 33 & 10.4 & 9.6 & 8 \\
Corroding Metal - & 10 & 11.8 & 5.2 & 77 \\
Aluminum & 19 & 6.5 & 6.8 & 4 \\
Non-Corroding Metal & 33 & 22.5 & 21.9 & 3 \\
Solid Inorganic Wastes & & & & \\
\hline
\end{tabular}

SOURCE: Personal Communication with Dr. Michael Connolloy, Idaho National Engineering Laboratory, Idaho Falls, Idaho. 
waste material parameters determined by, this characterization method. Table 4 illustrates that visual examination provides a lower weight estimate for waste materials composed of cellulosics and aluminum. The weights estimated by visual examination of other waste materials were similar to those determined by radiography. The relative percent difference (RPD) between the reported weights was determined as follows:

$$
R P D=\frac{C_{1}-C_{2}}{\frac{\left(C_{1}+C_{2}\right)}{2}} * 100
$$

where

$$
\begin{array}{lll}
C_{1} \text { and } C_{2} & =\text { two values obtained by the different procedures } \\
C_{1} & = & \text { larger of the two obtained values }
\end{array}
$$

\subsection{References}

DOE. 1995a. Transuranic Waste Characterization Quality Assurance Program Plan. CAO-94-1010, Carlsbad, New Mexico, Carlsbad Area Office, U.S. Department of Energy.

DOE. 1995b. Waste Isolation Pilot Plant Transuranic Waste Baseline Inventory Report. CAO-94-1005, Carlsbad, New Mexico, Carlsbad Area Office, U.S. Department of Energy. 


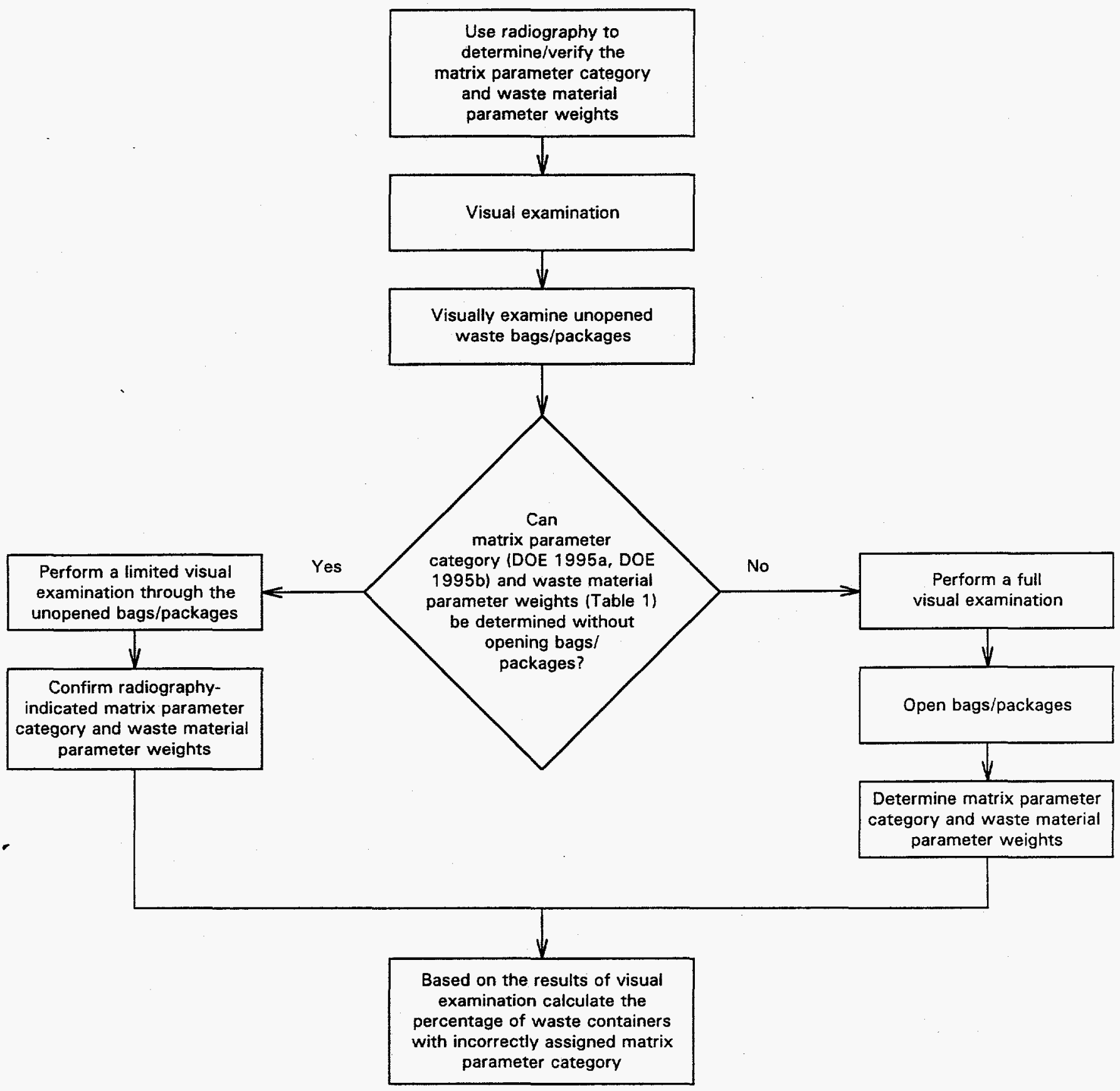

FIGURE 1

Overall Approach to Visual Examination of the Waste 

PROCEDURE 430.1

\author{
MODIFIED METHOD TO-14 FOR \\ THE GAS CHROMATOGRAPHY/MASS SPECTROMETRY \\ DETERMINATION OF VOLATILE ORGANIC COMPOUNDS \\ IN WASTE CONTAINER HEADSPACE
}

\title{
1.0 Scope and Application
}

1.1 This procedure is for the analysis of volatile organic compounds (VOCs) listed in Table 1 in the range from $1 \mathrm{ppmv}$ (ppm, volume/volume) to volume percent, that have been collected from waste container headspace using SUMMA ${ }^{\oplus}$ passivated canisters. Table 1 also lists the quality assurance objectives for this procedure. This procedure is based on the Environmental Protection Agency (EPA) "Compendium Method TO-14, The Determination of Volatile Organic Compounds (VOCs) in Ambient Air Using SUMMA Passivated Canister Sampling and Gas Chromatographic Analysis" (EPA 1988a), and modifications made to this procedure by the EPA Region IV laboratory (Stephenson et al. 1990). This procedure is also based on Method 8240A, "Volatile Organics by Gas Chromatography/Mass Spectrometry (GC/MS)" found in Test Methods for Evaluating Solid Waste, Physical/Chemical Methods, SW-846, Third Edition, Final Update I (SW-846)(EPA 1995). The procedures described in this document for the analysis of VOCs collected in SUMMA ${ }^{\circledR}$ canisters restrict the analytical options to Gas Chromatography/Mass Spectrometry (GC/MS). The sampling procedures are described in Procedure 110.1 through 110.4 of this Methods Manual. This procedure must be implemented with a sitespecific standard operating procedure (SOP).

1.2 The modifications to Method TO-14 described in this procedure are designed to enable the analyst to have more flexibility when analyzing high concentrations of VOCs (>1 ppmv), and the ability to analyze polar compounds (alcohols and ketones). Modifications included in this procedure are those developed and implemented by the EPA Region IV laboratory (Stephenson et al. 1990). These modifications include using internal standards and solid sorbent traps. Solid sorbent trapping is achieved using commercially available purge-andtrap systems (Tekmar LSC-2 or equivalent). Solid sorbent traps containing only hydrophobic materials (activated carbon and carbon molecular sieves) allow the use of a dry purge cycle to remove trapped water from the trap prior to thermal desorption of trapped VOCs. Nafion dryers will not be used to remove water prior to the trapping of VOCs because of the possibility of removing polar compounds.

1.3 The procedures described herein are applicable to the VOCs specified in Table 1. The EPA has not determined the stability of alcohols and ketones when stored in pressurized or subambient SUMMA ${ }^{\oplus}$ canisters. It is anticipated that no adverse problems will be encountered with these types of VOCs when stored in SUMMA ${ }^{\circ}$ canisters due to the concentration levels at which these VOCs are expected to be found.

1.4 This procedure is based upon cryogenic or solid sorbent trapping followed by GC/MS analysis and should be restricted to use by or under the supervision of analysts experienced in these areas. In addition, analysts should be skilled in the interpretation of mass spectra and their use as a quantitative tool.

1.5 The program required quantitation limits (PROLs) specified in Table 1 are based on a $10 \mathrm{~mL}$ sample aliquot. PROLs will be proportionally higher for samples that require dilution or reduced sample size to avoid instrument saturation. 
TABLE 1

Gas Volatile Organic Compounds Target Analyte List and Quality Assurance Objectives

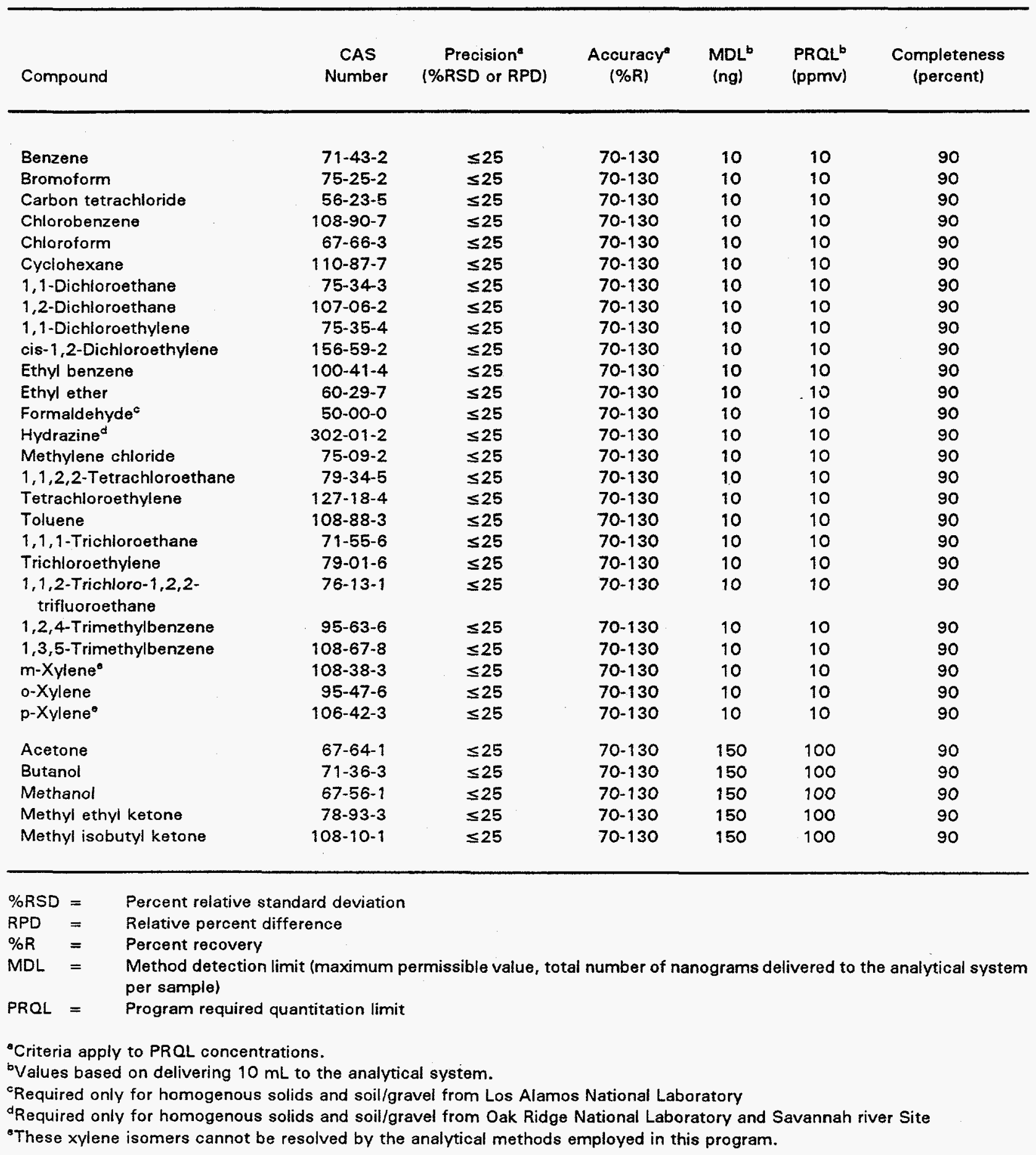


1.6 For the purposes of the TRU Waste Characterization Program, samples are to be analyzed in analytical batches. An analytical batch is defined as a suite of samples that is processed as a unit, using the same analytical method, within a specific time period. An analytical batch can be up to 20 samples (excluding laboratory $Q \mathrm{C}$ samples), all of which must be received by the laboratory within 14 days of the validated time of sample receipt (VTSR) of the first sample in the batch.

\subsection{Summary of Procedure}

2.1 Upon receipt at the laboratory, a canister containing a sample is attached to the sample manifold (Figure 1). The sample is then diluted, either by direct pressurization or the transfer of an aliquot to an evacuated laboratory canister, and pressurized. The canister containing the diluted sample is attached to the analytical system (Figure 2). The VOCs are then concentrated by collection in a cryogenically cooled trap or in a solid sorbent trap. The temperature of the trap is raised and the VOCs are desorbed into the gas chromatograph. The VOCs are separated on a widebore ( $\geq 0.53 \mathrm{~mm}$ I.D.) capillary column and detected using a mass spectrometer operating in the full scan mode. The combination of GC/MS allows for the qualitative and quantitative analysis of the VOCs contained in the SUMMA ${ }^{\circ}$ canister. The chromatographic conditions as well as typical mass spectrometer operating parameters are given in this procedure.

2.2 If a cryogenic trap is used, the trap is cooled with a cryogen and the gas sample is metered through the trap. The VOCs are condensed out and concentrated in the trap. The cryogen is then removed and the trap temperature is rapidly raised to revolatilize the retained VOCs directly onto the gas chromatographic column. When a solid sorbent trap is used, the gas sample is metered through the solid sorbent where the VOCs are trapped. The solid sorbent trap is backflushed with inert gas to remove water and, after this dry purge cycle, the trap is heated and backflushed with inert gas to desorb the VOCs onto the gas chromatographic column.

\subsection{Interferences}

3.1 Contamination may occur in the analytical system if the canisters are not properly cleaned prior to use. Instructions for cleaning the canisters are described in Procedure 210.1 of this Method's Manual.

3.2 Cross-contamination can occur whenever high-concentration and low-concentration samples are analyzed sequentially. Whenever a high-concentration sample is analyzed, it should be followed by the analysis of a blank of ultra pure air or nitrogen.

3.3 The laboratory where $\mathrm{VOC}$ analysis is performed should be free of solvents.

3.4 In the analytical system, the use of non-Teflon plastic coating, non-Teflon thread sealants, or flow controllers with rubber components should be avoided.

\subsection{Safety}

This procedure may involve the use of hazardous materials, operations, and equipment. It is the responsibility of whoever uses this procedure to consult appropriate site personnel concerning health and safety issues and establish appropriate health and safety practices. Consideration should be given to safety concerns regarding chemical and radiation exposure. Training regarding proper storage, usage, and disposal of chemicals is recommended.

Samples and reference materials may be flammable. Keep away from heat, sparks, and open flame. Provide adequate ventilation. 


\subsection{Apparatus and Materials}

\subsection{Analytical System.}

\subsubsection{GC/MS System:}

5.1.1.1 Gas Chromatograph: Capable of sub-ambient to $>160^{\circ} \mathrm{C}$ oven temperature programming. Optional capabilities for direct injection of liquid standards onto megabore columns. (Hewlett Packard, Model 5890 or equivalent).

5.1.1.2 Column: 30 meter $\times 0.53 \mathrm{~mm}$ I.D. DB-624 (J\&W Scientific) or equivalent.

5.1.1.3 Mass Spectrometer: Capable of scanning from 29-260 amu every 3 seconds or less, using 70 volts (nominal) electron energy in the electron impact mode and producing a mass spectrum that meets all the criteria listed in Table 2 when $50 \mathrm{ng}$ of 4-bromofluorobenzene (BFB) are injected through the gas chromatograph inlet.

5.1.1.4 GC/MS Interface: Any GC-to-MS interface that gives acceptable calibration data at the PROLs specified in Table 1 for all analytes and achieves all acceptable instrument performance criteria (Table 2). Interfaces should be constructed of all glass materials or glass-lined materials.

5.1.1.5 Data System: A data system that allows the continuous acquisition and storage of all mass spectra obtained throughout the duration of the chromatographic program must be interfaced to the mass spectrometer. The data system must have software that allows the searching of a GC/MS data file for ions of a specified mass and plotting such ion abundances versus time or scan number. This type of plot is defined as an Extracted lon Current Profile (EICP). Software must also be available that allows integrating the abundances in any EICP between specified time or scan-number limits. Data reduction software must be available that enables the qualitative and quantitative determination of target analytes using user-built mass spectral libraries and response factors based on EICP peak areas. The data system must be capabie of forward library searching of the most recent NBS mass spectral library.

\subsubsection{Inlet/Trapping System: (Figures 3 through 6)}

5.1.2.1 Cryogenic Trap and Desorber: Cryogenic trap with temperature control assembly (Nutech Corporation, Durham, NC, Model 320-01 or equivalent).

5.1.2.2 Solid Sorbent Trap and Desorber: This system is based on purge-andtrap devices employed for the analysis of VOCs in aqueous samples. These devices are typically composed of a sample sparge vessel, a solid sorbent trap, and a desorber. Several complete systems are available commercially (Tekmar Company, Cincinnati, OH, Model LSC-2 with auto sampler or equivalent).

Solid Sorbent Trap: The solid sorbent trap must be at least $25 \mathrm{~cm}$ long and have an inside diameter of at least $0.27 \mathrm{~cm}$. The recommended 
TABLE 2

4-Bromofluorobenzene Key lons and Abundance Criteria

Mass

Ion Abundance Criteria

50

75

95

96

173

174

175

176

177
15 to $40 \%$ of mass 95

30 to $60 \%$ of mass 95

base peak, $100 \%$ relative abundance

5 to $9 \%$ of mass 95

less than $2 \%$ of mass 174

greater than $50 \%$ of mass 95

5 to $9 \%$ of mass 174

greater than $95 \%$ but less than $101 \%$ of mass 174

5 to $9 \%$ of mass 176 
trap should have the following amounts of adsorbents (starting at the trap inlet): $7.6 \mathrm{~cm}$ of Carbopack $B$ and $1.3 \mathrm{~cm}$ of Carbosieve S-III (Supelco, Supelco Park, Bellafonte, PA, Model 2-301 or equivalent). Before initial use, the trap should be conditioned for one hour at $260^{\circ} \mathrm{C}$ by backflushing with an inert gas flow of at least $30 \mathrm{~mL} / \mathrm{min}$. Vent the trap effluent to a hood, not to the analytical column. Prior to daily use, the trap should be conditioned for $10 \mathrm{~min}$ at $260^{\circ} \mathrm{C}$ with backflushing.

Desorber: The desorber should be capable of rapidly heating the trap to $220^{\circ} \mathrm{C}$ for desorption.

5.1.2.3 Electronic Mass Flow Controller: The electronic mass flow controller must maintain constant flow for sample gas. The controller should be capable of indicating total flow (totalizer) through the controller (Matheson Model $8200-0422,2-100 \mathrm{~mL} / \mathrm{min}$ or equivalent).

5.1.2.4 Vacuum Pump: A diaphragm vacuum pump capable of drawing desired sample volume through either the cryogenic or the solid sorbent trap (Scientific Instrument Specialists, Model N05SV or equivalent).

5.1.2.5 Valve: A six-port gas chromatographic valve (Vaico Instruments, Model EC6VWE or equivalent).

5.1.2.6 Regulator: A stainless steel, two-stage cylinder regulator with pressure gauges for helium GC carrier gas.

5.2 Sample and Calibration Standard Preparation Manifold. (Figure 1)

5.2.1 Vacuum Pump: A vacuum pump capable of evacuating sample canister(s) to an absolute pressure of $<0.05 \mathrm{~mm} \mathrm{Hg}$.

5.2.2 Manifold: Manifold constructed of 0.25 chromatographic grade stainless steel tubing (Scott Specialty Gas Aculife treated tubing - optional) and stainless steel fittings. All tubing should be capable of being heated to $90^{\circ} \mathrm{C}$.

5.2.3 Flow Controllers: One (1) 0 to $100 \mathrm{~mL} / \mathrm{min}$ electronic mass flow controller and one (1) 0 to $1 \mathrm{~L} / \mathrm{min}$ electronic mass flow controller capable of indicating total flow through the controller (Matheson Model 8200-0422 with totalizer or equivalent).

5.2.4 Pressure/vacuum sensors: Two (2) pressure/vacuum sensors capable of indicating vacuum down to $0.01 \mathrm{~mm} \mathrm{Hg}$ and pressure up to $2500 \mathrm{~mm} \mathrm{Hg}$ with 1 percent accuracy (MKS Baratron Model 390HA or equivalent).

5.2.5 Vacuum Gauge: Vacuum gauge capable of indicating vacuum down to $0.01 \mathrm{~mm} \mathrm{Hg}$.

5.2.6 Solenoid Valve: Three-way magnelatch or solenoid valve (Scientific Instrument Specialists or equivalent).

5.2.7 Valves: Bellows-type shut-off valves made of stainless steel (Nupro " $\mathrm{H}$ " series or equivalent).

5.2.8 Chronometer: Time meter capable of measuring elapsed time (Scientific Instrument Specialist Model SCT-1c or equivalent). 
5.2.9 Humidifier: Pressurizable water bubbler (humidifier) containing ASTM Type II water or equivalent capable of providing moisture to the zero air, nitrogen or calibration gases.

5.2.10 Sparge Vessel: Sparge vessel capable of holding $5 \mathrm{~mL}$ of ASTM Type II water, being heated to $70^{\circ} \mathrm{C}$, and being purged with zero air or nitrogen.

\subsection{Reagents}

6.1 Carrier and Dilution Gases. Gas cylinders of helium, nitrogen and zero air; all ultra-high purity grade.

\subsection{Stock Standards.}

6.2.1 Gases: Stock calibration gas standards of all analytes listed in Table 1 must be purchased commercially (Scott Specialty Gases or equivalent), or prepared according to "Compendium Method TO-2, Determination of Volatile Organic Compounds in Ambient Air by Carbon Molecular Sieve Adsorption and Gas Chromatography/Mass Spectrometry" (EPA 1988b). The standards must be traceable to a National Institute of Standards and Technology (NIST) Standard Reference Material (SRM) or to a NIST/EPA approved Certified Reference Material (CRM). All gas standards must be replaced after 12 months if comparison with check standards indicates a problem.

6.2.2 Liquids: Stock solutions must be prepared from pure standard materials or purchased as certified solutions. All standards must be replaced every six months or sooner if comparison with check standards indicates a problem. Prepare stock solutions in an appropriate solvent as follows:

1. Place about $9.8 \mathrm{~mL}$ of solvent (e.g., propanol) in a $10 \mathrm{~mL}$ tared ground glassstoppered volumetric flask. Propanol is used only if methanol is to be determined by this method. Allow the flask to stand, unstoppered for about $10 \mathrm{~min}$ or until all alcohol-wetted surfaces have dried. Weigh the flask to the nearest $0.1 \mathrm{mg}$.

2. Using a 100- $\mu \mathrm{L}$ syringe, immediately add two drops or more of assayed reference material to the flask and reweigh. The liquid must fall directly into the alcohol without contacting the neck of the flask. Alternatively, the density of the reference material may be used to determine the volume of material to be added to the flask.

3. Dilute to volume with solvent, stopper, and then mix by inverting the flask several times. Standards and $\mathrm{OC}$ samples must be inverted the same number of times. Calculate the concentration in micrograms per milliliter $(\mu \mathrm{g} / \mathrm{mL})$ from the net gain in weight. When standard VOC purity is 96 percent or greater, the weight may be used without correction to calculate the concentration of the stock standard. Commercially prepared stock standards may be used at any concentration if they are certified by the manufacturer or by an independent source.

4. Transfer the standard stock solution into a Teflon-sealed screw cap bottle. Store with minimal headspace at $-10^{\circ} \mathrm{C}$ to $20^{\circ} \mathrm{C}$ and protect from direct light. 


\subsection{Secondary Dilution Standards.}

6.3.1 Calibration Standards: Using the stock calibration gas standards, prepare secondary dilution standards containing all the analytes in Table 1 in SUMMA ${ }^{\circ}$ canisters. Alternatively, a stock solution containing all the analytes listed in Table 1 can be directly injected into an evacuated SUMMA ${ }^{\oplus}$ canister followed by dilution with humid zero air or nitrogen.

Calibration standards must be prepared at a minimum of five concentration levels by dilution of stock standards. The low concentration standard must be prepared near the PRQLs specified in Table 1. The four remaining standards should define the calibration range of the GC/MS system. Each calibration standard should contain all the analytes listed in Table 1 . All secondary dilution calibration standards must be replaced after 30 days or sooner if comparison with check standards indicates a problem.

The following is an example of how external secondary dilution calibration gas standards or internal secondary dilution calibration gas standards might be prepared using the optional calibration standard preparation manifold (Figure 1). This procedure is intended for guidance purposes only, and is based on the following assumptions:

1. The system is calibrated in nanograms instead of ppmv. This allows the determination of sample concentrations in ppmv when variable sample volumes are delivered to the GC/MS system.

2. The volume of a real (field) sample needed to meet PRQLs is $10 \mathrm{~mL}$.

3. The stock calibration gas standard cylinder(s) are at $20 \mathrm{mg} / \mathrm{m}^{3}\left(\mathrm{mg} / \mathrm{m}^{3}=\mu \mathrm{g} / \mathrm{L}\right.$ $=\mathrm{ng} / \mathrm{mL}$ ).

4. The volume of the secondary dilution standard to be delivered to the GC/MS system is $300 \mathrm{~mL}$.

5. The final secondary dilution calibration canister pressure will be three times the barometric pressure. This is equivalent to a total gas volume added to an evacuated canister of three canister volumes.

Using benzene as an example, the amount (ng) of benzene contained in a $10 \mathrm{~mL}$ aliquot of $1 \mathrm{ppmv}$ benzene standard can be determined as follows:

$$
p p m v=\frac{(R)(T)}{(P)(M W)} \frac{(m g)}{\left(m^{3}\right)}
$$

where

$\boldsymbol{R}=0.0821 \mathrm{~L} \mathrm{~atm} / \mathrm{mol}^{\circ} \mathrm{K}$ (at $760 \mathrm{~mm} \mathrm{Hg}$ and $273^{\circ} \mathrm{K}$ )

$T=$ temperature $\left({ }^{\circ} \mathrm{K}\right)$

$M W=$ molecular weight of compound $(\mathrm{g} / \mathrm{mol})$

$\boldsymbol{P} \quad=$ pressure (atm)

Note: At $25^{\circ} \mathrm{C}$ and $1 \mathrm{~atm}, \boldsymbol{R T} / P=24.5 \mathrm{~L} / \mathrm{mol}$

Therefore, 1 ppmv of benzene ( $M W=78.1 \mathrm{~g} / \mathrm{mol}$ ) contains approximately $0.0032 \mathrm{~g}$ benzene $/ \mathrm{m}^{3}$ or $3.2 \mathrm{ng}$ benzene $/ \mathrm{mL}$, and a $10 \mathrm{~mL}$ aliquot would deliver about $32 \mathrm{ng}$ of benzene. 
This shows that the amount of benzene needed to be delivered to the GC/MS system for the low concentration standards is about $32 \mathrm{ng}$. The amount of volume used to deliver the $32 \mathrm{ng}$ is not important, what is important is that sufficient gas volume be used in order to minimize analytical error.

Assumptions (4) and (5) can be coupled with the $32 \mathrm{ng}$ of benzene to determine how many total nanograms of benzene need to be transferred from the stock calibration gas cylinder $(s)$ to the evacuated $2.8 \mathrm{~L}$ canister. The $2.8 \mathrm{~L}$ canister will contain a total of $8.4 \mathrm{~L}$ of gas. The amount of benzene needed for calibration is $32 \mathrm{ng}$ in $300 \mathrm{~mL}$. Therefore, the total amount of benzene in the canister is 132 $\mathrm{ng} / 0.30 \mathrm{~L}) \times(8.4 \mathrm{~L})=896 \mathrm{ng}$. It has been assumed that the stock calibration gas standards are $20 \mathrm{mg} / \mathrm{m}^{3}$ or $20 \mathrm{ng} / \mathrm{mL}$. Transferring $44.8 \mathrm{~mL}$ from the stock gas standard to the evacuated $2.8 \mathrm{~L}$ canister followed by dilution with $8.355 \mathrm{~L}$ of humid zero air or nitrogen will produce a secondary dilution standard that has $32 \mathrm{ng} / 300 \mathrm{~mL}$ of benzene. Delivery of $300 \mathrm{~mL}$ of this secondary dilution standard will yield a calibration point that is equivalent to $10 \mathrm{~mL}$ of $1 \mathrm{ppmv}$ benzene.

6.3.1.1 External Secondary Dilution Calibration Standard: To prepare a $1 \mathrm{ppmv}$ benzene external calibration standard using stock calibration gas standards, refer to Figure 1.

1. Install evacuated $2.8 \mathrm{~L}$ canister in position (1).

2. Install stock gas cylinders as shown $(34,35,36,37)$.

3. Evacuate all manifold lines up to valves (2) and $(25)$ to $<0.05 \mathrm{~mm}$ $\mathrm{Hg}$. Keep valves (3), (19) through (22) and (24) closed. All other valves should be open at this step.

4. Close valves (7), (4) and (23); open valve (2), and record the initial canister vacuum. Close valves (2) and (5).

5. Set calibration cylinder (34) to deliver gas at 20 psig.

6. Close valve (29) and set three-way valve $(8)$ to vent position.

7. Open valve (19) and set mass flow controller $(10)$ to $10 \mathrm{~mL} / \mathrm{min}$. Condition manifold tubes and stabilize mass flow controller by letting gas flow to vent for 5 minutes.

8. Set timer (9) to deliver the volume of gas needed. For a $1 \mathrm{ppmv}$ benzene standard from a $20 \mathrm{ng} / \mathrm{mL}$ stock calibration standard, this is $44.8 \mathrm{~mL}$ at $10 \mathrm{~mL} / \mathrm{min}$ which gives 4.48 minutes (269 seconds).

9. After 5 minutes of flowing to vent, switch three-way valve (8), initiate timer (9), and open valves (2) and (7). These steps should be done as quickly as possible.

10. When the timer (9) elapses, immediately close valve (2).

11. Close valve (19), open valve (29) and evacuate the manifold until the vacuum gauge (32) reads less than $0.05 \mathrm{~mm} \mathrm{Hg}$.

12. Repeat steps (4) to (11) until aliquots from all stock gas cylinders have been transferred to the $2.8 \mathrm{~L}$ SUMMA ${ }^{\circ}$ canister. 
13. Set either the zero air or nitrogen cylinder to deliver gas at 60 psig. Set three-way valve $(40)$ to vent position and set mass flow controller to $750 \mathrm{~mL} / \mathrm{min}$ and stabilize flow controller for 5 minutes.

14. Close valves (18) and (7). Switch three-way valve (40), open valves (25), (23), (4) and (2).

15. Transfer nitrogen or humid zero air until the $2.8 \mathrm{~L}$ canister has the equivalent of 3 canister volumes of gas $(8.4 \mathrm{~L})$. The volume of dilution gas (nitrogen/humid zero air) is determined by $8.4 \mathrm{~L}$ minus the amount of stock gas calibration standard added. The time required for this volume to be transferred is the volume needed divided by the flow rate. For the $1 \mathrm{ppmv}$ benzene standard, this would be $8355 \mathrm{~mL} / 750 \mathrm{~mL} / \mathrm{min}=11.14 \mathrm{~min}=668$ seconds.

16. After the required time has elapsed, immediately close valve (2). Then close valves (4), (23), and (25), and switch three-way valve $(40)$ to vent. Close nitrogen/humid zero air tank valve.

17. Open valves (29), (7), and (5), and evacuate to $<0.05 \mathrm{~mm} \mathrm{Hg}$.

18. Close valve (7), open valve (2), and record final canister pressure.

As an alternative to using accurate time and mass flow to transfer stock gas standards, continuous pressure/vacuum monitoring can be used. This can be accomplished by modifying steps 4 to 12 as follows:

4. Close valves (4), (7) and (23); open valve (2), and record the canister vacuum. Close valves (2) and (7).

5. Set calibration cylinder (34) to deliver gas at 20 psig.

6. Close valve (29) and set three-way valve (8) to vent position.

7. Open valve (19) and set mass flow controller (10) to $10 \mathrm{~mL} / \mathrm{min}$. Condition manifold tubes and stabilize mass flow controller by allowing gas flow to vent for 5 minutes.

8. Set timer (9) to deliver 25 percent more volume of gas then what is needed to transfer. For a $1 \mathrm{ppmv}$ benzene standard, $44.8 \mathrm{~mL}$ are required; 25 percent above the requirement would be $56.0 \mathrm{~mL}$. At $10 \mathrm{~mL} / \mathrm{min}$, the timer would be set to 5.6 minutes (336 seconds).

9. After 5 minutes of flowing to vent, switch three-way valve (8), initiate timer (9), and open valves (7) and (2). These steps should be done as quickly as possible.

10. To determine when to close valve (7) and stop the transfer of the stock calibration gas, the desired final pressure/vacuum needs to be calculated. The pressure/vacuum at the end of transfer is calculated as follows: 


$$
\frac{P_{f}-P_{i}}{P_{a}} V_{t}=V_{d}
$$

where

$$
\begin{aligned}
& \boldsymbol{P}_{a}=\text { atmospheric pressure }(\mathrm{mm} \mathrm{Hg}) \\
& \boldsymbol{P}_{f}=\text { final pressure }(\mathrm{mm} \mathrm{Hg}) \\
& \boldsymbol{P}_{i}=\text { initial pressure }(\mathrm{mm} \mathrm{Hg}) \\
& \boldsymbol{V}_{d}=\text { volume of gas to be delivered }(\mathrm{mL}) \\
& V_{t}=\text { SUMMA }
\end{aligned}
$$

If $44.8 \mathrm{~mL}$ are to be delivered to a $2.8 \mathrm{~L}$ canister with an initial pressure of $0.05 \mathrm{~mm} \mathrm{Hg}$, the final pressure will be $12.2 \mathrm{~mm} \mathrm{Hg}$. Therefore, when the pressure/vacuum sensor (6) approaches 12.2 $\mathrm{mm} \mathrm{Hg}$, reduce the flow with valve (7). When the desired pressure is reached, completely close valve (7) and document the final pressure. Close valve (2) and use the above equation to determine the volume of stock calibration gas standard transferred. After closing valve (2), reset the timer and switch three-way valve (8) to the vent position.

11. Close valve (19), open valves (7) and (29), and evacuate the manifold until vacuum gauge (5) reads less than $0.05 \mathrm{~mm} \mathrm{Hg}$.

12. Repeat steps (4) to (11) until aliquots from all stock gas cylinders have been transferred to the $2.8 \mathrm{~L} \mathrm{SUMMA}^{\circ}$ canister. When repeating step (4) it is not necessary to record the initial pressure because this is the final pressure of the previous stock calibration gas standard transfer. When repeating step (9), it is important that the pressure read at sensor (6) be greater than the final pressure of the previous transfer before opening valve (2).

6.3.1.2 Internal Standard Secondary Dilution Calibration Standard: The preparation of a $1 \mathrm{ppmv}$ internal standard is identical to the preparation of an external standard except for the addition of internal standards. In this procedure, internal standards are added to the $2.8 \mathrm{~L}$ SUMMA canister after transferring all stock calibration gas standards and prior to the final dilution with humid zero air or nitrogen. Several procedures may be used to introduce internal standards into calibration standard and/or sample canisters:

1. Direct injection of a stock solution of internal standards into the canister

2. Direct gas transfer from a stock gas standard into the canister

3. Injecting the internal standards into ASTM Type II water followed by purging them into the canister with nitrogen or ultra air at $100 \mathrm{~mL} / \mathrm{min}$ for 10 minutes while maintaining the sparge vessel at $70^{\circ} \mathrm{C}$.

The amount (ng) of each internal standard added to the $2.8 \mathrm{~L}$ canister must give $250 \mathrm{ng}$ per secondary dilution gas standard aliquot $(300 \mathrm{~mL})$ delivered to the GC/MS. For a $2.8 \mathrm{~L}$ canister with a total gas volume of $8.4 \mathrm{~L}$ and using a $300 \mathrm{~mL}$ aliquot, a total of 
$7000 \mathrm{ng}$ of each internal standard must be added to each calibration standard canister. Alternatively, internal standards may be added directly to the trap prior to calibration standard or sample loading. If this procedure is employed, $250 \mathrm{~mL}$ of a gas standards containing the internal standard should be used to deliver the $250 \mathrm{ng}$ of each internal standard.

6.3.2 Laboratory Control Samples: The preparation of laboratory control samples is identical to the preparation of calibration standards. Commercially purchased gas standards will be used to prepare laboratory control samples. Laboratory control samples will be prepared in the laboratory by attaching the gas standard to the laboratory sample preparation manifold and filling evacuated canisters. The gas standard used to prepare the laboratory control samples must be independent of those used for instrument calibration. Laboratory control samples must contain at least six of the analytes listed in Table 1 at concentrations in the calibration range of the GC/MS system.

6.4 Internal Standards. The recommended internal standards are fluorobenzene and chlorobenzene- $d_{5}$. Prepare internal standard stock and secondary dilution standards in an appropriate solvent. Each calibration standard or sample aliquot delivered to the GC/MS system must have $250 \mathrm{ng}$ of each internal standard.

6.5 4-Bromofluorobenzene (BFB) Standard. A standard solution containing $25 \mathrm{ng} / \mathrm{mL}$ of BFB in an appropriate solvent should be prepared if direct injection is used. A gas standard may be purchased commercially (Scott specialty Gases or equivalent) if gas introduction is used. Alternatively, a gas standard may be prepared according to Section 6.3.1.2. The concentrations of gas standards must be such that a total of $50 \mathrm{ng}$ is delivered to the GC/MS system.

6.6 Reagent Water. Reagent water is defined as water in which an interferant is not observed at the method detection limit (MDL) of the parameters of interest. Reagent water which is prepared must meet the performance specifications for ASTM Type II water. To prepare reagent water, either:

1. Pass tap water through a carbon filter bed containing $500 \mathrm{~g}$ of activated carbon (Calgon Corp., Filtrasorb-300 or equivalent); or,

2. Pass the water through a purification system (Millipore Super- $Q$ or equivalent); or,

3. Boil tap water for 15 minutes and then, while maintaining the water temperature at $85^{\circ} \mathrm{C}$ to $95^{\circ} \mathrm{C}$, bubble a contaminant-free inert gas through the water for one hour. While still hot, transfer the water to a narrow mouth screw-top bottle seal with a Teflon-lined septum and cap.

6.7 Liquid Nitrogen. (or other appropriate coolant)

\subsection{Sample Collection, Preservation, and Handling}

7.1 Samples will be collected using the sampling procedures described in Procedure 110.1 through 110.4 of this Methods Manual.

7.2 Sample canisters must be stored at room temperature. Sample holding times cannot exceed 28 days. 
7.3 All sample handling and chain-of-custody procedures described in Section 6.0 of the Transuranic Waste Characterization Quality Assurance Program Plan (QAPP)(DOE 1995b) must be followed.

\subsection{Procedure}

\subsection{Recommended GC/MS System Operating Conditions.}

$\begin{array}{ll}\text { Electron energy: } & 70 \text { volts (nominal) } \\ \text { Mass range: } & 29 \text { to } 260 \text { amu } \\ \text { Scan time: } & \text { One scan/second or enough to give } 5 \text { scans/peak } \\ \text { Column type: } & \mathrm{DB}-624,30 \mathrm{~m} \times 0.53 \mathrm{~mm} \text { I.D. } \\ \text { Initial column temperature: } & 10^{\circ} \mathrm{C} \\ \text { Initial column holding time: } & 5 \text { minutes } \\ \text { Column temperature program: } & 6^{\circ} \mathrm{C} / \text { minute } \\ \text { Final column temperature: } & 160^{\circ} \mathrm{C} \\ \text { Final column holding time: } & \text { Until all compounds have eluted } \\ \text { Injector temperature: } & 200 \text { to } 225^{\circ} \mathrm{C} \\ \text { Source temperature: } & \text { According to manufacturer specifications } \\ \text { Transfer line temperatures: } & 250 \text { to } 300^{\circ} \mathrm{C} \\ \text { Carrier gas: } & \text { Helium at } 15 \mathrm{~mL} / \text { minute }\end{array}$

8.2 Analytical System Options. Four possible inlet/trapping GC/MS systems are shown in Figures 3 to 6 . The hardware requirements for these systems have been given in Section 5.1. All SUMMA ${ }^{\circ}$ canisters used for GC/MS system calibration, quality control samples, and analytical purposes must first be cleaned and certified in accordance with Procedure 210.1 of this Methods Manual.

8.2.1 Cryogenic Trapping: Mass flow controller and evacuated canister systems for delivery and measurement of sample volumes are shown in Figures 3 and 4.

8.2.1.1 Mass Flow Controller System: The analytical system should be properly assembled using Figure 3 for guidance and certified using humid zero air or nitrogen (Section 8.3.2).

Mass flow controllers should be checked and adjusted to provide correct flow rates for the system. The small diaphragm pump is used to maintain a constant pressure differential across the mass flow controller.

The sample canister is connected to the system as shown in Figure 3. Calibration standards and samples that have been diluted by a factor of 30 or more are delivered to the cryogenic trap at $30 \mathrm{~mL} / \mathrm{min}$. Samples that have not been diluted to this extent can still be analyzed with this system but the mass flow controller needs to be set to deliver the sample over 10 minutes. Sub-ambient pressure samples can also be analyzed by this procedure.

With the system in the desorb/precondition mode (Figure 3B), the gas chromatographic oven and cryogenic trap are set to their initial set points of $10^{\circ} \mathrm{C}$ and $-150^{\circ} \mathrm{C}$, respectively. When the cryogenic trap reaches its set point, the canister valve is opened and the transfer lines and mass flow controller are conditioned and stabilized. After one to two minutes, the six-way chromatographic valve is turned to its fill (trapping) position (Figure 3A). A ten-minute collection period of 
canister sample is utilized. [Note: $30 \mathrm{~mL} / \mathrm{min} \times 10 \mathrm{~min}=300 \mathrm{~mL}$ sampled]. After 10 minutes, the sample canister valve is closed.

After the sample is preconcentrated in the cryogenic trap, the six-way chromatographic valve is cycled to the inject position and the cryogenic trap is heated to $120^{\circ} \mathrm{C}$. The trapped analytes are thermally desorbed onto the head of the DB-624 capillary column. Upon sample desorption, the GC/MS data system is signaled to begin mass spectrometric data acquisition and to begin the chromatographic temperature program. Qualitative and quantitative identification procedures will be discussed in Section 9.0, "Calibrations."

8.2.1.2 Evacuated Canister System: The analytical system should be properly assembled using Figure 4 for guidance and certified using humid zero air or nitrogen (Section 8.3.2). This system should be employed when the available volume of sample is limited and it is not possible to deliver $300 \mathrm{~mL}$.

The volume of the evacuated canister used to collect the trap effluent should be known to within 2 percent of the actual volume. The change in pressure of the evacuated canister as trap effluent is collected is used to determine how much sample has been delivered to the trap. The volume of gas delivered can be calculated using equation (1) from Section 6.3. The sample canister and evacuated canister are connected as shown in Figure 4. The flow controller is used to regulate sample flow so that the desired final pressure is achieved. With the system in the sample trapping mode (Figure 4A), the gas chromatographic oven and cryogenic trap are set to their initial set points of $10^{\circ} \mathrm{C}$ and $-150^{\circ} \mathrm{C}$, respectively.

When the cryogenic trap reaches its set point, the trap vent valve is closed isolating the vacuum pump. The pressure in the evacuated canister is measured and recorded. The isolation valves on both canisters are opened allowing sample to flow through the trap and into the evacuated canister. The sample canister valve is closed when the evacuated canister pressure indicates the desired volume of sample has been delivered.

After the sample is preconcentrated in the cryogenic trap, the six-way chromatographic valve is cycled to the inject position and the cryogenic trap is heated to $120^{\circ} \mathrm{C}$. The trapped analytes are thermally desorbed onto the head of the DB-624 capillary column. Upon sample desorption, the GC/MS data system is signaled to begin mass spectrometric data acquisition and to begin the chromatographic temperature program. Qualitative and quantitative identification procedures will be discussed in Section 9.0, "Calculations."

8.2.2 Solid Sorbent Trapping: Mass flow controller and evacuated canister systems for delivery and measurement of sample volumes are shown in Figures 5 and 6 . If a commercial purge-and-trap system or equivalent is used (Tekmar LSC-2/ALS), then the vacuum pump and mass flow controller are connected to the trap vent bulk head fitting and the sample canister is connected to the purge flow inlet on the LSC-2 or the ALS. If connected to the ALS with 1/16 inch stainless steel heated transfer line, the line is connected to the purge flow outlet. 
8.2.2.1 Mass Flow Controller System: The analytical system should be properly assembled using Figure 5 for guidance and certified using humid zero air or nitrogen (Section 8.3.2). Mass flow controllers should be checked and adjusted to provide correct flow rates for the system. The small diaphragm pump is used to maintain a constant pressure differential across the mass flow controller.

The sample canister is connected to the system as shown in Figure 5. Calibration standards and samples that have been diluted by a factor of 30 or more are delivered to the solid sorbent trap at $30 \mathrm{~mL} / \mathrm{min}$. Samples that have not been diluted to this extent can still be analyzed with this system but the mass flow controller needs to be set to deliver the sample over 10 minutes. Sub-ambient pressure samples can also be analyzed by this procedure.

With the system in the desorb/precondition mode (Figure 5B), the gas chromatographic oven and solid sorbent trap are set to their initial set points of $10^{\circ} \mathrm{C}$ and $22^{\circ} \mathrm{C}$, respectively.

When the solid sorbent trap reaches its set point, the canister valve is opened and the transfer line and mass flow controller are conditioned and stabilized. This step is omitted when a commercial purge-and-trap system is used. After one to two minutes, the six-way chromatographic valve is turned to its fill (trapping) mode (Figure 5A). With a commercial purge-and-trap, the system would be set to the purge mode and the sample canister would be opened. A 10-minute collection period of canister sample is utilized. [Note: $30 \mathrm{~mL} / \min \times 10 \mathrm{~min}=300 \mathrm{~mL}$ sampled]. After 10 minutes, the sample canister valve is closed.

After the sample is preconcentrated in the solid sorbent trap, the sixway chromatographic valve is cycled to the inject position and the solid sorbent trap is heated to $260^{\circ} \mathrm{C}$. The trapped analytes are thermally desorbed onto the head of the DB-624 capillary column. Using commercial purge-and-trap hardware, the system would advance to the desorb mode after the time set for purging had elapsed.

Upon sample desorption, the GC/MS data system is signaled to begin mass spectrometric data acquisition and to begin the chromatographic temperature program. Qualitative and quantitative identification procedures will be discussed in Section 9.0, "Calculations."

8.2.2.2 Evacuated Canister System: The analytical system should be properly assembled using Figure 6 for guidance and certified using humid zero air or nitrogen (Section 8.3.2). If a commercial purge-and-trap system is used, the vacuum pump and evacuated canister are connected to the trap vent bulkhead fitting and the sample canister is connected to the purge flow inlet on the LSC-2 or the ALS. The volume of the evacuated canister used to collect the trap effluent should be known to within 2 percent of the actual volume. The change in pressure of the evacuated canister as trap effluent is used to determine how much sample has been delivered to the trap. The volume of gas delivered can be calculated using equation (1) from Section 6.3. The sample canister and evacuated canister are connected as shown in Figure 6 . The flow controller is used to regulate sample flow so that desired final pressure is achieved. 
With the system in the sample trapping mode (Figure 6A), the gas chromatographic oven and solid sorbent trap are set to their initial temperature set points of $10^{\circ} \mathrm{C}$ and $22^{\circ} \mathrm{C}$, respectively. A commercial purge-and-trap system would be in the purge mode. When the solid sorbent trap reaches its set point, the trap vent valve is closed isolating the vacuum pump. The pressure in the evacuated canister is measured and recorded. The isolation valves on both canisters are opened allowing sample to flow through the trap and into the evacuated canister. The sample canister valve is closed when the evacuated canister pressure indicates the desired volume of sample has been delivered.

After the sample is preconcentrated in the solid sorbent trap, the sixway chromatographic valve is cycled to the inject position and the solid sorbent trap is heated to $260^{\circ} \mathrm{C}$. The trapped analytes are thermally desorbed onto the head of the DB-624 capillary column. A commercial purge-and-trap system would be advanced to the desorb mode.

Upon sample desorption, the GC/MS data system is signaled to begin mass spectrometric data acquisition and to begin the chromatographic temperature program. Qualitative and quantitative identification procedures will be discussed in Section 9.0, "Calculations."

\subsection{GC/MS Instrument Performance Testing.}

8.3.1 4-Bromofluorobenzene Tune. Every 12 hour shift or prior to a calibration, it must be demonstrated that the GC/MS system meets the BFB criteria specified in Table 2. Instrument hardware tuning may be performed with perfluorotributylamine (PFTBA). Instrument performance criteria must be met by injecting $50 \mathrm{ng}$ of BFB on-column. The BFB may be introduced to the GC/MS system via a gas standard or a liquid standard.

8.3.2 Humid Zero Air/Nitrogen Test. The sample and calibration preparation manifold (Figure 1) is connected to the inlet of the GC/MS system, and humid zero air or nitrogen passes through the manifold to the GC/MS system. The amount of gas delivered to the analytical trap should be the same as that used while analyzing samples. The humid zero air or nitrogen is trapped and subsequently analyzed by the GC/MS system. In order to pass the humid zero air/nitrogen test, the concentration of the targeted VOCs (Table 1) cannot exceed 3 time their MDLs.

\subsection{Calibration Table 3 provides a summary of calibration requirements}

8.4.1 Initial Calibration Procedures. Initially, a five-point calibration is performed on the GC/MS system. Section 6.0 provides guidance on how to prepare both internal and external standard calibration mixtures at the required concentration (ng) levels. Sections 8.1 and 8.2 provide suggested GC/MS operating parameters and GC/MS analytical system options. After the secondary dilution gas standards have been prepared and the GC/MS analytical system has been configured, initial calibration can begin. Prior to the analysis of any samples (including standards), instrument performance criteria must be satisfied (Section 8.3). The initial calibration curve for each compound listed in Table 1 must be checked and verified once during every 12-hour shift and prior to the analysis of any samples. This is accomplished by analyzing a continuing calibration standard that contains amounts (ng) of compounds near the midpoint amounts of the initial calibration. 
TABLE 3

Summary of GC/MS Calibration Requirements for Gas Volatile Organic Compounds Analysis

Technique Procedure Frequency of Procedure Acceptance Criteria

\begin{tabular}{llll} 
GC/MS & BFB Tune & Every 12 hours & $\begin{array}{l}\text { Abundance criteria for all key ions are } \\
\text { met (see Table 2) }\end{array}$ \\
$\begin{array}{lll}5 \text {-pt initial calibration } \\
\text { (5 standards) }\end{array}$ & Initially, and as needed & $\begin{array}{l}\% R S D \text { of response factor for each } \\
\text { analyte }<35\end{array}$ \\
Continuing calibration & Every 12 hours & $\begin{array}{l}\% \mathrm{D} \text { for all compounds } \leq 30 \text { of initial } \\
\text { calibration }\end{array}$ \\
\hline
\end{tabular}

BFB = 4-Bromofluorbenzene

$\% \mathrm{D}=$ Percent difference

$\% \mathrm{RSD}=$ Percent relative standard deviation 
8.4.1.1 Internal Standard Calibration: Tabulate the area response of the characteristic ion used, found in Table 4 (primary or secondary), against the amount (ng) for each compound and for each internal standard for the five calibration standards. Calculate the relative response factor (RRF) for each compound using the assignments made in Table 5. When the analytical system is calibrated in nanograms, the RRF is calculated as follows:

$$
R R F=\frac{\left(A_{x} C_{I S}\right)}{\left(A_{t 5} C_{x}\right)}
$$

where

$$
\begin{aligned}
& A_{x}=\text { area of the characteristic ion for the compound being } \\
& \text { measured } \\
& A_{I S}=\text { area of the characteristic ion for the specific internal } \\
& C_{I S}=\quad \begin{array}{l}
\text { standard } \\
\text { amount of the specific internal standard (ng) }
\end{array} \\
& C_{x}=\text { amount of the compound being measured (ng) }
\end{aligned}
$$

The average RRF must also be calculated for each compound. Using the RRFs and standard deviation, calculate the percent relative standard deviation (\%RSD) for all compounds listed in Table 1. The \%RSD is calculated as follows:

$$
\% R S D=\frac{s}{\bar{x}} * 100
$$

where

$$
\begin{array}{lll}
s & = & \text { standard deviation of RRFs for a compound } \\
\bar{x} & = & \text { mean of } 5 \text { initial RRFs for a compound (average RRF) }
\end{array}
$$

The standard deviation $(s)$ is defined as follows:

$$
s=\sqrt{\sum_{i=1}^{n} \frac{\left(x_{i}-\bar{x}\right)^{2}}{n-1}}
$$

where

$$
\begin{aligned}
& x_{i}=\quad \begin{array}{l}
\text { measured value of the } i^{\text {th }} \text { replicate sample analysis } \\
n=
\end{array} \quad \text { number of replicate analyses }
\end{aligned}
$$

The \%RSD for each individual compound must be less than 35 percent. For those compounds having a \%RSD greater than 35 percent, the GC/MS data system may be used to generate a second or third order regression calibration curve. Sample analysis cannot proceed until the \%RSD requirement for each compound is met.

8.4.1.2 External Standard Calibration: Tabulate the area response of the characteristic ions (Table 3) against amount (ng) for each compound from the five calibration standards. Calculate the response factor (RF) for each compound. When the analytical system is calibrated in nanograms, the RF is calculated as follows: 
TABLE 4

Characteristic lons for Volatile Organic Compounds

Volatile Organic Compound
Primary lon

43

78

173

56

117

112

83

56

63

63

96

96

106

31

31

72

43

84

83

164

92

97

130

101

- 105

105

106

106

106

117

96
Secondary Ion

58

52,77

$171,175,252$

31

119,121

114,77

85,47

65,83

65,83

61,98

61,98

91

59

57,43

58,100

$49,51,86$

$85,131,166$

$129,131,166$

91,65

99,117

$95,97,132$

155,85

120

120

91

91

91

82,119

77

"Sites required to analyze these compounds must determine the applicable primary and secondary ions.

IS = Internal Standard 
TABLE 5

Volatile Organic Internal Standards with Corresponding Analytes Assigned for Quantitation

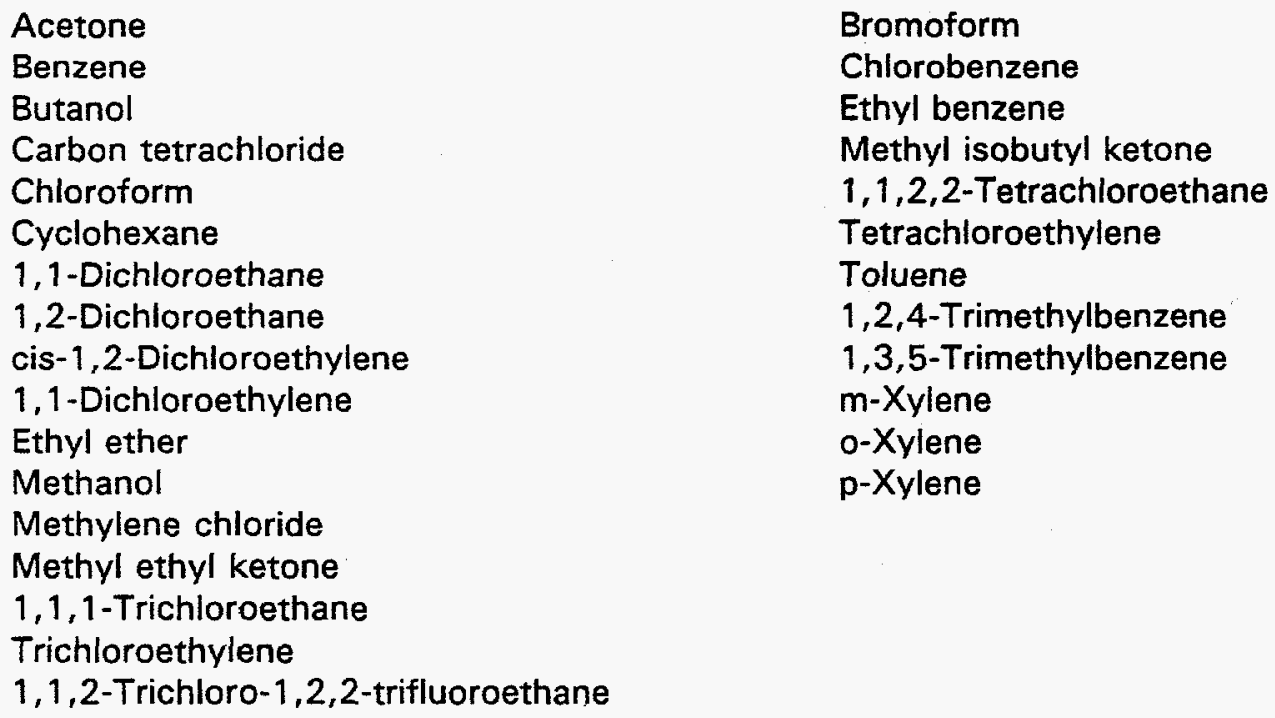

Bromoform

Chlorobenzene

Ethyl benzene

Methyl isobutyl ketone

1,1,2,2-Tetrachloroethane

Tetrachloroethylene

Toluene

1,2,4-Trimethylbenzene

1,3,5-Trimethylbenzene

$\mathrm{m}$-Xylene

$0-X y l e n e$

p-Xylene

Sites required to analyze samples for formaldehyde and hydrazine must determine the appropriate internal standard for each. 


$$
R F=\frac{A_{x}}{C_{x}}
$$

where

$$
\begin{aligned}
& A_{x}=\text { area of the characteristic ion for the compound being } \\
& \text { measured } \\
& C_{x} \quad=\quad \text { amount of the compound being measured (ng) }
\end{aligned}
$$

The average RF, standard deviation, and the \%RSD must be calculated for all compounds listed in Table 1 . The \%RSD is calculated as in equation (4). The same acceptance criteria apply to RFs as to RRFs.

8.4.2 Continuing Calibration: The continuing calibration procedure for internal and external calibration standards is identical. The validity of the initial calibration curve is checked by calculating the percent difference $(\% D)$ for each compound as follows:

$$
\% D=\frac{\left|\left(R F_{l}\right)-\left(R F_{C}\right)\right|}{\left(R F_{l}\right)} * 100
$$

where

$$
\begin{aligned}
& R F_{I}=\begin{array}{l}
\text { average response factor or average relative response factor from } \\
\text { initial calibration (area/ng) }
\end{array} \\
& R F_{c}=\begin{array}{l}
\text { response factor or relative response factor from current midpoint } \\
\text { standard (area/ng) }
\end{array}
\end{aligned}
$$

The \%D for all compounds must be less than or equal to 30 percent. For those compounds where a higher order regression curve was used, the point from the continuing calibration standard for the compound must fall within 30 percent of the curve value from the initial calibration. If the $\% D$ criterion is not met, corrective action must be taken. If no source of the problem can be determined after corrective action has been taken, a new five-point calibration must be generated. This criterion must be met before quantitative sample analysis begins.

The internal standard area responses in the continuing calibration standard must be evaluated immediately after or during sample analysis. If the retention time (RT) of any internal standard changes by more than 30 seconds from the last continuing calibration, the chromatographic system must be checked for malfunctions and corrections must be made. If the EICP area for any internal standard changes by a factor of two or more from the last continuing calibration standard, the GC/MS system must be checked for malfunctions and corrections must be made. Samples analyzed while the system was malfunctioning must be reanalyzed.

\subsection{Sample Analysis.}

8.5.1 Figure 7 illustrates the sequence of events that should occur at the laboratory upon receipt of a sample canister. Details of chain-of-custody requirements and example forms are given in Section 6.0 of the QAPP. The $250 \mathrm{~mL}$ SUMMA ${ }^{\oplus}$ canisters used to collect the headspace samples have pressure/vacuum gauges attached. The canister pressure as received from the field must be documented.

8.5.2 The minimum volume of sample required to meet the PROLs specified in Table 1 is $10 \mathrm{~mL}$. The analytical systems described in Section 8.2 are for sample volumes greater than $20 \mathrm{~mL}$. Sections 8.2.1.1 and 8.2.2.1 describe analytical systems that can be used when the volume of gas delivered is $>100 \mathrm{~mL}$. Sections 8.2 .1 .2 and 
8.2.2.2 describe analytical systems that can be used when the volume of gas delivered is in the range of $20-80 \mathrm{~mL}$. Given that a $10 \mathrm{~mL}$ sample aliquot is sufficient to meet the PROLs but not sufficient for the analytical systems described, it will be necessary to dilute the sample prior to analysis. Section 6.3 presents procedures for transferring and diluting gas samples.

8.5.3 Figure 2 illustrates how a sample can be diluted by a factor of 30 or more. Dilution of a $10 \mathrm{~mL}$ sample by a factor of 30 would allow delivery of $300 \mathrm{~mL}$ to the analytical system and would be the same as described for calibration standards (Section 6.3). Transferring a $50 \mathrm{~mL}$ sample aliquot to a $500 \mathrm{~mL}$ evacuated canister followed by the addition of $1.45 \mathrm{~L}$ of humid zero air will result in a thirty-fold dilution of the sample. If the field canister is pressurized before transferring the sample aliquot, the resultant dilution must be taken into account before transferring to the $500 \mathrm{~mL}$ canister, i.e., the aliquot volume would increase. The analytical systems described in Sections 8.2.1.1 and 8.2.2.1 should be used for dilution factors of ten or greater. The analytical systems described in Sections 8.2.1.2 and 8.2.2.2 should be used for dilution factors of ten or less.

\subsubsection{Guidance for the Preparation and Analysis of a Diluted (30x) Sample:}

1. Attach a $500 \mathrm{~mL}$ evacuated laboratory canister (1) as shown in Figure 1.

2. Attach a $250 \mathrm{~mL}$ field canister (16) as shown in Figure 1 .

3. Evacuate the manifold to $<0.05 \mathrm{~mm} \mathrm{Hg}$ with valves (5), (7), (12), (13), (18), (28), (29), and (31) open.

4. Measure and document the field canister pressure by closing valve (12) and briefly opening valve (15). Open valve (12) and evacuate to $<0.05 \mathrm{~mm} \mathrm{Hg}$.

5. Pressurize the field canister using humid zero air or nitrogen by a factor of two. Set three-way valve $(40)$ to vent, set mass-flow controller $(27)$ to $75 \mathrm{~mL} / \mathrm{min}$, and let stabilize for one minute. Close valves (29) and (28), switch three-way valve (40), and open valves (25), (12) and (15). When pressure/vacuum sensor (14) approaches two times the initial pressure measured in step 4, close valves $(12)$ and $(25)$ and switch three-way valve $(40)$ to vent. Document the final field canister pressure. The dilution factor (DF) is calculated as follows:

$$
D F=\frac{C_{p 2}}{C_{p 1}}
$$

where

$$
\begin{aligned}
& C_{p 2}=\quad \text { canister pressure after dilution }(\mathrm{mm} \mathrm{Hg}) \\
& C_{p 1}=\quad \text { canister pressure before dilution }(\mathrm{mm} \mathrm{Hg})
\end{aligned}
$$

6. Transfer $(50 \times$ DF) $\mathrm{mL}$ from the field canister to the $500 \mathrm{~mL}$ evacuated laboratory canister (Section 6.3).

7. Final dilution is achieved by delivering $[(500-50) \times D F] \mathrm{mL}$ of humid zero air or nitrogen to the $500 \mathrm{~mL}$ laboratory canister. Section 6.3 gives details on how to add internal standards prior to the final dilution. A total of $250 \mathrm{ng}$ of each internal standard must be delivered to the analytical system during the sample analysis sequence. 
8. The final dilution factor is determined by:

- Multiplying the dilution factors calculated using equation (8) for steps 4, 5, and 6 . The final DF is the product of the individual dilution factors.

- The ratio of the total gas volume to the gas volume of sample transferred. The volume of sample transferred has to be corrected for any dilutions before the ratio is calculated.

9. The sample is analyzed by attaching the $500 \mathrm{~mL}$ canister to one of the analytical GC/MS systems described in Section 8.2.

8.6 Samples are analyzed by attaching the appropriate sample canister to the inlet of one of the analytical systems described in Section 8.2. If the initial analysis of a sample detects any analytes with concentrations that exceed the initial calibration range, the sample must either be diluted and reanalyzed, or reanalyzed with a lower sample volume.

\subsection{Calculations}

9.1 Qualitative Analysis. An analyte listed in Table 1 is identified by comparison of the sample mass spectrum with the mass spectrum of a standard of the suspected compound (standard reference mass spectrum). Standard reference mass spectra are obtained through analysis of calibration standards. Two criteria must be satisfied to verify identification:

1. A compound must elute within either the specified gas chromatographic RT or the relative retention time (RRT) window of the analyte in the reference standard.

2. A compound's mass spectrum must match the mass spectrum of the analyte in the reference standard.

The RT or RRT of compounds must be determined by comparison with a reference standard analyzed on the same day as the sample. The compound RRT must compare within \pm 0.06 RRT units of the RRT of the analyte in the reference standard. The RT must compare within plus or minus three standard deviations of the individual RT of the analyte from the most recent initial calibration. If co-elution or interference prohibits accurate assignment of the compound RT or RRT from the total ion chromatogram, the RT or RRT should be assigned by using the EICP for the primary characteristic ions listed in Table 4.

All ions present in the standard mass spectrum at a relative intensity greater than 10 percent (most abundant ion in the spectrum equals 100 percent) must be present in the sample spectrum. The relative intensities of all ions present at $>10$ percent must agree to within \pm 20 percent between the standard and sample spectra.

For samples containing compounds not listed in Table 1, with total ion current peak areas greater than 10 percent of the nearest (RT) internal standards, a forward library search of the latest NIST mass spectral data base must be performed. With external standard quantitation, unknown compounds with total ion current peak areas greater than 10 percent of the largest target compound identified or ten times greater than the standard deviation of the background must be searched. Guidelines for making tentative identification of these unknown compounds are:

1. The relative intensities of major ions in the reference spectrum (ions $>10$ percent of the most abundant ion) should be present in the sample spectrum. 
2. The relative intensities of major ions should agree within \pm 20 percent.

3. Molecular ions present in the reference spectrum should be present in the sample spectrum.

4. lons present in the sample spectrum but not in the reference spectrum should be reviewed for possible contamination or presence of co-eluting compounds.

Only after visual comparison of the sample spectrum with the results of the library searches will the mass spectral interpretation specialist assign a tentative identification.

9.2 Quantitative Analysis. Once a compound has been qualitatively identified, the quantitation of that compound will be based on the integrated abundance from the EICP of the primary characteristic ion (Table 4). Quantitation will be performed using the internal standard technique or the external standard technique. The internal standard used for quantitation of an analyte must be the same internal standard assigned to the analyte in Table 5 .

When the analytical system is calibrated in nanograms, the concentration (in ppmv) of each identified analyte in the sample is calculated as follows.

\section{Internal standard quantitation}

$$
\text { concentration (ppmv) }=\frac{\left(A_{x}\right)\left(C_{I S}\right)(R)(T)}{\left(A_{I S}\right)(R R F)(P)(M W)\left(V_{0}\right)}
$$

where

$$
V_{o}=\left(\frac{V_{a}}{D F}\right)\left(\frac{P_{a}}{760 m m H g}\right)\left(\frac{298^{\circ} \mathrm{K}}{273+T_{a}}\right)
$$

and
$A_{x} \quad=\quad$ area of the characteristic ion for compound being measured
$A_{I S}=$ area of the characteristic ion for the internal standard
$C_{I S}=$ amount of internal standard (ng)
$\boldsymbol{R} \boldsymbol{R F}=$ average relative response factor for compound being measured (Section 8.4)
$\boldsymbol{R}=0.0821 \mathrm{~L} \mathrm{~atm} / \mathrm{mol}^{\circ} \mathrm{K}$ (at $760 \mathrm{~mm} \mathrm{Hg}$ and $273^{\circ} \mathrm{K}$ )
$T=$ canister temperature $\left({ }^{\circ} \mathrm{K}\right)$
$\boldsymbol{P}=$ canister pressure (atm)
$M W=$ molecular weight of compound being measured $(\mathrm{g} / \mathrm{mol})$
$V_{o}=$ volume of sample delivered to the analytical system, corrected for dilution and standard conditions $(\mathrm{mL})$
$V_{k}=$ volume during analysis $(\mathrm{mL})$
$\boldsymbol{P}_{a} \quad=\quad$ barometric pressure during analysis $(\mathrm{mm} \mathrm{Hg})$
$T_{\mathrm{a}}=$ temperature during analysis $\left({ }^{\circ} \mathrm{C}\right)$
$D F=$ dilution factor resulting from pressurization (see equation (8)1)

Note: At $25^{\circ} \mathrm{C}$ and $1 \mathrm{~atm}, \boldsymbol{R T} / \boldsymbol{P}=24.5 \mathrm{~L} / \mathrm{mol}$

\section{External standard quantitation}

$$
\text { concentration }(p p m v)=\frac{\left(A_{x}\right)(R)(T)}{(R F)(P)(M W)\left(V_{o}\right)}
$$

where 
$A_{x}, R, T, P, M W$, and $V_{o}$ are the same as for internal standard quantitation

$\boldsymbol{R} \boldsymbol{F}=$ average response factor for the compound being measured (Section 8.4) (area/ng)

An estimate of the concentration of tentatively identified compounds (TICs) in the sample should be made. The equations given above should be used to determine the estimated concentrations of TICs with the following modifications:

1. The areas $A_{x}$ and $A_{I S}$ should be from the total ion chromatograms.

2. The RRF for the TIC is assumed to be one.

3. The nearest internal standard free of interference should be used.

4. The RF for a compound similar in structure should be used.

\subsection{Quality Control}

10.1 Each laboratory that uses this procedure is required to operate a formal quality control program. The laboratory must retain records to document the quality of the data generated. Each laboratory must have SOPs documenting and describing activities involved in utilizing this procedure. Specific quality control practices will include, but are not limited to, the analysis of quality control samples. The types of quality control samples, their associated frequency of analysis, acceptance criteria, and corrective action required if samples do not meet the acceptance criteria, is summarized in Table 6. Laboratory SOPs shall address requirements for preparing laboratory blanks, laboratory duplicates, and laboratory control samples.

10.2 All laboratories using this procedure must demonstrate acceptable performance prior to the analysis of actual samples. Demonstration of acceptable performance will be achieved by analyzing method performance samples (Table 6). These samples can be either commercially purchased or laboratory prepared, but must contain all of the analytes listed in Table 1 at concentrations appropriate (2-5 times the PROLs) to meet the quality assurance objectives specified in Table 1. The analysis of seven samples must meet the criteria specified for precision, accuracy and MDL in Table 1. Demonstration of acceptable method and analyst performance must be repeated (by analyzing four samples) at a minimum of every six months. Method performance should be conducted over a period of several days to account for long-term variation. Precision will be determined as follows:

- For duplicate measurements, the precision expressed as the relative percent difference (RPD) is calculated as

$$
R P D=\frac{C_{1}-C_{2}}{\frac{\left(C_{1}+C_{2}\right)}{2}} * 100
$$

where

$$
\begin{array}{ll}
C_{1} \text { and } C_{2} & =\text { two values obtained by analyzing the duplicate samples } \\
C_{1} & =\text { the larger of the two observed values }
\end{array}
$$


TABLE 6

\section{Summary of Laboratory Quality Control Samples and Frequencies} for Gas Volatile Organic Compounds Analysis

\begin{tabular}{|c|c|c|c|}
\hline QC Sample & Minimum Frequency & $\begin{array}{l}\text { Acceptance } \\
\text { Criteria }\end{array}$ & $\begin{array}{l}\text { Corrective } \\
\text { Action }^{a}\end{array}$ \\
\hline $\begin{array}{l}\text { Method performance } \\
\text { samples }\end{array}$ & $\begin{array}{l}\text { Seven (7) samples } \\
\text { initially and four (4) } \\
\text { semiannually }\end{array}$ & Meet Table 1 QAOs & $\begin{array}{l}\text { Repeat until } \\
\text { acceptable }\end{array}$ \\
\hline Laboratory duplicates & $\begin{array}{l}\text { One (1) per analytical } \\
\text { batch }\end{array}$ & $\mathrm{RPD} \leq 25^{\mathrm{b}}$ & $\begin{array}{l}\text { Nonconformance if } \\
R P D>25\end{array}$ \\
\hline Laboratory blanks & $\begin{array}{l}\text { Daily prior to sample } \\
\text { analysis }\end{array}$ & $\begin{array}{l}\text { Analyte amounts } \\
<3 \times \text { MDLs }\end{array}$ & $\begin{array}{l}\text { Nonconformance if } \\
\text { analyte amounts } \\
>3 \times \text { MDLs }\end{array}$ \\
\hline $\begin{array}{l}\text { Laboratory control } \\
\text { samples }\end{array}$ & $\begin{array}{l}\text { One (1) per analytical } \\
\text { batch }\end{array}$ & $70-130 \% R$ & $\begin{array}{l}\text { Nonconformance if } \\
\% R<70 \text { or }> \\
130\end{array}$ \\
\hline Blind audit samples & $\begin{array}{l}\text { Samples and frequency } \\
\text { controlled by the Gas } \\
\text { PDP Plan }\end{array}$ & $\begin{array}{l}\text { Specified in the Gas } \\
\text { PDP Plan }\end{array}$ & $\begin{array}{l}\text { Specified in the } \\
\text { Gas PDP Plan }\end{array}$ \\
\hline
\end{tabular}

${ }^{a}$ Corrective Action when $Q C$ samples do not meet the acceptance criteria; Nonconformance procedures are outlined in Section 2.1.2.1 of the QAPP.

${ }^{\mathrm{b}}$ Applies only to concentrations greater than the PROLs listed in Table 1.

MDL $=$ Method detection limit

PDP $=$ Performance Demonstration Program

$\mathrm{QAO}=$ Quality assurance objective

$\% R=$ Percent recovery

$\mathrm{RPD}=$ Relative percent difference 
- For three or more replicate measurements, the precision expressed as the \%RSD is calculated as

$$
\% R S D=\frac{s}{\bar{y}} * 100
$$

where

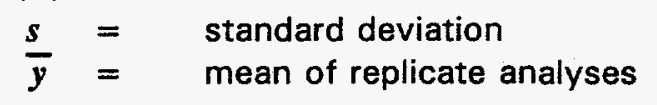

- The standard deviation (s) is defined as

$$
s=\sqrt{\sum_{i=1}^{n} \frac{\left(y_{i}-\bar{y}\right)^{2}}{n-1}}
$$

where

$$
\begin{array}{ll}
y_{i} & =\text { measured value of the } i^{\text {th }} \text { replicate sample analysis measurement } \\
n & =\text { number of replicate analyses }
\end{array}
$$

Accuracy will be determined as the percent recovery $(\% R)$ as follows:

$$
\% R=\frac{C_{m}}{C_{s m}} * 100
$$

where

$$
\begin{array}{lll}
C_{m} & =\text { measured concentration } \\
C_{\text {srm }} & = & \text { true concentration }
\end{array}
$$

MDL will be determined as follows:

$$
M D L=t_{(n-1,1-\alpha=0.99)} * S
$$

where

$$
\begin{array}{lll}
s & = & \text { standard deviation } \\
t_{(n-1,1-\alpha=0.99)=} & t \text {-distribution value appropriate to a } 99 \text {-percent confidence level and } \\
& \text { a standard deviation estimate with } n-1 \text { degrees of freedom }
\end{array}
$$

10.3 The laboratory must analyze blanks at the frequency specified in Table 6 . Using the sample preparation manifold, blanks will be generated in the laboratory by filling SUMMA ${ }^{\circledR}$ canisters with humid zero air or nitrogen (99.999-percent pure). The same procedure used to prepare and analyze field samples will be used to prepare and analyze the laboratory blank's. Laboratory blank results are acceptable if the concentration of individual analytes listed in Table 1 are less than 3 times the MDL. Corrective action must be implemented if blanks exceed this level.

10.4 The laboratory must analyze individual field samples in duplicate at the frequency specified in Table 6. Single canisters analyzed in duplicate will be used to assess laboratory precision. Laboratory duplicate results will be considered acceptable if the RPD is $\leq 25$ percent. RPD is determined as in Equation (11). Duplicates which do not meet these criteria should be flagged.

10.5 The laboratory must analyze laboratory control samples at the frequency specified in Table 6. Commercially purchased gas standards will be used to prepare laboratory control 
samples. Laboratory control samples will be prepared in the laboratory by attaching the gas standard to the laboratory sample preparation manifold and filling evacuated canisters (see Section 6.3). The gas standard used to prepare the laboratory control samples must be independent of those used for instrument calibration. Laboratory control samples must contain at least six of the analytes listed in Table 1 at concentrations in the linear calibration range of the GC/MS system. The laboratory control sample analysis is designed to ensure that the laboratory's performance is in control for each analytical batch of samples processed. Laboratory control sample results will be considered acceptable if the $\% R$ is 70 to 130 percent of the known value. \%R is determined as in Equation (14). If the results are outside of this range, then the source of error must be determined, and any problems corrected.

10.6 The laboratory will be required to analyze blind audit samples. These audit samples are part of the Performance Demonstration Program. Details of this program are in the Performance Demonstration Program Plan for the Analysis of Simulated Headspace Gases for the Transuranic Waste Characterization Program (Gas PDP Plan)(DOE 1995a).

\subsection{Procedure Performance}

As part of the Performance Demonstration Program (PDP), three blind audit samples containing analytes listed in Table 1 were distributed to three laboratories. The laboratories analyzed four replicates of each sample. The results of the replicate analyses were used to determine precision and accuracy for each analyte in each sample, by laboratory. \%RSD was determined as in Equation (12), \%R was determined as in Equation (14). The precision and accuracy was then averaged for each analyte in each sample. Overall procedure performance is presented in Tables 7 through 9.

A separate laboratory determined precision and accuracy for this procedure through replicate analysis of a laboratory control samples. Precision (as \%RSD) was determined as in Equation (12), accuracy (as \%R) was determined as in Equation (14). Procedure performance for these analyses is presented in Table 10.

\subsection{References}

DOE 1995a. Performance Demonstration Program Plan for the Analysis of Simulated Headspace Gases for the Transuranic Waste Characterization Program. CAO-95-1076, Current Revision, Carlsbad, New Mexico, Carlsbad Area Office, U.S. Department of Energy.

DOE 1995b. Transuranic Waste Characterization Quality Assurance Program Plan. CAO-941010, Current Revision, Carlsbad, New Mexico, Carlsbad Area Office, U.S. Department of Energy.

EPA. 1988a. "Compendium Method TO-14, The Determination of Volatile Organic Compounds - in Ambient Air Using SUMMA Passivated Canister Sampling and Gas Chromatographic Analyses," Compendium of Methods for the Determination of Toxic Organic Compounds in Ambient Air, Research Triangle Park, North Carolina, Quality Assurance Division, Environmental Monitoring Systems Laboratory, U.S. Environmental Protection Agency.

EPA. 1988b. "Compendium Method TO-2, The Determination of Volatile Organic Compounds in Ambient Air by Carbon Molecular Sieve Adsorption and Gas Chromatography/Mass Spectrometry (GC/MS)." Compendium of Methods for the Determination of Toxic Organic Compounds in Ambient Air, Research Triangle Park, North Carolina, Quality Assurance Division, Environmental Monitoring Systems Laboratory, U.S. Environmental Protection Agency. 
Table 7

Procedure Precision and Accuracy for PDP Sample \#1

\begin{tabular}{|c|c|c|c|c|c|c|c|c|c|}
\hline \multirow[b]{2}{*}{ Analyte } & \multirow{2}{*}{$\begin{array}{c}\text { True } \\
\text { Concentration } \\
\text { (ppmv) }\end{array}$} & \multicolumn{2}{|c|}{ Laboratory 1} & \multicolumn{2}{|c|}{ Laboratory 2} & \multicolumn{2}{|c|}{ Laboratory 3} & \multirow{2}{*}{$\begin{array}{c}\text { Average } \\
\text { Precision } \\
\text { (\%RSD) }\end{array}$} & \multirow{2}{*}{$\begin{array}{c}\text { Average } \\
\text { Accuracy } \\
\text { (\%R) }\end{array}$} \\
\hline & & $\%$ RSD & $\% R$ & $\%$ RSD & $\% R$ & $\%$ RSD & $\% R$ & & \\
\hline Acetone & 349 & 0.36 & 118.98 & 1.99 & 119.27 & 8.32 & 72.89 & 3.56 & 103.71 \\
\hline Benzene & 150 & 1.04 & 95.83 & 1.24 & 92.52 & 2.50 & 82.86 & 1.60 & 90.40 \\
\hline Butanol & 200 & 0.90 & 135.50 & 4.79 & 78.38 & 1.64 & 82.00 & 2.44 & 98.63 \\
\hline Carbon tetrachloride & 197 & 1.93 & 86.93 & 2.33 & 106.40 & 2.73 & 85.78 & 2.33 & 93.04 \\
\hline Chlorobenzene & 151 & 0.39 & 83.94 & 2.83 & 101.62 & 2.02 & 81.59 & 1.75 & 89.05 \\
\hline Cyclohexane & 492 & 1.32 & 93.50 & 3.12 & 99.41 & 2.64 & 84.56 & 2.36 & 92.49 \\
\hline Ethyl benzene & 177 & 0.00 & 82.49 & 1.58 & 101.78 & 2.20 & 80.60 & 1.26 & 88.29 \\
\hline Methylene chloride & 301 & 0.83 & 105.23 & 2.41 & 101.20 & 3.87 & 86.48 & 2.37 & 97.64 \\
\hline Toluene & 295 & 0.74 & 87.29 & 1.84 & 98.72 & 2.78 & 85.44 & 1.79 & 90.48 \\
\hline 1,1,1-Trichloroethane & 249 & 1.17 & 99.00 & 2.93 & 106.70 & 2.60 & 84.55 & 2.23 & 96.75 \\
\hline Trichloroethylene & 197 & 0.91 & 83.63 & 3.05 & 107.17 & 2.28 & 82.88 & 2.08 & 91.23 \\
\hline
\end{tabular}


Table 8

Procedure Precision and Accuracy for PDP Sample \#2

\begin{tabular}{|c|c|c|c|c|c|c|c|c|c|}
\hline \multirow[b]{2}{*}{ Analyte } & \multirow{2}{*}{$\begin{array}{c}\text { True } \\
\text { Concentration } \\
\text { (ppmv) }\end{array}$} & \multicolumn{2}{|c|}{ Laboratory 1} & \multicolumn{2}{|c|}{ Laboratory 2} & \multicolumn{2}{|c|}{ Laboratory 3} & \multirow{2}{*}{$\begin{array}{c}\text { Average } \\
\text { Precision } \\
\text { (\%RSD) }\end{array}$} & \multirow{2}{*}{$\begin{array}{c}\text { Average } \\
\text { Accuracy } \\
(\% \mathrm{R})\end{array}$} \\
\hline & & $\%$ RSD & $\% R$ & $\%$ RSD & $\% R$ & $\%$ RSD & $\% R$ & & \\
\hline Acetone & 300 & 0.99 & 120.42 & 0.51 & 110.07 & 9.63 & 63.38 & 3.71 & 97.95 \\
\hline Butanol & 250 & 2.17 & 128.40 & 6.31 & 88.66 & 5.31 & 87.43 & 4.60 & 101.50 \\
\hline Cyclohexane & 351 & 0.42 & 95.44 & 1.22 & 99.01 & 3.69 & 86.63 & 1.78 & 93.69 \\
\hline Ethyl ether & 103 & 1.37 & 97.06 & 2.49 & 102.38 & 5.14 & 94.54 & 3.00 & 98.00 \\
\hline Methanol & 151 & 5.25 & 92.88 & 0.85 & 106.74 & 9.24 & 143.49 & 5.11 & 114.37 \\
\hline Methyl isobutyl ketone & 200 & 0.39 & 106.00 & 1.51 & 118.89 & 6.14 & 85.72 & 2.68 & 103.54 \\
\hline Toluene & 101 & 0.17 & 81.58 & 1.68 & 94.63 & 3.60 & 88.14 & 1.82 & 88.12 \\
\hline p-Xylene & 151 & 0.42 & 78.31 & 2.12 & 85.53 & 4.00 & 80.19 & 2.18 & 81.34 \\
\hline
\end{tabular}


Table 9

Procedure Precision and Accuracy for PDP Sample \#3

\begin{tabular}{|c|c|c|c|c|c|c|c|c|c|}
\hline \multirow[b]{2}{*}{ Analyte } & \multirow{2}{*}{$\begin{array}{c}\text { True } \\
\text { Conconcentration } \\
\text { (ppmv) }\end{array}$} & \multicolumn{2}{|c|}{ Laboratory 1} & \multicolumn{2}{|c|}{ Laboratory 2} & \multicolumn{2}{|c|}{ Laboratory 3} & \multirow{2}{*}{$\begin{array}{c}\text { Average } \\
\text { Precision } \\
\text { (\%RSD) }\end{array}$} & \multirow{2}{*}{$\begin{array}{c}\text { Average } \\
\text { Accuracy } \\
(\% \mathrm{R})\end{array}$} \\
\hline & & $\%$ RSD & $\% R$ & $\%$ RSD & $\%$ R & $\%$ RSD & $\% R$ & & \\
\hline Benzene & 4.9 & 1.32 & 95.41 & 1.03 & 99.49 & 2.68 & 91.07 & 1.68 & 95.32 \\
\hline Carbon tetrachloride & 10.2 & 0.98 & 83.48 & 1.13 & 109.56 & 2.38 & 92.82 & 1.49 & 95.29 \\
\hline Chlorobenzene & 2.0 & 0.49 & 98.13 & 0.00 & 110.00 & 5.43 & 104.00 & 1.97 & 104.04 \\
\hline Chloroform & 1.9 & 0.47 & 106.71 & 0.00 & 105.26 & 2.67 & 93.03 & 1.05 & 101.67 \\
\hline Cyclohexane & 10.0 & 0.49 & 102.75 & 0.48 & 103.25 & 2.36 & 89.20 & 1.11 & 98.40 \\
\hline 1,1-Dichloroethane & 2.0 & 0.68 & 104.00 & 2.46 & 117.50 & 3.65 & 99.13 & 2.26 & 106.88 \\
\hline 1,2-Dichloroethane & 10.0 & 0.92 & 84.10 & 0.92 & 103.75 & 1.54 & 91.03 & 1.13 & 92.96 \\
\hline cis-1,2-Dichloroethylene & 9.9 & 0.00 & 105.05 & 1.34 & 97.47 & 2.08 & 87.37 & 1.14 & 96.63 \\
\hline Ethyl benzene & 5.0 & 0.81 & 94.00 & 0.97 & 103.50 & 4.35 & 97.10 & 2.05 & 98.20 \\
\hline Methylene chloride & 19.8 & 0.38 & 107.58 & 0.62 & 103.16 & 3.14 & 87.69 & 1.38 & 99.47 \\
\hline 1,1,2,2-Tetrachloroethane & 4.9 & 1.09 & 91.89 & 0.99 & 103.57 & 3.18 & 87.50 & 1.75 & 94.32 \\
\hline Toluene & 2.1 & 0.78 & 91.79 & 2.35 & 101.19 & 4.03 & 106.90 & 2.39 & 99.96 \\
\hline $1,1,1$-Trichloroethane & 14.8 & 0.36 & 94.43 & 0.51 & 108.78 & 2.89 & 91.99 & 1.25 & 98.40 \\
\hline Trichlor oethylene & 14.8 & 0.67 & 81.76 & 0.77 & 110.64 & 3.20 & 94.27 & 1.55 & 95.56 \\
\hline $\begin{array}{l}\text { 1,1,2-Trichloro-1,2,2- } \\
\text { trifluoroethane }\end{array}$ & 9.9 & 1.02 & 91.77 & 1.55 & 97.73 & 1.21 & 96.01 & 1.26 & 95.17 \\
\hline p-Xylene & 10.0 & 0.83 & 94.65 & 1.43 & 104.75 & 4.79 & 93.38 & 2.35 & 97.59 \\
\hline
\end{tabular}


Table 10

Procedure Precision and Accuracy from Laboratory Control Sample Analysis

\begin{tabular}{|c|c|c|c|}
\hline Analyte & $\begin{array}{c}\text { True } \\
\text { Concentration } \\
\text { (ppmv) }\end{array}$ & $\begin{array}{c}\text { Precision } \\
\text { (\%RSD) }\end{array}$ & $\begin{array}{c}\text { Accuracy } \\
(\% R)\end{array}$ \\
\hline Benzene ${ }^{a}$ & 10.13 & 4.23 & 98.9 \\
\hline Bromoform ${ }^{b}$ & 9.75 & 3.48 & 191.8 \\
\hline Carbon tetrachloride ${ }^{b}$ & 10.50 & 2.99 & 86.8 \\
\hline Chlorobenzene $^{a}$ & 9.67 & 3.78 & 103.2 \\
\hline Chloroform ${ }^{b}$ & 10.50 & 2.13 & 93.0 \\
\hline Cyclohexane $^{a}$ & 10.95 & 5.84 & 94.6 \\
\hline 1,1-Dichloroethane ${ }^{a}$ & 10.47 & 3.89 & 92.2 \\
\hline 1,2-Dichloroethane ${ }^{a}$ & 9.81 & 3.58 & 100.3 \\
\hline 1,1-Dichloroethylene ${ }^{a}$ & 10.21 & 3.78 & 100.1 \\
\hline cis-1,2-Dichloroethylene & 10.37 & 1.90 & 93.8 \\
\hline Ethyl benzene ${ }^{a}$ & 9.72 & 3.32 & 97.6 \\
\hline Ethyl ether ${ }^{\mathrm{a}}$ & 10.43 & 7.49 & 91.2 \\
\hline Methylene chloride ${ }^{b}$ & 10.12 & 5.16 & 103.2 \\
\hline $1,1,2,2$-Tetrachloroethane $e^{b}$ & 9.78 & 4.15 & 102.8 \\
\hline Tetrachloroethylene ${ }^{b}$ & 9.84 & 3.07 & 95.7 \\
\hline Toluene $^{a}$ & 9.73 & 4.14 & 98.8 \\
\hline $1,1,1$-Trichloroethane $e^{b}$ & 10.65 & 2.61 & 85.6 \\
\hline Trichloroethylene $^{b}$ & 10.04 & 2.34 & 99.3 \\
\hline 1,1,2-Trichloro-1,2,2-trifluoroethane $e^{b}$ & 10.50 & 2.73 & 92.7 \\
\hline $1,2,4$-Trimethylbenzene $e^{a}$ & 5.22 & 3.67 & 93.3 \\
\hline 1,3,5-Trimethylbenzene $\mathrm{e}^{a}$ & 5.11 & 3.98 & 90.9 \\
\hline $\mathrm{m} / \mathrm{p}-$ Xylene $^{a}$ & 10.06 & 5.27 & 106.5 \\
\hline $0-X y l e n e^{a}$ & 10.11 & 3.35 & 101.1 \\
\hline
\end{tabular}

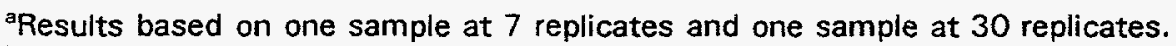

${ }^{b}$ Results based on one sample at 30 replicates. 
EPA. 1995. Test Methods for Evaluating Solid Waste, Physical/Chemical Method. SW-846, Third Edition, Final Update I, and Final Update II, Washington D.C., Office of Solid Waste and Emergency Response, U.S. Environmental Protection Agency.

Stephenson, J. H. M., F. Allen, and T. Slagle. 1990. EPA/Air and Waste Management Association-International Symposium on Measurement of Toxic and Related Air Pollutants. 


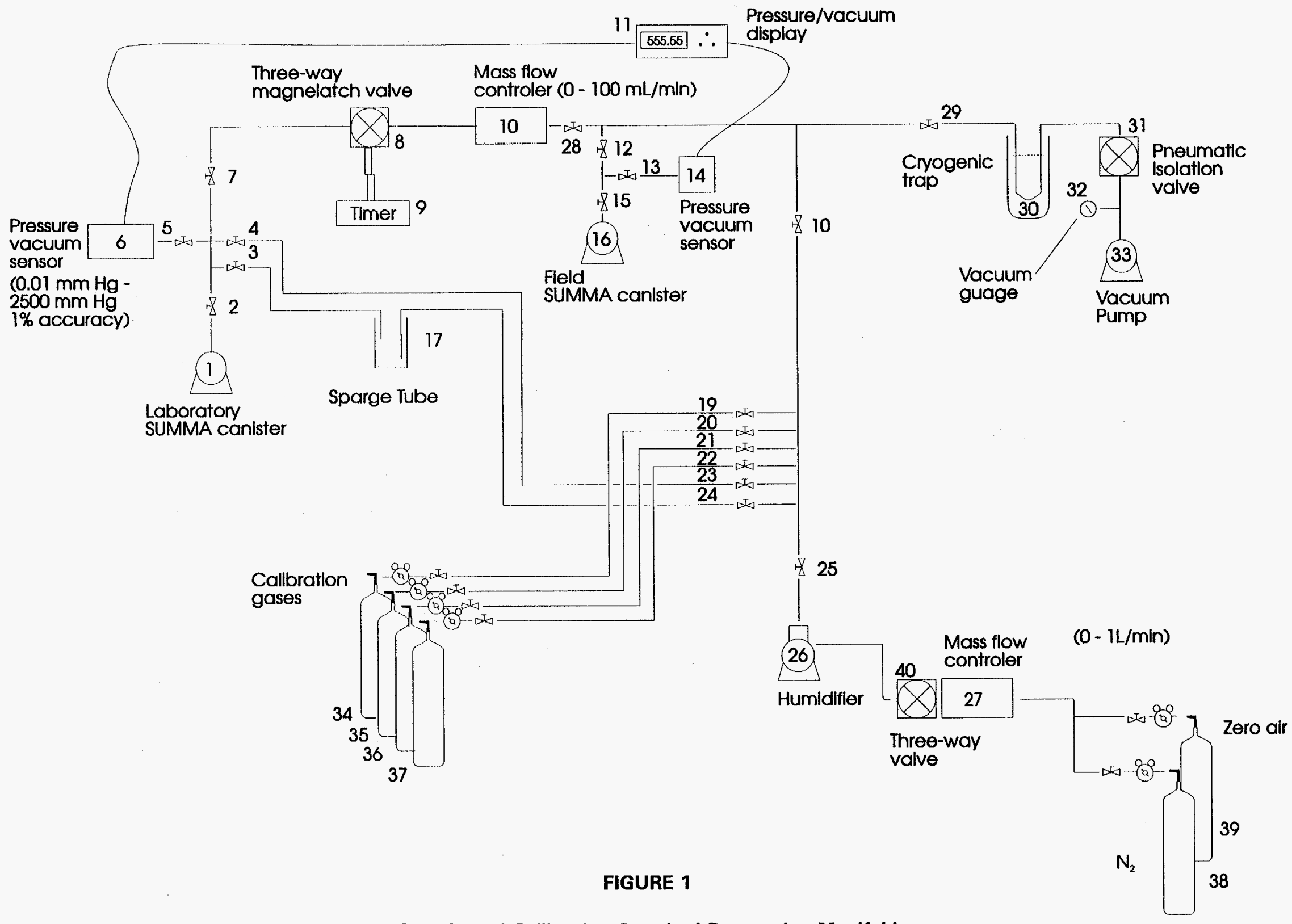




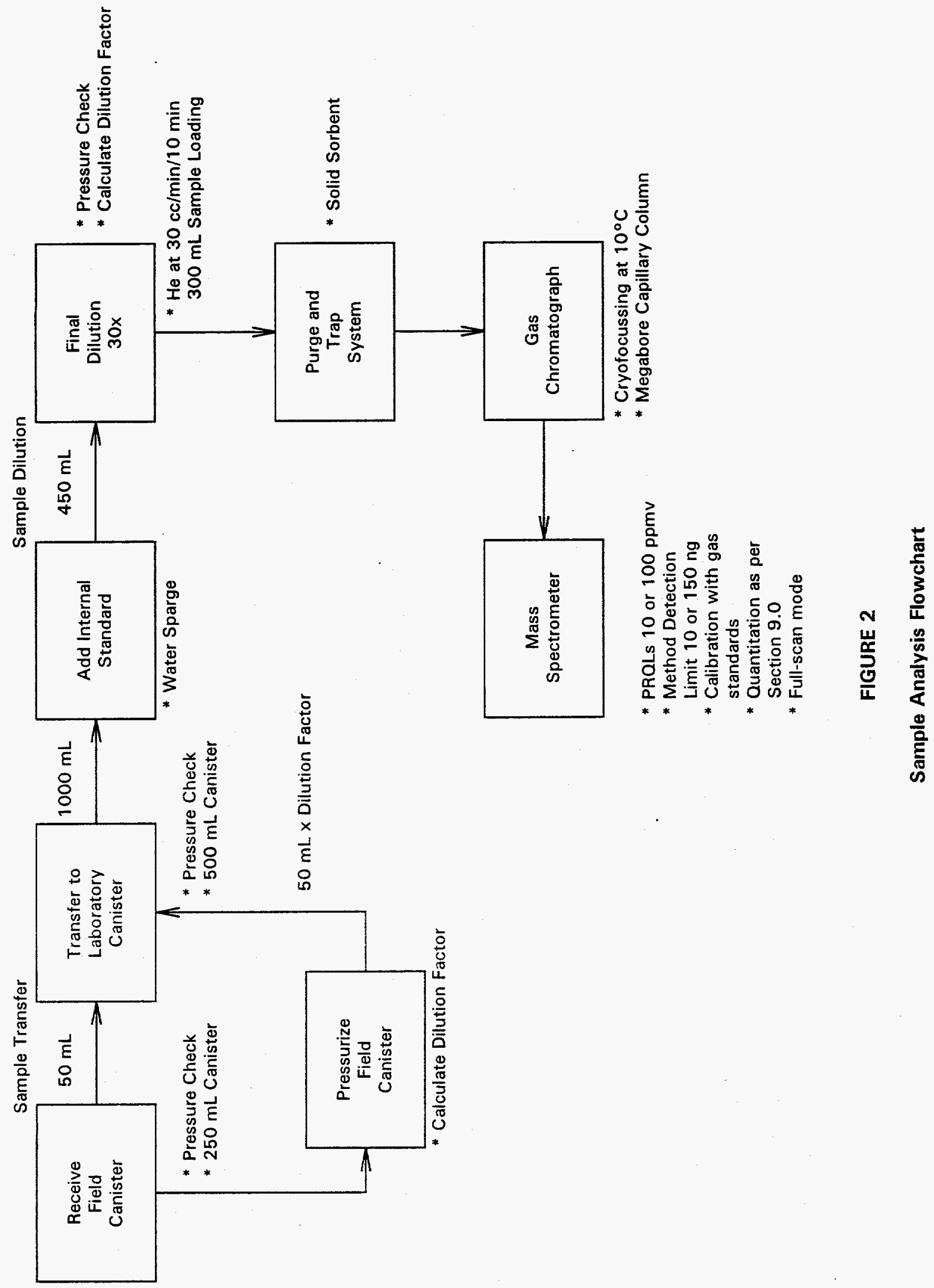




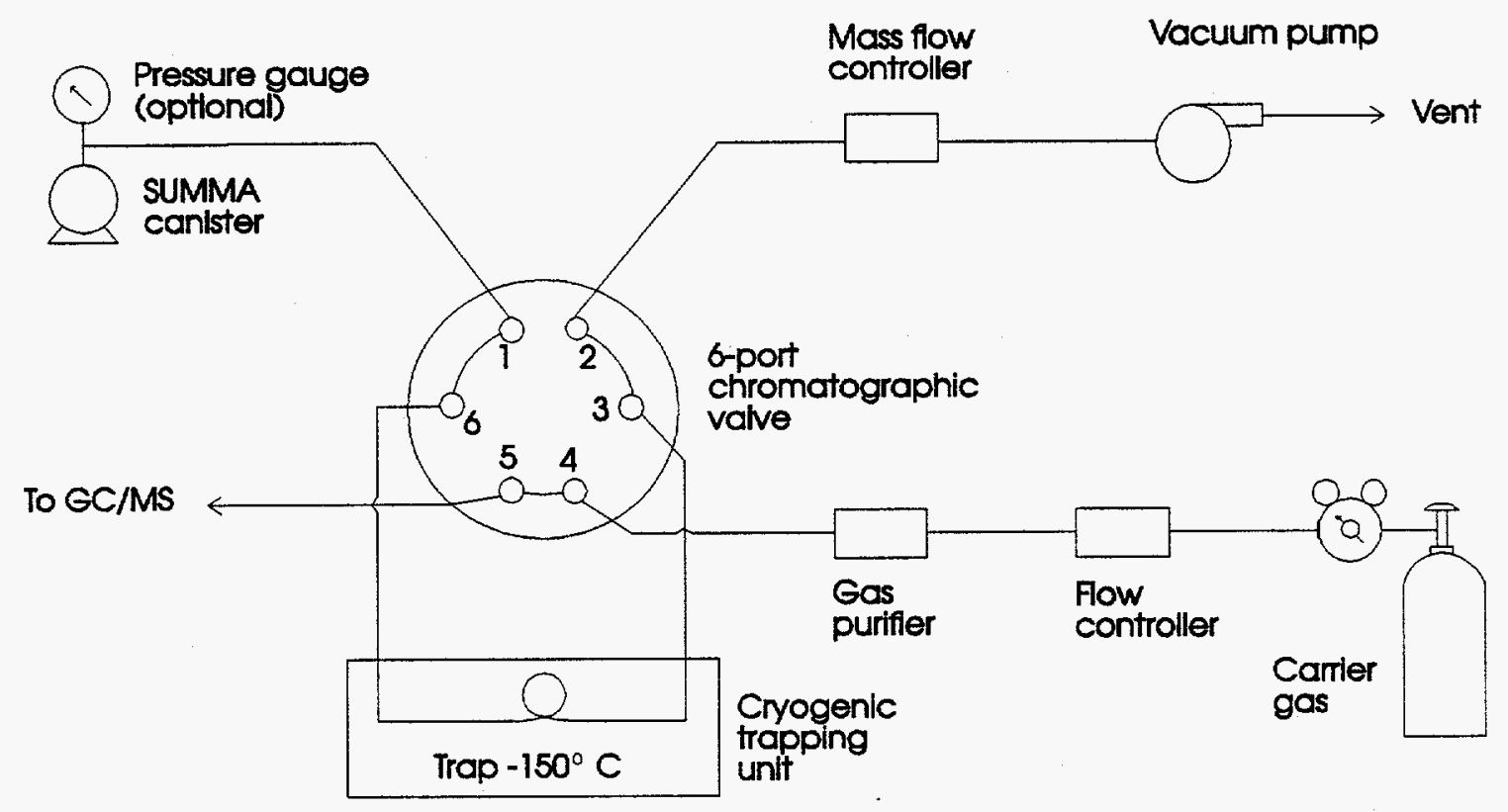

A. Sample trapping mode

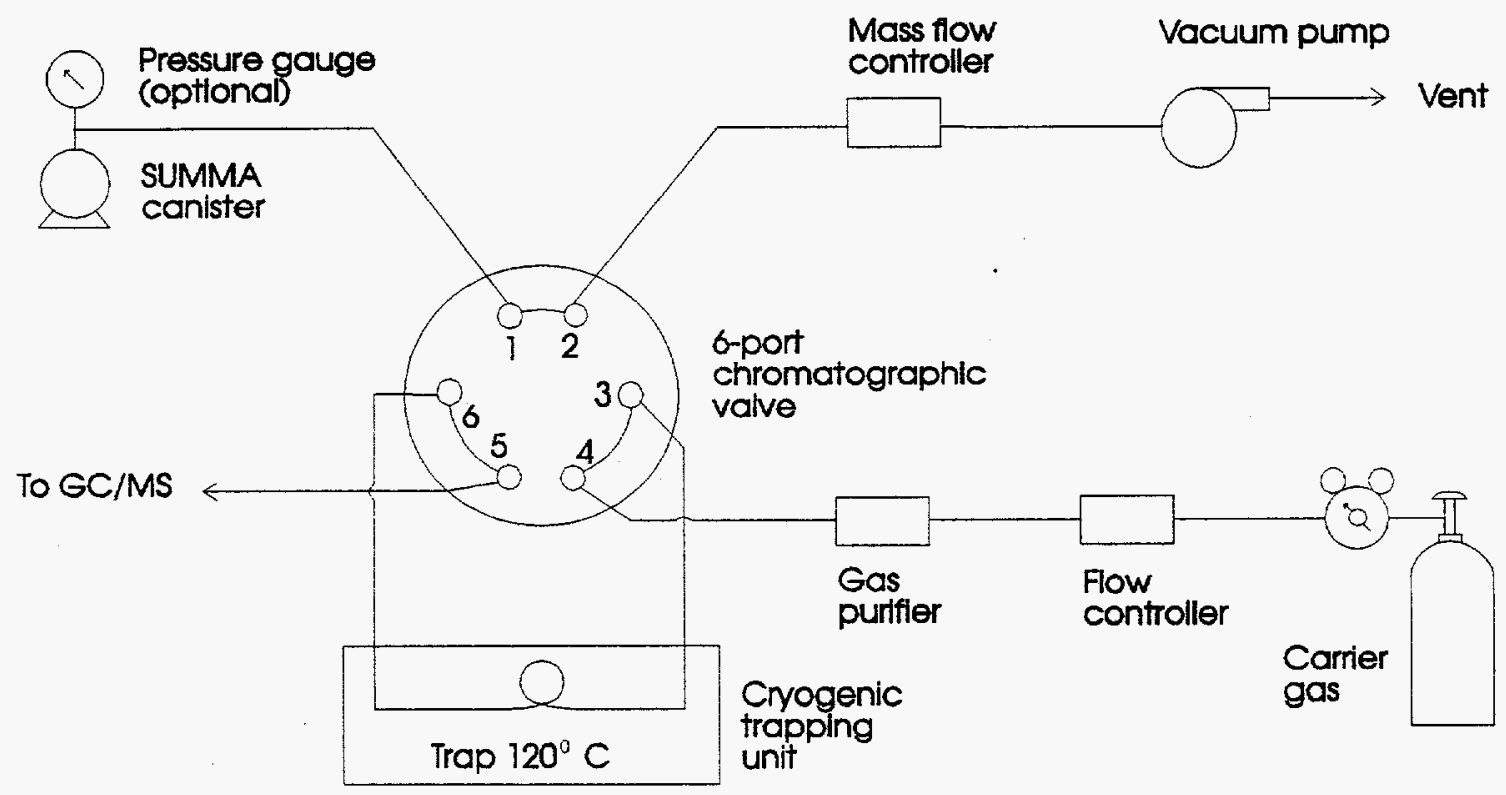

B. Sample desorption mode

FIGURE 3

Cryogenic Trapping With Mass Flow Control 


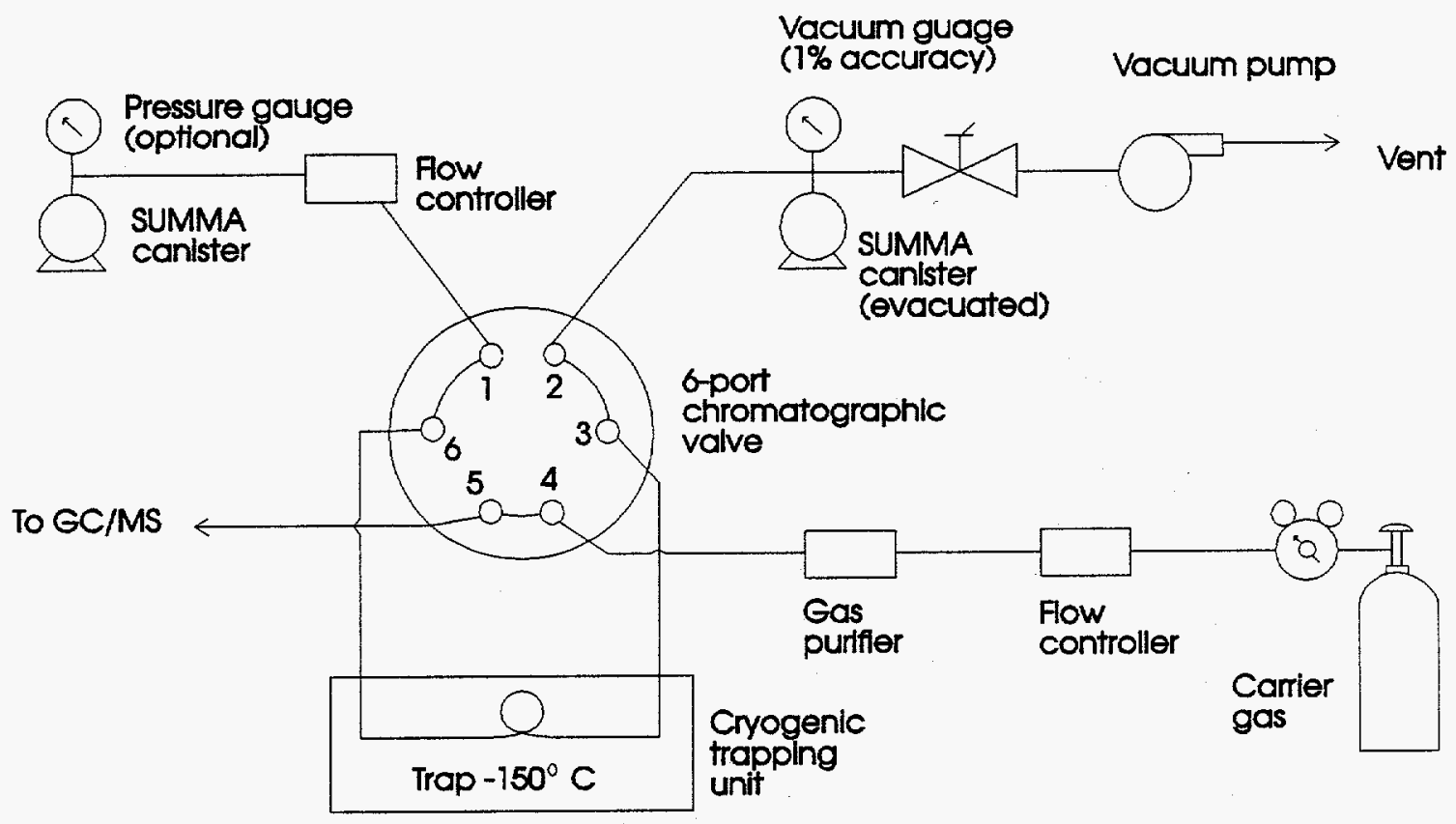

A. Sample trapping mode

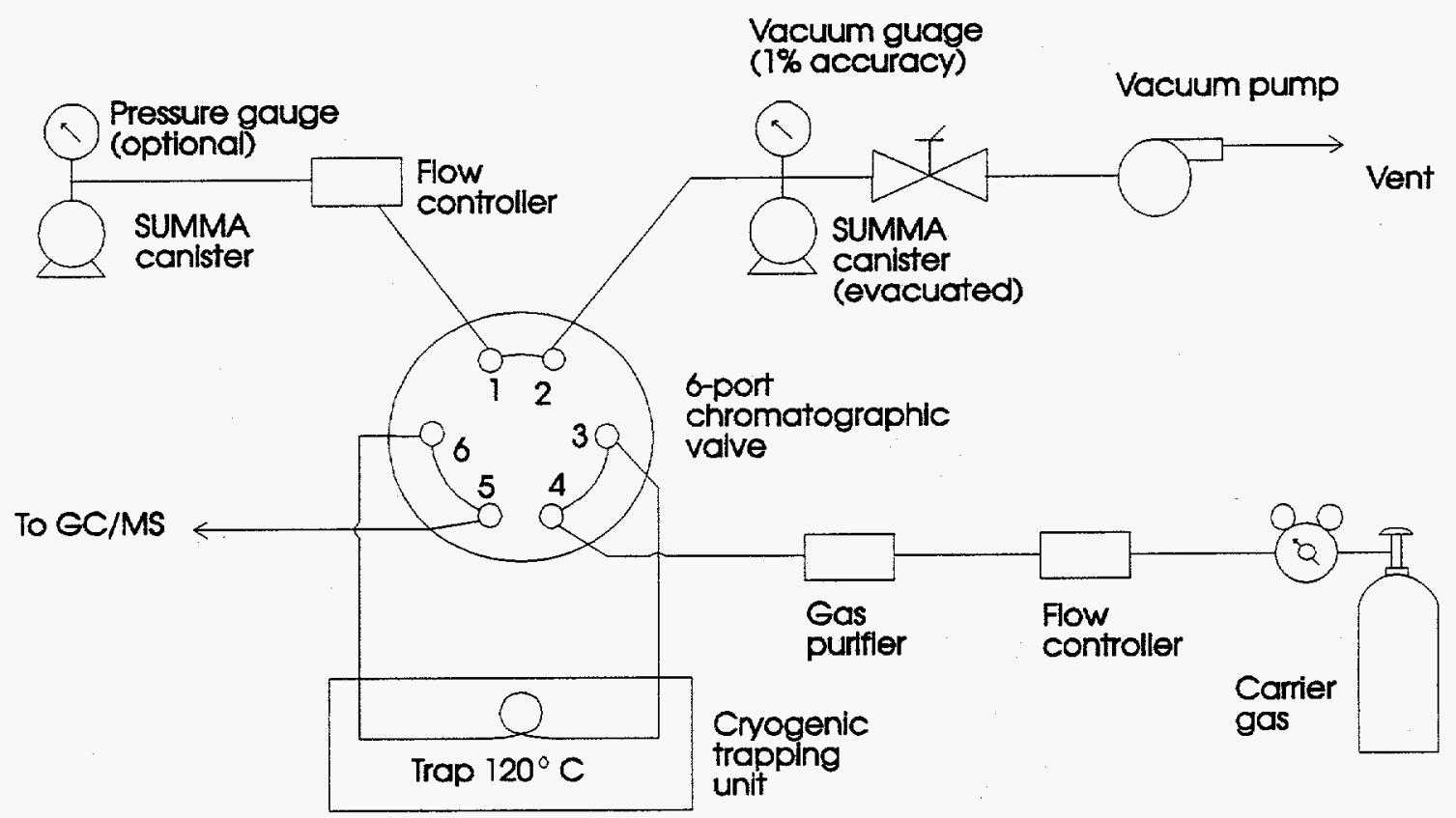

B. Sample desorption mode

FIGURE 4

Cryogenic Trapping With Evacuated Canister 


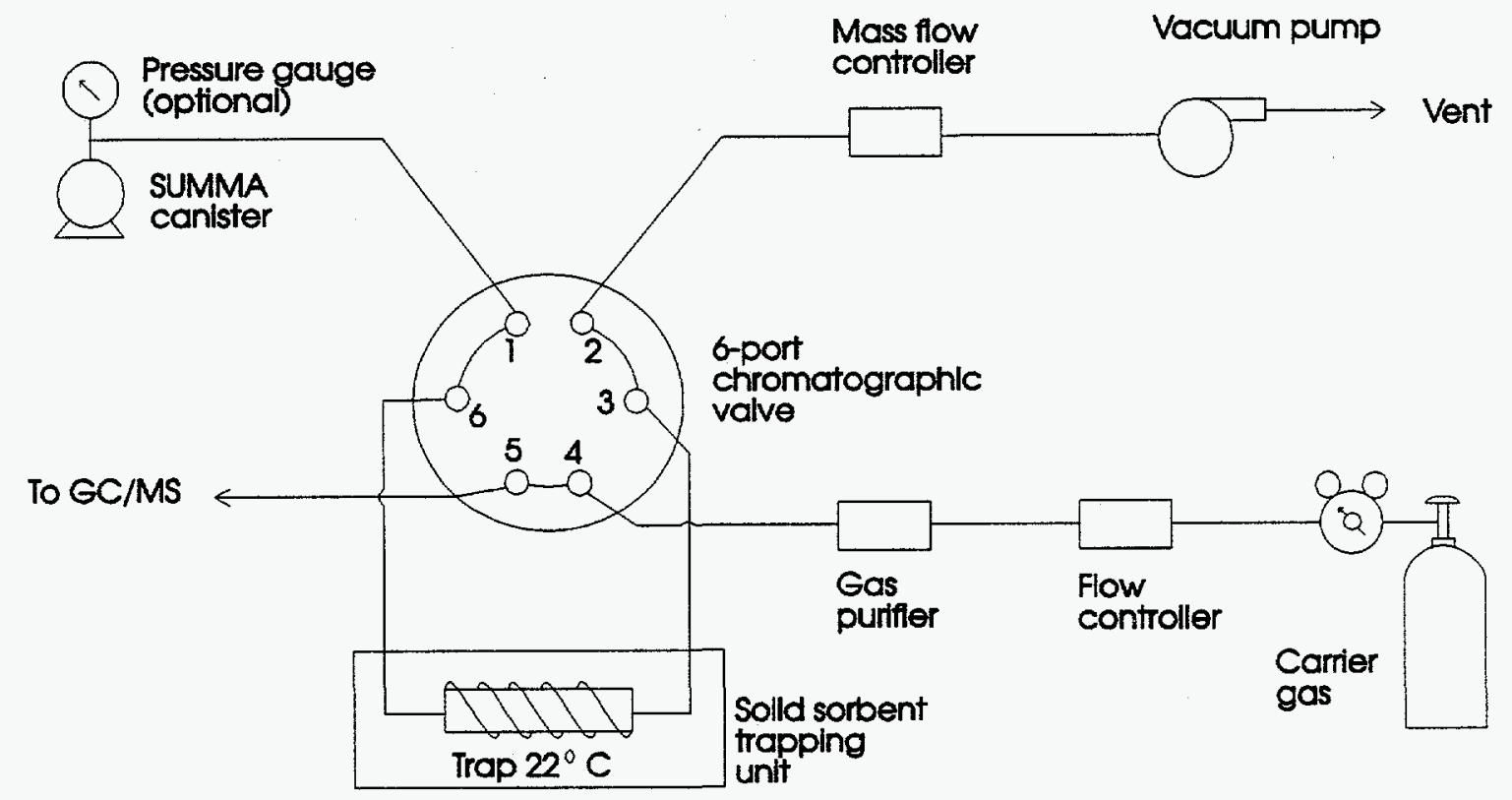

A. Sample trapping mode

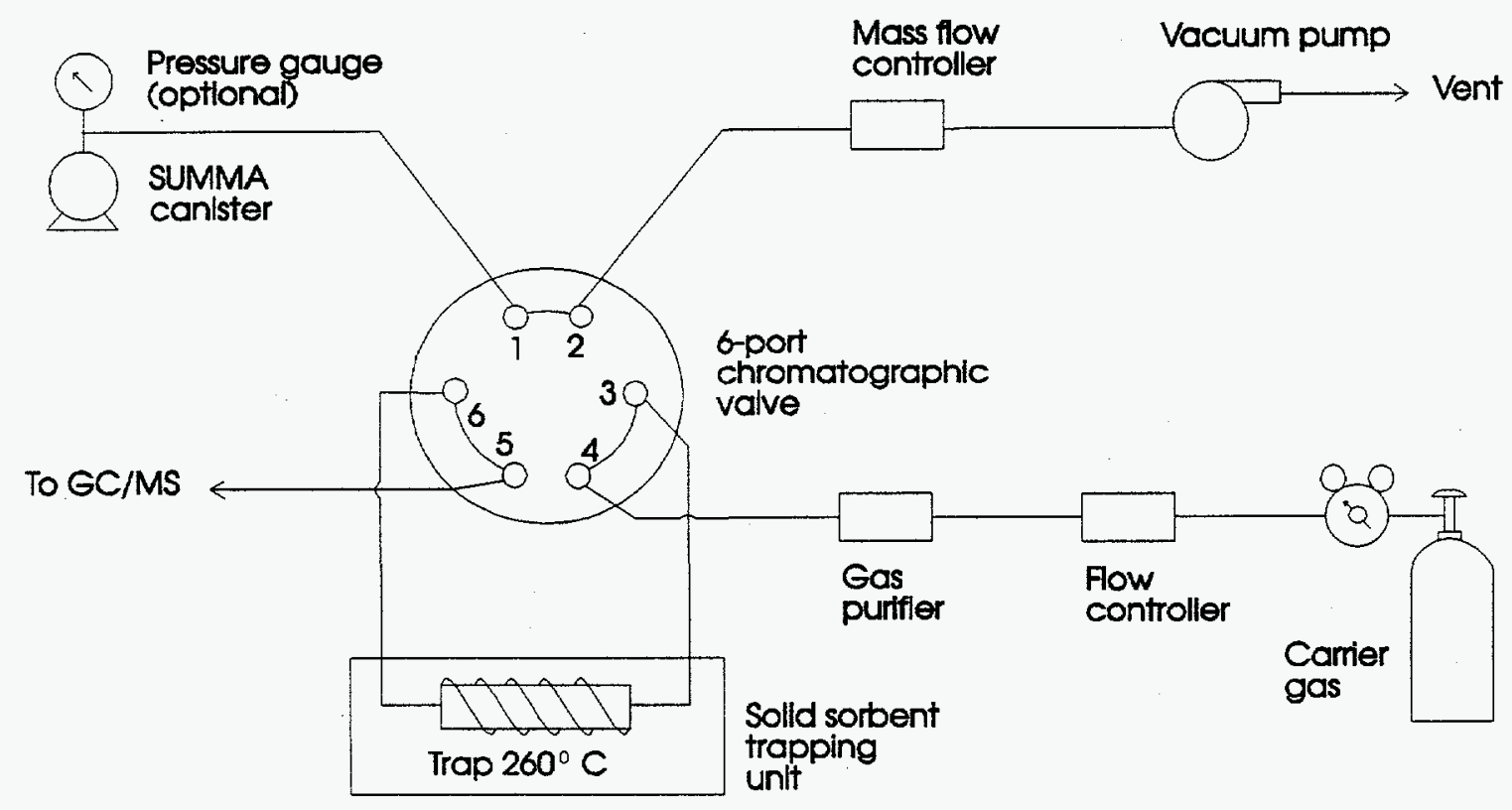

B. Sample desorption mode

FIGURE 5

Solid Sorbent Trapping With Mass Flow Control 


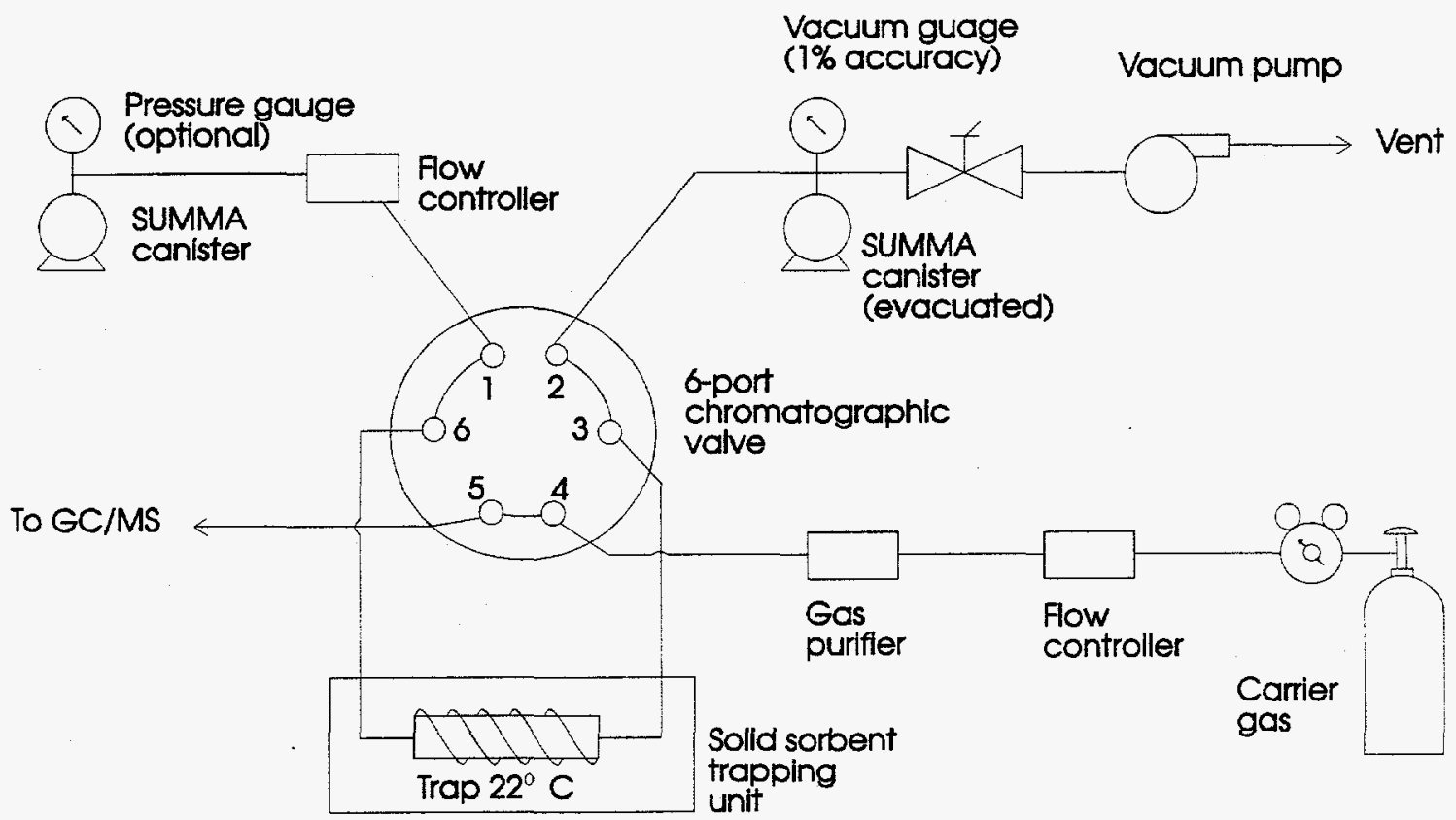

A. Sample trapping mode

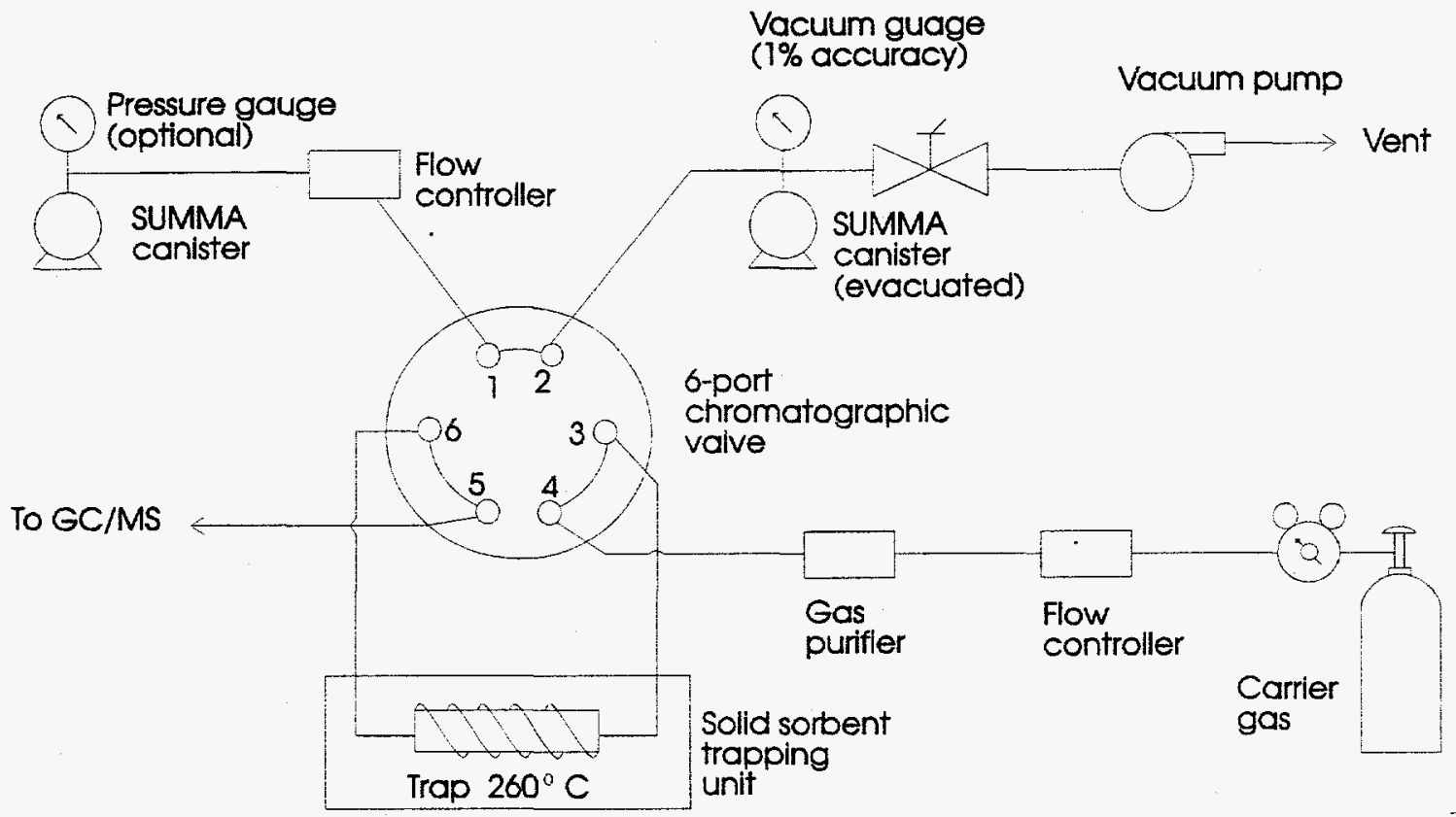

B. Sample desorption mode

FIGURE 6

Solid Sorbent Trapping With Evacuated Canister 


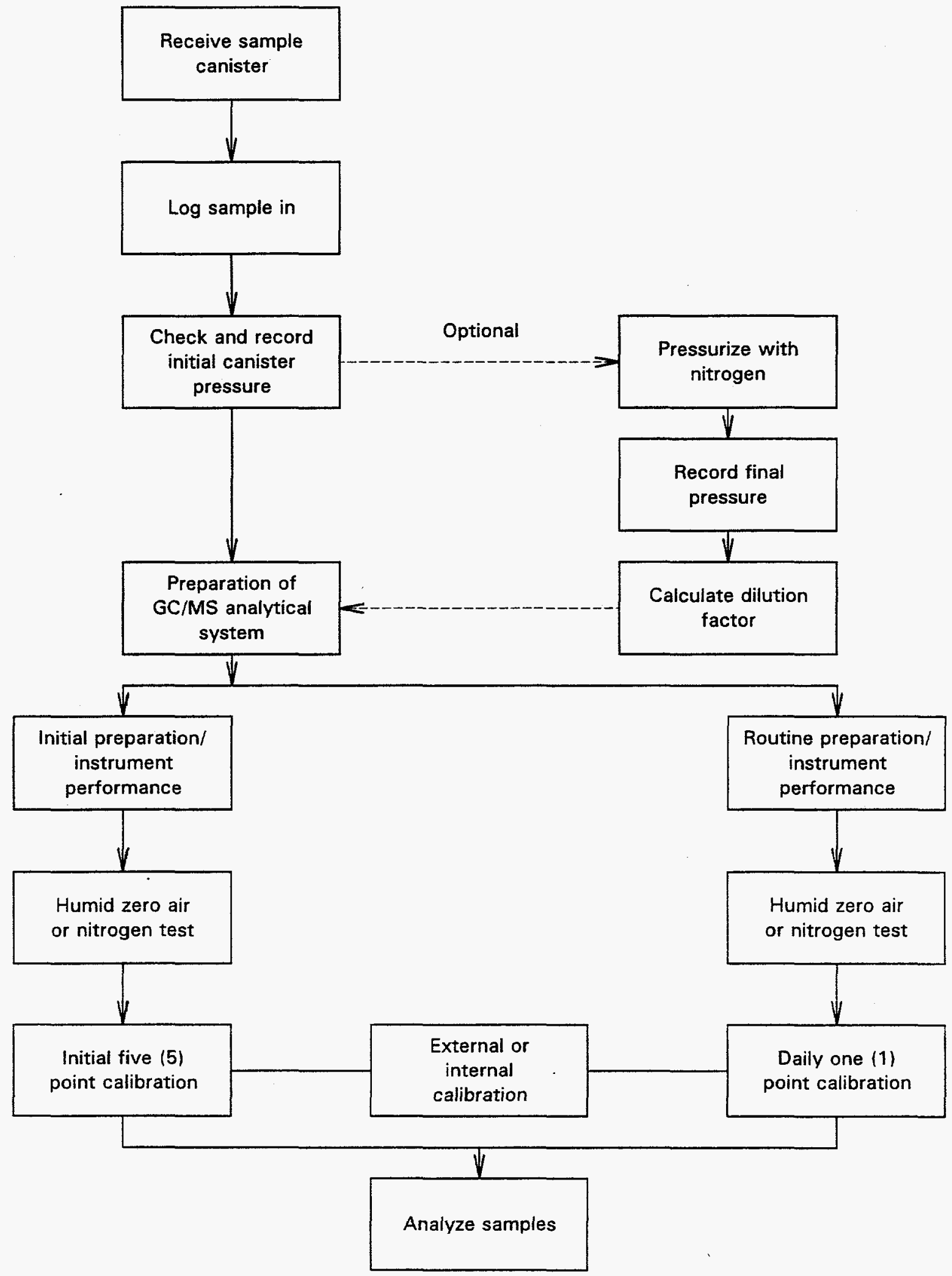

FIGURE 7

Analytical System Preparation 


\section{MODIFIED METHOD 8240/8260 FOR THE \\ DETERMINATION OF VOLATILE ORGANIC COMPOUNDS IN WASTE CONTAINER HEADSPACE}

\subsection{Scope and Application}

1.1 This procedure is for the analysis of the volatile organic compounds (VOCs), listed in Table 1 in concentrations ranging from $1 \mathrm{ppmv}$ (ppm, volume/volume) to volume percent, that have been collected from waste container headspace using SUMMA ${ }^{\oplus}$ passivated canisters. Table 1 also lists the quality assurance objectives for this procedure. This procedure is based on Method 8240 , "Volatile Organics by Gas Chromatography/Mass Spectrometry (GC/MS)" and Method 8260, "Volatile Organic Compounds by Gas Chromatography/Mass Spectrometry (GC/MS): Capillary Column Technique," in Test Methods for Evaluating Solid Waste, Physical/Chemical Methods, SW-846, Third Edition, Final Update I (SW-846) (EPA 1995). The sampling procedures are described in Procedures 110.1 through 110.4 of this Methods Manual. This procedure must be implemented with a site-specific standard operating procedure (SOP).

1.2 Modifications to SW-846 Methods 8240 and 8260 described in this procedure enable the analyst to analyze vaporized VOCs at concentrations $>1$ ppmv in gas samples up to 25 $\mathrm{mL}$ in volume that have been collected using SUMMA passivated canisters. Modifications in this procedure include the use of solid sorbent traps. These traps allow the use of a dry purge cycle to remove water from the solid sorbent column prior to the thermal desorption of trapped VOCs. Solid sorbent trapping is achieved by the use of solid sorbent columns containing only hydrophobic materials (activated carbon and carbon molecular sieves). This procedure only details the sections of SW-846 Methods 8240 and 8260 that need to be modified to allow the analysis of gas samples. Analysts must refer to SW-846 Methods 8240 and 8260 for additional guidance.

1.3 The Environmental Protection Agency (EPA) has not determined the stability of alcohols and ketones when stored in pressurized or sub-ambient pressure SUMMA ${ }^{\oplus}$ canisters. It is anticipated that no adverse problems will be encountered with these types of VOCs when stored in SUMMA ${ }^{\circ}$ canisters due to the concentration levels at which these VOCs are expected to be found.

1.4 This procedure is based upon solid sorbent trapping followed by Gas Chromatography/ Mass Spectrometry (GC/MS) analysis and should be restricted to use by or under the supervision of analysts experienced in these areas. In addition, analysts should be skilled in the interpretation of mass spectra and their use as a quantitative tool.

1.5 The program required quantitation limits (PROLs) specified in Table 1 are based on a $10 \mathrm{~mL}$ sample aliquot. PROLs will be proportionally higher for samples that require dilution or reduced sample size to avoid instrument saturation.

1.6 For the purposes of the TRU Waste Characterization Program, samples are to be analyzed in analytical batches. An analytical batch is defined as a suite of samples that is processed as a unit, using the same analytical method, within a specific time period. An analytical batch can be up to 20 samples (excluding laboratory OC samples), all of which must be received by the laboratory within 14 days of the validated time of sample receipt (VTSR) of the first sample in the batch. 
TABLE 1

Gas Volatile Organic Compounds Target Analyte List and Quality Assurance Objectives

\begin{tabular}{|c|c|c|c|c|c|c|}
\hline Compound & $\begin{array}{c}\text { CAS } \\
\text { Number }\end{array}$ & $\begin{array}{c}\text { Precision }^{a} \\
\text { (\%RSD or RPD) }\end{array}$ & $\begin{array}{l}\text { Accuracy } \\
(\% R)\end{array}$ & $\begin{array}{l}\mathrm{MDL}^{\mathrm{b}} \\
\text { (ng) }\end{array}$ & $\begin{array}{l}\text { PRQLb } \\
\text { (ppmv) }\end{array}$ & $\begin{array}{l}\text { Completeness } \\
\text { (percent) }\end{array}$ \\
\hline Benzene & $71-43-2$ & $\leq 25$ & $70-130$ & 10 & 10 & 90 \\
\hline Bromoform & $75-25-2$ & $\leq 25$ & $70-130$ & 10 & 10 & 90 \\
\hline Carbon tetrachloride & $56-23-5$ & $\leq 25$ & $70-130$ & 10 & 10 & 90 \\
\hline Chlorobenzene & $108-90-7$ & $\leq 25$ & $70-130$ & 10 & 10 & 90 \\
\hline Chloroform & $67-66-3$ & $\leq 25$ & $70-130$ & 10 & 10 & 90 \\
\hline Cyclohexane & $110-87-7$ & $\leq 25$ & $70-130$ & 10 & 10 & 90 \\
\hline 1,1-Dichloroethane & $75-34-3$ & $\leq 25$ & $70-130$ & 10 & 10 & 90 \\
\hline 1,2-Dichloroethane & $107-06-2$ & $\leq 25$ & $70-130$ & 10 & 10 & 90 \\
\hline 1,1-Dichloroethylene & $75-35-4$ & $\leq 25$ & $70-130$ & 10 & 10 & 90 \\
\hline cis-1,2-Dichloroethylene & $156-59-2$ & $\leq 25$ & $70-130$ & 10 & 10 & 90 \\
\hline Ethyl benzene & $100-41-4$ & $\leq 25$ & $70-130$ & 10 & 10 & 90 \\
\hline Ethyl ether & $60-29-7$ & $\leq 25$ & $70-130$ & 10 & 10 & 90 \\
\hline Formaldehyde $e^{c}$ & $50-00-0$ & $\leq 25$ & $70-130$ & 10 & 10 & 90 \\
\hline Hydrazine ${ }^{d}$ & $302-01-2$ & $\leq 25$ & $70-130$ & 10 & 10 & 90 \\
\hline Methylene chloride & $75-09-2$ & $\leq 25$ & $70-130$ & 10 & 10 & 90 \\
\hline $1,1,2,2$-Tetrachloroethane & $79-34-5$ & $\leq 25$ & $70-130$ & 10 & 10 & 90 \\
\hline Tetrachloroethylene & $127-18-4$ & $\leq 25$ & $70-130$ & 10 & 10 & 90 \\
\hline Toluene & $108-88-3$ & $\leq 25$ & $70-130$ & 10 & 10 & 90 \\
\hline $1,1,1$-Trichloroethane & $71-55-6$ & $\leq 25$ & $70-130$ & 10 & 10 & 90 \\
\hline Trichloroethylene & $79-01-6$ & $\leq 25$ & $70-130$ & 10 & 10 & 90 \\
\hline $\begin{array}{l}\text { 1,1,2-Trichloro-1,2,2- } \\
\text { trifluoroethane }\end{array}$ & $76-13-1$ & $\leq 25$ & $70-130$ & 10 & 10 & 90 \\
\hline 1,2,4-Trimethylbenzene & $95-63-6$ & $\leq 25$ & $70-130$ & 10 & 10 & 90 \\
\hline $1,3,5$-Trimethylbenzene & $108-67-8$ & $\leq 25$ & $70-130$ & 10 & 10 & 90 \\
\hline $\mathrm{m}$-Xylene $\mathrm{e}^{\mathrm{e}}$ & $108-38-3$ & $\leq 25$ & $70-130$ & 10 & 10 & 90 \\
\hline $0-X y l e n e$ & $95-47-6$ & $\leq 25$ & $70-130$ & 10 & 10 & 90 \\
\hline$p-X y l e n e^{e}$ & $106-42-3$ & $\leq 25$ & $70-130$ & 10 & 10 & 90 \\
\hline Acetone & $67-64-1$ & $\leq 25$ & $70-130$ & 150 & 100 & 90 \\
\hline Butanol & $71-36-3$ & $\leq 25$ & $70-130$ & 150 & 100 & 90 \\
\hline Methanol & $67-56-1$ & $\leq 25$ & $70-130$ & 150 & 100 & 90 \\
\hline Methyl ethyl ketone & $78-93-3$ & $\leq 25$ & $70-130$ & 150 & 100 & 90 \\
\hline Methyl isobutyl ketone & $108-10-1$ & $\leq 25$ & $70-130$ & 150 & 100 & 90 \\
\hline
\end{tabular}

$\%$ RSD $=$ Percent relative standard deviation

$\mathrm{RPD}=$ Relative percent difference

$\% R=$ Percent recovery

$\mathrm{MDL}=$ Method detection limit (maximum permissible value, total number of nanograms delivered to the analytical system per sample)

PROL $=$ Program required quantitation limit

${ }^{a}$ Criteria apply to PROL concentrations.

b $V$ alues based on delivering $10 \mathrm{~mL}$ to the analytical system.

cRequired only for homogenous solids and soil/gravel from Los Alamos National Laboratory

${ }^{d}$ Required only for homogenous solids and soil/gravel from Oak Ridge National Laboratory and Savannah river Site

'These xylene isomers cannot be resolved by the analytical methods employed in this program. 


\subsection{Summary of Procedure}

2.1 Gas samples are analyzed by injecting an aliquot of the gas sample into $5 \mathrm{~mL}$ of reagent water that has been spiked with internal standards. A purge-and-trap system transfers any VOCs in the sample to a calibrated GC/MS system.

2.2 Upon receipt at the laboratory, a canister containing a sample is attached to the sample manifold (Figure 1). The sample is diluted by direct pressurization with humid zero air or nitrogen and the canister is sampled by removing an aliquot with a gas-tight syringe (Figure 2). The volume of the sample aliquot is determined by multiplying the dilution factor from pressurization times $10 \mathrm{~mL}$. The sample aliquot is then slowly injected into a $25 \mathrm{~mL}$ sparge tube that contains $5 \mathrm{~mL}$ reagent water spiked with internal standards. The VOCs are introduced into the gas chromatograph by the purge-and-trap method. The VOCs are separated on a megabore capillary column and detected using a mass spectrometer operating in the full scan mode. The combination of purge-and-trap introduction with analysis by GC/MS allows for the qualitative and quantitative analysis of the VOCs contained in the SUMMA ${ }^{\oplus}$ canister.

2.3 The VOCs in the gas sample form a solution when injected into the $5 \mathrm{~mL}$ of reagent water. An inert gas is bubbled through the resulting solution at ambient temperature, and the VOCs are efficiently transferred (purged) from the solution. The purged VOCs are swept through a sorbent column where the VOCs are trapped. After purging is complete, the sorbent column is heated and backflushed with inert gas to desorb the VOCs onto the head of a megabore capillary column.

\subsection{Interference}

3.1 Contamination of samples may occur if the sample canisters are not properly cleaned prior to use. Instructions for cleaning the canisters are described in Procedure 210.1 of this Methods Manual.

3.2 Cross-contamination can occur whenever high-concentration and low-concentration samples are analyzed sequentially. Whenever a high-concentration sample is analyzed, it should be followed by the analysis of a blank of ultra-pure air or nitrogen.

3.3 For additional information concerning interferences, consult Section 3.0 of SW-846 Methods 8240 and 8260 .

\subsection{Safety}

This procedure may involve the use of hazardous materials, operations, and equipment. It is the responsibility of whoever uses this procedure to consult appropriate site personnel concerning health and safety issues and establish appropriate health and safety practices. Consideration should be given to safety concerns regarding chemical and radiation exposure. Training regarding proper storage, usage, and disposal of chemicals is recommended.

Samples and reference materials may be flammable. Keep away from heat, sparks, and open flame. Provide adequate ventilation.

\subsection{Apparatus and Materials}

\subsection{Analytical System.}

5.1.1 GC/MS System: Section 4.3 of SW-846 Method 8260 gives a detailed description of the minimum requirements for the gas chromatograph, the GC/MS interface, the 
gas chromatographic column, the mass spectrometer, and the data system to be used by this method.

5.1.2 Purge-and-Trap System: A purge-and-trap device consists of three separate components: a sparge vessel, a trap, and a desorber. Several complete devices are commercially available. Section 4.1 of SW-846 Method 8260 and Section 4.11 of SW-846 Method 8240 present more detailed descriptions of purge-and-trap devices.

Sparge Vessel: A $25 \mathrm{~mL}$ sparge vessel is required by this method.

Trap: The trap must be at least $25 \mathrm{~cm}$ long and have an inside diameter of at least $0.27 \mathrm{~cm}$. The recommended trap should have the following amounts of adsorbents (starting at the trap inlet): $7.6 \mathrm{~cm}$ of Carbopack $B$ and $1.3 \mathrm{~cm}$ of Carbosieve S-III (Supelco, Supelco Park, Bellafonte, PA, Model 2-301 or equivalent.) Before initial use, the trap should be conditioned for one hour at $260^{\circ} \mathrm{C}$ by backflushing with an inert gas flow of at least $30 \mathrm{~mL} / \mathrm{min}$. Vent the trap effluent to a hood, not to the analytical column. Prior to daily use, the trap should be conditioned for 10 minutes at $260^{\circ} \mathrm{C}$ with backflushing.

Desorber: The desorber should be capable of rapidly heating the trap to $220^{\circ} \mathrm{C}$ for desorption.

5.2 Sample and Calibration Standard Preparation Manifold. Section 5.2 of Procedure 430.1 (Modified Method TO-14) of this Methods Manual gives a detailed description of the requirements for the sample and calibration standard preparation manifold to be used by this method.

5.3 Syringes. Sections 4.5 through 4.7 of SW-846 Method 8260 give detailed descriptions of the syringes required by this method.

\subsection{Reagents}

6.1 Methanol, $\mathrm{CH}_{3} \mathrm{OH}$, or Propanol, $\mathrm{CH}_{3} \mathrm{CH}_{2} \mathrm{CH}_{2} \mathrm{OH}$. HPLC grade or equivalent, demonstrated to be free of analytes (less than 3 times the MDLs in Table 1).

6.2 Reagent Water. Section 5.2 of SW-846 Method 8260 addresses the preparation of organic-free reagent water.

6.3 Carrier and Dilution Gases. Helium, nitrogen, and zero air; all ultra-high purity grade.

6.4 Liquid Nitrogen. (or other appropriate coolant)

6.5 Stock Standards.

6.5.1 Gases: Stock calibration gas standards containing all the anaiytes listed in Table 1 must be purchased commercially (Scott Specialty Gases or equivalent.) The gas standards must be traceable to a National Institute of Standards and Technology (NIST) Standard Reference Material (SRM) or to a NIST/EPA approved Certified Reference Material (CRM). All gas standards must be replaced after 12 months if comparison with check standards indicates a problem.

6.5.2 Liquids: Stock solutions must be prepared from pure standard materials or purchased as certified solutions. Prepare stock solutions in the appropriate solvent. 
The preparation of liquid stock standards is described in Section 5.7 of SW-846 Method 8260. As an alternative to weighing the flask, the density of the reference material may be used to determine the volume of material to be added to the flask.

6.6 Secondary Dilution Standards. Using stock standard solutions, prepare secondary dilution standards in an appropriate solvent, containing the compounds of interest, either singly or mixed together. Secondary dilution standards must be prepared fresh every week and stored with minimal headspace.

6.7 Internal Standards. The recommended internal standards are fluorobenzene and chlorobenzene- $d_{5}$. Prepare internal stock standards and secondary dilution standards in an appropriate solvent. It is recommended that the secondary dilution internal standard be prepared at a concentration of $25 \mathrm{ng} / \mu \mathrm{L}$ for each internal standard. Addition of $10 \mu \mathrm{L}$ of this standard to $5 \mathrm{~mL}$ of water or calibration standard would be equivalent to $250 \mathrm{ng}$ of each internal standard.

6.8 4-Bromofluorobenzene (BFB) Standard. A standard solution containing $25 \mathrm{ng} / \mu \mathrm{L}$ of BFB should be prepared in an appropriate solvent.

6.9 Calibration Standards. Calibration standards must be prepared at a minimum of five concentration levels from secondary dilution of stock standards. Prepare these solutions in reagent water. One of the concentration levels should be at a concentration that delivers an amount (ng) of each analyte equal to a $10 \mathrm{~mL}$ gas sample at the PROLs listed in Table 1. As an example, a $10 \mathrm{~mL}$ sample of benzene at $1 \mathrm{ppmv}$ contains about $32 \mathrm{ng}$ of benzene at one atmosphere pressure and $25^{\circ} \mathrm{C}$ (Section 6.3 of Procedure 430.1 , Modified Method TO-14, of this Methods Manual). The remaining concentration levels should define the calibration range of the GC/MS system.

6.10 Laboratory Control Samples. Laboratory control samples are prepared from stock calibration gas standards in the same way as secondary dilution standards. The preparation of secondary dilution standards is described in Section 6.3 in Procedure 430.1 (Modified Method TO-14) of this Methods Manual.

\subsection{Sample Collection, Preservation, and Handling}

7.1 Samples will be collected using the sampling procedures described in Procedures 110.1 through 110.4 of this Methods Manual.

7.2 Sample canisters must be stored at room temperature. Sample holding times cannot exceed 28 days.

7.3 All sample handling and chain-of-custody procedures described in Section 6.0 of the Transuranic Waste Characterization Quality Assurance Program Plan (QAPP) (DOE 1995b) must be followed.

\subsection{Procedure}

\subsection{Recommended GC/MS System Operating Conditions.}

Electron energy:

Mass Range:

Scan Time:

Column type:

Initial column temperature:
70 volts (nominal)

29 to 260 amu

One scan/second or enough to give 5 scans/peak

DB-624, $30 \mathrm{~m} \times 0.53 \mathrm{~mm}$ ID

$10^{\circ} \mathrm{C}$ 
Initial column holding time:

Column temperature program:

Final column temperature:

Final column holding time:

Injector temperature:

Source temperature:

Transfer line temperature:

Carrier gas:
5 minutes

$6^{\circ} \mathrm{C} /$ minute

$160^{\circ} \mathrm{C}$

Until all compounds have eluted

200 to $225^{\circ} \mathrm{C}$

According to manufacturer specifications

250 to $300^{\circ} \mathrm{C}$

Helium at $15 \mathrm{~mL} /$ minute

8.2 All SUMMA ${ }^{\circ}$ canisters used for GC/MS system calibration, quality control samples, and analytical purposes must first be cleaned and certified in accordance with Procedure 210.1 of this Methods Manual.

8.3 GC/MS Instrument Performance Testing. Every 12 hour shift, or prior to a calibration, the GC/MS system must meet the BFB criteria specified in Table 2. Instrument performance criteria must be met by injecting $50 \mathrm{ng}$ of BFB on column. Instrument hardware tuning may be performed with perfluorotributylamine (PFTBA).

8.4 Purge-and-Trap Assembly. Assemble a purge-and-trap device that meets the specifications given in Section 5.1. Connect the purge-and-trap device to the gas chromatograph of the GC/MS system.

8.5 Calibration. Table 3 provides a summary of calibration requirements.

8.5.1 Initial Calibration. Initially, a five-point calibration is performed on the GC/MS system. Each calibration standard must contain all of the analytes in Table 1 and $250 \mathrm{ng}$ of each internal standard. One calibration standard must be set below the PRQLs specified in Table 1. Sections 7.3.4 and 7.3.5 of SW-846 Method 8260 or Sections 7.2.5 and 7.2.6 of SW-846 Method 8240 give the specific details for performing initial calibrations.

Prior to the analysis of any samples (including standards), instrument performance criteria must be satisfied (Section 8.3). The initial calibration curve for each compound listed in Table 1 must be checked and verified once during every 12 hour shift and prior to the analysis of any samples. This is accomplished by analyzing a continuing calibration standard that contains amounts (ng) of compounds near the midpoint of the initial calibration.

8.5.1.1 An Extracted Ion Current Profile (EICP) plots ions of a specified mass versus time or scan number. Tabulate the area response of the EICP characteristic ion used, found in Table 4 (primary or secondary), against the amount (ng) for each compound and for each internal standard from the five calibration standards. Calculate the relative response factor (RRF) for each compound using the assignments made in Table 5. When the analytical system is calibrated in nanograms, the RRF is calculated as follows:

$$
R R F=\frac{\left(A_{x} C_{I S}\right)}{\left(A_{I S} C_{x}\right)}
$$

where

$$
\begin{array}{ll}
A_{x}= & \begin{array}{l}
\text { area of the characteristic ion for the compound } \\
\text { being measured }
\end{array} \\
A_{I S}= & \begin{array}{l}
\text { area of the characteristic ion for the specific } \\
\text { internal standard }
\end{array}
\end{array}
$$




\section{TABLE 2}

\section{4-Bromofluorobenzene Key lons and Abundance Criteria}

\begin{tabular}{ll}
\hline Mass & Ion Abundance Criteria \\
\hline 50 & 15 to $40 \%$ of mass 95 \\
75 & 30 to $60 \%$ of mass 95 \\
95 & base peak, $100 \%$ relative abundance \\
96 & 5 to $9 \%$ of mass 95 \\
173 & less than $2 \%$ of mass 174 \\
174 & greater than $50 \%$ of mass 95 \\
175 & 5 to $9 \%$ of mass 174 \\
176 & greater than $95 \%$ but less than $101 \%$ of mass 174 \\
177 & 5 to $9 \%$ of mass 176 \\
\hline
\end{tabular}


TABLE 3

Summary of GC/MS Calibration Requirements for Gas Volatile Organic Compounds Analysis

$\begin{array}{lll}\text { Technique Procedure } & \text { Frequency of Procedure Acceptance Criteria }\end{array}$

\begin{tabular}{|c|c|c|c|}
\hline \multirow[t]{3}{*}{ GC/MS } & BFB Tune & Every 12 hours & $\begin{array}{l}\text { Abundance criteria for all key ions are } \\
\text { met (see Table 2) }\end{array}$ \\
\hline & $\begin{array}{l}\text { 5-pt initial calibration } \\
(5 \text { standards) }\end{array}$ & Initially, and as needed & $\begin{array}{l}\text { \%RSD of response factor for each } \\
\text { analyte }<35\end{array}$ \\
\hline & Continuing calibration & Every 12 hours & $\begin{array}{l}\% \mathrm{D} \text { for all compounds } \leq 30 \text { of initial } \\
\text { calibration }\end{array}$ \\
\hline
\end{tabular}

$\mathrm{BFB}=4$-Bromofluorbenzene

$\% \mathrm{D}=$ Percent difference

$\%$ RSD $=$ Percent relative standard deviation 
TABLE 4

Characteristic lons for Volatile Organic Compounds

\section{Acetone}

Benzene

Bromoform

Butanol

Carbon tetrachloride

Chlorobenzene

Chloroform

Cyclohexane

1,1-Dichloroethane

1,2-Dichloroethane

1,1-Dichloroethylene

cis-1,2-Dichloroethylene

Ethyl benzene

Ethyl ether

Formaldehyde ${ }^{a}$

Hydrazine ${ }^{a}$

Methanol

Methyl ethyl ketone

Methyl isobutyl ketone

Methylene chloride

1,1,2,2-Tetrachlorothane

Tetrachloroethylene

Toluene

1,1,1-Trichloroethane

Trichloroethylene

1,1,2-Trichloro-1,2,2-

trifluoroethane

1,2,4-Trimethylbenzene

1,3,5-Trimethylbenzene

m-Xylene

o-Xylene

p-Xylene

Chlorobenzene- $\mathrm{d}_{5}$ (IS)

Fluorobenzene (IS)

\section{3}

78

173

56

117

112

83

56

63

63

96

96

106

31

31

72

43

84

83

164

92

97

130

101

105

105

106

106

106

117

96

\section{8}

52,77

$171,175,252$

31

119,121

114,77

85,47

65,83

65,83

61,98

61,98

91

59

57,43

58,100

$49,51,86$

$85,131,166$

$129,131,166$

91,65

99,117

$95,97,132$

155,85

120

120

91

91

91

82,119

77

"Sites required to analyze these compounds must determine the applicable primary and secondary ions.

IS = Internal Standard 
TABLE 5

Volatile Organic Internal Standards with Corresponding Analytes Assigned for Quantitation

\section{Acetone}

Benzene

Butanol

Carbon tetrachloride.

Chloroform

Cyclohexane

1,1-Dichloroethane

1,2-Dichloroethane

cis-1,2-Dichloroethylene

1,1-Dichloroethylene

Ethyl ether

Methanol

Methylene chloride

Methyl ethyl ketone

1,1,1-Trichloroethane

Trichloroethylene

1,1,2-Trichloro-1,2,2-trifluoroethane

\section{Bromoform}

Chlorobenzene

Ethyl benzene

Methyl isobutyl ketone

1,1,2,2-Tetrachloroethane

Tetrachloroethylene

Toluene

1,2,4-Trimethylbenzene

1,3,5-Trimethylbenzene

$\mathrm{m}$-Xylene

0 -Xylene

p-Xylene

Sites required to analyze samples for formaldehyde and hydrazine must determine the appropriate internal standard for each. 


$$
\begin{array}{lll}
C_{I S} & = & \text { amount of the specific internal standard }(\mathrm{ng}) \\
C_{x} & = & \text { amount of the compound being measured }(\mathrm{ng})
\end{array}
$$

8.5.1.2 The average RRF and standard deviation .must be calculated for each compound. Calculate the percent relative standard deviation (\%RSD) of the RRF from the five calibration points for all compounds listed in Table 1. The \%RSD is calculated as follows:

$$
\% R S D=\frac{s}{\bar{x}} * 100
$$

where

$$
\begin{array}{lll}
s & = & \text { standard deviation of RRFs for a compound } \\
\bar{x} & = & \text { mean of } 5 \text { initial RRFs for a compound (average } \\
\text { RRF) }
\end{array}
$$

The standard deviation $(s)$ is defined as follows:

$$
s=\sqrt{\sum_{i=1}^{n} \frac{\left(x_{i}-\bar{x}\right)^{2}}{n-1}}
$$

where

$$
\begin{array}{lll}
x_{i} & = & \begin{array}{l}
\text { measured value of the } i^{t h} \text { replicate sample } \\
\text { analysis measurement }
\end{array} \\
n & = & \text { number of replicate analyses }
\end{array}
$$

The \%RSD for each individual compound must be less than 35 percent. For those compounds having a \%RSD greater than 35 percent, the GC/MS data system may be used to generate a second or third order regression calibration curve. Sample analysis cannot proceed until the \%RSD requirement for each compound is met.

\subsubsection{For external calibration, refer to Procedure 430.1.}

8.5.2 Continuing Calibration: The validity of the initial calibration curve is checked by calculating the percent difference $(\% D)$ for each compound as follows:

$$
\% D=\frac{\left|\left(R R F_{l}\right)-\left(R R F_{c}\right)\right|}{\left(R R F_{l}\right)} * 100
$$

where

$$
\begin{array}{ll}
R R F_{I}= & \text { average relative response factor from initial calibration } \\
R R F_{C}= & \text { relative response factor from current midpoint standard }
\end{array}
$$

The \%D for all compounds must be less than or equal to 30 percent. For those compounds where a higher order regression curve was used, the point from the continuing calibration standard for the compound must fall within 30 percent of the curve value from the initial calibration. If the \%D criterion is not met, corrective action must be taken. If no source of the problem can be determined after corrective action has been taken, a new five-point initial calibration must be generated. This criterion must be met before quantitative sample analysis begins.

The internal standard area responses in the continuing calibration standard must be evaluated immediately after or during sample analysis. If the retention time of 
any internal standard changes by more than 30 seconds from the last continuing calibration, the chromatographic system must be checked for malfunctions and corrections must be made. If the EICP area for any internal standard changes by a factor of two from the last continuing calibration standard, the GC/MS system must be checked for malfunctions and corrections must be made. Samples analyzed while the system was malfunctioning must be reanalyzed.

\subsection{Sample Analysis.}

8.6.1 The volume of sample required to meet the PRQLs specified in Table 1 is $10 \mathrm{~mL}$. This method is designed to analyze gas volumes less than $25 \mathrm{~mL}$.

8.6.2 Pressurize the field canister using zero air or nitrogen by a factor of two. This will facilitate syringe sampling of SUMMA ${ }^{\oplus}$ canisters.

Refer to Section 8.5.4 of Procedure 430.1 (Modified Method TO-14) of this Methods Manual for details on pressurizing the field canister.

8.6.3 All sampies and standard solutions must be allowed to warm to ambient temperature before analysis.

1. Remove the plunger from a 5-mL syringe and attach a closed syringe valve. Carefully pour reagent water that has been allowed to come to ambient temperature into the syringe barrel to just short of overflowing. Replace the syringe plunger and compress the sample. Open the syringe valve and vent any residual air while adjusting the sample volume to $5.0 \mathrm{~mL}$. Care must be taken to prevent air from leaking into the syringe.

2. Add $10 \mu \mathrm{L}$ of internal standard spiking solution through the valve bore of the syringe; then close the valve. The addition of $10 \mu \mathrm{L}$ of the internal standard spiking solution to $5 \mathrm{~mL}$ of sample is equivalent to $250 \mathrm{ng}$ of each internal standard.

3. Attach the syringe-syringe valve assembly to the syringe valve on the purging device. Open the syringe valves and inject the sample into the purging chamber.

4. SUMMA ${ }^{\circledast}$ canisters can be sampled with gas-tight syringes by first securing a Teflon-faced septum to the valve inlet using a Swagelok ${ }^{\circledast}$ nut.

Equip the appropriate Luerlock gas-tight syringe with a syringe valve and needle. Place the syringe plunger into the barrel of the syringe. Open the syringe valve and compress the plunger, forcing the air from the syringe. To flush the syringe, insert the needle of the syringe into the septum of the sample canister. Open the syringe valve and either allow the pressure inside the canister to push the plunger back to volume or pull back on the plunger to fill it to volume. Close the syringe valve and remove the needle from the sample canister. Open the syringe valve and compress the plunger to empty the syringe. If there is adequate sample volume, repeat the process. If not, insert the syringe needle with the syringe valve open into the sample canister and fill the syringe with the desired volume of sample plus $5 \mathrm{~mL}$. Close the syringe valve and remove the needle from the sample canister. Open the syringe valve and compress the plunger to the desired volume and close the syringe valve. Remove the needle from the syringe. 
5. Attach the syringe valve assembly to the syringe valve on the purging device. Open the syringe valves and inject the sample into the purging chamber containing $5 \mathrm{~mL}$ water and $250 \mathrm{ng}$ of each internal standard.

6. Close both valves, remove the syringe, and purge the sample for a total of 19 minutes $(8$ minutes wet purge and 11 minutes dry purge).

7. At the conclusion of the purge cycle, the trap should be preheated to $100^{\circ} \mathrm{C}$ and the sample desorbed for 4 minutes at $225^{\circ} \mathrm{C}$ while being backflushed with helium.

8. After desorbing the sample for 4 minutes, recondition the trap by baking for 15 minutes at $260^{\circ} \mathrm{C}$. When the trap temperature has returned to less than $30^{\circ} \mathrm{C}$, it is ready for the next sample.

8.6.4 If the initial analysis of a sample detects any analytes with concentrations that exceed the initial calibration range, the sample must be either diluted and reanalyzed or reanalyzed at a lower sample volume.

8.6.5 When a sample is analyzed that has saturated the detector or shows concentrations of analytes greater than 10 times the upper limit of the initial calibration, the analysis must be followed by a humid zero air blank analysis. If the analysis is not free of interferences (target analytes $<3$ times the MDL), the system must be decontaminated. Sample analysis may not resume until a blank can be analyzed that is free of interferences.

\subsection{Calculations}

9.1 Qualitative Analysis. An analyte listed in Table 1 is identified by comparison of the sample mass spectrum with the mass spectrum of a standard of the suspected compound (standard reference mass spectrum). Standard reference mass spectra are obtained through analysis of calibration standards. Two criteria must be satisfied to verify identification:

1. A compound must elute within either the specified GC retention time (RT) window or the specified relative retention time (RRT) window of the analyte in the standard, and

2. A compound's mass spectrum must match the mass spectrum of the analyte in the standard.

The RT or RRT of compounds must be determined by comparison with a reference standard analyzed on the same day as the sample. The compound RRT must compare within \pm 0.06 RRT units of the RRT of the analyte in the reference standard. The RT must compare within plus or minus three standard deviations of the individual retention times from the most recent initial calibration. If co-elution or interference prohibits the accurate assignment of the compound RT or RRT from the total ion chromatogram, the RT or RRT should be assigned by using the EICP for the primary characteristic ions listed in Table 4.

All ions present in the standard mass spectrum at a relative intensity greater than 10 percent (most abundant ion in the spectrum equals 100 percent) must be present in the sample spectrum. The relative intensities of all ions present at $>10$ percent must agree to within \pm 20 percent between the standard and sample spectra.

For samples containing compounds not listed in Table 1, with total ion current peak areas greater than 10 percent of the nearest (RT) internal standards, a forward library search of 
the latest NIST mass spectral data base must be performed. Guidelines for making tentative identification of these unknown compounds are:

1. The relative intensities of major ions in the reference spectrum (ions $>10$ percent of the most abundant ion) should be present in the sample spectrum.

2. The relative intensities of major ions should agree within \pm 20 percent.

3. Molecular ions present in the reference spectrum should be present in the sample spectrum.

4. lons present in the sample spectrum but not in the reference spectrum should be reviewed for possible contamination or presence of co-eluting compounds.

Only after visual comparison of the sample spectrum with the results of the library searches will the mass spectral interpretation specialist assign a tentative identification.

9.2 Quantitative Analysis. Once a compound has been qualitatively identified, the quantitation of that compound will be based on the integrated abundance from the EICP of the primary characteristic ion (Table 4). Quantitation will be performed using the internal standard technique. The internal standard used for the quantitation of an analyte must be the same internal standard assigned to the analyte in Table 5.

When the analytical system is calibrated in nanograms, calculate the concentration (in ppmv) of each analyte identified in the sample as follows:

\section{Internal standard quantitation}

$$
\text { concentration }(p p m v)=\frac{\left(A_{x}\right)\left(C_{I S}\right)(R)(T)}{\left(A_{I S}\right)(R R F)\left(V_{o}\right)(M W)(P)}
$$

where

$$
V_{o}=\left(\frac{V_{a}}{D F}\right)\left(\frac{P_{a}}{760 m m H g}\right)\left(\frac{298^{\circ} K}{273+T_{a}}\right)
$$

and

\begin{tabular}{|c|c|c|}
\hline $\begin{array}{l}A_{x} \\
A_{I S} \\
C_{I S}\end{array}$ & $\begin{array}{l}= \\
= \\
=\end{array}$ & $\begin{array}{l}\text { area of the characteristic ion for compound being measured } \\
\text { area of the characteristic ion for the internal standard } \\
\text { amount of the internal standard (ng) }\end{array}$ \\
\hline $\boldsymbol{R R F}$ & $=$ & $\begin{array}{l}\text { average relative response factor for compound being measured } \\
\text { (Section } 8.5 \text { ) }\end{array}$ \\
\hline $\boldsymbol{R}$ & $=$ & $0.0821 \mathrm{~L} \mathrm{~atm} / \mathrm{mol}^{\circ} \mathrm{K}$ (at $760 \mathrm{~mm} \mathrm{Hg}$ and $273^{\circ} \mathrm{K}$ ) \\
\hline$T$ & $=$ & canister temperature $\left({ }^{\circ} \mathrm{K}\right)$ \\
\hline $\boldsymbol{P}$ & $=$ & canister pressure (atm) \\
\hline$M W$ & $=$ & molecular weight of compound being measured $(\mathrm{g} / \mathrm{mol})$ \\
\hline$V_{o}$ & $=$ & $\begin{array}{l}\text { volume of sample delivered to the analytical system, corrected for } \\
\text { dilution and standard conditions }(\mathrm{mL})\end{array}$ \\
\hline$V_{a}$ & $=$ & volume during analysis $(\mathrm{mL})$ \\
\hline $\boldsymbol{P}_{a}$ & $=$ & barometric pressure during analysis $(\mathrm{mm} \mathrm{Hg})$ \\
\hline
\end{tabular}

$$
D F=\frac{C_{P 2}}{C_{P 1}}
$$




$\begin{array}{lll}T_{a} & = & \text { temperature during analysis }\left({ }^{\circ} \mathrm{C}\right) \\ D F & = & \text { dilution factor resulting from pressurization } \\ C_{P 2} & = & \text { canister pressure after dilution }(\mathrm{mm} \mathrm{Hg}) \\ C_{P 1} & = & \text { canister pressure before dilution }(\mathrm{mm} \mathrm{Hg})\end{array}$

Note: At $25^{\circ} \mathrm{C}$ and $1 \mathrm{~atm}, \boldsymbol{R T} / \boldsymbol{P}=24.5 \mathrm{~L} / \mathrm{mol}$

9.3 An estimate of the concentration of tentatively identified compounds (TICs) in the sample should be made. The equation given above should be used to determine the estimated concentrations of TICs with the following modifications:

1. The areas $A_{x}$ and $A_{I s}$ should be from the total ion chromatograms.

2. The RRF for the TIC is assumed to be one.

3. The nearest internal standard free of interference should be used.

\subsection{Quality Control}

10.1 Each laboratory that uses this procedure is required to operate a formal quality control program. The laboratory must retain records to document the quality of the data generated. Each laboratory must have SOPs documenting and describing activities involved in using this procedure. Specific quality control practices will include, but are not limited to, the analysis of quality control samples. The types of quality control samples, their associated frequency of analysis, acceptance criteria, and corrective action required if samples do not meet the acceptance criteria, is summarized in Table 6. Laboratory SOPs shall address requirements for preparing laboratory blanks, laboratory duplicates, and laboratory control samples.

10.2 All laboratories using this procedure must demonstrate acceptable performance prior to the analysis of actual samples. Demonstration of acceptable performance will be achieved by analyzing method performance samples (Table 6). These samples can either be commercially purchased or laboratory prepared, but must contain all of the analytes listed in Table 1 at concentrations appropriate (2-5 times the PROLs) to meet the quality assurance objectives specified in Table 1 . The analysis of seven samples must meet the criteria specified for precision, accuracy, and MDL in Table 1. Demonstration of acceptable method and analyst performance must be repeated (by analyzing four samples) at a minimum of every six months. Method performance should be conducted over a period of several days to account for long-term variation. Corrective action will be implemented when acceptable method performance is not met. Precision will be determined as follows:

- For duplicate measurements, the precision expressed as the relative percent difference (RPD) is calculated as

$$
R P D=\frac{C_{1}-C_{2}}{\frac{\left(C_{1}+C_{2}\right)}{2}} * 100
$$

where

$$
\begin{array}{lll}
C_{1} \text { and } C_{2} & = & \text { two values obtained by analyzing the duplicate samples } \\
C_{1} & = & \text { larger of the two observed values }
\end{array}
$$

- For three or more replicate measurements, the precision expressed as the \%RSD is calculated as 
TABLE 6

\section{Summary of Laboratory Quality Control Samples and Frequencies for Gas Volatile Organic Compounds Analysis}

\begin{tabular}{|c|c|c|c|}
\hline QC Sample & Minimum Frequency & $\begin{array}{l}\text { Acceptance } \\
\text { Criteria }\end{array}$ & $\begin{array}{l}\text { Corrective } \\
\text { Action }^{\mathbf{a}}\end{array}$ \\
\hline $\begin{array}{l}\text { Method performance } \\
\text { samples }\end{array}$ & $\begin{array}{l}\text { Seven (7) samples initially } \\
\text { and four (4) semiannually }\end{array}$ & Meet Table 1 QAOs & $\begin{array}{l}\text { Repeat until } \\
\text { acceptable }\end{array}$ \\
\hline Laboratory duplicates & $\begin{array}{l}\text { One (1) per analytical } \\
\text { batch }\end{array}$ & $\mathrm{RPD} \leq 25^{\mathrm{b}}$ & $\begin{array}{l}\text { Nonconformance if } \\
\mathrm{RPD}>25\end{array}$ \\
\hline Laboratory blanks & $\begin{array}{l}\text { Daily prior to sample } \\
\text { analysis }\end{array}$ & $\begin{array}{l}\text { Analyte amounts } \\
<3 \times \mathrm{MDLs}\end{array}$ & $\begin{array}{l}\text { Nonconformance if } \\
\text { analyte amounts }> \\
3 \times \text { MDLs }\end{array}$ \\
\hline $\begin{array}{l}\text { Laboratory control } \\
\text { samples }\end{array}$ & $\begin{array}{l}\text { One (1) per analytical } \\
\text { batch }\end{array}$ & $70-130 \% R$ & $\begin{array}{l}\text { Nonconformance if } \\
\% R<70 \text { or }>130\end{array}$ \\
\hline Blind audit samples & $\begin{array}{l}\text { Samples and frequency } \\
\text { controlled by the Gas PDP } \\
\text { Plan }\end{array}$ & $\begin{array}{l}\text { Specified in the Gas } \\
\text { PDP Plan }\end{array}$ & $\begin{array}{l}\text { Specified in the Gas } \\
\text { PDP Plan }\end{array}$ \\
\hline
\end{tabular}

${ }^{a}$ Corrective Action when $Q C$ samples do not meet the acceptance criteria; Nonconformance procedures are outlined in Section 2.1.2.1 of the QAPP.

Applies only to concentrations greater than the PROLs listed in Table 1.

MDL $=$ Method detection limit
PDP $=$ Performance Demonstration Program
QAO $=$ Quality assurance objective
$\% \mathrm{R}=$ Percent recovery
$\mathrm{RPD}=$ Relative percent difference




$$
\% R S D=\frac{s}{y} * 100
$$

where

$$
\begin{array}{lll}
\boldsymbol{s} & = & \text { standard deviation } \\
\boldsymbol{y} & = & \text { mean of replicate analyses }
\end{array}
$$

- The standard deviation (s) is defined as

$$
s=\sqrt{\sum_{i=1}^{n} \frac{\left(y_{i}-\bar{y}\right)^{2}}{n-1}}
$$

where

$$
\begin{aligned}
& y_{i}=\quad \text { measured value of the } i^{\text {th }} \text { replicate sample analysis measurement } \\
& n=\text { the number of replicate analyses }
\end{aligned}
$$

Accuracy will be determined as the percent recovery $(\% R)$ as follows:

$$
\% R=\frac{C_{m}}{C_{s m m}} * 100
$$

where

$$
\begin{array}{ll}
C_{m m} & =\text { measured concentration } \\
C_{s m} & =\quad \text { true concentration }
\end{array}
$$

MDL will be determined as follows:

$$
M D L=t_{(n-1,1-\alpha=0.99)} * s
$$

where

$$
\begin{array}{lll}
s & = & \text { standard deviation } \\
t_{(n-1,1-\alpha=0.99)}= & t \text {-distribution value appropriate to a 99-percent confidence level } \\
& \text { and a standard deviation estimate with } n-1 \text { degrees of freedom }
\end{array}
$$

10.3 The laboratory must analyze blanks at the frequency specified in Table 6 . Using the sample preparation manifold, blanks will be generated in the laboratory by filling SUMMA ${ }^{\circledR}$ canisters with humid zero air or nitrogen (99.999-percent pure). The same procedures used to prepare and analyze field samples will be used to prepare and analyze the laboratory blanks. Laboratory blank results will be acceptable if the concentration of individual analytes listed in Table 1 are less than 3 times the MDLs. Corrective action must be implemented if blanks exceed this level.

10.4 The laboratory must analyze individual field samples in duplicate at the frequency specified in Table 6. Single canisters analyzed in duplicate will be used to assess laboratory precision. Laboratory duplicate results will be considered acceptable if the RPD is $\leq 25$ percent. RPD is determined as in equation (6). Duplicates which do not meet these criteria should be flagged.

10.5 The laboratory must analyze laboratory control samples at the frequency specified in Table 6. Commercially purchased gas standards will be used to prepare laboratory control samples. Laboratory control samples will be prepared in the laboratory by attaching the gas standard to the laboratory sample preparation manifold and filling evacuated canisters. For guidance on preparing laboratory control samples, see Section 6.3 of Procedure 430.1 
(Modified Method TO-14) of this Methods Manual. The gas standard used to prepare the laboratory control samples must be independent of those used for instrument calibration. Laboratory control samples must contain at least six of the analytes listed in Table 1 at concentrations in the calibration range of the GC/MS system. The laboratory control sample analysis is designed to ensure that the laboratory's performance is in control for each analytical batch of samples processed. Laboratory control sample results will be considered acceptable if the \%R is 70 to 130 percent of the known value. $\% R$ is determined as in equation (9). If the results are outside of this range, the source of error must be determined and the problem corrected.

10.6 The laboratory will also be required to analyze blind audit samples. These audit samples are part of the Performance Demonstration Program. Details of this program are in the Performance Demonstration Program Plan for the Analysis of Simulated Headspace Gases for the Transuranic Waste Characterization Program (Gas PDP Plan)(DOE 1995a).

\subsection{Procedure Performance}

The INEL Environmental Chemistry Laboratory determined precision and accuracy for this procedure through replicate analysis of laboratory control samples. Three separate laboratory control samples were analyzed. Each sample underwent 30 replicate analyses for each analyte. The precision and accuracy was determined for each analyte in each sample. Precision (as $\%$ RSD) was determined as in Equation (7), accuracy (as \%R) was determined as in Equation (9). Procedure performance for these analyses is presented in Tables 7 through 9.

\subsection{References}

DOE. 1995a. Performance Demonstration Program Plan for the Analysis of Simulated Headspace Gases for the Transuranic Waste Characterization Program. CAO-95-1076, Current Revision, Carlsbad, New Mexico, Carlsbad Area Office, U.S. Department of Energy.

DOE. 1995b. Transuranic Waste Characterization Quality Assurance Program Plan. CAO-941010, Current Revision, Carlsbad, New Mexico, Carlsbad Area Office, U.S. Department of Energy.

EPA. 1995. Test Methods for Evaluating Solid Waste, Physical/Chemical Methods. SW-846, Third Edition, Final Update I and Final Update II, Washington D.C., Office of Solid Waste and Emergency Response, U.S. Environmental Protection Agency. 
Table 7

Procedure Performance for Laboratory Control Sample \#1

\begin{tabular}{|c|c|c|c|}
\hline Analyte & $\begin{array}{l}\text { Concentration } \\
\text { (ppmv) }\end{array}$ & $\begin{array}{l}\text { Precision } \\
\text { (\%RSD) }\end{array}$ & $\begin{array}{c}\text { Accuracy } \\
(\% R)\end{array}$ \\
\hline Benzene & 10.1 & 5.1 & 89.4 \\
\hline Bromoform & 9.8 & 8.2 & 99.6 \\
\hline Carbon tetrachloride & 10.5 & 8.1 & 120.6 \\
\hline Chlorobenzene & 9.7 & 5.7 & 87.6 \\
\hline Chloroform & 10.5 & 8.2 & 105.9 \\
\hline Cyclohexane & 11.0 & 6.6 & 110.5 \\
\hline 1,1-Dichloroethane & 10.5 & 8.4 & 90.2 \\
\hline 1,2-Dichloroethane & 9.8 & 8.6 & 105.7 \\
\hline 1,1-Dichloroethylene & 10.2 & 7.5 & 112.0 \\
\hline cis-1,2-Dichloroethylene & 10.4 & 5.6 & 91.9 \\
\hline Ethyl benzene & 9.7 & 5.8 & 79.1 \\
\hline Ethyl ether & 10.4 & 6.5 & 115.7 \\
\hline Methylene chloride & 10.1 & 4.4 & 109.0 \\
\hline $1,1,2,2$-Tetrachloroethane & 9.8 & 8.8 & 74.8 \\
\hline Tetrachloroethylene & 9.8 & 5.6 & 93.2 \\
\hline Toluene & 9.7 & 5.4 & 90.3 \\
\hline $1,1,1$-Trichloroethane & 10.7 & 7.8 & 95.0 \\
\hline Trichloroethylene & 10.0 & 5.0 & 93.8 \\
\hline $\begin{array}{l}\text { 1,1,2-Trichloro-1,2,2- } \\
\text { trifluoroethane }\end{array}$ & 10.5 & 6.5 & 136.8 \\
\hline 1,2,4-Trimethylbenzene & 5.2 & 7.9 & 74.3 \\
\hline 1,3,5-Trimethylbenzene & 5.1 & 7.0 & 80.3 \\
\hline $\mathrm{m} / \mathrm{p}$-Xylene & 10.1 & 5.7 & 81.7 \\
\hline o-Xylene & 10.1 & 6.4 & 81.2 \\
\hline
\end{tabular}


Table 8

Procedure Performance for Laboratory Control Sample \#2

\begin{tabular}{lccc}
\hline Analyte & $\begin{array}{c}\text { Concentration } \\
\text { (ppmv) }\end{array}$ & $\begin{array}{c}\text { Precision } \\
\text { (\%RSD) }\end{array}$ & $\begin{array}{c}\text { Accuracy } \\
\text { (\%R) }\end{array}$ \\
\hline Bromoform & 0.6 & 9.1 & 111.7 \\
Carbon tetrachloride & 1.0 & 7.3 & 108.0 \\
Chloroform & 1.3 & 5.9 & 112.1 \\
Methylene chloride & 1.8 & 5.8 & 103.3 \\
1,1,2,2-Tetrachloroethane & 0.9 & 9.4 & 100.8 \\
Tetrachloroethylene & 1.0 & 8.5 & 108.4 \\
1,1,1-Trichloroethane & 1.2 & 7.0 & 107.8 \\
Trichloroethylene & 1.2 & 4.9 & 110.8 \\
1,1,2-Trichloro-1,2,2- & 0.8 & 7.1 & 121.5 \\
trifluoroethane & & & \\
\hline
\end{tabular}

Table 9

Procedure Performance for Laboratory Control Sample \#3

\begin{tabular}{lccc}
\hline Analyte & $\begin{array}{c}\text { Concentration } \\
\text { (ppmv) }\end{array}$ & $\begin{array}{c}\text { Precision } \\
\text { (\%RSD) }\end{array}$ & $\begin{array}{c}\text { Accuracy } \\
\text { (\%R) }\end{array}$ \\
\hline Benzene & 2.0 & 6.2 & 99.5 \\
Chlorobenzene & 1.4 & 5.6 & 100.5 \\
Cyclohexane & 1.9 & 9.8 & 98.2 \\
1,1-Dichloroethane & 1.6 & 5.8 & 98.3 \\
1,2-Dichloroethane & 1.6 & 8.4 & 110.6 \\
1,1-Dichloroethylene & 1.6 & 7.3 & 118.1 \\
cis-1,2-Dichloroethylene & 1.6 & 5.7 & 102.3 \\
Ethyl benzene & 1.5 & 6.3 & 102.7 \\
Ethyl ether & 2.1 & 7.0 & 111.0 \\
Toluene & 1.7 & 6.1 & 99.4 \\
1,2,4-Trimethylbenzene & 1.3 & 5.8 & 103.6 \\
1,3,5-Trimethylbenzene & 1.3 & 6.3 & 104.4 \\
m/p-Xylene & 1.5 & 6.6 & 97.6 \\
o-Xylene & 1.5 & 6.6 & 100.2 \\
& & & \\
\hline
\end{tabular}




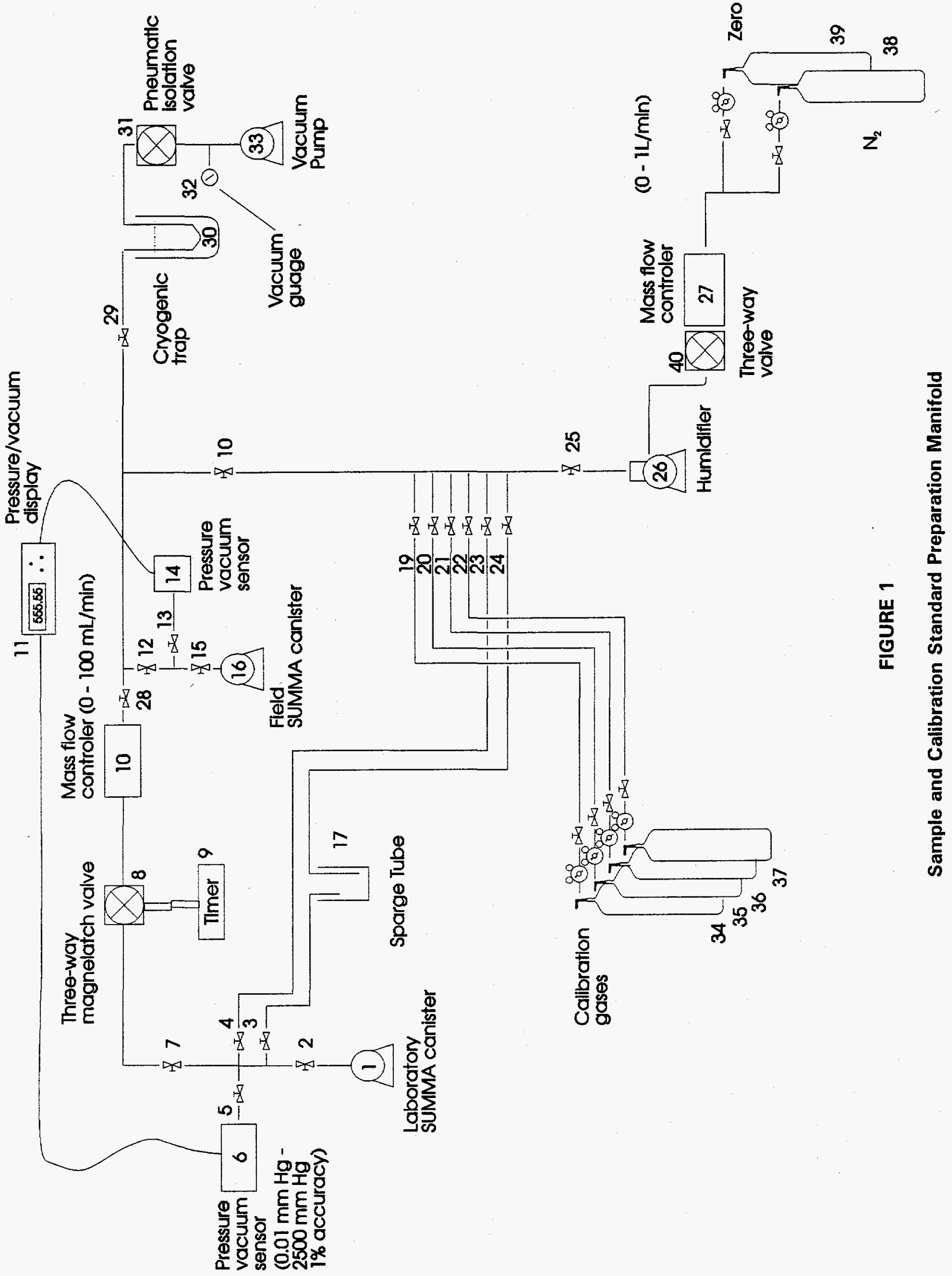




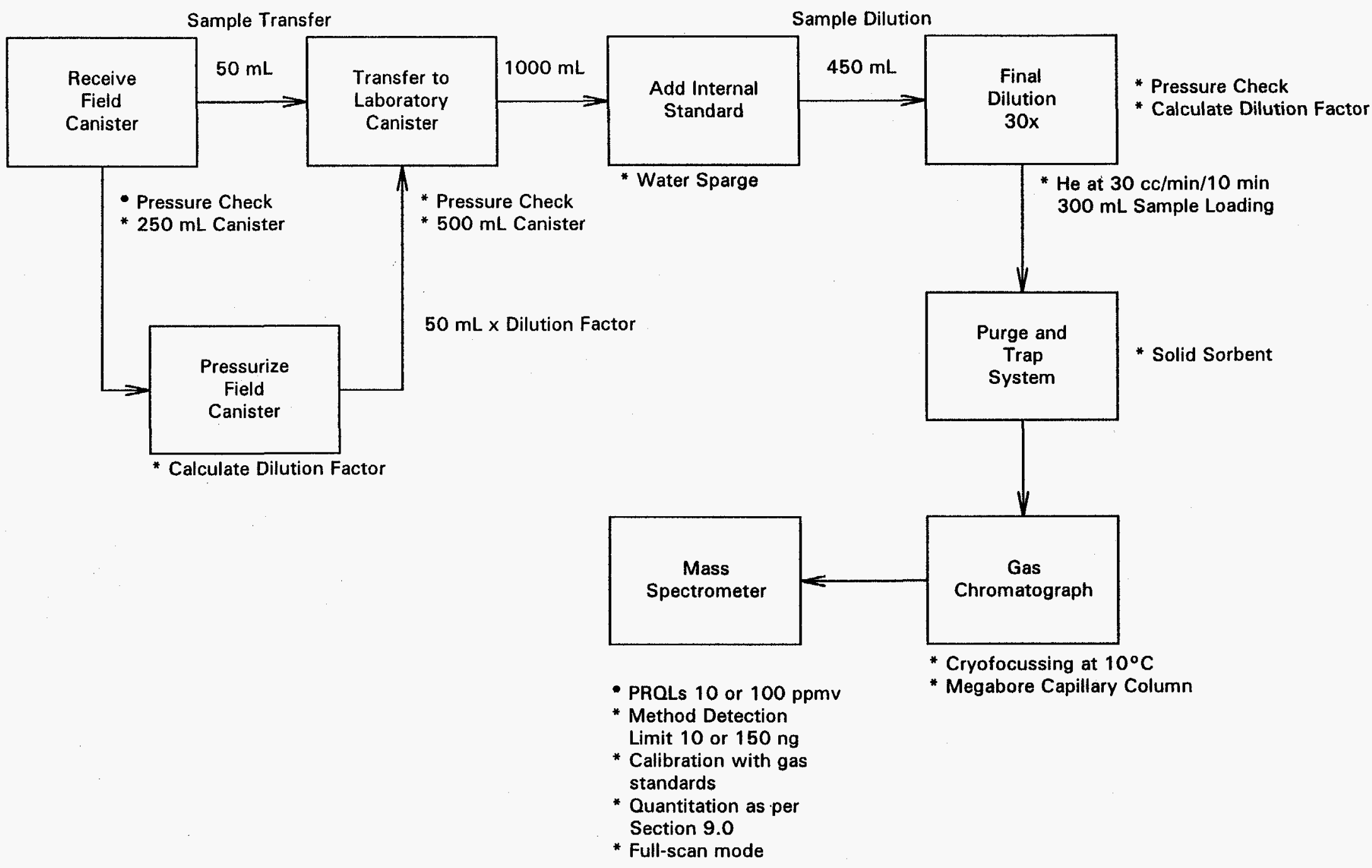


PROCEDURE 430.3

\author{
METHOD 8240B FOR THE DETERMINATION \\ OF TOTAL VOLATILE ORGANIC COMPOUNDS \\ IN HOMOGENOUS SOLIDS AND SOIL/GRAVEL
}

\title{
1.0 Scope and Application
}

1.1 This procedure is for the determination of total volatile organic compounds (VOCs) in samples of homogenous solids and soil/gravel. The procedure employs Gas Chromatography/Mass Spectrometry (GC/MS) for the quantitation of total VOCs. Table 1 lists the target analytes and quality assurance objectives for this procedure. A sampling procedure for homogenous solids and soil/gravel is described in Procedure 120.1 of this Methods Manual. This procedure must be implemented with a sitespecific standard operating procedure (SOP).

1.2 The analyst is referred to Method $8240 \mathrm{~B}$ of Test Methods for Evaluating Solid Waste, Physical/Chemical Methods, SW-846, Third Edition, Final Update II (SW-846) (EPA 1995) for the specific requirements of this procedure. The analyst is referred to Section 1.0 of SW-846 Method $8240 B$ for additional information concerning the scope and application of this procedure.

1.3 For the purposes of the TRU Waste Characterization Program, samples are to be analyzed in analytical batches. An analytical batch is defined as a suite of samples that is processed as a unit, using the same analytical method, within a specific time period. An analytical batch can be up to 20 samples (excluding laboratory $\mathrm{OC}$ samples), all of which must be received by the laboratory within 14 days of validated time of sample receipt (VTSR) of the first sample in the batch.

\subsection{Summary of Procedure}

The analyst is referred to Section 2.0 of SW-846 Method $8240 \mathrm{~B}$ for a summary of this procedure.

\subsection{Interferences}

The analyst is referred to Section 3.0 of SW-846 Method $8240 B$ for an explanation of potential interferences.

\subsection{Safety}

This procedure may involve the use of hazardous materials, operations, and equipment. It is the responsibility of whoever uses this procedure to consult appropriate site personnel concerning health and safety issues and establish appropriate health and safety practices. Consideration should be given to safety concerns regarding chemical and radiation exposure. Training regarding proper storage, usage, and disposal of chemicals is recommended.

\subsection{Apparatus and Materials}

The analyst is referred to Section 4.0 of SW-846 Method $8240 B$ for a description and listing of the required apparatus and materials for this procedure. 
TABLE 1

Total Volatile Organic Compounds Target Analyte List and Quality Assurance Objectives

\begin{tabular}{|c|c|c|c|c|c|c|}
\hline Compound & $\begin{array}{c}\text { CAS } \\
\text { Number }\end{array}$ & $\begin{array}{c}\text { Precision }{ }^{a} \\
\text { (\%RSD or RPD) }\end{array}$ & $\begin{array}{c}\text { Accuracy } \\
(\% \mathrm{R})\end{array}$ & $\begin{array}{c}\dot{M D L} \\
(\mathrm{mg} / \mathrm{kg})\end{array}$ & $\begin{array}{l}\text { PRQL } \\
(\mathrm{mg} / \mathrm{kg})\end{array}$ & $\begin{array}{c}\text { Completeness } \\
(\%)\end{array}$ \\
\hline Benzene & $71-43-2$ & $\leq 45$ & $37-151$ & 1 & 10 & 90 \\
\hline Bromoform & $75-25-2$ & $\leq 47$ & $45-169$ & 1 & 10 & 90 \\
\hline Carbon disulfide & $75-15-0$ & $\leq 50$ & $60-150$ & 1 & 10 & 90 \\
\hline Carbon tetrachloride & $56-23-5$ & $\leq 30$ & $70-140$ & 1 & 10 & 90 \\
\hline Chlorobenzene & $108-90-7$ & $\leq 38$ & $37-160$ & 1 & 10 & 90 \\
\hline Chloroform & $67-66-3$ & $\leq 44$ & $51-138$ & 1 & 10 & 90 \\
\hline 1,4-Dichlorobenzene ${ }^{b}$ & $106-46-7$ & $\leq 60$ & $18-190$ & 1 & 10 & 90 \\
\hline ortho-Dichlorobenzene $^{b}$ & $95-50-1$ & $\leq 60$ & $18-190$ & 1 & 10 & 90 \\
\hline 1,2-Dichloroethane & $107-06-2$ & $\leq 42$ & $49-155$ & 1 & 10 & 90 \\
\hline 1,1-Dichloroethylene & $75-35-4$ & $\leq 250$ & $D-234^{C}$ & 1 & 10 & 90 \\
\hline Ethyl benzene & $100-41-4$ & $\leq 43$ & $37-162$ & 1 & 10 & 90 \\
\hline Methylene chloride & $75-09-2$ & $\leq 50$ & $D-221^{C}$ & 1 & 10 & 90 \\
\hline $1,1,2,2$-Tetrachloroethane & $79-34-5$ & $\leq 55$ & $46-157$ & 1 & 10 & 90 \\
\hline Tetrachloroethylene & $127-18-4$ & $\leq 29$ & $64-148$ & 1 & 10 & 90 \\
\hline Toluene & $108-88-3$ & $\leq 29$ & $47-150$ & 1 & 10 & 90 \\
\hline 1,1,1-Trichloroethane & $71-55-6$ & $\leq 33$ & $52-162$ & 1 & 10 & 90 \\
\hline 1,1,2-Trichloroethane & $79-00-5$ & $\leq 38$ & $52-150$ & 1 & 10 & 90 \\
\hline Trichloroethylene & $79-01-6$ & $\leq 36$ & $71-157$ & 1 & 10 & 90 \\
\hline Trichlorofluoromethane & $75-69-4$ & $\leqslant 110$ & $17-181$ & 1 & 10 & 90 \\
\hline $\begin{array}{l}\text { 1,1,2-Trichloro-1,2,2- } \\
\text { trifluoroethane }\end{array}$ & $76-13-1$ & $\leq 50$ & $60-150$ & 1 & 10 & 90 \\
\hline Vinyl chloride & $75-01-4$ & $\leq 200$ & $D-251^{C}$ & 1 & 4 & 90 \\
\hline m-xylene & $108-38-3$ & $\leq 50$ & $60-150$ & 1 & 10 & 90 \\
\hline o-xylene & $95-47-6$ & $\leq 50$ & $60-150$ & 1 & 10 & 90 \\
\hline p-xylene & $106-42-3$ & $\leq 50$ & $60-150$ & 1 & 10 & 90 \\
\hline Acetone & $67-64-1$ & $\leq 50$ & $60-150$ & $10^{d}$ & 100 & 90 \\
\hline Butanol & $71-36-3$ & $\leq 50$ & $60-150$ & $10^{\mathrm{d}}$ & 100 & 90 \\
\hline Ethyl ether & $60-29-7$ & $\leq 50$ & $60-150$ & $10^{d}$ & 100 & 90 \\
\hline Formaldehyde ${ }^{\theta}$ & $50-00-0$ & $\leq 50$ & $60-150$ & $10^{d}$ & 100 & 90 \\
\hline Hydrazine $^{f}$ & $302-01-2$ & $\leq 50$ & $60-150$ & $10^{d}$ & 100 & 90 \\
\hline Isobutanol & 78-83-1 & $\leq 50$ & $60-150$ & $10^{d}$ & 100 & 90 \\
\hline Methanol & $67-56-1$ & $\leq 50$ & $60-150$ & $10^{d}$ & 100 & 90 \\
\hline Methyl ethyl ketone & $78-93-3$ & $\leq 50$ & $60-150$ & $10^{d}$ & 100 & 90 \\
\hline Pyridine $^{b}$ & $110-86-1$ & $\leq 50$ & $60-150$ & $10^{d}$ & 100 & 90 \\
\hline
\end{tabular}

$\%$ RSD $=$ Percent relative standard deviation

$\mathrm{RPD} \quad=\quad$ Relative percent difference

$\% \mathrm{R}=$ Percent recovery

$\mathrm{MDL} \quad=\quad$ Method detection limit (maximum permissible value)

PRQL = Program required quantitation limit; calculated from the toxicity characteristic level for benzene assuming a $25 \mathrm{~g}$ sample, $0.5 \mathrm{~L}$ of extraction fluid, and 100 percent analyte extraction

${ }^{a}$ Criteria apply to PROL concentrations.

${ }^{b}$ Can also be analyzed as a semi-volatile organic compound.

'Detected; result must be greater than zero.

¿Estimate, to be determined.

${ }^{e}$ Required only for homogenous solids and soil/gravel from Los Alamos National Laboratory.

${ }^{f}$ Required only for homogenous solids and soil/gravel from Oak Ridge National Laboratory and Savannah River Site. 


\subsection{Reagents}

The analyst is referred to Section 5.0 of SW-846 Method $8240 B$ for a description and listing of the required reagents, standard stock solutions, and standard solutions for this procedure.

\subsection{Sample Collection, Preservation, and Handling}

7.1 Samples will be collected using the sampling procedures described in Procedure 120.1 of this Methods Manual.

7.2 All sample handling and chain-of-custody procedures described in Section 6.0 of the Transuranic Waste Characterization Quality Assurance Program Plan (QAPP) (DOE 1995b) must be followed.

\subsection{Procedure}

Each site must decide, based on the nature of its waste stream, what preparation methods are appropriate for its waste types. Use of preparation methods in SW-846, or other nationally recognized standard methods (e.g., ASTM) is acceptable.

The analyst is referred to Sections 7.2, 7.3, and 7.4.3 of SW-846 Method 8240B for a description of the procedural requirements. Table 2 of this procedure provides a summary of required calibration and analysis quality control.

\subsection{Calculations}

The analyst is referred to Section 7.5 of SW-846 Method 8240 B for a description of the steps required for qualitative and quantitative analysis. Note that all results must be reported in $\mathrm{mg} / \mathrm{kg}$ (weight/wet-weight basis).

\subsection{Quality Control}

10.1 Each laboratory that uses this procedure is required to operate a formal quality control program. The laboratory must retain records to document the quality of the data generated. Each laboratory must have SOPs documenting and describing activities involved in using this procedure. Specific quality control practices will include, but are not limited to, the analysis of quality control samples. The types of quality control samples, their associated frequency of analysis, acceptance criteria, and corrective action required if samples do not meet the acceptance criteria, is summarized in Table 3. Laboratory SOPs shall address requirements for preparing matrix spikes, matrix spike duplicates, laboratory blanks, and laboratory control samples.

10.2 All laboratories using this procedure must demonstrate acceptable performance prior to the analysis of actual samples. Demonstration of acceptable performance will be achieved by analyzing method performance samples (Table 3). These samples will contain all of the analytes listed in Table 1 at concentrations appropriate (2-5 times the PROLs) to determine the quality assurance objectives specified in Table 1 . The analysis of seven samples must meet the criteria specified for precision, accuracy, and method detection limit (MDL) in Table 1. Demonstration of acceptable method and analyst performance must be repeated (by analyzing four samples) at a minimum of every six months. Method performance should be conducted over a period of several days to account for long-term variability. Corrective action must be implemented when acceptable method performance is not met. Precision will be determined as follows: 
TABLE 2

Summary of Calibration Requirements for Total Volatile Organic Compounds Analysis

Technique Procedure Frequency of Procedure Acceptance Criteria

\begin{tabular}{|c|c|c|c|}
\hline \multirow[t]{3}{*}{ GC/MS } & BFB Tune & Every 12 hours & $\begin{array}{l}\text { Abundance criteria for all key ions are } \\
\text { met (see SW-846 Method } 8240 B \text { ) }\end{array}$ \\
\hline & $\begin{array}{l}\text { 5-pt initial calibration } \\
\text { ( } 5 \text { standards) }\end{array}$ & Initially, and as needed & $\begin{array}{l}\text { Response factor \%RSD for CCCs } \leq \\
30 ; \text { response factor for SPCCs } \\
\geq 0.30^{\mathrm{a}} \text {; average relative response } \\
\text { factor is used if \%RSD } \leq 15 \text {; linear } \\
\text { regression equation is generated if } \\
\% \text { RSD }>15\end{array}$ \\
\hline & Continuing calibration & Every 12 hours & $\begin{array}{l}\text { Response factor or conc. } \% D \text { for CCCs } \\
\leq 20 ; \text { response factor for SPCCs } \geq \\
0.30^{\mathrm{a}} \text {; RT for internal standards must } \\
\text { be } \pm 30 \text { seconds from last daily } \\
\text { calibration check; internal standard } \\
\text { area count must be }>50 \% \text { or }< \\
200 \% \text { of the area counts from the last } \\
\text { daily calibration check; surrogate } \\
\text { compound \%R must be met (see } \\
\text { SW-846 Method } 8240 \mathrm{~B} \text { ) }\end{array}$ \\
\hline
\end{tabular}

aromoform $\geq 0.25$

BFB = 4-Bromofluorbenzene

$\mathrm{CCC}=$ Calibration check compounds

$\% \mathrm{D}=$ Percent difference

$\%$ RSD $=$ Percent relative standard deviation

RT $=$ Retention time

SPCC $=$ System performance check compounds 
TABLE 3

\section{Summary of Laboratory Quality Control Samples and Frequencies for Total Volatile Organic Compound Analysis}

\begin{tabular}{|c|c|c|c|}
\hline OC Sample & Minimum Frequency & $\begin{array}{l}\text { Acceptance } \\
\text { Criteria }\end{array}$ & $\begin{array}{l}\text { Corrective } \\
\text { Action }^{2}\end{array}$ \\
\hline $\begin{array}{l}\text { Method performance } \\
\text { samples }\end{array}$ & $\begin{array}{l}\text { Seven (7) samples initially } \\
\text { and four (4) semiannually }\end{array}$ & Meet Table 1 QAOs & Repeat until acceptable \\
\hline Laboratory duplicates $^{b}$ & $\begin{array}{l}\text { One (1) per analytical } \\
\text { batch }\end{array}$ & Meet Table 1 RPDs & $\begin{array}{l}\text { Nonconformance if RPDs } \\
>\text { Table } 1 \text { values }\end{array}$ \\
\hline Laboratory blanks & $\begin{array}{l}\text { One (1) per analytical } \\
\text { batch }\end{array}$ & $\begin{array}{l}\text { Analyte concentrations } \\
<3 \times \text { MDLs }\end{array}$ & $\begin{array}{l}\text { Nonconformance if } \\
\text { analyte concentrations } \\
>3 \times \text { MDLs }\end{array}$ \\
\hline Matrix spikes & $\begin{array}{l}\text { One (1) per analytical } \\
\text { batch }\end{array}$ & Meet Table $1 \%$ Rs & $\begin{array}{l}\text { Nonconformance if \%Rs } \\
\text { are outside the range } \\
\text { specified in Table } 1\end{array}$ \\
\hline Matrix spike duplicates & $\begin{array}{l}\text { One (1) per analytical } \\
\text { batch }\end{array}$ & $\begin{array}{l}\text { Meet Table } 1 \text { RPDs and } \\
\% \text { Rs }\end{array}$ & $\begin{array}{l}\text { Nonconformance if RPDs } \\
\text { and } \% \text { Rs }>\text { Table } 1 \\
\text { values }\end{array}$ \\
\hline $\begin{array}{l}\text { Laboratory control } \\
\text { samples }\end{array}$ & $\begin{array}{l}\text { One (1) per analytical } \\
\text { batch }\end{array}$ & $80-120 \% R$ & $\begin{array}{l}\text { Nonconformance if } \% R \\
<80 \text { or }>120\end{array}$ \\
\hline Surrogate compounds & Each analytical sample & $\begin{array}{l}\text { Average \%R from } \\
\text { minimum of } 30 \text { samples } \\
\text { for a given matrix } \pm 3 \\
\text { standard deviations }\end{array}$ & $\begin{array}{l}\text { Nonconformance if } \% \mathrm{R} \\
<\text { (average } \% \mathrm{R}-3 \\
\text { standard deviation) or } \\
>\text { (average } \% \mathrm{R}+3 \\
\text { standard deviation) }\end{array}$ \\
\hline Blind audit samples & $\begin{array}{l}\text { Samples and frequency } \\
\text { controlled by the Solid } \\
\text { PDP Plan }\end{array}$ & $\begin{array}{l}\text { Specified in the Solid } \\
\text { PDP Plan }\end{array}$ & $\begin{array}{l}\text { Specified in the Solid } \\
\text { PDP Plan }\end{array}$ \\
\hline
\end{tabular}

${ }^{a}$ Corrective Action when QC samples do not meet the acceptance criteria; Nonconformance procedures are outlined in Section 2.1.2.1 of the QAPP.

bMay be satisfied using matrix spike duplicate; acceptance criteria applies only to concentrations greater than the PRQLs listed in Table 1.

$\begin{array}{lll}\text { MDL } & =\text { Method detection limit } \\ \text { QAO } & \text { Quality assurance objective } \\ \text { PDP } & =\text { Performance Demonstration Program } \\ \% R & = & \text { Percent recovery } \\ \text { RPD } & =\text { Relative percent difference }\end{array}$


- For duplicate measurements, the precision expressed as the relative percent difference (RPD) is calculated as

$$
R P D=\frac{C_{1}-C_{2}}{\frac{\left(C_{1}+C_{2}\right)}{2}} * 100
$$

where

$C_{1}$ and $C_{2}=$ two values obtained by analyzing the duplicate samples

$C_{I}=$ larger of the two observed values

- For three or more replicate measurements, the precision expressed as the percent relative standard deviation (\%RSD) is calculated as

$$
\% R S D=\frac{s}{\bar{y}} * 100
$$

where

$$
\begin{aligned}
& \dot{s}=\text { standard deviation } \\
& y=\text { mean of replicate analyses }
\end{aligned}
$$

- The standard deviation $(s)$ is defined as

$$
s=\sqrt{\sum_{i=1}^{n} \frac{\left(y_{i}-\bar{y}\right)^{2}}{n-1}}
$$

where

$y_{i}=$ measured value of the $i^{\text {th }}$ replicate sample analysis measurement

$\boldsymbol{n}=$ number of replicate analyses

Accuracy will be determined as the percent recovery (\%R) as follows:

$$
\% R=\frac{C_{m}}{C_{s r m}} * 100
$$

where

$$
\begin{array}{ll}
C_{m} & =\text { measured concentration } \\
C_{s r m} & =\text { true concentration }
\end{array}
$$

MDL will be determined as follows:

$$
M D L=t_{(n-1,1-\alpha=0.99)} * s
$$

where

$$
\begin{aligned}
& s \quad=\text { standard deviation } \\
& \left.t_{(n-1,1-\alpha}=0.99\right)=t \text {-distribution value appropriate to a 99-percent confidence level and }
\end{aligned}
$$

10.3 The laboratory must analyze blanks at the frequency specified in Table 3 . The same analytical method used to prepare and analyze field samples will be used to prepare and analyze the laboratory blanks. Laboratory blank results will be acceptable if the concentration of target analytes is less than 3 times the MDL specified in Table 1 for each compound. Corrective action must be implemented if blanks exceed this level. 
10.4 The laboratory must analyze matrix spikes at the frequency specified in Table 3 . Matrix spike results shall be considered acceptable if the $\% R$ is within the range specified for each compound in Table 1. Matrix spikes which do not meet these criteria should be flagged.

$\% R$ will be determined as follows:

$$
\% R=\frac{S-U}{C_{s a}} * 100
$$

where

$$
\begin{aligned}
& S=\text { measured concentration in the spiked aliquot } \\
& U=\text { measured concentration in the unspiked aliquot } \\
& C_{s a}=\text { actual concentration of the spike added }
\end{aligned}
$$

10.5 The laboratory must analyze laboratory duplicates or matrix spike duplicates at the frequency specified in Table 3. Duplicate results shall be considered acceptable if the RPD for each compound is within the range specified in Table 1. RPD is determined as in Equation (1). Duplicates which do not meet these criteria should be flagged.

10.6 The laboratory must analyze laboratory control samples at the frequency specified in Table 3. Laboratory control samples must contain at least ten of the compounds listed in Table 1 and shall be prepared, in water, at a concentration of $20 \mu \mathrm{g} / \mathrm{L}$ per laboratory control analyte from standards independent of those used for instrument calibration. The laboratory control samples must be quantitated within the calibration range of the instrument. The laboratory control sample analysis is designed to ensure that laboratory performance is in control for each analytical batch of samples processed. If the laboratory control sample \%R is outside the range specified in Table 3 for the ten compounds, then new laboratory control samples must be prepared. \%R is determined as in Equation (4).

10.7 The laboratory will be required to analyze blind audit samples. These audit samples are part of the Performance Demonstration Program. Details of this program are in the Performance Demonstration Program Plan for the Analysis of Solidified Wastes for the Transuranic Waste Characterization Program (Solid PDP Plan)(DOE 1995a).

\subsection{Procedure Performance}

The analyst is referred to Section 9.0 of SW-846 Method $8240 B$ for a summary of method performance.

\subsection{References}

DOE. 1995a. Performance Demonstration Program Plan for the Analysis of Solidified Wastes for the Transuranic Waste Characterization Program. CA0-95-1077, Current Revision, Carlsbad, New Mexico, Carlsbad Area Office, U.S. Department of Energy.

DOE. 1995b. Transuranic Waste Characterization Quality Assurance Program Plan. CAO-941010 Current Revision, Carlsbad, New Mexico, Carisbad Area Office, U.S. Department of Energy.

EPA. 1995. Test Methods for Evaluating Solid Waste, Physical/Chemical Methods. SW-846, Third Edition, Final Update I, and Final Update II, Washington D.C., Office of Solid Waste and Emergency Response, U.S. Environmental Protection Agency. 


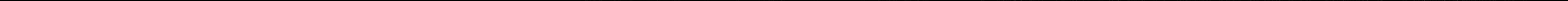


PROCEDURE 430.4

\section{METHOD 8260A FOR THE DETERMINATION OF TOTAL VOLATILE ORGANIC COMPOUNDS IN HOMOGENOUS SOLIDS AND SOIL/GRAVEL}

\subsection{Scope and Application}

1.1 This procedure is for the determination of total volatile organic compounds (VOCs) in samples of homogenous solids and soil/gravel. The procedure employs Gas Chromatography/Mass Spectrometry (GC/MS) for the quantitation of total VOCs. Table 1 lists the target analytes and quality assurance objectives for this procedure. A sampling procedure for homogenous solids and soil/gravel is described in Procedure 120.1 of this Methods Manual. This procedure must be implemented with a sitespecific standard operating procedure (SOP).

1.2 The analyst is referred to Method 8260A of Test Methods for Evaluating Solid Waste, Physical/Chemical Methods, SW-846, Third Edition, Final Update II (SW-846) (EPA $1995)$ for the specific requirements of this procedure. The analyst is referred to Section 1.0 of SW-846 Method 8260A for additional information concerning the scope and application of this procedure.

1.3 For the purposes of the TRU Waste Characterization Program, samples are to be analyzed in analytical batches. An analytical batch is defined as a suite of samples that is processed as a unit, using the same analytical method, within a specific time period. An analytical batch can be up to 20 samples (excluding laboratory $O C$ samples), all of which must be received by the laboratory within 14 days of validated time of sample receipt (VTSR) of the first sample in the batch.

\subsection{Summary of Procedure}

The analyst is referred to Section 2.0 of SW-846 Method $8260 \mathrm{~A}$ for a summary of this procedure.

\subsection{Interferences}

The analyst is referred to Section 3.0 of SW-846 Method $8260 \mathrm{~A}$ for an explanation of potential interferences.

\subsection{Safety}

This procedure may involve the use of hazardous materials, operations, and equipment. It is the responsibility of whoever uses this procedure to consult appropriate site personnel concerning health and safety issues and establish appropriate health and safety practices. Consideration should be given to safety concerns regarding chemical and radiation exposure. Training regarding proper storage, usage, and disposal of chemicals is recommended.

\subsection{Apparatus and Materials}

The analyst is referred to Section 4.0 of SW-846 Method 8260 A for a description and listing of the required apparatus and materials for this procedure. 
TABLE 1

Total Volatile Organic Compounds Target Analyte List and Quality Assurance Objectives

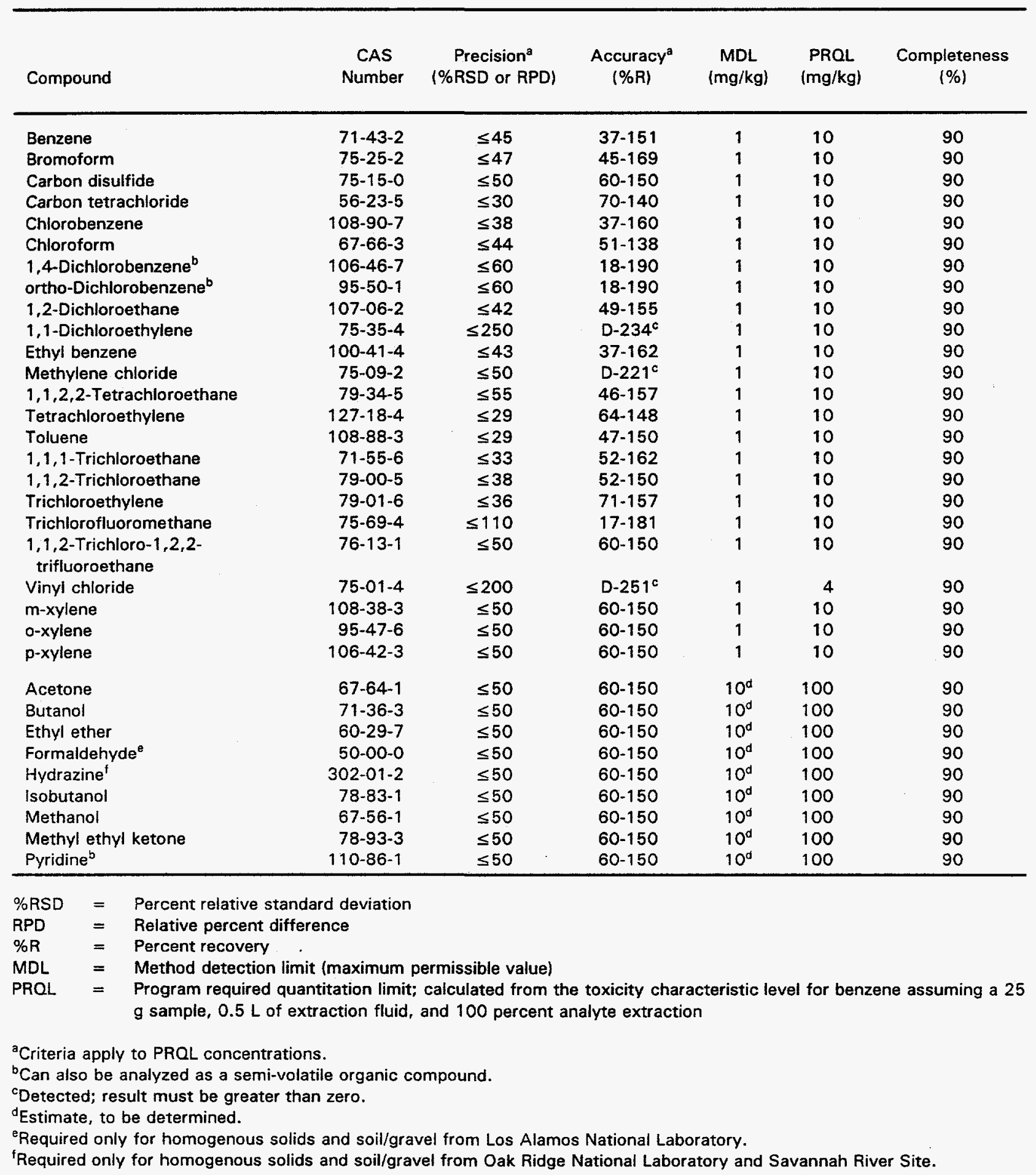




\subsection{Reagents}

The analyst is referred to Section 5.0 of SW-846 Method 8260A for a description and listing of the required reagents, standard stock solutions, and standard solutions for this procedure.

\subsection{Sample Collection, Preservation, and Handling}

7.1 Samples will be collected using the sampling procedures described in Procedure 120.1 of this Methods Manual.

7.2 All sample handling and chain-of-custody procedures described in Section 6.0 of the Transuranic Waste Characterization Quality Assurance Program Plan (OAPP) (DOE 1995b) must be followed.

\subsection{Procedure}

Each site must decide, based on the nature of its waste stream, what preparation methods are appropriate for its waste types. Use of preparation methods in SW-846, or other nationally recognized standard methods (e.g., ASTM) is acceptable.

The analyst is referred to Sections 7.1 through 7.5 of SW-846 Method $8260 \mathrm{~A}$ for a description of the procedural requirements. Table 2 of this procedure provides a summary of required calibration and analysis quality control.

\subsection{Calculations}

The analyst is referred to Section 7.6 of SW-846 Method 8260A for a description of the steps required for qualitative and quantitative analysis. Note that all results must be reported in $\mathrm{mg} / \mathrm{kg}$ (weight/wet-weight basis).

\subsection{Quality Control}

10.1 Each laboratory that uses this procedure is required to operate a formal quality control program. The laboratory must retain records to document the quality of the data generated. Each laboratory must have SOPs documenting and describing activities involved in using this procedure. Specific quality control practices will include, but are not limited to, the analysis of quality control samples. The types of quality control samples, their associated frequency of analysis, acceptance criteria, and corrective action required if samples do not meet the acceptance criteria, is summarized in Table 3. Laboratory SOPs shall address requirements for preparing matrix spikes, matrix spike duplicates, laboratory blanks, and laboratory control samples.

10.2 All laboratories using this procedure must demonstrate acceptable performance prior to the analysis of actual samples. Demonstration of acceptable performance will be achieved by analyzing method performance samples (Table 3). These samples will contain all of the analytes listed in Table 1 at concentrations appropriate $12-5$ times the PROLs) to determine the quality assurance objectives specified in Table 1 . The analysis of seven samples must meet the criteria specified for precision, accuracy, and method detection limit (MDL) in Table 1. Demonstration of acceptable method and analyst performance must be repeated (by analyzing four samples) at a minimum of every six months. Method performance should be conducted over a period of several days to account for long-term variability. Corrective action must be implemented when acceptable method performance is not met. Precision will be determined as follows: 
TABLE 2

\section{Summary of Calibration Requirements for Total Volatile Organic Compounds Analysis}

Technique Procedure

GC/MS

BFB Tune

Every 12 hours

5-pt initial calibration

(5 standards)

Continuing calibration

Every 12 hours
Frequency of Procedure

Acceptance Criteria
Initially, and as needed

30; response factor for SPCCs $\geq 0.30^{\mathrm{a}}$; average relative response factor is used if \%RSD $\leq 15$; linear regression equation is generated if \%RSD > 15

Response factor or conc. \%D for CCCs $\leq 20$; response factor for SPCCs $\geq$ $0.30^{\mathrm{a}} ; \mathrm{RT}$ for internal standards must be \pm 30 seconds from last daily calibration check; internal standard area count must be $>50 \%$ or < $200 \%$ of the area counts from the last daily calibration check; surrogate compound \%R must be met (see SW-846 Method 8260A)

${ }^{\text {a Bromoform }} \geq 0.25$

BFB $=$ 4-Bromofluorbenzene

CCC $=$ Calibration check compounds

$\% \mathrm{D}=$ Percent difference

$\% \mathrm{RSD}=$ Percent relative standard deviation

RT $=$ Retention time

SPCC $=$ System performance check compounds 
TABLE 3

\section{Summary of Laboratory Quality Control Samples and Frequencies for Total Volatile Organic Compound Analysis}

\begin{tabular}{|c|c|c|c|}
\hline QC Sample & Minimum Frequency & $\begin{array}{l}\text { Acceptance } \\
\text { Criteria }\end{array}$ & $\begin{array}{l}\text { Corrective } \\
\text { Action }^{\mathrm{a}}\end{array}$ \\
\hline $\begin{array}{l}\text { Method performance } \\
\text { samples }\end{array}$ & $\begin{array}{l}\text { Seven (7) samples initially } \\
\text { and four (4) semiannually }\end{array}$ & Meet Table 1 QAOs & Repeat until acceptable \\
\hline Laboratory duplicates ${ }^{b}$ & $\begin{array}{l}\text { One (1) per analytical } \\
\text { batch }\end{array}$ & Meet Table 1 RPDs & $\begin{array}{l}\text { Nonconformance if RPDs } \\
>\text { Table } 1 \text { values }\end{array}$ \\
\hline Laboratory blanks & $\begin{array}{l}\text { One (1) per analytical } \\
\text { batch }\end{array}$ & $\begin{array}{l}\text { Analyte concentrations } \\
<3 \times \text { MDLs }\end{array}$ & $\begin{array}{l}\text { Nonconformance if } \\
\text { analyte concentrations } \\
>3 \times M D L s\end{array}$ \\
\hline Matrix spikes & $\begin{array}{l}\text { One (1) per analytical } \\
\text { batch }\end{array}$ & Meet Table $1 \%$ Rs & $\begin{array}{l}\text { Nonconformance if \%Rs } \\
\text { are outside the range } \\
\text { specified in Table } 1\end{array}$ \\
\hline Matrix spike duplicates & $\begin{array}{l}\text { One (1) per analytical } \\
\text { batch }\end{array}$ & $\begin{array}{l}\text { Meet Table } 1 \text { RPDs and } \\
\% \text { Rs }\end{array}$ & $\begin{array}{l}\text { Nonconformance if RPDs } \\
\text { and } \% \text { Rs }>\text { Table } 1 \\
\text { values }\end{array}$ \\
\hline $\begin{array}{l}\text { Laboratory control } \\
\text { samples }\end{array}$ & $\begin{array}{l}\text { One (1) per analytical } \\
\text { batch }\end{array}$ & $80-120 \% R$ & $\begin{array}{l}\text { Nonconformance if } \% R \\
<80 \text { or }>120\end{array}$ \\
\hline Surrogate compounds & Each analytical sample & $\begin{array}{l}\text { Average } \% R \text { from } \\
\text { minimum of } 30 \text { samples } \\
\text { for a given matrix } \pm 3 \\
\text { standard deviations }\end{array}$ & $\begin{array}{l}\text { Nonconformance if } \% R \\
<\text { (average } \% R-3 \\
\text { standard deviation) or } \\
>\text { (average } \% R+3 \\
\text { standard deviation) }\end{array}$ \\
\hline Blind audit samples & $\begin{array}{l}\text { Samples and frequency } \\
\text { controlled by the Solid } \\
\text { PDP Plan }\end{array}$ & $\begin{array}{l}\text { Specified in the Solid } \\
\text { PDP Plan }\end{array}$ & $\begin{array}{l}\text { Specified in the Solid } \\
\text { PDP Plan }\end{array}$ \\
\hline
\end{tabular}

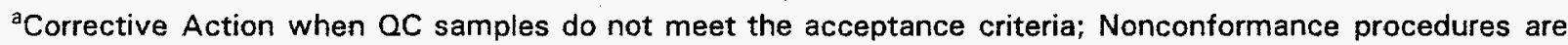
outlined in Section 2.1.2.1 of the QAPP.

bMay be satisfied using matrix spike duplicate; acceptance criteria applies only to concentrations greater than the PROLs listed in Table 1.

MDL = Method detection limit

QAO = Quality assurance objective

$\mathrm{PDP}=$ Performance Demonstration Program

$\% \mathrm{R}=$ Percent recovery

RPD $=$ Relative percent difference 
- For duplicate measurements, the precision expressed as the relative percent difference (RPD) is calculated as

$$
R P D=\frac{C_{1}-C_{2}}{\frac{\left(C_{1}+C_{2}\right)}{2}} * 100
$$

where

$C_{1}$ and $C_{2}=$ two values obtained by analyzing the duplicate samples

$C_{1}=$ larger of the two observed values

- For three or more replicate measurements, the precision expressed as the percent relative standard deviation (\%RSD) is calculated as

$$
\% R S D=\frac{s}{\bar{y}} * 100
$$

where

$$
\begin{aligned}
& s=\text { standard deviation } \\
& \bar{y}=\text { mean of replicate analyses }
\end{aligned}
$$

- The standard deviation $(s)$ is defined as

$$
s=\sqrt{\sum_{i=1}^{n} \frac{\left(y_{i}-\bar{y}\right)^{2}}{n-1}}
$$

where

$$
\begin{aligned}
& y_{i}=\text { measured value of the } i^{\text {th }} \text { replicate sample analysis measurement } \\
& n=\text { number of replicate analyses }
\end{aligned}
$$

Accuracy will be determined as the percent recovery $(\% \mathrm{R})$ as follows:

$$
\% R=\frac{C_{m}}{C_{s r m}} * 100
$$

and

$$
C_{m} \quad=\text { measured concentration }
$$

$C_{s r m}=$ true concentration

MDL will be determined as follows:

$$
M D L=t_{(n-1,1-\alpha=0.99)} * S
$$

where

$$
\begin{array}{lll}
s & = & \text { standard deviation } \\
t_{(n-1,1-\alpha=0.99)}= & t \text {-distribution value appropriate to a } 99-\text { percent confidence level } \\
& \text { and a standard deviation estimate with } n-1 \text { degrees of freedom }
\end{array}
$$

10.3 The laboratory must analyze blanks at the frequency specified in Table 3 . The same analytical method used to prepare and analyze field samples will be used to prepare and analyze the laboratory blanks. Laboratory blank results will be acceptable if the concentration of target analytes is less than 3 times the MDL specified in Table 1 for each compound. Corrective action must be implemented if blanks exceed this level. 
10.4 The laboratory must analyze matrix spikes at the frequency specified in Table 3 . Matrix spike results shall be considered acceptable if the \%R is within the range specified for each compound in Table 1. Matrix spikes which do not meet these criteria should be flagged.

$\% R$ will be determined as follows:

$$
\% R=\frac{S-U}{C_{s a}} * 100
$$

$$
\begin{gathered}
\text { where } \\
S=\text { measured concentration in the spiked aliquot } \\
U=\text { measured concentration in the unspiked aliquot } \\
C_{s a}=\text { actual concentration of the spike added }
\end{gathered}
$$

10.5 The laboratory must analyze laboratory duplicates or matrix spike duplicates at the frequency specified in Table 3. Duplicate results shall be considered acceptable if the RPD for each compound is within the range specified in Table 1. RPD is determined as in Equation (1). Duplicates which do not meet these criteria should be flagged.

10.6 The laboratory must analyze laboratory control samples at the frequency specified in Table 3. Laboratory control samples must contain at least ten of the compounds listed in Table 1 and shall be prepared, in water, at a concentration of $20 \mu \mathrm{g} / \mathrm{L}$ per laboratory control analyte from standards independent of those used for instrument calibration. The laboratory control samples must be quantitated within the calibration range of the instrument. The laboratory control sample analysis is designed to ensure that laboratory performance is in control for each analytical batch of samples processed. If the laboratory control sample $\% R$ is outside the range specified in Table 3 for the ten compounds, then new laboratory control samples must be prepared. \%R is determined as in Equation (4).

10.7 The laboratory will be required to analyze blind audit samples. These audit samples are part of the Performance Demonstration Program. Details of this program are in the Performance Demonstration Program Plan for the Analysis of Solidified Waste for the Transuranic Waste Characterization Program (Solid PDP Plan)(DOE 1995a).

\subsection{Procedure Performance}

The analyst is referred to Section 9.0 of SW-846 Method 8260 A for a summary of method performance.

\section{References}

DOE. 1995a. Performance Demonstration Program Plan for the Analysis of Solidified Waste for the Transuranic Waste Characterization Program. CA0-95-1077, Current Revision, Carlsbad, New Mexico, Carlsbad Area Office, U.S. Department of Energy.

DOE. 1995b. Transuranic Waste Characterization Quality Assurance Program Plan. CA0-941010, Current Revision, Carlsbad, New Mexico, Carisbad Area Office, U.S. Department of Energy.

EPA. 1995. Test Methods for Evaluating Solid Waste, Physical/Chemical Methods. SW-846, Third Edition, Final Update 1, and Final Update II, Washington D.C., Office of Solid Waste and Emergency Response, U.S. Environmental Protection Agency. 
PROCEDURE 430.5

\author{
METHOD 8250A FOR THE DETERMINATION \\ OF TOTAL SEMI-VOLATILE ORGANIC COMPOUNDS \\ IN HOMOGENOUS SOLIDS AND SOIL/GRAVEL
}

\title{
1.0 Scope and Application
}

1.1 This procedure is for the determination of total semi-volatile organic compounds (semiVOCs) in samples of homogenous solids and soil/gravel. The procedure employs Gas Chromatography/Mass Spectrometry (GC/MS) for the quantitation of total semi-VOCs. Table 1 lists the target analytes and quality assurance objectives for this procedure. A sampling procedure for homogenous solids and soil/gravel is described in Procedure 120.1 of this Methods Manual. This procedure must be implemented with a sitespecific standard operating procedure (SOP).

1.2 The analyst is referred to Method 8250A of Test Methods for Evaluating Solid Waste, Physical/Chemical Methods, SW-846, Third Edition, Final Update II (SW-846) (EPA 1995) for the specific requirements of this procedure. The analyst is referred to Section 1.0 of SW-846 Method 8250A for additional information concerning the scope and application of this procedure.

1.3 For the purposes of the TRU Waste Characterization Program, samples are to be analyzed in analytical batches. An analytical batch is defined as a suite of samples that is processed as a unit, using the same analytical method, within a specific time period. An analytical batch can be up to 20 samples (excluding laboratory $Q C$ samples), all of which must be received by the laboratory within 14 days of validated time of sample receipt (VTSR) of the first sample in the batch.

\subsection{Summary of Procedure}

The analyst is referred to Section 2.0 of SW-846 Method 8250 A for a summary of this procedure.

3.0 Interferences

The analyst is referred to Section 3.0 of SW-846 Method $8250 \mathrm{~A}$ for an explanation of potential interferences.

\subsection{Safety}

This procedure may involve the use of hazardous materials, operations, and equipment. It is the responsibility of whoever uses this procedure to consult appropriate site personnel concerning health and safety issues and establish appropriate health and safety practices. Consideration should be given to safety concerns regarding chemical and radiation exposure. Training regarding proper storage, usage, and disposal of chemicals is recommended.

\subsection{Apparatus and Materials}

The analyst is referred to Section 4.0 of SW-846 Method $8250 \mathrm{~A}$ for a description and listing of the required apparatus and materials for this procedure. 
TABLE 1

\section{Semi-Volatile Organic Compound Target Analyte List and Quality Assurance Objectives}

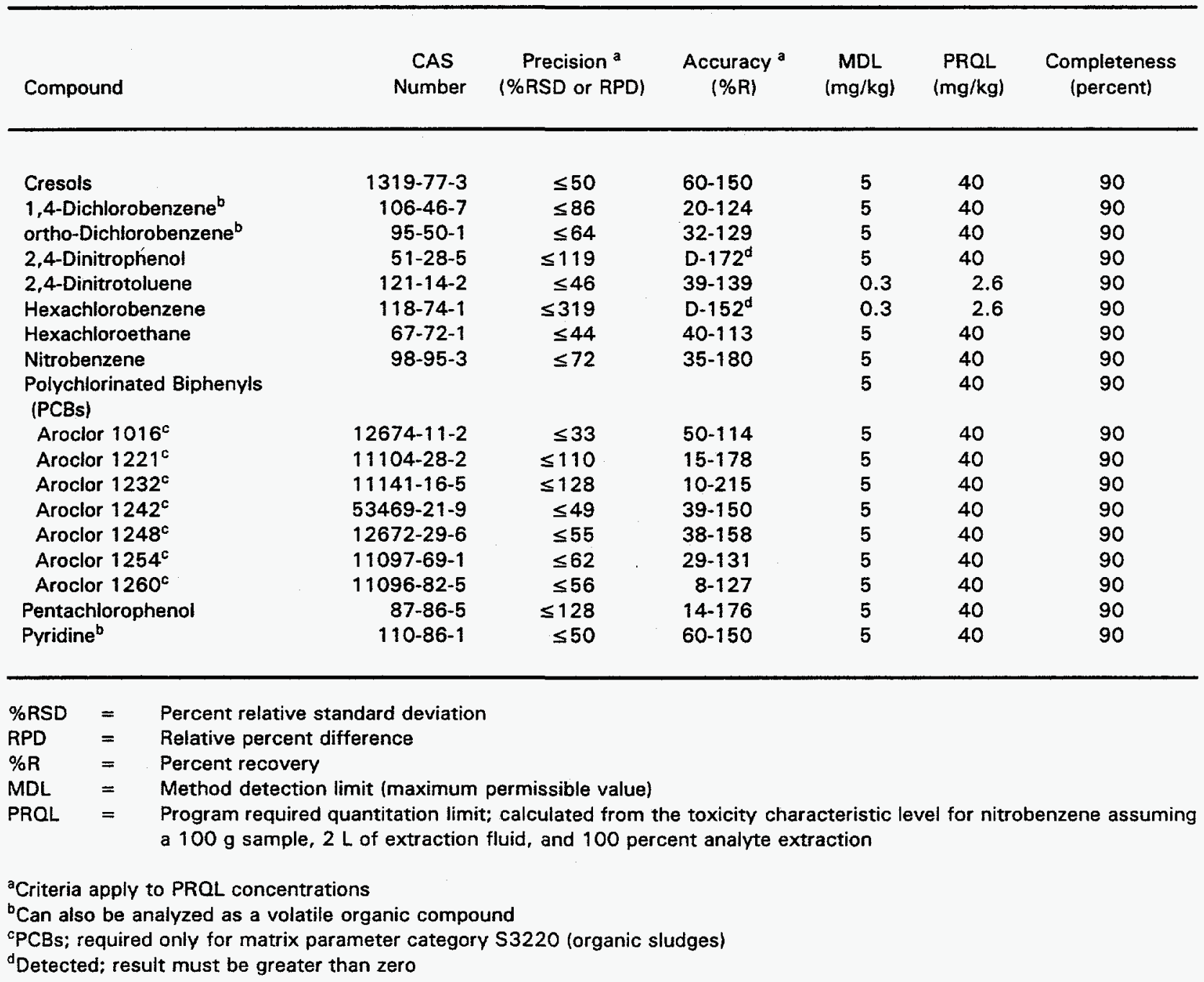




\subsection{Reagents}

The analyst is referred to Section 5.0 of SW-846 Method $8250 \mathrm{~A}$ for a description and listing of the required reagents, standard stock solutions, and standard solutions for this procedure.

\subsection{Sample Collection, Preservation, and Handling}

7.1 Samples will be collected using the sampling procedures described in Procedure 120.1 of this Methods Manual.

7.2 All sample handling and chain-of-custody procedures described in Section 6.0 of the Transuranic Waste Characterization Quality Assurance Program Plan (QAPP) (DOE 1995b) must be followed.

\subsection{Procedure}

Each site must decide, based on the nature of their waste stream, what preparation and cleanup methods are appropriate for their waste types. Use of preparation and cleanup methods in SW-846, or other nationally recognized standard methods (e.g., ASTM) is acceptable. The analyst is referred to Sections 7.3 through 7.6 of SW-846 Method 8250 A for a description of the procedural requirements for analysis. Table 2 of this procedure provides a summary of required calibration and analysis quality control.

\subsection{Calculations}

The analyst is referred to Section 7.7 of SW-846 Method 8250 A for a description of the steps required for qualitative and quantitative analysis. Note that all results must be reported in $\mathrm{mg} / \mathrm{kg}$ (weight/wet-weight basis).

\subsection{Quality Control}

10.1 Each laboratory that uses this procedure is required to operate a formal quality control program. The laboratory must retain records to document the quality of the data generated. Each laboratory must have SOPs documenting and describing activities involved in using this procedure. Specific quality control practices will include, but are not limited to, the analysis of quality control samples. The types of quality control samples, their associated frequency of analysis, acceptance criteria, and corrective action required if samples do not meet the acceptance criteria, is summarized in Table 3. Laboratory SOPs shall address requirements for preparing matrix spikes, matrix spike duplicates, laboratory blanks, and laboratory control samples.

10.2 All laboratories using this procedure must demonstrate acceptable performance prior to the analysis of actual samples. Demonstration of acceptable performance will be achieved by analyzing method performance samples (Table 3). These samples will contain all of the analytes listed in Table 1 at concentrations appropriate $12-5$ times the PROLs) to determine the quality assurance objectives specified in Table 1 . The analysis of seven samples must meet the criteria specified for precision, accuracy, and method detection limit (MDL) in Table 1. Demonstration of acceptable method and analyst performance must be repeated (by analyzing four samples) at a minimum of every six months. Method performance should be conducted over a period of several days to account for long-term variability. Corrective action must be implemented when acceptable method performance is not met. Precision will be determined as follows: 
TABLE 2

Summary of Calibration Requirements for Semi-Volatile Organic Compounds Analysis

Technique Procedure Frequency of Procedure Acceptance Criteria

GC/MS

DFTPP Tune

5-pt initial calibration

(5 standards)

Continuing calibration
Every 12 hours

Initially and as needed

Every 12 hours
Abundance criteria for all key ions are met (see SW-846 Method 8250A)

Response factor \%RSD for CCCs $\leq$ 30; response factor for SPCCs $\geq$ 0.05 ; average relative response factor used if \%RSD $\leq 15$; linear regression equation generated if \%RSD > 15

$\% \mathrm{D} \leq 20$ for all analytes; response factor for SPCCs $\geq 0.05$; RT for internal standards must be \pm 30 seconds from last daily calibration check; internal standards area count must be $>50 \%$ or $<200 \%$ of the area count from daily calibration check; surrogate compound \%R must be met (see SW-846 Method 8250A)

$\mathrm{CCC}=$ Calibration check compounds

$\% \mathrm{D}=$ Percent difference

DFTPP $=$ Decafluorotriphenylphosphine

$\%$ RSD $=$ Percent relative standard deviation

RT $\quad=\quad$ Retention time

SPCC $=$ System performance check compounds 
TABLE 3

\section{Summary of Laboratory Quality Control Samples and Frequencies for Total Semi-Volatile Organic Compounds Analysis}

\begin{tabular}{|c|c|c|c|}
\hline QC Sample & Minimum Frequency & $\begin{array}{l}\text { Acceptance } \\
\text { Criteria }\end{array}$ & $\begin{array}{l}\text { Corrective } \\
\text { Action }^{\mathrm{a}}\end{array}$ \\
\hline $\begin{array}{l}\text { Method performance } \\
\text { samples }\end{array}$ & $\begin{array}{l}\text { Seven (7) samples } \\
\text { initially and four (4) } \\
\text { semiannually }\end{array}$ & Meet Table 1 OAOs & Repeat until acceptable \\
\hline Laboratory duplicates ${ }^{b}$ & $\begin{array}{l}\text { One (1) per analytical } \\
\text { batch }\end{array}$ & Meet Table 1 RPDs & $\begin{array}{l}\text { Nonconformance if RPDs } \\
>\text { Table } 1 \text { values }\end{array}$ \\
\hline Laboratory blanks & $\begin{array}{l}\text { One (1) per analytical } \\
\text { batch }\end{array}$ & $\begin{array}{l}\text { Analyte concentrations } \\
<3 \times \text { MDLs }\end{array}$ & $\begin{array}{l}\text { Nonconformance if } \\
\text { analyte concentrations }> \\
3 \times \text { MDLs }\end{array}$ \\
\hline Matrix spikes & $\begin{array}{l}\text { One (1) per analytical } \\
\text { batch }\end{array}$ & Meet Table $1 \%$ Rs & $\begin{array}{l}\text { Nonconformance if } \% \text { Rs } \\
\text { are outside the range } \\
\text { specified in Table } 1\end{array}$ \\
\hline $\begin{array}{l}\text { Matrix spike } \\
\text { duplicates }\end{array}$ & $\begin{array}{l}\text { One (1) per analytical } \\
\text { batch }\end{array}$ & $\begin{array}{l}\text { Meet Table } 1 \text { RPDs } \\
\text { and } \% \text { Rs }\end{array}$ & $\begin{array}{l}\text { Nonconformance if RPDs } \\
\text { and } \% \text { Rs }>\text { Table } 1 \\
\text { values }\end{array}$ \\
\hline $\begin{array}{l}\text { Laboratory control } \\
\text { samples }\end{array}$ & $\begin{array}{l}\text { One (1) per analytical } \\
\text { batch }\end{array}$ & $80-120 \%$ Rs & $\begin{array}{l}\text { Nonconformance if } \% R \\
<80 \text { or }>120\end{array}$ \\
\hline Surrogate compounds & Each analytical sample & $\begin{array}{l}\text { Average } \% R \text { from minimum } \\
\text { of } 30 \text { samples from a given } \\
\text { matrix } \pm 3 \text { standard } \\
\text { deviations }\end{array}$ & $\begin{array}{l}\text { Nonconformance if } \% R \\
<\text { (average } \% R-3 \\
\text { standard deviations) or } \\
>\text { (average } \% R+3 \\
\text { standard deviations) }\end{array}$ \\
\hline Blind audit samples & $\begin{array}{l}\text { Samples and frequency } \\
\text { controlled by the Solid } \\
\text { PDP Plan }\end{array}$ & $\begin{array}{l}\text { Specified in the Solid PDP } \\
\text { Plan }\end{array}$ & $\begin{array}{l}\text { Specified in the Solid } \\
\text { PDP Plan }\end{array}$ \\
\hline
\end{tabular}

${ }^{a}$ Corrective Action when QC samples do not meet the acceptance criteria; Nonconformance procedures are outlined in Section 2.1.2.1 of the QAPP.

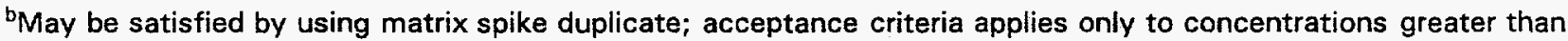
the PROLs listed in Table 1.

MDL = Method detection limit

$\mathrm{QAO}=$ Quality assurance objective

PDP $=$ Performance Demonstration Program

$\% R=$ Percent recovery

RPD $\approx$ Relative percent difference 
- For duplicate measurements, the precision expressed as the relative percent difference (RPD) is calculated as

$$
R P D=\frac{C_{1}-C_{2}}{\frac{\left(C_{1}+C_{2}\right)}{2}} * 100
$$

where

$C_{1}$ and $C_{2}=$ two values obtained by analyzing the duplicate samples

$C_{1} \quad=$ larger of the two observed values

- For three or more replicate measurements, the precision expressed as the percent relative standard deviation (\%RSD) is calculated as

$$
\% R S D=\frac{s}{\bar{y}} * 100
$$

where

$$
\begin{array}{ll}
s & =\text { standard deviation } \\
y & =\text { mean of replicate analyses }
\end{array}
$$

- The standard deviation $(s)$ is defined as

$$
s=\sqrt{\sum_{i=1}^{n} \frac{\left(y_{i}-\bar{y}\right)^{2}}{n-1}}
$$

where

$$
\begin{aligned}
& y_{i}=\text { measured value of the } i^{\text {th }} \text { replicate sample analysis measurement } \\
& n=\text { number of replicate analyses }
\end{aligned}
$$

Accuracy will be determined as the percent recovery $(\% R)$ as follows:

$$
\% R=\frac{C_{m}}{C_{s r m}} * 100
$$

where

$$
\begin{array}{ll}
C_{m} & =\text { measured concentration } \\
C_{s r m} & =\text { true concentration }
\end{array}
$$

MDL will be determined as follows:

$$
M D L=t_{(n-1,1-\alpha=0.99)} * S
$$

where

$$
\begin{array}{lll}
s & \text { standard deviation } \\
t_{(n-1,1-\alpha=0.99)}= & t \text {-distribution value appropriate to a 99-percent confidence level } \\
& \text { and a standard deviation estimate with } n-1 \text { degrees of freedom }
\end{array}
$$

10.3 The laboratory must analyze blanks at the frequency specified in Table 3 . The same analytical method used to prepare and analyze field samples will be used to prepare and analyze the laboratory blanks. Laboratory blank results will be acceptable if the 
concentration of target analytes is less than 3 times the MDL specified in Table 1 for each compound. Corrective action must be implemented if blanks exceed this level.

10.4 The laboratory must analyze matrix spikes at the frequency specified in Table 3. Matrix spike results shall be considered acceptable if the $\% R$ is within the range specified for each compound in Table 1. Matrix spikes which do not meet these criteria should be flagged.

$\% R$ will be determined as follows:

$$
\% R=\frac{S-U}{C_{s a}} * 100
$$

where

$S=$ measured concentration in the spiked aliquot

$U=$ measured concentration in the unspiked aliquot

$C_{s a}=$ actual concentration of the spike added

10.5 The laboratory must analyze laboratory duplicates or matrix spike duplicates at the frequency specified in Table 3. Duplicate results shall be considered acceptable if the RPD for each compound is within the range specified in Table 1. RPD is determined as in Equation (1). Duplicates which do not meet these criteria should be flagged.

10.6 The laboratory must analyze laboratory control samples at the frequency specified in Table 3. Laboratory control samples must contain 1,4-dichlorobenzene, 2,4-dinitrotoluene, and hexachloroethane and shall be prepared, in water, at a concentration of $100 \mu \mathrm{g} / \mathrm{L}$ per laboratory control analyte from standards independent of those used for instrument calibration. The laboratory control samples must be quantitated within the calibration range of the instrument. The laboratory control sample analysis is designed to ensure that laboratory performance is in control for each analytical batch of samples processed. If the laboratory control sample $\% R$ is outside the range specified in Table 3 for the three mandatory compounds, then new laboratory control samples must be prepared. \% $\mathrm{R}$ is determined as in Equation (4).

10.7 The laboratory will be required to analyze blind audit samples. These audit samples are part of the Performance Demonstration Program. Details of this program are in the Performance Demonstration Program Plan for the Analysis of Solidified Waste for the Transuranic Waste Characterization Program (Solid PDP Plan)(DOE 1995a).

\subsection{Procedure Performance}

The analyst is referred to Section 9.0 of SW-846 Method 8250 A for a summary of method performance.

\subsection{References}

DOE. 1995a. Performance Demonstration Program Plan for the Analysis of Solidified Waste for the Transuranic Waste Characterization Program. CA0-95-1077, Current Revision, Carlsbad, New Mexico, Carisbad Area Office, U.S. Department of Energy.

DOE. 1995b. Transuranic Waste Characterization Quality Assurance Program Plan. CAO-941010, Current Revision, Carisbad, New Mexico, Carlsbad Area Office, U.S. Department of Energy. 
EPA. 1995. Test Methods for Evaluating Solid Waste, Physical/Chemical Methods. SW-846, Third Edition, Final Update I, and Final Update II, Washington D.C., Office of Solid Waste and Emergency Response, U.S. Environmental Protection Agency. 
PROCEDURE 430.6

\author{
METHOD 8270B FOR THE DETERMINATION \\ OF TOTAL SEMI-VOLATILE ORGANIC COMPOUNDS \\ IN HOMOGENOUS SOLIDS AND SOIL/GRAVEL
}

1.0 Scope and Application

1.1 This procedure is for the determination of total semi-volatile organic compounds (semiVOCs) in samples of homogenous solids and soil/gravel. The procedure employs Gas Chromatography/Mass Spectrometry (GC/MS) for the quantitation of total semi-VOCs. Table 1 lists the target analytes and quality assurance objectives for this procedure. A sampling procedure for homogenous solids and soil/gravel is described in Procedure 120.1 of this Methods Manual. This procedure must be implemented with a sitespecific standard operating procedure (SOP).

1.2 The analyst is referred to Method $8270 \mathrm{~B}$ of Test Methods for Evaluating Solid Waste, Physical/Chemical Methods, SW-846, Third Edition, Final Update II (SW-846) (EPA $1995)$ for the specific requirements of this procedure. The analyst is referred to Section 1.0 of SW-846 Method 8270 B for additional information concerning the scope and application of this procedure.

1.3 For the purposes of the TRU Waste Characterization Program, samples are to be analyzed in analytical batches. An analytical batch is defined as a suite of samples that is processed as a unit, using the same analytical method, within a specific time period. An analytical batch can be up to 20 samples (excluding laboratory OC samples), all of which must be received by the laboratory within 14 days of validated time of sample receipt (VTSR) of the first sample in the batch.

\title{
2.0 Summary of Procedure
}

The analyst is referred to Section 2.0 of SW-846 Method $8270 \mathrm{~B}$ for a summary of this procedure.

3.0 Interferences

The analyst is referred to Section 3.0 of SW-846 Method $8270 \mathrm{~B}$ for an explanation of potential interferences.

4.0 Safety

This procedure may involve the use of hazardous materials, operations, and equipment. It is the responsibility of whoever uses this procedure to consult appropriate site personnel concerning health and safety issues and establish appropriate health and safety practices. Consideration should be given to safety concerns regarding chemical and radiation exposure. Training regarding proper storage, usage, and disposal of chemicals is recommended.

5.0 Apparatus and Materials

The analyst is referred to Section 4.0 of SW-846 Method $8270 \mathrm{~B}$ for a description and listing of the required apparatus and materials for this procedure. 
TABLE 1

Semi-Volatile Organic Compound Target Analyte List and Quality Assurance Objectives

\begin{tabular}{|c|c|c|c|c|c|c|}
\hline Compound & $\begin{array}{c}\text { CAS } \\
\text { Number }\end{array}$ & $\begin{array}{c}\text { Precision * } \\
\text { (\%RSD or RPD) }\end{array}$ & $\begin{array}{c}\text { Accuracy } \\
(\% R)\end{array}$ & $\begin{array}{c}\text { MDL } \\
(\mathrm{mg} / \mathrm{kg})\end{array}$ & $\begin{array}{l}\text { PROL } \\
(\mathrm{mg} / \mathrm{kg})\end{array}$ & $\begin{array}{l}\text { Completeness } \\
\text { (percent) }\end{array}$ \\
\hline Cresols & $1319-77-3$ & $\leq 50$ & $60-150$ & 5 & 40 & 90 \\
\hline 1,4-Dichlorobenzene ${ }^{b}$ & $106-46-7$ & $\leq 86$ & $20-124$ & 5 & 40 & 90 \\
\hline ortho-Dichlorobenzene ${ }^{b}$ & $95-50-1$ & $\leq 64$ & $32-129$ & 5 & 40 & 90 \\
\hline 2,4-Dinitrophenol & $51-28-5$ & $\leq 119$ & $0-172^{d}$ & 5 & 40 & 90 \\
\hline 2,4-Dinitrotoluene & $121-14-2$ & $\leq 46$ & $39-139$ & 0.3 & 2.6 & 90 \\
\hline Hexachlorobenzene & $118-74-1$ & $\leq 319$ & $D-152^{d}$ & 0.3 & 2.6 & 90 \\
\hline Hexachloroethane & $67-72-1$ & $\leq 44$ & $40-113$ & 5 & 40 & 90 \\
\hline Nitrobenzene & $98-95-3$ & $\leq 72$ & $35-180$ & 5 & 40 & 90 \\
\hline $\begin{array}{l}\text { Polychlorinated Biphenyls } \\
\text { (PCBs) }\end{array}$ & - & & & 5 & 40 & 90 \\
\hline Aroclor $1016^{\circ}$ & $12674-11-2$ & $\leq 33$ & $50-114$ & 5 & 40 & 90 \\
\hline Aroclor $1221^{\circ}$ & $11104-28-2$ & $\leq 110$ & $15-178$ & 5 & 40 & 90 \\
\hline Aroclor $1232^{\circ}$ & $11141-16-5$ & $\leq 128$ & $10-215$ & 5 & 40 & 90 \\
\hline Aroclor $1242^{\circ}$ & $53469-21-9$ & $\leq 49$ & $39-150$ & 5 & 40 & 90 \\
\hline Aroclor $1248^{c}$ & $12672-29-6$ & $\leq 55$ & $38-158$ & 5 & 40 & 90 \\
\hline Aroclor $1254^{\circ}$ & $11097-69-1$ & $\leq 62$ & $29-131$ & 5 & 40 & 90 \\
\hline Aroclor $1260^{\circ}$ & $11096-82-5$ & $\leq 5.6$ & $8-127$ & 5 & 40 & 90 \\
\hline Pentachlorophenol & $87-86-5$ & $\leq 128$ & $14-176$ & 5 & 40 & 90 \\
\hline Pyridine $^{\mathrm{b}}$ & $110-86-1$ & $\leq 50$ & $60-150$ & 5 & 40 & 90 \\
\hline
\end{tabular}

$\%$ RSD $=$ Percent relative standard deviation

RPD $=$ Relative percent difference

$\% R=$ Percent recovery

MDL = Method detection limit (maximum permissible value)

PRQL = Program required quantitation limit; calculated from the toxicity characteristic level for nitrobenzene assuming a $100 \mathrm{~g}$ sample, $2 \mathrm{~L}$ of extraction fluid, and 100 percent analyte extraction

${ }^{a}$ Criteria apply to PRQL concentrations

${ }^{b} \mathrm{C}$ an also be analyzed as a volatile organic compound

'PCBs; required only for matrix parameter category $\$ 3220$ (organic sludges)

DDetected; result must be greater than zero 


\subsection{Reagents}

The analyst is referred to Section 5.0 of SW-846 Method $8270 \mathrm{~B}$ for a description and listing of the required reagents, standard stock solutions, and standard solutions for this procedure.

7.0 Sample Collection, Preservation, and Handling

7.1 Samples will be collected using the sampling procedures described in Procedure 120.1 of this Methods Manual.

7.2 All sample handling and chain-of-custody procedures described in Section 6.0 of the Transuranic Waste Characterization Quality Assurance Program Plan (QAPP) (DOE 1995 b) must be followed.

\subsection{Procedure}

Each site must decide, based on the nature of their waste stream, what preparation and cleanup methods are appropriate for their waste types. Use of preparation and cleanup methods in SW-846, or other nationally recognized standard methods (e.g., ASTM) is acceptable. The analyst is referred to Sections 7.3 through 7.5 of SW-846 Method $8270 B$ for a description of the procedural requirements for analysis. Table 2 of this procedure provides a summary of required calibration and analysis quality control.

\subsection{Calculations}

The analyst is referred to Section 7.6 of SW-846 Method $8270 B$ for a description of the steps required for qualitative and quantitative analysis. Note that all results must be reported in $\mathrm{mg} / \mathrm{kg}$ (weight/wet-weight basis).

\subsection{Quality Control}

10.1 Each laboratory that uses this procedure is required to operate a formal quality control program. The laboratory must retain records to document the quality of the data generated. Each laboratory must have SOPs documenting and describing activities involved in using this procedure. Specific quality control practices will include, but are not limited to, the analysis of quality control samples. The types of quality control samples, their associated frequency of analysis, acceptance criteria, and corrective action required if samples do not meet the acceptance criteria, is summarized in Table 3. Laboratory SOPs shall address requirements for preparing matrix spikes, matrix spike duplicates, laboratory blanks, and laboratory control samples.

10.2 All laboratories using this procedure must demonstrate acceptable performance prior to the analysis of actual samples. Demonstration of acceptable performance will be achieved by analyzing method performance samples (Table 3). These samples will contain all of the analytes listed in Table 1 at concentrations appropriate $(2-5$ times the PROLs) to determine the quality assurance objectives specified in Table 1 . The analysis of seven samples must meet the criteria specified for precision, accuracy, and method detection limit (MDL) in Table 1. Demonstration of acceptable method and analyst performance must be repeated (by analyzing four samples) at a minimum of every six months. Method performance should be conducted over a period of several days to account for long-term variability. Corrective action must be implemented when acceptable method performance is not met. Precision will be determined as follows: 
TABLE 2

Summary of Calibration Requirements for Semi-Volatile Organic Compounds Analysis

Technique Procedure Frequency of Procedure Acceptance Criteria

GC/MS

DFTPP Tune

Every 12 hours

5-pt initial calibration

(5 standards)

Continuing calibration
Every 12 hours

Initially and as needed

Every 12 hours

Abundance criteria for all key ions are met (see SW-846 Method 8270B)

Response factor \%RSD for CCCs $\leq$ 30; response factor for SPCCs $\geq$ 0.05 ; average relative response factor used if \%RSD $\leq 15$; linear regression equation generated if \%RSD > 15

$\% D \leq 20$ for all analytes; response factor for SPCCs $\geq 0.05$; RT for internal standards must be \pm 30 seconds from last daily calibration check; internal standards area count must be $>50 \%$ or $<200 \%$ of the area count from daily calibration check; surrogate compound \%R must be met (see SW-846 Method $8270 B$ )
$\mathrm{CCC}=$
$\% \mathrm{D}=$
DFTPP $=$
Calibration check compounds
$\%$ RSD $=$
Percent difference
$\begin{array}{ll}\% R S D & = \\ R T & =\end{array}$
Decafluorotriphenylphosphine
SPCC $=$
Percent relative standard deviation
Retention time
System performance check compounds 
TABLE 3

\section{Summary of Laboratory Quality Control Samples and Frequencies for Total Semi-Volatile Organic Compounds Analysis}

\begin{tabular}{|c|c|c|c|}
\hline OC Sample & Minimum Frequency & $\begin{array}{l}\text { Acceptance } \\
\text { Criteria }\end{array}$ & $\begin{array}{l}\text { Corrective } \\
\text { Action }\end{array}$ \\
\hline $\begin{array}{l}\text { Method performance } \\
\text { samples }\end{array}$ & $\begin{array}{l}\text { Seven (7) samples } \\
\text { initially and four (4) } \\
\text { semiannually }\end{array}$ & Meet Table 1 OAOs & Repeat until acceptable \\
\hline Laboratory duplicates $^{b}$ & $\begin{array}{l}\text { One (1) per analytical } \\
\text { batch }\end{array}$ & Meet Table 1 RPDs & $\begin{array}{l}\text { Nonconformance if RPDs } \\
>\text { Table } 1 \text { values }\end{array}$ \\
\hline Laboratory blanks & $\begin{array}{l}\text { One (1) per analytical } \\
\text { batch }\end{array}$ & $\begin{array}{l}\text { Analyte concentrations } \\
<3 \times \text { MDLs }\end{array}$ & $\begin{array}{l}\text { Nonconformance if } \\
\text { analyte concentrations > } \\
3 \times \text { MDLs }\end{array}$ \\
\hline Matrix spikes & $\begin{array}{l}\text { One (1) per analytical } \\
\text { batch }\end{array}$ & Meet Table $1 \%$ Rs & $\begin{array}{l}\text { Nonconformance if } \% \text { Rs } \\
\text { are outside the range } \\
\text { specified in Table } 1\end{array}$ \\
\hline $\begin{array}{l}\text { Matrix spike } \\
\text { duplicates }\end{array}$ & $\begin{array}{l}\text { One (1) per analytical } \\
\text { batch }\end{array}$ & $\begin{array}{l}\text { Meet Table } 1 \text { RPDs and } \\
\% \text { Rs }\end{array}$ & $\begin{array}{l}\text { Nonconformance if RPDs } \\
\text { and } \% \text { Rs }>\text { Table } 1 \\
\text { values }\end{array}$ \\
\hline $\begin{array}{l}\text { Laboratory control } \\
\text { samples }\end{array}$ & $\begin{array}{l}\text { One (1) per analytical } \\
\text { batch }\end{array}$ & $80-120 \% R s$ & $\begin{array}{l}\text { Nonconformance if } \% R \\
<80 \text { or }>120\end{array}$ \\
\hline Surrogate compounds & Each analytical sample & $\begin{array}{l}\text { Average } \% R \text { from minimum } \\
\text { of } 30 \text { samples from a given } \\
\text { matrix } \pm 3 \text { standard } \\
\text { deviations }\end{array}$ & $\begin{array}{l}\text { Nonconformance if } \% \mathrm{R} \\
<\text { (average } \% \mathrm{R}-3 \\
\text { standard deviations) or } \\
>\text { (average } \% \mathrm{R}+3 \\
\text { standard deviations) }\end{array}$ \\
\hline Blind audit samples & $\begin{array}{l}\text { Samples and frequency } \\
\text { controlled by the Solid } \\
\text { PDP Plan }\end{array}$ & $\begin{array}{l}\text { Specified in the Solid PDP } \\
\text { Plan }\end{array}$ & $\begin{array}{l}\text { Specified in the Solid } \\
\text { PDP Plan }\end{array}$ \\
\hline
\end{tabular}

${ }^{a}$ Corrective Action when $\mathrm{QC}$ samples do not meet the acceptance criteria; Nonconformance procedures are outlined in Section 2.1.2.1 of the QAPP.

\footnotetext{
the PRQLs listed in Table 1.

MDL $=\quad$ Method detection limit

$\mathrm{QAO}=$ Quality assurance objective

PDP $=$ Performance Demonstration Program

$\% R=$ Percent recovery

RPD $=$ Relative percent difference
}

${ }^{b}$ May be satisfied by using matrix spike duplicate; acceptance criteria applies only to concentrations greater than 
- For duplicate measurements, the precision expressed as the relative percent difference (RPD) is calculated as

$$
R P D=\frac{C_{1}-C_{2}}{\frac{\left(C_{1}+C_{2}\right)}{2}} * 100
$$

where

$$
\begin{array}{ll}
C_{1} \text { and } C_{2} & =\text { two values obtained by analyzing the duplicate samples } \\
C_{1} & =\text { larger of the two observed values }
\end{array}
$$

- For three or more replicate measurements, the precision expressed as the percent relative standard deviation (\%RSD) is calculated as

$$
\% R S D=\frac{s}{\bar{y}} * 100
$$

where

$$
\begin{array}{ll}
s & =\text { standard deviation } \\
y & =\text { mean of replicate analyses }
\end{array}
$$

- The standard deviation ( $s$ ) is defined as

$$
s=\sqrt{\sum_{i=1}^{n} \frac{\left(y_{i}-\bar{y}\right)^{2}}{n-1}}
$$

where

$$
\begin{aligned}
& y_{i}=\text { measured value of the } i^{\text {th }} \text { replicate sample analysis measurement } \\
& n=\text { number of replicate analyses }
\end{aligned}
$$

Accuracy will be determined as the percent recovery $(\% R)$ as follows:

$$
\% R=\frac{C_{m}}{C_{s m}} * 100
$$

where

$$
\begin{array}{ll}
C_{m} & =\text { measured concentration } \\
C_{s r m} & =\text { true concentration }
\end{array}
$$

MDL will be determined as follows:

$$
M D L=t_{(n-1,1-\alpha=0.99)} * s
$$

where

$$
\begin{aligned}
& s \quad=\text { standard deviation } \\
& t_{(n-1,1-\alpha=0.99)}=t \text {-distribution value appropriate to a 99-percent confidence level and }
\end{aligned}
$$

10.3 The laboratory must analyze blanks at the frequency specified in Table 3 . The same analytical method used to prepare and analyze field samples will be used to prepare and analyze the laboratory blanks. Laboratory blank results will be acceptable if the concentration of target analytes is less than 3 times the MDL specified in Table 1 for each compound. Corrective action must be implemented if blanks exceed this level. 
10.4 The laboratory must analyze matrix spikes at the frequency specified in Table 3 . Matrix spike results shall be considered acceptable if the \% $R$ is within the range specified for each compound in Table 1. Matrix spikes which do not meet these criteria should be flagged.

$\% R$ will be determined as follows:

$$
\% R=\frac{S-U}{C_{s a}} * 100
$$

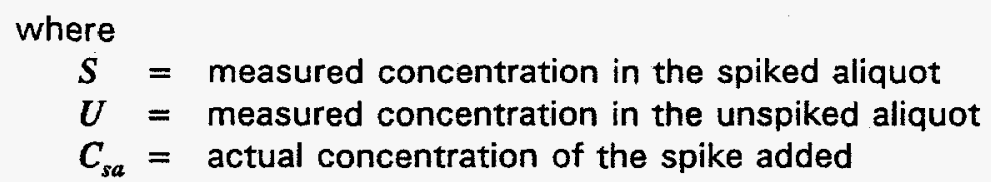

10.5 The laboratory must analyze laboratory duplicates or matrix spike duplicates at the frequency specified in Table 3. Duplicate results shall be considered acceptable if the RPD for each compound is within the range specified in Table 1. RPD is determined as in Equation (1). Duplicates which do not meet these criteria should be flagged.

10.6 The laboratory must analyze laboratory control samples at the frequency specified in Table 3. Laboratory control samples must contain 1,4-dichlorobenzene, 2,4dinitrotoluene, and hexachloroethane and shall be prepared, in water, at a concentration of $100 \mu \mathrm{g} / \mathrm{L}$ per laboratory control analyte from standards independent of those used for instrument calibration. The laboratory control samples must be quantitated within the calibration range of the instrument. The laboratory control sample analysis is designed to ensure that laboratory performance is in control for each analytical batch of samples processed. If the laboratory control sample recovery is outside the range specified in Table 3 for the three mandatory compounds, then new laboratory control samples must be prepared. \%R is determined as in Equation (4).

10.7 The laboratory will be required to analyze blind audit samples. These audit samples are part of the Performance Demonstration Program. Details of this program are in the Performance Demonstration Program Plan for the Analysis of Solidified Waste for the Transuranic Waste Characterization Program (Solid PDP Plan)(DOE 1995a).

\subsection{Procedure Performance}

The analyst is referred to Section 9.0 of SW-846 Method $8270 B$ for a summary of method performance.

\subsection{References}

DOE. 1995a. Performance Demonstration Program Plan for the Analysis of Solidified Waste for the Transuranic Waste Characterization Program. CA0-95-1077, Current Revision, Carlsbad, New Mexico, Carlsbad Area Office, U.S. Department of Energy.

DOE. 1995b. Transuranic Waste Characterization Quality Assurance Program Plan. CAO-941010, Current Revision, Carlsbad, New Mexico, Carisbad Area Office, U.S. Department of Energy. 
EPA. 1995. Test Methods for Evaluating Solid Waste, Physical/Chemical Methods. SW-846, Third Edition, Final Update I, and Final Update II, Washington D.C., Office of Solid Waste and Emergency Response, U.S. Environmental Protection Agency. 
PROCEDURE 440.1

\section{GAS CHROMATOGRAPHY/FLAME IONIZATION DETECTOR \\ DETERMINATION OF ALCOHOLS AND KETONES IN \\ WASTE CONTAINER HEADSPACE}

\subsection{Scope and Application}

1.1 This procedure is for the Gas Chromatography/Flame Ionization Detector (GC/FID) determination of selected alcohols and ketones in the range from $100 \mathrm{ppmv}(\mathrm{ppm}$, volume/volume) to volume percent, in samples that have been collected from waste container headspace using SUMMA ${ }^{\oplus}$ passivated canisters. This procedure is restricted to use by or under the supervision of analysts experienced in Gas Chromatography (GC). The target analytes and quality assurance objectives for this procedure are listed in Table 1. The sampling procedures are described in Procedures 110.1 through 110.4 of this Methods Manual. This procedure must be implemented with a site-specific standard operating procedure (SOP).

1.2 This procedure may also be used to screen samples for the volatile organic compounds (VOCs) listed in Table 2 prior to the analysis of these compounds by Gas Chromatography/Mass Spectrometry (GC/MS). The screening data may be used to determine appropriate dilution factors for samples prior to analysis by GC/MS.

1.3 The Environmental Protection Agency (EPA) has not determined the stability of the alcohols and ketones listed in Table 1 when stored in pressurized or sub-ambient pressure SUMMA ${ }^{\oplus}$ canisters. It is anticipated that no problems will be encountered with these compounds when collected and stored in SUMMA ${ }^{\oplus}$ canisters due to the concentration levels at which these compounds are to be detected.

1.4 For the purposes of the TRU Waste Characterization Program, samples are to be analyzed in analytical batches. An analytical batch is defined as a suite of samples that is processed as a unit, using the same analytical method, within a specific time period. An analytical batch can be up to 20 samples (excluding laboratory OC samples), all of which must be received by the laboratory within 14 days of the validated time of sample receipt (VTSR) of the first sample in the batch.

\subsection{Summary of Procedure}

Gas samples are analyzed for the analytes listed in Table 1 by separating components on two different wide-bore capillary columns followed by detection with flame ionization detectors (FIDs). Samples are introduced to the GC/FID system by attaching SUMMA ${ }^{\oplus}$ canisters directly to heated gas injection valves with integral sample loops via a heated transfer line. Prior to sample analysis, the SUMMA ${ }^{\oplus}$ canisters must be pressurized to a minimum of two times ambient atmospheric pressure. This will allow the positive pressure of the sample canister to fill the sample loop. Analyte identification is made by comparison of sample analyte peak retention times (RTs) to peak RTs of reference standards analyzed under identical operating conditions (RT windows). Analyte RT confirmation must be performed on both analytical columns. Quantitation of a given analyte shall be performed on one of the wide-bore capillary columns by the technique of external quantitation. The dilution factor resulting from pressurization must be used when calculating analyte concentrations. The column used for quantitation must be interferant free in the RT window corresponding to the analyte. 
TABLE 1

Alcohol and Ketone Target Analyte List and Quality Assurance Objectives

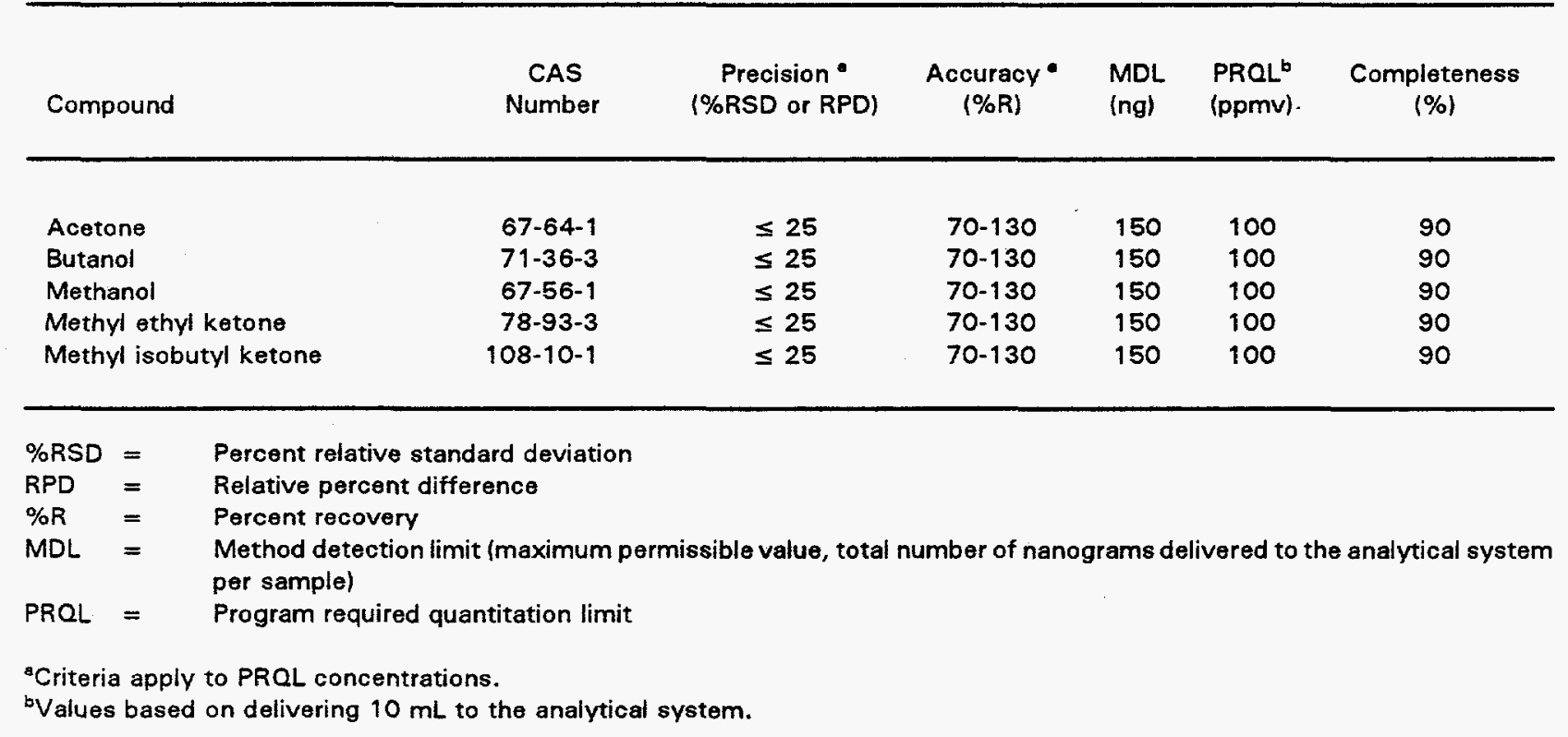


TABLE 2

Potential Interferant Compounds

CAS Number

Benzene

Bromoform

Carbon tetrachloride

Chlorobenzene

Chloroform

Cyclohexane

1,1-Dichloroethane

1,2-Dichloroethane

cis-1,2-Dichloroethylene

1,1-Dichloroethylene

Ethyl benzene

Ethyl ether

Formaldehyde

Hydrazine

Methylene chloride

1,1,2,2-Tetrachloroethane

Tetrachloroethylene

Toluene

1,1,1-Trichloroethane

Trichloroethylene

1,1,2-Trichloro-1,2,2-trifluoroethane

$1,2,4$-Trimethylbenzene

1,3,5-Trimethylbenzene

$\mathrm{m}$-Xylene

o-Xylene

$p$-Xylene
$71-43-2$

75-25-2

56-23-5

108-90-7

67-66-3

$110-87-7$

75-34-3

107-06-2

540-59-0

75-35-4

100-41-4

60-29-7

50-00-0

302-01-2

75-09-2

79-34-5

$127-18-4$

108-88-3

71-55-6

79-01-6

76-13-1

95-63-6

$108-67-8$

108-38-3

95-47-6

106-42-3 


\subsection{Interferences}

3.1 Contamination may occur in the analytical system if the canisters are not properly cleaned prior to use. Instructions for cleaning the canisters are given in Procedure 210.1 of this Methods Manual.

3.2 Cross-contamination can occur whenever high-concentration and low-concentration samples are analyzed sequentially. Whenever a highly concentrated sample is analyzed, it should be followed by the analysis of ultra-high purity air or nitrogen.

3.3 The use of non-Teflon plastic coating, non-Teflon thread sealants, or flow controllers with rubber components in the analytical system should be avoided.

3.4 The compounds listed in Table 2 may be present in samples. The chromatographic conditions employed must minimize any potential interference of Table 2 compounds with the target analytes listed in Table 1.

\subsection{Safety}

This procedure may involve the use of hazardous materials, operations, and equipment. It is the responsibility of whoever uses this procedure to consult appropriate site personnel concerning health and safety issues and establish appropriate health and safety practices. Consideration should be given to safety concerns regarding chemical and radiation exposure. Training regarding proper storage, usage, and disposal of chemicals is recommended.

Samples and reference materials may be flammable. Keep away from heat, sparks, and open flame. Provide adequate ventilation.

\subsection{Apparatus and Materials}

\subsection{GC/FID System.}

5.1.1 Sample Introduction System: System must be capable of attaching SUMMA ${ }^{\oplus}$ canisters directly to the gas chromatograph inlet. The introduction system must be capable of heating and maintaining the SUMMA ${ }^{\circledR}$ canisters and transfer line to the gas chromatograph inlet at $100^{\circ} \mathrm{C}$. The introduction system must be constructed of materials that minimize adsorption of sample components and minimize the loss of sample due to excessive transfer line volume.

5.1.2 Sample Inlet System: Sample inlet system must consist of a gas injection valve with integral fixed sample loop (Valco Instruments ECGUWE or equivalent). The gas injection valve and fixed volume sample loop must be capable of being heated to $100^{\circ} \mathrm{C}$ and must be constructed of materials that minimize adsorption of sample components. The sample injection valves may be configured such that the sample is swept by carrier gas directly onto the gas chromatographic column or into a split/splitless injection port. Two gas injection valves with fixed sample loops may be connected in series or parallel to enable simultaneous injection on two different capillary columns.

5.1.3 Oven Temperature Control: The oven must be capable of sub-ambient to > $150^{\circ} \mathrm{C}$ temperature programming with multiple temperature ramps in one program method. Isothermal temperature control to $\pm 0.5^{\circ} \mathrm{C}$ is recommended.

5.1.4 Detectors: The detectors must be FIDs with sufficient sensitivity to meet the quality assurance objectives listed in Table 1. 
5.1.5 Carrier Gas Flow: The system must be capable of regulating the flow of carrier gas through the gas injection valve and capillary column, regulating split flows, and providing make-up gas for the detector.

5.1.6 Analytical Columns: Wide-bore $(\geq 0.32 \mathrm{~mm}$ ) capillary columns are required. The recommended columns are 30-meter DB-624 and DB-1701 megabore columns (J\&W Scientific or equivalent).

5.1.7 Data System: The data system must be capable of receiving and displaying signal output from the GC/FID system for the entire chromatographic program. The data system must be capable of reprocessing data, performing external standard calculations, and archiving data for future processing.

\subsection{Gas Standard Preparation Equipment.}

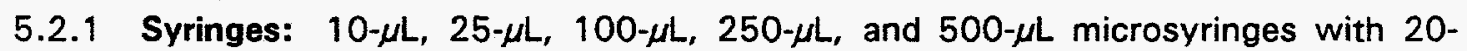
gauge needle; $1-\mathrm{mL}, 5-\mathrm{mL}, 10-\mathrm{mL}$, and $25-\mathrm{mL}$ gas tight syringes with shut-off valves.

5.2.2 Static Dilution Bottle: Static dilution bottle (glass) with shut-off valve and septum (Tekmar Company or equivalent).

5.2.3 Canisters: SUMMA ${ }^{\oplus}$ passivated stainless steel canisters (Scientific Instrument Specialist or equivalent).

\subsection{Reagents}

6.1 Carrier, Detector, and Dilution Gases. Gas cylinders of helium, nitrogen, hydrogen, and zero air; all ultra-high purity grade.

\subsection{Liquid Nitrogen. (or other appropriate coolant)}

\subsection{Stock Standards.}

6.3.1 Gases: Stock gas calibration cylinder(s) of all analytes listed in Table 1 must be purchased commercially (Scott Specialty Gas or equivalent). The standards must be traceable to National Institute of Standards and Technology (NIST) Standard Reference Material (SRM) or to a NIST/EPA approved Certified Reference Material (CRM). All standards must be replaced after 12 months if comparison with a check standard indicates a problem.

6.3.2 Liquids: Stock solutions of Table 1 analytes may be prepared from pure standard materials or purchased as certified solutions. Solutions may be prepared neat or in an appropriate solvent.

Stock solutions should be prepared in $10 \mathrm{~mL}$ volumetric flasks. Solutions may be prepared by weighing or transferring known volumes using microliter syringes. If known volumes are used, the density of the pure material is used to determine the final concentration.

Stock standards should be stored in Teflon-sealed screw cap bottles. Store with minimum headspace at $-10^{\circ} \mathrm{C}$ to $20^{\circ} \mathrm{C}$ and protected from direct sunlight.

All standards must be replaced at a minimum of every six months or sooner if comparison with check standards indicates a problem. 


\subsection{Secondary Dilution Standards.}

6.4.1 Using the stock calibration gas standards, prepare secondary dilution standards containing all the analytes in Table 1 in SUMMA ${ }^{\circledR}$ canisters. Secondary dilution standards prepared in SUMMA ${ }^{\circledR}$ canisters must be humidified to a minimum of 20-percent relative humidity. Humid secondary dilution standards may be prepared by either directly injecting reagent water (ASTM Type II or equivalent) into an evacuated SUMMA ${ }^{\circledR}$ canister or by passing the dilution gas (zero air or $\mathrm{N}_{2}$ ) through a humidifier.

6.4.2 Secondary dilution standards containing all the analytes listed in Table 1 may be prepared directly in dilution bottles or evacuated SUMMA ${ }^{\circledR}$ canisters by injecting aliquots of neat standards or solutions prepared in an appropriate solvent. Secondary dilution standards prepared in SUMMA ${ }^{\circledR}$ canisters must be humidified to a minimum of 20 -percent relative humidity.

The amount of each standard to be injected into the dilution bottle or SUMMA ${ }^{\circledast}$ canister can be calculated from the desired injection quantity and volume using the following equation:

$$
W_{T}=\frac{W_{I}}{V_{I}} \times V_{B}
$$

where

$$
\begin{aligned}
& W_{T}=\quad \text { total quantity of analyte to be injected into the dilution bottle/canister } \\
& \text { (ng) } \\
& W_{I}=\quad \text { desired amount of analyte to be injected onto the gas chromatographic } \\
& \text { column (ng) } \\
& V_{B}=\text { total volume of the dilution bottle or the total gas volume in the } \\
& \text { SUMMA }{ }^{\oplus} \text { canister after pressurization }(\mathrm{mL}) \\
& V_{I}=\quad \text { volume of sample delivered to the gas chromatographic column }(\mathrm{mL})
\end{aligned}
$$

The volume of the neat standard to be injected into the dilution bottle or SUMMA ${ }^{\circledast}$ canister is calculated as follows:

$$
V_{T}=\frac{W_{T}}{d}
$$

where

$V_{T}=\quad$ total volume of neat liquid or solution to be injected $(\mathrm{mL})$

$d=$ density of the neat standard or the concentration of the stock solution (ng/mL)

6.4.3 Calibration Standards: Calibration standards must be prepared at a minimum of three concentration levels according to the procedures described above. One of the calibration standards must be prepared below the PROLs specified in Table 1. The remaining standards must define the calibration range of the GC/FID system. Each calibration standard must contain all of the analytes listed in Table 1.

\subsection{Sample Collection, Preservation, and Handling}

7.1 Samples will be collected according to the sampling procedures described in Procedures 110.1 through 110.4 of this Methods Manual. 
7.2 Sample canisters will be stored at room temperature. Sample holding times cannot exceed 28 days.

7.3 All sample handling and chain-of-custody procedures described in Section 6.0 of the Transuranic Waste Characterization Quality Assurance Program Plan (QAPP) (DOE 1995b) must be followed.

\subsection{Procedure}

\subsection{Recommended GC/FID System Operating Conditions.}

Column types:

Initial column temperature: Initial column holding time:

Column temperature program:

Final column temperature:

Final column holding time:

Injector temperature:

Transfer line temperature:

Carrier gas:

Detector make up gas:
1) $\mathrm{DB} 624,30 \mathrm{~m} \times 0.53 \mathrm{~mm}$ l.D.

2) $D B 1701,30 \mathrm{~m} \times 0.53 \mathrm{~mm}$ l.D.

$-10^{\circ} \mathrm{C}$

5 minutes

$1.5^{\circ} \mathrm{C} /$ minute to $25^{\circ} \mathrm{C}$, then $3.0^{\circ} \mathrm{C} /$ minute to $50^{\circ} \mathrm{C}$, then $5.0^{\circ} \mathrm{C} /$ minute to $150^{\circ} \mathrm{C}$ $150^{\circ} \mathrm{C}$

3.5 minutes, or until all compounds have eluted 175 to $225^{\circ} \mathrm{C}$

$\geq 75^{\circ} \mathrm{C}$

Helium at $10 \mathrm{~mL} /$ minute

Helium at $15 \mathrm{~mL} / \mathrm{minute}$

The FID burner air, hydrogen, and helium carrier flow rates should be set to manufacturers' instructions to maintain as stable a flame as possible through the program cycle.

Calibration standard canisters and sample canisters should be maintained at $65^{\circ} \mathrm{C}$ for 30 minutes prior to sample analysis and during sample transfer to the gas chromatograph. The sample transfer line to the gas chromatograph should be maintained at a minimum of $10^{\circ} \mathrm{C}$ above the canister temperature. The transfer line should be purged with carrier gas between samples. The gas injection valve and fixed volume sample loop should be maintained at the same temperature as the sample canisters. The minimum sample loop volume should be $0.5 \mathrm{~mL}$.

8.2 All SUMMA ${ }^{\oplus}$ canisters used for GC/FID system calibration, quality control samples, and analytical purposes must first be cleaned and certified in accordance with Procedure 210.1 of this Methods Manual.

8.3 Calibration. Table 3 provides a summary of calibration requirements.

8.3.1 Initial Calibration: Initially a three-point calibration is performed on the GC/FID system. Each calibration standard must contain all of the analytes in Table 1. One calibration standard must be set below the PROLs listed in Table 1.

Inject each calibration standard using a gas injection valve with a fixed volume sample loop. All standards and samples must be analyzed under the same operating conditions. Tabulate peak area response of analyte against the amount injected. Calculate a response factor (RF) for each compound as follows: 
TABLE 3

Summary of GC Calibration Requirements for Gas Volatile Organic Compounds Analysis

\begin{tabular}{llll} 
Technique & Procedure & Frequency of Procedure & Acceptance Criteria \\
\hline GC/FID & $\begin{array}{l}\text { 3-pt initial calibration } \\
\text { (3 standards) }\end{array}$ & Initially, and as needed & $\begin{array}{l}\text { \%RSD of response factor for each } \\
\text { analyte }<30\end{array}$ \\
Continuing calibration & Every 12 hours & $\begin{array}{l}\text { linear regression plot yields straight } \\
\text { line and \%R is } 70-130 \text { for each } \\
\text { standard analyte }\end{array}$ & $\begin{array}{l}\text { \%D for all compounds } \leq 30 \text { of initial } \\
\text { calibration; RTs } \pm 3 \text { standard } \\
\text { deviations of initial calibration }\end{array}$
\end{tabular}

$\% \mathrm{D}$

$\%$ RSD

RT
Percent difference

Percent relative standard deviation

Retention time 


$$
R F=\frac{A_{x}}{C_{x}}
$$

where

$$
\begin{array}{lll}
\boldsymbol{A}_{\boldsymbol{x}} & = & \text { total area response for the compound } \\
\boldsymbol{C}_{\boldsymbol{x}} & = & \text { amount of the compound being measured (ng) }
\end{array}
$$

RF must be determined for all compounds on both analytical columns.

Alternately, a linear regression equation can be calculated for each compound:

$$
A_{x}=m C_{x}+b
$$

where

$$
\begin{array}{lll}
A_{x} & = & \text { total area response for the compound } \\
C_{x} & = & \text { amount (or concentration) of the compound being measured } \\
\boldsymbol{m} & = & \text { proportionality constant (slope) } \\
\boldsymbol{b} & = & \text { regression constant }
\end{array}
$$

Values of $\boldsymbol{m}$ and $\boldsymbol{b}$ are calculated using the method of least squares for linear regression.

$$
\begin{gathered}
m=\frac{\Sigma C_{x} \Sigma A_{x}-n \Sigma C_{x} A_{x}}{\left(\Sigma C_{x}\right)^{2}-n \Sigma C_{x}^{2}} \\
b=\frac{\Sigma C_{x} \Sigma C_{x} A_{x}-\Sigma C_{x}^{2} \Sigma A_{x}}{\left(\Sigma C_{x}\right)^{2}-n \Sigma C_{x}^{2}}
\end{gathered}
$$

where

$$
n \quad=\quad \text { number of data points }
$$

Using the RFs, the percent relative standard deviation (\%RSD) for each compound listed in Table 1 is calculated as follows:

$$
\% R S D=\frac{s}{\bar{x}} * 100
$$

where

$\bar{s}=$ standard deviation of the RFs for a compound

$=\quad$ mean of the 3 initial RFs for a compound

$$
s=\sqrt{\sum_{i=1}^{n} \frac{\left(x_{i}-\bar{x}\right)^{2}}{n-1}}
$$

where

$$
\begin{array}{ll}
x_{i} & =\begin{array}{l}
\text { measured value of the } i^{\text {th }} \text { replicate sample analysis } \\
\text { measurement }
\end{array} \\
n & =\text { number of replicate analyses }
\end{array}
$$

The \%RSD for each individual compound must be less than 30 percent. For those compounds having a \%RSD greater than 30 percent, the data system may be 
used to generate a second or third order regression curve. Sample analysis must not proceed until the \%RSD requirement is satisfied.

If using a linear regression equation, the initial calibration is considered valid if the resulting calibration plot yields a straight line and the calculated percent recovery (\%R) for each standard is within 30 percent of the known values when their area responses are entered into the regression equations. Sample analysis must not proceed until a valid initial calibration is achieved.

8.3.2 Continuing Calibration: The working calibration curve or response factor and the RT window (Section 8.4) must be verified every 12 hours of operation, and prior to the analysis of any samples. Verification is accomplished by analyzing a calibration standard that contains amounts (ng) of each target analyte that are near the midpoint amounts of the initial calibration curve.

The verification procedure (if using the RF method) involves calculating the $R F$ for each compound in the calibration verification standard and comparing these to the average RFs for each compound from the initial calibration sequence. The validity of the initial calibration RF is checked by calculating the percent difference (\%D) for individual compounds as follows:

$$
\% D=\frac{\left|\left(R F_{l}\right)-\left(R F_{C}\right)\right|}{\left(R F_{l}\right)} * 100
$$

where

$$
\begin{array}{ll}
R F_{I}= & \text { average } R F \text { from initial calibration (area/ng) } \\
R F_{C}= & \text { RF from the current midpoint calibration verification standard } \\
\text { (area/ng) }
\end{array}
$$

The \%D for each compound must be less than or equal to 30 percent. For compounds where a higher order regression calibration curve was used, the point from the calibration verification standard for the compound must fall within 30 percent of the curve value from the initial calibration. If the \%D criterion is not met, corrective action must be taken. If no source of the problem can be determined after corrective action has been taken, a new three-point initial calibration curve must be generated. This criterion must be satisfied before sample analysis begins.

If a linear regression equation was generated, the verification procedure involves calculating the measured value for each analyte in the continuing calibration verification standard and comparing these values to the known values.

The $\% \mathrm{D}$ is calculated as follows:

$$
\% D=\frac{\left|C_{k}-C_{m}\right|}{C_{k}} * 100
$$

where

$$
\begin{array}{lll}
C_{m} & = & \text { measured value } \\
C_{k} & = & \text { known value }
\end{array}
$$

The $\% \mathrm{D}$ must be $\leq 30$ for all analytes. If the $\% \mathrm{D}$ criterion is not met, corrective action must be taken. 
The absolute RT of each component must fall within the RT window (Section 8.4) established for that compound during the most recent initial calibration. If the RT window criterion is not met, corrective action must be taken. If no source of the problem can be determined after corrective action has been taken, a new threepoint initial calibration curve must be generated.

\subsection{Retention Time Windows.}

8.4.1 The RT window for each analyte is determined by calculating the standard deviation of the absolute RT for each analyte. RT windows are determined by injecting a minimum of three standards over a period of 72 hours. RT windows shall be calculated as the mean plus or minus three times the standard deviation of the individual RTs for each calibration standard analyzed in the 72-hour period.

8.4.2 The RT window for each analyte must be determined whenever a new initial calibration curve is generated or when a new gas chromatographic column is installed. RT windows must be established for all analytes in Table 1 for both analytical columns.

8.4.3 For the calibration verification standard, the midpoint of the RT window shall be the average absolute RT for each compound from the most recent initial calibration.

8.4.4 The midpoint of the 12-hour RT window shall be the absolute RT for each analyte from the calibration verification standard for that calibration period.

\subsection{Sample Analysis.}

8.5.1 The minimum sample volume required to meet the PROLs specified in Table 1 is $0.5 \mathrm{~mL}$.

8.5.2 All samples must be pressurized to a minimum of 1 to 3 times atmospheric pressure. Samples should be pressurized with humid zero air or nitrogen. The resulting dilution factor $(D F)$ is calculated as follows:

$$
D F=\frac{C_{p 2}}{C_{p 1}}
$$

where

$$
\begin{array}{lll}
C_{p 2} & = & \text { canister pressure after dilution }(\mathrm{mm} \mathrm{Hg}) \\
C_{p 1} & = & \text { canister pressure before dilution }(\mathrm{mm} \mathrm{Hg})
\end{array}
$$

1. After reaching thermal equilibrium, sample is allowed to flow through the sample introduction system and sweep through the fixed volume sample loop. A minimum of 10 fixed loop volume equivalents should be allowed to sweep through the sample loop. A flow restriction device (mass flow controller or mass flow meter) should be placed on the outlet of the sample loop. The transfer line should be purged with carrier gas between samples.

2. After the fixed volume sample loop has been conditioned, the gas injection valve should be rotated to the inject position. The gas chromatographic temperature program must be started at this time. The gas injection valve should have an electronic event module that automatically starts the gas chromatograph upon sample injection. 
8.5.3 If the initial analysis of a sample detects any analytes with concentrations that exceed the initial calibration range, the sample must be diluted and reanalyzed.

8.5.4 When a sample has been analyzed that saturates the detector or shows concentrations of analytes greater than ten times the upper limit of the initial calibration, the analysis must be followed by a humid zero air or nitrogen blank analysis. If the blank analysis is not free of interferences (target analytes $<50$ percent of the PROLs) the system must be decontaminated. Sample analysis may not resume until a blank can be analyzed that is free of contamination.

\subsection{Calculations}

9.1 Qualitative Analysis. An analyte listed in Table 1 is identified by RT confirmation on both analytical columns. For positive analyte identification, the sample component peak must fall within the RT window set by the most recent initial calibration for each analytical column.

9.2 Quantitative Analysis. Once a compound has been qualitatively identified, the quantitation of that compound will be based on the area response. Quantitation of a given compound shall be performed on one of the two analytical columns. The column used for quantitation should be interferant free in the RT window corresponding to the analyte. Calculate the concentration in ppmv of each identified analyte in the sample as follows:

where

$$
\text { concentration }(p p m v)=\frac{\left(A_{x}\right)(R)(T)}{(R F)(P)(M W)\left(V_{0}\right)}
$$

$$
V_{o}=\left(\frac{V_{a}}{D F}\right)\left(\frac{P_{a}}{760 m m H g}\right)\left(\frac{298^{\circ} K}{273+T_{a}}\right)
$$

and

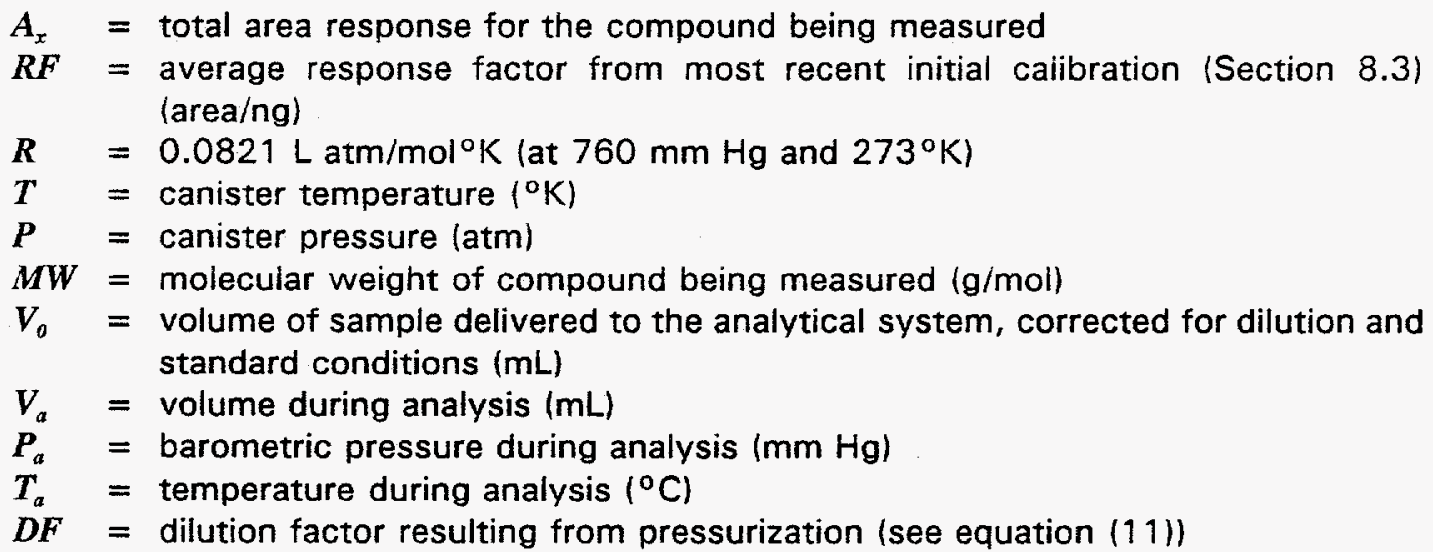

NOTE: At $25^{\circ} \mathrm{C}$ and $1 \mathrm{~atm}, R T / P=24.5 \mathrm{~L} / \mathrm{mol}$ 
If a linear regression equation is used, the following are substituted in Equation (12):

$$
\begin{aligned}
& A_{x} \text { is replaced by } A_{x}-b \text { and } \\
& R F \text { is replaced by } m \\
& \text { where } \\
& \qquad \begin{array}{l}
b= \\
m=\quad \text { linear regression constant (intercept) }
\end{array}
\end{aligned}
$$

\subsection{Quality Control}

10.1 Each laboratory that uses this procedure is required to operate a formal quality control program. The laboratory must retain records to document the quality of the data generated. Each laboratory must have SOPs documenting and describing activities involved in utilizing this procedure. Specific quality control practices will include, but are not limited to, the analysis of quality control samples. The types of quality control samples, their associated frequency of analysis, acceptance criteria, and corrective action required if samples do not meet the acceptance criteria, is summarized in Table 4. Laboratory SOPs shall address requirements for preparing laboratory duplicates, laboratory blanks, and laboratory control samples.

10.2 All laboratories using this procedure must demonstrate acceptable performance prior to the analysis of actual samples. Demonstration of acceptable performance will be achieved by analyzing method performance samples (Table 4). These samples can either be commercially purchased or laboratory prepared, but must contain all of the analytes listed in Table 1 at concentrations appropriate (two to five times the PROLs) to determine the quality assurance objectives specified in Table 1 . The analysis of seven samples must meet the criteria specified for precision, accuracy, and method detection limit (MDL) in Table 1. Demonstration of acceptable method and analyst performance must be repeated (by analyzing four samples) at a minimum of every six months. Method performance should be conducted over a period of several days to account for long-term variation. Corrective action must be implemented when acceptable method performance is not met. Precision will be determined as follows:

- For duplicate measurements, the precision expressed as the relative percent difference (RPD) is calculated as

$$
R P D=\frac{C_{1}-C_{2}}{\frac{\left(C_{1}+C_{2}\right)}{2}} * 100
$$

where

$$
\begin{array}{lll}
C_{1} \text { and } C_{2} & = & \text { two values obtained by analyzing the duplicate samples } \\
C_{t} & = & \text { larger of the two observed values }
\end{array}
$$

- For three or more replicate measurements, the precision expressed as the \%RSD is calculated as

$$
\% R S D=\frac{s}{\bar{y}} * 100
$$

where

$$
\begin{array}{lll}
s & = & \text { standard deviation } \\
\bar{y} & = & \text { mean of replicate analyses }
\end{array}
$$


TABLE 4

\section{Summary of Laboratory Quality Control Samples and Frequencies for Gas Volatile Organic Compounds Analysis}

\begin{tabular}{llll}
\hline OC Sample & Minimum Frequency & $\begin{array}{l}\text { Acceptance } \\
\text { Criteria }\end{array}$ & $\begin{array}{l}\text { Corrective } \\
\text { Action }\end{array}$ \\
\hline $\begin{array}{l}\text { Method performance } \\
\text { samples } \\
\text { Laboratory duplicates }\end{array}$ & $\begin{array}{l}\text { Seven (7) samples initially } \\
\text { and four (4) semiannually } \\
\text { One (1) per analytical } \\
\text { batch }\end{array}$ & Meet Table 1 QAOs & $\begin{array}{l}\text { Repeat until } \\
\text { acceptable }\end{array}$ \\
$\begin{array}{l}\text { Daily prior to sample } \\
\text { analysis }\end{array}$ & $\begin{array}{l}\text { Analyte amounts } \\
<3 \times \text { MDLs }\end{array}$ & $\begin{array}{l}\text { Nonconformance if } \\
\text { RPD }>25\end{array}$ \\
$\begin{array}{l}\text { Labonconformance if } \\
\text { analyte amounts }>3 \\
\text { X MDLs }\end{array}$ \\
$\begin{array}{l}\text { One (1) per analytical } \\
\text { Blind audit samples }\end{array}$ & $\begin{array}{l}70-130 \% R \\
\text { batch } \\
\text { Samples and frequency } \\
\text { controlled by the Gas PDP } \\
\text { Plan }\end{array}$ & $\begin{array}{l}\text { Specified in the Gas } \\
\text { PDP Plan }\end{array}$ & $\begin{array}{l}\text { \%R }<70 \text { or }>130 \\
\text { Specified in the Gas } \\
\text { PDP Plan }\end{array}$ \\
\hline
\end{tabular}

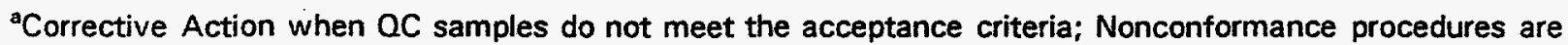
outlined in Section 2.1.2.1 of the QAPP.

${ }^{b}$ Applies only to concentrations greater than the PROLs listed in Table 1.

MDL $=$ Method detection limit
PDP $=$ Performance Demonstration Program
OAO $=$ Quality assurance objective
$\% R=$ Percent recovery
RPD $=$ Relative percent difference


- The standard deviation $(s)$ is defined as

$$
s=\sqrt{\sum_{i=1}^{n} \frac{\left(y_{i}-\bar{y}\right)^{2}}{n-1}}
$$

where

$y_{i}=$ measured value of the $i^{\text {th }}$ replicate sample analysis measurement

$n=$ the number of replicate analyses

Accuracy will be determined as the percent recovery $(\% R)$ as follows:

$$
\% R=\frac{C_{m}}{C_{s m m}} * 100
$$

where

$$
\begin{aligned}
& C_{m}=\text { measured concentration } \\
& C_{s m}=\text { true concentration }
\end{aligned}
$$

MDL will be determined as follows:

$$
\left.M D L=t_{(n-1,1-\alpha}=0.99\right) * S
$$

where

$$
\begin{array}{lll}
s & = & \text { standard deviation } \\
t_{(n-1,1-\alpha=0.99)}= & t \text {-distribution value appropriate to a 99-percent confidence level } \\
& \text { and a standard deviation estimate with } n-1 \text { degrees of freedom }
\end{array}
$$

10.3 The laboratory must analyze blanks at the frequency specified in Table 4. Using the sample preparation manifold, blanks will be generated in the laboratory by filling SUMMA ${ }^{\circ}$ canisters with humid zero air or nitrogen (99.999-percent pure). The same procedures used to prepare and analyze field samples will be used to prepare and analyze the laboratory blanks. Laboratory blank results are acceptable if the amounts of individuai analytes listed in Table 1 are less than 3 times the MDL. Corrective action must be implemented if blanks exceed this level.

10.4 The laboratory must analyze individual field samples in duplicate at the frequency specified in Table 4. Single canisters analyzed in duplicate will be used to assess laboratory precision. Laboratory duplicate results will be considered acceptable if the RPD is $\leq 25$ percent. RPD is determined as in equation (13). Duplicates which do not meet these criteria should be flagged.

10.5 The laboratory must analyze laboratory control samples at the frequency specified in Table 4. Commercially purchased gas standards will be used to prepare laboratory control samples. Laboratory control samples will be prepared in the laboratory by attaching the gas standard to the laboratory sample preparation manifold and filling evacuated canisters. For guidance on preparing laboratory control samples, see Section 6.3 of Procedure 430.1 (Modified Method TO-14) of this Methods Manual. The gas standard used to prepare the laboratory control samples must be independent of those used for instrument calibration and must contain all of the analytes listed in Table 1 at concentration in the calibration range of the GC system. The laboratory control sample analysis is designed to ensure that the laboratory's performance is in control with each analytical batch of samples processed. Laboratory control sample results will be considered acceptable if the $\% R$ is 70 to 130 percent of the known value. \%R is determined as in equation (16). If results 
are outside of this range, then the source of the error must be determined and the problem corrected.

10.6 The laboratory will be required to analyze blind audit samples. These audit samples are part of the Performance Demonstration Program. Details of this program are in the Performance Demonstration Program Plan for the Analysis of Simulated Headspace Gases for the Transuranic Waste Characterization Program (Gas PDP Plan)(DOE 1995a).

\subsection{Procedure Performance}

The INEL Environmental Chemistry Laboratory determined precision and accuracy for this procedure through replicate analysis of laboratory control samples. Three laboratory control samples were analyzed. Two samples underwent 30 replicate analyses for each analyte, one sample underwent seven replicate analyses for each analyte. The precision and accuracy was determined for each analyte in each sample. Precision (as \%RSD) was determined as in Equation (9), accuracy (as \%R) was determined as in Equation (16). Procedure performance for these analyses is presented in Table 5 and Table 6 for samples with 30 replicate analyses and Table 7 for the sample with seven replicate analyses.

\subsection{References}

DOE. 1995a. Performance Demonstration Program Plan for the Analysis of Simulated Headspace Gases for the Transuranic Waste Characterization Program. CA0-95-1076, Current Revision, Carlsbad, New Mexico, Carlsbad Area Office, U.S. Department of Energy.

DOE. 1995b. Transuranic Waste Characterization Quality Assurance Program Plan. CAO-941010, Current Revision, Carlsbad, New Mexico, Carlsbad Area Office, U.S. Department of Energy. 
Table 5

Procedure Performance for Sample \#1 (30 replicate analyses)

\begin{tabular}{lccc}
\hline Analyte & $\begin{array}{c}\text { Concentration } \\
\text { (ppmv) }\end{array}$ & $\begin{array}{c}\text { Precision } \\
\text { (\%RSD) }\end{array}$ & $\begin{array}{c}\text { Accuracy } \\
\text { (\%R) }\end{array}$ \\
\hline Acetone & 189.7 & 1.4 & 98.7 \\
Butanol & 152.2 & 1.3 & 98.8 \\
Methanol & 173.2 & 1.3 & 100.5 \\
Methyl ethyl ketone & 154.7 & 1.3 & 99.5 \\
Methyl isobutyl ketone & 168.1 & 1.3 & 99.7 \\
\hline
\end{tabular}

Table 6

Procedure Performance for Sample \#2 (30 replicate analyses)

\begin{tabular}{lccc}
\hline Analyte & $\begin{array}{c}\text { Concentration } \\
\text { (ppmv) }\end{array}$ & $\begin{array}{c}\text { Precision } \\
\text { (\%RSD) }\end{array}$ & $\begin{array}{c}\text { Accuracy } \\
\text { (\%R) }\end{array}$ \\
\hline Acetone & 190.6 & 1.2 & 99.3 \\
Butanol & 152.9 & 1.7 & 107.5 \\
Methanol & 218.1 & 1.3 & 103.6 \\
Methyl ethyl ketone & 154.7 & 1.2 & 100.1 \\
Methyl isobutyl ketone & 227.8 & 1.3 & 100.7 \\
\hline
\end{tabular}

Table 7

Procedure Performance for Sample \#3 (7 replicate analyses)

\begin{tabular}{lccc}
\hline Analyte & $\begin{array}{c}\text { Concentration } \\
\text { (ppmv) }\end{array}$ & $\begin{array}{c}\text { Precision } \\
\text { (\%RSD) }\end{array}$ & $\begin{array}{c}\text { Accuracy } \\
\text { (\%R) }\end{array}$ \\
\hline Acetone & 98.0 & 2.8 & 98.2 \\
Butanol & 98.0 & 3.9 & 107.7 \\
Methanol & 99.0 & 4.5 & 87.5 \\
Methyl ethyl ketone & 100.0 & 2.6 & 95.0 \\
Methyl isobutyl ketone & 102.0 & 2.3 & 93.7 \\
\hline
\end{tabular}



PROCEDURE 440.2

\section{DIRECT INJECTION GAS CHROMATOGRAPHY/FLAME IONIZATION DETECTOR DETERMINATION OF NONHALOGENATED VOLATILE ORGANIC COMPOUNDS IN HOMOGENOUS SOLIDS AND SOIL/GRAVEL}

\subsection{Scope and Application}

1.1 This procedure is for the Gas Chromatography/Flame lonization Detector (GC/FID) analysis of nonhalogenated volatile organic compounds (VOCs) in the range from $100 \mathrm{mg} / \mathrm{kg}$ to volume percent in samples of homogenous solids and soil/gravel. This procedure is restricted to use by or under the supervision of analysts experienced in Gas Chromatography (GC). The target analytes and quality assurance objectives for this procedure are listed in Table 1 . Sampling procedures for homogenous solids and soil/gravel are described in Procedure 120.1 of this Methods Manual. This procedure must be implemented with a site-specific standard operating procedure (SOP).

1.2 This method is similar to EPA Methods 8000, 8015A (EPA 1995), and DOE Method OGO15R (DOE 1994). It is based on the direct injection of an aqueous sample into a gas chromatograph equipped with a flame ionization detector for the determination of nonhalogenated VOCs that perform poorly by purge and trap methods. This technique was specifically developed for the determination of alcohols in aqueous extracts of radioactively contaminated solid materials. However, it should be applicable to the analysis of nonhalogenated VOCs in any aqueous solution.

1.3 For the purposes of the TRU Waste Characterization Program, samples are to be analyzed in analytical batches. An analytical batch is defined as a suite of samples that is processed as a unit, using the same analytical method, within a specific time period. An analytical batch can be up to 20 samples (excluding laboratory $\mathrm{OC}$ samples), all of which must be received by the laboratory within 14 days of validated time of sample receipt (VTSR) of the first sample in the batch.

\subsection{Summary of Procedure}

This procedure describes the dual column gas chromatographic separation and detection of nonhalogenated VOCs in potentially radioactively contaminated aqueous extracts. Quantitative analyses of the analytes shall be performed on a primary column. A different stationary phase chromatography column shall be used as a confirmation column.

\subsection{Interferences}

3.1 The water and sample preparation hardware may contain contaminants or interfering compounds. All processing hardware must be demonstrated to be clean by using method blanks during the sample preparation. Only freshly prepared ASTM Type I water should be used and it also should be periodically checked to insure purity.

3.2 Raw GC data from all blanks, samples, and spikes must be reviewed for possible co-contaminants and interferences. GC/FID analysis of aqueous extracts of organic sludges containing slightly soluble halogenated hydrocarbons has demonstrated that these compounds are partially co-extracted. Under appropriate chromatography conditions these compounds are separated from the analytes of interest. Example chromatograms of standards and extracted organic sludges for both recommended columns are shown in Figures 1 and 2 . Compounds that have been investigated for potential interferences are listed in Table 2. 
TABLE 1

Target Analyte List and Quality Assurance Objectives

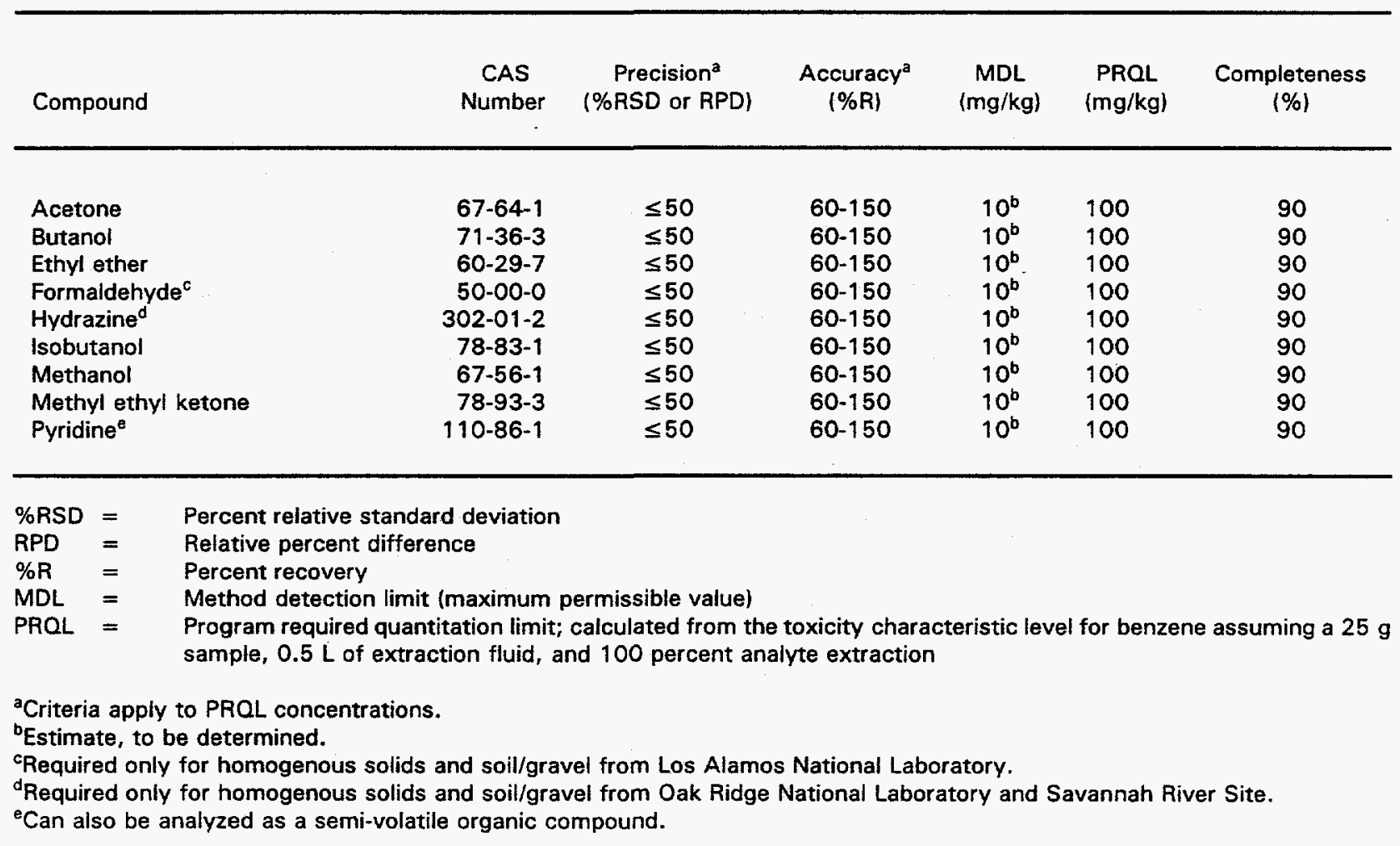


TABLE 2

Compounds Investigated for Potential Interferences ${ }^{a}$

Compound

CAS Number

1,1,1-Trichloroethane

1,1,2-Trichlorotrifluoroethane

Carbon tetrachloride

Dichloromethane

Trichloroethylene
$71-55-6$

76-13-1

56-23-5

75-09-2

79-01-6

aPartially soluble halogenated hydrocarbons evaluated for interferences for direct injection method. 
3.3 Cross contamination by carry over may occur anytime high- and low-concentration samples are sequentially analyzed. Care must be taken to rinse syringes between sample injections. Whenever a high-concentration sample is analyzed, it should be followed by an analysis of reagent water to check for cross-contamination.

3.4 The laboratory where volatile analysis is performed should be free of solvents that could potentially contaminate the samples.

\subsection{Safety}

This procedure may involve the use of hazardous materials, operations, and equipment. It is the responsibility of whoever uses this procedure to consult appropriate site personnel concerning health and safety issues and establish appropriate health and safety practices. Consideration should be given to safety concerns regarding chemical and radiation exposure. Training regarding proper storage, usage, and disposal of chemicals is recommended.

\subsection{Apparatus and Materials}

\subsection{Sample Extraction.}

5.1.1 $16 \mathrm{~mm}$ OD by $125 \mathrm{~mm}$ or similar screw cap glass tubes with PTFE lined caps.

5.1.2 Centrifuge capable of approximately $1000 \mathrm{rpm}$ with appropriately sized tube holders.

5.1.3 Mechanical test tube shaker is recommended.

5.1.4 Small crimp top gas chromatography sample vials.

\subsection{GC/FID System.}

5.2.1 Oven Temperature Control: The GC oven must be capable of temperature programming from sub-ambient to $>100^{\circ} \mathrm{C}$ with multiple temperature ramps in one program method. Isothermal temperature control to $\pm-0.5^{\circ} \mathrm{C}$ is recommended.

5.2.2 Detector: The detector must be a flame ionization detector with sufficient sensitivity to meet the quality assurance objectives listed in Table 1.

5.2.3 Carrier Gas Flow: The system must have the capability to regulate the flow of carrier gas, helium, through the gas injection valve and analytical capillary column, regulate spilt flows, and provide make-up gas for the detector.

5.2.4 Analytical Columns: Fused silica capillary columns with the following coatings are recommended.

- 1st (Quantitation) Column: Crossbonded Carbowax

- Length: 30 meters

- Film thickness: $0.25 \mu \mathrm{m}$

- Internal Diameter: $0.25 \mathrm{~mm}$ 
- 2nd (Confirmation) Column: 95 percent dimethyl 5 percent diphenyl polysiloxane

- Length: 30 meters

- Film thickness: $0.5 \mu \mathrm{m}$

- Internal Diameter: $0.25 \mathrm{~mm}$

5.2.5 Data System: A computer or integrator system that interfaces with the GC to accurately determine peak areas. The data system must be capable of receiving and displaying signal output from the GC/FID system for the entire chromatographic program. The data system must be capable of reprocessing data, external standard calculations, and archiving data for future use.

5.3 Volumetric Flasks. Assorted class A volumetric flasks including 10-, 50-, 100-, 250-, 500-, and 1000-mL flasks with ground glass stoppers are recommended.

5.4 Syringes. Several assorted syringes are needed for preparation of calibration standards. $100 \mu \mathrm{L}, 250 \mu \mathrm{L}, 500 \mu \mathrm{L}$, and $1 \mathrm{~mL}$ glass syringes are recommended.

5.5 Microsyringes. $10 \mu \mathrm{L}$ syringes or an automated injection system for the GC.

5.6 The splitless glass injection liners should be periodically cleaned with concentrated nitric acid to dissolve salts deposited from the injection of the aqueous samples. Failure to clean the liners can result in poor chromatographic performance. These liners are potentially contaminated with radiological materials and should be handied accordingly.

\subsection{Reagents}

6.1 High grade reagent water shall be used for all extraction and analytical purposes. The quality of the water shall meet ASTM Type I requirements for organic trace impurities.

6.2 Stock Standard Solutions. Stock standard solutions of the individual analytes can be prepared from pure (96 percent or better) standard material or purchased as certified solutions.

6.2.1 Prepare stock standard solutions by accurately weighing or transferring known quantities using microliter syringes. Dissolve the analytes in water and dilute to a known volume in a volumetric flask. If known volumes are used, the density of the pure material is used to determine the final concentration. When purity of the analyte is assayed to be 96 percent or better, the weight may be used without correction to calculate the concentration of the stock standard. Commercially prepared stock standard may be used at any concentration if they are certified by the manufacturer or by an independent source.

6.2.2 Transfer the stock solution into Teflon-sealed screw cap bottles. Store at $4^{\circ} \mathrm{C}$ and protect from light. Stock standard solutions shall be checked frequently for degradation or evaporation.

6.2.3 Stock standard solutions must be replaced after every six months or sooner if comparison with check samples indicates a problem.

6.3 Independent Verification Standard. Stock standard solutions of the analytes made from a second manufacturing source will be purchased or prepared according to the guidelines in 6.2. These independent stock standards will be the external check and verification for the analytes stock standard solutions. 
6.4 Calibration standards at a minimum of three concentration levels for each analyte of interest shall be prepared through dilution of the stock standards with water. One of the standards must be set below the program required quantitation limit (PROL). The remaining concentrations should correspond to the expected range of the concentrations in real samples or should define the working range of the GC. All standards will be stored at $4^{\circ} \mathrm{C}$ and should be prepared once a month or when $\mathrm{OC}$ samples indicate problem.

6.5 Daily continuing calibration check standard should be prepared by diluting the Stock Standard Solution. The daily continuing calibration verification standard shall be prepared weekly and stored at $4^{\circ} \mathrm{C}$.

6.6 Spiking Solution. An analyte spiking solution should be prepared with the concentrations of the spiked analytes near the concentration range expected in the extracted sample solutions. When no information is available on a sample type, spikes should be prepared at ten times the method detection limit (MDL). Store the spiking solution in a glass bottle with a PTFE lined screw cap in a refrigerator at $4^{\circ} \mathrm{C}$.

\subsection{Sample Collection, Preservation, and Handling}

7.1 Samples will be collected using the sampling procedures described in Procedure 120.1 of this Methods Manual.

7.2 All sample handling and chain-of-custody procedures described in Section 6.0 of the Transuranic Waste Characterization Quality Assurance Program Plan (QAPP) (DOE 1995c) must be followed.

\subsection{Procedure}

\subsection{Sample Preparation.}

8.1.1 Weigh a screw cap sample tube with cap installed on an analytical balance. Record the tare weight to the nearest $0.1 \mathrm{mg}$.

Note: Steps 8.1.2 and 8.1.3 have the greatest chance for loss of analytes. These steps should be completed as quickly as possible.

8.1.2 Remove the sample from the storage refrigerator and remove an aliquot using an appropriate sub-sampling device. The sample mass should be approximately 1-2 grams. Transfer the sample to the tared tube attach the cap and reweigh the vial. Record mass to $0.1 \mathrm{mg}$.

8.1.3 Duplicate samples, matrix spike samples, and matrix spike duplicate samples are prepared in the same way. The spiking solution should be added directly on the solid material. If the spike is prepared using microliter amounts of the spiking solution, the error in the mass caused by the addition of the spike will be negligible. The spiked concentration should be based on 0.5 to 1.5 times the expected concentration in the sample, when known. Otherwise spike at 10 times the MDL. Never spike at less than 10 times the MDL.

8.1.4 Remove the cap and fill the sample tube with reagent water leaving very little head space. Cap and reweigh the tube.

8.1.5 Shake the tube for at least 10 minutes and continue shaking until the sample is finely divided and evenly distributed throughout the solution. Oily samples may separate but partial emulsions should be achieved. 
8.1.6 Place the tubes into a centrifuge and spin at approximately $1000 \mathrm{rpm}$ for 10 minutes.

8.1.7 Remove an aliquot of the aqueous solution using a glass pipette and fill a small gas chromatography crimp top sample vial so that no head space exists and install the crimp on the cap.

8.1.8 Refrigerate the samples at $4^{\circ} \mathrm{C}$ until the direct injection GC/FID analysis can be performed.

\subsection{Recommended GC/FID System Operating Conditions.}

$\begin{array}{ll}\text { Column types: } & \text { 1.) Crossbonded Carbowax } 30 \mathrm{~m} \times 0.25 \mathrm{~mm} \text { ID } \\ & \begin{array}{l}\text { 2.) } 95 \text { percent dimethyl } 5 \text { percent diphenyl } \\ \text { polysiloxane } 30 \mathrm{~m} \times 0.25 \mathrm{~mm} \text { ID }\end{array} \\ \text { Initial column temperature: } & 20^{\circ} \mathrm{C} \\ \text { Initial column holding time: } & 3 \text { minutes } \\ \text { Column temperature program: } & 5^{\circ} \mathrm{C} / \text { minute } \\ \text { Final column temperature: } & 100^{\circ} \mathrm{C} \\ \text { Final column holding time: } & 1 \text { minute } \\ \text { Injector temperature: } & 80^{\circ} \mathrm{C} \\ \text { Detector temperature: } & 200^{\circ} \mathrm{C} \\ \text { Carrier gas: } & \text { Helium at } 1.0 \mathrm{~mL} / \text { minute } \\ \text { Detector make up gas: } & \text { Helium at } 3.0 \mathrm{~mL} / \text { minute }\end{array}$

The FID burner air, hydrogen, and helium carrier flow rates should be set to manufacturer's instructions to maintain as stable a flame as possible through the program cycle.

8.3 Calibration. Table 3 provides a summary of calibration requirements.

8.3.1 Initial Calibration Procedure: Initially a three-point calibration is performed in triplicate on the GC/FID system. Each calibration standard must contain all of the analytes listed in Table 1 . One calibration standard must be set below the PROL (Table 1) for each analyte. Inject in triplicate, 1 to $3 \mu \mathrm{L}$ of each calibration standard onto the GC/FID system. All standards and samples must be analyzed under the same injection and operating conditions. Tabulate peak area (peak heights may also be used) response against the mass injected. The results are used to prepare a calibration curve for each analyte.

An alternative calibration method can be used in which a response factor (RF) is calculated for the standards. If the percent relative standard deviation (\%RSD) of the $R F$ is less than 20 percent over the working range, linearity through the origin can be assumed, and the average RF can be used in place of a calibration curve.

$$
R F=\frac{A_{x}}{C_{x}}
$$

where

$A_{\boldsymbol{x}}=$ total areas response for the analyte

$C_{x}=$ amount of the analyte being measured (ng)

RF must be determined for all analytes on both analytical columns. 
TABLE 3

Summary of Calibration Requirements for Total Nonhalogenated Volatile Organic Compounds Analysis

\begin{tabular}{llll}
\hline Technique & Procedure & Frequency of Procedure & Acceptance Criteria \\
\hline GC/FID & $\begin{array}{l}\text { 3-pt initial calibration } \\
\text { (3 standards) }\end{array}$ & Initially, and as needed & $\begin{array}{l}\text { Correlation coefficient } \geq 0.93 \\
\text { (calibration curves) or \%RSD for } \\
\text { response factors }<35 \text { for all analytes } \\
\text { Response factor or measured } \\
\text { concentration \%D for all analytes } \\
\leq 15 \text { of initial calibration; RT } \pm 3 \\
\text { standard deviations from initial } \\
\text { calibration }\end{array}$ \\
\hline
\end{tabular}

$\% D$

$\%$ RSD

RT
$=$ Percent difference

$=$ Percent relative standard deviation

$=$ Retention time 
Note: This method may not provide the required accuracy for low concentrations of analytes, i.e., concentrations near the PROL. This occurs because offsets that are observed in a calculated calibration curve are ignored using the response factor method.

Using the RFs, the \%RSD for each analyte listed in Table 1 is calculated as follows:

$$
\% R S D=\frac{s}{\bar{x}} * 100
$$

where

$$
\begin{aligned}
& s=\text { standard deviation of RFs for an analyte } \\
& \bar{x}=\text { mean of } 3 \text { initial RFs for an analyte }
\end{aligned}
$$

The standard deviation $(s)$ is defined as follows:

$$
s=\sqrt{\sum_{i=1}^{n} \frac{\left(x_{i}-\bar{x}\right)^{2}}{n-1}}
$$

where

$$
\begin{aligned}
& x_{i}=\text { measured value of the } i^{\text {th }} \text { replicate sample analysis measurement } \\
& n=\text { number of replicate analyses }
\end{aligned}
$$

The \%RSD for each individual analyte must be less than 35 percent. For those analytes having a \%RSD greater than 35 percent, the data system may be used to generate a second or third order regression curve. Sample analysis must not proceed until the \%RSD requirement is satisfied for both columns.

8.3.2 Continuing Calibration: The working calibration curve or RF and the retention time (RT) windows (Section 8.4 ) must be verified on each working day or every 12 hours of operation, whichever is smaller, and prior to the analysis of any samples. Verification is accomplished by analyzing a continuing calibration standard that contains amounts (ng) of target analytes that are near midpoint range of the initial calibration curve.

The verification procedure involves calculating the RF for each compound in the calibration verification standard and comparing these to the average RFs for each compound from the initial calibration sequence. The validity of the initial calibration RF is checked by calculating the percent difference (\%D) for individual compounds as follows:

$$
\% D=\frac{\left|\left(R F_{1}\right)-\left(R F_{2}\right)\right|}{\left(R F_{1}\right)} * 100
$$

where:

$$
\begin{aligned}
& R F_{1}=\text { average response factor from initial calibration (area/ng) } \\
& \boldsymbol{R F}_{2}=\text { response factor from the current midpoint calibration } \\
& \text { verification standard (area/ng) }
\end{aligned}
$$

The \%D for all compounds must be less than or equal to 15 percent. For compounds where a higher order regression calibration curve was used, the point from the calibration verification standard for the compound must fall within 15 percent of the curve value from the initial calibration. If the $\% \mathrm{D}$ criterion is not 
met, corrective action must be taken. If no source of the problem can be determined after corrective action has been taken, a new three-point initial calibration curve must be generated. This criterion must be satisfied on both columns before sample analysis begins.

8.4 Retention Time Windows. The absolute RT of each analyte must fall within the RT window established for that analyte during the most recent initial calibration. If the RT window criterion is not met, corrective action must be taken (see Section 8.6). If no source of the problem can be determined after corrective action has been taken, a new initial calibration curve must be generated. RT windows for each analyte are determined by either of two approaches.

8.4.1 Calculate the standard deviation of the absolute RT for each analyte over each initial calibration standard. The RT window shall be defined as plus-or-minus three times the standard deviation of the absolute RT of each analyte determined from the most recent initial calibration curve.

8.4.2 RT windows for each analyte must be determined whenever a new initial calibration curve is generated or when a new GC column is installed. RT windows must be established for all analytes for both analytical columns.

8.4.3 For the calibration verification standard, the midpoint of the RT window shall be the average absolute $\mathrm{RT}$ for each analyte from the most recent initial calibration.

8.4.4 The midpoint of the daily or 12-hour calibration verification standard RT window shall be the absolute RT window for each analyte for that calibration period.

\subsection{Sample Analysis.}

8.5.1 The minimum sample volume required to meet the PROLs specified in Table 1 is $1.5 \mu \mathrm{L}$ using splitless injection.

8.5.2 Samples are analyzed in a set referred to as an analytical batch. The analytical batch begins with a continuing calibration standard and laboratory blank. The batch ends when the set of samples, containing all of the samples and sample preparation quality control samples, have been injected and when qualitative and/or quantitative quality control criteria are met as stated in Section 10.

8.5.3 If initial analysis of a sample detects any analytes with concentrations that exceed the initial calibration range, the sample must be diluted and reanalyzed.

8.5.4 When a sample has been analyzed that saturates the detector or shows concentrations of analytes greater than ten times the upper limit of the initial calibration, the analysis must be followed by a blank analysis. If the blank analysis is not free of interferences (target analytes $<3$ time the MDL) the system must be decontaminated. Sample analysis may not resume until a blank can be analyzed that is free of contamination.

\subsection{Calculations}

9.1 Qualitative Analysis. For positive analyte identification, the sample peak must fall within the RT window set by the most recent continuing calibration for each analytical column.

9.2 Quantitative Analysis. Once an analyte has been qualitatively identified, the quantitation of that analyte will be based on the peak area response (peak height may also be used). 
Quantitation of a given analyte shall be performed on one of the two analytical columns. The column used must be interferant free in the RT window corresponding to the analyte. The recommended column for quantitation is the first column listed in Section 5.2.4. Example chromatograms are shown in Figures 1 and 2. Calculate the concentration in $\mathrm{mg} / \mathrm{kg}$ of each identified analyte in the sample as follows:

$$
\text { Concentration }=\frac{\left(A_{x}\right)(D F)\left(V_{t}\right)}{(R F)\left(W_{x}\right)\left(V_{i}\right)}
$$

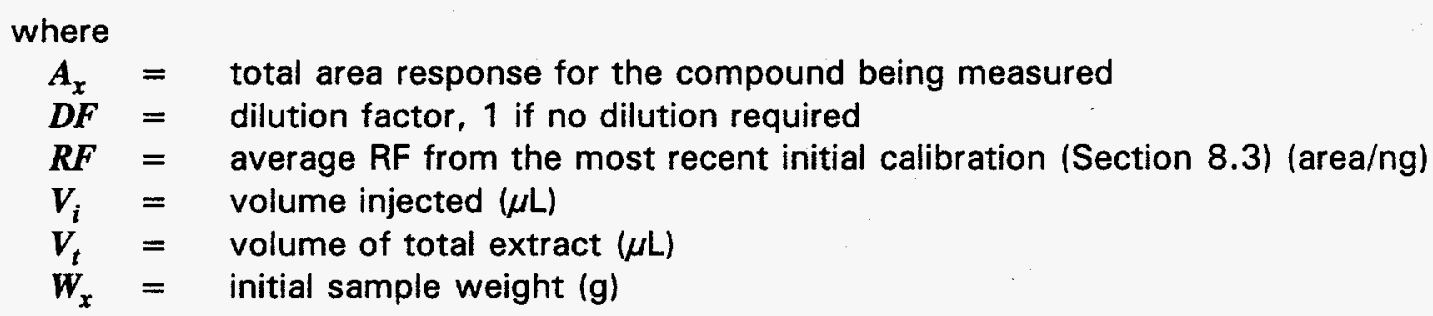

\subsection{Quality Control}

10.1 Each laboratory that uses this procedure is required to operate a formal quality control program. The laboratory must retain records to document the quality of the data generated. Each laboratory must have SOPs documenting and describing activities involved in using this procedure. Specific quality control practices will include, but are not limited to, the analysis of quality control samples. The types of quality control samples, their associated frequency of analysis, acceptance criteria, and corrective action required if samples do not meet the acceptance criteria, is summarized in Table 4. Laboratory SOPs shall address requirements for preparing matrix spikes, matrix spike duplicates, laboratory blanks, and laboratory control samples.

10.2 All laboratories using this procedure must demonstrate acceptable performance prior to the analysis of actual samples. Demonstration of acceptable performance will be achieved by analyzing method performance samples (Table 4). These samples will contain all of the analytes listed in Table 1 at concentrations appropriate (2-5 times the PROLs) to determine the quality assurance objectives specified in Table 1 . The analysis of seven samples must meet the criteria specified for precision, accuracy, and method detection limit (MDL) in Table 1. Demonstration of acceptable method and analyst performance must be repeated (by analyzing four samples) at a minimum of every six months. Method performance should be conducted over a period of several days to account for long-term variability. Corrective action must be implemented when acceptable method performance is not met. Precision will be determined as follows:

- For duplicate measurements, the precision expressed as the relative percent difference (RPD) is calculated as

$$
R P D=\frac{C_{1}-C_{2}}{\frac{\left(C_{1}+C_{2}\right)}{2}} * 100
$$

where

$$
\begin{array}{lll}
C_{I} \text { and } C_{2} & = & \text { two values obtained by analyzing the duplicate samples } \\
C_{1} & = & \text { larger of the two observed values }
\end{array}
$$




\begin{tabular}{|c|c|c|c|}
\hline OC Sample & Minimum Frequency & $\begin{array}{l}\text { Acceptance } \\
\text { Criteria }\end{array}$ & $\begin{array}{l}\text { Corrective } \\
\text { Action }^{\mathrm{a}}\end{array}$ \\
\hline $\begin{array}{l}\text { Method performance } \\
\text { samples }\end{array}$ & $\begin{array}{l}\text { Seven (7) samples initially } \\
\text { and four (4) semiannually }\end{array}$ & Meet Table 1 QAOs & Repeat until acceptable \\
\hline Laboratory duplicates $^{\mathbf{b}}$ & $\begin{array}{l}\text { One (1) per analytical } \\
\text { batch }\end{array}$ & Meet Table 1 RPDs & $\begin{array}{l}\text { Nonconformance if RPDs } \\
>\text { Table } 1 \text { values }\end{array}$ \\
\hline Laboratory blanks & $\begin{array}{l}\text { One (1) per analytical } \\
\text { batch }\end{array}$ & $\begin{array}{l}\text { Analyte concentrations } \\
<3 \times \text { MDLs }\end{array}$ & $\begin{array}{l}\text { Nonconformance if } \\
\text { analyte concentrations } \\
>3 \times \text { MDLs }\end{array}$ \\
\hline Matrix spikes & $\begin{array}{l}\text { One (1) per analytical } \\
\text { batch }\end{array}$ & Meet Table $1 \%$ Rs & $\begin{array}{l}\text { Nonconformance if \%Rs } \\
\text { are outside the range } \\
\text { specified in Table } 1\end{array}$ \\
\hline Matrix spike duplicates & $\begin{array}{l}\text { One (1) per analytical } \\
\text { batch }\end{array}$ & $\begin{array}{l}\text { Meet Table } 1 \text { RPDs and } \\
\% \text { Rs }\end{array}$ & $\begin{array}{l}\text { Nonconformance if RPDs } \\
\text { and } \% \text { Rs }>\text { Table } 1 \\
\text { values }\end{array}$ \\
\hline $\begin{array}{l}\text { Laboratory control } \\
\text { samples }\end{array}$ & $\begin{array}{l}\text { One (1) per analytical } \\
\text { batch }\end{array}$ & $80-120 \% R$ & $\begin{array}{l}\text { Nonconformance if } \% R \\
<80 \text { or }>120\end{array}$ \\
\hline Surrogate compounds & Each analytical sample & $\begin{array}{l}\text { Average } \% R \text { from } \\
\text { minimum of } 30 \text { samples } \\
\text { for a given matrix } \pm 3 \\
\text { standard deviations }\end{array}$ & $\begin{array}{l}\text { Nonconformance if } \% R \\
<\text { (average } \% R-3 \\
\text { standard deviation) or } \\
>\text { (average } \% R+3 \\
\text { standard deviation) }\end{array}$ \\
\hline Blind audit samples & $\begin{array}{l}\text { Samples and frequency } \\
\text { controlled by the Solid } \\
\text { PDP Plan }\end{array}$ & $\begin{array}{l}\text { Specified in the Solid } \\
\text { PDP Plan }\end{array}$ & $\begin{array}{l}\text { Specified in the Solid } \\
\text { PDP Plan }\end{array}$ \\
\hline
\end{tabular}

${ }^{a}$ Corrective Action when $\mathrm{QC}$ samples do not meet the acceptance criteria; Nonconformance procedures are outlined in Section 2.1.2.1 of the QAPP.

${ }^{b}$ May be satisfied using matrix spike duplicate; acceptance criteria applies only to concentrations greater than the PROLs listed in Table 1.

$\mathrm{MDL}=$ Method detection limit

$\mathrm{QAO}=$ Quality assurance objective

$\mathrm{PDP}=$ Performance Demonstration Program

$\% R=$ Percent recovery

$\mathrm{RPD}=$ Relative percent difference 
- For three or more replicate measurements, the precision expressed as \%RSD is calculated as

$$
\% R S D=\frac{s}{\bar{y}} * 100
$$

where

$$
\begin{array}{ll}
s & =\quad \text { standard deviation } \\
y & =\text { mean of replicate analyses }
\end{array}
$$

- The standard deviation $(s)$ is defined as

$$
s=\sqrt{\sum_{i=1}^{n} \frac{\left(y_{i}-\bar{y}\right)^{2}}{n-1}}
$$

where

$y_{i}=$ measured value of the $i^{\text {th }}$ replicate sample analysis measurement

$n=$ number of replicate analyses

Accuracy will be determined as the percent recovery $(\% \mathrm{R})$ as follows:

$$
\% R=\frac{C_{m}}{C_{s r m}} * 100
$$

where

$$
\begin{aligned}
& C_{m}=\text { measured concentration } \\
& C_{s r m}=\text { true concentration }
\end{aligned}
$$

MDL will be determined as follows:

$$
M D L=t_{(n-1,1-\alpha=0.99)} * S
$$

where

$$
\begin{array}{lll}
s & = & \text { standard deviation } \\
t_{(n-1,1-\alpha=0.99)} & & t \text {-distribution value appropriate to a 99-percent confidence level } \\
& \text { and a standard deviation estimate with } n-1 \text { degrees of freedom }
\end{array}
$$

10.3 The laboratory must analyze blanks at the frequency specified in Table 4. The same procedures used to prepare and analyze field samples will be used to prepare and analyze the laboratory blanks. Laboratory blank results will be acceptable if the concentration of target analytes is less 3 times the MDL specified in Table 1 . Corrective action must be implemented if blanks exceed this level.

10.4 The laboratory must analyze matrix spikes at the frequency specified in Table 4 . Matrix spike results shall be considered acceptable if the $\% R$ is within the range specified in Table 1. Matrix spikes which do not meet these criteria should be flagged. 
$\% \mathrm{R}$ will be determined as follows:

$$
\% R=\frac{S-U}{C_{s a}} * 100
$$

where

$S=$ measured concentration in the spiked aliquot

$U=$ measured concentration in the unspiked aliquot

$C_{s a}=$ actual concentration of the spike added

10.5 The laboratory shall analyze laboratory duplicates or matrix spike duplicates at the frequency specified in Table 4. Duplicate results shall be considered acceptable if the RPD is within the range specified in Table 1. RPD is determined as in equation (6). Duplicates which do not meet these criteria should be flagged.

10.6 The laboratory must analyze laboratory control samples at the frequency specified in Table 4. Laboratory control samples must contain all of the analytes listed in Table 1 and shall be prepared, in water, at a concentration of $20 \mu \mathrm{g} / \mathrm{L}$ per laboratory control analyte from standards independent of those used for instrument calibration. The laboratory control samples must be quantitated within the calibration range of the instrument. The laboratory control sample analysis is designed to ensure that laboratory performance is in control for each analytical batch of samples processed. If the laboratory control sample $\% R$ is outside the range specified in Table 4, then new laboratory control samples must be prepared. \%R is determined as in Equation (9).

10.7 The laboratory will be required to analyze blind audit samples. These audit samples are part of the Performance Demonstration Program. Details of this program are in the Performance Demonstration Program Plan for the Analysis of Solidified Waste for the Transuranic Waste Characterization Program (Solid PDP Plan)(DOE 1995b).

\subsection{Procedure Performance}

11.1 The MDL is defined as the minimum concentration of a substance that can be measured and reported with 99-percent confidence that the value is above zero. MDL values for three alcohols were determined in a single laboratory and are reported in Table 5 . The actual MDLs will vary depending upon the equipment used, and the consistency of an analyst or sample injection device to reproduce sample injection volumes.

11.2 Average spike recoveries for three alcohols in aqueous extracts of inorganic sludges, organic setups (oils and solvents stabilized in calcium silicate), and alcohol solutions solidify in Portland Cement were determined in a single laboratory. These results are given in Table 6. The extracted alcohol concentrations were approximately $20 \mu \mathrm{L} / \mathrm{L}$. Recoveries for matrix spikes are typically above 90 percent.

\subsection{References}

DOE. 1994. DOE Methods for Evaluating Environmental and Waste Management Samples. DOE/EM-0089T, Revision 1, Richland, Washington, Pacific Northwest Laboratory, U.S. Department of Energy.

DOE. 1995a. Performance Demonstration Program Plan for the Analysis of Solidified Waste for the Transuranic Waste Characterization Program. CA0-95-1077, Current Revision, Carlsbad, New Mexico, Carlsbad Area Office, U.S. Department of Energy. 


\section{TABLE 5}

\section{Method Performance for Selected Alcohols}

MDL (ppm)

\begin{tabular}{lccccc} 
Compound & $\begin{array}{c}\text { Precision } \\
\text { (\%RSD/RPD) }\end{array}$ & $\begin{array}{c}\text { Accuracy } \\
(\% \mathrm{R})\end{array}$ & Col 1 & Col 2 & $\begin{array}{c}\text { Completeness } \\
(\%)\end{array}$ \\
\hline Methanol & $\pm 25 \%$ & $70-130 \%$ & $2.0^{\mathrm{a}}$ & $0.9^{\mathrm{a}}$ & $90 \%$ \\
Isobutanol & $\pm 25 \%$ & $70-130 \%$ & $0.4^{\mathrm{a}}$ & $0.6^{\mathrm{a}}$ & $90 \%$ \\
n-Butyl alcohol & $\pm 25 \%$ & $70-130 \%$ & $0.6^{\mathrm{a}}$ & $0.9^{\mathrm{a}}$ & $90 \%$ \\
\hline
\end{tabular}

abed on 99-percent confidence level, $n=5$

$\%$ RSD $=$ Percent relative standard deviation

RPD $\quad=$ Relative percent difference

$\% \mathrm{R} \quad=$ Percent recovery

MDL $\quad=\quad$ Method detection limit 
TABLE 6

\section{Average Spike Recoveries for Selected Alcohols}

Compound Organic Setup $\quad$ Inorganic Sludge ${ }^{b} \quad \begin{gathered}\text { Cemented Aqueous } \\ \text { Solution }^{c}\end{gathered}$

Rt-5 Col. Stablewax Rt-5 Col. Stablewax Rt.-5 Col. Stablewax

$\begin{array}{lllllll}\text { Methanol } & 88.3 \pm 5.9 & 84.6 \pm 2.8 & 93.0 \pm 1.1 & 96.1 \pm 2.6 & 83.9 \pm 9.5 & 89.6 \pm 9.8 \\ \text { 2-Methyl-1 Propanol } & 90.6 \pm 1.5 & 88.8 \pm 3.4 & 89.9 \pm 0.8 & 92.9 \pm 0.2 & 84.6 \pm 13.2 & 94.5 \pm 15.5 \\ \text { n-Butyl alcohol } & 93.3 \div 1.4 & 98.1 \pm 3.3 & 89.1 \pm 1.5 & 91.7 \pm 0.4 & 88.2 \pm 13.5 & 96.0 \pm 14.7\end{array}$

Aliphatic hydrocarbon oil w/ 1,1,1-trichloroethane, carbon tetrachloride and 1,2,2-trichlorotrifluoroethane absorbed in calcium silicate.

'Inorganic sludge consisting of hydroxide and phosphate precipitated metals including iron, nickel, calcium, and magnesium. Sodium, potassium, fluoride, chloride, nitrate, and chromate salts are also entrained in the sludge.

${ }^{\mathrm{C} A q u e o u s ~ s o l u t i o n ~ c o n t a i n i n g ~ a l c o h o l s ~ s o l i d i f i e d ~ w i t h ~ P o r t l a n d ~ c e m e n t . ~}$

Sample extractions were performed in triplicate. The tolerances represent one standard deviation of the mean.

Rt-5 is a 95-percent dimethyl 5-percent diphenyl polysiloxane $0.25 \mathrm{~mm} \times 30 \mathrm{~m}$ capillary column. Stablewax is a crossbonded carbowax $0.25 \mathrm{~mm} \times 30 \mathrm{~m}$ capillary column. 
DOE. 1995b. Transuranic Waste Characterization Quality Assurance Program Plan. CAO-941010, Current Revision, Carlsbad, New Mexico, Carlsbad Area Office, U.S. Department of Energy.

EPA. 1995. Test Methods for Evaluating Solid Waste, Physical/Chemical Methods. SW-846, Third Edition, Final Update I, and Final Update II, Washington D.C., Office of Solid Waste and Emergency Response, U.S. Environmental Protection Agency. 


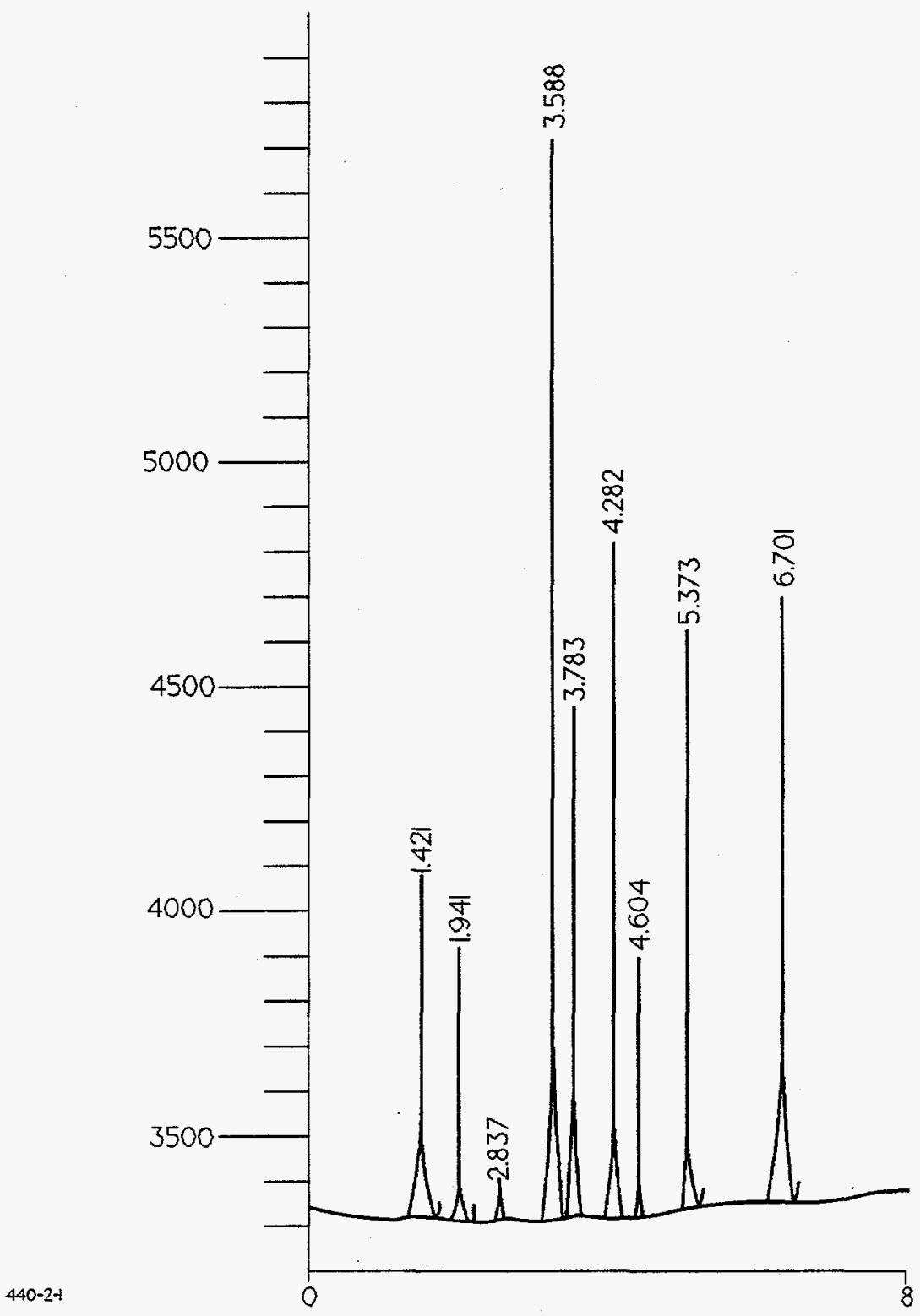

Aqueous extraction of alcohols spiked onto an organic sludge containing aliphatic hydrocarbons, 1,1,1trichloroethane, 1,2,2-trichlorotrifluoroethane, and carbon tetrachloride solidified with calcium silicate to form a paste like material. Methanol at $1.421 \mathrm{~min} ., 2$-methyl-1-propanol at $4.282 \mathrm{~min}$., butanol at $5.373 \mathrm{~min}$.

\section{FIGURE 1}

Alcohols Determined in an Organic Sludge Using a 95-Percent Dimethyl 5-Percent Diphenyl Polysiloxane Column 


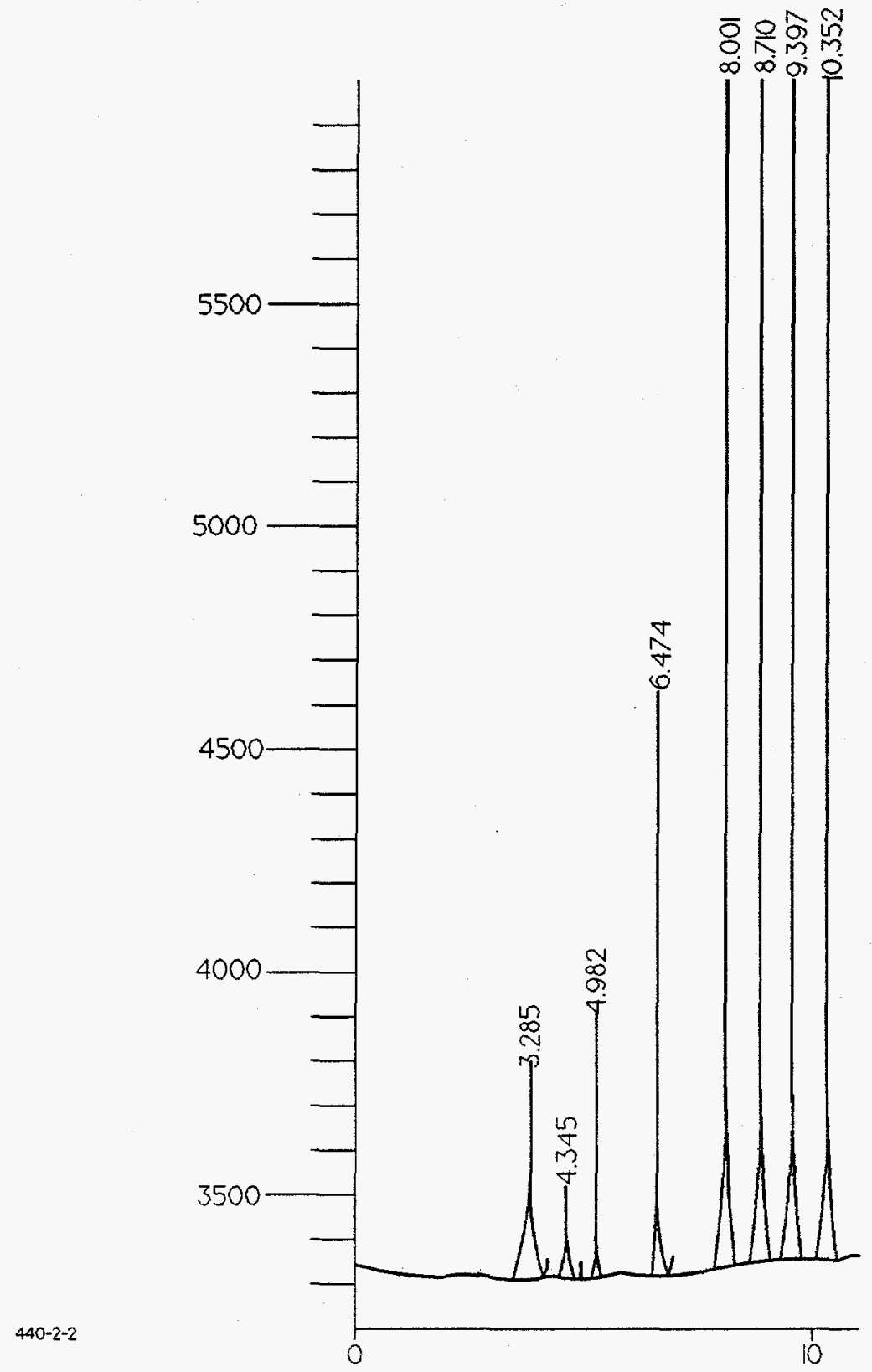

Aqueous extraction of alcohols spiked onto an organic sludge containing aliphatic hydrocarbons, 1,1,1trichloroethane, 1,2,2-trichlorotrifluoroethane, and carbon tetrachloride solidified with calcium silicate to form a paste like material. Methanol at $3.285 \mathrm{~min}$., 2-methyl-1-propanol at $9.397 \mathrm{~min}$., butanol at $10.352 \mathrm{~min}$.

FIGURE 2

Alcohols Determined in an Organic Sludge Using a Crossbonded Carbowax Column 


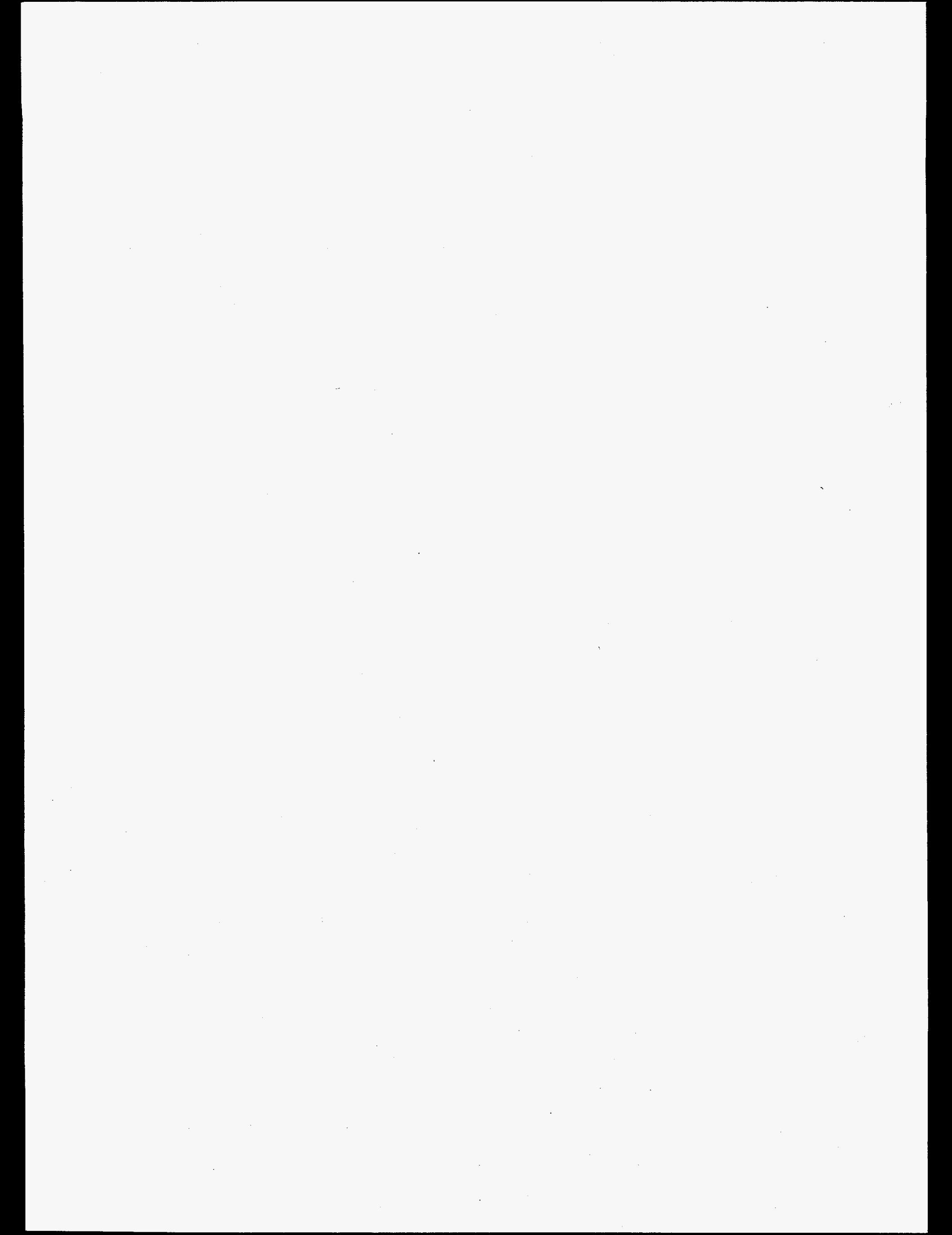


PROCEDURE 440.3

\section{GAS CHROMATOGRAPHY/ELECTRON CAPTURE DETECTION DETERMINATION OF PCBS IN ORGANIC SLUDGE}

\subsection{Scope and Application}

1.1 This procedure is for the determination of polychlorinated biphenyls (PCBs) in samples of organic sludge that have been collected from containers of transuranic (TRU) waste using rotational or non-rotational coring equipment. Sampling procedures for organic sludge are described in Procedure 120.1 of this Methods Manual. The procedure employs Gas Chromatography/Electron Capture Detection (GC/ECD) for the quantitation of PCBs. Table 1 lists the individual Aroclors that must be quantitated and the quality assurance objectives for this procedure. This procedure must be implemented with a site-specific standard operating procedure (SOP).

1.2 This procedure was developed using samples of simulated Rocky Flats Type IV sludge. Type IV sludge consists of organic solvents, degreasers, cutting oils, and TRU waste mixed with MicroCel $E^{\top}$ (calcium silicate) and Oil Dri to form a grease or paste-like material. The simulated Type IV sludge was a non-radioactive simulated waste material of composition similar to that expected in field samples. The simulated sludge was spiked with Aroclors 1254 and 1260, the most commonly expected PCB contaminants in Rocky Flats Type IV sludge.

1.3 The procedure was also tested with simulated sludge spiked with plutonium in solid and liquid forms. Reduction of radioactivity in the final extract versus the spiked sample ranged from a factor of $10^{5}$ to $10^{7}$. If radioactivity is not carried over into the purified extracts, samples can be analyzed in laboratories suitable for nonradioactive work. Although this procedure was developed using simulated sludge spiked only with Aroclors 1254 and 1260, it should be suitable for the determination of all the Aroclors listed in Table 1.

1.4 This procedure is adapted from three methods found in Test Methods for Evaluating Solid Waste, Physical/Chemical Methods, SW-846, Third Edition, Final Update 1, and Final Update II (SW-846) (EPA 1995). The three methods are: Method 3550, "Ultrasonic Extraction," Method 3620, "Florisil Column Cleanup," and Method 8081, "Organochlorine Pesticides, Halowaxes and PCBs as Aroclors by Gas Chromatography: Capillary Column Technique." The procedure has been optimized for the determination of PCBs in radioactive organic sludges. The analyst is referred to Method 8081, as well as SW-846 Method $8000 \mathrm{~A}$, "Gas Chromatography," for certain detailed requirements of this procedure.

1.5 For the purposes of the TRU Waste Characterization Program, samples are to be analyzed in analytical batches. An analytical batch is defined as a suite of samples that is processed as a unit, using the same analytical method, within a specific time period. An analytical batch can be up to 20 samples (excluding laboratory QC samples), all of which must be received by the laboratory within 14 days of validated time of sample receipt (VTSR) of the first sample in the batch.

\subsection{Summary of Procedure}

Simulated sludge samples are weighed and transferred to small vials. Surrogate standard solutions are added to all samples and blanks. The samples are extracted with $10 \mathrm{~mL}$ of hexane using vortex mixing. Extracts undergo a Florisil cleanup step. The Aroclor content is measured and quantitated using GC/ECD. 
TABLE 1

Total PCB Target Analyte List and Quality Assurance Objectives

\begin{tabular}{|c|c|c|c|c|c|c|}
\hline Compound & $\begin{array}{c}\text { CAS } \\
\text { Number }\end{array}$ & $\begin{array}{c}\text { Precision } \\
\text { (\%RSD or RPD) }\end{array}$ & $\begin{array}{c}\text { Accuracy } \\
(\% R)\end{array}$ & $\underset{(\mathrm{mg} / \mathrm{kg})}{\mathrm{MDL}}$ & $\begin{array}{l}\text { PRQL } \\
\text { (mg/kg) }\end{array}$ & $\begin{array}{c}\text { Completeness } \\
\text { (percent) }\end{array}$ \\
\hline \multicolumn{7}{|c|}{$\begin{array}{l}\text { Polychlorinated Biphenyls } \\
\text { (PCBs) }\end{array}$} \\
\hline Aroclor 1016 & $12674-11-2$ & $\leq 33$ & $50-114$ & 5 & 40 & 90 \\
\hline Arocior 1221 & $11104-28-2$ & $\leq 110$ & $15-178$ & 5 & 40 & 90 \\
\hline Aroclor 1232 & $11141-16-5$ & $\leq 128$ & $10-215$ & 5 & 40 & 90 \\
\hline Aroclor 1242 & $53469-21-9$ & $\leq 49$ & $39-150$ & 5 & 40 & 90 \\
\hline Arocior 1248 & $12672-29-6$ & $\leq 55$ & $38-158$ & 5 & 40 & 90 \\
\hline Aroclor 1254 & $11097-69-1$ & $\leq 62$ & $29-131$ & 5 & 40 & 90 \\
\hline Aroclor 1260 & $11096-82-5$ & $\leq 56$ & $8-127$ & 5 & 40 & 90 \\
\hline
\end{tabular}

$\%$ RSD $=\quad$ Percent relative standard deviation

$\mathrm{RPD}=$ Relative percent difference

$\% R=$ Percent recovery

$\mathrm{MDL}=$ Method detection limit (maximum permissible value)

PRQL = Program required quantitation limit; calculated from the toxicity characteristic level for nitrobenzene assuming a $100 \mathrm{~g}$ sample, $2 \mathrm{~L}$ of extraction fluid, and 100 percent analyte extraction

${ }^{a}$ Criteria apply to PROL concentrations 


\subsection{Interferences}

3.1 Organic constituents other than PCBs that are present in the extract may not be totally removed by Florisil cleanup. Whether these compounds interfere with the PCB determination depends upon their retention times and the relative response of the electron capture detector to these contaminants.

3.2 A major interference in the Aroclor 1260 region of the chromatogram is spindle oil. Gearbox and hydraulic oils elute earlier and would interfere most with less-chlorinated Aroclors. These interferences are adequately removed by the Florisil cleanup, as described in Section 8.2.

3.3 Analytical interferences may be caused by contaminants in solvents, reagents, glassware, and other sample processing hardware. All of these materials must be routinely demonstrated to be free of contaminants under the conditions of the analysis by running laboratory reagent blanks.

3.4 Refer to Section 3.0 of SW-846 Method 8081 for more information on possible interferences.

\subsection{Safety}

This procedure may involve the use of hazardous materials, operations, and equipment. It is the responsibility of whoever uses this procedure to consult appropriate site personnel concerning health and safety issues and establish appropriate health and safety practices. Consideration should be given to safety concerns regarding chemical and radiation exposure. Training regarding proper storage, usage, and disposal of chemicals is recommended.

\subsection{Apparatus and Materials}

5.1 Gas chromatograph equipped with electron capture detection, automatic sample injection system, and chromatograph data acquisition system (as listed in SW-846 Method 8081 or equivalent). The gas chromatograph should be equipped with a DB-5 (or equivalent) column: $30-\mathrm{m}$ length $\times 0.32-\mathrm{mm}$ ID, $0.25-\mu \mathrm{m}$ film thickness.

5.2 Super Mixer, Lab-Line Instruments, inc., Cat. No. 1290, or equivalent.

5.3 Mettler PT320 Top Loader Balance, or equivalent.

5.4 Glass extraction vials $(20 \mathrm{~mL})$ with Teflon-lined screwcaps.

5.5 Florisil Sep-Pak Light Cartridges, WATERS, Millipore Corp., Cat. No. 23543, or equivalent.

5.6 Glass syringe capable of delivering $50.0 \mu \mathrm{L}$ liquid.

5.7 Glass or polypropylene syringe $(1-\mathrm{mL})$ with Luer tip for use with Florisil cartridges. Use polypropylene syringes as disposables, but check batches for contaminants before using.

\subsection{Reagents}

6.1 Chemicals. Refer to Section 5.1 of SW-846 Method 8081 for a description of reagent grade chemicals to use during extraction and cleanup. 


\subsection{Stock Standard Solutions.}

6.2.1 Commercially prepared stock standards can be used if they are certified by the manufacturer or by an independent source. The following stock standards supplied by Supelco Inc. were used in the development of this procedure:

1. Pesticide Surrogate Spike Mix containing decachlorobiphenyl (DCBP) and 2, $4,5,6$,-tetrachloro-m-xylene (TCMX), at a concentration of $200 \mu \mathrm{g} / \mathrm{mL}$ in acetone.

2. Aroclor 1254 , at a concentration of $1 \mu \mathrm{g} / \mu \mathrm{L}$ in isooctane.

3. Arocior 1260 , at a concentration of $1 \mu \mathrm{g} / \mu \mathrm{L}$ in isooctane.

Additional stock standards containing all the Aroclors listed in Table 1 must also be commercially purchased or laboratory prepared.

6.2.2 Transfer the stock standard solutions into bottles with Teflon-lined screwcaps. Store at $4^{\circ} \mathrm{C}$ and protect from light. Check the stock standards frequently for signs of degradation or evaporation, especially just before preparing calibration standards.

6.2.3 Replace the stock standard solutions after six months, or sooner if comparison with check standards indicates a problem.

6.3 Calibration Standards. Prepare calibration standards for all Aroclors both individually and as a mixture. The calibration levels are $0.05,0.25,0.50,1.00$, and $5.00 \mathrm{ppm}$. Since samples are diluted 100 times during the Florisil cleanup step, these levels accommodate a sample concentration range of $5-500 \mathrm{ppm}$. Replace the calibration solutions after two months, or sooner if comparison with check standards indicates a problem.

6.4 Surrogate Standards. The analyst should monitor the performance of the extraction, cleanup, and analytical system and of the effectiveness of the method for each sample matrix by spiking each sample, standard, and blank with surrogates. The primary surrogate is DCBP and should be used whenever possible. If recovery is low or if compounds interfere with DCBP, then try TCMX, the secondary surrogate.

If both surrogates are out of limits for a sample, follow Section 8.3 of SW-846 Method 8081 . Add $50 \mu \mathrm{L}$ of the commercial Pesticides Surrogate stock standard solution to sludge samples and blanks. The resultant concentration of surrogates in the sludge should be $5 \mathrm{ppm}$, and surrogate concentration in the final extract after cleanup should be $0.05 \mathrm{ppm}$.

\subsection{Sample Collection, Preservation, and Handling}

7.1 Samples will be collected using the sampling procedures described in Procedure 120.1 of this Methods Manual.

7.2 All sample handling and chain-of-custody procedures described in Section 6.0 of the Transuranic Waste Characterization Quality Assurance Program Plan (QAPP) (DOE 1995b) must be followed. 


\subsection{Procedure}

8.1 Sample Extraction. Weigh approximately $2.0 \mathrm{~g}$ (measured to $0.01 \mathrm{~g}$ ) of a sludge sample into a $20-\mathrm{mL}$ extraction vial in a radiochemical hood or a glove box. Add $50 \mu \mathrm{L}$ of surrogate stock standard solution to each sample and blank so the final extract concentration of each surrogate is $0.05 \mu \mathrm{g} / \mathrm{mL}$. Then add $10 \mathrm{~mL}$ of hexane to each sample and vortex mix for at least 30 seconds.

8.2 Sample Cleanup. After vortexing, allow the sample to settle. Remove a $50-\mu \mathrm{L}$ portion of the supernatant and add it to a $1-\mathrm{mL}$ glass or polypropylene syringe connected to a Florisil Cartridge pre-wet with hexane. Add approximately $0.4 \mathrm{~mL}$ of hexane to the syringe. Apply pressure or vacuum to move the solution through the cartridge without introducing air into it. Then add approximately $0.5 \mathrm{~mL}$ of hexane to the syringe as a rinse for a final volume of $1.0 \mathrm{~mL}$. Collect the extract and rinses in an autosampler vial calibrated to $1.0 \mathrm{~mL}$.

\subsection{Recommended GC/ECD System Operating Conditions.}

Column Type:

Initial column temperature:

Initial column holding time:

DB-5, $30 \mathrm{~m} \times 0.32 \mathrm{~mm}$ I.D.

$100^{\circ} \mathrm{C}$

Column temperature program:

Final column temperature:

1 minute

$25^{\circ} \mathrm{C} / \mathrm{min}$ to $200^{\circ} \mathrm{C}$, then $2{ }^{\circ} \mathrm{C} / \mathrm{min}$ to $240^{\circ} \mathrm{C}$ and hold for 6 minutes, then $5^{\circ} \mathrm{C} / \mathrm{min}$ to $260^{\circ} \mathrm{C}$

Final column holding time:

$260^{\circ} \mathrm{C}$

2 minutes

Injector temperature:

$270^{\circ} \mathrm{C}$

$280^{\circ} \mathrm{C}$

Detector temperature:

Carrier gas:

Helium at $6 \mathrm{~mL} / \mathrm{min}$

Detector make up gas:

Nitrogen at $50 \mathrm{~mL} / \mathrm{min}$

8.4 Calibration. Refer to Section 7.4.2 of SW-846 Method 8000A for instructions on calibrating the chromatographic system using the external standard technique. Table 2 provides a summary of calibration requirements.

8.5 Sample Analysis. Refer to Section 7.6 of SW-846 Method 8000A for general instructions on analysis sequence, appropriate dilutions, establishment of daily retention-time windows, and identification criteria. Refer to Section 7.5 of SW-846 Method 8081 for more detailed instructions. An example of a GC/ECD chromatogram generated by this procedure is presented in Figure 1.

\subsection{Calculations}

9.1 If a single Aroclor is present in the sample, then quantitate by summing the areas of the five major peaks due to the Aroclor.

9.2 If a mixture of Aroclors is present, then quantitate by summing the areas of at least three peaks that are exclusive to each Aroclor. Additionally, prepare a standard mixture with approximately the same concentration of Aroclors as found in the sample.

\subsection{Quality Control}

10.1 All of the quality control requirements specified in Section 8.4 and 8.5 of SW-846 Method 8081 are mandatory. In instances where the quality control specifications in this procedure differ from those in SW-846 Method 8081, this procedure takes precedence. 
TABLE 2.

\section{Summary of Calibration Requirements for PCB Analysis}

$\begin{array}{llll}\text { Technique } & \text { Procedure } & \text { Frequency of Procedure } & \text { Acceptance Criteria } \\ \text { GC/ECD } & \begin{array}{l}\text { 3-pt initial calibration } \\ (3 \text { standards })\end{array} & \text { Initially and as needed } & \text { Response factor \%RS } 520 \\ & \text { Continuing calibration } & \begin{array}{l}\text { Every } 12 \text { hours as } \\ \text { needed }\end{array} & \begin{array}{l}\% D \leq 15 \text { for all analytes compared to } \\ \text { initial calibration }\end{array}\end{array}$

$\% \mathrm{D} \quad=\quad$ Percent difference

\%RSD $\quad=\quad$ Percent relative standard deviation 
10.2 Each laboratory that uses this procedure is required to operate a formal quality control program. The laboratory must retain records to document the quality of the data generated. Each laboratory must have SOPs documenting and describing activities involved in using this procedure. Specific quality control practices will include, but are not limited to, the analysis of quality control samples. The types of quality control samples, their associated frequency of analysis, acceptance criteria, and corrective action required if samples do not meet the acceptance criteria, is summarized in Table 3. Laboratory SOPs shall address requirements for preparing matrix spikes, matrix spike duplicates, laboratory blanks, and laboratory control samples.

10.3 All laboratories using this procedure must demonstrate acceptable performance prior to the analysis of actual samples. Demonstration of acceptable performance will be achieved by analyzing method performance samples (Table 3). These samples will contain all of the analytes listed in Table 1 at concentrations appropriate (2-5 times the PROLs) to determine the quality assurance objectives specified in Table 1 . The analysis of seven samples must meet the criteria specified for precision, accuracy, and method detection limit (MDL) in Table 1. Demonstration of acceptable method and analyst performance must be repeated (by analyzing four samples) at a minimum of every six months. Method performance should be conducted over a period of several days to account for long-term variability. Corrective action must be implemented when acceptable method performance is not met. Precision will be determined as follows:

- For duplicate measurements, the precision expressed as the relative percent difference (RPD) is calculated as

$$
R P D=\frac{C_{1}-C_{2}}{\frac{\left(C_{1}+C_{2}\right)}{2}} * 100
$$

where

$$
\begin{array}{lll}
C_{1} \text { and } C_{2} & =\text { two values obtained by analyzing the duplicate samples } \\
C_{1} & = & \text { larger of the two observed values }
\end{array}
$$

- For three or more replicate measurements, the precision expressed as the percent relative standard deviation (\%RSD) is calculated as

$$
\% R S D=\frac{s}{\bar{y}} * 100
$$

where

$$
\begin{array}{lll}
s & = & \text { standard deviation } \\
y & = & \text { mean of replicate analyses }
\end{array}
$$

- The standard deviation $(s)$ is defined as

$$
s=\sqrt{\sum_{i=1}^{n} \frac{\left(y_{i}-\bar{y}\right)^{2}}{n-1}}
$$

where

$$
\begin{aligned}
& y_{i}=\text { measured value of the } i^{\text {th }} \text { replicate sample analysis measurement } \\
& n=\text { number of replicate analyses }
\end{aligned}
$$


TABLE 3

\section{Summary of Laboratory Quality Control Samples and Frequencies for Total PCB Analysis}

\begin{tabular}{|c|c|c|c|}
\hline OC Sample & Minimum Frequency & $\begin{array}{l}\text { Acceptance } \\
\text { Criteria }\end{array}$ & $\begin{array}{l}\text { Corrective } \\
\text { Action }^{\mathrm{a}}\end{array}$ \\
\hline $\begin{array}{l}\text { Method performance } \\
\text { samples }\end{array}$ & $\begin{array}{l}\text { Seven (7) samples } \\
\text { initially and four (4) } \\
\text { semiannually }\end{array}$ & Meet Table 1 QAOs & Repeat until acceptable \\
\hline Laboratory duplicates $^{b}$ & $\begin{array}{l}\text { One (1) per analytical } \\
\text { batch }\end{array}$ & Meet Table 1 RPDs & $\begin{array}{l}\text { Nonconformance if RPDs } \\
>\text { Table } 1 \text { values }\end{array}$ \\
\hline Laboratory blanks & $\begin{array}{l}\text { One (1) per analytical } \\
\text { batch }\end{array}$ & $\begin{array}{l}\text { Analyte concentrations } \\
<3 \times \text { MDLs }\end{array}$ & $\begin{array}{l}\text { Nonconformance if } \\
\text { analyte concentrations > } \\
3 \times \text { MDLs }\end{array}$ \\
\hline Matrix spikes & $\begin{array}{l}\text { One (1) per analytical } \\
\text { batch }\end{array}$ & Meet Table $1 \%$ Rs & $\begin{array}{l}\text { Nonconformance if \%Rs } \\
\text { are outside the range } \\
\text { specified in Table } 1\end{array}$ \\
\hline $\begin{array}{l}\text { Matrix spike } \\
\text { duplicates }\end{array}$ & $\begin{array}{l}\text { One }\{1\} \text { per analytical } \\
\text { batch }\end{array}$ & $\begin{array}{l}\text { Meet Table } 1 \text { RPDs } \\
\text { and \%Rs }\end{array}$ & $\begin{array}{l}\text { Nonconformance if RPDs } \\
\text { and } \% \text { Rs }>\text { Table } 1 \\
\text { values }\end{array}$ \\
\hline $\begin{array}{l}\text { Laboratory control } \\
\text { samples }\end{array}$ & $\begin{array}{l}\text { One (1) per analytical } \\
\text { batch }\end{array}$ & $80-120 \% R s$ & $\begin{array}{l}\text { Nonconformance if } \% R \\
<80 \text { or }>120\end{array}$ \\
\hline Surrogate compounds & Each analytical sample & $\begin{array}{l}\text { Average \%R from minimum } \\
\text { of } 30 \text { samples from a given } \\
\text { matrix } \pm 3 \text { standard } \\
\text { deviations }\end{array}$ & $\begin{array}{l}\text { Nonconformance if } \% \mathrm{R} \\
<\text { (average } \% \mathrm{R}-3 \\
\text { standard deviations) or } \\
>\text { (average } \% \mathrm{R}+3 \\
\text { standard deviations) }\end{array}$ \\
\hline Blind audit samples & $\begin{array}{l}\text { Samples and frequency } \\
\text { controlled by the Solid } \\
\text { PDP Plan }\end{array}$ & $\begin{array}{l}\text { Specified in the Solid PDP } \\
\text { Plan }\end{array}$ & $\begin{array}{l}\text { Specified in the Solid } \\
\text { PDP Plan }\end{array}$ \\
\hline
\end{tabular}

${ }^{a}$ Corrective Action when $O C$ samples do not meet the acceptance criteria; Nonconformance procedures are outined in Section 2.1.2.1 of the QAPP.

bMay be satisfied by using matrix spike duplicate; acceptance criteria applies only to concentrations greater than the PRQLs listed in Table 1.

$\begin{array}{ll}M D L & =\text { Method detection limit } \\ \text { QAO } & =\text { Quality assurance objective } \\ \text { PDP } & =\text { Performance Demonstration Program } \\ \% R & =\text { Percent recovery } \\ \text { RPD } & =\quad \text { Relative percent difference }\end{array}$


Accuracy. will be determined as the percent recovery (\%R) as follows:

$$
\% R=\frac{C_{m}}{C_{s m}} * 100
$$

where

$$
\begin{array}{ll}
C_{m}= & \text { measured concentration } \\
C_{s r m}= & \text { true concentration }
\end{array}
$$

MDL will be determined as:

$$
M D L=t_{(n-1,1-\alpha=0.99)} * s
$$

where

$$
\begin{array}{lll}
s & = & \text { standard deviation } \\
t_{(n-1,1-\alpha=0.99)}= & \begin{array}{l}
t \text {-distribution value appropriate to a 99-percent } \\
\text { confidence level and a standard deviation estimate with } \\
n-1 \text { degrees of freedom }
\end{array}
\end{array}
$$

10.4 The laboratory must analyze blanks at the frequency specified in Table 3 . The same analytical method used to prepare and analyze field samples will be used to prepare and analyze the laboratory blanks. Laboratory blank results will be acceptable if the concentration of target analytes is less than 3 times the MDL specified in Table 1 for each compound. Corrective action must be implemented if blanks exceed this level.

10.5 The laboratory must analyze matrix spikes at the frequency specified in Table 3 . Matrix spike results shall be considered acceptable if the \%R is within the range specified for each compound in Table 1. Matrix spikes which do not meet these criteria should be flagged.

$\% R$ will be determined as follows:

$$
\% R=\frac{S-U}{C_{s a}} * 100
$$

where

$S=$ measured concentration in the spiked aliquot

$U=$ measured concentration in the unspiked aliquot

$C_{s a}=$ actual concentration of the spike added

10.6 The laboratory shall analyze laboratory duplicates or matrix spike duplicates at the frequency specified in Table 3. Duplicate results shall be considered acceptable if the RPD for each compound is within the range specified in Table 1. RPD is determined as in Equation (1). Duplicates which do not meet these criteria should be flagged.

10.7 The laboratory must analyze laboratory control samples at the frequency specified in Table 3. Laboratory control samples must contain Aroclor 1254 and 1260, and shall be prepared, in hexane, at a concentration of $100 \mu \mathrm{g} / \mathrm{L}$ per laboratory control analyte from standards independent of those used for instrument calibration. The laboratory control samples must be quantitated within the calibration range of the instrument. The laboratory control sample analysis is designed to ensure that laboratory performance is in control for each analytical batch of samples processed. If the laboratory control sample $\% R$ is outside the range specified in Table 3 for the two aroclors, then new laboratory control samples must be prepared. \%R is determined as in Equation (4). 
10.8 The laboratory will be required to analyze blind audit samples. These audit samples are part of the Performance Demonstration Program. Details of this program are in the Performance Demonstration Program Plan for the Analysis of Solidified Waste for the Transuranic Waste Characterization Program (Solid PDP Plan)(DOE 1995a).

\subsection{Procedure Performance}

11.1 Analyte and Surrogate Recoveries. The extraction technique was applied to a simulated Rocky Flats Type IV sludge that consisted of an organic liquid mixed with MicroCel $E^{\circledR}$ (calcium silicate) and Oil Driø to solidify the waste. The organic liquid portion was spiked with Arocior 1254 to a concentration of $50 \mathrm{ppm}$ after mixing with MicroCel $E^{\oplus}$ and Oil Dri ${ }^{\oplus}$. Surrogates (TCMX and DCBP) were added to the sample sludges $(\sim 2.0 \mathrm{~g})$ to achieve a concentration of $5 \mathrm{ppm}$. Extractions were performed using Procedure 440.3 and SW-846 Method 3550 Medium-Level Ultrasonic Extractions. The recoveries of Aroclors 1254, 1260, TCMX, and DCBP are summarized in Tables 4, 5, and 6.

\subsection{Precision.}

11.2.1 Instrument Precision: Ten autosampler vials were filled with the cleaned extract of a single simulated sludge spiked with $50 \mathrm{ppm}$ Aroclor 1254 and $5 \mathrm{ppm}$ surrogate standards, TCMX and DCBP. Similarly, another ten autosampler vials were filled with the cleaned extract of a single simulated sludge spiked with $50 \mathrm{ppm}$ Aroclor 1260 and $5 \mathrm{ppm}$ surrogate standards. Each set of ten samples was analyzed with the GC/ECD. The \%RSD was calculated. Results are shown in Tables 7 and 8.

11.2.2 Method Precision: Extractions were performed according to Procedure 440.3 and SW-846 Method 3550 Medium-Level Ultrasonic Extraction. Method Precision was determined for $50 \mathrm{ppm}$-Aroclor 1254-spiked simulated sludge. Five replicate samples and a blank (not spiked with Aroclor 1254) were extracted according to each method. Results are shown in Table 9.

Method Precision was also determined for $50 \mathrm{ppm}$-Aroclor 1260 -spiked simulated sludge. Five replicate samples and a blank (not spiked with Aroclor 1260) were extracted according to each method. Results are shown in Table 10.

11.3 Method Detection Limits. MDLs were determined from the analysis of five replicate aliquots of each matrix processed through the entire analytical method (extraction, cleanup, and GC/ECD analysis). The MDL values were determined using Equation (5). The results are presented in Table 11.

11.4 Chromatograms. Figure 1 is a chromatogram of a simulated sludge extract spiked with a mixture of $50 \mathrm{ppm}$ Aroclor 1254 and $50 \mathrm{ppm}$ Aroclor 1260. The Aroclors were extracted according to Procedure 440.3.

11.5 Sources of Variation. Tables 7 and 8 show the relative standard deviations of the instrumental method. Tables 9 and 10 show the relative standard deviations of both extraction methods for the simulated sludge and of the instrumental method. Table 12 summarizes the magnitude of the sources of variation.

\subsection{References}

Argonne National Laboratory. 1993. Determination of PCBs in Rocky Flats Type IV Waste Sludge by Gas Chromatography/Electron Capture Detection. ANL/ACL-93/1, Argonne, Illinois, Argonne National Laboratory, University of Chicago, U.S. Department of Energy. 
TABLE 4

Comparison of Extraction Recoveries (\%)

Aroclor 1254 in 50 ppm-Spiked Sludge

Procedure 440.3

SW-846 Method 3550

$\begin{array}{ccc}\text { Surrogate } 1^{\mathrm{a}} & & \\ \text { Blank } & 77 & 82 \\ 1 & 76 & 79 \\ 2 & 80 & 75 \\ 3 & 80 & 73 \\ 4 & 78 & 79 \\ 5 & 81 & 80 \\ \text { Average } & 79 & 78 \\ \text { \%RSD } & 2.5 \% & 4.3 \%\end{array}$

Surrogate $2^{b}$

$\begin{array}{lcc}\text { Blank } & 74 & 86 \\ 1 & 83 & 69 \\ 2 & 86 & 83 \\ 3 & 76 & 77 \\ 4 & 67 & 82 \\ 5 & 79 & 86 \\ \text { Average } & 79 & 81 \\ \text { \%RSD } & 5.9 \% & 8.1 \%\end{array}$

Aroclor 1254

$\begin{array}{lcc}\text { Blank } & -- & -- \\ 1 & 73 & 71 \\ 2 & 77 & 70 \\ 3 & 75 & 69 \\ 4 & 73 & 72 \\ 5 & 77 & 72 \\ \text { Average } & 75 & 71 \\ \text { \%RSD } & 2.5 \% & 1.8 \%\end{array}$

a Surrogate 1 is tetrachloro-meta-xylene.

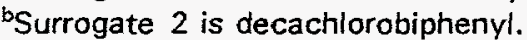

Source: Determination of PCBs in Rocky Flats Type N Waste S/udge by Gas Chromatography/Electron Capture Detection. (Argonne National Laboratory 1993). 
TABLE 5

\section{Comparison of Extraction Recoveries (\%) \\ Aroclor 1260 in 50 ppm-Spiked Sludge}

Procedure 440.3

SW-846 Method 3550

$\begin{array}{lll}\text { Surrogate } 1^{\mathbf{a}} & & \\ \text { Blank } & 94 & 80 \\ 1 & 88 & 84 \\ 2 & 79 & 81 \\ 3 & 80 & 81 \\ 4 & 90 & 84 \\ 5 & 88 & 82 \\ \text { Average } & 87 & 82 \\ \text { \%RSD } & 6.7 \% & 2.0 \%\end{array}$

Surrogate $2^{b}$

Blank

83

67

1

2

79

72

71

73

3

68

65

77

75

5

75

70

Average

\%RSD

76

70

$7.1 \%$

$5.4 \%$

Aroclor 1260

Blank

1

2

79

72

69

71

3

68

65

75

74

4

75

73

71

Average

\%RSD

$6.3 \%$

71

$4.7 \%$

asurrogate 1 is tetrachloro-meta-xylene.

bSurrogate 2 is decachlorobiphenyl.

Source: Determination of PCBs in Rocky Flats Type IV Waste S/udge by Gas Chromatography/Electron Capture Detection. (Argonne National Laboratory 1993). 


\section{TABLE 6}

Extraction Recoveries (\%) for Procedure 440.3

Aroclors 1254 and 1260 in 50 ppm (each) Spiked Sludge

\section{$\begin{array}{llll}\text { Surrogate } 1^{\mathrm{a}} & \text { Surrogate } 2^{\mathrm{b}} & \text { Aroclor } 1254 & \text { Aroclor } 1260\end{array}$}

\begin{tabular}{lcccc}
\hline & & & \\
Blank & 77 & 74 & 66 & - \\
1 & 79 & 78 & 67 & 69 \\
2 & 80 & 80 & 64 & 72 \\
3 & 79 & 76 & 66 & 72 \\
4 & 79 & 81 & 64 & 68 \\
5 & 79 & 76 & 65 & 70 \\
Average & 78 & 78 & $2.1 \%$ & $2.7 \%$ \\
\%RSD & $1.2 \%$ & $3.4 \%$ & & \\
\hline
\end{tabular}

asurrogate 1 is tetrachloro-meta-xylene.

burrogate 2 is decachlorobiphenyl.

Source: $\quad$ Determination of PCBs in Rocky Flats Type IV Waste Sludge by Gas Chromatography/Electron Capture Detection. (Argonne National Laboratory 1993). 
TABLE 7

Instrument Precision Results for 50 ppm-Aroclor 1254-Spiked Simulated Sludge Extract

$\begin{array}{ll}\text { Compound(s) } & \text { Precision }^{\circ} \\ \text { (\% RSD) }\end{array}$

$\begin{array}{ll}\text { Aroclor } 1254 & \pm 1.8 \\ \begin{array}{l}\text { Surrogate 1 } \\ \text { (TCMX). }\end{array} & \pm 3.3 \\ \text { Surrogate 2 } & \pm 3.0\end{array}$

${ }^{a}$ Ten replicates.

Source: Determination of PCBs in Rocky Flats Type IV Waste Sludge by Gas Chromatography/ Electron Capture Detection. (Argonne National Laboratory 1993).

TABLE 8

Instrument Precision Results for 50 ppm-Aroclor 1260-Spiked Simulated Sludge Extract

\begin{tabular}{|c|c|}
\hline Compound(s) & $\begin{array}{l}\text { - } \\
\text { Precision }^{a} \\
\text { (\% RSD) }\end{array}$ \\
\hline Aroclor 1260 & \pm 2.2 \\
\hline $\begin{array}{l}\text { Surrogate } 1 \\
\text { (TCMX) }\end{array}$ & \pm 1.5 \\
\hline $\begin{array}{l}\text { Surrogate } 2 \\
\text { (DCBP) }\end{array}$ & \pm 2.3 \\
\hline
\end{tabular}

${ }^{a}$ Ten replicates.

Source: Determination of PCBs in Rocky Flats Type IV Waste Sludge by Gas Chromatography/ Electron Capture Detection. (Argonne National Laboratory 1993). 
TABLE 9

Method Precision Results for 50 ppm-Aroclor 1254-Spiked Simulated Sludge Extract

\begin{tabular}{lcc}
\hline & \multicolumn{2}{c}{ Precision (\%RSD) } \\
\cline { 2 - 3 } Compound(s) & Procedure 440.3 & SW-846 Method 3550 \\
\hline & $\pm 2.4^{\mathrm{a}}$ & $\pm 1.8^{\mathrm{a}}$ \\
Aroclor 1254 & $\pm 2.7^{\mathrm{b}}$ & $\pm 4.3^{\mathrm{b}}$ \\
$\begin{array}{l}\text { Surrogate 1 } \\
\text { (TCMX) }\end{array}$ & $\pm 5.8^{\mathrm{b}}$ & $\pm 8.1^{\mathrm{b}}$ \\
$\begin{array}{l}\text { Surrogate 2 } \\
\text { (DCBP) }\end{array}$ & & \\
\hline
\end{tabular}

aFive replicates.

bSix replicates.

Source: Determination of PCBs in Rocky Flats Type IV Waste Sludge by Gas Chromatography/ Electron Capture Detection. (Argonne National Laboratory 1993).

TABLE 10

Method Precision Results for 50 ppm-Aroclor 1260-Spiked Simulated Sludge Extract

\begin{tabular}{|c|c|c|}
\hline \multirow[b]{2}{*}{ Compound(s) } & \multicolumn{2}{|c|}{ Precision (\%RSD) } \\
\hline & Procedure 440.3 & SW-846 Method 3550 \\
\hline Arocior 1260 & $\pm 6.3^{a}$ & $\pm 4.7^{a}$ \\
\hline $\begin{array}{l}\text { Surrogate } 1 \\
\text { (TCMX) }\end{array}$ & $\pm 6.7^{b}$ & $\pm 2.0^{b}$ \\
\hline $\begin{array}{l}\text { Surrogate } 2 \\
\text { (DCBP) }\end{array}$ & $\pm 7.1^{b}$ & $\pm 5.4^{b}$ \\
\hline
\end{tabular}

aFive replicates.

bSix replicates.

Source: Determination of PCBs in Rocky Flats Type IV Waste Sludge by Gas Chromatography/ Electron Capture Detection. (Argonne National Laboratory 1993). 
TABLE 11

Method Detection Limits for Procedure 440.3

and SW-846 Method 3550 Extractions

$\operatorname{MDL}(\mu g / g)$

Compound(s) Procedure 440.3 SW-846 Method 3550
Aroclor 1254
1.2
0.54
Aroclor 1260
1.0
0.83

Source: Determination of PCBs in Rocky Flats Type IV Waste Sludge by Gas Chromatography/Electron Capture Detection. (Argonne National Laboratory 1993).

TABLE 12

Relative Standard Deviations of the Instrumental and Both Extraction Methods

$$
\begin{gathered}
\begin{array}{c}
\text { Instrumental Method } \\
(\% \text { RSD) }
\end{array} \\
\cline { 2 - 3 }
\end{gathered}
$$

\begin{tabular}{llll} 
Aroclor 1254, & $\pm 1.8^{\mathrm{b}}$ & $\pm 2.5^{\mathrm{c}}$ & $\pm 1.8^{\mathrm{c}}$ \\
Aroclor 1260 & $\pm 2.2^{\mathrm{b}}$ & $\pm 6.3^{\mathrm{c}}$ & $\pm 4.7^{\mathrm{c}}$ \\
$\begin{array}{l}\text { Surrogate } 1 \text { of Aroclor } \\
1254 \text { and } 1260\end{array}$ & $\pm 2.4^{\mathrm{d}}$ & $\pm 4.6^{\mathrm{e}}$ & $\pm 3.2^{\mathrm{e}}$ \\
Surrogate 2 of Aroclor & $\pm 2.7^{\mathrm{d}}$ & & \\
\hline
\end{tabular}

\footnotetext{
aExtraction was performed on 50-ppm spiked simulated sludge matrices. Extractions were performed according to Procedure 440.3 and the SW-846 Method 3550 Medium-Level Ultrasonic Extraction.

${ }^{\mathrm{b}}$ Ten replicates.

${ }^{\circ}$ Five replicates.

${ }^{\mathrm{d}}$ Twenty replicates.

${ }^{ } \mathrm{T}$ welve replicates.

Source: Determination of PCBs in Rocky Flats Type IV Waste Sludge by Gas Chromatography/Electron Capture Detection. (Argonne National Laboratory 1993).
} 
DOE. 1995a. Performance Demonstration Program Plan for the Analysis of Solidified Waste for the Transuranic Waste Characterization Program. CAO-95-1077, Current Revision, Carlsbad, New Mexico, Carlsbad Area Office, U.S. Department of Energy.

DOE. 1995b. Transuranic Waste Characterization Quality Assurance Program Plan. CAO-941010, Current Revision, Carlsbad, New Mexico, Carlsbad Area Office, U.S. Department of Energy.

EPA. 1995. Test Methods for Evaluating Solid Waste, Physical/Chemical Methods. SW-846, Third Edition, Final Update I, and Final Update II, Washington, D.C., Office of Solid Waste and Emergency Response, U.S. Environmental Protection Agency. 


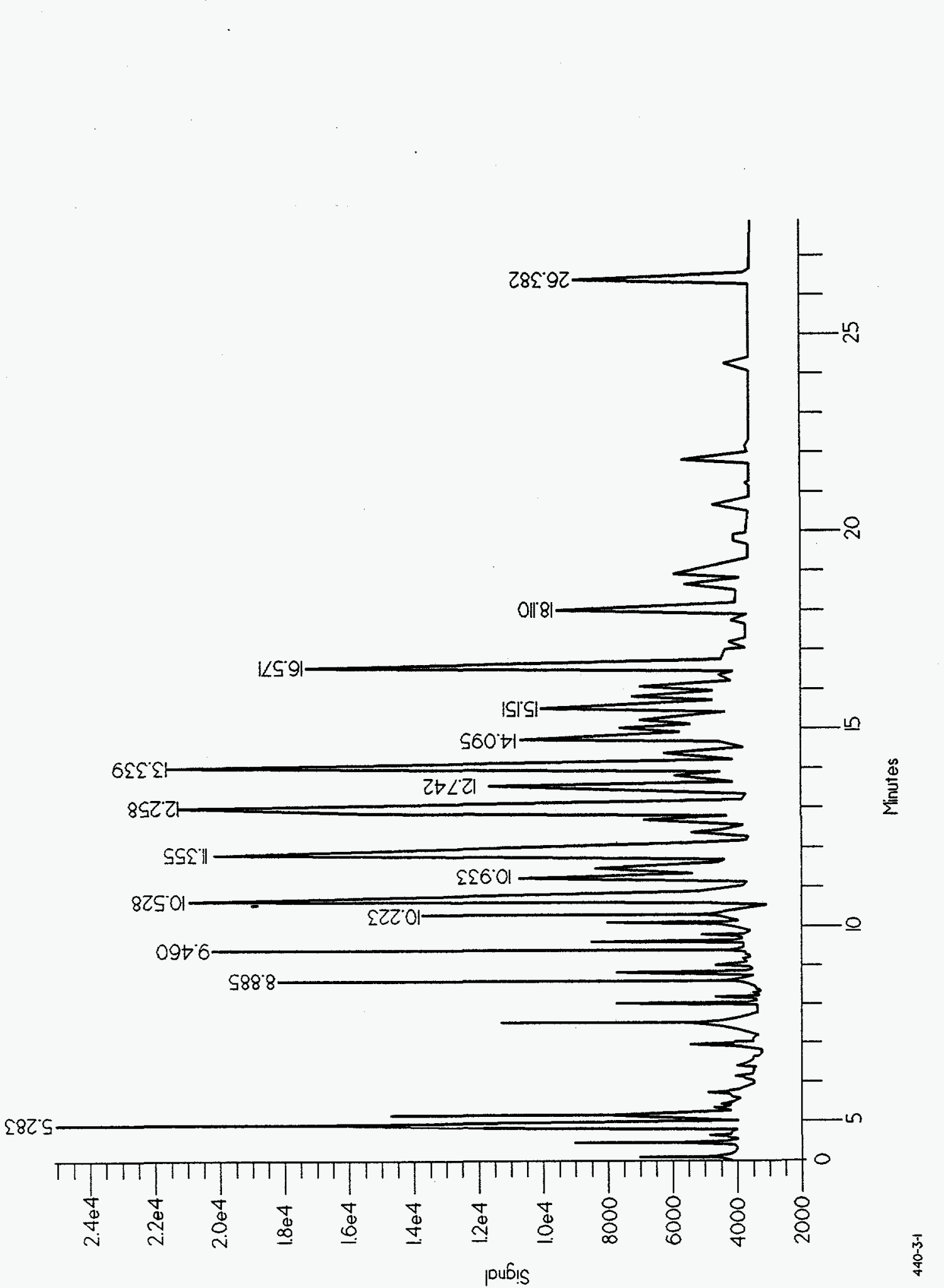


PROCEDURE 510.1

\section{MASS SPECTROMETRY DETERMINATION OF HYDROGEN AND METHANE IN WASTE CONTAINER HEADSPACE}

\subsection{Scope and Application}

1.1 This procedure is based on ASTM Method D2650-83, "Test Method for Chemical Composition of Gases by Mass Spectrometry" (ASTM 1983), and can be used for the qualitative and quantitative determination of hydrogen and methane in headspace gas samples. This procedure is based on mass spectrometric procedures and is restricted to use by, or under the supervision of, analysts experienced in the use of Mass Spectrometry (MS) and skilled in the interpretation of mass spectra. The quality assurance objectives for this procedure are listed in Table 1. The sampling procedures are described in Procedures 110.1 through 110.4 of this Methods Manual. This procedure must be implemented with a site-specific standard operating procedure (SOP).

1.2 For the purposes of the TRU Waste Characterization Program, samples are to be analyzed in analytical batches. An analytical batch is defined as a suite of samples that is processed as a unit, using the same analytical method, within a specific time period. An analytical batch can be up to 20 samples (excluding laboratory OC samples), all of which must be received by the laboratory within 14 days of the validated time of sample receipt (VTSR) of the first sample in the batch.

\subsection{Summary of Procedure}

2.1 The molecular species which make up a gaseous mixture are ionized and may be dissociated by electron bombardment. The positive ions of different masses thus formed are accelerated in an electrostatic field and separated in a magnetic field. The abundance of each mass present is determined from the signal at the corresponding mass-to-charge ratio. This allows the identification and quantitation of the individual constituents of the gas mixture.

\subsection{Definitions.}

2.2.1 Mass Number or $\mathrm{m} / \mathrm{e}$ Value of an lon: The quotient of the mass of that ion (given in atomic mass units) and its positive charge (number of electrons lost during ionization).

2.2.2 Relative Intensity: The ratio of a peak at any $\mathrm{m} / \mathrm{e}$ relative to its base peak (usually, its parent peak).

2.2.3 Sensitivity: The height of the base peak in the spectrum of the pure analyte divided by the pressure prevailing in the inlet system of the mass spectrometer immediately before opening the expansion bottle to leak.

2.2.4 Partial Pressure: The pressure of an analyte in the inlet system before opening the expansion bottle to leak. 
TABLE 1

Hydrogen and Methane Analysis Quality Assurance Objectives

\begin{tabular}{lcccccc}
\hline Analyte & CAS Number & $\begin{array}{c}\text { Precision } \\
\text { (\%RSD or RPD) }\end{array}$ & $\begin{array}{c}\text { Accuracy } \\
(\% \mathrm{R})\end{array}$ & $\begin{array}{c}\text { MDL } \\
(\mathrm{Vol} \%)\end{array}$ & $\begin{array}{c}\text { PROL } \\
\text { (vol\%) }\end{array}$ & $\begin{array}{c}\text { Completeness } \\
\text { (percent) }\end{array}$ \\
\hline Hydrogen & $1333-74-0$ & $\leq 25$ & $70-130$ & 0.05 & 0.1 & 90 \\
Methane & $74-82-8$ & $\leq 25$ & $70-130$ & 0.05 & 0.1 & 90 \\
\hline
\end{tabular}

\%RSD $=$ Percent relative standard deviation

RPD $=$ Relative percent difference

$\% R=$ Percent recovery

MDL = Method detection limit

PRQL = Program required quantitation limit

acriteria apply to PROL concentrations 
3.0 Interferences

3.1 Gases which appear to desensitize the filament (e.g., $\mathrm{NO}_{2}, \mathrm{SiF}_{4}$ ) may introduce uncertainties in the analysis.

3.2 Trace amounts of a gas may be undetected in the presence of larger amounts of a gas having an ion fragment which coincides with the principal peak of the trace gas (e.g., $\mathrm{CH}_{4}$ in $\mathrm{C}_{2} \mathrm{H}_{6}$ ).

4.0 Safety

This procedure may involve the use of hazardous materials, operations, and equipment. It is the responsibility of whoever uses this procedure to consult appropriate site personnel concerning health and safety issues and establish appropriate health and safety practices. Consideration should be given to safety concerns regarding chemical and radiation exposure. Training regarding proper storage, usage, and disposal of chemicals is recommended.

Samples and reference materials may be flammable. Keep away from heat, sparks, and open flame. Provide adequate ventilation.

\subsection{Apparatus and Materials}

5.1 Mass Spectrometer. The mass spectrometer must be capable of resolving the doublet, $\mathrm{NO}^{+} / \mathrm{C}_{2} \mathrm{H}_{6}{ }^{+}$, at mass 30 . Additional performance tests described herein and in ASTM E137-82, "Practice for Evaluation of Mass Spectrometers for Quantitative Analysis from a Batch Inlet" (ASTM 1982), must be considered.

If the parent peak is not used to determine a given analyte, details of using the alternate masses should be outlined in the SOP.

5.2 Data System. A data processing system must be interfaced to the mass spectrometer and must allow acquisition of data at all masses of interest. At a minimum, it should be capable of reprocessing data and external standard calculations and archiving for future processing.

5.3 Sample Inlet. The sample inlet system must be configured to permit attaching SUMMA ${ }^{\oplus}$ canisters directly to the mass spectrometer inlet. A series of expansion reservoirs should allow the introduction of the samples at a predetermined pressure (as recommended by the manufacturer).

\subsection{Reagents}

Reference Standards: The mass spectrometer must be calibrated with hydrogen and methane. The calibrating gases should be of high purity ( $>99.9$ percent). Pure gases and certified mixtures may be used. Contribution of ion fragments for all appropriate masses must be measured. In general, the mass spectrometer is capable of detecting impurities in calibrants and the contribution of such impurities to the calibration spectrum must be accounted for. This is done by measuring the concentration of the impurity and correcting measured signals for contributions due to ions in its spectrum.

\subsection{Sample Collection, Preservation, and Handling}

7.1 Samples will be collected using the sampling procedures described in Procedures 110.1 through 110.4 of this Methods Manual. 
7.2 There are no method-specific requirements for sample preservation or holding times prior to analysis. However, to facilitate timely reporting of the analysis data, sample holding times should not exceed 28 days.

7.3 All sample handling and chain-of-custody procedures described in Section 6.0 of the Transuranic Waste Characterization Quality Assurance Program Plan (QAPP) (DOE 1995b) must be followed.

\subsection{Procedure}

\subsection{Recommended MS System Operating Conditions}

Obtain the mass spectrum of the sample under the same conditions as the calibration spectra. Instrument settings are established to provide optimum performance for the gas mixture to be analyzed. Specific instrument settings must be listed in an SOP. Suggested instrument parameters are as follows:

Inlet system temperature:

Ion source temperature:

Trap current:

Electron energy:

Deflection plate voltages:

YD:

HP:

ZD:

Source slit:

Ion repeller voltage:

Ion collectors:

Axial collection slit:

Electron multiplier voltage:

Daily electrode supply

(Bradenburg) voltage:

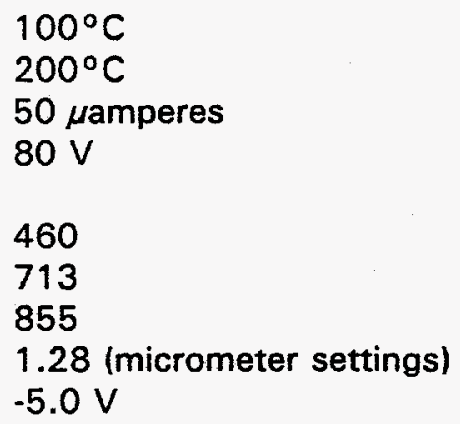

$8 \mathrm{kV}$

The above instrument conditions are for a VG Model 30-01. These values may vary or be set automatically on different instruments.

The general procedure may involve a continuous or peak-hopping mass scan to determine if there are unexpected compounds in the gas mixture. This is followed by a slower scan or high precision peak-hopping mode, which allows precise measurement of the intensities of specific peaks for quantification of hydrogen and methane in the mixture.

It shall be the responsibility of the analyst to verify and document that the instrument configuration and operating conditions (scanning rate, resolution, ion voltages, detector voltages) satisfy the analytical requirements, and to maintain quality control data confirming instrument performance and analytical results.

8.2 All SUMMA ${ }^{\circ}$ canisters used for MS system calibration, quality control samples, and analytical purposes must first be cleaned and certified in accordance with Procedure 210.1 of this Methods Manual.

8.3 Calibration. A summary of calibration requirements is provided in Table 2.

8.3.1 Apparatus: Determine whether operating conditions remain normal by making certain tests periodically, following instructions furnished by the manufacturer 


\section{TABLE 2}

Summary of MS Calibration Requirements for Hydrogen and Methane Analysis

Technique

Procedure

Initial mass calibration

Initial sensitivity

calibration

Continuing mass

calibration

Continuing sensitivity

calibration
Frequency of Procedure

Initially and as needed

Initially and as needed

Beginning and end of each analytical batch

Beginning and end of each analytical batch
Acceptance Criteria

Base peak of 2 and 16 for hydrogen and methane, respectively

$\% D \leq 15$ for each analyte from two consecutive sensitivity measurements

RPD $\leq 10$ for each analyte

Sum of partial pressure for all components in the standard gas cylinder equals total pressure of introduced sample $\pm 3 \%$
$\% \mathrm{D}=$ Percent difference

RPD $=$ Relative percent difference 
of the apparatus. Include in these tests rate of leak, magnet current, and ionbeam control settings. These tests should be performed during initial instrument setup and as specified by the manufacturer. For example, these tests may need to be performed after changing a leak valve or filament, or performing other maintenance functions.

8.3.2 Reference Standards: To calibrate the mass spectrometer, a known quantity of the calibration gas is introduced into the mass spectrometer. The field position and sensitivity are determined for hydrogen and methane.

8.3.3 Initial Calibration: The mass spectrometer must be calibrated for mass position and sensitivity. Mass position calibration establishes a general relationship between mass and magnetic (or electric) fields over the useful mass range. Mass calibration must be done with at least four calibration gases designed to cover the useful mass range. Typical calibrations masses include mass 2.016 (hydrogen), mass 4.003 (helium), mass 14 (nitrogen), mass 28 (nitrogen), and mass 40 (argon).

8.3.4 Continuing Calibration: Continuing calibration checks may be done with the laboratory control sample. If a gas standard other than the laboratory control sample is used for continuing calibration, then the laboratory control sample must be run as a sample during the analytical sequence. The continuing calibration gas must be from a separate source than that used for the initial instrument calibration. The calibration check must be run at the beginning and end of each analytical batch. The concentration of each of the analytes in the mixture is measured. If the RPD between this concentration and the certified concentration is less than 10 percent, then the mass spectrometer will be considered calibrated. Calibration is also verified by showing that the sum of the measured partial pressures of the compounds in the standard gas equals the total pressure of the introduced sample (within \pm 3 percent).

\subsection{Calculations}

The partial pressure of hydrogen and methane may be calculated automatically by the instrument or manually as follows:

$$
P_{i}=\frac{P k H t}{S e n s}
$$

where

$$
\begin{array}{lll}
P_{i} & = & \text { the partial pressure of the analyte } \mathrm{i}(\mathrm{m} \text { Torr }) \\
P k \boldsymbol{H} t= & \text { the peak height }(\mathrm{mV}) \\
\text { Sens } & =\text { the sensitivity of the analyte in } \mathrm{mV} \text { per unit pressure (m Torr) }
\end{array}
$$

The sum of the partial pressures of measured compounds should agree within \pm 3 percent of the pressure measured in the expansion reservoir of the mass spectrometer. If water vapor is present in the sample, poorer agreement may be expected by the analyst when the data is reviewed. Poor agreement between calculated and measured pressure may indicate that a major mixture compound was overlooked or that the instrument needs recalibration. 
Volume percent is calculated as follows:

$$
\text { Volume } \%=\frac{P_{i}}{\Sigma P_{i}} * 100
$$

where

$\Sigma P_{i} \quad=\quad$ the sum of partial pressures of all the gases

\subsection{Quality Control}

10.1 Each laboratory that uses this procedure is required to operate a formal quality control program. The laboratory must retain records to document the quality of the data generated. Each laboratory must have SOPs documenting and describing activities involved in utilizing this procedure. Specific quality control practices will include, but are not limited to, the analysis of quality control samples. The types of quality control samples, their associated frequency of analysis, acceptance criteria, and corrective action required if samples do not meet the acceptance criteria, is summarized in Table 3. Laboratory SOPs shall address requirements for preparing laboratory blanks, laboratory duplicates, and laboratory control samples.

10.2 All laboratories using this procedure must demonstrate acceptable performance prior to the analysis of actual samples. Demonstration of acceptance performance will be achieved by analyzing method performance samples (Table 3). These samples can be either commercially purchased or laboratory prepared, but must contain hydrogen and methane at concentrations appropriate ( 2 to 5 times the PROLs) to determine the quality assurance objectives specified in Table 1. The analysis of seven samples must meet the criteria specified for precision, accuracy, and method detection limit (MDL) in Table 1. Demonstration of acceptable method and analyst performance must be repeated (by analyzing four samples) at a minimum of every six months. Method performance should be conducted over a period of several days to account for longterm variation. Corrective action must be implemented when acceptable method performance is not met. Precision will be determined as follows:

- For duplicate measurements, the precision expressed as the relative percent difference $(R P D)$ is calculated as

$$
R P D=\frac{C_{1}-C_{2}}{\frac{\left(C_{1}+C_{2}\right)}{2}} * 100
$$

where

$$
\begin{array}{lll}
C_{1} \text { and } C_{2} & = & \text { two values obtained by analyzing the duplicate samples } \\
C_{1} & = & \text { larger of the two observed values }
\end{array}
$$


TABLE 3

\section{Summary of Laboratory Quality Control Samples and Frequencies for Hydrogen and Methane Analysis}

\begin{tabular}{|c|c|c|c|}
\hline OC Sample & Minimum Frequency & $\begin{array}{l}\text { Acceptance } \\
\text { Criteria }\end{array}$ & $\begin{array}{l}\text { Corrective } \\
\text { Action }^{\text {a }}\end{array}$ \\
\hline $\begin{array}{l}\text { Method performance } \\
\text { samples }\end{array}$ & $\begin{array}{l}\text { Seven (7) samples initially } \\
\text { and four (4) semiannually }\end{array}$ & Meet Table 1 QAOs & $\begin{array}{l}\text { Repeat until } \\
\text { acceptable }\end{array}$ \\
\hline Laboratory duplicates & $\begin{array}{l}\text { One (1) per analytical } \\
\text { batch }\end{array}$ & $\mathrm{RPD} \leq 25^{b}$ & $\begin{array}{l}\text { Nonconformance if } \\
\text { RPD }>25\end{array}$ \\
\hline Laboratory blanks & $\begin{array}{l}\text { One (1) per analytical } \\
\text { batch }\end{array}$ & $\begin{array}{l}\text { Analyte concentrations } \\
<3 \times \mathrm{MDL}\end{array}$ & $\begin{array}{l}\text { Nonconformance if } \\
\text { analyte concentrations } \\
>3 \times \mathrm{MDL}\end{array}$ \\
\hline $\begin{array}{l}\text { Laboratory control } \\
\text { samples }\end{array}$ & $\begin{array}{l}\text { One (1) per analytical } \\
\text { batch }\end{array}$ & $70-130 \% R$ & $\begin{array}{l}\text { Nonconformance if } \\
\% R<70 \text { or }>130\end{array}$ \\
\hline Blind audit samples & $\begin{array}{l}\text { Samples and frequency } \\
\text { controlled by the Gas PDP } \\
\text { Plan }\end{array}$ & $\begin{array}{l}\text { Specified in the } \\
\text { Gas PDP Plan }\end{array}$ & $\begin{array}{l}\text { Specified in the } \\
\text { Gas PDP Plan }\end{array}$ \\
\hline
\end{tabular}

${ }^{a}$ Corrective Action when $\mathrm{OC}$ samples do not meet the acceptance criteria; Nonconformance procedures are outlined in Section 2.1.2.1 of the QAPP.

${ }^{b}$ Applies only to concentrations greater than the PROL listed in Table 1.

MDL = Method detection limit

PDP = Performance Demonstration Program

$\mathrm{OAO}=$ Quality assurance objective

$\% \mathrm{R}=$ Percent recovery

RPD = Relative percent difference 
- For three or more replicate measurements, the precision expressed as the \%RSD is calculated as

$$
\% R S D=\frac{s}{\bar{y}} * 100
$$

where

$$
\begin{array}{lll}
s & = & \text { standard deviation } \\
y & = & \text { mean of replicate analyses }
\end{array}
$$

- The standard deviation (s) is defined as

$$
s=\sqrt{\sum_{i=1}^{n} \frac{\left(y_{i}-\bar{y}\right)^{2}}{n-1}}
$$

where

$$
\begin{array}{lll}
y_{i} & = & \text { measured value of the } i^{\text {th }} \text { replicate sample analysis } \\
n & = & \text { measurement } \\
n & \text { the number of replicate analyses }
\end{array}
$$

Accuracy will be determined as the percent recovery $(\% R)$ as follows:

$$
\% R=\frac{C_{m}}{C_{s m}} * 100
$$

where

$$
\begin{array}{lll}
C_{m} & = & \text { measured concentration } \\
C_{s m m} & = & \text { true concentration }
\end{array}
$$

MDL will be determined as follows:

$$
\left.M D L=t_{(r-1,1-\alpha}=0.99\right) * s
$$

where

$$
\begin{array}{ll}
s & =\quad \begin{array}{l}
\text { standard deviation } \\
t_{(n-1,1-\alpha=0.99)}= \\
\text {-distribution value appropriate to a 99-percent } \\
\text { confidence level and a standard deviation estimate with } \\
n-1 \text { degrees of freedom }
\end{array}
\end{array}
$$

10.3 The laboratory must analyze blanks at the frequency specified in Table 3 . The laboratory blanks will consist of ultra-high purity nitrogen or helium $(99.999$ percent purel. The same procedures used to prepare and analyze field samples will be used to prepare and analyze the laboratory blanks. Laboratory blank results will be acceptable if the concentrations of hydrogen and methane are less than 3 times the MDL specified in Table 1. Corrective action must be implemented for blanks which exceed this level.

10.4 The laboratory must analyze field samples in duplicate. Single canisters shall be analyzed in duplicate at a frequency specified in Table 3. Single canisters analyzed in duplicate shall be used to assess laboratory precision. Laboratory duplicate results 
shall be considered acceptable if the RPD is $\leq 25$ percent. RPD is determined as in Equation (3). Duplicates which do not meet these criteria should be flagged.

10.5 The laboratory must analyze laboratory control samples at the frequency specified in Table 3. A laboratory control sample consists of a certified standard gas mixture and must be from sources independent of those used for instrument calibration. These samples must contain hydrogen and methane at concentrations in the calibration range of the instrument. The laboratory control sample analysis is designed to ensure that the laboratory's performance is in control for each analytical batch of samples processed. Laboratory control sample results will be considered acceptable if the \%R is 70 to 130 percent of the known value. \%R is determined as in Equation (6). If results are outside of this range, then new laboratory control samples must be prepared.

10.6 The laboratory will be required to analyze blind audit samples. These audit samples are part of the Performance Demonstration Program. Details of this program are in the Performance Demonstration Program Plan for the Analysis of Simulated Headspace Gases for the Transuranic Waste Characterization Program (Gas PDP Plan)(DOE 1995a).

\subsection{Procedure Performance}

The characteristics of hydrogen and methane measurement using MS were investigated using two data sources. The first is the repeated measurement of a hydrogen standard and a methane standard over time as part of initial calibration verification and continuing calibration verification activities. The second is groups of four repeated measurements made over time using several hydrogen and methane standards as part of the Performance Demonstration Program (PDP). The results are shown in Table 4 for hydrogen and in Table 5 for methane. Accuracy was determined as in Equation (6), and precision was determined as in Equation (4).

\subsection{References}

ASTM. 1982. "Practice for Evaluation of Mass Spectrometers for Quantitative Analysis from a Batch Inlet." ASTM E137-82, Annual Book of ASTM Standards, Philadelphia, Pennsylvania, American Society for Testing and Materials.

ASTM. 1983. "Test Method for Chemical Composition of Gases by Mass Spectrometry." ASTM D2650-83, Annual Book of ASTM Standards, Philadelphia, Pennsylvania, American Society for Testing and Materiais.

DOE. 1995a. Performance Demonstration Program Plan for the Analysis of Simulated Headspace Gases for the Tranisuranic Waste Characterization Program. CA0-95-1076, Current Revision, Carlsbad, New Mexico, Carisbad Area Office, U.S. Department of Energy.

DOE. 1995b. Transuranic Waste Characterization Quality Assurance Program Plan. CAO-941010, Current Revision, Carlsbad, New Mexico, Carisbad Area Office, U.S. Department of Energy. 
TABLE 4

Method Performance of Gas Mass Spectrometry for Hydrogen Analysis

\begin{tabular}{lccc}
\hline $\begin{array}{c}\text { Known Value } \\
\text { (vol \%) }\end{array}$ & $\begin{array}{c}\text { Number of } \\
\text { Measurements }\end{array}$ & $\begin{array}{c}\text { Accuracy } \\
\text { (Mean \%R) }\end{array}$ & $\begin{array}{c}\text { Precision } \\
\text { (\%RSD) }\end{array}$ \\
\hline 0.119 & 8 & 106.95 & 4.056 \\
0.149 & 8 & 102.86 & 0.538 \\
0.404 & 8 & 97.26 & 3.315 \\
0.437 & 4 & 102.46 & 2.33 \\
0.512 & 8 & 94.58 & 0.786 \\
0.89 & 4 & 98.65 & 1.213 \\
0.997 & 4 & 95.24 & 1.048 \\
0.982 & 190 & 99.52 & 2.682 \\
1.21 & 4 & 99.26 & 1.061 \\
1.32 & 8 & 99.92 & 0.539 \\
1.98 & 4 & 97.34 & 1.036 \\
\hline
\end{tabular}




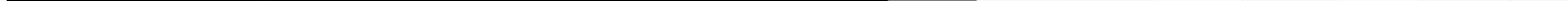


PROCEDURE 520.1

\section{GAS CHROMATOGRAPHY DETERMINATION OF HYDROGEN AND METHANE IN WASTE CONTAINER HEADSPACE}

1.1 This procedure is based on ASTM Method 1946-82, "Standard Method for Analysis of Reformed Gas by Gas Chromatograph" (ASTM 1982), and can be used for the qualitative and quantitative determination of hydrogen and methane in headspace gas samples. This procedure is based on gas chromatographic procedures and is restricted to use by, or under the supervision of, analysts experienced in the use of Gas Chromatography (GC) instrumentation and skilled in the interpretation of gas chromatograms. The quality assurance objectives for this procedure are listed in Table 1. The sampling procedures are described in Procedures 110.1 through 110.4 of this Methods Manual. This procedure must be implemented with a site-specific standard operating procedure (SOP).

1.2 For the purposes of the TRU Waste Characterization Program, samples are to be analyzed in analytical batches. An analytical batch is defined as a suite of samples that is processed as a unit, using the same analytical method, within a specific time period. An analytical batch can be up to 20 samples (excluding laboratory $O C$ samples), all of which must be received by the laboratory within 14 days of the validated time of sample receipt (VTSR) of the first sample in the batch.

\subsection{Summary of Procedure}

Hydrogen and methane in a headspace gas sample are physically separated by GC and compared to a reference standard of known composition separated under identical operating conditions. The hydrogen and methane concentration of the headspace gas is calculated by comparison of either the peak height or area response of hydrogen and methane with the corresponding value of hydrogen and methane in the reference standard.

3.0 Interferences

Helium can interfere with the hydrogen determination.

4.0 Safety

This procedure may involve the use of hazardous materials, operations, and equipment. It is the responsibility of whoever uses this procedure to consult appropriate site personnel concerning health and safety issues and establish appropriate health and safety practices. Consideration should be given to safety concerns regarding chemical and radiation exposure. Training regarding proper storage, usage, and disposal of chemicals is recommended.

Samples and reference materials may be flammable. Keep away from heat, sparks, and open flame. Provide adequate ventilation.

\subsection{Apparatus and Materials}

5.1 Gas Chromatograph. Chromatographic instrument and required accessaries including detectors, column supplies, a data system, a pressure measuring device, and a fixed volume sample loop. The data system must be interfaced to the gas chromatograph and must allow continuous acquisition of data throughout the duration of the 
TABLE 1

Hydrogen and Methane Analysis Quality Assurance Objectives

\begin{tabular}{lcccccc}
\hline Analyte & CAS Number & $\begin{array}{c}\text { Precision } \\
\text { (\%RSD or RPD) }\end{array}$ & $\begin{array}{c}\text { Accuracy } \\
(\% \mathrm{R})\end{array}$ & $\begin{array}{c}\text { MDL } \\
(\text { vol\%) }\end{array}$ & $\begin{array}{c}\text { PRQL } \\
\text { (vol\%) }\end{array}$ & $\begin{array}{c}\text { Completeness } \\
\text { (percent) }\end{array}$ \\
\hline Hydrogen & $1333-74-0$ & $\leq 25$ & $70-130$ & 0.05 & 0.1 & 90 \\
Methane & $74-82-8$ & $\leq 25$ & $70-130$ & 0.05 & 0.1 & 90 \\
\hline
\end{tabular}

$\%$ RSD $=$ Percent relative standard deviation

RPD $=$ Relative percent difference

$\% R=$ Percent recovery

MDL = Method detection limit

PROL = Program required quantitation limit

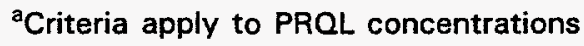


chromatographic program. At a minimum, it must be capable of reprocessing data and external standard calculations and archiving for future processing.

5.2 Detector. The detector shall be a thermal conductivity type, or its equivalent in stability and sensitivity for the target analytes. The thermal conductivity detector must be sufficiently sensitive to produce a signal of at least $0.5 \mathrm{mV}$ for 1 mole-percent methane in a $0.5 \mathrm{~mL}$ sample.

5.3 Detector Temperature Control. The detector temperature shall be maintained at a constant temperature $\pm 0.3^{\circ} \mathrm{C}$ (as specified by the manufacturer) during the course of the sample run and the corresponding reference run. The detector temperature shall be greater than or equal to, the maximum column temperature.

5.4 Sample Inlet System. Constant Volume Gas Sampling Loop.

5.4.1 The system must be capable of attaching SUMMA ${ }^{\circ}$ canisters directly to the sample inlet. The sample inlet system must be constructed of materials that are inert and nonadsorptive with respect to the gas compounds in the sample. The preferred material of construction is stainless steel. Cooper and copperbearing alloys are not acceptable: Loss of sample due to excessive transfer line volume should be minimized. The inlet system should allow pressure measurements to $0.1 \mathrm{kPa}$ and temperature measurements to $1^{\circ} \mathrm{C}$. The inlet system should be operated at ambient temperature.

5.4.2 Provision must be made to introduce into the carrier gas ahead of the analyzing column a gas-phase sample that has been entrapped in either a fixed volume loop or tubular section. The sample injection valves may be configured such that the sample is swept by carrier gas directly onto the gas chromatograph column or into a split/splitless injection port. The temperature, pressure, and volume of the sample must be known. The sample loop and tubing to the sample loop must be capable of being evacuated or purged for the purpose of decontamination.

\subsection{Gas Chromatographic Columns.}

5.5.1 The columns shall be constructed of materials that are inert and nonadsorptive with respect to the gas compounds in the sample. The preferred material of construction is stainless steel. Copper and copper-bearing alloys are not acceptable.

5.5.2 An adsorption-type column (e.g., molecular sieve) may be used to conduct the analysis. Cooled capillary columns may also be used. See ASTM Standard E260-85, "Recommended Practice for General Gas Chromatography Procedures" (ASTM 1985), for general GC procedures.

The column must completely separate hydrogen and methane. Demonstration of separation is required. Figure 1 is an example chromatogram obtained with an adsorption column.

Nitrogen or argon carrier gas is suitable for both percent and parts per million quantities of hydrogen.

Another technique for isolating the hydrogen in a sample is to use a palladium transfer tube at the end of the adsorption column; this will permit only 
hydrogen to be transferred to a stream of argon or nitrogen carrier gas for analysis in a second thermal conductivity detector.

5.5.3 General: Those column materials operated either isothermally or with temperature programming, or both, may be utilized if they provide satisfactory separation of hydrogen and methane. If complete separation is not possible with packed columns, multiple injections using different columns and/or conditions may be used. Capillary columns at reduced temperatures may also be used.

\subsection{Column Temperature Control}

5.6.1 Isothermal: When isothermal operation is utilized, the analytical columns shall be maintained at a temperature $\pm 0.3^{\circ} \mathrm{C}$ (as specified by the manufacturer) during the course of the sample run and the corresponding reference run.

5.6.2 Temperature Programming: Temperature programming may be used, as appropriate. The oven temperature shall not be below or above the recommended temperature limits for the materials in the column.

5.7 Carrier Gas. The instrument shall be equipped to provide flow of carrier gas through the analyzer and detector under isothermal conditions at a flow rate that is constant to 1 percent throughout the analysis of the sample and the reference standard. The purity of the carrier gas may be improved by allowing it to flow through selective filters prior to its entry into the chromatograph.

\subsection{Reagents}

Reference Standards: Certified gas mixtures of known hydrogen and methane composition are required for making a standard calibration curve. Hydrogen and methane in the reference standard must be homogeneous in the vapor state at the time of use and should be traceable to a nationally recognized standard.

7.0 Sample Collection, Preservation, and Handling

7.1 Samples will be collected using the sampling procedures described in Procedures 110.1 through 110.4 of this Methods Manual.

7.2 There are no method-specific requirements for sample preservation or holding times prior to analysis. However, to facilitate timely reporting of the analysis data, sample holding times should not exceed 28 days.

7.3 All sample handling and chain-of-custody procedures described in Section $6.0^{\circ}$ of the Transuranic Waste Characterization Quality Assurance Program Plan (QAPP) (DOE 1995b) must be followed.

\subsection{Procedure}

\subsection{Recommended GC System Operating Conditions}

Prepacked or capillary columns are generally recommended (mole sieve $5 \mathrm{a}$ or equivalent). Variations in column material, dimensions, and mesh sizes of packing are permissible if the columns produce the desired separations as shown in Figure 1. 
Columns:

Sample loop volume:

Injection temperature:

Column temperature:

Carrier gas:

Gas Flow Rates:

Through columns:

Split flow:

Septum purge flow:

Column + auxiliary flow:

Column + auxiliary + reference flow:
Mole sieve $5 \mathrm{a}$ (or equivalent)

$200 \mu \mathrm{L}$

ambient

$-20^{\circ} \mathrm{C}$

nitrogen or argon

5.4 to $6.0 \mathrm{~mL} /$ minute

4.0 to $4.2 \mathrm{~mL} /$ minute

1.0 to $1.1 \mathrm{~mL} /$ minute

7.9 to $8.1 \mathrm{~mL} /$ minute

21.4 to $22 \mathrm{~mL} /$ minute

The above operating conditions are for a HP 5890 gas chromatograph. These values vary depending on the gas chromatograph used.

It shall be the responsibility of the analyst to verify and document the instrument configuration and operating conditions (sample volume, column type, temperatures, flow rates) used to satisfy the analytical requirements and to maintain quality control data confirming instrument performance and analytical results.

8.2 All SUMMA ${ }^{\oplus}$ canisters used for GC system calibration, quality control samples, and analytical purposes must first be cleaned and certified in accordance with Procedure 210.1 of this Methods Manual.

8.3 Calibration. A summary of calibration requirements is provided in Table 2.

8.3.1 Initial Calibration: A calibration curve is constructed from a minimum of three certified gas standards. The resulting calibration curve must yield a straight line with correlation coefficient $(r) \geq 0.995$. The lowest standard should set below the PROL and the highest standard will be such that it brackets the expected sample concentrations and remains in the calibration range of the instrument.

All standards and samples must be analyzed under the same operating conditions. Tabulate peak area response of the analyte against the amount injected. Calculate a response factor (RF) for hydrogen and methane as follows:

$$
R F=\frac{A_{x}}{C_{x}}
$$

where

$$
\begin{array}{lll}
A_{x} & = & \text { total area response for the analyte } \\
C_{x} & = & \text { amount (mole) of the analyte being measured (mole) }
\end{array}
$$

To calculate the number of moles of each gas, the following equation may be used:

$$
C_{x}=\frac{\left(\text { Vol\%) } V_{s} P_{s}\right.}{R T_{s}}
$$

where:

$$
\begin{aligned}
& \text { Vol\% }=\text { volume percent of the gas in the standard } \\
& V_{s}=\text { volume of the sample loop }(\mathrm{mL})
\end{aligned}
$$


TABLE 2

Summary of GC Calibration Requirements for Hydrogen and Methane Analysis

\begin{tabular}{llll}
\hline Technique & Procedure & Frequency of Procedure & Acceptance Criteria \\
\hline GC & $\begin{array}{l}\text { 3-pt initial calibration } \\
\text { (3 standards) }\end{array}$ & Initially and as needed & $\begin{array}{l}\% \text { RSD of response factor for each } \\
\text { analyte } \leq 35\end{array}$ \\
& & & $\begin{array}{l}\text { Linear regression plot yields straight } \\
\text { line and \%R for each analyte is } \\
70-130\end{array}$ \\
& Continuing calibration & Every 12 hours & \begin{tabular}{l}
$\% 0 \leq 30$ for each analyte \\
\hline
\end{tabular}
\end{tabular}

$\% \mathrm{D}=$ Percent difference

$\% \mathrm{R} \quad=\quad$ Percent recovery

$\% \mathrm{RSD}=$ Percent relative standard deviation 


$$
\begin{array}{ll}
\boldsymbol{P}_{\boldsymbol{s}} & =\text { pressure of the sampling loop (atm) } \\
\boldsymbol{R} & =82.057 \mathrm{~mL} \text { atm } /{ }^{\circ} \mathrm{K} \text { mole } \\
\boldsymbol{T}_{\boldsymbol{s}} & =\text { temperature of the sampling loop }\left({ }^{\circ} \mathrm{K}\right)
\end{array}
$$

Alternately, the standards may be converted to values at standard temperature and pressure. The sample inlet pressure may vary, and this variation must be compensated.

8.3.2 Continuing Calibration: Calibration check standards must be analyzed at the beginning of every 12 hours of operation and prior to the analysis of any samples to verify working curves and retention time (RT) windows. The RF (or concentration) of the continuing calibration check and RT window (Section 8.3) will be compared to the corresponding values from the most recent valid calibration.

The verification procedure involves calculating the $R F$ for hydrogen and methane in the calibration verification standard and comparing these to the average RFs for hydrogen and methane from the initial calibration sequence. The validity of the initial calibration RF is checked by calculating the percent difference $(\% D)$ for hydrogen and methane as follows:

$$
\% D=\frac{\left|\left(R F_{1}\right)-\left(R F_{c}\right)\right|}{\left(R F_{1}\right)} * 100
$$

where

$$
\begin{aligned}
& \boldsymbol{R} F_{1}=\text { average } \mathrm{RF} \text { from initial calibration (area/mole) } \\
& \boldsymbol{R} F_{c}=\mathrm{RF} \text { from the check standard (area/mole) }
\end{aligned}
$$

Alternately, the value of the calibration verification may be calculated and the percent recovery $(\% R)$ of the expected value determined.

The absolute RT for each analyte must fall within the RT window (Section 8.3) established for that analyte during the most recent initial calibration. If the RT window criterion is not met, corrective action must be taken. If no source of the problem can be determined after corrective action has been taken, a new three-point initial calibration curve must be generated.

\subsection{Retention Time Window}

8.4.1 The RT window for each analyte is determined by calculating the standard deviation of the absolute RT for each analyte. RT windows are determined by injecting a minimum of three standards over a period of 72 hours. RT windows shall be calculated as the mean plus or minus three times the standard deviation of the individual RTs for each calibration standard analyzed in the 72-hour period.

8.4.2 The RT window for hydrogen and methane must be determined whenever a new initial calibration curve is generated or when a new gas chromatographic column is installed.

8.4.3 For the calibration verification standard, the midpoint of the RT window shall be the average absolute RT for hydrogen and methane from the most recent initial calibration. 
8.4.4 The midpoint of the RT shall be the absolute RT for hydrogen and methane from the calibration verification standard for that calibration period.

\subsection{Calculations}

9.1 Qualitative Analysis. Hydrogen and methane are identified by RT. The RT must be within the RT window for the most recent calibration verification standard.

9.2 Quantitative Analysis. Once an analyte has been qualitatively identified, the quantitation of that analyte will be based on the area response. The column used for quantitation should be interferant-free in the RT window corresponding to the analyte.

Calculate the concentration in volume percent for hydrogen and methane in the sample as follows:

$$
\text { Concentration (vol. \%) }=\frac{A_{x}(R)\left(T_{s}\right)}{(R F)\left(V_{s}\right)\left(P_{s}\right)}
$$

with the variables as described in Equations (1) and (2).

If Equations ( 1 ) and (2) were not used to calculate the response factors, the samples must be adjusted for sample loop pressure in the same manner as the standards.

\subsection{Quality Control}

10.1 Each laboratory that uses this procedure is required to operate a formal quality control program. The laboratory must retain records to document the quality of the data generated. Each laboratory must have SOPs documenting and describing activities involved in utilizing this procedure. Specific quality control practices will include, but are not limited to, the analysis of quality control samples. The types of quality control samples, their associated frequency of analysis, acceptance criteria, and corrective action required if samples do not meet the acceptance criteria, is summarized in Table 3. Laboratory SOPs shall address requirements for preparing laboratory blanks, laboratory duplicates, and laboratory control samples.

10.2 All laboratories using this procedure must demonstrate acceptable performance prior to the analysis of actual samples. Demonstration of acceptance performance will be achieved by anaiyzing method performance samples (Table 3). These samples can be either commercially purchased or laboratory prepared, but must contain hydrogen and methane at concentrations appropriate (2 to 5 times the PRQLs) to determine the quality assurance objectives specified in Table 1. The analysis of seven samples must meet the criteria specified for precision, accuracy, and method detection limit (MDL) in Table 1. Demonstration of acceptable method and analyst performance must be repeated (by analyzing four samples) at a minimum of every six months. Method performance should be conducted over a period of several days to account for longterm variation. Corrective action must be implemented when acceptable method performance is not met. Precision will be determined as follows: 
TABLE 3

\section{Summary of Laboratory Quality Control Samples and Frequencies} for Hydrogen and Methane Analysis

\begin{tabular}{|c|c|c|c|}
\hline QC Sample & Minimum Frequency & $\begin{array}{l}\text { Acceptance } \\
\text { Criteria }\end{array}$ & $\begin{array}{l}\text { Corrective } \\
\text { Action }^{\mathrm{a}}\end{array}$ \\
\hline $\begin{array}{l}\text { Method performance } \\
\text { samples }\end{array}$ & $\begin{array}{l}\text { Seven (7) samples initially } \\
\text { and four (4) semiannually }\end{array}$ & Meet Table 1 QAOs & $\begin{array}{l}\text { Repeat until } \\
\text { acceptable }\end{array}$ \\
\hline Laboratory duplicates & $\begin{array}{l}\text { One (1) per analytical } \\
\text { batch }\end{array}$ & $R P D \leq 25^{b}$ & $\begin{array}{l}\text { Nonconformance if } \\
R P D>25\end{array}$ \\
\hline Laboratory blanks & $\begin{array}{l}\text { One (1) per analytical } \\
\text { batch }\end{array}$ & $\begin{array}{l}\text { Analyte concentrations } \\
<3 \times \mathrm{MDL}\end{array}$ & $\begin{array}{l}\text { Nonconformance if } \\
\text { analyte concentrations } \\
>3 \times \mathrm{MDL}\end{array}$ \\
\hline $\begin{array}{l}\text { Laboratory control } \\
\text { samples }\end{array}$ & $\begin{array}{l}\text { One (1) per analytical } \\
\text { batch }\end{array}$ & $70-130 \% R$ & $\begin{array}{l}\text { Nonconformance if } \\
\% R<70 \text { or }>130\end{array}$ \\
\hline Blind audit samples & $\begin{array}{l}\text { Samples and frequency } \\
\text { controlled by the Gas PDP } \\
\text { Plan }\end{array}$ & $\begin{array}{l}\text { Specified in the } \\
\text { Gas PDP Plan }\end{array}$ & $\begin{array}{l}\text { Specified in the } \\
\text { Gas PDP Plan }\end{array}$ \\
\hline
\end{tabular}

${ }^{a}$ Corrective Action when QC samples do not meet the acceptance criteria; Nonconformance procedures are outlined in Section 2,1.2.1 of the QAPP.

${ }^{b}$ Applies only to concentrations greater than the PRQL listed in Table 1.

MDL = Method detection limit

$\mathrm{PDP}=$ Performance Demonstration Program

$\mathrm{QAO}=$ Quality assurance objective

$\% \mathrm{R}=$ Percent recovery

$\mathrm{RPD}=$ Relative percent difference 
- For duplicate measurements, the precision expressed as the relative percent difference (RPD) is calculated as

$$
R P D=\frac{C_{1}-C_{2}}{\frac{\left(C_{1}+C_{2}\right)}{2}} * 100
$$

where

$$
\begin{array}{lll}
C_{1} \text { and } C_{2} & = & \text { two values obtained by analyzing the duplicate samples } \\
C_{1} & = & \text { larger of the two observed values }
\end{array}
$$

- For three or more replicate measurements, the precision expressed as the \%RSD is calculated as

$$
\% R S D=\frac{s}{\bar{y}} * 100
$$

where

$$
\begin{array}{lll}
s & = & \text { standard deviation } \\
y & = & \text { mean of replicate analyses }
\end{array}
$$

- The standard deviation (s) is defined as

$$
s=\sqrt{\sum_{i=1}^{n} \frac{\left(y_{i}-\bar{y}\right)^{2}}{n-1}}
$$

where

$$
\begin{array}{ll}
y_{i} & =\begin{array}{l}
\text { measured value of the } i^{\text {th }} \text { replicate sample analysis } \\
\text { measurement }
\end{array} \\
n & =\text { the number of replicate analyses }
\end{array}
$$

Accuracy will be determined as the percent recovery $(\% R)$ as follows:

$$
\% R=\frac{C_{m}}{C_{s m m}} * 100
$$

where

$$
\begin{array}{lll}
C_{m} & = & \text { measured concentration } \\
C_{s m m} & = & \text { true concentration }
\end{array}
$$

MDL will be determined as follows:

$$
\left.M D L=t_{(n-1,1-\alpha}=0.99\right) * s
$$

where

$$
\begin{array}{lll}
s & = & \text { standard deviation } \\
t_{(n-1,1-\alpha=0.99)}= & t \text {-distribution value appropriate to a 99-percent confidence level } \\
& \text { and a standard deviation estimate with } n-1 \text { degrees of freedom }
\end{array}
$$

10.3 The laboratory must analyze blanks at the frequency specified in Table 3 . The laboratory blanks will consist of ultra-high purity nitrogen or helium $(99.999$ percent pure). The same procedures used to prepare and analyze field samples will be used to 
prepare and analyze the laboratory blanks. Laboratory blank results will be acceptable if the concentrations of hydrogen and methane are less than 3 times the MDL specified in Table 1. Corrective action must be implemented for blanks which exceed this level.

10.4 The laboratory shall analyze field samples in duplicate. Single canisters shall be analyzed in duplicate at a frequency specified in Table 3 . Single canisters analyzed in duplicate shall be used to assess laboratory precision. Laboratory duplicate results shall be considered acceptable if the RPD is $\leq 25$ percent. RPD is determined as in equation (5). Duplicates which do not meet these criteria should be flagged.

10.5 The laboratory must analyze laboratory control samples at the frequency specified in Table 3. A laboratory control sample consists of a certified standard gas mixture and must be from sources independent of those used for instrument calibration. These samples must contain hydrogen and methane at concentrations in the calibration range of the instrument. The laboratory control sample analysis is designed to ensure that the laboratory's performance is in control for each analytical batch of samples processed. Laboratory control sample results will be considered acceptable if the \% $R$ is 70 to 130 percent of the certified known value. \%R is determined as in Equation (8). If the results are outside of this range, then new laboratory control samples must be prepared.

10.6 The laboratory will be required to analyze blind audit samples. These audit samples are part of the Performance Demonstration Program. Details of this program are in the Performance Demonstration Program Plan for the Analysis of Simulated Headspace Gases for the Transuranic Waste Characterization Program (Gas PDP Plan)(DOE 1995a).

\subsection{Procedure Performance}

As part of the Performance Demonstration Program (PDP), three blind audit samples containing the analytes listed in Table 1 were distributed to three laboratories. The laboratories analyzed four replicates of each sample. The results of the replicate analyses were used to determine precision and accuracy for each analyte in each sample, by laboratory. \%RSD was determined as in Equation (6), \%R was determined as in Equation (8). The precision and accuracy was then averaged for each analyte in each sample. Overall procedure performance is presented in Table 4.

\subsection{References}

ASTM. 1982. "Standard Method for Analysis of Reformed Gas by Gas Chromatography." ASTM 1946-82, Annual Book of ASTM Standards, Philadelphia, Pennsylvania, American Society for Testing and Materials.

ASTM. 1985. "Recommended Practice for General Gas Chromatography Procedures." ASTM E260-85, Annual Book of ASTM Standards, Philadelphia, Pennsylvania, American Society for Testing and Materials.

DOE. 1995a. Performance Demonstration Program Plan for the Analysis of Simulated Headspace Gases for the Transuranic Waste Characterization Program. CA0-95-1076, Current Revision, Carlsbad, New Mexico, Carlsbad Area Office, U.S. Department of Energy.

DOE. 1995b. Transuranic Waste Characterization Quality Assurance Program Plan. CAO-941010, Current Revision, Carisbad, New Mexico, Carlsbad Area Office, U.S. Department of Energy. 
Procedure Performance for Gas Chromatography

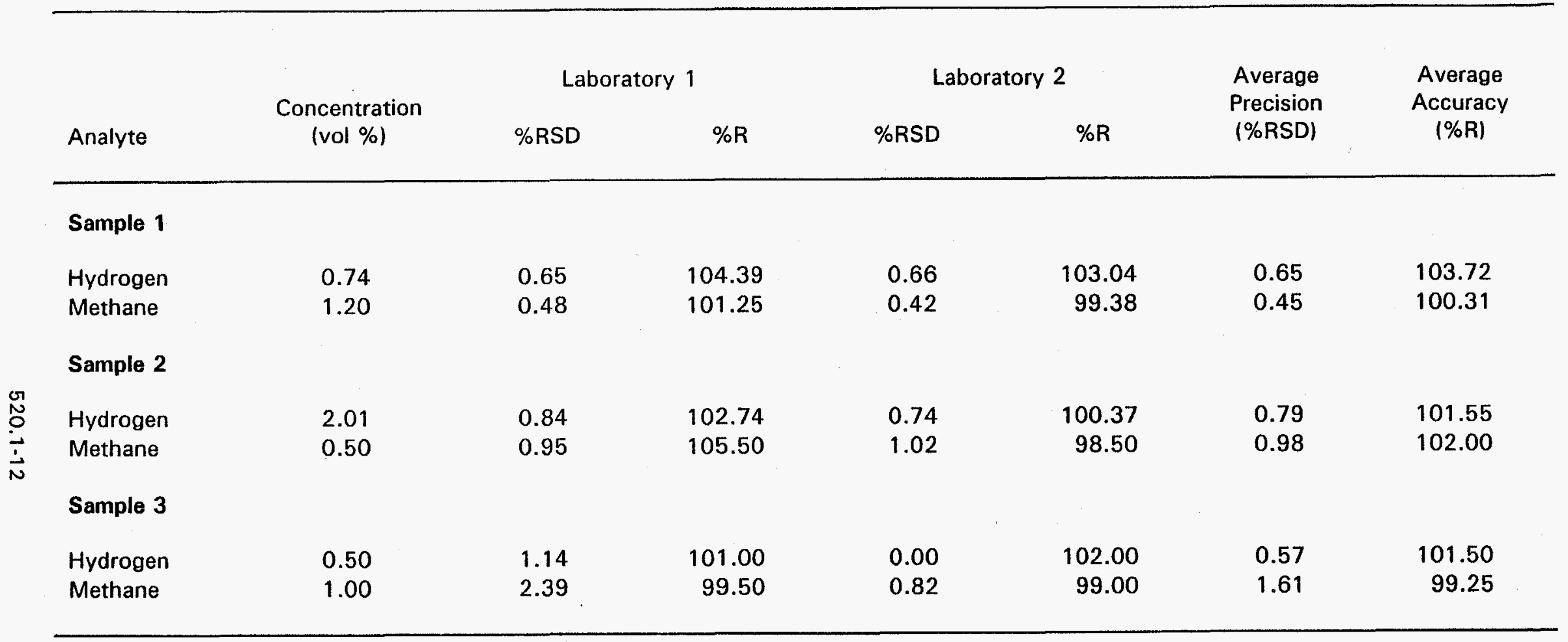




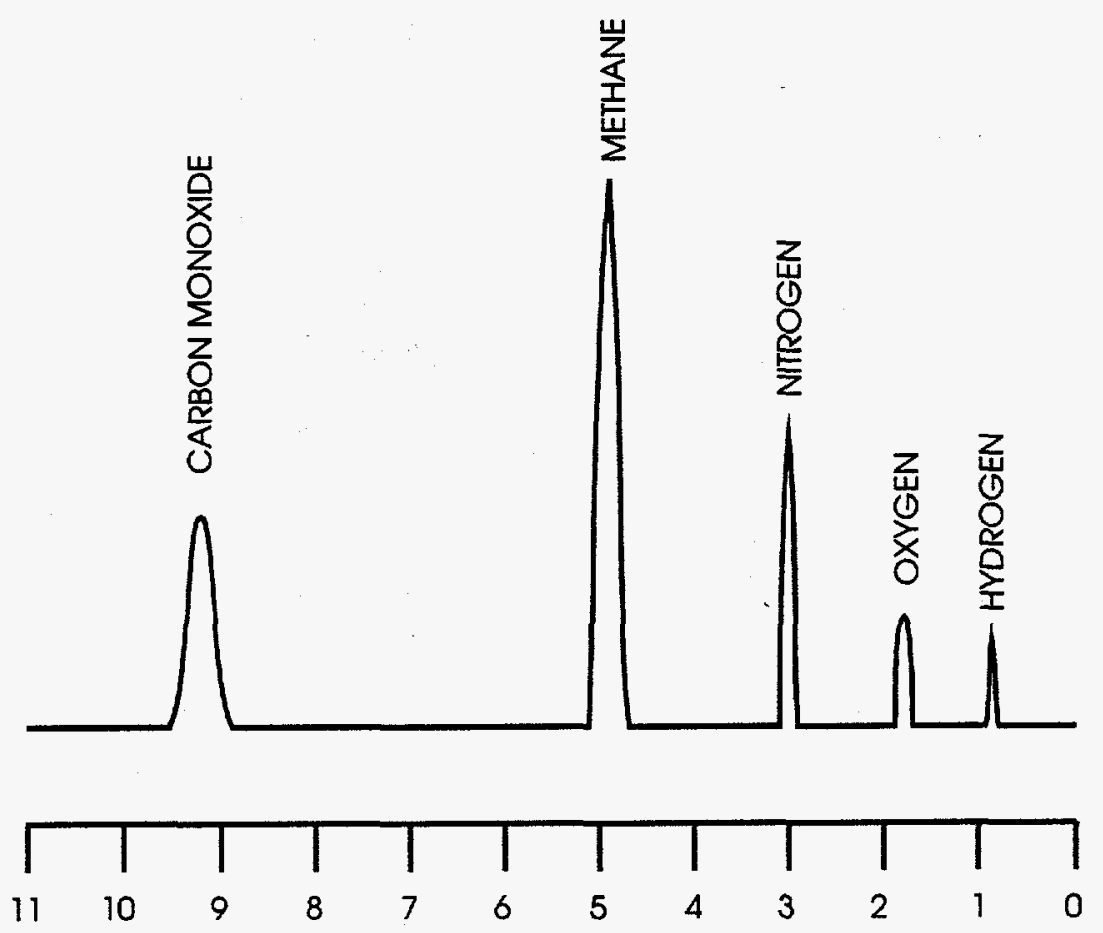

Column: $2 \mathrm{~m}$ by $6 \mathrm{~mm}$ inside dameter Type $13 x$ molecular sieves, 14 to 30 mesh Temperature: $35^{\circ} \mathrm{C}$

Flow rate: $60 \mathrm{~mL}$ hellum $/ \mathrm{min}$

Sample size: $0.5 \mathrm{~mL}$

FIGURE 1

Chromatogram of Reformed Gas on Molecular Sieve Column

(reproduced from ASTM Method 1946-82) 

PROCEDURE 610.1

\section{MICROWAVE ASSISTED ACID DIGESTION OF HOMOGENOUS SOLIDS AND SOIL/GRAVEL}

\section{Scope and Application}

1.1 This procedure is for the microwave assisted acid digestion of samples of homogenous solids and soil/gravel undergoing analysis for the following program-required total metals: arsenic, barium, beryllium, cadmium, chromium, lead, nickel, selenium, silver, and thallium (DOE 1995). This digestion procedure is only applicable to samples undergoing the following analytical procedures found in this Methods Manual: Inductively Coupled Plasma-Mass Spectrometry (ICP-MS), Inductively Coupled PlasmaAtomic Emission Spectroscopy (ICP-AES), Flame Atomic Absorption Spectroscopy (FLAA), and Graphite Furnace Atomic Absorption Spectroscopy (GFAA) (Procedures $630.1,640.1,650.1$, and 650.2, respectively, of this Methods Manual). Other total metals analytical procedures found in this Methods Manual include the appropriate digestion and sample preparation procedures. This procedure must be implemented with a site-specific standard operating procedure (SOP).

1.2 The analyst is referred to Method 3051 of Test Methods for Evaluating Solid Waste, Physical/Chemical Methods, SW-846, Third Edition, Final Update II (SW-846) (EPA 19951 for the specific requirements of this procedure.

1.3 For the purposes of the TRU Waste Characterization Program, samples are to be analyzed in analytical batches. An analytical batch is defined as a suite of samples that is processed as a unit, using the same analytical method, within a specific time period. An analytical batch can be up to 20 samples (excluding laboratory OC samples), all of which must be received by the laboratory within 14 days of the validated time of sample receipt (VTSR) of the first sample in the batch.

\subsection{Summary of Procedure}

The analyst is referred to Section 2.0 of SW-846 Method 3051 for a summary of this procedure.

3.0 Interferences

The analyst is referred to Section 3.0 of SW-846 Method 3051 for an explanation of potential interferences.

\subsection{Safety}

This procedure may involve the use of hazardous materials, operations, and equipment. It is the responsibility of whoever uses this procedure to consult appropriate site personnel concerning health and safety issues and establish appropriate health and safety practices. Consideration should be given to safety concerns regarding chemical and radiation exposure. Training regarding proper storage, usage, and disposal of chemicals is recommended.

\subsection{Apparatus and Materials}

The analyst is referred to Section 4.0 of SW-846 Method 3051 for a listing of the required apparatus and materials for this procedure. 


\subsection{Reagents}

The analyst is referred to Section 5.0 of SW-846 Method 3051 for description and listing of the required reagents for this procedure.

7.0 Sample Collection, Preservation, and Handling

7.1 Samples will be collected using the sampling procedures described in Procedure 120.1 of this Methods Manual.

7.2 All sample handling and chain-of-custody procedures described in Section 6.0 of the Transuranic Waste Characterization Quality Assurance Program Plan (QAPP) (DOE 1995) must be followed.

\subsection{Procedure}

The analyst is referred to Section 7.0 of SW-846 Method 3051 for a description of the procedural requirements. If Procedure 620.1 of this Methods Manual is to be used for sample cleanup, the final acid concentration of the digestates should be $4 M$ nitric acid.

\subsection{Calculations}

Does not apply to this procedure.

\subsection{Quality Control}

A laboratory blank, matrix spike, matrix spike duplicate, and a laboratory control sample must be included in each analytical batch. The analyst is referred to Section 10.0 of the specific analytical procedure found in this Methods Manual for appropriate quality control requirements.

\subsection{Procedure Performance}

The analyst is referred to Section 9.0 of SW-846 Method 3051 for a summary of method performance.

\subsection{References}

DOE. 1995. Transuranic Waste Characterization Quality Assurance Program Plan. CAO-941010, Current Revision, Carlsbad, New Mexico, Carlsbad Area Office, U.S. Department of Energy.

EPA. 1995. Test Methods for Evaluating Solid Waste, Physical/Chemical Methods. SW-846, Third Edition, Final Update I, and Final Update II, Washington D.C., Office of Solid Waste and Emergency Response, U.S. Environmental Protection Agency. 
PROCEDURE 620.1

\author{
EXTRACTION CHROMATOGRAPHY CLEANUP OF \\ HOMOGENOUS SOLIDS AND SOIL/GRAVEL SAMPLES \\ UNDERGOING TOTAL METALS ANALYSIS
}

\title{
1.0 Scope and Application
}

1.1 This procedure is for the cleanup of radioactive waste samples to be analyzed for the target analyte metals by Inductively Coupled Plasma-Mass Spectrometry (ICP-MS), Inductively Coupled Plasma-Atomic Emission Spectroscopy (ICP-AES), Flame Atomic Absorption Spectroscopy (FLAA), and Graphite Furnace Atomic Absorption Spectroscopy (GFAA) (Procedures 630.1, 640.1, 650.1, and 650.2, respectively, of this Methods Manual). This procedure applies extraction chromatography using an EIChroM ${ }^{\text {Tw }}$ transuranic specific (TRU•Spec) column which absorbs transuranic elements at high acid concentrations, but eludes them under low acid conditions. The TRU・Spec can be obtained as a pre-packed column consisting of an extractant, octyl(phenyl)-N-Ndiisobutyl-carbamoyl-methylphosphine oxide, (CMPO), dissolved in tri-butyl phosphate supported on an inert substrate. Its use is important for the elimination of emission interferences by removing the actinides, uranium, and thorium, or for easier sample handling by removing most alpha emitters. Samples prepared by this method may be analyzed by ICP-MS, ICP-AES, FLAA, or GFAA for the following total metals:

$\begin{array}{lll}\text { Antimony } & \text { Chromium } & \text { Silver } \\ \text { Arsenic } & \text { Lead } & \text { Thallium } \\ \text { Barium } & \text { Nickel } & \text { Vanadium } \\ \text { Beryllium } & \text { Selenium } & \text { Zinc } \\ \text { Cadmium } & & \end{array}$

1.2 For the purposes of the TRU Waste Characterization Program, samples are to be analyzed in analytical batches. An analytical batch is defined as a suite of samples that is processed as a unit, using the same analytical method, within a specific time period. An analytical batch can be up to 20 samples (excluding laboratory $\mathrm{OC}$ samples), all of which must be received by the laboratory within 14 days of the validated time of sample receipt (VTSR) of the first sample in the batch.

\subsection{Summary of Procedure}

Samples to be analyzed are first digested by the microwave assisted acid digestion procedure (Procedure 610.1 of this Methods Manual). The pre-packed column is conditioned by passing a high acid solution through it. A sample aliquot corresponding to the working capacity of the column is loaded on the column in a small amount of the high acid solution followed by a larger volume "wash" of high acid solution. All of the eluate is collected. An aliquot of the eluate is brought to a known volume using a $5 \%$ nitric acid solution. If an internal standard is employed, it is added at the time the sample is brought to a known volume. The sample can now be analyzed for the target analytes.

\subsection{Interferences}

3.1 To achieve efficient extraction of the actinides, the sample aliquot should correspond to the working capacity (maximum possible loading) of the column. The maximum capacity is 2 to $4 \mathrm{mg}$ of actinide based on americium-241 studies. Literature recommends loadings of 10 to 20 percent of capacity to ensure minimum analyte loss. However, if recovery of the actinides is not necessary, and only separation from the 
actinides is desired, 80 to 90 percent of the maximum loading capacity could be applied to the column. If capacity is a concern for a particular sample, a dilution of the sample should be performed and analyzed by ICP-MS or ICP-AES to determine if the capacity of the actinides has been met.

3.2 According to literature, breakthrough of the actinides can occur after passage of 65 free column volumes through the column ( 1 free column volume $=1.3 \mathrm{~mL} /$ column). To prevent breakthrough, the total volume passed through the column should be maintained below $65 \mathrm{~mL}$.

3.3 Ferric iron is absorbed by the TRU・Spec column and inhibits the retention of the actinides. Ferric iron should be reduced to ferrous iron to prevent this effect. This can be achieved by titrating the sample to endpoint with $1 M$ ascorbic acid using ammonium thiocyanate as an indicator. Thiocyanate forms a red complex with ferric iron. Reduction of ferric iron is indicated when the red color dissipates and the sample is colorless.

3.4 The use of hydrochloric acid is avoided here due to extensive use of stainless steel in radiochemical laboratories and glove box fabrication. Only nitric acid is used throughout this procedure.

\subsection{Safety}

This procedure may involve the use of hazardous materials, operations, and equipment. It is the responsibility of whoever uses this procedure to consult appropriate site personnel concerning health and safety issues and establish appropriate health and safety practices. Consideration should be given to safety concerns regarding chemical and radiation exposure. Training regarding proper storage, usage, and disposal of chemicals is recommended.

5.0 Apparatus and Materials

5.1 EIChroM ${ }^{m}$ TRU•Spec Pre-packed Column. (EIChroM Industries Inc., cat. no. TR-C50 or TR-C200).

5.2 Column Rack.

5.3 Volumetric Glassware.

5.4 Pipettes.

6.0 Reagents

6.1 $4 M$ Nitric Acid. Prepare this solution by adding $254 \mathrm{~mL}$ of concentrated nitric acid to $500 \mathrm{~mL}$ of distilled water and diluting to $1 \mathrm{~L}$ with ASTM Type II water.

$6.25 \%$ (wt\%) Nitric Acid. Add $72 \mathrm{~mL}$ of concentrated nitric acid to $500 \mathrm{~mL}$ of distilled water in a $1 \mathrm{~L}$ volumetric flask. Dilute to volume with ASTM Type II water.

7.0 Sample Collection, Preservation, and Handling

7.1 Samples will be collected using the sampling procedures described in Procedure 120.1 of this Methods Manual. 
7.2 All sample handling and chain-of-custody procedures described in Section 6.0 of the Transuranic Waste Characterization Quality Assurance Program Plan (QAPP) (DOE 1995) must be followed.

\subsection{Procedure}

8.1 Using Procedure 610.1 of the Methods Manual, digest the sample in nitric acid alone. Avoid using hydrochloric acid (see Section 3.4). Adjust the digested sample to $4 M$ nitric acid.

8.2 Remove the tip and the cap of the TRU•Spec column. Lower the frit to just above the top of the resin. Condition the column by passing 10 to $15 \mathrm{~mL}$ of $4 M$ nitric acid through the column.

8.3 Load on the column an amount of sample which meets the restrictions stated in Section 3.1. Follow with a wash of $4 M$ nitric acid being sure to maintain a total volume of less than $65 \mathrm{~mL}$ (see Section 3.2). Collect all of the eluate.

8.4 Pipet into a $25.0 \mathrm{~mL}$ volumetric flask a sample aliquot which is expected to be within the detection range of the instrument to be used for analysis. Dilute to volume with the acid matrix specified in the appropriate instrument analysis method. The sample may now be analyzed by ICP-MS, ICP-AES, FLAA, or GFAA for target analyte concentration.

\subsection{Calculations}

An overall dilution for each sample must be calculated which considers volume of aliquots(s), titrant, and acid washes.

\subsection{Quality Control}

10.1 All analytical batch quality control samples (i.e., laboratory blanks, matrix spikes, matrix spike duplicates, laboratory control samples) must undergo the extraction column cleanup.

10.2 An extraction blank consisting of nitric acid media alone should be carried through this procedure and used in the instrument calibration along with appropriate standards. Instrument calibration and subtraction of blanks should be followed as instructed in the particular analytical procedure being used. This will account for effects of the TRU・Spec matrix.

\subsection{Procedure Performance}

11.1 In a single laboratory evaluation, the recoveries of the elements being eluted from the TRU・Spec under high acid conditions were determined. In this study a $5 \mathrm{~mL} 4 M$ nitric acid solution was spiked with an analyte and loaded on the column followed by a $15 \mathrm{~mL}$ wash with $4 M$ nitric acid. This was performed in duplicate at room temperature under gravitational flow. The recoveries of the analytes in the $4 M$ nitric acid eluate were determined and averaged. These averages along with their relative standard deviations are shown in Table 1. None of the analytes were found to be retained by the TRU・Spec column.

11.2 Table 2 is a reconstruction of a table presented in a product brochure by ElChroM Industries, Inc. titled "Extraction Chromatographic Material for Rapid Separation of the Transuranic Elements." 
TABLE 1

Recovery of Elements from TRU•Spec Extraction

\begin{tabular}{|c|c|c|c|}
\hline Element $^{*}$ & $\begin{array}{l}\text { Radioactive } \\
\text { Tracer }\end{array}$ & $\begin{array}{c}\text { TRU•Spec } \\
4 M \text { nitric acid } \\
\% \text { recovery (\% RSD) }\end{array}$ & $\begin{array}{l}\text { TRU・Spec } \\
2 M \text { nitric acid } \\
\% \text { recovery }\end{array}$ \\
\hline Aluminum & - & $101(9)$ & 100 \\
\hline Antimony & ${ }^{124} \mathrm{Sb}$ & $100(<1)$ & NA \\
\hline Arsenic & ${ }^{76}$ As & $100(<1)$ & NA \\
\hline Barium & ${ }^{133} \mathrm{Ba}$ & $94(2)$ & 100 \\
\hline Beryllium & - & $101(11)$ & NA \\
\hline Cadmium & ${ }^{109} \mathrm{Cd}$ & $104(5)$ & 100 \\
\hline Calcium & - & $100(9)$ & 100 \\
\hline Chromium & ${ }^{51} \mathrm{Cr}$ & $93(2)$ & 100 \\
\hline Cobalt & ${ }^{80} \mathrm{Co}$ & $103(3)$ & 100 \\
\hline Copper & - & $101(10)$ & 100 \\
\hline Iron & ${ }^{59} \mathrm{Fe}$ & NA & 100 \\
\hline Lead & ${ }^{210} \mathrm{~Pb}$ & $108(6)$ & 100 \\
\hline Magnesium & - & $99(10)$ & 100 \\
\hline Manganese & ${ }^{54} \mathrm{Mn}$ & $99(<1)$ & 100 \\
\hline Mercury & ${ }^{203} \mathrm{Hg}$ & $101(19)$ & - \\
\hline Nickel & ${ }^{63} \mathrm{Ni}$ & $86(5)$ & 100 \\
\hline Potassium & ${ }^{42} \mathrm{~K}$ & $95(6)$ & 82 \\
\hline Selenium & ${ }^{75} \mathrm{Se}$ & 100 (NA) & NA \\
\hline Silver & ${ }^{110 m} \mathrm{Ag}$ & $96(4)$ & 100 \\
\hline Sodium & ${ }^{24} \mathrm{Na}$ & $96(5)$ & 93 \\
\hline Thallium & ${ }^{204} \mathrm{TI}$ & $99(1)$ & NA \\
\hline Vanadium & $\cdot$ & $99(11)$ & NA \\
\hline Zinc & ${ }^{65} \mathrm{Zn}$ & $97(2)$ & 100 \\
\hline
\end{tabular}

aElements in bold are Transuranic Waste Characterization Program required metals.

bData obtained from Oak Ridge National Laboratory with radioactive tracers and inactive tracers.

'Data obtained from Argonne National Laboratory by ICP-AES. 
TABLE 2

Elution of Selected Elements on a TRU•Spec Column

Fraction Eluting $(\%)$ by number of free column volumes

Element ${ }^{a}$

$\begin{array}{lllllll}1-5 & 6-10 & 11-15 & 16-20 & 21-25 & 26-30 & 31-40\end{array}$

\begin{tabular}{|c|c|c|c|c|c|c|c|}
\hline Aluminum & 99.8 & $<2.9$ & - & - & - & - & - \\
\hline Americium $^{b}$ & - & - & - & - & - & - & $<99$ \\
\hline Cerium & - & - & - & - & - & $<25$ & 75.0 \\
\hline Europium & - & - & - & - & - & - & $<99$ \\
\hline Iron & 102 & 12.3 & - & - & - & - & - \\
\hline Lanthanum & - & - & - & - & - & 30.0 & 72.0 \\
\hline Lithium & 98.4 & $<19$ & - & - & - & - & - \\
\hline Mercury & $(100)$ & $(60)$ & (19) & - & - & - & - \\
\hline Neodymium & - & - & - & - & - & - & 96.0 \\
\hline Potassium & 81.8 & 40.9 & - & - & - & - & - \\
\hline Praseodymium & - & - & - & - & - & - & 100 \\
\hline Ruthenium & 82.6 & $<19$ & - & - & - & - & - \\
\hline Samarium & - & - & - & $\because$ & - & - & 100 \\
\hline Sodium & 92.8 & $<1.2$ & - & - & - & - & - \\
\hline$x^{c}$ & 100 & - & - & - & - & - & . \\
\hline Yttrium & 23.4 & 76.8 & - & - & - & - & - \\
\hline Zirconium & - & - & - & - & - & - & 75 \\
\hline
\end{tabular}

aElements in bold are Transuranic Waste Characterization Program required metals.

bradiometric.

' $X$ represents the following elements: barium, cadmium, calcium, chromium, cobalt, copper, lead, magnesium, manganese, nickel, rhodium, silver, strontium, and zinc.

1-30 free column volume: $2 N$ nitric acid

31-40 free column volume: $0.05 \mathrm{~N}$ nitric acid

NOTE: These results were obtained from ICP-AES. Due to uncertainties inherent in this analysis, the fractions for each element may not total 100 percent. Values in parenthesis hold considerable uncertainty and should only be used as a rough guide. 


\subsection{References}

DOE. 1994. "Cleanup of Transuranic Liquid Wastes Using Extraction Chromatography." MP110R, DOE Methods for Evaluating Environmental and Waste Management Samples, Richland, Washington, Pacific Northwest Laboratory, U.S. Department of Energy.

DOE. 1995. Transuranic Waste Characterization Quality Assurance Program Plan. CAO-941010, Current Revision, Carlsbad, New Mexico, Carlsbad Area Office, U.S. Department of Energy. 
PROCEDURE 630.1

\section{METHOD 6020 FOR THE INDUCTIVELY COUPLED PLASMA-MASS SPECTROMETRY DETERMINATION OF TOTAL METALS IN HOMOGENOUS SOLIDS AND SOIL/GRAVEL}

\subsection{Scope and Application}

1.1 This procedure is for the determination of total metals in samples of homogenous solids and soil/gravel. The procedure employs Inductively Coupled Plasma-Mass Spectrometry (ICP-MS) for the quantitation of the analytes listed in Table 1. The quality assurance objectives for this procedure are also listed in Table 1. A sampling procedure for homogenous solids and soil/gravel is described in Procedure 120.1 of this Methods Manual. Use of this procedure is restricted to spectroscopists who are knowledgeable in the recognition, and correction, of spectral, chemical, and physical interferences in ICPMS. This procedure must be implemented with a site-specific standard operating procedure (SOP).

1.2 The analyst is referred to Method 6020 of Test Methods for Evaluating Solid Waste, Physical/Chemical Methods SW-846, Third Edition, Final Update II (SW-846) (EPA 1995) for the specific requirements of this procedure.

1.3 For the purposes of the TRU Waste Characterization Program, samples are to be analyzed in analytical batches. An analytical batch is defined as a suite of samples that is processed as a unit, using the same analytical method, within a specific time period. An analytical batch can be up to 20 samples (excluding laboratory OC samples), all of which must be received by the laboratory within 14 days of the validated time of sample receipt (VTSR) of the first sample in the batch.

\subsection{Summary of Procedure}

The analyst is referred to Section 2.2 of SW-846 Method 6020 for a summary of this procedure.

\subsection{Interferences}

The analyst is referred to Section 3.0 of SW-846 Method 6020 for an explanation of potential interferences.

\subsection{Safety}

This procedure may involve the use of hazardous materials, operations, and equipment. It is the responsibility of whoever uses this procedure to consult appropriate site personnel concerning health and safety issues and establish appropriate health and safety practices. Consideration should be given to safety concerns regarding chemical and radiation exposure. Training regarding proper storage, usage, and disposal of chemicals is recommended.

\subsection{Apparatus and Materials}

The analyst is referred to Section 4.0 of SW-846 Method 6020 for a listing of the required apparatus and materials for this procedure. 
TABLE 1

Target Analyte List and Quality Assurance Objectives for ICP-MS

\begin{tabular}{lcccccc}
\hline Analyte & CAS Number & $\begin{array}{c}\text { Precision } \\
(\% \text { RSD or RPD) }\end{array}$ & $\begin{array}{c}\text { Accuracy } \\
(\% \mathrm{R})^{\mathrm{b}}\end{array}$ & $\begin{array}{c}\text { PRDL } \\
(\mu \mathrm{g} / \mathrm{L})\end{array}$ & $\begin{array}{c}\text { PRQL } \\
(\mathrm{mg} / \mathrm{kg})\end{array}$ & $\begin{array}{c}\text { Completeness } \\
(\%)\end{array}$ \\
\hline Antimony & $7440-36-0$ & $\leq 30$ & $80-120$ & 100 & 100 & 90 \\
Arsenic & $7440-38-2$ & $\leq 30$ & $80-120$ & 100 & 100 & 90 \\
Barium & $7440-39-3$ & $\leq 30$ & $80-120$ & 2000 & 2000 & 90 \\
Beryllium & $7440-41-7$ & $\leq 30$ & $80-120$ & 100 & 100 & 90 \\
Cadmium & $7440-43-9$ & $\leq 30$ & $80-120$ & 20 & 20 & 90 \\
Chromium & $7440-47-3$ & $\leq 30$ & $80-120$ & 100 & 100 & 90 \\
Lead & $7439-92-1$ & $\leq 30$ & $80-120$ & 100 & 100 & 90 \\
Nickel & $7440-02-0$ & $\leq 30$ & $80-120$ & 100 & 100 & 90 \\
Silver & $7440-22-4$ & $\leq 30$ & $80-120$ & 100 & 100 & 90 \\
Thallium & $7440-28-0$ & $\leq 30$ & $80-120$ & 100 & 100 & 90 \\
Vanadium & $7440-62-2$ & $\leq 30$ & $80-120$ & 100 & 100 & 90 \\
Zinc & $7440-66-6$ & $\leq 30$ & $80-120$ & 100 & 100 & 90 \\
\hline
\end{tabular}

$\%$ RSD $\quad=$ Percent relative standard deviation

$\mathrm{RPD} \quad=$ Relative percent difference

$\% \mathrm{R} \quad=$ Percent recovery

PRDL $\quad$ = Program required detection limit (i.e., maximum permissible value for instrument detection limit)

PROL $\quad=$ Program required quantitation limit

a $30 \%$ control limits apply when sample and duplicate concentrations are $\geq 100 \times$ instrument detection limit. If less than this limit, the absolute difference between the two values shall be less than or equal to the PRDL.

${ }^{b}$ Applies to laboratory control samples. If a solid laboratory control sample material which has established statistical control limits is used, then the established control limits for that material should be used for accuracy requirements.

'PRDL set such that it is a factor of 10 below the PRQL for $100 \%$ solid samples, assuming a $100 \times$ dilution during digestion. 


\subsection{Reagents}

The analyst is referred to Section 5.0 of SW-846 Method 6020 for a description and listing of the required reagents, standard stock solutions, standard solutions, blanks, check standards, interference check solutions, and quality control samples for this procedure.

\subsection{Sample Collection, Preservation, and Handling}

7.1 Samples will be collected using the sampling procedures described in Procedure 120.1 of this Methods Manual.

7.2 All sample handling and chain-of-custody procedures described in Section 6.0 of the Transuranic Waste Characterization Quality Assurance Program Plan (QAPP) (DOE 1995b) must be followed.

\subsection{Procedure}

8.1 The sample preparation procedures described in Procedure 610.1 of this Methods Manual must be followed.

8.2 The sample clean up procedures described in Procedure 620.1 of this Methods Manual may be used.

8.3 The analyst is referred to Section 7.2 through 7.11 of SW-846 Method 6020 for a description of the procedural requirements. Table 2 of this procedure provides a summary of required calibration and analysis quality control.

\subsection{Calculations}

The analyst is referred to Section 7.12 of SW-846 Method 6020 for a description of the required calculations for this procedure. Any sample dilution must be taken into account. Note that all results must be reported in $\mathrm{mg} / \mathrm{kg}$ (wet weight basis). To convert analytical results into $\mathrm{mg} / \mathrm{kg}$ dry weight, the percent solid content must be determined on a separate sample aliquot from that used for digestion and analysis.

\subsection{Quality Control}

10.1 All of the quality control requirements specified in Section 8.1 through 8.5 of SW-846 Method 6020 are mandatory, as well as the requirements specified below. In instances where quality control specifications in this procedure differ from those in SW-846 Method 6020 , this procedure shall take precedence.

10.2 Each laboratory that uses this procedure is required to operate a formal quality control program. The laboratory must retain records to document the quality of the data generated. Each laboratory must have SOPs documenting and describing activities involved in using this procedure. Specific quality control practices will include, but are not limited to, the analysis of quality control samples. The types of quality control samples, their associated frequency of analysis, acceptance criteria, and corrective action required if samples do not meet the acceptance criteria, is summarized in Table 3. Laboratory SOPs shall address requirements for preparing matrix spikes, matrix spike duplicates, laboratory blanks, and laboratory control samples.

10.3 All laboratories using this procedure must demonstrate acceptable performance prior to the analysis of actual samples. Demonstration of acceptable performance will be achieved by analyzing method performance samples (Table 3). These samples can be either 


\begin{tabular}{|c|c|c|c|c|}
\hline Technique & Procedure & Frequency of Procedure & Acceptance Criteria & Corrective Action \\
\hline \multirow[t]{8}{*}{ ICP-MS } & Mass Tune & Daily & $\begin{array}{l}4 \text { Replicate } \% \text { RSD }<10 \text {; mass } \\
\text { calibration within } 0.1 \text { amu; } \\
\text { resolution }<1.0 \text { amu full width at } \\
10 \% \text { peak height }\end{array}$ & $\begin{array}{l}\text { Manufacturer } \\
\text { recommendations }\end{array}$ \\
\hline & \multirow[t]{2}{*}{$\begin{array}{l}1 \text {-pt. initial calibration } \\
\text { (1 standard and a blank) }\end{array}$} & \multirow[t]{2}{*}{ Daily } & $\begin{array}{l}90-110 \% R \text { for initial calibration } \\
\text { verification solution }\end{array}$ & \multirow[t]{2}{*}{$\begin{array}{l}\text { Repeat tune and initial } \\
\text { calibration }\end{array}$} \\
\hline & & & $\begin{array}{l}95-105 \% \mathrm{R} \text { for highest calibration } \\
\text { standard }\end{array}$ & \\
\hline & Continuing calibration & $\begin{array}{l}\text { Every } 10 \text { samples plus beginning and } \\
\text { end of run }\end{array}$ & $\begin{array}{l}90-110 \% R \text { for check standard; } \\
\text { blank must measure } \leq 5 \times 1 D L ; \\
\text { internal standard areas must be } \\
\leq 20 \% \mathrm{D} \text { of calibration blank } \\
\text { internal standard areas }\end{array}$ & $\begin{array}{l}\text { Correct problem, recalibrate } \\
\text { and rerun last } 10 \text { samples }\end{array}$ \\
\hline & $\begin{array}{l}\text { Interference correction } \\
\text { verification }\end{array}$ & $\begin{array}{l}\text { Beginning and end of run or every } 12 \\
\text { hours, whichever is more frequent }\end{array}$ & $\begin{array}{l}\text { Solution containing interferants } \\
\text { only must measure } \leq 5 \times 1 D L \text { for } \\
\text { interferants; solution containing } \\
\text { interferants plus analytes must be } \\
\text { within } 80-120 \% R \text { for all analytes }\end{array}$ & $\begin{array}{l}\text { Correct problem and } \\
\text { recalibrate }\end{array}$ \\
\hline & Serial difution & $\begin{array}{l}\text { once per analytical batch or per matrix } \\
\text { within an analytical batch }\end{array}$ & $\begin{array}{l}5 x \text { dilution of sample which is }> \\
100 x \text { IDL must be } \leq 10 \% D \text { of } \\
\text { initial value }\end{array}$ & Define in Laboratory SOPs \\
\hline & Post-digestion spike & $\begin{array}{l}\text { once per analytical batch or per matrix } \\
\text { within an analytical batch if serial } \\
\text { dilution, matrix spike, or matrix spike } \\
\text { duplicate does not meet acceptance } \\
\text { criteria }\end{array}$ & $75-125 \% R$ & Define in Laboratory SOPs \\
\hline & $\begin{array}{l}\text { Internal standard area } \\
\text { verification }\end{array}$ & Every sample & $\begin{array}{l}\text { Area must be } \leq 30 \% \mathrm{D} \text { of } \\
\text { calibration blank internal standard } \\
\text { areas }\end{array}$ & $\begin{array}{l}\text { Dilute sample } 5 x \text { and } \\
\text { reanalyze }\end{array}$ \\
\hline $\begin{array}{l}\% D \\
\% R\end{array}$ & $\begin{array}{l}=\text { Percent difference } \\
=\text { Percent recovery }\end{array}$ & $\begin{array}{l}\% R \\
\text { IDL }\end{array}$ & $\begin{array}{l}=\text { Percent relative standa } \\
=\text { Instrument detection lir }\end{array}$ & deviation \\
\hline
\end{tabular}


TABLE 3

\section{Summary of Laboratory Quality Control Samples and Frequencies for ICP-MS Total Metals Analyses}

\begin{tabular}{|c|c|c|c|}
\hline QC Samples & Minimum Frequency & $\begin{array}{l}\text { Acceptance } \\
\text { Criteria }\end{array}$ & Corrective Actions $^{a}$ \\
\hline $\begin{array}{l}\text { Method performance } \\
\text { samples }\end{array}$ & $\begin{array}{l}\text { Seven (7) samples initially, } \\
\text { and four (4) semiannually }\end{array}$ & Meet Table 1 QAOs & Repeat until acceptable \\
\hline Laboratory blanks & $\begin{array}{l}\text { One (1) per analytical } \\
\text { batch }\end{array}$ & $\leq 5 \times \mathrm{IDL}$ & $\begin{array}{l}\text { Redigest and reanalyze } \\
\text { any samples with } \\
\text { analyte concentrations } \\
\text { which are } \\
\leq 10 \times \text { blank value and } \\
\geq 0.5 \times \text { PROL }\end{array}$ \\
\hline Matrix spikes & $\begin{array}{l}\text { One (1) per analytical } \\
\text { batch }\end{array}$ & $80-120 \% R$ & $\begin{array}{l}\text { Nonconformance if } \% R \\
<80 \text { or }>120\end{array}$ \\
\hline Matrix spike duplicates & $\begin{array}{l}\text { One (1) per analytical } \\
\text { batch }\end{array}$ & $\begin{array}{l}R P D \leq 30 \\
80-120 \% R\end{array}$ & $\begin{array}{l}\text { Nonconformance if } \\
\text { RPD }>30 \text { or if } \% R< \\
80 \text { or }>120\end{array}$ \\
\hline $\begin{array}{l}\text { Laboratory control } \\
\text { samples }\end{array}$ & $\begin{array}{l}\text { One (1) per analytical } \\
\text { batch }\end{array}$ & $80-120 \% R$ & $\begin{array}{l}\text { Redigest and reanalyze } \\
\text { for affected analytes }\end{array}$ \\
\hline Blind audit samples & $\begin{array}{l}\text { Samples and frequency } \\
\text { controlled by the Solid } \\
\text { PDP Plan }\end{array}$ & $\begin{array}{l}\text { Specified in the Solid } \\
\text { PDP Plan }\end{array}$ & $\begin{array}{l}\text { Specified in the Solid } \\
\text { PDP Plan }\end{array}$ \\
\hline
\end{tabular}

${ }^{a}$ Corrective Actions when OC samples do not meet acceptance criteria; Nonconformance procedures are outlined in Section 2.1.2.1 of the QAPP.

$\mathrm{IDL}=$ Instrument detection limit

PDP $=$ Performance Demonstration Program

PROL $=$ Program required detection limit

$\% \mathrm{R}=$ Percent recovery

RPD $=$ Relative percent difference 
commercially purchased or laboratory prepared, but must contain all of the analytes listed in Table 1 at concentrations appropriate (2-5 times the PROLs) to determine the quality assurance objectives specified in Table 1. The analysis of seven samples must meet the criteria specified for precision, accuracy, and program required detection limit (PRDL) in Table 1. Note that the instrument detection limit (IDL) must be less than or equal to the PRDL. Demonstration of acceptable method and analyst performance must be repeated (by analyzing four samples) at a minimum of every six months. Method performance should be conducted over a period of several days to account for long-term variability. Corrective action must be implemented when acceptable method performance is not met. Precision will be determined as follows:

- For duplicate measurements, the precision expressed as the relative percent difference (RPD) is calculated as

$$
R P D=\frac{C_{1}-C_{2}}{\frac{\left(C_{1}+C_{2}\right)}{2}} * 100
$$

where

$\begin{array}{lll}C_{1} \text { and } C_{2} & = & \text { two values obtained by analyzing the duplicate samples } \\ C_{1} & = & \text { larger of the two observed values }\end{array}$

- For three or more replicate measurements, the precision expressed as the percent relative standard deviation (\%RSD) is calculated as

$$
\% R S D=\frac{s}{\bar{y}} * 100
$$

where

$$
\begin{array}{lll}
\frac{s}{y} & = & \text { standard deviation } \\
& = & \text { mean of replicate analyses }
\end{array}
$$

- The standard deviation $(s)$ is defined as

$$
s=\sqrt{\sum_{i=1}^{n} \frac{\left(y_{i}-\bar{y}\right)^{2}}{n-1}}
$$

where

$$
\begin{array}{ll}
y_{i} \quad & \begin{array}{l}
\text { measured value of the } i^{\text {th }} \text { replicate sample analysis } \\
\text { measurement }
\end{array} \\
n & =\quad \text { number of replicate analyses }
\end{array}
$$

Accuracy will be determined as the percent recovery $(\% \mathrm{R})$ as follows:

$$
\% R=\frac{C_{m}}{C_{s r m}} * 100
$$

where

$$
\begin{array}{lll}
C_{m} & = & \text { measured concentration } \\
C_{s r m} & = & \text { true concentration }
\end{array}
$$


IDL will be determined as follows:

$$
I D L=t_{(n-1,1-\alpha=0.99)} * s
$$

where

$$
\begin{array}{lll}
s & = & \text { standard deviation } \\
\left.t_{(n-1,1-\alpha}=0.99\right) & = & t \text {-distribution value appropriate to a 99-percent confidence level } \\
& \text { and a standard deviation estimate with } n-1 \text { degrees of freedom }
\end{array}
$$

10.4 The laboratory must analyze blanks at the frequency specified in Table 3 . The same procedures used to prepare and analyze field samples will be used to prepare and analyze the laboratory blanks. Laboratory blank results will be acceptable if the concentration of target analytes is less than or equal to 5 times the IDL. Corrective action must be implemented if blanks exceed this level.

10.5 The laboratory must analyze matrix spikes at the frequency specified in Table 3 . Matrix spike results shall be considered acceptable if the \%R is 80 to 120 percent of the true added value. Matrix spikes which do not meet these criteria should be flagged. \%R will be determined as follows:

$$
\% R=\frac{S-U}{C_{s a}} * 100
$$
where
$S=$
$U=$
$C_{s a}=$
measured concentration in the spiked aliquot measured concentration in the unspiked aliquot actual concentration of the spike added

10.6 The laboratory must analyze matrix spike duplicates at the frequency specified in Table 3 . Matrix spike duplicate results shall be considered acceptable if the RPD is $\leq 30$ and the $\% R$ is $80-120$. RPD is determined as in Equation (1), \%R is determined as in Equation (6). Duplicates which do not meet these criteria should be flagged.

10.7 The laboratory must analyze laboratory control samples at the frequency specified in Table 3. Solid laboratory control samples (e.g., characterized surrogate sludges) should be used whenever it is possible to match the matrix of the waste samples. Otherwise, commercially purchased standards shall be used to prepare laboratory control samples. The laboratory control samples must contain the metals being analyzed, must undergo the same preparation and clean-up methods as the samples, and be quantitated within the calibration range of the instrument. The laboratory control sample analysis is designed to ensure that the laboratory's performance is in control for each analytical batch of samples processed. Laboratory control sample results will be considered acceptable if the \% $R$ is 80 to 120 percent of the known value. $\% R$ is calculated as in Equation (4). If the results are outside of this range, then the associated samples must be reprepared and reanalyzed for the affected analytes.

10.8 The laboratory will be required to analyze blind audit samples. These audit samples are part of the Performance Demonstration Program. Details of this program are in the Performance Demonstration Program Plan for the Analysis of Solidified Wastes for the Transuranic Waste Characterization Program (Solid PDP Plan)(DOE 1995a).

\subsection{Procedure Performance}

The analyst is referred to Section 9.0 of SW-846 Method 6020 for a summary of method performance associated with this procedure. 


\subsection{References}

DOE. 1995a. Performance Demonstration Program Plan for the Analysis of Solidified Wastes for the Transuranic Waste Characterization Program. CAO-95-1077, Current Revision, Carlsbad, New Mexico, Carisbad Area Office, U.S. Department of Energy.

DOE. 1995b. Transuranic Waste Characterization Quality Assurance Program Plan. CAO-941010, Current Revision, Carlsbad, New Mexico, Carlsbad Area Office, U.S. Department of Energy.

EPA. 1995. Test Methods for Evaluating Solid Waste, Physical/Chemical Methods. SW-846, Third Edition, Final Update I, and Final Update II, Washington D.C., Office of Solid Waste and Emergency Response, U.S. Environmental Protection Agency. 
PROCEDURE 640.1

\section{METHOD 6010A FOR THE INDUCTIVELY COUPLED PLASMA-ATOMIC EMISSION SPECTROSCOPY DETERMINATION OF TOTAL METALS IN HOMOGENOUS SOLIDS AND SOIL/GRAVEL}

\subsection{Scope and Application}

1.1 This procedure is for the determination of total metals in samples of homogenous solids and soil/gravel. The procedure employs Inductively Coupled Plasma-Atomic Emission Spectroscopy (ICP-AES) for the quantitation of the analytes listed in Table 1 . The quality assurance objectives for this procedure are also listed in Table 1. A sampling procedure for homogenous solids and soil/gravel is described in Procedure 120.1 of this Methods Manual. Use of this procedure is restricted to spectroscopists who are knowledgeable in the recognition, and correction, of spectral, chemical, and physical interferences in ICPAES. This procedure must be implemented with a site-specific standard operating procedure (SOP).

1.2 The analyst is referred to Method 6010A of Test Methods for Evaluating Solid Waste, Physical/Chemical Methods, SW-846, Third Edition, Final Update I (SW-846) (EPA 1995) for the specific requirements of this procedure.

1.3 For the purposes of the TRU Waste Characterization Program, samples are to be analyzed in analytical batches. An analytical batch is defined as a suite of samples that is processed as a unit, using the same analytical method, within a specific time period. An analytical batch can be up to 20 samples (excluding laboratory $Q C$ samples), all of which must be received by the laboratory within 14 days of the validated time of sample receipt (VTSR) of the first sample in the batch.

\subsection{Summary of Procedure}

The analyst is referred to Section 2.2 of SW-846 Method 6010A for a summary of this procedure.

\subsection{Interferences}

The analyst is referred to Section 3.0 of SW-846 Method 6010A for an explanation of potential interferences.

\subsection{Safety}

This procedure may involve the use of hazardous materials, operations, and equipment. It is the responsibility of whoever uses this procedure to consult appropriate site personnel concerning health and safety issues and establish appropriate health and safety practices. Consideration should be given to safety concerns regarding chemical and radiation exposure. Training regarding proper storage, usage, and disposal of chemicals is recommended.

\subsection{Apparatus and Materials}

The analyst is referred to Section 4.0 of SW-846 Method 6010A for a listing of the required apparatus and materials for this procedure. 


\section{TABLE 1}

Target Analyte List and Quality Assurance Objectives for ICP-AES

\begin{tabular}{lcccccc}
\hline Analyte & CAS Number & $\begin{array}{c}\text { Precision } \\
(\% R S D \text { or RPD })^{\mathrm{a}}\end{array}$ & $\begin{array}{c}\text { Accuracy } \\
(\% \mathrm{R})^{\mathrm{b}}\end{array}$ & $\begin{array}{c}\text { PRDL } \\
(\mu \mathrm{g} / \mathrm{L})\end{array}$ & $\begin{array}{c}\text { PROL } \\
(\mathrm{mg} / \mathrm{kg})\end{array}$ & $\begin{array}{c}\text { Completeness } \\
(\%)\end{array}$ \\
\hline Antimony & $7440-36-0$ & $\leq 30$ & $80-120$ & 100 & 100 & 90 \\
Arsenic & $7440-38-2$ & $\leq 30$ & $80-120$ & 100 & 100 & 90 \\
Barium & $7440-39-3$ & $\leq 30$ & $80-120$ & 2000 & 2000 & 90 \\
Beryllium & $7440-41-7$ & $\leq 30$ & $80-120$ & 100 & 100 & 90 \\
Cadmium & $7440-43-9$ & $\leq 30$ & $80-120$ & 20 & 20 & 90 \\
Chromium & $7440-47-3$ & $\leq 30$ & $80-120$ & 100 & 100 & 90 \\
Lead & $7439-92-1$ & $\leq 30$ & $80-120$ & 100 & 100 & 90 \\
Nickel & $7440-02-0$ & $\leq 30$ & $80-120$ & 100 & 100 & 90 \\
Selenium & $7782-49-2$ & $\leq 30$ & $80-120$ & 20 & 20 & 90 \\
Silver & $7440-22-4$ & $\leq 30$ & $80-120$ & 100 & 100 & 90 \\
Thallium & $7440-28-0$ & $\leq 30$ & $80-120$ & 100 & 100 & 90 \\
Vanadium & $7440-62-2$ & $\leq 30$ & $80-120$ & 100 & 100 & 90 \\
Zinc & $7440-66-6$ & $\leq 30$ & $80-120$ & 100 & 100 & 90 \\
\hline
\end{tabular}

\footnotetext{
$\%$ RSD $\quad=$ Percent relative standard deviation

RPD $\quad=$ Relative percent difference

$\% R \quad \quad=$ Percent recovery

PRDL $\quad=$ Program required detection limit (i.e., maximum permissible value for instrument detection limit)

PROL $\quad=$ Program required quantitation limit
}

$a \leq 30 \%$ control limits apply when sample and duplicate concentrations are $\geq 10 \times$ the instrument detection limit. If less than this limit, the absolute difference between the two values shall be less than or equal to the PRDL.

${ }^{b}$ Applies to laboratory control samples. If a solid laboratory control sample material which has established statistical control limits is used, then the established control limits for that material should be used for accuracy requirements.

CPROL set such that it is a factor of 10 below the PROL for $100 \%$ solid samples, assuming a $100 \mathrm{X}$ dilution during digestion. 


\subsection{Reagents}

The analyst is referred to Section 5.0 of SW-846 Method 6010A for a description and listing of the required reagents, standard stock solutions, standard solutions, blanks, check standards, interference check solutions, and quality control samples for this procedure.

\subsection{Sample Collection, Preservation, and Handling}

7.1 Samples will be collected using the sampling procedures described in Procedure 120.1 of this Methods Manual.

7.2 All sample handling and chain-of-custody procedures described in Section 6.0 of the Transuranic Waste Characterization Quality Assurance Program Plan (QAPP) (DOE 1995b) must be followed.

\subsection{Procedure}

8.1 The sample preparation procedures described in Procedure 610.1 of this Methods Manual must be followed.

8.2 The sample clean up procedures described in Procedure 620.1 may be used.

8.3 The analyst is referred to Section 7.2 through 7.5 of SW-846 Method 6010A for a description of the procedural requirements. Table 2 of this procedure provides a summary of required calibration and analysis quality control.

\subsection{Calculations}

Read the metal analyte concentration from the calibration curve or directly from the instrument readout. Any sample dilution must be taken into account. Note that all results must be reported in $\mathrm{mg} / \mathrm{kg}$ (wet weight basis). To convert analytical results into $\mathrm{mg} / \mathrm{kg}$ dry weight, the percent solid content must be determined on a separate sample aliquot from that used for digestion and analysis.

\subsection{Quality Control}

10.1 All of the quality control requirements specified in Section 8.1 through 8.5 of SW-846 Method 6010A are mandatory as well as the requirements specified below. In instances where quality control specifications in this procedure differ from those in SW-846 Method $6010 \mathrm{~A}$, this procedure shall take precedence.

10.2 Each laboratory that uses this procedure is required to operate a formal quality control program. The laboratory must retain records to document the quality of the data generated. Each laboratory must have SOPs documenting and describing activities involved in using this procedure. Specific quality control practices will include, but are not limited to, the analysis of quality control samples. The types of quality control samples, their associated frequency of analysis, acceptance criteria, and corrective action required if samples do not meet the acceptance criteria, is summarized in Table 3. Laboratory SOPs shall address requirements for preparing matrix spikes, matrix spike duplicates, laboratory blanks, and laboratory control samples.

10.3 All laboratories using this procedure must demonstrate acceptable performance prior to the analysis of actual samples. Demonstration of acceptable performance will be achieved by analyzing method performance samples (Table 3). These samples can be either commercially purchased or laboratory prepared, but must contain all of the analytes listed 
TABLE 2

Summary of Calibration Requirements and Analysis Quality Control for ICP-AES Total Metals Analysis

\begin{tabular}{|c|c|c|c|c|}
\hline Technique & Procedure & Frequency of Procedure & Acceptance Criteria & Corrective Action \\
\hline \multirow[t]{5}{*}{ ICP-AES } & $\begin{array}{l}\text { 1-pt. initial } \\
\text { calibration } \\
(1 \text { standard and a } \\
\text { blank) }\end{array}$ & Daily & $\begin{array}{l}90-110 \% R \text { for initial calibration } \\
\text { verification solution } \\
95-105 \% R \text { for highest calibration } \\
\text { standard }\end{array}$ & $\begin{array}{l}\text { Correct problem and repeat } \\
\text { initial calibration }\end{array}$ \\
\hline & $\begin{array}{l}\text { Continuing } \\
\text { calibration }\end{array}$ & $\begin{array}{l}\text { Every } 10 \text { samples plus beginning } \\
\text { and end of run }\end{array}$ & $\begin{array}{l}90-110 \% R \text { for check standard; blank } \\
\text { must measure } \leq 3 \times 1 D L\end{array}$ & $\begin{array}{l}\text { Recalibrate and rerun last } \\
10 \text { samples }\end{array}$ \\
\hline & $\begin{array}{l}\text { Interference } \\
\text { correction } \\
\text { verification }\end{array}$ & $\begin{array}{l}\text { Beginning and end of run or } \\
\text { twice per } 8 \text { hours, whichever is } \\
\text { more frequent }\end{array}$ & $\begin{array}{l}\text { Solution containing interferants only must } \\
\text { measure } \leq 3 \times 1 D L \text { for interferants; solution } \\
\text { containing interferants plus analytes must } \\
\text { be } 80-120 \% \text { for all analytes }\end{array}$ & $\begin{array}{l}\text { Correct problem and } \\
\text { recalibrate }\end{array}$ \\
\hline & Serial dilution & $\begin{array}{l}\text { once per analytical batch or per } \\
\text { matrix within an analytical batch }\end{array}$ & $\begin{array}{l}5 x \text { dilution of sample which is }>10 \times 1 D L \\
\text { must be } \leq 10 \% D \text { of initial value }\end{array}$ & Define in Laboratory SOPs \\
\hline & $\begin{array}{l}\text { Post-digestion } \\
\text { spike }\end{array}$ & $\begin{array}{l}\text { once per analytical batch or per } \\
\text { matrix within an analytical batch } \\
\text { if serial dilution, matrix spike, or } \\
\text { matrix spike duplicate does not } \\
\text { meet acceptance criteria }\end{array}$ & $75-125 \% R$ & Define in Laboratory SOPs \\
\hline
\end{tabular}


TABLE 3

\section{Summary of Laboratory Quality Control Samples and Frequencies for ICP-AES Total Metals Analyses}

\begin{tabular}{|c|c|c|c|}
\hline OC Samples & Minimum Frequency & $\begin{array}{l}\text { Acceptance } \\
\text { Criteria }\end{array}$ & Corrective Actions ${ }^{a}$ \\
\hline $\begin{array}{l}\text { Method performance } \\
\text { samples }\end{array}$ & $\begin{array}{l}\text { Seven (7) samples initially, } \\
\text { and four (4) semiannually }\end{array}$ & Meet Table 1 QAOs & Repeat until acceptable \\
\hline Laboratory blanks & $\begin{array}{l}\text { One (1) per analytical } \\
\text { batch }\end{array}$ & $\leq 3 \times \mathrm{IDL}$ & $\begin{array}{l}\text { Redigest and reanalyze } \\
\text { any samples with } \\
\text { analyte concentrations } \\
\text { which are } \\
\leq 10 \times \text { blank value and } \\
\geq 0.5 \times \text { PROL }\end{array}$ \\
\hline Matrix spikes & $\begin{array}{l}\text { One (1) per analytical } \\
\text { batch }\end{array}$ & $80-120 \% R$ & $\begin{array}{l}\text { Nonconformance if } \% R \\
<80 \text { or }>120\end{array}$ \\
\hline Matrix spike duplicates & $\begin{array}{l}\text { One (1) per analytical } \\
\text { batch }\end{array}$ & $\begin{array}{l}\text { RPD } \leq 30 \\
80-120 \% R\end{array}$ & $\begin{array}{l}\text { Nonconformance if } \\
\text { RPD }>30 \text { or if } \% R< \\
80 \text { or }>120\end{array}$ \\
\hline $\begin{array}{l}\text { Laboratory control } \\
\text { samples }\end{array}$ & $\begin{array}{l}\text { One (1) per analytical } \\
\text { batch }\end{array}$ & $80-120 \% R$ & $\begin{array}{l}\text { Redigest and reanalyze } \\
\text { for affected analytes }\end{array}$ \\
\hline Blind audit samples & $\begin{array}{l}\text { Samples and frequency } \\
\text { controlled by the Solid } \\
\text { PDP Plan }\end{array}$ & $\begin{array}{l}\text { Specified in the Solid } \\
\text { PDP Plan }\end{array}$ & $\begin{array}{l}\text { Specified in the Solid } \\
\text { PDP Plan }\end{array}$ \\
\hline
\end{tabular}

${ }^{a}$ Corrective Actions when $\mathrm{OC}$ samples do not meet acceptance criteria; Nonconformance procedures are outlined in Section 2.1.2.1 of the QAPP.

IDL $=$ Instrument detection limit

PDP $=$ Performance Demonstration Program

PROL $=$ Program required detection limit

$\% \mathrm{R}=$ Percent recovery

$\mathrm{RPD}=$ Relative percent difference 
in Table 1 at concentrations appropriate (2-5 times the PROLs) to determine the quality assurance objectives specified in Table 1 . The analysis of seven samples must meet the criteria specified for precision, accuracy, and program required detection limit (PRDL) in Table 1. Note that the instrument detection limit (IDL) must be less than or equal to the PRDL. Demonstration of acceptable method and analyst performance must be repeated (by analyzing four samples) at a minimum of every six months. Method performance should be conducted over a period of several days to account for long-term variability. Corrective action must be implemented when acceptable method performance is not met. Precision will be determined as follows:

- For duplicate measurements, the precision expressed as the relative percent difference (RPD) is calculated as

$$
R P D=\frac{C_{1}-C_{2}}{\frac{\left(C_{1}+C_{2}\right)}{2}} * 100
$$

where

$$
\begin{array}{lll}
C_{1} \text { and } C_{2} & = & \text { two values obtained by analyzing the duplicate samples } \\
C_{1} & = & \text { larger of the two observed values }
\end{array}
$$

- For three or more replicate measurements, the precision expressed as the percent relative standard deviation (\%RSD) is calculated as

$$
\% R S D=\frac{s}{\bar{y}} * 100
$$

where

$$
\begin{array}{lll}
\frac{s}{y} & = & \text { standard deviation } \\
& = & \text { mean of replicate analyses }
\end{array}
$$

- The standard deviation $(s)$ is defined as

$$
s=\sqrt{\sum_{i=1}^{n} \frac{\left(y_{i}-\bar{y}\right)^{2}}{n-1}}
$$

where

$$
\begin{array}{ll}
y_{i} & =\begin{array}{l}
\text { measured value of the } i^{\text {th }} \text { replicate sample analysis } \\
\text { measurement }
\end{array} \\
n & =\text { number of replicate analyses }
\end{array}
$$

Accuracy will be determined as the percent recovery $(\% R)$ as follows:

$$
\% R=\frac{C_{m}}{C_{s m m}} * 100
$$

where

$$
\begin{array}{lll}
C_{m} & = & \text { measured concentration } \\
C_{s r m} & = & \text { true concentration }
\end{array}
$$


IDL will be determined as follows:

$$
I D L=t_{(n-1,1-\alpha=0.99)} * s
$$

where

$$
\begin{array}{lll}
s & = & \text { standard deviation } \\
t_{(n-1,1-\alpha=0.99)}= & \begin{array}{l}
t \text {-distribution value appropriate to a 99-percent confidence level } \\
\text { and a standard deviation estimate with } n-1 \text { degrees of freedom }
\end{array}
\end{array}
$$

10.4 The laboratory must analyze blanks at the frequency specified in Table 3 . The same procedures used to prepare and analyze field samples will be used to prepare and analyze the laboratory blanks. Laboratory blank results will be acceptable if the concentration of target analytes is less than or equal to 3 times the IDL. Corrective action must be implemented if blanks exceed this level.

10.5 The laboratory must analyze matrix spikes at the frequency specified in Table 3 . Matrix spike results shall be considered acceptable if the \%R is 80 to 120 percent of the true value. Matrix spikes which do not meet these criteria should be flagged. \%R will be determined as follows:

$$
\% R=\frac{S-U}{C_{s a}} * 100
$$

where
$S=$
$U=$
$C_{s a}=$

measured concentration in the spiked aliquot measured concentration in the unspiked aliquot actual concentration of the spike added

10.6 The laboratory must analyze matrix spike duplicates at the frequency specified in Table 3. Matrix spike duplicate results shall be considered acceptable if the RPD is $\leq 30$ and the $\% R$ is 80 to 120. RPD is determined as in Equation (1), \%R is determined as in Equation (6). Duplicates which do not meet these criteria should be flagged.

10.7 The laboratory must analyze laboratory control samples at the frequency specified in Table 3. Solid laboratory control samples (e.g., characterized surrogate sludges) should be used whenever it is possible to match the matrix of the waste samples. Otherwise, commercially purchased standards shall be used to prepare laboratory control samples. The laboratory control samples must contain the metals being analyzed, must undergo the same preparation and cleanup methods as the samples, and be quantitated within the calibration range of the instrument. The laboratory control sample analysis is designed to ensure that laboratory performance is in control for each analytical batch of samples processed. Laboratory control sample results will be considered acceptable if the \% $R$ is 80 to 120 percent of the known value. \%R is calculated as in Equation (4). If the results are outside of this range, then the associated samples must be reprepared and reanalyzed for the affected analytes.

10.8 The laboratory will be required to analyze blind audit samples. These audit samples are part of the Performance Demonstration Program. Details of this program are in the Performance Demonstration Program Plan for the Analysis of Solidified Waste for the Transuranic Waste Characterization Program (Solid PDP Plan)(DOE 1995a).

\subsection{Procedure Performance}

The analyst is referred to Section 9.0 of SW-846 Method 6010A for a summary of method performance. 


\subsection{References}

DOE. 1995a. Performance Demonstration Program Plan for the Analysis of Solidified Waste for the Transuranic Waste Characterization Program. CA0-95-1077, Current Revision, Carlsbad, New Mexico, Carlsbad Area Office, U.S. Department of Energy.

DOE. 1995b. Transuranic Waste Characterization Quality Assurance Program Plan. CAO-941010, Current Revision, Carlsbad, New Mexico, Carlsbad Area Office, U.S. Department of Energy.

EPA. 1995. Test Methods for Evaluating Solid Waste, Physical/Chemical Methods. SW-846, Third Edition, Final Update I, and Final Update II, Washington D.C., Office of Solid Waste and Emergency Response, U.S. Environmental Protection Agency. 
PROCEDURE 650.1.

\section{FLAME ATOMIC ABSORPTION SPECTROSCOPY DETERMINATION OF TOTAL METALS IN HOMOGENOUS SOLIDS AND SOIL/GRAVEL}

\subsection{Scope and Application}

1.1 This procedure is for the determination of total metals in samples of homogenous solids and soil/gravel. The procedure is actually a combination of eight separate analytical methods found in Test Methods for Evaluating Solid Waste, Physical/Chemical Methods, SW-846, Third Edition, Final Update I, and Final Update II (SW-846) (EPA 1995). Table 1 lists the individual SW-846 method that applies to the specific metal analyte of interest. In addition to the analyte-specific methods listed in Table 1 , the analyst is referred to SW846 Method 7000A for a general discussion of Flame Atomic Absorption Spectroscopy (FLAA) methods. The procedure employs FLAA for the quantitation of analytes listed in Table 2. The quality assurance objectives for this procedure are also listed in Table 2. A sampling procedure for homogenous solids and soil/gravel is described in Procedure 120.1 of this Methods Manual. This procedure must be implemented with a site-specific standard operating procedure (SOP).

1.2 For the purposes of the TRU Waste Characterization Program, samples are to be analyzed in analytical batches. An analytical batch is defined as a suite of samples that is processed as a unit, using the same analytical method, within a specific time period. An analytical batch can be up to 20 samples (excluding laboratory OC samples), all of which must be received by the laboratory within 14 days of the validated time of sample receipt (VTSR) of the first sample in the batch.

\subsection{Summary of Procedure}

The analyst is referred to Section 2.0 of the analyte-specific SW-846 method as indicated in Table 1 for a summary of the procedure, except that the sample preparation procedure described in Procedure 610.1 of this Methods Manual must be followed.

\subsection{Interferences}

The analyst is referred to Section 3.0 of the analyte-specific SW-846 method as indicated in Table 1 for an explanation of potential interferences.

\subsection{Safety}

This procedure may involve the use of hazardous materials, operations, and equipment. It is the responsibility of whoever uses this procedure to consult appropriate site personnel concerning health and safety issues and establish appropriate health and safety practices. Consideration should be given to safety concerns regarding chemical and radiation exposure. Training regarding proper storage, usage, and disposal of chemicals is recommended.

\subsection{Apparatus and Materials}

The analyst is referred to Section 4.0 of the analyte-specific SW-846 method as indicated in Table 1 for a description and listing of the required apparatus and materials for this procedure. 
TABLE 1

Total Metal FLAA Analytical Methods

\begin{tabular}{lcc}
\hline Analyte & CAS Number & SW-846 \\
& & Analytical Method \\
\hline Antimony & $7440-36-0$ & 7040 \\
Barium & $7440-39-3$ & $7080 A^{\mathrm{c}}$ \\
Beryllium & $7440-41-7$ & 7090 \\
Cadmium & $7440-43-9$ & 7130 \\
Chromium & $7440-47-3$ & 7190 \\
Lead & $7439-92-1$ & 7420 \\
Nickel & $7440-02-0$ & 7520 \\
Silver & $7440-22-4$ & $7760 A^{\mathrm{b}}$ \\
Thallium & $7440-28-0$ & 7840 \\
Vanadium & $7440-62-2$ & 7910 \\
Zinc & $7440-66-6$ & 7950 \\
\hline
\end{tabular}

${ }^{a}$ Test Methods for Evaluating Solid Waste, Physical/Chemical Methods, Third Edition, Final Update I and Final Update II (SW-846) (EPA 1995)

${ }^{b}$ Method contained in SW-846, Final Update I

"Method contained in SW-846, Final Update II 
TABLE 2

Target Analyte List and Quality Assurance Objectives for FLAA

\begin{tabular}{lcccccc}
\hline Analyte & CAS Number & $\begin{array}{c}\text { Precision } \\
\text { (\%RSD or RPD) }\end{array}$ & $\begin{array}{c}\text { Accuracy } \\
(\% R)^{b}\end{array}$ & $\begin{array}{c}\text { PRDLc } \\
(\mu g / L)\end{array}$ & $\begin{array}{c}\text { PROL } \\
(\mathbf{m g} / \mathrm{kg})\end{array}$ & $\begin{array}{c}\text { Completeness } \\
(\%)\end{array}$ \\
\hline Antimony & $7440-36-0$ & $\leq 30$ & $80-120$ & 100 & 100 & 90 \\
Barium & $7440-39-3$ & $\leq 30$ & $80-120$ & 2000 & 2000 & 90 \\
Beryllium & $7440-41-7$ & $\leq 30$ & $80-120$ & 100 & 100 & 90 \\
Cadmium & $7440-43-9$ & $\leq 30$ & $80-120$ & 20 & 20 & 90 \\
Chromium & $7440-47-3$ & $\leq 30$ & $80-120$ & 100 & 100 & 90 \\
Lead & $7439-92-1$ & $\leq 30$ & $80-120$ & 100 & 100 & 90 \\
Nickel & $7440-02-0$ & $\leq 30$ & $80-120$ & 100 & 100 & 90 \\
Silver & $7440-22-4$ & $\leq 30$ & $80-120$ & 100 & 100 & 90 \\
Thallium & $7440-28-0$ & $\leq 30$ & $80-120$ & 100 & 100 & 90 \\
Vanadium & $7440-62-2$ & $\leq 30$ & $80-120$ & 100 & 100 & 90 \\
Zinc & $7440-66-6$ & $\leq 30$ & $80-120$ & 100 & 100 & 90 \\
\hline
\end{tabular}

$\begin{array}{lll}\% R S D & = & \text { Percent relative standard deviation } \\ \text { RPD } & = & \text { Relative percent difference } \\ \% R & = & \text { Percent recovery } \\ \text { PRDL } & = & \begin{array}{l}\text { Program required detection limit (i.e., maximum permissible value for instrument detection } \\ \text { limit) }\end{array} \\ \text { PROL } & = & \text { Program required quantitation limit }\end{array}$

a $\leq 30 \%$ control limits apply when sample and duplicate concentrations are $\geq 10 x$ instrument detection limit.

If less than this limit, the absolute difference between the two values shall be less than or equal to the PRDL.

${ }^{b}$ Applies to laboratory control samples. If a solid laboratory control sample material which has established statistical control limits is used, then the established control limits for that material should be used for accuracy requirements.

CPRDL set such that it is a factor of 10 below the PROL for $100 \%$ solid samples, assuming a $100 \times$ dilution during digestion. 


\subsection{Reagents}

The analyst is referred to Section 5.0 of the analyte-specific SW-846 method as indicated in Table 1 for a description and listing of the required reagents, standard stock solutions, and standard solutions for this procedure.

7.0 Sample Collection, Preservation, and Handling

7.1 Samples will be collected using the sampling procedures described in Procedure 120.1 of this Methods Manual.

7.2 All sample handling and chain-of-custody procedures described in Section 6.0 of the Transuranic Waste Characterization Quality Assurance Program Plan (QAPP) (DOE 1995b) must be followed.

\subsection{Procedure}

8.1 The sample preparation procedures described in Procedure 610.1 of this Methods Manual must be followed.

8.2 The sample cleanup procedures described in Procedure 620.1 of this Methods Manual may be used.

8.3 The analyst is referred to Section 7.2 of the analyte-specific SW-846 method as indicated in Table 1, except for the following:

- Silver: see Section 7.4 through 7.6 of SW-846 Method 7760A

8.4 Use of proven matrix modifiers other than those specified in SW-846 is acceptable. The choice of matrix modifiers must be documented in laboratory SOPs.

8.5 Table 3 of this procedure provides a summary of required calibration and analysis quality control.

\subsection{Calculations}

Read the metal analyte concentration from the calibration curve or directly from the instrument readout. Any sample dilution must be taken into account. Note that all results must be reported in $\mathrm{mg} / \mathrm{kg}$ (wet weight basis). To convert analytical results into $\mathrm{mg} / \mathrm{kg}$ dry weight, the percent solid content must be determined on a separate sample aliquot from that used for digestion and analysis.

\subsection{Quality Control}

10.1 All of the quality control requirements specified in Section 8.6 of SW-846 Method 7000A are mandatory. If the method of standard additions is needed to overcome interferences, see Section 8.7 of SW-846 Method 7000A. In instances where the quality control specifications in this procedure differ from those in SW-846 Method 7000A, this procedure shall take precedence.

10.2 Each laboratory that uses this procedure is required to operate a formal quality control program. The laboratory must retain records to document the quality of the data generated. Each laboratory must have SOPs documenting and describing activities involved in using this procedure. Specific quality control practices will include, but are not limited to, the analysis of quality control samples. The types of quality control samples, 


\section{TABLE 3}

Summary of Calibration Requirements and Analysis Quality Control for FLAA Total Metals Analysis

\begin{tabular}{|c|c|c|c|c|}
\hline Technique & Procedure & Frequency of Procedure & Acceptance Criteria & Corrective Action \\
\hline \multirow[t]{4}{*}{ FLAA } & $\begin{array}{l}\text { 3-pt. initial calibration } \\
\text { ( } 3 \text { standards and a } \\
\text { blank). }\end{array}$ & Daily & $\begin{array}{l}90-110 \% R \text { for initial calibration verification } \\
\text { solution } \\
95-105 \% R \text { for highest calibration standard } \\
\text { Regression coefficient }\left(r^{2}\right) \text { must be } \geq 0.995\end{array}$ & $\begin{array}{l}\text { Correct problem and repeat initial } \\
\text { calibration }\end{array}$ \\
\hline & Continuing calibration & $\begin{array}{l}\text { Every } 10 \text { samples plus beginning and } \\
\text { end of run }\end{array}$ & $\begin{array}{l}80-120 \% R \text { for check standard; blank must } \\
\text { measure } \leq 3 \times 1 D L\end{array}$ & Recalibrate and rerun last 10 samples \\
\hline & Serial dilution & $\begin{array}{l}\text { once per analytical batch or per matrix } \\
\text { within an analytical batch }\end{array}$ & $\begin{array}{l}5 x \text { dilution of sample which is }>25 \times I D L \\
\text { must be } \leq 10 \% \mathrm{D} \text { of initial value }\end{array}$ & $\begin{array}{l}\text { Use MSA to quantitate samples of like } \\
\text { matrix }\end{array}$ \\
\hline & Post-digestion spike & $\begin{array}{l}\text { once per analytical batch or per matrix } \\
\text { within an analytical batch if serial } \\
\text { dilution, matrix spike, or matrix spike } \\
\text { duplicate does not meet acceptance } \\
\text { criteria }\end{array}$ & $85-115 \% R$ & $\begin{array}{l}\text { Use MSA to quantitate samples of like } \\
\text { matrix }\end{array}$ \\
\hline
\end{tabular}

$\mathrm{IDL} \quad=\quad$ Instrument detection limit

$\% D=$ Percent difference

MSA = Method of standard additions

$\% R=$ Percent recovery 
their associated frequency of analysis, acceptance criteria, and corrective action required if samples do not meet the acceptance criteria, is summarized in Table 4 . Laboratory SOPs shall address requirements for preparing matrix spikes, matrix spike duplicates, laboratory blanks, and laboratory control samples.

10.3 All laboratories using this procedure must demonstrate acceptable performance prior to the analysis of actual samples. Demonstration of acceptable performance will be achieved by analyzing method performance samples (Table 4). These samples can be either commercially purchased or laboratory prepared, but must contain all of the analytes listed in Table 1 at concentrations appropriate (2-5 times the PROLs) to determine the quality assurance objectives specified in Table 1 . The analysis of seven samples must meet the criteria specified for precision, accuracy, and program required detection limit (PRDL) in Table 1. Note that the instrument detection limit (IDL) must be less than or equal to the PRDL. Demonstration of acceptable method and analyst performance must be repeated (by analyzing four samples) at a minimum of every six months. Method performance should be conducted over a period of several days to account for long-term variability. Corrective action must be implemented when acceptable method performance is not met. Precision will be determined as follows:

- For duplicate measurements, the precision expressed as the relative percent difference (RPD) is calculated as

$$
R P D=\frac{C_{1}-C_{2}}{\frac{\left(C_{1}+C_{2}\right)}{2}} * 100
$$

where

$$
\begin{array}{lll}
C_{1} \text { and } C_{2} & = & \text { two values obtained by analyzing the duplicate samples } \\
C_{1} & = & \text { larger of the two observed values }
\end{array}
$$

- For three or more replicate measurements, the precision expressed as the percent relative standard deviation (\%RSD) is calculated as

$$
\% \text { RSD }=\frac{s}{\bar{y}} * 100
$$

where

$$
\begin{array}{lll}
\frac{s}{y} & = & \text { standard deviation } \\
& = & \text { mean of replicate analyses }
\end{array}
$$

- The standard deviation $(s)$ is defined as

$$
\text { where } s=\sqrt{\sum_{i=1}^{n} \frac{\left(y_{i}-\bar{y}\right)^{2}}{n-1}}
$$

$\begin{array}{ll}y_{i} & =\begin{array}{l}\text { measured value of the } i^{t h} \text { replicate sample analysis } \\ \text { measurement }\end{array} \\ n & =\quad \begin{array}{l}\text { number of replicate analyses }\end{array}\end{array}$


TABLE 4

\section{Summary of Laboratory Quality Control Samples and Frequencies for FLAA Total Metals Analyses}

\section{ac Samples \\ Method performance samples}

Laboratory blanks

Matrix spikes

Matrix spike duplicates

Laboratory control samples

Blind audit samples
Seven (7) samples initially, Meet Table 2 QAOs and four (4) semiannually

One (1) per analytical $\leq 3 \times$ IDL batch

One (1) per analytical batch

One (1) per analytical batch

One (1) per analytical batch

Samples and frequency controlled by the Solid PDP Plan
$\mathrm{RPD} \leq 30$

80-120\%R

$80-120 \% R$

Specified in the Solid PDP Plan
Acceptance

Criteria

Corrective Actions ${ }^{a}$

Repeat until acceptable

Redigest and reanalyze

any samples with analyte concentrations which are

$\leq 10 \times$ blank value and $\geq 0.5 \times$ PROL

Nonconformance if \%R $<80$ or $>120$

Nonconformance if RPD > 30 or if $\% \mathrm{R}<$ 80 or $>120$

Redigest and reanalyze for affected analytes

Specified in the Solid PDP Plan

${ }^{a}$ Corrective Actions when OC samples do not meet acceptance criteria; Nonconformance procedures are outlined in Section 2.1.2.1 of the QAPP.

$I D L=$ Instrument detection limit
PDP $=$ Performance Demonstration Program
PRQL $=$ Program required detection limit
$\% R=$ Percent recovery
RPD $=$ Relative percent difference


Accuracy will be determined as the percent recovery $(\% \mathrm{R})$ as follows:

$$
\% R=\frac{C_{m}}{C_{s r m}} * 100
$$

where

$$
\begin{array}{lll}
C_{m} & = & \text { measured concentration } \\
C_{s r m} & = & \text { true concentration }
\end{array}
$$

IDL will be determined as follows:

$$
I D L=t_{(n-1,1-\alpha=0.99)} * s
$$

where

$$
\begin{array}{lll}
s & = & \text { standard deviation } \\
t_{(n-1,1-\alpha=0.99)} & = & t \text {-distribution value appropriate to a 99-percent confidence level } \\
& \text { and a standard deviation estimate with } n-1 \text { degrees of freedom }
\end{array}
$$

10.4 The laboratory must analyze blanks at the frequency specified in Table 4. The same procedures used to prepare and analyze field samples will be used to prepare and analyze the laboratory blanks. Laboratory blank results will be acceptable if the concentration of target analytes is less than or equal to 3 times the IDL. Corrective action must be implemented if blanks exceed this level.

10.5 The laboratory must analyze matrix spikes at the frequency specified in Table 3 . Matrix spike results shall be considered acceptable if the $\% R$ is 80 to 120 percent of the true value. Matrix spikes which do not meet these criteria should be flagged. \%R will be determined as follows:

$$
\% R=\frac{S-U}{C_{s a}} * 100
$$

$$
\begin{gathered}
\text { where } \\
S= \\
U= \\
C_{s a}=
\end{gathered}
$$

\begin{abstract}
measured concentration in the spiked aliquot measured concentration in the unspiked aliquot actual concentration of the spike added
\end{abstract}

10.6 The laboratory must analyze matrix spike duplicates at the frequency specified in Table 4 . Matrix spike duplicate results shall be considered acceptable if the RPD is $\leq 30$ and the $\% R$ is 80 to 120. RPD is determined as in Equation (1), \%R is determined as in Equation (6). Duplicates which do not meet these criteria should be flagged.

10.7 The laboratory must analyze laboratory control samples at the frequency specified in Table 4. Solid laboratory control samples (e.g., characterized surrogate sludges) should be used whenever it is possible to match the matrix of the waste samples. Otherwise, commercially purchased standards shall be used to prepare laboratory control samples. The laboratory control samples must contain the metals being analyzed, must undergo the same preparation and cleanup methods as the samples, and be quantitated within the calibration range of the instrument. The laboratory control sample analysis is designed to ensure that laboratory performance is in control for each analytical batch of samples processed. Laboratory control sample results will be considered acceptable if the $\% R$ is 80 to 120 percent of the known value. \%R is calculated as in Equation (4). If the results are outside this range, then the associated samples must be reprepared and reanalyzed for the affected analytes. 
10.8 The laboratory will be required to analyze blind audit samples. These audit samples are part of the Performance Demonstration Program. Details of this program are in the Performance Demonstration Program Plan for the Analysis of Solidified Waste for the Transuranic Waste Characterization Program (Solid PDP Plan)(DOE 1995a).

\subsection{Procedure Performance}

The analyst is referred to Section 9.0 of the analyte-specific SW-846 method as indicated in Table 1 for a summary of method performance.

\subsection{References}

DOE. 1995a. Performance Demonstration Program Plan for the Analysis of Solidified Waste for the Transuranic Waste Characterization Program. CA0-95-1077, Current Revision, Carlsbad, New Mexico, Carlsbad Area Office, U.S. Department of Energy.

DOE. 1995b. Transuranic Waste Characterization Quality Assurance Program Plan. CAO-941010, Current Revision, Carlsbad, New Mexico, Carlsbad Area Office, U.S. Department of Energy.

EPA. 1995. Test Methods for Evaluating Solid Waste, Physical/Chemical Methods. SW-846, Third Edition, Final Update I, and Final Update II, Washington D.C., Office of Solid Waste and Emergency Response, U.S. Environmental Protection Agency. 

PROCEDURE 650.2

\section{GRAPHITE FURNACE ATOMIC ABSORPTION \\ SPECTROSCOPY DETERMINATION OF TOTAL METALS IN HOMOGENOUS SOLIDS AND SOIL/GRAVEL}

\subsection{Scope and Application}

1.1 This procedure is for the determination of total metals in samples of homogenous solids and soil/gravel. The procedure is actually a combination of nine separate analytical methods found in Test Methods for Evaluating Solid Waste, Physical/Chemical Methods, SW-846, Third Edition, Final Update I, and Final Update II (SW-846) (EPA 1995). Table 1 lists the individual SW-846 method that applies to the specific metal analyte of interest. In addition to the analyte-specific methods listed in Table 1, the analyst is referred to SW846 Method 7000A for a general discussion of Graphite Furnace Atomic Absorption Spectroscopy (GFAA) methods. The procedure employs GFAA for the quantitation of analytes listed in Table 2. The quality assurance objectives for this procedure are also listed in Table 2. A sampling procedure for homogenous solids and soil/gravel is described in Procedure 120.1 of this Methods Manual. This procedure must be implemented with a site-specific standard operating procedure (SOP).

1.2 For the purposes of the TRU Waste Characterization Program, samples are to be analyzed in analytical batches. An analytical batch is defined as a suite of samples that is processed as a unit, using the same analytical method, within a specific time period. An analytical batch can be up to 20 samples (excluding laboratory QC samples), all of which must be received by the laboratory within 14 days of the validated time of sample receipt (VTSR) of the first sample in the batch.

\subsection{Summary of Procedure}

The analyst is referred to Section 2.0 of the analyte-specific SW-846 method as indicated in Table 1 for a summary of the procedure, except that the sample preparation procedure described in Procedure 610.1 of this Methods Manual must be followed.

\subsection{Interferences}

The analyst is referred to Section 3.0 of the analyte-specific SW-846 method as indicated in Table 1 for an explanation of potential interferences.

\subsection{Safety}

This procedure may involve the use of hazardous materials, operations, and equipment. It is the responsibility of whoever uses this procedure to consult appropriate site personnel concerning health and safety issues and establish appropriate health and safety practices. Consideration should be given to safety concerns regarding chemical and radiation exposure. Training regarding proper storage, usage, and disposal of chemicals is recommended.

\subsection{Apparatus and Materials}

The analyst is referred to Section 4.0 of the analyte-specific SW-846 method as indicated in Table 1 for a description and listing of the required apparatus and materials for this procedure. 
TABLE 1

Total Metal GFAA Analytical Methods

\begin{tabular}{lcc} 
Analyte & CAS Number & SW-846 \\
& & Analytical Method \\
\hline Antimony & $7440-36-0$ & 7041 \\
Barium & $7440-39-3$ & $7080 A^{\mathrm{c}}$ \\
Beryllium & $7440-41-7$ & 7090 \\
Cadmium & $7440-43-9$ & 7130 \\
Chromium & $7440-47-3$ & 7190 \\
Lead & $7439-92-1$ & 7420 \\
Nickel & $7440-02-0$ & 7520 \\
Silver & $7440-22-4$ & $7760 A^{\mathrm{b}}$ \\
Thallium & $7440-28-0$ & 7840 \\
Vanadium & $7440-62-2$ & 7911 \\
Zinc & $7440-66-6$ & $7951^{\mathrm{b}}$ \\
\hline
\end{tabular}

${ }^{a}$ Test Methods for Evaluating Solid Waste, Physical/Chemical Methods, Third Edition, Final Update I and Final Update II (SW-846) (EPA 1995)

${ }^{b}$ Method contained in SW-846, Final Update I

${ }^{c}$ Method contained in SW-846, Final Update II 
TABLE 2

Target Analyte List and Quality Assurance Objectives for GFAA

\begin{tabular}{lcccccc}
\hline Analyte & CAS Number & $\begin{array}{c}\text { Precision } \\
(\% R S D \text { or RPD })^{a}\end{array}$ & $\begin{array}{c}\text { Accuracy } \\
(\%)^{\mathrm{b}}\end{array}$ & $\begin{array}{c}\text { PRDL } \\
(\mu \mathrm{L} / \mathrm{L})\end{array}$ & $\begin{array}{c}\text { PROL } \\
(\mathrm{mg} / \mathrm{kg})\end{array}$ & $\begin{array}{c}\text { Completeness } \\
(\%)\end{array}$ \\
\hline Antimony & $7440-36-0$ & $\leq 30$ & $80-120$ & 100 & 100 & 90 \\
Arsenic & $7440-38-2$ & $\leq 30$ & $80-120$ & 100 & 100 & 90 \\
Barium & $7440-39-3$ & $\leq 30$ & $80-120$ & 2000 & 2000 & 90 \\
Beryllium & $7440-41-7$ & $\leq 30$ & $80-120$ & 100 & 100 & 90 \\
Cadmium & $7440-43-9$ & $\leq 30$ & $80-120$ & 20 & 20 & 90 \\
Chromium & $7440-47-3$ & $\leq 30$ & $80-120$ & 100 & 100 & 90 \\
Lead & $7439-92-1$ & $\leq 30$ & $80-120$ & 100 & 100 & 90 \\
Selenium & $7782-49-2$ & $\leq 30$ & $80-120$ & 20 & 20 & 90 \\
Silver & $7440-22-4$ & $\leq 30$ & $80-120$ & 100 & 100 & 90 \\
Thallium & $7440-28-0$ & $\leq 30$ & $80-120$ & 100 & 100 & 90 \\
Vanadium & $7440-62-2$ & $\leq 30$ & $80-120$ & 100 & 100 & 90 \\
Zinc & $7440-66-6$ & $\leq 30$ & $80-120$ & 100 & 100 & 90 \\
\hline & & & & & & 900 \\
\hline
\end{tabular}

$\begin{array}{ll}\% \mathrm{RSD} & =\text { Percent relative standard deviation } \\ \text { RPD } & =\text { Relative percent difference } \\ \% \mathrm{R} & =\text { Percent recovery } \\ \text { PRDL } & =\text { Program required detection limit (i.e., maximum permissible value for instrument detection limit) } \\ \text { PRQL } & =\text { Program required quantitation limit }\end{array}$

a $\leq 30 \%$ control limits apply when sample and duplicate concentrations are $\geq 10 x$ the instrument detection limit. If less than this limit, the absolute difference between the two values shall be less than or equal to the PRDL.

${ }^{b}$ Applies to laboratory control samples. If a solid laboratory control sample material which has established statistical control limits is used, then the established control limits for that material should be used for accuracy requirements.

'PRDL set such that it is a factor of 10 below the PROL for $100 \%$ solid samples, assuming a $100 \times$ dilution during digestion. 


\subsection{Reagents}

The analyst is referred to Section 5.0 of the analyte-specific SW-846 method as indicated in Table 1 for a description and listing of the required reagents, standard stock solutions, and standard solutions for this procedure.

\subsection{Sample Collection, Preservation, and Handling}

7.1 Samples will be collected using the sampling procedures described in Procedure 120.1 of this Methods Manual.

7.2 All sample handling and chain-of-custody procedures described in Section 6.0 of the Transuranic Waste Characterization Quality Assurance Program Plan (QAPP) (DOE 1995b) must be followed.

\subsection{Procedure}

8.1 The sample preparation procedures described in Procedure 610.1 of this Methods Manual must be followed.

8.2 The sample clean up procedures described in Procedure 620.1 of this Methods Manual may be used.

8.3 The analyst is referred to Section 7.2 of the analyte-specific SW-846 method as indicated in Table 1, except for the following:

- Arsenic: see Section 7.2 through 7.4 of SW-846 Method 7060A

- Selenium: see Section 7.2 through 7.6 of SW-846 Method 7740

- Silver: see Section 7.4 through 7.9 of SW-846 Method 7761

8.4 Use of proven matrix modifiers other than those specified in SW-846 is acceptable. The choice of matrix modifiers must be documented in laboratory SOPs.

8.5 Table 3 of this procedure provides a summary of required calibration and analysis quality contror.

\subsection{Calculations}

Read the metal analyte concentration from the calibration curve or directly from the instrument readout. Any sampie dilution must be taken into account. Note that all results must be reported in $\mathrm{mg} / \mathrm{kg}$ (wet weight basis). To convert analytical results into $\mathrm{mg} / \mathrm{kg}$ dry weight, the percent solid content must be determined on a separate sample aliquot from that used for digestion and analysis.

\subsection{Quality Control}

10.1 All of the quality control requirements specified in Section 8.6 of SW-846 Method 7000A are mandatory. If the method of standard additions is needed to overcome interferences, see Section 8.7 of SW-846 Method 7000A. In instances where the quality control specifications in this procedure differ from those in SW-846 Method 7000A, this procedure shall take precedence.

10.2 Each laboratory that uses this procedure is required to operate a formal quality control program. The laboratory must retain records to document the quality of the data generated. Each laboratory must have SOPs documenting and describing activities 


\section{TABLE 3}

Summary of Calibration Requirements and Analysis Quality Control for GFAA Total Metals Analysis

\begin{tabular}{|c|c|c|c|c|}
\hline Technique & Procedure & Frequency of Procedure & Acceptance Criteria & Corrective Action \\
\hline \multirow[t]{6}{*}{ GFAA } & $\begin{array}{l}\text { 3-pt. initial calibration } \\
\text { ( } 3 \text { standards and a blank) }\end{array}$ & Daily & $\begin{array}{l}90-110 \% R \text { for initial calibration verification } \\
\text { solution }\end{array}$ & $\begin{array}{l}\text { Correct problem and repeat initial } \\
\text { calibration }\end{array}$ \\
\hline & & & $95-105 \% R$ for highest calibration standard & \\
\hline & & & Regression coefficient $\left(r^{2}\right)$ must be $\geq 0.995$ & \\
\hline & Continuing calibration & $\begin{array}{l}\text { Every } 10 \text { sample injections plus } \\
\text { beginning and end of run }\end{array}$ & $\begin{array}{l}80-120 \% R \text { for check standard (mandatory); } \\
\text { blank (optional) should measure } \leq 3 \times 1 D L\end{array}$ & $\begin{array}{l}\text { Recalibrate and rerun last } 10 \\
\text { samples }\end{array}$ \\
\hline & Serial dilution & $\begin{array}{l}\text { once per analytical batch or per } \\
\text { matrix within an analytical batch }\end{array}$ & $\begin{array}{l}5 x \text { dilution of sample which is }>25 \times 1 D L \text { must } \\
\text { be } \leq 10 \% \mathrm{D} \text { of initial value }\end{array}$ & $\begin{array}{l}\text { Use MSA to quantitate samples of } \\
\text { like matrix }\end{array}$ \\
\hline & Post-digestion spike & $\begin{array}{l}\text { once per analytical batch or per } \\
\text { matrix within an analytical batch }\end{array}$ & $85 \cdot 115 \% R$ & $\begin{array}{l}\text { Use MSA to quantitate sample of like } \\
\text { matrix }\end{array}$ \\
\hline
\end{tabular}

is matrix within an analytical batch matrix

$I D L=$ Instrument detection limit

$\% \mathrm{D}=$ Percent difference

MSA $=$ Method of standard additions

$\% R=$ Percent recovery 
involved in using this procedure. Specific quality control practices will include, but are not limited to, the analysis of quality control samples. The types of quality control samples, their associated frequency of analysis, acceptance criteria, and corrective action required if samples do not meet the acceptance criteria, is summarized in Table 4. Laboratory SOPs shall address requirements for preparing matrix spikes, matrix spike duplicates, laboratory blanks, and laboratory control samples.

10.3 All laboratories using this procedure must demonstrate acceptable performance prior to the analysis of actual samples. Demonstration of acceptable performance will be achieved by analyzing method performance samples (Table 4). These samples can be either commercially purchased or laboratory prepared, but must contain all of the analytes listed in Table 1 at concentrations appropriate (2-5 times the PRQLs) to determine the quality assurance objectives specified in Table 1. The analysis of seven samples must meet the criteria specified for precision, accuracy, and program required detection limit (PRDL) in Table 1. Note that the instrument detection limit (IDL) must be less than or equal to the PRDL. Demonstration of acceptable method and analyst performance must be repeated (by analyzing four samples) at a minimum of every six months. Method performance should be conducted over a period of several days to account for long-term variability. Corrective action must be implemented when acceptable method performance is not met. Precision will be determined as follows:

- For duplicate measurements, the precision expressed as the relative percent difference (RPD) is calculated as

$$
R P D=\frac{C_{1}-C_{2}}{\frac{\left(C_{1}+C_{2}\right)}{2}} * 100
$$

where

$$
\begin{array}{lll}
C_{1} \text { and } C_{2} & = & \text { two values obtained by analyzing the duplicate samples } \\
C_{1} & = & \text { larger of the two observed values }
\end{array}
$$

- For three or more replicate measurements, the precision expressed as the percent relative standard deviation (\%RSD) is calculated as

$$
\% R S D=\frac{s}{\bar{y}} * 100
$$

where

$$
\begin{array}{lll}
\boldsymbol{s} & = & \text { standard deviation } \\
\boldsymbol{y} & = & \text { mean of replicate analyses }
\end{array}
$$

- The standard deviation $(s)$ is defined as

$$
s=\sqrt{\sum_{i=1}^{n} \frac{\left(y_{i}-\bar{y}\right)^{2}}{n-1}}
$$

$\begin{array}{cl}\begin{array}{cl}\text { where } \\ y_{i}\end{array} & \begin{array}{l}\text { measured value of the } i^{\text {at }} \text { replicate sample analysis } \\ \text { measurement }\end{array} \\ n & =\text { number of replicate analyses }\end{array}$


TABLE 4

\section{Summary of Laboratory Quality Control Samples and Frequencies for GFAA Total Metals Analyses}

$\begin{array}{llll}\text { OC Samples } & \text { Minimum Frequency } & \begin{array}{l}\text { Acceptance } \\ \text { Criteria }\end{array} & \text { Corrective Actions } \\ \end{array}$

Method performance samples

Laboratory blanks

Matrix spikes

Matrix spike duplicates

Laboratory control samples

Blind audit samples
Seven (7) samples initially, Meet Table 2 QAOs and four (4) semiannually

One (1) per analytical batch

One (1) per analytical batch

One (1) per analytical batch

One (1) per analytical batch

Samples and frequency controlled by the Solid PDP Plan
Repeat until acceptable

$\leq 3 \times I D L$

Redigest and reanalyze any samples with analyte concentrations which are

$\leq 10 \times$ blank value and

$\geq 0.5 \times$ PROL

Nonconformance if $\% R$

$<80$ or $>120$

Nonconformance if RPD $>30$ or if $\% R<$ 80 or $>120$

Redigest and reanalyze for affected analytes

Specified in the Solid PDP Plan
Specified in the Solid PDP Plan

${ }^{a}$ Corrective Actions when QC samples do not meet acceptance criteria; Nonconformance procedures are outlined in Section 2.1.2.1 of the OAPP.

$\mathrm{IDL}=$ Instrument detection limit

PDP $=$ Performance Demonstration Program

PRQL $=$ Program required detection limit

$\% R=$ Percent recovery

$\mathrm{RPD}=$ Relative percent difference 
Accuracy will be determined as the percent recovery $(\% \mathrm{R})$ as follows:

$$
\% R=\frac{C_{m}}{C_{s m m}} * 100
$$

where

$$
\begin{array}{lll}
C_{m} & = & \text { measured concentration } \\
C_{s r m} & = & \text { true concentration }
\end{array}
$$

IDL will be determined as follows:

$$
I D L=t_{(n-1,1-\alpha=0.99)} * s
$$

where

$$
\begin{array}{lll}
s & = & \text { standard deviation } \\
\left.t_{(n-1,1-\alpha}=0.99\right) & = & t \text {-distribution value appropriate to a 99-percent confidence level } \\
& & \text { and a standard deviation estimate with } n-1 \text { degrees of freedom }
\end{array}
$$

10.4 The laboratory must analyze blanks at the frequency specified in Table 4 . The same procedures used to prepare and analyze field samples will be used to prepare and analyze the laboratory blanks. Laboratory blank results will be acceptable if the concentration of target analytes is less than or equal to 3 times the IDL. Corrective action must be implemented if blanks exceed this level.

10.5 The laboratory must analyze matrix spikes at the frequency specified in Table 3 . Matrix spike results shall be considered acceptable if the \%R is 80 to 120 percent of the true value. Matrix spikes which do not meet these criteria should be flagged. \%R will be determined as follows:

$$
\% R=\frac{S-U}{C_{s a}} * 100
$$

where

$\begin{array}{lll}S & = & \text { measured concentration in the spiked aliquot } \\ U & = & \text { measured concentration in the unspiked aliquot } \\ C_{s a} & = & \text { actual concentration of the spike added }\end{array}$

10.6 The laboratory must analyze matrix spike duplicates at the frequency specified in Table 4 . Matrix spike duplicate results shall be considered acceptable if the RPD is $\leq 30$ and the $\% R$ is 80 to 120 . RPD is determined as in Equation (1), \%R is determined as in Equation (6). Duplicates which do not meet these criteria should be flagged.

10.7 The laboratory must analyze laboratory control samples at the frequency specified in Table 4. Solid laboratory control samples (e.g., characterized surrogate sludges) should be used whenever it is possible to match the matrix of the waste samples. Otherwise, commercially purchased standards shall be used to prepare laboratory control samples. The laboratory control samples must contain the metals being analyzed, must undergo the same preparation and cleanup methods as the samples, and be quantitated within the calibration range of the instrument. The laboratory control sample analysis is designed to ensure that laboratory performance is in control for each analytical batch of samples processed. Laboratory control sample results will be considered acceptable if the \% $R$ is 80 to 120 percent of the known value. \%R is calculated as in Equation (4). If the results are outside of this range, then the associated samples must be reprepared and reanalyzed for the affected analytes. 
10.8 The laboratory will be required to analyze blind audit samples. These audit samples are part of the Performance Demonstration Program. Details of this program are in the Performance Demonstration Program Plan for the Analysis of Solidified Waste for the Transuranic Waste Characterization Program (Solid PDP Plan)(DOE 1995a).

\subsection{Procedure Performance}

The analyst is referred to Section 9.0 of the analyte-specific SW-846 method as indicated in Table 1 for a summary of method performance.

\subsection{References}

DOE. 1995a. Performance Demonstration Program Plan for the Analysis of Solidified Waste for the Transuranic Waste Characterization Program. CA0-95-1077, Current Revision, Carlsbad, New Mexico, Carlsbad Area Office, U.S. Department of Energy.

DOE. 1995b. Transuranic Waste Characterization Quality Assurance Program Plan. CAO-941010, Current Revision, Carlsbad, New Mexico, Carlsbad Area Office, U.S. Department of Energy.

EPA. 1995. Test Methods for Evaluating Solid Waste, Physical/Chemical Methods. SW-846, Third Edition, Final Update I, and Final Update II, Washington D.C., Office of Solid Waste and Emergency Response, U.S. Environmental Protection Agency. 

PROCEDURE 650.3

\section{COLD VAPOR ATOMIC ABSORPTION \\ SPECTROSCOPY DETERMINATION OF TOTAL MERCURY IN HOMOGENOUS SOLIDS AND SOIL/GRAVEL}

\subsection{Scope and Application}

1.1 This procedure is for the determination of total mercury in samples of homogenous solids and soil/gravel. The procedure employs Cold Vapor Atomic Absorption Spectroscopy (CVAA) for the quantitation of mercury. The quality assurance objectives for this procedure are listed in Table 1. A sampling procedure for homogenous solids and soil/gravel is described in Procedure 120.1 of this Methods Manual. This procedure must be implemented with a site-specific standard operating procedure (SOP).

1.2 The analyst is referred to Method 7471A of Test Methods for Evaluating Solid Waste, Physical/Chemical Methods, SW-846, Third Edition, Final Update II, (SW-846) (EPA 1995) for the specific requirements of this procedure. The analyst is also referred to SW-846 Method 7000A for a general discussion of atomic absorption spectroscopy.

1.3 For the purposes of the TRU Waste Characterization Program, samples are to be analyzed in analytical batches. An analytical batch is defined as a suite of samples that is processed as a unit, using the same analytical method, within a specific time period. An analytical batch can be up to 20 samples (excluding laboratory $Q C$ samples), all of which must be received by the laboratory within 14 days of the validated time of sample receipt (VTSR) of the first sample in the batch.

\subsection{Summary of Procedure}

The analyst is referred to Section 2.0 of SW-846 Method 7471A for a summary of this procedure.

\subsection{Interferences}

The analyst is referred to Section 3.0 of SW-846 Method 7471A for an explanation of potential interferences.

\subsection{Safety}

This procedure may involve the use of hazardous materials, operations, and equipment. It is the responsibility of whoever uses this procedure to consult appropriate site personnel concerning health and safety issues and establish appropriate health and safety practices. Consideration should be given to safety concerns regarding chemical and radiation exposure. Training regarding proper storage, usage, and disposal of chemicals is recommended.

\subsection{Apparatus and Materials}

The analyst is referred to Section 4.0 of SW-846 Method 7471A for a description and listing of the required apparatus and materials for this procedure.

\subsection{Reagents}

The analyst is referred to Section 5.0 of SW-846 Method 7471A for a description and listing of the required reagents, stock standard solution, and working standard solutions for this procedure. 
TABLE 1

Quality Assurance Objectives for Total Mercury Analysis

\begin{tabular}{|c|c|c|c|c|c|c|}
\hline Analyte & CAS Number & $\begin{array}{c}\text { Precision } \\
(\% \text { RSD or RPD })^{a}\end{array}$ & $\begin{array}{c}\text { Accuracy } \\
(\% R)^{b}\end{array}$ & $\begin{array}{l}\mathrm{PRDL}^{\mathrm{c}} \\
(\mu \mathrm{g} / \mathrm{L})\end{array}$ & $\begin{array}{c}\text { PRQL } \\
\text { (mg/kg) }\end{array}$ & $\begin{array}{c}\text { Completeness } \\
(\%)\end{array}$ \\
\hline Mercury & $7439-97-6$ & $\leq 30$ & $80-120$ & 4.0 & 4.0 & 90 \\
\hline
\end{tabular}

\%RSD $=\quad$ Percent relative standard deviation

RPD $=$ Relative percent difference

$\% R=$ Percent recovery

PRDL = Program required detection limit (i.e., maximum permissible value for instrument detection limit)

$P R Q L=$ Program required quantitation limit

$a \leq 30 \%$ control limits apply when sample and duplicate concentrations are $\geq 10 \times$ instrument detection limit. If less than $10 \mathrm{x}$ instrument detection limit, the absolute difference between the two values shall be less than or equal to the PRDL.

${ }^{b}$ Applies to laboratory control samples. If a solid laboratory control sample material which has established statistical control limits is used, then the established control limits for that material should be used for accuracy requirements.

'PRDL set such that it is a factor of 10 below the PRQL for 100 percent solid samples, assuming a $100 \mathrm{X}$ dilution during digestion. 


\subsection{Sample Collection, Preservation, and Handling}

7.1 Samples will be collected using the sampling procedures described in Procedure 120.1 of this Methods Manual.

7.2 All sample handling and chain-of-custody procedures described in Section 6.0 of the Transuranic Waste Characterization Quality Assurance Program Plan (QAPP) (DOE 1995b) must be followed.

\subsection{Procedure}

The analyst is referred to Section 7.1 through 7.4 of SW-846 Method 7471A for a description of the procedural requirements. Use of fluorescence detection instead of absorption is acceptable as long as all other method requirements are met and the choice of detector is documented in laboratory SOPs. Table 2 provides a summary of required calibration and analysis quality control.

\subsection{Calculations}

Read the mercury concentration from the calibration curve or directly from the instrument readout. Any sample dilution must be taken into account. Note that all results must be reported in $\mathrm{mg} / \mathrm{kg}$ (wet weight basis). To convert analytical results into $\mathrm{mg} / \mathrm{kg}$ dry weight, the percent solid content must be determined on a separate sample aliquot from that used for digestion and analysis.

\subsection{Quality Control}

10.1 All of the quality control requirements specified in Section 8.5 and 8.6 of SW-846 Method $7000 \mathrm{~A}$ are mandatory. If the method of standard additions is neressary to overcome interferences, this information is presented in Section 8.7 of SW-846 Method 7000A. In instances where quality control specifications in this procedure differ with those in SW-846 Method 7000A, this procedure shall take precedence.

10.2 Each laboratory that uses this procedure is required to operate a formal quality control program. The laboratory must retain records to document the quality of the data generated. Each laboratory must have SOPs documenting and describing activities involved in using this procedure. Specific quality control practices will include, but are not limited to, the analysis of quality control samples. The types of quality control samples, their associated frequency of analysis, acceptance criteria, and corrective action required if samples do not meet the acceptance criteria, is summarized in Table 3. Laboratory SOPs shall address requirements for preparing matrix spikes, matrix spike duplicates, laboratory blanks, and laboratory control samples.

10.3 All laboratories using this procedure must demonstrate acceptable performance prior to the analysis of actual samples. Demonstration of acceptable performance will be achieved by analyzing method performance samples (Table 3). These samples can be either commercially purchased or laboratory prepared, but must contain mercury at concentrations appropriate $(2-5$ times the PROLs) to determine the quality assurance objectives specified in Table 1 . The analysis of seven samples must meet the criteria specified for precision, accuracy, and program required detection limit (PRDL) in Table 1. Note that the instrument detection limit (IDL) must be less than or equal to the PRDL. Demonstration of acceptable method and analyst performance must be repeated (by analyzing four samples) at a minimum of every six months. Method performance should be conducted over a period of several days to account for long-term variability. Corrective action must be implemented when acceptable method performance is not met. Precision will be determined as follows: 


\section{Summary of Calibration Requirements and Analysis Quality Control for Total Mercury Analysis}

\begin{tabular}{|c|c|c|c|c|}
\hline Technique & Procedure & Frequency of Procedure & Acceptance Criteria & Corrective Action \\
\hline \multirow[t]{6}{*}{ CVAA } & $\begin{array}{l}\text { 5-pt. initial calibration } \\
\text { (5 standards and a blank) }\end{array}$ & Daily & $\begin{array}{l}90-110 \% \mathrm{R} \text { for initial calibration verification } \\
\text { solution }\end{array}$ & $\begin{array}{l}\text { Correct problem and repeat initial } \\
\text { calibration }\end{array}$ \\
\hline & & & 95-105\%R for highest calibration standard & \\
\hline & & & Regression coefficient $\left(r^{2}\right)$ must be $\geq 0.995$ & \\
\hline & Continuing calibration & $\begin{array}{l}\text { Every } 10 \text { samples plus beginning and end } \\
\text { of run }\end{array}$ & $\begin{array}{l}80-120 \% R \text { for check standard (mandatory); } \\
\text { blank (optional) should measure } \leq 3 \times 1 D L\end{array}$ & Recalibrate and rerun last 10 samples \\
\hline & Serial dilution & $\begin{array}{l}\text { once per analytical batch or per matrix } \\
\text { within an analytical batch }\end{array}$ & $\begin{array}{l}5 x \text { dilution of sample which is }>25 x I D L \\
\text { must be } \leq 10 \% \mathrm{D} \text { of initial value }\end{array}$ & $\begin{array}{l}\text { Use MSA to quantitate samples of like } \\
\text { matrix }\end{array}$ \\
\hline & Post-digestion spike & $\begin{array}{l}\text { once per analytical batch or per matrix } \\
\text { within an analytical batch }\end{array}$ & $85-115 \% R$ & $\begin{array}{l}\text { Use MSA to quantitate samples of like } \\
\text { matrix }\end{array}$ \\
\hline
\end{tabular}

$\begin{array}{lll}I D L & = & \text { Instrument detection limit } \\ \% D & = & \text { Percent difference } \\ M S A & = & \text { Method of standard additions } \\ \% R & = & \text { Percent recovery }\end{array}$


TABLE 3

\section{Summary of Laboratory Quality Control Samples and Frequencies for Total Mercury Analyses}

\begin{tabular}{|c|c|c|c|}
\hline QC Samples & Minimum Frequency & $\begin{array}{l}\text { Acceptance } \\
\text { Criteria }\end{array}$ & Corrective Actions ${ }^{a}$ \\
\hline $\begin{array}{l}\text { Method performance } \\
\text { samples }\end{array}$ & $\begin{array}{l}\text { Seven (7) samples initially, } \\
\text { and four (4) semiannually }\end{array}$ & Meet Table 1 QAOs & Repeat until acceptable \\
\hline Laboratory blanks & $\begin{array}{l}\text { One (1) per analytical } \\
\text { batch }\end{array}$ & $\leq 3 \times 1 D L$ & $\begin{array}{l}\text { Redigest and reanalyze } \\
\text { any samples with } \\
\text { analyte concentrations } \\
\text { which are } \\
\leq 10 \times \text { blank value and } \\
\geq 0.5 \times \text { PRQL }\end{array}$ \\
\hline Matrix spikes & $\begin{array}{l}\text { One (1) per analytical } \\
\text { batch }\end{array}$ & $80-120 \% R$ & $\begin{array}{l}\text { Nonconformance if } \% R \\
<80 \text { or }>120\end{array}$ \\
\hline Matrix spike duplicates & $\begin{array}{l}\text { One ( } 1 \text { ) per analytical } \\
\text { batch }\end{array}$ & $\begin{array}{l}R P D \leq 30 \\
80-120 \% R\end{array}$ & $\begin{array}{l}\text { Nonconformance if } \\
\text { RPD }>30 \text { or if } \% R< \\
80 \text { or }>120\end{array}$ \\
\hline $\begin{array}{l}\text { Laboratory control } \\
\text { samples }\end{array}$ & $\begin{array}{l}\text { One (1) per analytical } \\
\text { batch }\end{array}$ & $80-120 \% R$ & $\begin{array}{l}\text { Redigest and reanalyze } \\
\text { for affected analytes }\end{array}$ \\
\hline Blind audit samples & $\begin{array}{l}\text { Samples and frequency } \\
\text { controlled by the Solid } \\
\text { PDP Plan }\end{array}$ & $\begin{array}{l}\text { Specified in the Solid } \\
\text { PDP Plan }\end{array}$ & $\begin{array}{l}\text { Specified in the Solid } \\
\text { PDP Plan }\end{array}$ \\
\hline
\end{tabular}

${ }^{a}$ Corrective Actions when QC samples do not meet acceptance criteria; Nonconformance procedures are outlined in Section 2.1.2.1 of the QAPP.

IDL $=$ Instrument detection limit

PDP = Performance Demonstration Program

$\mathrm{PROL}=$ Program required detection limit

$\% R=$ Percent recovery

$\mathrm{RPD}=$ Relative percent difference 
- For duplicate measurements, the precision expressed as the relative percent difference (RPD) is calculated as

$$
R P D=\frac{C_{1}-C_{2}}{\frac{\left(C_{1}+C_{2}\right)}{2}} * 100
$$

where

$$
\begin{array}{lll}
C_{1} \text { and } C_{2} & = & \text { two values obtained by analyzing the duplicate samples } \\
C_{I} & = & \text { larger of the two observed values }
\end{array}
$$

- For three or more replicate measurements, the precision expressed as the percent relative standard deviation (\%RSD) is calculated as

$$
\% R S D=\frac{S}{\bar{y}} * 100
$$

$$
\begin{array}{cll}
\text { where } & = & \text { standard deviation } \\
\frac{s}{y} & = & \text { mean of replicate analyses }
\end{array}
$$

- The standard deviation (s) is defined as

$$
s=\sqrt{\sum_{i=1}^{n} \frac{\left(y_{i}-\bar{y}\right)^{2}}{n-1}}
$$

where

$\begin{array}{ll}y_{i} & =\begin{array}{l}\text { measured value of the } i^{\text {th }} \text { replicate sample analysis } \\ \text { measurement }\end{array} \\ n & =\quad \text { number of replicate analyses }\end{array}$

Accuracy will be determined as the percent recovery (\%R) as follows:

$$
\% R=\frac{C_{m}}{C_{s m m}} * 100
$$

where

$$
\begin{array}{lll}
C_{m} & = & \text { measured concentration } \\
C_{s r m} & = & \text { true concentration }
\end{array}
$$

IDL will be determined as follows:

$$
\left.I D L=t_{(n-1,1-\alpha}=0.99\right) * S
$$

where

$$
\begin{array}{lll}
s & = & \text { standard deviation } \\
t_{(n-1,1-\alpha=0.99)} & \quad & t \text {-distribution value appropriate to a 99-percent confidence level } \\
\text { and a standard deviation estimate with } n-1 \text { degrees of freedom }
\end{array}
$$

10.4 The laboratory must analyze blanks at the frequency specified in Table 3 . The same procedures used to prepare and analyze field samples will be used to prepare and analyze the laboratory blanks. Laboratory blank results will be acceptable if the concentration of 
target analytes is less than or equal to 3 times the IDL. Corrective action must be implemented if blanks exceed this level.

10.5 The laboratory must analyze matrix spikes at the frequency specified in Table 3. Matrix spike results shall be considered acceptable if the \%R is 80 to 120 percent of the true value. Matrix spikes which do not meet these criteria should be flagged. \%R will be determined as follows:

$$
\% R=\frac{S-U}{C_{s a}} * 100
$$

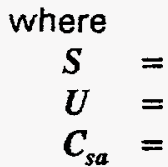

measured concentration in the spiked aliquot measured concentration in the unspiked aliquot actual concentration of the spike added

10.6 The laboratory must analyze matrix spike duplicates at the frequency specified in Table 3 . Matrix spike duplicate results shall be considered acceptable if the RPD is $\leq 30$ and the $\% R$ is 80 to 120 . RPD is determined as in Equation (1), \%R is determined as in Equation (6). Duplicates which do not meet these criteria should be flagged.

10.7 The laboratory must analyze laboratory control samples at the frequency specified in Table 3. Solid laboratory control samples (e.g., characterized surrogate sludges) should be used whenever it is possible to match the matrix of the waste samples. Otherwise, commercially purchased standards shall be used to prepare laboratory control samples. The laboratory control samples must contain the metals being analyzed, must undergo the same preparation and cleanup methods as the samples, and be quantitated within the calibration range of the instrument. The laboratory control sample analysis is designed to ensure that laboratory performance is in control for each analytical batch of samples processed. Laboratory control sample results will be considered acceptable if the $\% R$ is 80 to 120 percent of the known value. \%R is calculated as in Equation (4). If the results are outside of this range, then the associated samples must be reprepared and reanalyzed.

10.8 The laboratory will be required to analyze blind audit samples. These audit samples are part of the Performance Demonstration Program. Details of this program are in the Performance Demonstration Program Plan for the Analysis of Solidified Waste for the Transuranic Waste Characterization Program (Solid PDP Plan)(DOE 1995a).

\subsection{Procedure Performance}

The analyst is referred to Section 9.0 of SW-846 Method 7471A for a summary of method performance.

\subsection{References}

DOE. 1995a. Performance Demonstration Program Plan for the Analysis of Solidified Waste for the Transuranic Waste Characterization Program. CA0-95-1077, Current Revision, Carisbad, New Mexico, Carlsbad Area Office, U.S. Department of Energy.

DOE. 1995b. Transuranic Waste Characterization Quality Assurance Program Plan. CAO-941010, Current Revision, Carlsbad, New Mexico, Carlsbad Area Office, U.S. Department of Energy.

EPA. 1995. Test Methods for Evaluating Solid Waste, Physical/Chemical Methods. SW-846, Third Edition, Final Update I, and Proposed Update II, Washington D.C., Office of Solid Waste and Emergency Response, U.S. Environmental Protection Agency. 

PROCEDURE 650.4

\section{HYDRIDE GENERATION ATOMIC ABSORPTION \\ SPECTROSCOPY DETERMINATION OF TOTAL ARSENIC IN HOMOGENOUS SOLIDS AND SOIL/GRAVEL}

\subsection{Scope and Application}

1.1 This procedure is for the determination of total arsenic in samples of homogenous solids and soil/gravel. The procedure employs gaseous Hydride Generation Atomic Absorption Spectroscopy (HAA) for the quantitation of total arsenic. This procedure is not appropriate for samples which contain high concentrations of chromium, cobalt, copper, mercury, molybdenum, nickel, and silver. If high concentrations of these metals are known or suspected to be present in samples, Procedure 650.5 of this Methods Manual should be used. The quality assurance objectives for this procedure are listed in Table 1. A sampling procedure for homogenous solids and soil/gravel is described in Procedure 120.1 of this Methods Manual. This procedure must be implemented with a site-specific standard operating procedure (SOP).

1.2 The analyst is referred to Method 7061A of Test Methods for Evaluating Solid Waste, Physical/Chemical Methods, SW-846. Third Edition, Final Update I (SW-846) (EPA 1995) for the specific requirements of this procedure. The analyst is also referred to SW-846 Method 7000A for a general discussion of atomic absorption spectroscopy.

1.3 For the purposes of the TRU Waste Characterization Program, samples are to be analyzed in analytical batches. An analytical batch is defined as a suite of samples that is processed as a unit, using the same analytical method, within a specific time period. An analytical batch can be up to 20 samples (excluding laboratory OC samples), all of which must be received by the laboratory within 14 days of the validated time of sample receipt (VTSR) of the first sample in the batch.

\subsection{Summary of Procedure}

The analyst is referred to Section 2.0 of SW-846 Method 7061A for a summary of this procedure.

\subsection{Interferences}

The analyst is referred to Section 3.0 of SW-846 Method 7061A for an explanation of potential interferences.

\subsection{Safety}

This procedure may involve the use of hazardous materials, operations, and equipment. It is the responsibility of whoever uses this procedure to consult appropriate site personnel concerning health and safety issues and establish appropriate health and safety practices. Consideration should be given to safety concerns regarding chemical and radiation exposure. Training regarding proper storage, usage, and disposal of chemicals is recommended.

\subsection{Apparatus and Materials}

The analyst is referred to Section 4.0 of SW-846 Method 7061A for a description and listing of the required apparatus and materials for this procedure. 
TABLE 1

Quality Assurance Objectives for Total Arsenic Analysis

\begin{tabular}{lcccccc} 
Analyte & CAS Number & $\begin{array}{c}\text { Precision } \\
(\% R S D \text { or RPD })^{a}\end{array}$ & $\begin{array}{c}\text { Accuracy } \\
(\% R)^{b}\end{array}$ & $\begin{array}{c}\text { PRDL } \\
(\mu \mathrm{g} / \mathrm{L})\end{array}$ & $\begin{array}{c}\text { PRQL } \\
(\mathrm{mg} / \mathrm{kg})\end{array}$ & $\begin{array}{c}\text { Completeness } \\
(\%)\end{array}$ \\
\hline Arsenic & $7740-38-2$ & $\leq 30$ & $80-120$ & 100 & 100 & 90 \\
\hline
\end{tabular}

$\%$ RSD $=$ Percent relative standard deviation

RPD = Relative percent difference

$\% R=$ Percent recovery

PRDL = Program required detection limit (i.e., maximum permissible value for instrumental detection limit)

PRQL = Program required quantitation limit

$a 30 \%$ control limits apply when sample and duplicate concentrations are $\geq 10 \times$ instrument detection limit. If less than $10 \mathrm{x}$ instrumental detection limit, the absolute difference between the two values shall be less than or equal to the PRDL.

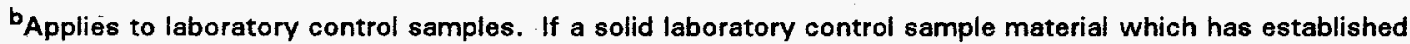
statistical control limits is used, then the established control limits for that material should be used for accuracy requirements.

CPRDL set such that it is a factor of 10 below the PRQL for 100 percent solid samples, assuming a $100 \mathrm{X}$ dilution during digestion. 


\subsection{Reagents}

The analyst is referred to Section 5.0 of SW-846 Method 7061A for a description and listing of the required reagents, standard stock solutions, and standard solutions for this procedure.

\subsection{Sample Collection, Preservation, and Handling}

7.1 Samples will be collected using the sampling procedures described in Procedure 120.1 of this Methods Manual.

7.2 All sample handling and chain-of-custody procedures described in Section 6.0 of the Transuranic Waste Characterization Quality Assurance Program Plan (QAPP) (DOE 1995b) must be followed.

\subsection{Procedure}

The analyst is referred to Section 7.1 through 7.7 of SW-846 Method 7061A for a description of the procedural requirements. Table 2 of this procedure provides a summary of required calibration and analysis quality control.

\subsection{Calculations}

Read the arsenic concentration from the calibration curve or directly from the instrument readout. Any sample dilution must be taken into account. Note that all results must be reported in $\mathrm{mglkg}$ (wet weight basis). To convert analytical results into $\mathrm{mg} / \mathrm{kg}$ dry weight, the percent solid content must be determined on a separate sample aliquot from that used for digestion and analysis.

\subsection{Quality Control}

10.1 All of the quality control requirements specified in Section 8.5 through 8.6 of SW-846 Method $7000 \mathrm{~A}$ are mandatory. If the method of standard additions is needed to overcome interferences, this information is presented in Section 8.7 of SW-846 Method $7000 \mathrm{~A}$. In instances where the quality control specifications in this procedure differ with those in SW-846 Method 7000A, this procedure shall take precedence.

10.2 Each laboratory that uses this procedure is required to operate a formal quality control program. The laboratory must retain records to document the quality of the data generated. Each laboratory must have SOPs documenting and describing activities involved in using this procedure. Specific quality control practices will include, but are not limited to, the analysis of quality control samples. The types of quality control samples, their associated frequency of analysis, acceptance criteria, and corrective action required if samples do not meet the acceptance criteria, is summarized in Table 3. Laboratory SOPs shall address requirements for preparing matrix spikes, matrix spike duplicates, laboratory blanks, and laboratory control samples.

10.3 All laboratories using this procedure must demonstrate acceptable performance prior to the analysis of actual samples. Demonstration of acceptable performance will be achieved by analyzing method performance samples (Table 3). These samples can be either commercially purchased or laboratory prepared, but must contain arsenic at concentrations appropriate (2-5 times the PRQLs) to determine the quality assurance objectives specified in Table 1. The analysis of seven samples must meet the criteria specified for precision, accuracy, and program required detection limit (PRDL) in Table 1. Note that the instrument detection limit (IDL) must be less than or equal to the PRDL. Demonstration of acceptable method and analyst performance must be repeated (by analyzing four samples) 
TABLE 2

Summary of Calibration Requirements and Analysis Quality Control for Total Arsenic Analysis

\begin{tabular}{|c|c|c|c|c|}
\hline Technique & Procedure & Frequency of Procedure & Acceptance Criteria & Corrective Action \\
\hline \multirow[t]{6}{*}{ HAA } & $\begin{array}{l}\text { 5-pt. initial calibration } \\
\text { ( } 5 \text { standards and a blank) }\end{array}$ & Daily & $\begin{array}{l}90-110 \% R \text { for initial calibration verification } \\
\text { solution }\end{array}$ & $\begin{array}{l}\text { Correct problem and repeat initial } \\
\text { calibration }\end{array}$ \\
\hline & & & 95-105\%R for highest calibration standard & \\
\hline & & & Regression coefficient $\left(r^{2}\right)$ must be $\geq 0.995$ & \\
\hline & Continuing calibration & $\begin{array}{l}\text { Every } 10 \text { samples plus beginning and end } \\
\text { of run }\end{array}$ & $\begin{array}{l}80-120 \% \mathrm{R} \text { for check standard (mandatory); } \\
\text { blank (optional) should measure } \leq 3 \times 1 \mathrm{DL}\end{array}$ & Recalibrate and rerun last 10 samples \\
\hline & Serial dilution & $\begin{array}{l}\text { once per analytical batch or per matrix } \\
\text { within an analytical batch }\end{array}$ & $\begin{array}{l}5 \times \text { dilution of sample which is }>25 \times 1 D L \\
\text { must be } \leq 10 \% D \text { of initial value }\end{array}$ & $\begin{array}{l}\text { Use MSA to quantitate samples of like } \\
\text { matrix }\end{array}$ \\
\hline & Post-digestion spike & $\begin{array}{l}\text { once per analytical batch or per matrix } \\
\text { within an analytical batch }\end{array}$ & $85-115 \% R$ & $\begin{array}{l}\text { Use MSA to quantitate samples of like } \\
\text { matrix }\end{array}$ \\
\hline
\end{tabular}

$\begin{array}{lll}I D L & = & \text { Instrument detection limit } \\ \% D & = & \text { Percent difference } \\ \text { MSA } & =\text { Method of standard additions } \\ \% R & =\quad \text { Percent recovery }\end{array}$

$\% R=$ Percent recovery 
TABLE 3

\section{Summary of Laboratory Quality Control Samples and Frequencies for Total Arsenic Analyses}

\begin{tabular}{|c|c|c|c|}
\hline QC Samples & Minimum Frequency & $\begin{array}{l}\text { Acceptance } \\
\text { Criteria }\end{array}$ & Corrective Actions ${ }^{a}$ \\
\hline $\begin{array}{l}\text { Method performance } \\
\text { samples }\end{array}$ & $\begin{array}{l}\text { Seven (7) samples initially, } \\
\text { and four (4) semiannually }\end{array}$ & Meet Table 1 QAOs & Repeat until acceptable \\
\hline Laboratory blanks & $\begin{array}{l}\text { One (1) per analytical } \\
\text { batch }\end{array}$ & $\leq 3 \times \mathrm{IDL}$ & $\begin{array}{l}\text { Redigest and reanalyze } \\
\text { any samples with } \\
\text { analyte concentrations } \\
\text { which are } \\
\leq 10 \times \text { blank value and } \\
\geq 0.5 \times \text { PRQL }\end{array}$ \\
\hline Matrix spikes & $\begin{array}{l}\text { One (1) per analytical } \\
\text { batch }\end{array}$ & $80-120 \% R$ & $\begin{array}{l}\text { Nonconformance if } \% R \\
<80 \text { or }>120\end{array}$ \\
\hline Matrix spike duplicates & $\begin{array}{l}\text { One (1) per analytical } \\
\text { batch }\end{array}$ & $\begin{array}{l}R P D \leq 30 \\
80-120 \% R\end{array}$ & $\begin{array}{l}\text { Nonconformance if } \\
\mathrm{RPD}>30 \text { or if } \% \mathrm{R}< \\
80 \text { or }>120\end{array}$ \\
\hline $\begin{array}{l}\text { Laboratory control } \\
\text { samples }\end{array}$ & $\begin{array}{l}\text { One (1) per analytical } \\
\text { batch }\end{array}$ & $80-120 \% R$ & $\begin{array}{l}\text { Redigest and reanalyze } \\
\text { for affected analytes }\end{array}$ \\
\hline Blind audit samples & $\begin{array}{l}\text { Samples and frequency } \\
\text { controlled by the Solid } \\
\text { PDP Plan }\end{array}$ & $\begin{array}{l}\text { Specified in the Solid } \\
\text { PDP Plan }\end{array}$ & $\begin{array}{l}\text { Specified in the Solid } \\
\text { PDP Plan }\end{array}$ \\
\hline
\end{tabular}

${ }^{a}$ Corrective Actions when QC samples do not meet acceptance criteria; Nonconformance procedures are outlined in Section 2.1.2.1 of the QAPP.

$\mathrm{IDL}=$ Instrument detection limit
PDP $=$ Performance Demonstration Program
PRQL $=$ Program required detection limit
$\% R=$ Percent recovery
RPD $=$ Relative percent difference


at a minimum of every six months. Method performance should be conducted over a period of several days to account for long-term variability. Corrective action must be implemented when acceptable method performance is not met. Precision will be determined as follows:

- For duplicate measurements, the precision expressed as the relative percent difference (RPD) is calculated as

$$
R P D=\frac{C_{1}-C_{2}}{\frac{\left(C_{1}+C_{2}\right)}{2}} * 100
$$

where

$$
\begin{array}{lll}
C_{1} \text { and } C_{2} & = & \text { two values obtained by analyzing the duplicate samples } \\
C_{1} & = & \text { larger of the two observed values }
\end{array}
$$

- For three or more replicate measurements, the precision expressed as the percent relative standard deviation (\%RSD) is calculated as

$$
\% R S D=\frac{s}{\bar{y}} * 100
$$

where

$$
\begin{array}{lll}
s & = & \text { standard deviation } \\
y & = & \text { mean of replicate analyses }
\end{array}
$$

- The standard deviation, $(s)$ is defined as

$$
s=\sqrt{\sum_{i=1}^{n} \frac{\left(y_{i}-\bar{y}\right)^{2}}{n-1}}
$$

where

$$
\begin{array}{ll}
y_{i} & =\begin{array}{l}
\text { measured value of the } i^{t h} \text { replicate sample analysis } \\
\text { measurement }
\end{array} \\
n & =\text { number of replicate analyses }
\end{array}
$$

Accuracy will be determined as the percent recovery $(\% \mathrm{R})$ as follows:

$$
\% R=\frac{C_{m}}{C_{s m}} * 100
$$

where

$$
\begin{array}{ll}
C_{m}= & \text { measured concentration } \\
C_{s r m}= & \text { true concentration }
\end{array}
$$

IDL will be determined as follows:

$$
I D L=t_{(n-1,1-\alpha=0.99)} * s
$$

where

$$
\begin{array}{lll}
s & = & \text { standard deviation } \\
t_{(n-1,1-\alpha=0.99)} & = & t \text {-distribution value appropriate to a 99-percent confidence level } \\
\text { and a standard deviation estimate with } n-1 \text { degrees of freedom }
\end{array}
$$


10.4 The laboratory must analyze blanks at the frequency specified in Table 3 . The same procedure used to prepare and analyze field samples will be used to prepare and analyze the laboratory blanks. Laboratory blank results will be acceptable if the concentration of target analytes is less than or equal to 3 times the IDL. Corrective action must be implemented if blanks exceed this level.

10.5 The laboratory must analyze matrix spikes at the frequency specified in Table 3 . Matrix spike results shall be considered acceptable if the \%R is 80 to 120 percent of the true value. Matrix spikes which do not meet these criteria should be flagged. \%R will be determined as follows:

$$
\% R=\frac{S-U}{C_{s a}} * 100
$$

where
$S=$
$U=$
$C_{s a}=$ measured concentration in the spiked aliquot measured concentration in the unspiked aliquot actual concentration of the spike added

10.6 The laboratory must analyze matrix spike duplicates at the frequency specified in Table 3 . Matrix spike duplicate results shall be considered acceptable if the RPD is $\leq 30$ and $\% R$ is 80 to 120. RPD is determined as in Equation (1), \%R is determined as in Equation (6). Duplicates which do not meet these criteria should be flagged.

10.7 The laboratory must analyze laboratory control samples at the frequency specified in Table 3. Solid laboratory control samples (e.g., characterized surrogate sludges) should be used whenever it is possible to match the matrix of the waste samples. Otherwise, commercially purchased standards shall be used to prepare laboratory control samples. The laboratory control samples must contain the metals being analyzed, must undergo the same preparation and clean-up methods as the samples, and be quantitated within the calibration range of the instrument. The laboratory control sample analysis is designed to ensure that laboratory performance is in control for each analytical batch of samples processed. Laboratory control sample results will be considered acceptable if the \%R is 80 to 120 percent of the known value. \%R is calculated as in Equation (4). If the results are outside of this range, then the associated samples must be reprepared and reanalyzed.

10.8 The laboratory will be required to analyze blind audit samples. These audit samples are part of the Performance Demonstration Program. Details of this program are in the Performance Demonstration Program Plan for the Analysis of Solidified Waste for the Transuranic Waste Characterization Program (Solid PDP Plan)(DOE 1995a).

\subsection{Procedure Performance}

The analyst is referred to Section 9.0 of SW-846 Method 7061A for a summary of method performance.

\subsection{References}

DOE. 1995a. Performance Demonstration Program Plan for the Analysis of Solidified Waste for the Transuranic Waste Characterization Program. CA0-95-1077, Current Revision, Carlsbad, New Mexico, Carlsbad Area Office, U.S. Department of Energy. 
DOE. 1995b. Transuranic Waste Characterization Quality Assurance Program Plan. CAO-941010, Current Revision, Carlsbad, New Mexico, Carlsbad Area Office, U.S. Department of Energy.

EPA. 1995. Test Methods for Evaluating Solid Waste, Physical/Chemicai Methods. SW-846, Third Edition, Final Update I, and Final Update II, Washington D.C., Office of Solid Waste and Emergency Response, U.S. Environmental Protection Agency. 


\section{PROCEDURE 650.5}

\section{BOROHYDRIDE GENERATION ATOMIC ABSORPTION SPECTROSCOPY DETERMINATION OF TOTAL ANTIMONY AND ARSENIC IN HOMOGENOUS SOLIDS AND SOIL/GRAVEL}

\subsection{Scope and Application}

1.1 This procedure is for the determination of total antimony and arsenic in samples of homogenous solids and soil/gravel. The procedure employs gaseous Borohydride Generation Atomic Absorption Spectroscopy (HAA) for the quantitation of total metals. This procedure should be used for samples which contain high concentrations of chromium, cobalt, copper, mercury, molybdenum, nickel, and silver which may cause interferences when using Procedure 650.4 of this Methods Manual. Analytical samples can contain concentrations of up to $4000 \mathrm{mg} / \mathrm{L}$ of cobalt, copper, iron, mercury, and nickel and still be successfully analyzed by this procedure. Note that solid samples can contain up to 40 percent by weight of these metals before exceeding $4000 \mathrm{mg} / \mathrm{L}$ in a digested sample. The quality assurance objectives for this procedure are listed in Table 1. A sampling procedure for homogenous solids and soil/gravel is described in Procedure 120.1 of this Methods Manual. This procedure must be implemented with a site-specific standard operating procedure (SOP).

1.2 The analyst is referred to Method 7062 of Test Methods for Evaluating Solid Waste, Physical/Chemical Methods, SW-846, Third Edition, Final Update II (SW-846) (EPA 1995) for the specific requirements of this procedure. The analyst is also referred to SW-846 Method 7000A for a general discussion of atomic absorption spectroscopy.

1.3 For the purposes of the TRU Waste Characterization Program, samples are to be analyzed in analytical batches. An analytical batch is defined as a suite of samples that is processed as a unit, using the same analytical method, within a specific time period. An analytical batch can be up to 20 samples (excluding laboratory OC samples), all of which must be received by the laboratory within 14 days of the validated time of sample receipt (VTSR) of the first sample in the batch.

\subsection{Summary of Procedure}

The analyst is referred to Section 2.0 of SW-846 Method 7062 for a summary of this procedure, except that the sample preparation procedures described in Procedure 610.1 of this Methods Manual must be followed prior to evaporating the samples to near dryness at the end of the digestion.

\subsection{Interferences}

The analyst is referred to Section 3.0 of SW-846 Method 7062 for an explanation of potential interferences.

\subsection{Safety}

This procedure may involve the use of hazardous materials, operations, and equipment. It is the responsibility of whoever uses this procedure to consult appropriate site personnel concerning health and safety issues and establish appropriate health and safety practices. Consideration should be given to safety concerns regarding chemical and radiation exposure. Training regarding proper storage, usage, and disposal of chemicals is recommended. 


\section{TABLE 1}

\section{Quality Assurance Objectives for Total Antimony and Arsenic Analysis}

\begin{tabular}{lcccccc} 
Analyte & CAS Number & $\begin{array}{c}\text { Precision } \\
\text { (\%RSD or RPD) }\end{array}$ & $\begin{array}{c}\text { Accuracy } \\
(\% R)^{\mathrm{b}}\end{array}$ & $\begin{array}{c}\text { PRDLC } \\
(\mu \mathrm{g} / \mathrm{L})\end{array}$ & $\begin{array}{c}\text { PRQL } \\
(\mathrm{mg} / \mathrm{kg})\end{array}$ & $\begin{array}{c}\text { Completeness } \\
(\%)\end{array}$ \\
\hline \multirow{2}{*}{ Antimony } & $7440-36-0$ & $\leq 30$ & $80-120$ & 100 & 100 & 90 \\
Arsenic & $7740-38-2$ & $\leq 30$ & $80-120$ & 100 & 100 & 90 \\
\hline
\end{tabular}

$\begin{array}{ll}\text { \%RSD } & =\text { Percent relative standard deviation } \\ \text { RPD } & =\text { Relative percent difference } \\ \% \text { R } & =\text { Percent recovery } \\ \text { PRDL } & =\text { Program required detection limit (i.e., maximum permissible value for instrument detection limit) } \\ \text { PRQL } & =\text { Program required quantitation limit }\end{array}$

${ }^{a} \leq 30 \%$ control limits apply when sample and duplicate concentrations are $\geq 10 x$ instrument detection limit. If less than $10 \times$ instrument detection limit, the absolute difference between the two values shall be less than or equal to the PRDL.

${ }^{b}$ Applies to laboratory control samples. If a solid laboratory control sample material which has established statistical control limits is used, then the established control limits for that material should be used for accuracy requirements.

cPRDL set such that it is a factor of 10 below the PROL for 100 percent solid samples, assuming a $100 \mathrm{X}$ dilution during digestion. 


\subsection{Apparatus and Materials}

The analyst is referred to Section 4.0 of SW-846 Method 7062 for a description and listing of the required apparatus and materials for this procedure.

\subsection{Reagents}

The analyst is referred to Section 5.0 of SW-846 Method 7062 for a description and listing of the required reagents, standard stock solutions, and standard solutions for this procedure.

\subsection{Sample Collection, Preservation, and Handling}

7.1 Samples will be collected using the sampling procedures described in Procedure 120.1 of this Methods Manual.

7.2 All sample handling and chain-of-custody procedures described in Section 6.0 of the Transuranic Waste Characterization Quality Assurance Program Plan (QAPP) (DOE 1995b) must be followed.

\subsection{Procedure}

The analyst is referred to Section 7.1 through 7.6 of SW-846 Method 7062 for a description of the procedural requirements. Table 2 of this procedure provides a summary of required calibration and analysis quality control.

\subsection{Calculations}

Read the analyte concentration from the calibration curve or directly from the instrument readout. Any sample dilution must be taken into account. Note that all results must be reported in $\mathrm{mg} / \mathrm{kg}$ (wet weight basis). To convert analytical results into $\mathrm{mglkg}$ dry weight, the percent solid content must be determined on a separate sample aliquot from that used for digestion and analysis.

\subsection{Quality Control}

10.1 All of the quality control requirements specified in Section 8.5 and 8.6 of SW-846 Method $7000 \mathrm{~A}$ are mandatory. If the method of standard additions is needed to overcome interferences, this information is presented in Section 8.7 of SW-846 Method 7000A. In instances where the quality control specifications in this procedure differ with those in SW-846 Method 7000A, this procedure shall take precedence.

10.2 Each laboratory that uses this procedure is required to operate a formal quality control program. The laboratory must retain records to document the quality of the data generated. Each laboratory must have SOPs documenting and describing activities involved in using this procedure. Specific quality control practices will include, but are not limited to, the analysis of quality control samples. The types of quality control samples, their associated frequency of analysis, acceptance criteria, and corrective action required if samples do not meet the acceptance criteria, is summarized in Table 3. Laboratory SOPs shall address requirements for preparing matrix spikes, matrix spike duplicates, laboratory blanks, and laboratory control samples.

10.3 All laboratories using this procedure must demonstrate acceptable performance prior to the analysis of actual samples. Demonstration of acceptable performance will be achieved by analyzing method performance samples (Table 3). These samples can be either commercially purchased or laboratory prepared, but must contain arsenic at concentrations 
TABLE 2

Summary of Calibration Requirements and Analysis Quality Control for Total Antimony and Arsenic Analysis

\begin{tabular}{|c|c|c|c|c|}
\hline Technique & Procedure & Frequency of Procedure & Acceptance Criteria & Corrective Action \\
\hline \multirow[t]{6}{*}{ HAA } & $\begin{array}{l}\text { 5-pt. initial calibration } \\
\text { (5 standards and a blank) }\end{array}$ & Daily & $\begin{array}{l}90-110 \% R \text { for initial calibration verification } \\
\text { solution }\end{array}$ & $\begin{array}{l}\text { Correct problem and repeat initial } \\
\text { calibration }\end{array}$ \\
\hline & & & $95-105 \% \mathrm{R}$ for highest calibration standard & \\
\hline & & & Regression coefficient $\left(r^{2}\right)$ must be $\geq 0.995$ & \\
\hline & Continuing calibration & $\begin{array}{l}\text { Every } 10 \text { samples plus beginning and end } \\
\text { of run }\end{array}$ & $\begin{array}{l}80-120 \% R \text { for check standard (mandatory); } \\
\text { blank (optional) should measure } \leq 3 \times 1 D L\end{array}$ & Recalibrate and rerun last 10 samples \\
\hline & Serial dilution & $\begin{array}{l}\text { once per analytical batch or per matrix } \\
\text { within an analytical batch }\end{array}$ & $\begin{array}{l}5 x \text { dilution of sample which is }>25 \times 1 D L \\
\text { must be } \leq 10 \% \mathrm{D} \text { of initial value }\end{array}$ & $\begin{array}{l}\text { Use MSA to quantitate samples of like } \\
\text { matrix }\end{array}$ \\
\hline & Post-digestion spike & $\begin{array}{l}\text { once per analytical batch or per matrix } \\
\text { within an analytical batch }\end{array}$ & $85-115 \% R$ & $\begin{array}{l}\text { Use MSA to quantitate samples of like } \\
\text { matrix }\end{array}$ \\
\hline
\end{tabular}

$\begin{array}{lll}I D L & = & \text { Instrument detection limit } \\ \% D= & \text { Percent difference } \\ \text { MSA }= & \text { Method of standard additions } \\ \% R= & \text { Percent recovery }\end{array}$


TABLE 3

\section{Summary of Laboratory Quality Control Samples and Frequencies for Total Antimony and Arsenic Analyses}

QC Samples

Minimum Frequency
Acceptance

Criteria
Corrective Actions ${ }^{a}$

Seven (7) samples initially, Meet Table 1 QAOs and four (4) semiannually

One (1) per analytical $\quad \leq 3 \times$ IDL batch

$80-120 \% R$

One (1) per analytical batch

Samples and frequency controlled by the Solid PDP Plan
$80-120 \% R$

RPD $\leq 30$

80-120\%R

Repeat until acceptable

Redigest and reanalyze any samples with analyte concentrations which are

$\leq 10 \times$ blank value and $\geq 0.5 \times$ PROL

Nonconformance if $\% R$ $<80$ or $>120$

Nonconformance if RPD > 30 or if \%R < 80 or $>120$

Redigest and reanalyze for affected analytes

Specified in the Solid PDP Plan
Specified in the Solid PDP Plan

${ }^{a}$ Corrective Actions when QC samples do not meet acceptance criteria; Nonconformance procedures are outlined in Section 2.1.2.1 of the QAPP.

IDL $=$ Instrument detection limit

PDP $=$ Performance Demonstration Program

$\mathrm{PROL}=$ Program required detection limit

$\% R=$ Percent recovery

$\mathrm{RPD}=$ Relative percent difference 
appropriate (2-5 times the PROLs) to determine the quality assurance objectives specified in Table 1. The analysis of seven samples must meet the criteria specified for precision, accuracy, and program required detection limit (PRDL) in Table 1. Note that the instrument detection limit (IDL) must be less than or equal to the PRDL. Demonstration of acceptable method and analyst performance must be repeated (by analyzing four samples) at a minimum of every six months. Method performance should be conducted over a period of several days to account for long-term variability. Corrective action must be implemented when acceptable method performance is not met. Precision will be determined as follows:

- For duplicate measurements, the precision expressed as the relative percent difference (RPD) is calculated as

$$
R P D=\frac{C_{1}-C_{2}}{\frac{\left(C_{1}+C_{2}\right)}{2}} * 100
$$

where

$$
\begin{array}{lll}
C_{1} \text { and } C_{2} & = & \text { two values obtained by analyzing the duplicate samples } \\
C_{1} & = & \text { larger of the two observed values }
\end{array}
$$

- For three or more replicate measurements, the precision expressed as the percent relative standard deviation (\%RSD) is calculated as

$$
\% R S D=\frac{s}{\bar{y}} * 100
$$

where

$$
\begin{array}{lll}
s & = & \text { standard deviation } \\
y & = & \text { mean of replicate analyses }
\end{array}
$$

- The standard deviation $(s)$ is defined as

$$
s=\sqrt{\sum_{i=1}^{n} \frac{\left(y_{i}-\bar{y}\right)^{2}}{n-1}}
$$

where

$$
\begin{array}{lll}
y_{i} & =\begin{array}{l}
\text { measured value of the } i^{\text {th }} \text { replicate sample analysis } \\
\text { measurement }
\end{array} \\
n & =\text { number of replicate analyses }
\end{array}
$$

Accuracy will be determined as the percent recovery $(\% R)$ as follows:

$$
\text { where }
$$$$
\% R=\frac{C_{m}}{C_{s r m}} * 100
$$ 
IDL will be determined as follows:

$$
I D L=t_{(n-1,1-\alpha=0.99)} * s
$$

where

$$
\begin{array}{lll}
s & = & \text { standard deviation } \\
t_{(n-1,1-\alpha=0.99)} & = & \begin{array}{l}
\text { t-distribution value appropriate to a 99-percent confidence level } \\
\text { and a standard deviation estimate with } n-1 \text { degrees of freedom }
\end{array}
\end{array}
$$

10.4 The laboratory must analyze blanks at the frequency specified in Table 3 . The same procedures used to prepare and analyze field samples will be used to prepare and analyze the laboratory blanks. Laboratory blank results will be acceptable if the concentration of target analytes is less than or equal to 3 times the IDL. Corrective action must be implemented if blanks exceed this level.

10.5 The laboratory must analyze matrix spikes at the frequency specified in Table 3. Matrix spike results shall be considered acceptable if the \%R is 80 to 120 percent of the true value. Matrix spikes which do not meet these criteria should be flagged. \%R will be determined as follows:

$$
\% R=\frac{S-U}{C_{s a}} * 100
$$

$$
\begin{array}{cll}
\text { where } & & \text { measured concentration in the spiked aliquot } \\
S & = & \text { measured concentration in the unspiked aliquot } \\
U & = & \text { actual concentration of the spike added }
\end{array}
$$

10.6 The laboratory must analyze matrix spike duplicates at the frequency specified in Table 3 . Matrix spike duplicate results shall be considered acceptable if the RPD is $\leq 30$ and the $\% R$ is 80 to 120 . RPD determined as in Equation (1), \%R is determined as in Equation (6). Duplicates which do not meet these criteria should be flagged.

10.7 The laboratory must analyze laboratory control samples at the frequency specified in Table 3. Solid laboratory control samples (e.g., characterized surrogate sludges) should be used whenever it is possible to match the matrix of the waste samples. Otherwise, commercially purchased standards shall be used to prepare laboratory control samples. The laboratory control samples must contain the metals being analyzed, must undergo the same preparation and clean-up methods as the samples, and be quantitated within the calibration range of the instrument. The laboratory control sample analysis is designed to ensure that laboratory performance is in control for each analytical batch of samples processed. Laboratory control sample results will be considered acceptable if the \% $R$ is 80 to 120 percent of the known value. \%R is calculated as in Equation (4). If the results are outside of this range, then the associated samples must be reprepared and reanlayzed.

10.8 The laboratory will be required to analyze blind audit samples. These audit samples are part of the Performance Demonstration Program. Details of this program are in the Performance Demonstration Program Plan for the Analysis of Solidified Waste for the Transuranic Waste Characterization Program (Solid PDP Plan)(DOE 1995a).

\subsection{Procedure Performance}

The analyst is referred to Section 9.0 of SW-846 Method 7062 for a summary of method performance. 


\subsection{References}

DOE. 1995a. Performance Demonstration Program Plan for the Analysis of Solidified Waste for the Transuranic Waste Characterization Program. CA0-95-1077, Current Revision, Carlsbad, New Mexico, Carlsbad Area Office, U.S. Department of Energy.

DOE. 1995b. Transuranic Waste Characterization Quality Assurance Program Plan. CAO-941010, Current Revision, Carlsbad, New Mexico, Carlsbad Area Office, U.S. Department of Energy.

EPA. 1995. Test Methods for Evaluating Solid Waste, Physical/Chemical Methods. SW-846, Third Edition, Final Update I, and Final Update II, Washington D.C., Office of Solid Waste and Emergency Response, U.S. Environmental Protection Agency. 
PROCEDURE 650.6

\author{
HYDRIDE GENERATION ATOMIC ABSORPTION \\ SPECTROSCOPY DETERMINATION OF TOTAL SELENIUM \\ IN HOMOGENOUS SOLIDS AND SOIL/GRAVEL
}

\title{
1.0 Scope and Application
}

1.1 This procedure is for the determination of total selenium in samples of homogenous solids and soil/gravel. The procedure employs gaseous Hydride Generation Atomic Absorption Spectroscopy (HAA) for the quantitation of total selenium. This procedure is not appropriate for samples which contain high concentrations of chromium, cobalt, copper, mercury, molybdenum, and silver. If high concentrations of these metals are known or suspected to be present in samples, Procedure 650.7 of this Methods Manual should be used. The quality assurance objectives for this procedure are listed in Table 1. A sampling procedure for homogenous solids and soil/gravel is described in Procedure 120.1 of this Methods Manual. This procedure must be implemented with a site-specific standard operating procedure (SOP).

1.2 The analyst is referred to Method 7741A of Test Methods for Evaluating Solid Waste, Physical/Chemical Methods, SW-846, Third Edition, Final Update II (SW-846) (EPA 1995) for the specific requirements of this procedure. The analyst is also referred to SW-846 Method 7000A for a general discussion of atomic absorption spectroscopy.

1.3 For the purposes of the TRU Waste Characterization Program, samples are to be analyzed in analytical batches. An analytical batch is defined as a suite of samples that is processed as a unit, using the same analytical method, within a specific time period. An analytical batch can be up to 20 samples (excluding laboratory $\mathrm{OC}$ samples), all of which must be received by the laboratory within 14 days of the validated time of sample receipt (VTSR) of the first sample in the batch.

\subsection{Summary of Procedure}

The analyst is referred to Section 2.0 of SW-846 Method 7741A for a summary of this procedure.

\subsection{Interferences}

The analyst is referred to Section 3.0 of SW-846 Method 7741A for an explanation of potential interferences.

\subsection{Safety}

This procedure may involve the use of hazardous materiais, operations, and equipment. It is the responsibility of whoever uses this procedure to consult appropriate site personnel concerning health and safety issues and establish appropriate health and safety practices. Consideration should be given to safety concerns regarding chemical and radiation exposure. Training regarding proper storage, usage, and disposal of chemicals is recommended.

\subsection{Apparatus and Materials}

The analyst is referred to Section 4.0 of SW-846 Method 7741 A for a description and listing of the required apparatus and materials for this procedure. 
TABLE 1

\section{Quality Assurance Objectives for Total Selenium Analysis}

\begin{tabular}{lcccccc}
\hline Analyte & CAS Number & $\begin{array}{c}\text { Precision } \\
(\% R S D \text { or RPD) }\end{array}$ & $\begin{array}{c}\text { Accuracy } \\
(\% R)^{\mathrm{b}}\end{array}$ & $\begin{array}{c}\text { PRDLc } \\
(\mu \mathrm{g} / \mathrm{L})\end{array}$ & $\begin{array}{c}\text { PRQL } \\
(\mathrm{mg} / \mathrm{kg})\end{array}$ & $\begin{array}{c}\text { Completeness } \\
(\%)\end{array}$ \\
\hline Selenium & $7782-49-2$ & $\leq 30$ & $80-120$ & 20 & 20 & 90 \\
\hline
\end{tabular}

\%RSD $=$ Percent relative standard deviation

$\mathrm{RPD}=$ Relative percent difference

$\% R=$ Percent recovery

PRDL = Program required detection limit (i.e., maximum permissible value for instrument detection limit) PROL $=$ Program required quantitation limit

$a \leq 30 \%$ control limits apply when sample and duplicate concentrations are $\geq 10 \times$ instrument detection limit. If either are less than $10 \mathrm{x}$ instrument detection limit, the absolute difference between the two values shall be less than or equal to the PRDL.

${ }^{b}$ Applies to laboratory control samples. If a solid laboratory control sample material which has established statistical control limits is used, then the established control limits for that material should be used for accuracy requirements.

CPRDL set such that it is a factor of 10 below the PROL for 100 percent solid samples, assuming a $100 \mathrm{X}$ dilution during digestion. 


\subsection{Reagents}

The analyst is referred to Section 5.0 of SW-846 Method 7741A for a description and listing of the required reagents and standard stock solutions for this procedure.

\subsection{Sample Collection, Preservation, and Handling}

7.1 Samples will be collected using the sampling procedures described in Procedure 120.1 of this Methods Manual.

7.2 All sample handling and chain-of-custody procedures described in Section 6.0 of the Transuranic Waste Characterization Quality Assurance Program Plan (QAPP) (DOE 1995b) must be followed.

\subsection{Procedure}

The analyst is referred to Section 7.1 through 7.6 of SW-846 Method 7741A for a description of the procedural requirements. Table 2 of this procedure provides a summary of required calibration and analysis quality control.

\subsection{Calculations}

Read the selenium concentration from the calibration curve or directly from the instrument readout. Any sample dilution must be taken into account. Note that all results must be reported in mglkg (wet weight basis). To convert analytical results into $\mathrm{mg} / \mathrm{kg}$ dry weight, the percent solid content must be determined on a separate sample aliquot from that used for digestion and analysis.

\subsection{Quality Control}

10.1 All of the quality control requirements specified in Section 8.5 through 8.6 of SW-846 Method $7000 \mathrm{~A}$ are mandatory. If the method of standard additions is needed to overcome interferences, this information is presented in Section 8.7 of SW-846 Method $7000 \mathrm{~A}$. In instances where the quality control specifications in this procedure differ with those in SW-846 Method 7000A, this procedure shall take precedence.

10.2 Each laboratory that uses this procedure is required to operate a formal quality control program. The laboratory must retain records to document the quality of the data generated. Each laboratory must have SOPs documenting and describing activities involved in using this procedure. Specific quality control practices will include, but are not limited to, the analysis of quality control samples. The types of quality control samples, their associated frequency of analysis, acceptance criteria, and corrective action required if samples do not meet the acceptance criteria, is summarized in Table 3. Laboratory SOPs shall address requirements for preparing matrix spikes, matrix spike duplicates, laboratory blanks, and laboratory control samples.

10.3 All laboratories using this procedure must demonstrate acceptable performance prior to the analysis of actual samples. Demonstration of acceptable performance will be achieved by analyzing method performance samples (Table 3). These samples can be either commercially purchased or laboratory prepared, but must contain selenium at concentrations appropriate (2-5 times the PROLs) to determine the quality assurance objectives specified in Table 1. The analysis of seven samples must meet the criteria specified for precision, accuracy, and program required detection limit (PRDL) in Table 1. Note that the instrument detection limit (IDL) must be less than or equal to the PRDL. Demonstration of acceptable method and analyst performance must be repeated (by 
TABLE 2

Summary of Calibration Requirements and Analysis Quality Control for Total Selenium Analysis

\begin{tabular}{|c|c|c|c|c|}
\hline Technique & Procedure & Frequency of Procedure & Acceptance Criteria & Corrective Action \\
\hline \multirow[t]{6}{*}{ HAA } & $\begin{array}{l}\text { 5-pt. initial calibration } \\
\text { ( } 5 \text { standards and a blank) }\end{array}$ & Daily & $\begin{array}{l}90-110 \% R \text { for initial calibration verification } \\
\text { solution }\end{array}$ & $\begin{array}{l}\text { Correct problem and repeat initial } \\
\text { calibration }\end{array}$ \\
\hline & & & 95-105\%R for highest calibration standard & \\
\hline & & & Regression coefficient $\left(r^{2}\right)$ must be $\geq 0.995$ & \\
\hline & Continuing calibration & $\begin{array}{l}\text { Every } 10 \text { samples plus beginning and end } \\
\text { of run }\end{array}$ & $\begin{array}{l}80-120 \% R \text { for check standard (mandatory); } \\
\text { blank (optional) should measure } \leq 3 \times 1 D L\end{array}$ & Recalibrate and rerun last 10 samples \\
\hline & Serial dilution & $\begin{array}{l}\text { once per analytical batch or per matrix } \\
\text { within an analytical batch }\end{array}$ & $\begin{array}{l}5 x \text { dilution of sample which is }>25 \times 1 D L \\
\text { must be } \leq 10 \% D \text { of initial value }\end{array}$ & $\begin{array}{l}\text { Use MSA to quantitate samples of like } \\
\text { matrix }\end{array}$ \\
\hline & Post-digestion spike & $\begin{array}{l}\text { once per analytical batch or per matrix } \\
\text { within an analytical batch }\end{array}$ & $85-115 \% R$ & $\begin{array}{l}\text { Use MSA to quantitate samples of like } \\
\text { matrix }\end{array}$ \\
\hline
\end{tabular}

$\begin{array}{lll}\text { IDL } & = & \text { Instrument detection limit } \\ \% D= & \text { Percent difference } \\ \text { MSA }= & \text { Method of standard additions } \\ \% \mathrm{R}= & \text { Percent recovery }\end{array}$

$\begin{array}{lll}\text { MSA } & =\text { Method of standard } \\ \% R=\text { Percent recovery }\end{array}$ 
TABLE 3

\section{Summary of Laboratory Quality Control Samples and Frequencies for Total Selenium Analyses}

\begin{tabular}{|c|c|c|c|}
\hline OC Samples & Minimum Frequency & $\begin{array}{l}\text { Acceptance } \\
\text { Criteria }\end{array}$ & Corrective Actions ${ }^{\mathrm{a}}$ \\
\hline $\begin{array}{l}\text { Method performance } \\
\text { samples }\end{array}$ & $\begin{array}{l}\text { Seven (7) samples initially, } \\
\text { and four (4) semiannually }\end{array}$ & Meet Table 1 OAOs & Repeat until acceptable \\
\hline Laboratory blanks & $\begin{array}{l}\text { One (1) per analytical } \\
\text { batch }\end{array}$ & $\leq 3 \times I D L$ & $\begin{array}{l}\text { Redigest and reanalyze } \\
\text { any samples with } \\
\text { analyte concentrations } \\
\text { which are } \\
\leq 10 \times \text { blank value and } \\
\geq 0.5 \times \text { PRQL }\end{array}$ \\
\hline Matrix spikes & $\begin{array}{l}\text { One (1) per analytical } \\
\text { batch }\end{array}$ & $80-120 \% R$ & $\begin{array}{l}\text { Nonconformance if } \% R \\
<80 \text { or }>120\end{array}$ \\
\hline Matrix spike duplicates & $\begin{array}{l}\text { One (1) per analytical } \\
\text { batch }\end{array}$ & $\begin{array}{l}\text { RPD } \leq 30 \\
80-120 \% R\end{array}$ & $\begin{array}{l}\text { Nonconformance if } \\
\text { RPD }>30 \text { or if } \% R< \\
80 \text { or }>120\end{array}$ \\
\hline $\begin{array}{l}\text { Laboratory control } \\
\text { samples }\end{array}$ & $\begin{array}{l}\text { One (1) per analytical } \\
\text { batch }\end{array}$ & $80-120 \% R$ & $\begin{array}{l}\text { Redigest and reanalyze } \\
\text { for affected analytes }\end{array}$ \\
\hline Blind audit samples & $\begin{array}{l}\text { Samples and frequency } \\
\text { controlled by the Solid } \\
\text { PDP Plan }\end{array}$ & $\begin{array}{l}\text { Specified in the Solid } \\
\text { PDP Plan }\end{array}$ & $\begin{array}{l}\text { Specified in the Solid } \\
\text { PDP Plan }\end{array}$ \\
\hline
\end{tabular}

${ }^{a}$ Corrective Actions when $\mathrm{OC}$ samples do not meet acceptance criteria; Nonconformance procedures are outlined in Section 2.1.2.1 of the QAPP.

$I D L=$ Instrument detection limit

PDP $=$ Performance Demonstration Program

PRQL $=$ Program required detection limit

$\% R=$ Percent recovery

$\mathrm{RPD}=$ Relative percent difference 
analyzing four samples) at a minimum of every six months. Method performance should be conducted over a period of several days to account for long-term variability. Corrective action must be implemented when acceptable method performance is not met. Precision will be determined as follows:

- For duplicate measurements, the precision expressed as the relative percent difference (RPD) is calculated as

$$
R P D=\frac{C_{1}-C_{2}}{\frac{\left(C_{1}+C_{2}\right)}{2}} * 100
$$

where

$$
\begin{array}{lll}
C_{1} \text { and } C_{2} & = & \text { two values obtained by analyzing the duplicate samples } \\
C_{1} & = & \text { larger of the two observed values }
\end{array}
$$

- For three or more replicate measurements, the precision expressed as the percent relative standard deviation (\%RSD) is calculated as

$$
\% R S D=\frac{s}{\bar{y}} * 100
$$

where

$$
\begin{array}{lll}
s & = & \text { standard deviation } \\
y & = & \text { mean of replicate analyses }
\end{array}
$$

- The standard deviation $(s)$ is defined as

$$
s=\sqrt{\sum_{i=1}^{n} \frac{\left(y_{i}-\bar{y}\right)^{2}}{n-1}}
$$

where

$$
\begin{array}{ll}
y_{i} & =\begin{array}{l}
\text { measured value of the } i^{\text {th }} \text { replicate sample analysis } \\
\text { measurement }
\end{array} \\
n & =\text { number of replicate analyses }
\end{array}
$$

Accuracy will be determined as the percent recovery $(\% R)$ as follows:

$$
\% R=\frac{C_{m}}{C_{s r m}} * 100
$$

where

$$
\begin{array}{lll}
C_{m} & = & \text { measured concentration } \\
C_{s r m} & = & \text { true concentration }
\end{array}
$$

IDL will be determined as follows:

$$
I D L=t_{(n-1,1-\alpha=0.99)} * s
$$

where

$$
\begin{array}{lll}
s & = & \text { standard deviation } \\
t_{(n-1,1-\alpha=0.99)} \quad & \begin{array}{l}
t \text {-distribution value appropriate to a 99-percent confidence level } \\
\text { and a standard deviation estimate with } n-1 \text { degrees of freedom }
\end{array}
\end{array}
$$


10.4 The laboratory must analyze blanks at the frequency specified in Table 3 . The same procedures used to prepare and analyze field samples will be used to prepare and analyze the laboratory blanks. Laboratory blank results will be acceptable if the concentration of target analytes is less than or equal to 3 times the IDL. Corrective action must be implemented if blanks exceed this level.

10.5 The laboratory must analyze matrix spikes at the frequency specified in Table 3 . Matrix spike results shall be considered acceptable if the \%R is 80 to 120 percent of the true value. Matrix spikes which do not meet these criteria should be flagged. \%R will be determined as follows:

$$
\% R=\frac{S-U}{C_{s a}} * 100
$$

where
$S=$
measured concentration in the spiked aliquot
$U=$ measured concentration in the unspiked aliquot
$C_{s a}=$ actual concentration of the spike added

10.6 The laboratory must analyze matrix spike duplicates at the frequency specified in Table 3 . Matrix spike duplicate results shall be considered acceptable if the RPD is $\leq 30$ and the $\% R$ is 80 to 120 . RPD is determined as in Equation (1), \% R is determined as in Equation (6). Duplicates which do not meet these criteria should be flagged.

10.7 The laboratory must analyze laboratory control samples at the frequency specified in Table 3. Solid laboratory control samples (e.g., characterized surrogate sludges) should be used whenever it is possible to match the matrix of the waste samples. Otherwise, commercially purchased standards shall be used to prepare laboratory control samples. The laboratory control samples must contain the metals being analyzed, must undergo the same preparation and clean-up methods as the samples, and be quantitated within the calibration range of the instrument. The laboratory control sample analysis is designed to ensure that laboratory performance is in control for each analytical batch of samples processed. Laboratory control sample results will be considered acceptable if the $\% R$ is 80 to 120 percent of the known value. \%R is calculated as in Equation (4). If the results are outside of this range, then the associated samples must be reprepared and reanalyzed.

10.8 The laboratory will be required to analyze blind audit samples. These audit samples are part of the Performance Demonstration Program. Details of this program are in the Performance Demonstration Program Plan for the Analysis of Solidified Waste for the Transuranic Waste Characterization Program (Solid PDP Plan)(DOE 1995a).

\subsection{Procedure Performance}

The analyst is referred to Section 9.0 of SW-846 Method 7741A for a summary of method performance.

\subsection{References}

DOE. 1995a. Performance Demonstration Program Plan for the Analysis of Solidified Waste for the Transuranic Waste Characterization Program. CAO-95-1077, Current Revision, Carlsbad, New Mexico, Carlsbad Area Office, U.S. Department of Energy.

DOE. 1995b. Transuranic Waste Characterization Quality Assurance Program Plan. CAO-941010, Current Revision, Carlsbad, New Mexico, Carlsbad Area Office, U.S. Department of Energy. 
EPA. 1995. Test Methods for Evaluating Solid Waste, Physical/Chemical Methods. SW-846, Third Edition, Final Update I, and Final Update II, Washington D.C., Office of Solid Waste and Emergency Response, U.S. Environmental Protection Agency. 
PROCEDURE 650.7

\author{
BOROHYDRIDE GENERATION ATOMIC ABSORPTION \\ SPECTROSCOPY DETERMINATION OF TOTAL SELENIUM \\ IN HOMOGENOUS SOLIDS AND SOIL/GRAVEL
}

\title{
1.0 Scope and Application
}

1.1 This procedure is for the determination of total selenium in samples of homogenous solids and soil/gravel. The procedure employs gaseous Borohydride Generation Atomic Absorption Spectroscopy (HAA) for the quantitation of total selenium. This procedure should be used for samples which contain high concentrations of chromium, cobalt, copper, mercury, molybdenum, nickel, and silver which may cause interferences when using Procedure 650.5 of this Methods Manual. Analytical samples can contain concentrations of up to $1000 \mathrm{mg} / \mathrm{L}$ of cobalt, copper, iron, mercury, and nickel and still be successfully analyzed by this procedure. Note that solid samples can contain up to 10 percent by weight of these metals before exceeding $1000 \mathrm{mg} / \mathrm{L}$ in a digested sample. The quality assurance objectives for this procedure are listed in Table 1. A sampling procedure for homogenous solids and soil/gravel is described in Procedure 120.1 of this Methods Manual. This procedure must be implemented with a site-specific standard operating procedure (SOP).

1.2 The analyst is referred to Method 7742 of Test Methods for Evaluating Solid Waste, Physical/Chemical Methods, SW-846, Third Edition, Final Update II (SW-846) (EPA 1995) for the specific requirements of this procedure. The analyst is also referred to SW-846 Method 7000A for a general discussion of atomic absorption spectroscopy.

1.3 For the purposes of the TRU Waste Characterization Program, samples are to be analyzed in analytical batches. An analytical batch is defined as a suite of samples that is processed as a unit, using the same analytical method, within a specific time period. An analytical batch can be up to 20 samples (excluding laboratory OC samples), all of which must be received by the laboratory within 14 days of the validated time of sample receipt (VTSR) of the first sample in the batch.

\subsection{Summary of Procedure}

The analyst is referred to Section 2.0 of SW-846 Method 7742 for a summary of this procedure, except that the sample preparation procedures described in Procedure 610.1 of this Methods Manual must be followed prior to evaporating the samples to near dryness at the end of the digestion.

\subsection{Interferences}

The analyst is referred to Section 3.0 of SW-846 Method 7742 for an explanation of potential interferences.

\subsection{Safety}

This procedure may involve the use of hazardous materials, operations, and equipment. It is the responsibility of whoever uses this procedure to consult appropriate site personnel concerning health and safety issues and establish appropriate health and safety practices. Consideration should be given to safety concerns regarding chemical and radiation exposure. Training regarding proper storage, usage, and disposal of chemicals is recommended. 


\section{TABLE 1}

\section{Quality Assurance Objectives for Total Selenium Analysis}

\begin{tabular}{lcccccc}
\hline Analyte & CAS Number & $\begin{array}{c}\text { Precision } \\
(\% R S D \text { or RPD) }\end{array}$ & $\begin{array}{c}\text { Accuracy } \\
(\% R)^{b}\end{array}$ & $\begin{array}{c}\text { PRDL }^{\mathrm{b}} \\
(\mu \mathrm{g} / \mathrm{L})\end{array}$ & $\begin{array}{c}\text { PRQL } \\
(\mathrm{mg} / \mathrm{kg})\end{array}$ & $\begin{array}{c}\text { Completeness } \\
(\%)\end{array}$ \\
\hline Selenium & $7782-49-2$ & $\leq 30$ & $80-120$ & 20 & 20 & 90 \\
\hline
\end{tabular}

$\begin{array}{lll}\% R S D= & \text { Percent relative standard deviation } \\ \text { RPD }= & \text { Relative percent difference } \\ \% R= & \text { Percent recovery } \\ \text { PRDL }= & \text { Program required detection limit (i.e., maximum permissible value for instrument detection limit) } \\ \text { PRQL }= & \text { Program required quantitation limit }\end{array}$

${ }^{a} \leq 30 \%$ control limits apply when sample and duplicate concentrations are $\geq 10 \times$ instrument detection limit. If less than $10 \times$ instrument detection limit, the absolute difference between the two values shall be less than or equal to the PRDL.

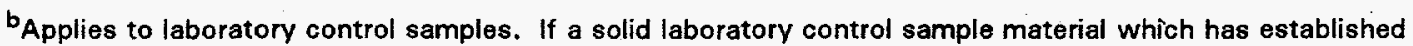
statistical control limits is used, then the established control limits for that material should be used for accuracy requirements.

CPRDL set such that it is a factor of 10 below the PRQL for 100 percent solid samples, assuming a $100 X$ dilution during digestion. 


\subsection{Apparatus and Materials}

The analyst is referred to Section 4.0 of SW-846 Method 7742 for a description and listing of the required apparatus and materials for this procedure.

\subsection{Reagents}

The analyst is referred to Section 5.0 of SW-846 Method 7742 for a description and listing of the required reagents, standard stock solutions, and standard solutions for this procedure.

\subsection{Sample Collection. Preservation, and Handling}

7.1 Samples will be collected using the sampling procedures described in Procedure 120.1 of this Methods Manual.

7.2 All sample handling and chain-of-custody procedures described in Section 6.0 of the Transuranic Waste Characterization Quality Assurance Program Plan (QAPP) (DOE 1995b) must be followed.

\subsection{Procedure}

The analyst is referred to Section 7.1 through 7.6 of SW-846 Method 7742 for a description of the procedural requirements. Table 2 of this procedure provides a summary of required calibration and analysis quality control.

\subsection{Calculations}

Read the selenium concentration from the calibration curve or directly from the instrument readout. Any sample dilution must be taken into account. Note that all results must be reported in mglkg (wet weight basis). To convert analytical results into mglkg dry weight, the percent solid content must be determined on a separate sample aliquot from that used for digestion and analysis.

\subsection{Quality Control}

10.1 All of the quality control requirements specified in Section 8.5 through 8.6 of SW-846 Method 7000A are mandatory. If the method of standard additions is needed to overcome interferences, this information is presented in Section 8.7 of SW-846 Method $7000 \mathrm{~A}$. In instances where the quality control specifications in this procedure differ with those in SW-846 Method 7000A, this procedure shall take precedence.

10.2 Each laboratory that uses this procedure is required to operate a formal quality control program. The laboratory must retain records to document the quality of the data generated. Each laboratory must have SOPs documenting and describing activities involved in using this procedure. Specific quality control practices will inciude, but are not limited to, the analysis of quality control samples. The types of quality control samples, their associated frequency of analysis, acceptance criteria, and corrective action required if samples do not meet the acceptance criteria, is summarized in Table 3. Laboratory SOPs shall address requirements for preparing matrix spikes, matrix spike duplicates, laboratory blanks, and laboratory control samples.

10.3 All laboratories using this procedure must demonstrate acceptable performance prior to the analysis of actual samples. Demonstration of acceptable performance will be achieved by analyzing method performance samples (Tabie 3 ). These samples can be either 


\section{TABLE 2}

Summary of Calibration Requirements and Analysis Quality Control for Total Selenium Analysis

\begin{tabular}{|c|c|c|c|c|}
\hline Technique & Procedure & Frequency of Procedure & Acceptance Criteria & Corrective Action \\
\hline \multirow[t]{6}{*}{ HAA } & $\begin{array}{l}\text { 5-pt. initial calibration } \\
\text { (5 standards and a blank) }\end{array}$ & Daily & $\begin{array}{l}90-110 \% R \text { for initial calibration verification } \\
\text { solution }\end{array}$ & $\begin{array}{l}\text { Correct problem and repeat initial } \\
\text { calibration }\end{array}$ \\
\hline & & & $95-105 \% \mathrm{R}$ for highest calibration standard & \\
\hline & & & Regression coefficient $\left(r^{2}\right)$ must be $\geq 0.995$ & \\
\hline & Continuing calibration & $\begin{array}{l}\text { Every } 10 \text { samples plus beginning and end } \\
\text { of run }\end{array}$ & $\begin{array}{l}80-120 \% R \text { for check standard (mandatory); } \\
\text { blank (optional) should measure } \leq 3 \times 1 D L\end{array}$ & Recalibrate and rerun last 10 samples \\
\hline & Serial dilution & $\begin{array}{l}\text { once per analytical batch or per matrix } \\
\text { within an analytical batch }\end{array}$ & $\begin{array}{l}5 x \text { dilution of sample which is }>25 \times 1 D L \\
\text { must be } \leq 10 \% \text { of initial value }\end{array}$ & $\begin{array}{l}\text { Use MSA to quantitate samples of like } \\
\text { matrix }\end{array}$ \\
\hline & Post-digestion spike & $\begin{array}{l}\text { once per analytical batch or per matrix } \\
\text { within an analytical batch }\end{array}$ & $85-115 \% R$ & $\begin{array}{l}\text { Use MSA to quantitate samples of like } \\
\text { matrix }\end{array}$ \\
\hline
\end{tabular}

$I D L=$ Instrument detection limit

$\% D=$ Percent difference

MSA $=$ Method of standard additions

$\% R=$ Percent recovery 
TABLE 3

\section{Summary of Laboratory Quality Control Samples and Frequencies for Total Selenium Analyses}

\begin{tabular}{|c|c|c|c|}
\hline OC Samples & Minimum Frequency & $\begin{array}{l}\text { Acceptance } \\
\text { Criteria }\end{array}$ & Corrective Actions ${ }^{\mathrm{a}}$ \\
\hline $\begin{array}{l}\text { Method performance } \\
\text { samples }\end{array}$ & $\begin{array}{l}\text { Seven (7) samples initially, } \\
\text { and four (4) semiannually }\end{array}$ & Meet Table 1 OAOs & Repeat until acceptable \\
\hline Laboratory blanks & $\begin{array}{l}\text { One (1) per analytical } \\
\text { batch }\end{array}$ & $\leq 3 \times \mathrm{IDL}$ & $\begin{array}{l}\text { Redigest and reanalyze } \\
\text { ariy samples with } \\
\text { analyte concentrations } \\
\text { which are } \\
\leq 10 \times \text { blank value and } \\
\geq 0.5 \times \text { PROL }\end{array}$ \\
\hline Matrix spikes & $\begin{array}{l}\text { One (1) per analytical } \\
\text { batch }\end{array}$ & $80-120 \% R$ & $\begin{array}{l}\text { Nonconformance if } \% R \\
<80 \text { or }>120\end{array}$ \\
\hline Matrix spike duplicates & $\begin{array}{l}\text { One (1) per analytical } \\
\text { batch }\end{array}$ & $\begin{array}{l}\text { RPD } \leq 30 \\
80-120 \% R\end{array}$ & $\begin{array}{l}\text { Nonconformance if } \\
R P D>30 \text { or if } \% R< \\
80 \text { or }>120\end{array}$ \\
\hline $\begin{array}{l}\text { Laboratory control } \\
\text { samples }\end{array}$ & $\begin{array}{l}\text { One (1) per analytical } \\
\text { batch }\end{array}$ & $80-120 \% R$ & $\begin{array}{l}\text { Redigest and reanalyze } \\
\text { for affected analytes }\end{array}$ \\
\hline Blind audit samples & $\begin{array}{l}\text { Samples and frequency } \\
\text { controlled by the Solid } \\
\text { PDP Plan }\end{array}$ & $\begin{array}{l}\text { Specified in the Solid } \\
\text { PDP Plan }\end{array}$ & $\begin{array}{l}\text { Specified in the Solid } \\
\text { PDP Plan }\end{array}$ \\
\hline
\end{tabular}

${ }^{a}$ Corrective Actions when OC samples do not meet acceptance criteria; Nonconformance procedures are outlined in Section 2.1.2.1 of the QAPP.

IDL = Instrument detection limit

PDP $=$ Performance Demonstration Program

$\mathrm{PROL}=$ Program required detection limit

$\% R=$ Percent recovery

$\mathrm{RPD}=$ Relative percent difference 
commercially purchased or laboratory prepared, but must contain selenium at concentrations appropriate (2-5 times the PROLs) to determine the quality assurance objectives specified in Table 1. The analysis of seven samples must meet the criteria specified for precision, accuracy, and program requirement detection limit (PRDL) in Table 1. Note that the instrument detection limit (IDL) must be less than or equal to the PRDL. Demonstration of acceptable method and analyst performance must be repeated (by analyzing four samples) at a minimum of every six months. Method performance should be conducted over a period of several days to account for long-term variability. Corrective action must be implemented when acceptable method performance is not met. Precision will be determined as follows:

- For duplicate measurements, the precision expressed as the relative percent difference (RPD) is calculated as

$$
R P D=\frac{C_{1}-C_{2}}{\frac{\left(C_{1}+C_{2}\right)}{2}} * 100
$$

where

$$
\begin{array}{lll}
C_{1} \text { and } C_{2} & =\text { two values obtained by analyzing the duplicate samples } \\
C_{1} & =\quad \text { larger of the two observed values }
\end{array}
$$

- For three or more replicate measurements, the precision expressed as the percent relative standard deviation (\%RSD) is calculated as

$$
\% R S D=\frac{s}{\bar{y}} * 100
$$

where

$$
\begin{array}{lll}
s & = & \text { standard deviation } \\
y & = & \text { mean of replicate analyses }
\end{array}
$$

- The standard deviation (s) is defined as

$$
s=\sqrt{\sum_{i=1}^{n} \frac{\left(y_{i}-\bar{y}\right)^{2}}{n-1}}
$$

where

$$
\begin{array}{ll}
y_{i} & =\begin{array}{l}
\text { measured value of the } i^{\text {th }} \text { replicate sample analysis } \\
\text { measurement }
\end{array} \\
n & =\text { number of replicate analyses }
\end{array}
$$

Accuracy will be determined as the percent recovery $(\% \mathrm{R})$ as follows:

$$
\% R=\frac{C_{m}}{C_{s m}} * 100
$$

$$
\begin{array}{cl}
\text { where } & \\
C_{m}= & \text { measured concentration } \\
C_{s r m}= & \text { true concentration }
\end{array}
$$


IDL will be determined as follows:

$$
I D L=t_{(n-1,1-\alpha=0.99)} * s
$$

where

$\begin{array}{ll}s & = \\ \left.t_{(n-1,1-\alpha}=0.99\right) & =\end{array}$

standard deviation

$t$-distribution value appropriate to a 99-percent confidence level and a standard deviation estimate with $n-1$ degrees of freedom

10.4 The laboratory must analyze blanks at the frequency specified in Table 3 . The same procedures used to prepare and analyze field samples will be used to prepare and analyze the laboratory blanks. Laboratory blank results will be acceptable if the concentration of target analytes is less than or equal to 3 times the IDL. Corrective action must be implemented if blanks exceed this level.

10.5 The laboratory must analyze matrix spikes at the frequency specified in Table 3. Matrix spike results shall be considered acceptable if the \%R is 80 to 120 percent of the true value. Matrix spikes which do not meet these criteria should be flagged. \%R will be determined as follows:

$$
\% R=\frac{S-U}{C_{s a}} * 100
$$

where
$S=$
$U=$
$C_{s a}=$

measured concentration in the spiked aliquot measured concentration in the unspiked aliquot actual concentration of the spike added

10.6 The laboratory must analyze matrix spike duplicates at the frequency specified in Table 3 . Matrix spike duplicate results shall be considered acceptable if the RPD is $\leq 30$ and the $\% R$ is 80 to 120 . RPD is determined as in Equation (1), \%R is determined as in Equation (6). Duplicates which do not meet these criteria should be flagged.

10.7 The laboratory must analyze laboratory control samples at the frequency specified in Table 3. Solid laboratory control samples (e.g., charactérized surrogate sludges) should be used whenever it is possible to match the matrix of the waste samples. Otherwise, commercially purchased standards shall be used to prepare laboratory control samples. The laboratory control samples must contain the metals being analyzed, must undergo the same preparation and clean-up methods as the samples, and be quantitated within the calibration range of the instrument. The laboratory control sample analysis is designed to ensure that laboratory performance is in control for each analytical batch of sampless processed. Laboratory control sample results will be considered acceptable if the $\% R$ is 80 to 120 percent of the known value. \%R is calculated as in Equation (4). If the results are outside of this range, then the associated samples must be reprepared and reanalyzed.

10.8 The laboratory will be required to analyze blind audit samples. These audit samples are part of the Performance Demonstration Program. Details of this program are in the Performance Demonstration Program Plan for the Analysis of Solidified Waste for the Transuranic Waste Characterization Program (Solid PDP Plan)(DOE 1995a).

\subsection{Procedure Performance}

The analyst is referred to Section 9.0 of SW-846 Method 7742 for a summary of method performance. 


\subsection{References}

DOE. 1995a. Performance Demonstration Program Plan for the Analysis of Solidified Waste for the Transuranic Waste Characterization Program. CAO-95-1077, Current Revision, Carlsbad, New Mexico, Carisbad Area Office, U.S. Department of Energy.

DOE. 1995b. Transuranic Waste Characterization Quality Assurance Program Plan. CAO-941010, Current Revision, Carlsbad, New Mexico, Carlsbad Area Office, U.S. Department of Energy.

EPA. 1995. Test Methods for Evaluating Solid Waste, Physical/Chemical Methods. SW-846, Third Edition, Final Update I, and Final Update II, Washington D.C., Office of Solid Waste and Emergency Response, U.S. Environmental Protection Agency. 